
June 1945

\title{
TERM ANALYSES OF THE FIRST TWO SPECTRA OF COLUMBIUM
}

\author{
By Curtis J. Humphreys and William F. Meggers
}

\section{ABSTRACT}

Published wavelengths and estimated intensities of lines characterizing the first two spectra of columbium (RP881) have been supplemented by observations of the arc spectrum (6500 to $8500 \mathrm{~A}$ ) in an atmosphere of helium, by observations of the spark spectrum (2000 to $2100 \mathrm{~A}$ ), and by measurements of the Zeeman patterns for 1,557 lines. All available data have been analyzed for the purpose of correcting and extending the information about the structures of $\mathrm{Cb}$ II and $\mathrm{Cb}$ I (RP793).

The $\mathrm{Cb}$ Ir table contains 1,723 lines (2002.41 to $7026.15 \mathrm{~A}), 1,494$ of which are explained as combinations of 183 ionic energy levels comprising 27 singlet, 40 triplet, and 9 quintet spectral terms. The terms arising from electron configurations $4 d^{4}, 4 d^{3} 5 s$, and $4 d^{3} 5 p$ have been almost completely established, but efforts to find spectral series for $\mathrm{Cb}$ II were futile. The lowest energy (normal state) of $\mathrm{Cb}^{+}$ions is represented by $\left(4 d^{4}\right) a^{5} \mathrm{D}_{0}$, but the strongest emission lines involve $\left(4 d^{3} 5 s\right) a^{5} \mathrm{~F}$, a metastable term. The most intense line of the $\mathrm{Cb}$ II spectrum is $\left(4 d^{3} 5 s\right) a^{5} \mathrm{~F}_{5}-\left(4 d^{3} 5 p\right) z^{5} \mathrm{G}_{6}^{\circ}$, with a wavelength of $3094.172 \mathrm{~A}$. Zeeman patterns observed for $646 \mathrm{Cb}$ Ir lines were most helpful in extending this analysis, which now includes 87 percent of the recognized lines and 95 percent of their total intensity.

The $\mathrm{Cb}$ I table contains 3,313 lines (2164.54 to $10920.7 \mathrm{~A}), 2,836$ of which have been interpreted as combinations of 364 atomic energy levels representing 58 doublet, 55 quartet, and 13 sextet spectral terms. The lowest term (normal state of neutral Cb atoms is $\left(4 d^{4} 5 s\right)^{6} a^{6} \mathrm{D}_{035}$, and the strongest line of the $\mathrm{Cb}$ I spectrum is the transition $\left(4 d^{4} 5 s\right) a^{6} \mathrm{D}_{41 / 2}-\left(4 d^{4} 5 p\right) y^{6} \mathrm{~F}^{\circ}{ }^{\circ} / 2$, with a wavelength of $4058.931 \mathrm{~A}$. The $\mathrm{Cb}$ I spectrum is largely accounted for by transitions between levels arising from $4 d^{4} 5 s$ or $4 d^{3} 5 s^{2}$ and $4 d^{4} 5 p$ or $4 d^{3} 5 s 5 p$ electron configurations. Zeeman patterns measured for $911 \mathrm{Cb}$ I lines have been 90 percent interpreted in this analysis, which now includes 86 percent of the known lines and over 93 percent of their intensity. Two members of the series $\left(4 d^{4} n s\right)^{6} \mathrm{D}$ and two each of $\left(4 d^{3} 5 s n p\right)^{4}(\mathrm{DFG})$ have been established. Extrapolation of these indicates a limit of $54,600 \mathrm{~cm}^{-1}$, which represents the energy difference between the normal states of $\mathrm{Cb}$ atoms and $\mathrm{Cb}^{+}$ions. The corresponding ionization potential for neutral columbium atoms is 6.77 volts.

\section{CONTENTS}

P 478

II. Observational data $\ldots \ldots$

1. Wavelengths .

2. Intensities and other line characteristics.

3. Temperature classes

4. Zeeman effect. .

III. Term analysis of $\mathrm{Cb}$ II

1. Lines of the $\mathrm{Cb}$ II spectrum... 481

2. Terms of the Cb II spectrum .............. 509

3. Electron configurations, theoretical and observed terms..... 514 
IV. Term analysis of $\mathrm{Cb}_{\mathrm{I}} \ldots \ldots \ldots$

1. Lines of the $\mathrm{Cb}$ I spectrum

2. Terms of the Cbi spectrum

3. Electron configurations, theoretical and observed terms

4. Series and ionization potential ....... 583

V. References_...

\section{INTRODUCTION}

Structural analyses of columbium spectra began 20 years ago, when one of the present authors [1] ${ }^{1}$ succeeded in finding three multiplets in the arc spectrum of columbium $(\mathrm{Cb} \mathrm{I})$. Two years later the first regularities in the spark spectrum of columbium (Cb II) were announced [2]. The impossibility of extending such analyses without improved and additional descriptive material was recognized, and steps were taken to obtain the desired data. At that time the precision of the published wavelengths was not sufficient to permit finding true "constant differences" in such complex spectra nor were the intensity estimates of arc and spark lines reliable enough for discrimination of ionization stages. The furnace spectra of columbium vapor at various temperatures had not been investigated, and although the published Zeeman-effect data gave the clue to the first regularities in these spectra, they were inadequate for the extension of these analyses.

In 1931 the furnace spectra of columbium were investigated by King [3]. In 1936, new descriptions of arc and spark spectra were published by Meggers and King [4]. These new data formed the basis for a report on multiplets and terms in the first two spectra of columbium published by Meggers and Scribner [5] in 1935. In that report about $400 \mathrm{Cb}$ I lines were accounted for as combinations of sextet and quartet terms, and about $250 \mathrm{Cb}$ Ir lines as combinations of quintet and triplet terms. Theoretically predicted doublet terms for $\mathrm{Cb}$ I and singlet terms for $\mathrm{Cb}$ II could not be established at that time. That work indicated that the term analyses of columbium spectra could not be concluded satisfactorily without more extensive and accurate observations of the Zeeman effect. Several years later the unique facilities of the Massachusetts Institute of Technology [6] for making such observations with high magnetic and spectrographic resolution became available, and several sets of Zeeman spectrograms of columbium were made for the Bureau by G. R. Harrison. These were measured, calculated, and interpreted during 1940-41, and the results served to correct and greatly amplify the term analyses of the first two spectra of columbium. Incidental to these analyses, additional observations were made at this Bureau on the arc spectrum in the red and infrared, on the spark spectrum in the ultraviolet, and on the Zeeman effect in the risible and near infrared. The present results for columbium spectra are comparable or superior to those published [7] for the corresponding spectra of analogous vanadium, and since there is now neither opportunity nor necessity for making further observations, this final report on the term analyses of the first two spectra of columbium has been prepared.

Although columbium was discovered 143 years ago, it is only during the past decade that this chemical element has lost its status as a

1 Figures in brackets indicate the literature references at the end of this paper. 
scientific curiosity and found important industrial application. Considerable quantities of columbium are now being used as a constituent of steel [8] and other alloys [9], and since the efficiency and popularity of spectrochemical analysis of such alloys is rapidly increasing, it may be assumed that this report on the structures of columbium spectra will be of interest to practical spectroscopists, as well as to theoretical physicists.

\section{OBSERVATIONAL DATA}

The analyses of columbium spectra are based on observed facts, consisting of wavelength measurements, intensity estimates and other line characteristics, temperature classification, and Zeemaneffect data, all of which are collected in tables 1 and 4 . The second spectrum (Cb II), associated with four valence electrons, is presented first because it is simpler than the first spectrum ( $\mathrm{Cb}$ I) associated with five electrons, and because the low energy states of $\mathrm{Cb}$ II are convergence limits of $\mathrm{Cb}$ I spectral series.

\section{WAVELENGTHS}

Most of the wavelengths shown in tables 1 and 4 are quoted from the description of arc and spark spectra of columbium published by Meggers and King [4]. Their claim that the majority of these values had an average probable error of less than $0.005 \mathrm{~A}$ was tested and confirmed by partial analyses [5] based mainly on the combination principle, and has been further verified by the present more complete analyses of these spectra. Supplementary measurements of wavelengths were made in the ultraviolet to extend the data for $\mathrm{Cb}$ II lines from 2100 to $2000 \mathrm{~A}$, and new observations were undertaken between 6500 and $8500 \mathrm{~A}$ to detect faint lines of $\mathrm{Cb}$ I masked by molecular spectra (presumably due to $\mathrm{CbO}$ ) when the arc-in-air is employed. For the latter purpose an inclosed arc was used, through which pure helium gas was drawn to remove the ambient oxygen. Although this device did not completely eliminate the molecular spectra, it reduced their intensity sufficiently to reveal a considerable number of previously undetected atomic lines. The latter observations were limited to the red and near infrared, because this is the range in which the background of masking molecular spectra is most intense and is also the region in which combinations of series-forming terms are to be expected. The finding of the expected series-forming terms led to a determination of the spectroscopic ionization potential for columbium atoms (see below).

\section{INTENSITIES AND OTHER LINE CHARACTERISTICS}

Intensity comparisons of columbium lines from arc and spark sources enabled Meggers and King [4] to make a satisfactory separation of $\mathrm{CbI}, \mathrm{Cb}$ II, and $\mathrm{Cb}$ III or Cbiv spectra. That assignment of lines to $\mathrm{CbI}$ and $\mathrm{CbII}$ spectra has been confirmed for all lines whose Zeeman patterns have been observed and interpreted. Although not sufficiently precise to test quantum rules, the estimated relative intensities in either spectrum were helpful in grouping lines into multiplets and in intrepreting levels. 
Other characteristics of spectral lines that proved useful in the analyses of these spectra are self-reversals ( $r$ or $R$ ), hyperfine complexes $(c$ or $c W)$ and haziness $(h$ or $H)$.

The absorption spectrum of columbium vapor has never been observed, and an attempt to find reversals in the underwater spark between columbium electrodes failed to give positive results. The only information of this type for columbium is that of partial selfreversals $(r$ or $R)$ observed for certain lines in arc or spark sources. That such lines involve the ground state (or low metastable state) of the emitter is true without exception in columbium spectra.

A large number of $\mathrm{Cb}$ I and $\mathrm{Cb}$ Ir lines exhibit hyperfine structure ( $c$ or $c W$ ) due to nuclear spin, and it was assumed by Meggers and Scribner [5] that most of the wide lines $(c W)$ involve low levels arising from electron configurations with a single penetrating $s$-type electron, as first mentioned by Meggers and Burns [10] in connection with other spectra. Some of the $\mathrm{Cb}$ levels were first found and interpreted on this assumption, and in every case of this kind, subsequent observation of the Zeeman effect has confirmed the interpretation. A value of $I=9 / 2$ for the nuclear moment of columbium was found from hyperfine structure by Ballard [11], who from observed intervals derived $J$ values of $5 / 2$ and $7 / 2$ for the upper levels of the transitions producing $5344 \mathrm{~A}$ and $6661 \mathrm{~A}$, respectively. This analysis establishes the $J$ values in question to be $7 / 2$ and $9 / 2$, respectively.

\section{TEMPERATURE CLASSES}

King [3] assigned temperature classes (III, IV, or V) to 646 of the stronger lines of columbium between 3100 and $6900 \mathrm{~A}$, by comparing electric-furnace spectra at temperatures of about $2,500^{\circ}$ and $2,900^{\circ} \mathrm{C}$ with are and spark spectra. Approximately 200 lines appeared in the furnace spectrum, but many $\mathrm{Cb}$ I and all $\mathrm{Cb}$ II lines require higher excitation. The present analyses of these spectra are consistent with these results.

\section{ZEEMAN EFFECT}

To this date the only published observations of the Zeeman patterns for $\mathrm{Cb}$ lines are those that Jack [12] made 32 years ago. These comprised about 100 lines between 2656 and $4700 \mathrm{~A}$, the majority being reported as pseudotriplets. Component separations of less than 0.6 Lorentz unit were not resolved and lines with patterns less than this in total width were said to be unaffected by the magnetic field. These observations are credited $[1,2]$ with having revealed the first regularities in $\mathrm{Cb}$ I and $\mathrm{Cb}$ II, but unfortunately they were not suitable for extending the analyses.

In 1939 and 1940 several sets of $\mathrm{Cb}$ spectrograms were made at the Bureau's request in the Spectroscopy Laboratory of the Massachusetts Institute of Technology, where high-power spectrographs were combined with a magnet producing field intensities up to 100,000 oersteds to observe Zeeman patterns surpassing any hitherto seen [6]. With this combination, separations of 0.1 Lorentz unit are readily resolved, and under favorable conditions the resolving power may attain 0.05 Lorentz unit.

These MIT spectrograms extend from 2200 to $6500 \mathrm{~A}$, and the magnetic field intensities range from 74,000 to 95,000 oersteds. The 

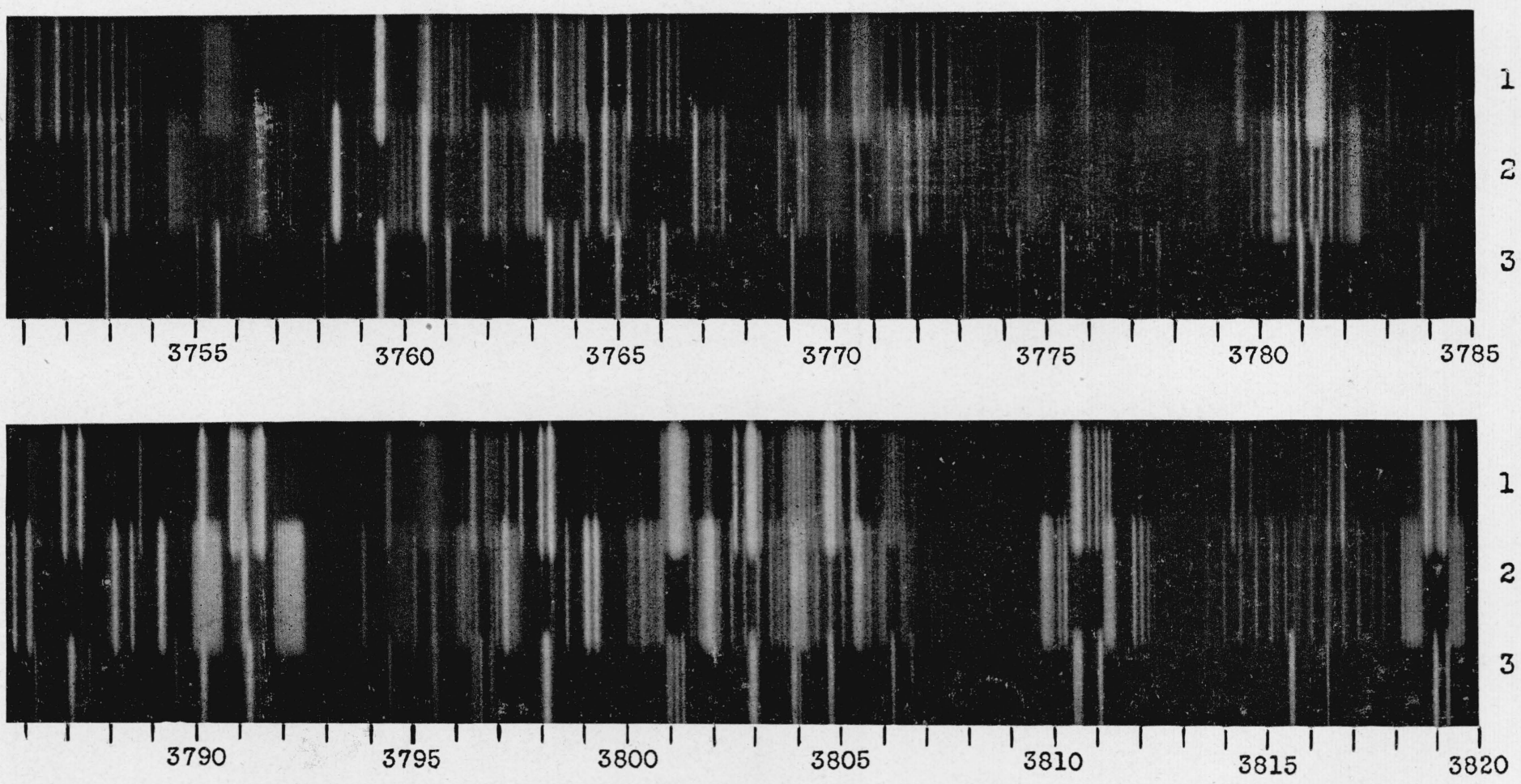

FigURe 1.-Zeeman patterns for $\mathrm{Cb}$ at 95,000 oersteds.

$1=$ parallel components, $2=$ perpendicular components, $3=$ spectrum without magnetic field. 

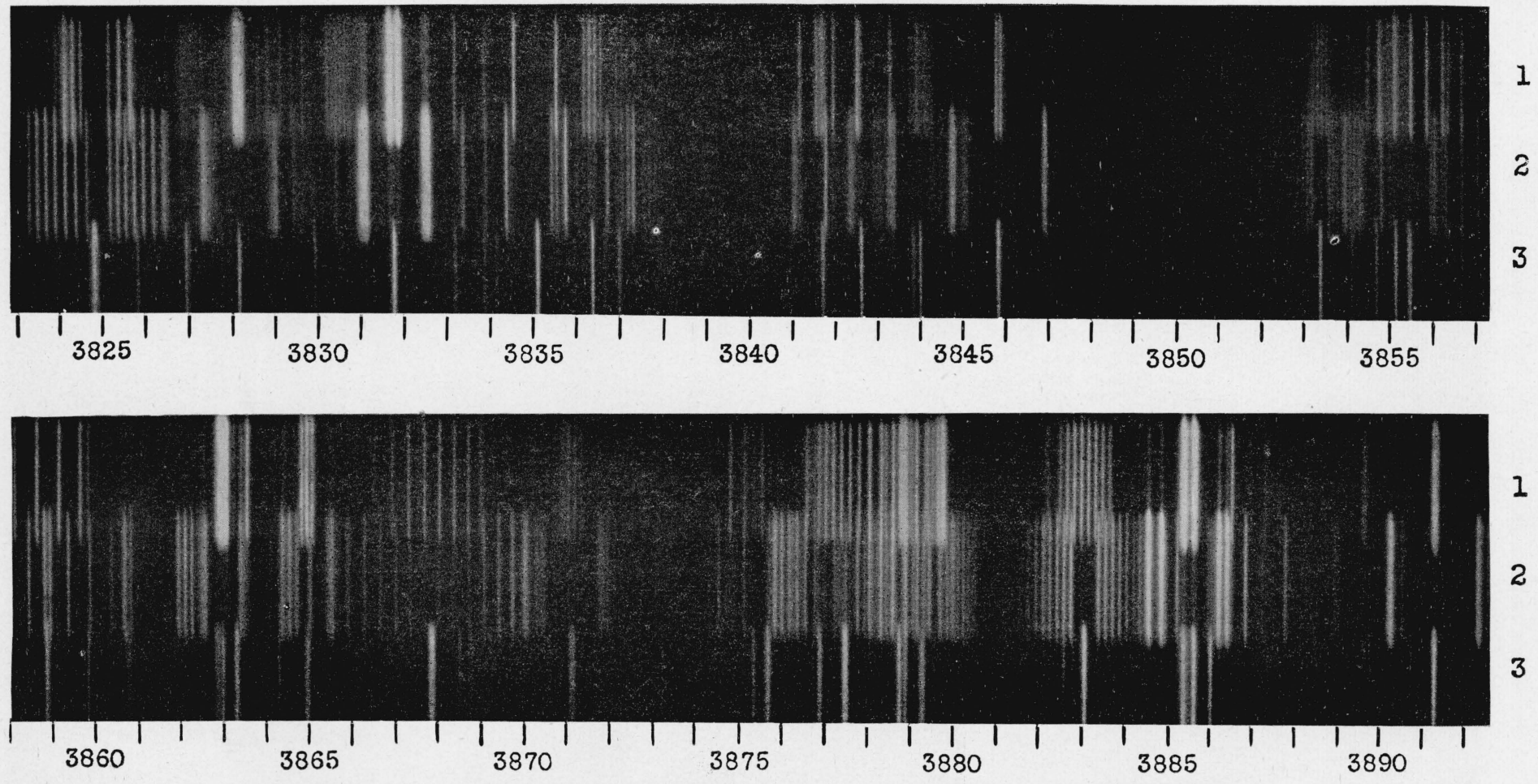

Figure 2.-Zeeman patterns for $\mathrm{Cb}$ at 95,000 oersteds. 

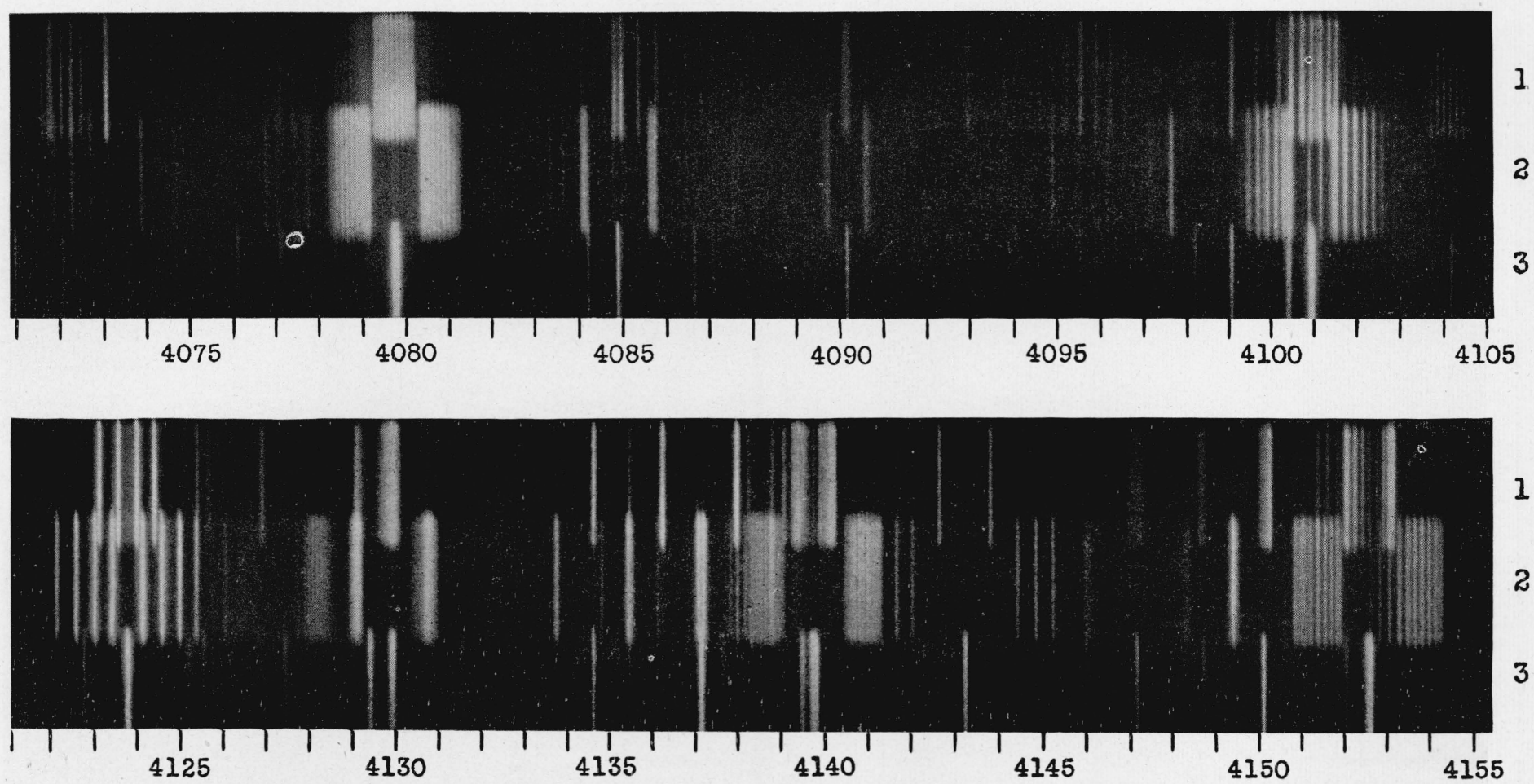

Figure 3.-Zeeman patterns for $\mathrm{Cb}$ at 95,000 oersteds.

$1=$ parallel components, 2 =perpendicular components, $3=$ spectrum without magnetic field. 


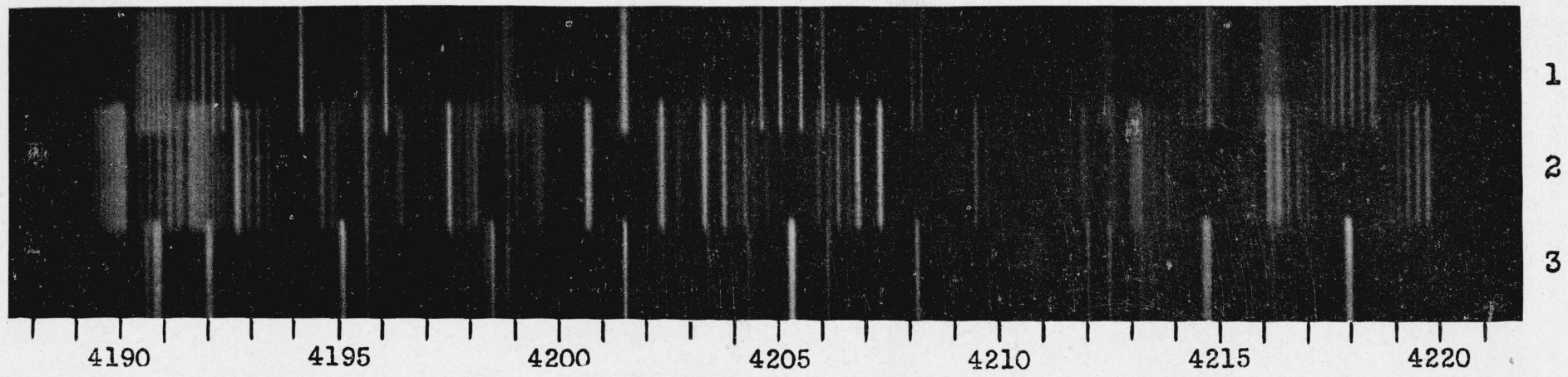

1

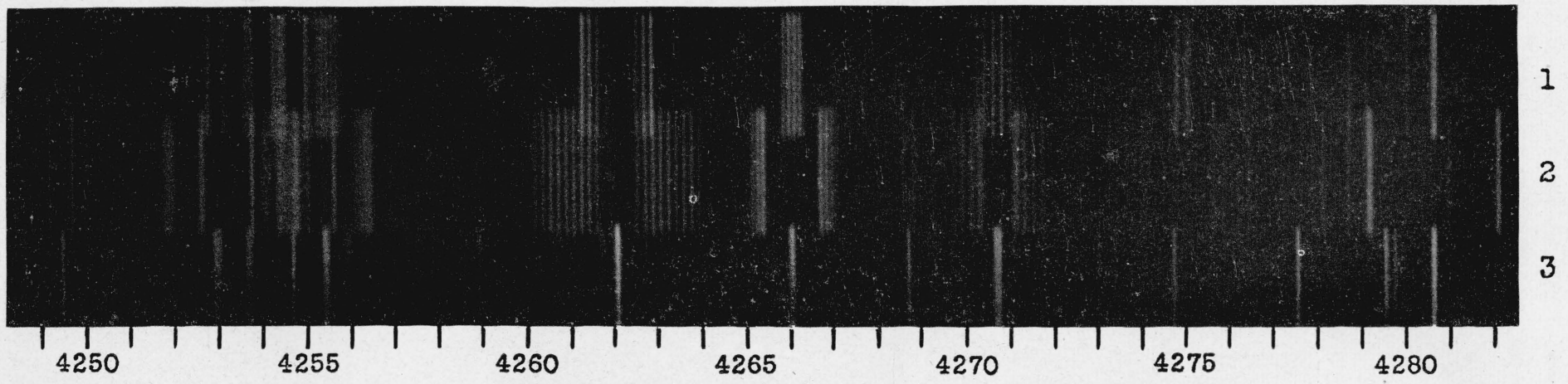

FIGURE 4.-Zeeman patterns for $\mathrm{Cb}$ at 95,000 oersteds. 
results for wavelengths less than $4500 \mathrm{~A}$, obtained with long focus concave gratings giving a scale of $0.4 \mathrm{~A} / \mathrm{mm}$, leave little or nothing to be desired, but most of the longer wave data were derived from spectrograms with a dispersion of $3.7 \mathrm{~A} / \mathrm{mm}$. The long wave region 5000 to $8000 \mathrm{~A}$ was photographed in the spectroscopy laboratory of this Bureau with a dispersion of $1.8 \mathrm{~A} / \mathrm{mm}$, the light source being an interrupted arc between $\mathrm{Cb}$ electrodes in an evacuated chamber between the pole pieces of a Weiss electromagnet yielding field intensities of 35,000 oersteds. These observations were extended to $8600 \mathrm{~A}$ by employing another grating with a dispersion of $5 \mathrm{~A} / \mathrm{mm}$.

All the Zeeman spectrograms were hand measured at the Bureau, and one set of MIT plates was machine measured [13]. Three decimal places were calculated for patterns measured on high-dispersion spectrograms, or when the results from different spectrograms were in good accord. All the measurements were Peduced to Lorentz units on the assumption that $\mathrm{Cu} 3247 \mathrm{~A}, \mathrm{Ag} 3280 \mathrm{~A}, \mathrm{Ca}^{+} 3933 \mathrm{~A}, \mathrm{Na}$ $5889 \mathrm{~A}$, or K $7664 \mathrm{~A}$ patterns are represented by $(0.333) 1.000,1.667$, and $\mathrm{Cu} 3273 \mathrm{~A}, \mathrm{Ag} 3382 \mathrm{~A}, \mathrm{Ca}^{+} 3968 \mathrm{~A}, \mathrm{Na} 5895$, or K $7699 \mathrm{~A}$ by (0.667) 1.333. Two or more of these lines appeared on each set of spectrograms.

Although hyperfine structure was obvious in many of the $\mathrm{Cb}$ patterns, and fully resolved in some, this phenomenon was ignored in the final compilation of results.

Resolved Zeeman patterns of $\mathrm{Cb}$ lines may exhibit from 1 to 30 or more components, but it is impractical and unnecessary to give all in minute detail. The important observed and derived facts concerning the Zeeman effect are presented in the last six columns of tables 1 and 4 ; the first of which indicates the type of pattern according to the classification of Back and Landé [14], the second reports the separation of resolved components, the third and fourth contain values for the strongest components of parallel and perpendicular polarization, respectively, and the last two exhibit the derived splitting factors of the low and high energy levels responsible for the spectral line. Values in parentheses are borrowed from other lines. All numerical values in the last five columns are expressed in Lorentz units $\left(L=H e / 4 \pi m c^{2}\right)$. Some typical Zeeman patterns of columbium lines are displayed in figures $1,2,3,4$ as $5 \times$ enlarged reproductions from original spectrograms. A few patterns are somewhat unsymmetrical (see $3824.88 \mathrm{~A}$ in fig. 2, 4152.58 A in fig. 3, and others marked " $u s$ " following the Zeeman-type number in tables 1 and 4), but since the splitting factors derived from these were in good accord, no further study was made of these dissymmetries.

\section{TERM ANALYSIS OF CbII}

\section{LINES OF THE CbII SPECTRUM}

The available data for $1,723 \mathrm{Cb}$ In lines, characteristic of singly ionized $\mathrm{Cb}$ atoms, are displayed in table 1 , where column 1 shows the estimated intensity and character, column 2 the measured wavelength, column 3 the vacuum wave number, column 4 the term combinations, and the last six columns contain data on the Zeeman effect. $\mathrm{Cb}$ spark lines definitely identified as belonging to $\mathrm{Cb}$ III [15] and $\mathrm{Cb}$ Iv[16] spectra are omitted, and also about 60 lines not observed in the are 
and appearing very hazy $(H)$ in the spark. The latter could not be classified as $\mathrm{Cb}$ II and some of them may belong to $\mathrm{Cb}$ irr. The total number of $\mathrm{Cb}$ II lines now interpreted as combinations of singlet, triplet, and quintet terms is 1,494 . This constitutes 87 percent of the total number listed in table 1 , and includes 95 percent of the total intensity. It may be noted that 22 of these lines are doubly classified. In these cases the first term combination is the more probable. The Zeeman patterns observed for $646 \mathrm{Cb}$ II lines constitute the strongest evidence for the correctness of this analysis and interpretation of the $\mathrm{Cb}$ II spectrum.

TABLE 1.-Second spectrum of columbium (Cb II)

\begin{tabular}{|c|c|c|c|c|c|c|c|c|c|}
\hline $\begin{array}{l}\text { Intensity } \\
\text { spark }\end{array}$ & $\lambda_{\text {air }} \mathbf{A}$ & $\begin{array}{l}\text { Wave } \\
\text { No. } \\
\text { vac. } \\
\mathrm{cm}^{-1}\end{array}$ & $\begin{array}{c}\text { Therm combi- } \\
\text { nation }\end{array}$ & $\begin{array}{c}\text { Zeeman } \\
\text { type }\end{array}$ & $\begin{array}{l}\text { Sepa- } \\
\text { ration }\end{array}$ & $\begin{array}{c}\text { Strong- } \\
\text { est } \\
p\end{array}$ & $\begin{array}{c}\text { Strongest } \\
n\end{array}$ & 01 & 0, \\
\hline 1 & 2 & 3 & 4 & 5 & 6 & 7 & 8 & 9 & 10 \\
\hline $\begin{array}{c}10 h \\
30 h \\
4 \\
2 \\
20 h\end{array}$ & $\begin{array}{l}7026.15 \\
6940.90 \\
6929.05 \\
6920.85 \\
6629.11\end{array}$ & $\begin{array}{l}14228.63 \\
14403.39 \\
14428.02 \\
14445.11 \\
15080.82\end{array}$ & $\begin{array}{l}c^{3} \mathrm{~F}_{2}-z^{3} \mathrm{D}_{1}^{1} \\
c^{3} \mathrm{~F}_{2}-z 2^{3} \mathrm{D}_{2}^{2} \\
d^{3} \mathrm{P}_{2}-z^{3} \mathrm{P}_{2}^{1} \\
c^{3} \mathrm{~F}_{4}-z^{3} \mathrm{D}_{3}^{\circ}\end{array}$ & ${ }_{7 b, 1}^{2}$ & 0.25 & $\begin{array}{l}0 \\
0 \\
0 \\
0\end{array}$ & $\begin{array}{l}0.93 \\
1.04 \\
1.32 \\
1.09+\end{array}$ & $\begin{array}{l}0.68 \\
(1.08)\end{array}$ & $\begin{array}{l}0.43 \\
1.10\end{array}$ \\
\hline $\begin{array}{c}10 \\
1 h \\
5 h \\
2 \\
8 h\end{array}$ & $\begin{array}{l}6435.53 \\
6331.51 \\
6285.81 \\
6249.68 \\
6183.24\end{array}$ & $\begin{array}{l}15534.45 \\
15789.66 \\
15904.46 \\
15996.40 \\
16168.29\end{array}$ & $\begin{array}{l}b^{3} \mathrm{D}_{2}-2{ }^{3} \mathrm{D}_{1}^{\mathrm{d}} \\
c^{8} \mathrm{~F}_{4}-2{ }^{3} \mathrm{~F}_{3}^{3} \\
c^{1} \mathrm{G}_{4}-2{ }^{1} \mathrm{~F}_{3}^{*}\end{array}$ & $\begin{array}{l}2 \\
7 b, 1 \\
3 \\
7 b, 2\end{array}$ & 0.74 & $\begin{array}{l}0 \\
0 \\
0 \\
.14 \\
0\end{array}$ & $\begin{array}{l}1.93 \\
1.31 \\
1.20 \\
1.06 \\
1.11\end{array}$ & $\begin{array}{l}1.19 \\
(1.24) \\
(1.09)\end{array}$ & $\begin{array}{l}0.45 \\
1.25 \\
1.08\end{array}$ \\
\hline $\begin{array}{l}2 h \\
2 h \\
5 h \\
5 h \\
5 h\end{array}$ & $\begin{array}{l}6175.01 \\
6157.83 \\
6119.68 \\
6091.80 \\
6062.17\end{array}$ & $\begin{array}{l}16189.84 \\
16235.00 \\
16336.21 \\
16410.98 \\
16491.19\end{array}$ & 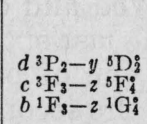 & $\begin{array}{l}3 \\
2 \\
7 b, 2\end{array}$ & .245 & $\begin{array}{l}0 \\
0 \\
.27 \\
0 \\
0\end{array}$ & $\begin{array}{l}1.14 \\
1.18 \\
1.43 \pm \\
2.08 \\
1.24\end{array}$ & $\begin{array}{l}(1.49) \\
1.10 \\
(1.01)\end{array}$ & $\begin{array}{l}1.35 \\
1.34 \\
1.07\end{array}$ \\
\hline $\begin{array}{r}15 h \\
2 h \\
6 h \\
2 h \\
8 h\end{array}$ & $\begin{array}{l}6039.95 \\
6022.41 \\
6000.25 \\
5979.56 \\
5973.26\end{array}$ & $\begin{array}{l}16551.86 \\
16600.06 \\
16661.37 \\
16719.02 \\
16736.65\end{array}$ & $\begin{array}{l}c^{3} \mathrm{~F}_{4}-2{ }^{5} \mathrm{~F}_{3} \\
c^{1} \mathrm{G}_{4}-y^{3} \mathrm{~F}^{4} \\
c^{3} \mathrm{~F}_{2}-2{ }^{5} \mathrm{~F}_{3}^{4}\end{array}$ & $\begin{array}{l}2 \\
7 b, 3\end{array}$ & & $\begin{array}{l}0 \\
0 \\
0 \\
0\end{array}$ & $\begin{array}{l}1.75- \\
1.12 \\
1.10 \\
.98\end{array}$ & $\begin{array}{l}(1.240) \\
(1.09)\end{array}$ & $\begin{array}{l}1.34 \\
1.11\end{array}$ \\
\hline $\begin{array}{r}6 h \\
4 h \\
30 h \\
20 h \\
3 h\end{array}$ & $\begin{array}{l}5970.67 \\
5828.85 \\
5753.06 \\
5746.90 \\
5690.78\end{array}$ & $\begin{array}{l}16743.91 \\
17151.30 \\
17377.24 \\
17395.87 \\
17567.42\end{array}$ & $\begin{array}{l}c^{3} \mathrm{~F}_{4}-2{ }^{8} \mathrm{D}_{3}^{3} \\
b^{1} \mathrm{D}_{2}-z^{3} \mathrm{P}_{3} \\
b^{1} \mathrm{I}_{3}-y^{1} \mathrm{I}_{4}\end{array}$ & $\begin{array}{l}1 \\
3 \\
3 \\
1 \\
3 \\
3\end{array}$ & $\begin{array}{l}.22 \\
.33\end{array}$ & $\begin{array}{l}0 \\
.71 \\
.66 \\
0.97 \\
.97\end{array}$ & $\begin{array}{r}.62+ \\
.98,1.31 \\
.86\end{array}$ & $\begin{array}{r}1.28 \\
.98 \\
(1.01)\end{array}$ & $\begin{array}{r}1.50 \\
1.31 \\
.97\end{array}$ \\
\hline $\begin{array}{c}2 h \\
20 h \\
2 \\
100 \\
3 h\end{array}$ & $\begin{array}{l}5644.66 \\
5635.48 \\
5546.35 \\
5545.63 \\
5510.19\end{array}$ & $\begin{array}{l}17710.96 \\
17739.81 \\
18024.88 \\
18027.22 \\
18143.17\end{array}$ & $\begin{array}{l}c^{1} \mathrm{G}_{1}-y^{3} \mathrm{H}_{4} \\
b^{3} \mathrm{D}_{2}-z^{5} \mathrm{~F}_{3} \\
c^{3} \mathrm{~F}_{2}-2^{3} \mathrm{G}_{3}^{3} \\
b^{3} \mathrm{D}_{1}-2^{3} \mathrm{~F}_{2}^{3}\end{array}$ & 2 & & .55 & .91 & $(.670)$ & 1.95 \\
\hline $\begin{array}{c}200 \\
30 \\
150 \\
20 h \\
25 h\end{array}$ & $\begin{array}{l}5487.60 \\
5455.03 \\
5365.89 \\
5363.07 \\
5356.84\end{array}$ & $\begin{array}{l}18217.85 \\
18326.63 \\
18631.07 \\
18640.86 \\
18662.53\end{array}$ & $\begin{array}{l}c^{3} \mathrm{~F}_{3}-2^{3} \mathrm{G}_{4} \\
c^{3} \mathrm{~F}_{2}-2^{3} \mathrm{~F}_{2}^{2} \\
c^{3} \mathrm{~F}_{4}-2^{3} \mathrm{G}_{3}^{3} \\
c^{3} \mathrm{~F}_{3}-2^{3} \mathrm{~F}_{3}^{3}\end{array}$ & $\begin{array}{l}7 b, 1 \\
7 b, 3 \\
7 b, 1 \\
7 b, 3\end{array}$ & & $\begin{array}{l}0 \\
0 \\
0 \\
0\end{array}$ & $\begin{array}{l}1.06 \\
.68 \\
1.19 \\
1.09\end{array}$ & $\begin{array}{c}(1.080) \\
(.670) \\
(1.240) \\
(1.08)\end{array}$ & $\begin{array}{l}1.075 \\
.690 \\
1.23 \\
1.10\end{array}$ \\
\hline $\begin{array}{c}10 h \\
5 h \\
5 c \\
4 h \\
4 h\end{array}$ & $\begin{array}{l}5331.19 \\
5273.486 \\
5237.34 \\
5165.368 \\
5147.94\end{array}$ & $\begin{array}{l}18752.34 \\
18957.53 \\
19088.36 \\
19354.33 \\
19419.85\end{array}$ & $\begin{array}{l}c^{3} \mathrm{P}_{1}-z^{1} \mathrm{~S}_{0} \\
b^{1} \mathrm{D}_{2}-y^{3} \mathrm{D}_{2} \\
c^{3} \mathrm{~F}_{1}-z^{3} \mathrm{D}_{4}^{8} \\
a^{1} \mathrm{P}_{1}-z^{1} \mathrm{~S}_{0}^{8} \\
b^{1} \mathrm{~F}_{3}-y^{1} \mathrm{D}_{2}^{2}\end{array}$ & $\begin{array}{l}7 a \\
3 \\
7 a\end{array}$ & .24 & $\begin{array}{l}{ }^{0} .48 \\
0\end{array}$ & 1.115 & $\begin{array}{l}1.21 \\
(.98) \\
1.115\end{array}$ & $\begin{array}{l}0 / 0 \\
1.22 \\
0 / 0\end{array}$ \\
\hline $\begin{array}{l}2 h \\
2 \\
2 \\
8 h \\
3 h\end{array}$ & $\begin{array}{l}5120.492 \\
5006.22 \\
4969.44 \\
4957.74 \\
4949.44\end{array}$ & $\begin{array}{l}19523.95 \\
19969.60 \\
20117.37 \\
20164.87 \\
20198.68\end{array}$ & $\begin{array}{l}c^{1}{ }^{1} G_{4}-y^{1} \mathrm{I}_{4}^{4} \\
b^{3} \mathrm{G}_{3}-2^{3} \mathrm{D}_{2}^{2} \\
c^{1} \mathrm{D}_{2}-y^{1} \mathrm{D}_{2}^{2} \\
b^{3} \mathrm{D}_{1}-z^{3} \mathrm{~F}^{2} \\
c^{3} \mathrm{P}_{2}-z^{1} \mathrm{D}_{2}^{2}\end{array}$ & 2 & & 0 & .70 & $(.68)$ & .69 \\
\hline $\begin{array}{c}4 h \\
15 h \\
2 \\
12 c \\
3 h\end{array}$ & $\begin{array}{l}4942.00 \\
4893.885 \\
4891.07 \\
4865.99 \\
4854.96\end{array}$ & $\begin{array}{l}20229.09 \\
20427.98 \\
20439.73 \\
20545.08 \\
20591.75\end{array}$ & $\begin{array}{c}b^{3} \mathrm{~F}_{3}-z^{5} \mathrm{G}_{3}^{0} \\
b^{3} \mathrm{D}_{2}-z^{3} \mathrm{D}_{3}^{3} \\
a^{3} \mathrm{D}_{2}-z^{3} \mathrm{~F}_{3}^{3} \\
b^{3} \mathrm{~F}_{2}-z^{5} \mathrm{G}_{2}^{2} \\
b^{1} \mathrm{D}_{2}-z^{3} \mathrm{P}_{2}^{3}\end{array}$ & 3 & .50 & .98 & , & .84 & $(.341)$ \\
\hline
\end{tabular}


TABLE 1.-Second spectrum of columbium (Cb II)-Continued

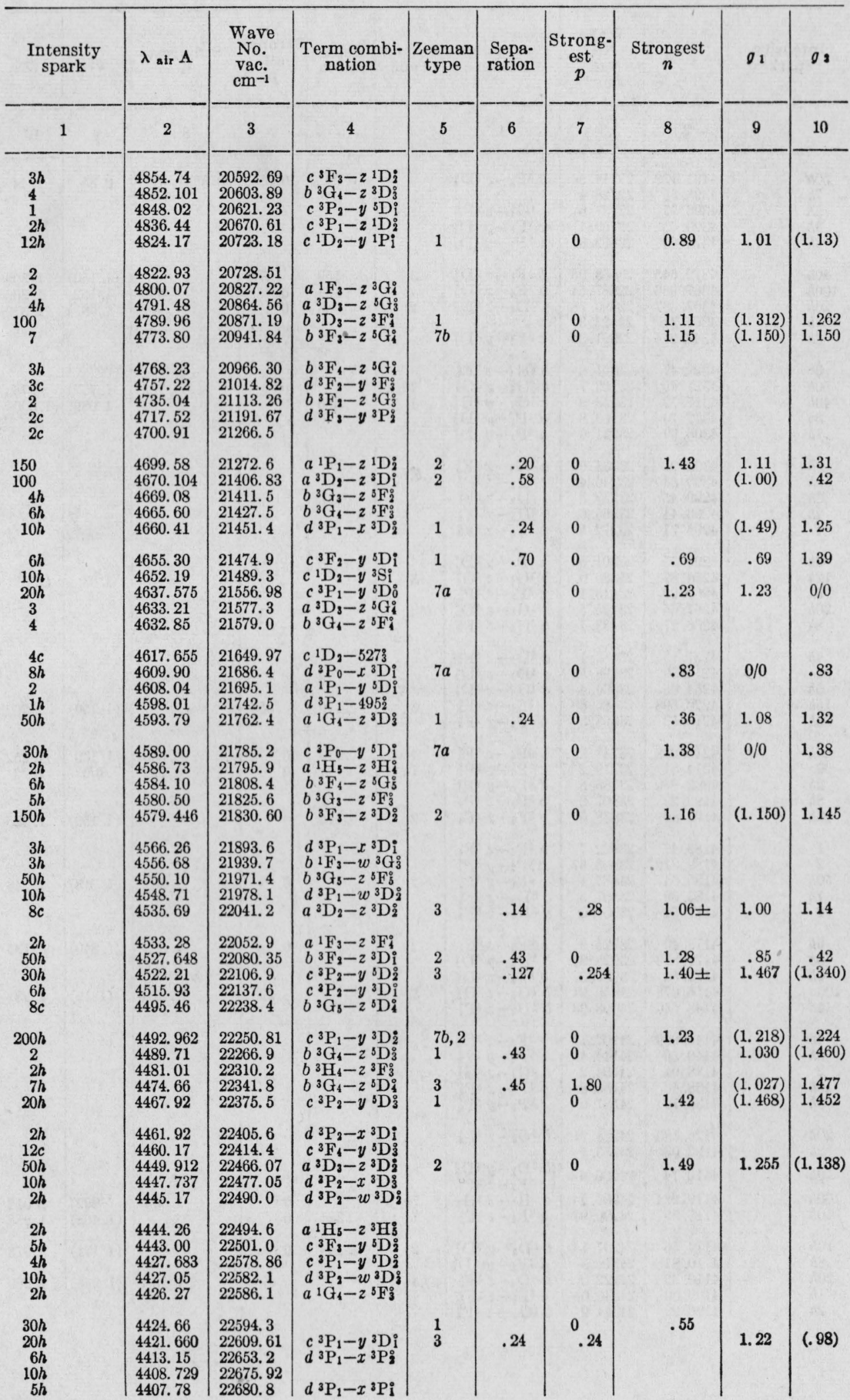


484 Journal of Research of the National Bureau of Standards

TABLE 1.-Second spectrum of columbium (Cb II)-Continued

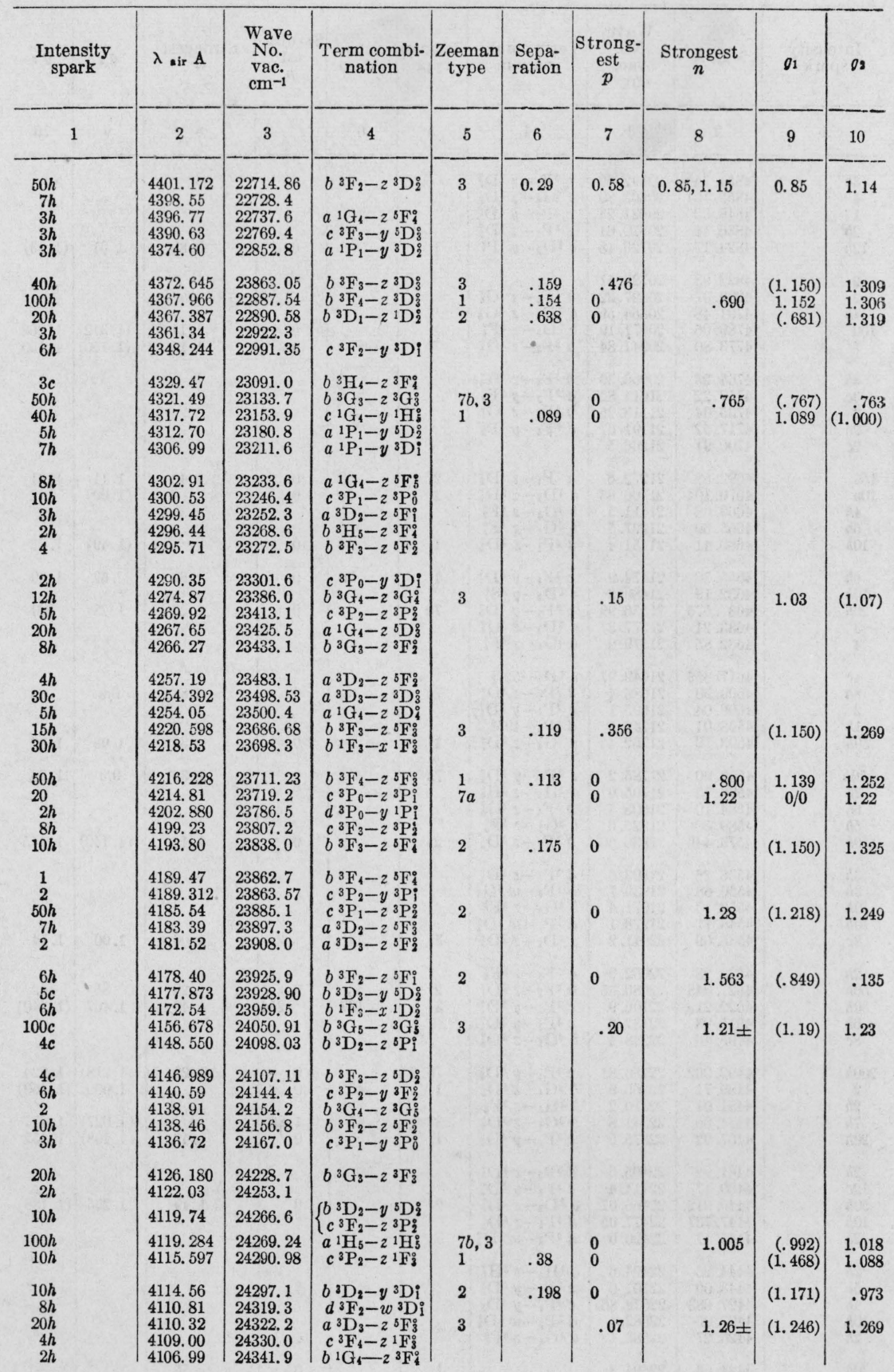


TABLE 1.-Second spectrum of columbium (Cb II)-Continued

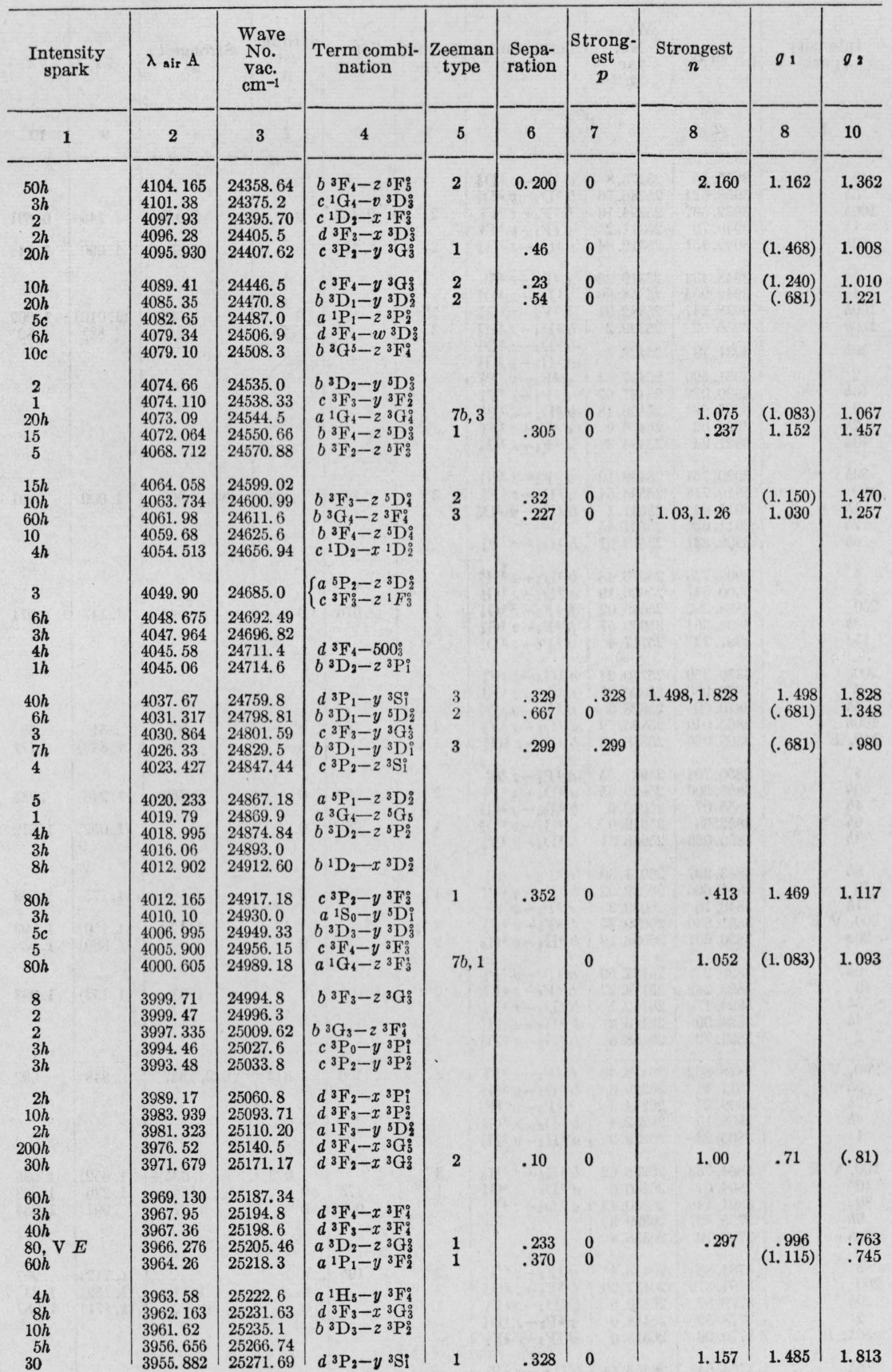


TABLE 1.-Second spectrum of columbium (Cb II)-Continued

\begin{tabular}{|c|c|c|c|c|c|c|c|c|c|}
\hline $\begin{array}{l}\text { Intensity } \\
\text { spark }\end{array}$ & $\lambda_{\text {sir }} A$ & $\begin{array}{l}\text { Wave } \\
\text { No. } \\
\text { vac. } \\
\mathrm{cm}^{-1}\end{array}$ & $\begin{array}{l}\text { Term combi- } \\
\text { nation }\end{array}$ & $\begin{array}{c}\text { Zeeman } \\
\text { type }\end{array}$ & $\begin{array}{l}\text { Sepa- } \\
\text { ration }\end{array}$ & $\begin{array}{c}\text { Strong- } \\
\text { est } \\
p\end{array}$ & $\underset{n}{\text { Strongest }}$ & $g_{1}$ & $g_{2}$ \\
\hline 1 & 2 & 3 & 4 & 5 & 6 & 7 & 8 & 9 & 10 \\
\hline $\begin{array}{r}3 \\
5 h \\
100 h \\
1 h \\
60 h\end{array}$ & $\begin{array}{l}3955.55 \\
3953.524 \\
3952.367 \\
3949.70 \\
3949.451\end{array}$ & $\begin{array}{l}25273.8 \\
25286.76 \\
25294.16 \\
25311.2 \\
25312.84\end{array}$ & $\begin{array}{l}a{ }^{3} \mathrm{G}_{3}-z{ }^{3} \mathrm{D}_{3}^{2} \\
b^{3} \mathrm{D}_{2}-y^{3} \mathrm{D}^{3} \\
b^{3} \mathrm{~F}_{3}-z^{3} \mathrm{~F}_{2}^{3} \\
c^{3} \mathrm{~F}_{3}-y^{3} \mathrm{~F}_{3}^{3} \\
a^{1} \mathrm{G}_{4}-z^{3} \mathrm{G}_{3}^{3}\end{array}$ & 2 & 0.458 & 0 & 2. 065 & 1.090 & $\begin{array}{l}0.691 \\
1.244\end{array}$ \\
\hline $\begin{array}{r}5 h \\
4 h \\
100 h \\
150 c\end{array}$ & $\begin{array}{l}3948.433 \\
3942.905 \\
3938.547 \\
3936.02\end{array}$ & $\begin{array}{l}25319.36 \\
25354.86 \\
25382.91 \\
25399.2\end{array}$ & $\begin{array}{c}c{ }^{3} \mathrm{P}_{1}-z{ }^{3} \mathrm{~S}_{1} \\
b^{1} \mathrm{D}_{2}-x \\
b^{3} \mathrm{D}_{1} \\
{ }^{1} \mathrm{~F}_{3}-x \\
b^{3} \mathrm{D}_{4} \mathrm{G}_{4}-z{ }^{3} \mathrm{H}_{4}\end{array}$ & $\begin{array}{l}7 b \\
3\end{array}$ & & $\begin{array}{l}0 \\
.24\end{array}$ & $\begin{array}{l}1.007 \\
.84 \pm\end{array}$ & $\begin{array}{c}(1.010) \\
(.88)\end{array}$ & $\begin{array}{c}1.009 \\
.82\end{array}$ \\
\hline $\begin{array}{c}30 h \\
2 \\
10 h \\
2 \\
2 h \\
10 h\end{array}$ & $\begin{array}{l}3931.79 \\
3931.590 \\
3930.022 \\
3925.622 \\
3924.02 \\
3921.34\end{array}$ & $\begin{array}{l}25426.5 \\
25427.83 \\
25437.97 \\
25466.48 \\
25476.9 \\
25494.3\end{array}$ & 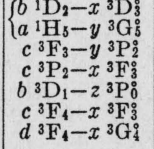 & & & & 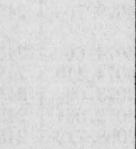 & & \\
\hline $\begin{array}{r}30 h \\
100 \\
1 h \\
2 h \\
6 h\end{array}$ & $\begin{array}{l}3920.754 \\
3919.718 \\
3915.64 \\
3911.036 \\
3909.331\end{array}$ & $\begin{array}{l}25498.10 \\
25504.84 \\
25531.4 \\
25561.45 \\
25572.61\end{array}$ & $\begin{array}{l}d^{3} \mathrm{~B}_{3}-x{ }^{3} \mathrm{G}_{4}^{0} \\
a^{3} \mathrm{D}_{2}-z{ }^{3} \mathrm{~F}_{2}^{0} \\
b{ }^{1} \mathrm{D}_{2}-w^{3} \mathrm{D}_{3}^{3} \\
b^{3} \mathrm{D}_{2}-z^{3} \mathrm{P}_{2}^{0}\end{array}$ & 3 & .309 & .620 & $.691,1.000$ & 1.000 & .691 \\
\hline $\begin{array}{r}4 \\
5 \\
200 \\
5 h \\
15 c\end{array}$ & $\begin{array}{l}3909.739 \\
3900.547 \\
3898.292 \\
3894.564 \\
3887.32\end{array}$ & $\begin{array}{l}25576.48 \\
25630.19 \\
25645.02 \\
25669.57 \\
25717.4\end{array}$ & $\begin{array}{c}b^{3} \mathrm{H}_{5}-z^{3} \mathrm{H}_{1} \\
a^{3} \mathrm{D}_{3}-z^{3} \mathrm{G}_{3} \\
b^{3} \mathrm{~F}_{3}-z^{3} \mathrm{G}_{4} \\
b^{3} \mathrm{~F}_{4}-z^{3} \mathrm{G}_{4} \\
a^{5} \mathrm{P}_{2}-z^{3} \mathrm{D}_{3}^{3}\end{array}$ & 1 & .076 & 0 & .843 & 1. 147 & 1.071 \\
\hline $\begin{array}{c}200 c \\
4 \\
6 h \\
100 h \\
150, E\end{array}$ & $\begin{array}{l}3879.350 \\
3870.936 \\
3870.60 \\
3865.019 \\
3863.056\end{array}$ & $\begin{array}{l}25770.24 \\
25826.25 \\
25828.5 \\
25865.79 \\
25878.93\end{array}$ & $\begin{array}{l}a{ }^{1} \mathrm{G}_{4}-z^{3} \mathrm{~F}^{\circ} \\
c{ }^{1} \mathrm{G}_{1}-x{ }^{1} \mathrm{~F}_{3}^{\circ} \\
d^{3} \mathrm{~F}_{3}-y{ }^{1} \mathrm{D}_{2}^{\circ} \\
a^{3} \mathrm{D}_{1}-z z^{3} \mathrm{~F}_{2}^{\circ} \\
b^{3} \mathrm{~F}_{2}-z^{3} \mathrm{G}_{3}^{\circ}\end{array}$ & $\begin{array}{l}2 \\
1\end{array}$ & .18 & $\begin{array}{l}0 \\
0\end{array}$ & .87 & $\begin{array}{l}.51 \\
(.849)\end{array}$ & $\begin{array}{l}.69 \\
.771\end{array}$ \\
\hline $\begin{array}{c}4 \\
50 h \\
4 h \\
6 h \\
3 h\end{array}$ & $\begin{array}{l}3856.704 \\
3855.500 \\
3855.07 \\
3852.63 \\
3850.056\end{array}$ & $\begin{array}{l}25921.55 \\
25929.65 \\
25932.5 \\
25949.0 \\
25966.31\end{array}$ & $\begin{array}{l}a{ }^{1} \mathrm{P}_{1}-z{ }^{3} \mathrm{~S}_{1}^{\circ} \\
a{ }^{3} \mathrm{D}_{3}-z{ }^{3} \mathrm{~F}_{2}^{2} \\
b{ }^{3} \mathrm{D}_{3}-y{ }^{3} \mathrm{G}_{4}^{\circ} \\
a^{3} \mathrm{G}_{4}-z{ }^{3} \mathrm{D}_{3}^{3} \\
b^{3} \mathrm{D}_{3}-y{ }^{3} \mathrm{~F}_{2}^{0}\end{array}$ & $\begin{array}{l}2 \\
1\end{array}$ & $\begin{array}{l}.560 \\
.26\end{array}$ & $\begin{array}{l}0 \\
0\end{array}$ & 2. 366 & $\begin{array}{c}1.246 \\
(1.052)\end{array}$ & $\begin{array}{r}.685 \\
1.312\end{array}$ \\
\hline $\begin{array}{c}8 h \\
10 \\
1 h\end{array}$ & $\begin{array}{l}3843.397 \\
3841.666 \\
3840.16\end{array}$ & $\begin{array}{l}\text { 26011. } 30 \\
26022.92 \\
26033.2\end{array}$ & $\begin{array}{c}c{ }^{3} \mathrm{P}_{0}-z{ }^{3} \mathrm{~S}_{1} \\
b^{3} \mathrm{D}_{2}-y{ }^{3} \mathrm{P}_{1} \\
c^{3} \mathrm{P}_{1}-z{ }^{5} \mathrm{~S}_{2}^{8}\end{array}$ & 1 & .587 & 0 & .588 & 1. 175 & 1.762 \\
\hline$\underset{20 c}{200, \mathrm{~V} E}$ & $\begin{array}{l}3831.840 \\
3830.601\end{array}$ & $\begin{array}{l}26089.75 \\
26098.19\end{array}$ & $\begin{array}{l}b{ }^{3} \mathrm{~F}_{3}-z^{3} \mathrm{~F}_{3}^{0} \\
b^{3} \mathrm{H}_{4}-z^{3} \mathrm{H}_{5}^{\circ}\end{array}$ & $\begin{array}{l}3 \\
2\end{array}$ & .15 & $0^{.17}$ & $1.12 \pm$ & $\begin{array}{r}(1.150) \\
(.880)\end{array}$ & $\begin{array}{l}1.093 \\
1.03\end{array}$ \\
\hline $\begin{array}{c}6 h \\
40 \\
5 h \\
4 h \\
2\end{array}$ & $\begin{array}{l}3829.221 \\
3828.242 \\
3824.17 \\
3824.00 \\
3821.72\end{array}$ & $\begin{array}{l}26107.59 \\
26114.27 \\
26142.1 \\
26143.2 \\
26158.8\end{array}$ & 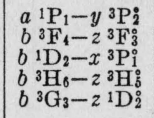 & 2 & & 0 & 1.33 & $(1.152)$ & 1.093 \\
\hline $\begin{array}{c}200, \mathrm{~V} E \\
5 h \\
2 h \\
4 h \\
1\end{array}$ & $\begin{array}{l}3818.862 \\
3811.41 \\
3809.27 \\
3808.10 \\
3805.23\end{array}$ & $\begin{array}{l}26178.40 \\
26229.6 \\
26244.3 \\
26252.4 \\
26272.2\end{array}$ & $\begin{array}{c}b{ }^{3} \mathrm{~F}_{2}-z{ }^{3} \mathrm{~F}_{2}^{\circ} \\
b^{3} \mathrm{D}_{3}-y^{3} \mathrm{G}_{3}^{3} \\
c^{3} \mathrm{P}_{2}-w^{3} \mathrm{~F}_{2}^{\circ} \\
b^{1} \mathrm{D}_{2}-x^{3} \mathrm{G}_{3}^{\circ} \\
a^{1}{ }^{1} \mathrm{H}_{5}-y^{3}{ }^{3} \mathrm{H}_{4}^{\circ}\end{array}$ & 3 & .156 & .312 & $.692, .847$ & .848 & .692 \\
\hline $\begin{array}{c}100, \mathrm{~V} E \\
10 \\
80 \\
6 h \\
30 h\end{array}$ & $\begin{array}{l}3804.733 \\
3804.01 \\
3801.146 \\
3795.527 \\
3792.79\end{array}$ & $\begin{array}{l}26275.62 \\
26280.6 \\
26300.42 \\
26339.35 \\
26358.4\end{array}$ & $\begin{array}{l}b{ }^{3} \mathrm{H}_{5}-z{ }^{3} \mathrm{H}_{5}^{0} \\
a{ }^{3} \mathrm{D}_{3}-z{ }^{3} \mathrm{GG}_{4}^{0} \\
a^{3} \mathrm{D}_{2}-z{ }^{3} \mathrm{~F}_{3}^{\circ}\end{array}$ & $\begin{array}{l}3 \\
1 \\
2\end{array}$ & $\begin{array}{l}.173 \\
.093\end{array}$ & $\begin{array}{l}0^{.078} \\
0\end{array}$ & $\begin{array}{l}1.035 \pm \\
.544 \\
1.27\end{array}$ & $\begin{array}{c}(1.052) \\
1.236 \\
.991\end{array}$ & $\begin{array}{l}1.036 \\
1.063 \\
1.084\end{array}$ \\
\hline $\begin{array}{c}5 \\
200 \\
20 h \\
2 \\
30 h, E\end{array}$ & $\begin{array}{l}3784.88 \\
3781.379 \\
3779.57 \\
3778.50 \\
3770.66\end{array}$ & $\begin{array}{l}26413.4 \\
26437.90 \\
26450.6 \\
26458.0 \\
26513.0\end{array}$ & $\begin{array}{l}a{ }^{3} \mathrm{~F}_{2}-z{ }^{5} \mathrm{G}_{3}^{\circ} \\
b^{3} \mathrm{~F}_{1}-z z^{3} \mathrm{G}_{5}^{3} \\
b^{3} \mathrm{D}_{2}-z{ }^{1} \mathrm{~F}_{3}^{0} \\
a^{5} \mathrm{P}_{3}-z{ }^{5} \mathrm{D}_{2}^{\circ} \\
d^{3} \mathrm{~F}_{2}-v^{3} \mathrm{~F}_{2}^{\circ}\end{array}$ & $\begin{array}{l}2 \\
2 \\
1\end{array}$ & .195 & $\begin{array}{l}0 \\
0 \\
0\end{array}$ & $\begin{array}{r}1.54 \\
.92\end{array}$ & $\begin{array}{l}(.712) \\
(1.152) \\
(1.171)\end{array}$ & $\begin{array}{l}.907 \\
1.23 \\
1.087\end{array}$ \\
\hline $\begin{array}{l}5 h \\
8 h \\
3 h \\
2 h \\
2 h\end{array}$ & $\begin{array}{l}3764.641 \\
3763.13 \\
3762.99 \\
3762.064 \\
3761.32\end{array}$ & $\begin{array}{l}26555.44 \\
26566.1 \\
26567.1 \\
26573.63 \\
26578.9\end{array}$ & $\begin{array}{l}b^{3}{ }^{3} \mathrm{D}_{1}-y^{3} \mathrm{P}_{1}^{\circ} \\
c^{3} \mathrm{P}_{2}-w^{3} \mathrm{~F}_{3}^{3} \\
b^{3} \mathrm{D}_{2}-y^{3} \mathrm{G}_{3}^{3} \\
d^{3} \mathrm{~F}_{3}-v^{3} \mathrm{~F}_{2}^{0} \\
d^{3} \mathrm{~F}_{4}-v^{3} \mathrm{~F}_{3}\end{array}$ & & & & & & \\
\hline
\end{tabular}


TABLE 1.-Second spectrum of columbium (Cb II)-Continued

\begin{tabular}{|c|c|c|c|c|c|c|c|c|c|}
\hline $\begin{array}{l}\text { Intensity } \\
\text { spark }\end{array}$ & $\lambda_{\text {sir }} \mathrm{A}$ & $\begin{array}{l}\text { Wave } \\
\text { No. } \\
\text { vac. } \\
\mathrm{cm}^{-1}\end{array}$ & $\begin{array}{l}\text { Term combi- } \\
\text { nation }\end{array}$ & $\begin{array}{c}\text { Zeeman } \\
\text { type }\end{array}$ & $\begin{array}{l}\text { Sepa- } \\
\text { ration }\end{array}$ & $\begin{array}{c}\text { Strong- } \\
\text { est } \\
p\end{array}$ & $\underset{n}{\text { Strongest }}$ & $\theta_{1}$ & 92 \\
\hline 1 & 2 & 3 & 4 & 5 & 6 & 7 & 8 & 9 & 10 \\
\hline $\begin{array}{c}15 h \\
1 h \\
3 h \\
1 \\
1\end{array}$ & $\begin{array}{l}3760.76 \\
3757.60 \\
3757.466 \\
3752.93 \\
3752.08\end{array}$ & $\begin{array}{l}26582.8 \\
26605.2 \\
26606.14 \\
26638.3 \\
26644.3\end{array}$ & $\begin{array}{l}d^{3} \mathrm{~F}_{3}-v^{3} \mathrm{~F}_{3}^{0} \\
c^{3} \mathrm{~F}_{4}-w^{3} \mathrm{~F}_{3}^{3} \\
b^{3} \mathrm{D}_{3}-y^{3} \mathrm{~F}_{4}^{3} \\
c^{3} \mathrm{~F}_{3}-w^{3} \mathrm{~F}_{2}^{2} \\
a^{5} \mathrm{P}_{2}-z^{5} \mathrm{D}_{1}^{3}\end{array}$ & & & & & & \\
\hline $\begin{array}{c}1 c \\
20 \\
3 \\
10\end{array}$ & $\begin{array}{l}3752.02 \\
3751.285 \\
3748.00 \\
3741.292\end{array}$ & $\begin{array}{l}26644.8 \\
26649.98 \\
26673.3 \\
26721.16\end{array}$ & $\begin{array}{l}a{ }^{3} \mathrm{P}_{1}-z{ }^{5} \mathrm{D}_{0}^{\circ} \\
b^{1} \mathrm{G}_{4}-z z^{3} \mathrm{H}_{4}^{\circ} \\
a^{3} \mathrm{D}_{1}-z{ }^{1} \mathrm{~S}_{0} \\
a^{1}{ }^{1} \mathrm{D}_{2}-z \\
{ }^{3} \mathrm{~F}_{2}^{\circ}\end{array}$ & 3 & 0.137 & 0.548 & & $(0.950)$ & 0.814 \\
\hline 200 , V $E$ & 3740.726 & 26725.21 & $a^{3} \mathrm{D}_{3}-z{ }^{3} \mathrm{~F}_{3}^{2}$ & 3 & .162 & .478 & 1. $093,1.252$ & 1. 252 & 1. 092 \\
\hline $\begin{array}{c}3 h \\
3 h \\
12 c \\
6 \\
30 h\end{array}$ & $\begin{array}{l}3736.49 \\
3728.03 \\
3726.58 \\
3725.25 \\
3723.44\end{array}$ & $\begin{array}{l}26755.5 \\
26816.2 \\
26826.7 \\
26836.2 \\
26849.3\end{array}$ & $\begin{array}{l}b^{3} \mathrm{G}_{5}-z{ }^{3} \mathrm{H}_{4}^{\circ} \\
a^{3} \mathrm{P}_{1}-z{ }^{3} \mathrm{D}_{1}^{0} \\
b^{3} \mathrm{D}_{1}-y{ }^{3} \mathrm{~F}_{2}^{0} \\
b^{1} \mathrm{D}_{2}-y{ }^{1} \mathrm{D}_{2}^{2}\end{array}$ & $7 b, 3$ & & 0 & .974 & $(.980)$ & .968 \\
\hline $\begin{array}{c}40 h \\
100 \\
30 c\end{array}$ & $\begin{array}{l}3722.548 \\
3720.456 \\
3719.63\end{array}$ & $\begin{array}{l}26855.71 \\
26870.81 \\
26876.8\end{array}$ & $\begin{array}{l}b^{3}{ }^{3} \mathrm{D}_{3}-y^{3} \mathrm{P}_{2}^{0} \\
b^{3} \mathrm{~F}_{3}-z^{3} \mathrm{~F}_{4}^{0} \\
a^{5} \mathrm{P}_{3}-z{ }^{5} \mathrm{D}_{3}^{\circ}\end{array}$ & $\begin{array}{l}1 \\
2\end{array}$ & $\begin{array}{l}.195 \\
.107\end{array}$ & $\begin{array}{l}0 \\
0\end{array}$ & $\begin{array}{r}.916 \\
1.582\end{array}$ & $\begin{array}{l}1.306 \\
1.154\end{array}$ & $\begin{array}{l}\text { 1. } 501 \\
1.261\end{array}$ \\
\hline $\begin{array}{c}300, V E \\
50 h, E\end{array}$ & $\begin{array}{l}3717.06 \\
3713.72\end{array}$ & $\begin{array}{l}26895.4 \\
26919.6\end{array}$ & $\begin{array}{l}b^{3} \mathrm{~F}_{4}-z^{3} \mathrm{~F}_{4}^{0} \\
b^{3} \mathrm{G}_{4}-z^{3} \mathrm{H}_{4}\end{array}$ & 3 & .104 & .417 & 1. $150,1.258$ & 1. 154 & 1. 258 \\
\hline $\begin{array}{l}25 h \\
100 c, E \\
60 c \\
3 \\
3\end{array}$ & $\begin{array}{l}3713.356 \\
3709.29 \\
3707.96 \\
3701.713 \\
3701.44\end{array}$ & $\begin{array}{l}26922.19 \\
26951.7 \\
26961.4 \\
27006.86 \\
27008.9\end{array}$ & $\begin{array}{l}d^{3} \mathrm{~F}_{4}-v^{3} \mathrm{~F}_{4}^{0} \\
a^{3} \mathrm{P}_{3}-z \\
a^{3} \mathrm{D}_{4}^{4} \\
b^{3} \mathrm{P}_{2}-z{ }^{3} \mathrm{D}_{2} \\
{ }^{3} \mathrm{D}_{2}-z \\
c^{3} \mathrm{~S}_{2}-z{ }^{1} \mathrm{P}_{1}^{\circ}\end{array}$ & & & & & & \\
\hline$\stackrel{2}{10 h}$ & $\begin{array}{l}3698.79 \\
3696.68\end{array}$ & $\begin{array}{l}27028.2 \\
27043.6\end{array}$ & $\begin{array}{l}c^{3} \mathrm{~F}_{1}-y^{3} \mathrm{H}_{3}^{\circ} \\
a^{3} \mathrm{H}_{4}-z^{3} \mathrm{D}_{3}^{3}\end{array}$ & & & & & & \\
\hline $\begin{array}{r}100 \\
10 \\
5\end{array}$ & $\begin{array}{l}3695.90 \\
3694.792 \\
3692.178\end{array}$ & $\begin{array}{l}27049.3 \\
27057.45 \\
27076.60\end{array}$ & $\begin{array}{l}b^{3} \mathrm{P}_{2}-z{ }^{1} \mathrm{D}_{2}^{0} \\
a^{1}{ }^{1} \mathrm{H}_{5}-z{ }^{3} \mathrm{I}_{3}^{0} \\
b^{3} \mathrm{D}_{2}-y^{3} \mathrm{~F}_{3}\end{array}$ & 3 & .166 & .333 & $1.312,1.477$ & 1.478 & 1.312 \\
\hline $\begin{array}{c}30 \\
3 \\
50 h\end{array}$ & $\begin{array}{l}3691.174 \\
3689.27 \\
3688.187\end{array}$ & $\begin{array}{l}27083.97 \\
27097.9 \\
27105.90\end{array}$ & $\begin{array}{l}b^{3} \mathrm{P}_{1}-z{ }^{1} \mathrm{D}_{2}^{0} \\
c^{3} \mathrm{~F}_{2}-w^{3} \mathrm{~F}_{2}^{0} \\
a^{3} \mathrm{G}_{5}-z^{5} \mathrm{~F}_{5}^{0}\end{array}$ & 1 & .196 & 0 & 1.110 & 1. 502 & 1. 306 \\
\hline $200 c, V E$ & $\begin{array}{l}3687.968 \\
3684.931\end{array}$ & $\begin{array}{l}27107.51 \\
27129.86\end{array}$ & $a^{3} \mathrm{H}_{6}-2{ }^{3} \mathrm{H}^{6} 6$ & $7 b, 3$ & & & $1.156 \pm$ & (1. 154) & 1.158 \\
\hline $\begin{array}{c}10 c \\
6 \\
2\end{array}$ & $\begin{array}{l}3683.02 \\
3681.690 \\
3681.57\end{array}$ & $\begin{array}{l}27143.9 \\
27153.74 \\
27154.6\end{array}$ & $\begin{array}{l}a{ }^{5} \mathrm{P}_{1}-z{ }^{5} \mathrm{D}_{2}^{2} \\
a^{3} \mathrm{~F}_{4}-z^{5} \mathrm{G}_{5}^{0} \\
c^{3} \mathrm{~F}_{4}-w^{3} \mathrm{~F}_{4}\end{array}$ & $\begin{array}{l}1 \\
2\end{array}$ & 1.000 & $\begin{array}{l}0 \\
0\end{array}$ & $\begin{array}{r}.465 \\
1.350\end{array}$ & $\begin{array}{c}2.465 \\
(1.230)\end{array}$ & $\begin{array}{l}1.465 \\
1.254\end{array}$ \\
\hline $10 h$ & 3679. 61 & 27169.1 & $b^{3} \mathrm{H}_{4}-y^{3} \mathrm{D}_{3}^{\circ}$ & 1 & .15 & 0 & & $(.880)$ & 1. 030 \\
\hline 40 & 3678.063 & 27180.51 & $a{ }^{1} \mathrm{H}_{5}-z{ }^{1} \mathrm{G}_{4}^{\circ}$ & 1 & .08 & 0 & .66 & .98 & 1. 06 \\
\hline $\begin{array}{l}15 \\
20 h \\
2 \\
60 h \\
4\end{array}$ & $\begin{array}{l}3676.335 \\
3670.05 \\
3664.467 \\
3663.751 \\
3662.73\end{array}$ & $\begin{array}{l}27193.29 \\
27239.8 \\
27281.36 \\
27286.69 \\
27294.3\end{array}$ & $\begin{array}{l}b{ }^{3} \mathrm{D}_{2}-y^{3} \mathrm{P}_{2}^{\circ} \\
b^{3} \mathrm{H}_{5}-z{ }^{3} \mathrm{H}_{6}^{8} \\
a^{3} \mathrm{G}_{3}-z{ }^{3} \mathrm{~F}_{4} \\
a^{1} \mathrm{~F}_{3}-z{ }^{1} \mathrm{~F}_{3}^{0}\end{array}$ & & & & & & \\
\hline $\begin{array}{l}300, \mathrm{~V} E \\
4 \\
8 h\end{array}$ & $\begin{array}{l}3659.602 \\
3654.270 \\
3652.25\end{array}$ & $\begin{array}{l}27317.62 \\
27357.48 \\
27372.6\end{array}$ & $\begin{array}{l}b^{3} \mathrm{G}_{3}-z^{3} \mathrm{H}_{4}^{\circ} \\
d^{3} \mathrm{~F}_{4}-527_{3}^{2} \\
a^{3} \mathrm{G}_{5}-z^{5} \mathrm{D}_{4}^{\circ}\end{array}$ & 2 & & 0 & .935 & $(.767)$ & .809 \\
\hline 200, V $E$ & $\begin{array}{l}3651.182 \\
3648.760\end{array}$ & $\begin{array}{l}27380.62 \\
27398.8\end{array}$ & $a^{3} \mathrm{~F}_{2}-z^{3} \mathrm{D}_{1}^{\mathrm{i}}$ & 2 & .296 & 0 & 1. 010 & .714 & .418 \\
\hline 20 & $\begin{array}{l}3645.94 \\
3641.382\end{array}$ & $\begin{array}{l}27420.0 \\
27454.30\end{array}$ & $\begin{array}{c}a{ }^{3} \mathrm{G}_{4}-z{ }^{5} \mathrm{~F}_{5}^{\circ} \\
b^{3} \mathrm{P}_{0}^{0}-y \\
{ }^{5} \mathrm{D}_{\mathrm{i}}^{\circ}\end{array}$ & $\begin{array}{l}1 \\
7 a\end{array}$ & .315 & $\begin{array}{l}0 \\
0\end{array}$ & & $\begin{array}{l}(1.052) \\
0 / 0\end{array}$ & $\begin{array}{l}1.367 \\
1.386\end{array}$ \\
\hline & 3641. 293 & 27454.98 & $b{ }^{1} \mathrm{D}_{2}-y{ }^{1} \mathrm{P}_{1}^{1}$ & 1 & .144 & 0 & $\begin{array}{r}1.080 \\
.839\end{array}$ & .983 & 1.127 \\
\hline $\begin{array}{l}30 \\
15 h, \mathrm{E}\end{array}$ & & $\begin{array}{l}27471.84 \\
27480.9\end{array}$ & $\begin{array}{l}b^{3} \mathrm{P}_{2}-y \\
c{ }^{3} \mathrm{D}_{1}^{\mathrm{i}} \\
{ }^{3} \mathrm{P}_{1}-z{ }^{1} \mathrm{P}_{1}\end{array}$ & 2 & .09 & 0 & 1. 552 & 1. 482 & 1.392 \\
\hline 40 & 3634.489 & 27506.37 & $\left\{\begin{array}{l}a^{3} \mathrm{D}_{3}-z^{3} \mathrm{~F}_{4} \\
b^{3} \mathrm{P}_{1}-y^{5} \mathrm{D}_{1}\end{array}\right.$ & & & & & & \\
\hline $\begin{array}{l}1 h \\
5 h\end{array}$ & $\begin{array}{l}3634.03 \\
3633.894\end{array}$ & $\begin{array}{l}27509.8 \\
27510.88\end{array}$ & $c^{3} \mathrm{~F}_{3}-w^{3} \mathrm{~F}^{3}$ & & & & & & \\
\hline $\begin{array}{r}100 \\
4\end{array}$ & $\begin{array}{l}3633.318 \\
3633.12\end{array}$ & $\begin{array}{l}27515.24 \\
27516.7\end{array}$ & $\begin{array}{l}b^{3} \mathrm{G}_{5}-z^{3} \mathrm{H}_{5}^{0} \\
a^{1} \mathrm{D}_{2}-z^{3} \mathrm{~F}_{3}^{0}\end{array}$ & 3 & .144 & .720 & & $(1.190)$ & 1.046 \\
\hline
\end{tabular}


488 Journal of Research of the National Bureau of Standards

TABLE 1.-Second spectrum of columbium (Cb II) - Continued

\begin{tabular}{|c|c|c|c|c|c|c|c|c|c|}
\hline $\begin{array}{l}\text { Intensity } \\
\text { spark }\end{array}$ & $\lambda_{\text {air }} \mathrm{A}$ & $\begin{array}{l}\text { Wave } \\
\text { No. } \\
\text { vac. } \\
\text { cm-1 }^{-1}\end{array}$ & $\begin{array}{l}\text { Term combi- } \\
\text { ation }\end{array}$ & $\begin{array}{c}\text { Zeeman } \\
\text { type }\end{array}$ & $\begin{array}{l}\text { Sepa- } \\
\text { ration }\end{array}$ & $\begin{array}{l}\text { Strong- } \\
\text { est } \\
p\end{array}$ & $\underset{n}{\text { Strongest }}$ & $\theta_{1}$ & $g_{2}$ \\
\hline 1 & 2 & 3 & 4 & 5 & 6 & 7 & 8 & 9 & 10 \\
\hline $\begin{array}{c}30 \\
2 \\
40 \\
3 h \\
3 h\end{array}$ & $\begin{array}{l}3629.47 \\
3628.70 \\
3628.180 \\
3622.90 \\
3622.49\end{array}$ & $\begin{array}{l}27544.4 \\
27550.2 \\
27554.20 \\
27594.4 \\
27597.5\end{array}$ & $\begin{array}{l}a{ }^{1} \mathrm{H}_{3}-z^{3} \mathrm{I}_{8}^{8} \\
a^{3} \mathrm{G}_{3}-z^{3} \mathrm{D}_{2} \\
a^{1} \mathrm{H}_{5}-w^{3} \mathrm{~F}^{4} \\
b^{1} \mathrm{D}_{2}-v^{3} \mathrm{~F}_{2} \\
b^{3} \mathrm{D}_{2}-x^{3} \mathrm{~F}_{3}^{8}\end{array}$ & 2 & 0.100 & 0 & $\begin{array}{r}1.280 \\
.584\end{array}$ & (0. 992) & 1.084 \\
\hline $\begin{array}{l}6 h \\
100 c, \mathrm{~V} E \\
200, \mathrm{~V} E \\
4 \\
3 h\end{array}$ & $\begin{array}{l}3620.57 \\
3619.729 \\
3619.514 \\
3618.89 \\
3614.88\end{array}$ & $\begin{array}{l}27612.1 \\
27618.53 \\
27620.17 \\
27624.9 \\
27655.6\end{array}$ & $\begin{array}{l}a^{3}{ }^{3} \mathrm{G}_{4}-2^{3} \mathrm{D}_{3}^{3} \\
b^{3} \mathrm{G}_{4}-2^{3} \mathrm{H}_{3}^{3} \\
a^{3} \mathrm{H}_{3}-2^{3} \mathrm{D}_{3}^{3} \\
a^{3} \mathrm{P}_{3}-2^{3} \mathrm{D}_{1}^{2} \\
b^{2} \mathrm{D}_{3}-y^{3} \mathrm{H}_{4}\end{array}$ & $\begin{array}{l}2 \\
1\end{array}$ & & $\begin{array}{l}0 \\
0\end{array}$ & $\begin{array}{l}1.06 \\
.933\end{array}$ & $\begin{array}{l}(1.027) \\
(1.070)\end{array}$ & $\begin{array}{l}1.034 \\
1.138\end{array}$ \\
\hline $\begin{array}{c}4 \\
3 \\
6 \\
15 \\
2 h\end{array}$ & $\begin{array}{l}3613.25 \\
3611.39 \\
3609.362 \\
3607.01 \\
3606.35\end{array}$ & $\begin{array}{l}27668.1 \\
27682.3 \\
27697.86 \\
2715.9 \\
27721.0\end{array}$ & 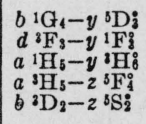 & $\begin{array}{l}2 \\
1\end{array}$ & $\begin{array}{l}.133 \\
.276\end{array}$ & $\begin{array}{l}0 \\
0\end{array}$ & & $\begin{array}{r}(.992) \\
(1.050)\end{array}$ & $\begin{array}{l}\text { 1. } 125 \\
1.326\end{array}$ \\
\hline $\begin{array}{c}8 h \\
40\end{array}$ & $\begin{array}{l}3604.66 \\
3591.197 \\
3588.02\end{array}$ & $\begin{array}{l}27734.0 \\
27837.95\end{array}$ & $\begin{array}{l}b^{3}{ }^{3} \mathrm{P}_{2}-x^{3} \mathrm{D}_{2}^{2} \\
a^{2} \mathrm{H}_{6}-z^{6} \mathrm{~F}_{3}^{3}\end{array}$ & 1 & .19 & 0 & .21 & 1.16 & 1.35 \\
\hline $\begin{array}{l}10 h \\
50 h \\
3 h\end{array}$ & $\begin{array}{l}3588.02 \\
3586.75 \\
3584.33\end{array}$ & $\begin{array}{l}27862.6 \\
27872.5 \\
27891.3\end{array}$ & $b^{3} \mathrm{~F}_{4}-z^{1} \mathrm{H}_{5}$ & 2 & .122 & 0 & 1. 506 & .896 & 1.018 \\
\hline $\begin{array}{l}4 h \\
2 \\
10 \\
30 \\
40 h\end{array}$ & $\begin{array}{l}3580.98 \\
3578.38 \\
3574.202 \\
3568.50 \\
3568.001\end{array}$ & $\begin{array}{l}27917.4 \\
27937.7 \\
27970.32 \\
28015.0 \\
28018.93\end{array}$ & 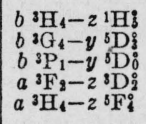 & 3 & .431 & .859 & $.709,1.142$ & .710 & 1.141 \\
\hline $\begin{array}{c}40 h \\
3 h \\
1 \\
15 h \\
4\end{array}$ & $\begin{array}{l}3566.10 \\
3565.68 \\
3565.23 \\
3564.075 \\
3561.88\end{array}$ & $\begin{array}{l}28033.9 \\
28037.2 \\
28040.7 \\
28049.79 \\
28067.1\end{array}$ & 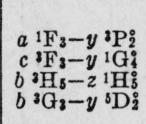 & & & & & & \\
\hline $\begin{array}{c}5 \\
6 h \\
60 \\
6 h \\
4\end{array}$ & $\begin{array}{l}3560.47 \\
3559.893 \\
3559.592 \\
3554.14 \\
3551.102\end{array}$ & $\begin{array}{l}28078.2 \\
28082.74 \\
28085.12 \\
28128.2 \\
28152.26\end{array}$ & $\begin{array}{l}a^{1} \mathrm{G}_{4}-2{ }^{3} \mathrm{H}_{4} \\
a^{1} \mathrm{P}_{1}-z^{1} \mathrm{Pi} \\
a^{1} \mathrm{H}_{5}-y^{1} \mathrm{G}_{4}^{8} \\
c^{3} \mathrm{~F}_{3}-x^{3} \mathrm{D}_{3}^{3} \\
b^{3} \mathrm{H}_{4}-y^{3} \mathrm{~B}_{4}\end{array}$ & 2 & & 0 & 1. 17 & $(.992)$ & .95 \\
\hline $\begin{array}{l}4 h, \dot{\mathrm{V}} E ? \\
15 h \\
50 \\
200, \mathrm{~V} E \\
6 \mathrm{~h}\end{array}$ & $\begin{array}{l}3548.08 \\
3544.346 \\
3541.247 \\
3540.961 \\
3539.11\end{array}$ & $\begin{array}{l}28176.2 \\
28205.92 \\
28230.68 \\
28232.88 \\
28247.6\end{array}$ & $\begin{array}{l}c^{3} \mathrm{P}_{2}-x^{3} \mathrm{D}_{1}^{\circ} \\
c^{3} \mathrm{P}_{1}-x^{3} \mathrm{D}_{2}^{3} \\
a^{3} \mathrm{D}_{2}-z^{1} \mathrm{D}_{2}^{2} \\
a^{3} \mathrm{~F}_{4}-z^{3} \mathrm{D}^{3} \\
c^{3} \mathrm{P}_{2}-x^{3} \mathrm{D}_{3}^{3}\end{array}$ & 1 & & 0 & .95 & (1. 230) & 1.323 \\
\hline $\begin{array}{l}40, \mathrm{~V} E \\
50 \\
20 \\
10 h \\
20\end{array}$ & $\begin{array}{l}3537.625 \\
3534.215 \\
3534.114 \\
3534.05 \\
3528.890\end{array}$ & $\begin{array}{l}28259.50 \\
28286.77 \\
28287.58 \\
2888.1 \\
28329.46\end{array}$ & $\begin{array}{l}a^{3} \mathrm{~s}_{2}-z^{3} \mathrm{~b}_{2}^{2} \\
c^{3} \mathrm{D}_{4}-x^{3} \mathrm{D}_{3}^{2} \\
d^{3} \mathrm{~F}_{2}-w^{3} \mathrm{G}_{3}^{8} \\
b^{3} \mathrm{H}_{5}-y^{3} \mathrm{~B}_{4}^{8}\end{array}$ & 3 & .312 & .621 & 1. $145,1.458$ & 1.457 & 1.145 \\
\hline $\begin{array}{c}30 \\
5 \\
8 c \\
50 h \\
10 h\end{array}$ & $\begin{array}{l}3528.474 \\
3528.09 \\
3527.024 \\
3525.986 \\
3522.368\end{array}$ & $\begin{array}{l}28332.79 \\
28335.9 \\
28344.44 \\
28352.79 \\
28381.90\end{array}$ & $\begin{array}{l}b^{3} \mathrm{H}_{4}-2{ }^{1} \mathrm{~F}_{3}^{\circ} \\
b^{3} \mathrm{G}_{3}-y^{3} \mathrm{D}_{3}^{3} \\
d^{3} \mathrm{~F}_{4}-w^{3} \mathrm{G}_{3}^{3} \\
c^{3} \mathrm{P}_{2}-w^{3} \mathrm{D}_{3}^{\circ} \\
b^{1} \mathrm{D}_{2}-527_{3}^{3}\end{array}$ & 1 & .157 & 0 & .266 & 1. 457 & 1.078 \\
\hline $\begin{array}{c}5 h \\
20 h \\
5 h \\
150 \\
200 c, \text { V } E\end{array}$ & $\begin{array}{l}3521.599 \\
3521.14 \\
3519.66 \\
3517.670 \\
3515.421\end{array}$ & $\begin{array}{l}28388.10 \\
28391.8 \\
28403.7 \\
2819.81 \\
28437.99\end{array}$ & $\begin{array}{l}b^{3} \mathrm{D}_{3}-w^{3} \mathrm{~F}_{3}^{\circ} \\
c^{3} \mathrm{~F}_{4}-w^{3} \mathrm{D}_{3}^{3} \\
b^{3} \mathrm{D}_{2}-w^{3} \mathrm{~F}_{2}^{2} \\
b^{1} \mathrm{G}_{4}-y^{3} \mathrm{D}_{3}^{3} \\
a^{3} \mathrm{G}_{3}-z^{3} \mathrm{G}_{3}^{3}\end{array}$ & $\frac{1}{7 b}$ & .083 & $\begin{array}{l}0 \\
0 \\
0\end{array}$ & $\begin{array}{l}.70 \\
.765\end{array}$ & $\begin{array}{r}(1.240) \\
.949 \\
.765\end{array}$ & $\begin{array}{l}1.30 \\
1.032 \\
.765\end{array}$ \\
\hline $\begin{array}{l}20 \mathrm{c} \\
400, \mathrm{~V} E \\
5 \mathrm{c} \\
15 \\
10 h\end{array}$ & $\begin{array}{l}3514.02 \\
3510.262 \\
3505.992 \\
3501.32 \\
3500.74\end{array}$ & $\begin{array}{l}28449.3 \\
28479.78 \\
28514.47 \\
28552.5 \\
28557.3\end{array}$ & $\begin{array}{l}b^{3} \mathrm{H}_{4}-y^{3} \mathrm{G}_{3}^{\circ} \\
b^{3} \mathrm{G}_{3}-z^{3} \mathrm{H}_{6}^{6} \\
a^{3} \mathrm{H}_{4}-z^{3} \mathrm{~F}^{5} \\
b^{1} \mathrm{G}_{4}-z^{5} \mathrm{P}^{3} \\
c^{3} \mathrm{P}_{2}-5000_{3}^{3}\end{array}$ & $\begin{array}{l}7 b, 2 \\
1\end{array}$ & .623 & $\begin{array}{l}0 \\
0\end{array}$ & $\begin{array}{r}1.118 \\
.930\end{array}$ & $\begin{array}{c}(1.19) \\
.939\end{array}$ & 1.18 \\
\hline $\begin{array}{l}30 c \\
30 c \\
20 \\
10 \\
90, \mathrm{~V} E\end{array}$ & $\begin{array}{l}3499.93 \\
3496.027 \\
3491.896 \\
3490.418 \\
3489.093\end{array}$ & $\begin{array}{l}28563.9 \\
28595.75 \\
28629.57 \\
28641.70 \\
28652.57\end{array}$ & $\begin{array}{l}b^{3} \mathrm{D}_{3}-z^{1} \mathrm{G} \\
c^{3} \mathrm{G}_{4}-5000_{3}^{8} \\
b^{3} \mathrm{P}_{2}-y^{3} \mathrm{D}_{2}^{2} \\
c^{3} \mathrm{~F}_{3}-x^{3} \mathrm{D}_{3}^{3} \\
a^{3} \mathrm{~F}_{3}-z^{3} \mathrm{D}_{3}^{3}\end{array}$ & $\begin{array}{l}1 \\
7 b \\
3\end{array}$ & $\begin{array}{l}.228 \\
.25\end{array}$ & $\begin{array}{l}0 \\
0 \\
.50\end{array}$ & $\begin{array}{ll} & .401 \\
1.24 & 1.49 \\
1.49\end{array}$ & $\begin{array}{l}\text { 1. } 313 \\
\text { 1. } 240 \\
\text { 1. } 49\end{array}$ & $\begin{array}{l}1.085 \\
1.240 \\
1.24\end{array}$ \\
\hline
\end{tabular}


TABLE 1.-Second spectrum of columbium (Cb iI)-Continued

\begin{tabular}{|c|c|c|c|c|c|c|c|c|c|}
\hline $\begin{array}{l}\text { Intensity } \\
\text { spark }\end{array}$ & $\lambda_{\text {sir }} \mathrm{A}$ & $\begin{array}{l}\text { Wave } \\
\text { No. } \\
\text { vac. } \\
\mathrm{cm}^{-1}\end{array}$ & $\begin{array}{l}\text { Term combi- } \\
\text { nation }\end{array}$ & $\begin{array}{c}\text { Zeeman } \\
\text { type }\end{array}$ & $\begin{array}{l}\text { Sepa- } \\
\text { ration }\end{array}$ & $\begin{array}{c}\text { Strong- } \\
\text { est } \\
p\end{array}$ & $\underset{n}{\text { Strongest }}$ & $g_{1}$ & $g_{2}$ \\
\hline 1 & 2 & 3 & 4 & 5 & 6 & 7 & 8 & 8 & 10 \\
\hline $\begin{array}{c}80 \\
10 \\
80, \mathrm{~V} E \\
100 \\
5 h\end{array}$ & $\begin{array}{l}3488.81 \\
3484.625 \\
3484.054 \\
3482.953 \\
3482.52\end{array}$ & $\begin{array}{l}28654.9 \\
28689.31 \\
28694.01 \\
28703.08 \\
28706.7\end{array}$ & $\begin{array}{l}c^{3} \mathrm{~F}_{3}-w^{3} \mathrm{D}_{2}^{2} \\
b^{3} \mathrm{G}_{4}-y^{3} \mathrm{D}_{3}^{3} \\
a^{3} \mathrm{~B}_{1}-z^{3} \mathrm{D}_{1}^{1} \\
b^{1} \mathrm{D}_{2}-y^{1} \mathrm{D}_{3}^{3} \\
a^{3} \mathrm{H}_{4}-z^{3} \mathrm{D}_{3}^{3}\end{array}$ & $\begin{array}{l}3 \\
2\end{array}$ & 1. 078 & $\begin{array}{l}1.078 \\
0\end{array}$ & $\begin{array}{c}0.416,1.495 \\
1.15\end{array}$ & $\begin{array}{l}1.495 \\
(.98)\end{array}$ & $\begin{array}{l}0.416 \\
1.04\end{array}$ \\
\hline $\begin{array}{l}80 \\
150, \mathrm{~V} E \\
100, \mathrm{~V} \\
6 \\
25 \mathrm{c}\end{array}$ & $\begin{array}{l}3480.213 \\
3479.567 \\
3478.79 \\
3476.28 \\
3474.68\end{array}$ & $\begin{array}{l}28725.68 \\
28731.01 \\
28737.4 \\
28758.2 \\
28771.4\end{array}$ & 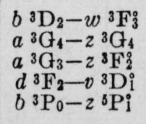 & $\begin{array}{l}1 \\
3 \\
2 \\
2 \\
2\end{array}$ & .112 & $\begin{array}{l}0 \\
0.110 \\
0\end{array}$ & $\begin{array}{l}.842 \\
1.056 \pm \\
.90\end{array}$ & $\begin{array}{l}1.178 \\
1.042 \\
(.765) \\
.70\end{array}$ & $\begin{array}{l}1.066 \\
1.070 \\
.698 \\
\text { (. } 53)\end{array}$ \\
\hline $\begin{array}{l}20 \\
50 \\
15 c \\
4 c \\
15\end{array}$ & $\begin{array}{l}3474.004 \\
3470.27 \\
3468.127 \\
3461.54 \\
3459.56\end{array}$ & $\begin{array}{l}28777.02 \\
28808.0 \\
28825.78 \\
28880.6 \\
28897.2\end{array}$ & $\begin{array}{l}a^{1}{ }^{1} \mathrm{G}_{4}-z^{3} \mathrm{H}_{5}^{3} \\
a^{1} \mathrm{P}_{1}-x^{3} \mathrm{D}_{2}^{2} \\
b^{3} \mathrm{H}_{3}-y^{3} \mathrm{~F}_{4}^{4}\end{array}$ & $\begin{array}{l}1 \\
2\end{array}$ & .143 & $\begin{array}{l}0 \\
0\end{array}$ & $\begin{array}{r}.919 \\
1.404 \\
.997\end{array}$ & $\begin{array}{c}(1.083) \\
1.118\end{array}$ & $\begin{array}{l}\text { 1. } 050 \\
\text { 1. } 261\end{array}$ \\
\hline $\begin{array}{c}10 \\
4 h \\
80 \\
60 \\
5 c\end{array}$ & $\begin{array}{l}3458.728 \\
3457.04 \\
3454.910 \\
3454.708 \\
3453.96\end{array}$ & $\begin{array}{l}28904.11 \\
28918.2 \\
28936.06 \\
28937.74 \\
28944.0\end{array}$ & $\begin{array}{l}b^{3} \mathrm{~F}_{2}-z^{1} \mathrm{D}_{2}^{0} \\
b^{3} \mathrm{D}_{5}-y^{6} \mathrm{D}_{4}^{8} \\
c^{3} \mathrm{P}_{2}-x^{3} \mathrm{P}_{2}^{2} \\
b^{3} \mathrm{D}_{3}-w^{3} \mathrm{~F}_{4}^{4} \\
a^{6} \mathrm{P}_{2}-z^{3} \mathrm{~F}_{3}^{0}\end{array}$ & $\begin{array}{l}3 \\
1\end{array}$ & $\begin{array}{l}.11 \\
.213\end{array}$ & $0^{.22}$ & $\begin{array}{r}1.36,1.47 \\
.470\end{array}$ & $\begin{array}{l}1.47 \\
1.322\end{array}$ & $\begin{array}{l}\text { 1. } 36 \\
\text { 1. } 109\end{array}$ \\
\hline $\begin{array}{l}40 \\
3 h \\
20 \\
60 \\
20\end{array}$ & $\begin{array}{l}3452.350 \\
3452.19 \\
3451.640 \\
3450.766 \\
3448.674\end{array}$ & $\begin{array}{l}28957.51 \\
28958.9 \\
28963.47 \\
28970.80 \\
28988.47\end{array}$ & $\begin{array}{l}b^{3} \mathrm{P}_{2}-y^{3} \mathrm{D}_{2}^{\circ} \\
b^{3} \mathrm{H}_{4}-y^{3} \mathrm{~F}_{3}^{3} \\
c^{3} \mathrm{P}_{2}-x^{3} \mathrm{P}_{1}^{1} \\
b^{3} \mathrm{P}_{0}-y^{3} \mathrm{D}_{1}^{1} \\
b^{3} \mathrm{P}_{2}-y^{3} \mathrm{D}_{1}^{1}\end{array}$ & $\begin{array}{l}2 \\
7 a \\
2\end{array}$ & \begin{tabular}{l|}
.13 \\
.270 \\
.498
\end{tabular} & $\begin{array}{l}.26 \\
0 \\
0 \\
0\end{array}$ & $\begin{array}{r}1.34,1.47 \\
1.746 \\
.976 \\
1.982\end{array}$ & $\begin{array}{l}1.47 \\
1.476 \\
0 / 0 \\
1.484\end{array}$ & $\begin{array}{r}1.34 \\
1.206 \\
.976 \\
.986\end{array}$ \\
\hline $\begin{array}{l}40 \\
5 \\
60 \\
20 \\
10 c\end{array}$ & $\begin{array}{l}3448.221 \\
3446.928 \\
3444.281 \\
3443.737 \\
3441.64\end{array}$ & $\begin{array}{l}28992.18 \\
29003.06 \\
29025.44 \\
29029.93 \\
29047.6\end{array}$ & $\begin{array}{l}b^{3} \mathrm{P}_{1}-y^{5} \mathrm{D}_{2}^{\circ} \\
b^{3} \mathrm{H}_{1}-y^{3} \mathrm{~F}_{4}^{2} \\
c^{3} \mathrm{H}_{4}-x^{3} \mathrm{G}_{3}^{6} \\
c^{3} \mathrm{~F}_{2}-x^{3} \mathrm{D}_{1}^{1} \\
a^{3} \mathrm{~F}_{2}-z^{3} \mathrm{D}_{3}^{6}\end{array}$ & $\begin{array}{l}1 \text { lus } \\
1 \\
1 \\
2\end{array}$ & $\begin{array}{l}.134 \\
.590\end{array}$ & $\begin{array}{l}.18 \\
0 \\
0 \\
0\end{array}$ & $\begin{array}{l}1.18 \\
1.05 \\
.540\end{array}$ & $\begin{array}{c}1.52 \\
(1.240) \\
.674 \\
(.712)\end{array}$ & $\begin{array}{l}1.35 \\
1.20 \\
.808 \\
1.302\end{array}$ \\
\hline $\begin{array}{l}200, \mathrm{~V} E \\
60, \mathrm{~V} \\
80 \\
80\end{array}$ & $\begin{array}{l}3440.589 \\
3439.925 \\
3438.41\end{array}$ & $\begin{array}{l}29056.50 \\
29062.10 \\
29074.9\end{array}$ & $\begin{array}{c}a^{3} \mathrm{~F}_{4}-z^{5} \mathrm{~F}_{3}^{0} \\
a^{3} \mathrm{~F}_{3}-z^{3} \mathrm{~F}_{2}^{2} \\
\left\{b^{3} \mathrm{H}_{6}-y^{3} \mathrm{C}_{3}^{3}\right.\end{array}$ & $\begin{array}{l}1 \\
2\end{array}$ & & $\begin{array}{l}0 \\
0\end{array}$ & $\begin{array}{l}\text { 1. } 14 \\
1.116\end{array}$ & $\begin{array}{l}(1.230) \\
(1.070)\end{array}$ & $\begin{array}{l}\text { 1. } 260 \\
1.047\end{array}$ \\
\hline${ }_{20}^{60,} E$ & $\begin{array}{l}3436.964 \\
3436.834\end{array}$ & $\begin{array}{l}29087.14 \\
29088.24\end{array}$ & $\begin{array}{c}b^{3} \mathrm{C}_{3}-y^{3} \mathrm{D}^{3} \\
a^{3} \mathrm{G}_{3}-z^{3} \mathrm{C}^{3} \mathrm{G}^{3}\end{array}$ & $3 u s$ & .270 & .810 & & (.767) & 1. 037 \\
\hline $\begin{array}{c}2 h \\
2 h \\
3 \\
5 h \\
400, \mathrm{~V} E\end{array}$ & $\begin{array}{l}3435.899 \\
3435.583 \\
3433.952 \\
3433.747 \\
3432.708\end{array}$ & $\begin{array}{l}29096.15 \\
29098.83 \\
29112.65 \\
29114.39 \\
29123.20\end{array}$ & $\begin{array}{l}a^{1} \mathrm{G}_{4}-y^{5} \mathrm{DD}^{\circ} \\
a^{1} \mathrm{P}_{1}-495_{2}^{\circ} \\
c^{3} \mathrm{P}_{4}-x^{3} \mathrm{G}_{3}^{3} \\
c^{3} \mathrm{~F}_{2}-w^{3} \mathrm{D}_{2}^{2} \\
b^{1} \mathrm{G}_{4}-z^{1} \mathrm{H}_{8}\end{array}$ & 2 & & 0 & 1. 25 & $(.950)$ & 1.01 \\
\hline $\begin{array}{l}4 h \\
2 h\end{array}$ & $\begin{array}{l}\text { 3431. } 18 \\
3430.912\end{array}$ & $\begin{array}{l}29136.2 \\
29138.45\end{array}$ & 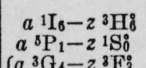 & & & & & & \\
\hline $250 c, \mathrm{~V} E$ & 3426. 562 & 29175.44 & $\left\{\begin{array}{l}a^{3} \mathrm{~B}_{4}-z^{3} \mathrm{~F}^{3} \\
a^{3} \mathrm{H}_{4} z^{3} \mathrm{G}_{3}\end{array}\right.$ & & & & & & \\
\hline$\underset{5 c}{300 c, \vee} E$ & $\begin{array}{l}3425.432 \\
3423.643\end{array}$ & $\begin{array}{l}29185.06 \\
29200.31\end{array}$ & $a^{3} \mathrm{G}_{5}-z^{3} \mathrm{G}^{3}$ & 3 & & .19 & 1. $21 \pm$ & 1. 19 & 1.23 \\
\hline $\begin{array}{c}10 \\
5 \\
3 \\
20 c \\
80, \mathrm{~V} E\end{array}$ & \begin{tabular}{|l|l|}
3422.85 \\
3422.77 \\
3421.318 \\
3421.161 \\
3420.633
\end{tabular} & $\begin{array}{l}29207.1 \\
29207.8 \\
29220.15 \\
29221.49 \\
29226.00\end{array}$ & $\begin{array}{l}b^{3} \mathrm{H}_{5}-y^{3} \mathrm{G}_{8}^{\circ} \\
a^{3} \mathrm{~F}_{4}-z^{3} \mathrm{~F}^{8} \\
b^{3} \mathrm{G}_{3}-z^{3} \mathrm{P}_{3}^{3} \\
a^{3} \mathrm{P}_{3}-z^{3} \mathrm{~F}_{4} \\
a^{3} \mathrm{~F}_{2}-z^{3} \mathrm{~F}_{1}\end{array}$ & 2 & .590 & 0 & 1. 302 & .712 & .122 \\
\hline $\begin{array}{c}2 \\
5 h \\
20 \\
150, \mathrm{~V} E \\
10 h\end{array}$ & $\begin{array}{l}3417.80 \\
3417.17 \\
3413.209 \\
3412.934 \\
3412.480\end{array}$ & $\begin{array}{l}29250.2 \\
29255.6 \\
29289.57 \\
29291.93 \\
29295.82\end{array}$ & $\begin{array}{l}a{ }^{1} \mathrm{P}_{1}-x{ }^{3} \mathrm{D}^{\mathrm{i}} \\
c^{3} \mathrm{P}_{1}-x^{3} \mathrm{P}_{0}^{\mathrm{i}} \\
b^{3} \mathrm{G}_{5}-z^{1} \mathrm{H}_{5}^{\mathrm{i}} \\
a^{3} \mathrm{P}_{2}-z^{3} \mathrm{D}_{3}^{3} \\
a^{1} \mathrm{P}_{1}-w^{3} \mathrm{D}_{1}^{\mathrm{i}}\end{array}$ & $\begin{array}{l}7 a \\
1\end{array}$ & .143 & $\begin{array}{l}0 \\
0\end{array}$ & 1. 022 & $\begin{array}{l}1.20 \\
1.451\end{array}$ & $\begin{array}{l}0 / 0 \\
1.308\end{array}$ \\
\hline $\begin{array}{l}100, \mathrm{~V} E \\
100, \mathrm{~V} E \\
3 h \\
10 \\
10 h\end{array}$ & $\begin{array}{l}3409.191 \\
3408.678 \\
3408.53 \\
3407.32 \\
3406.947\end{array}$ & $\begin{array}{l}29324.09 \\
29328.50 \\
29329.8 \\
29340.2 \\
29343.41\end{array}$ & $\begin{array}{l}a^{3} \mathrm{P}_{0}-z^{3} \mathrm{~B}_{1} \\
a^{3} \mathrm{P}_{1}-z^{3} \mathrm{D}_{2}^{2} \\
c^{3} \mathrm{~B}_{3}-x^{3} \mathrm{P}_{2}^{2} \\
c^{3} \mathrm{P}_{0}-x^{3} \mathrm{D}_{1}^{\mathrm{i}}\end{array}$ & $\begin{array}{l}7 a \\
1\end{array}$ & .357 & $\begin{array}{l}0 \\
0\end{array}$ & $\begin{array}{l}.423 \\
.788\end{array}$ & $\begin{array}{l}0 / 0 \\
1.502 \\
0 / 0\end{array}$ & $\begin{array}{r}.423 \\
\text { 1. } 145\end{array}$ \\
\hline
\end{tabular}


TABLE 1.-Second spectrum of columbium (Cb II)-Continued

\begin{tabular}{|c|c|c|c|c|c|c|c|c|c|}
\hline $\begin{array}{l}\text { Intensity } \\
\text { spark }\end{array}$ & $\lambda_{\text {air }} \mathrm{A}$ & $\begin{array}{l}\text { Wave } \\
\text { No. } \\
\text { vac } \\
\mathrm{cm}^{-1}\end{array}$ & $\begin{array}{l}\text { Term combi- } \\
\text { nation }\end{array}$ & $\begin{array}{c}\text { Zeeman } \\
\text { type }\end{array}$ & $\begin{array}{l}\text { Sepa- } \\
\text { raton }\end{array}$ & $\begin{array}{c}\text { Strong- } \\
\text { est } \\
p\end{array}$ & $\underset{n}{\text { Strongest }}$ & $g_{1}$ & 92 \\
\hline 1 & 2 & 3 & 4 & 5 & 6 & 7 & 8 & 9 & 10 \\
\hline $\begin{array}{l}3 h \\
2 h \\
20 \\
10 \\
30, \mathrm{~V} E\end{array}$ & $\begin{array}{l}3403.49 \\
3402.81 \\
3402.02 \\
3401.231 \\
3399.714\end{array}$ & $\begin{array}{l}29373.2 \\
29379.1 \\
29385.9 \\
29392.72 \\
29405.83\end{array}$ & $\begin{array}{l}b^{3} \mathrm{G}_{3}-z^{3} \mathrm{P}_{2}^{2} \\
c^{3} \mathrm{~F}_{1}-x^{3} \mathrm{G}_{4}^{8} \\
c^{3} \mathrm{P}_{0}-w^{3} \mathrm{D}_{1} \mathrm{i} \\
b^{3} \mathrm{G}_{4}-z z^{1} \mathrm{H}_{3}^{3} \\
b^{3} \mathrm{P}_{2}-z z^{3} \mathrm{P}_{1}\end{array}$ & $\begin{array}{l}7 a \\
7 b \\
2\end{array}$ & 0.244 & $\begin{array}{l}0 \\
0 \\
0\end{array}$ & $\begin{array}{l}0.613 \\
1.067 \\
1.732\end{array}$ & $\begin{array}{c}0 / 0 \\
(1.027) \\
1.488\end{array}$ & $\begin{array}{l}0.613 \\
1.035 \\
1.244\end{array}$ \\
\hline $\begin{array}{l}5 h \\
10 h \\
50 h \\
15 \\
60\end{array}$ & $\begin{array}{l}3397.516 \\
3397.319 \\
3396.365 \\
3395.72 \\
3394.978\end{array}$ & $\begin{array}{l}29424.85 \\
29426.56 \\
29434.82 \\
29440.4 \\
29446.85\end{array}$ & $\begin{array}{l}a{ }^{1} \mathrm{H}_{5}-x^{3} \mathrm{G}_{5} \\
c^{3} \mathrm{~F}_{3}-x^{3} \mathrm{~F}_{1}^{\mathrm{i}} \\
b^{3} \mathrm{3}_{1}-z^{3} \mathrm{P}_{1}^{1} \\
a^{1} \mathrm{D}_{2}-z^{1} \mathrm{D}_{2}\end{array}$ & $\begin{array}{l}3 \\
3\end{array}$ & $\begin{array}{l}.281 \\
.297\end{array}$ & $\begin{array}{l}.284 \\
.602\end{array}$ & $\begin{array}{l}1.224,1.501 \\
1.008,1.302\end{array}$ & $\begin{array}{l}1.503 \\
1.006\end{array}$ & $\begin{array}{l}1.222 \\
1.305\end{array}$ \\
\hline $\begin{array}{l}10 \\
4 h \\
9\end{array}$ & $\begin{array}{l}3393.810 \\
3392.475 \\
3391.594\end{array}$ & $\begin{array}{l}29456.98 \\
29468.57 \\
29476.23\end{array}$ & $\begin{array}{c}a^{3} \mathrm{~F}_{2}-2{ }^{5} \mathrm{~F}_{2}^{2} \\
b^{3} \mathrm{D}_{3}-y^{1} \mathrm{G}_{4} \\
a^{3} \mathrm{~F}_{3}-z^{5} \mathrm{~F}_{3}^{3}\end{array}$ & 3 & .314 & .628 & & $(.712)$ & 1.026 \\
\hline $250, \mathrm{~V} E$ & $\begin{array}{l}3389.939 \\
3386.243\end{array}$ & $\begin{array}{l}29499.32 \\
29522.81\end{array}$ & $\begin{array}{l}a^{3} \mathrm{G}_{4}-2^{3} \mathrm{G}_{5}^{3} \\
a^{3} \mathrm{H}_{5}-z^{3} \mathrm{G}_{4}\end{array}$ & $\begin{array}{l}2 \\
1\end{array}$ & .180 & $\begin{array}{l}0 \\
0\end{array}$ & $\begin{array}{r}1.960 \\
.962\end{array}$ & $\begin{array}{c}1.060 \\
(1.050)\end{array}$ & $\begin{array}{l}\text { 1. } 240 \\
\text { 1. } 072\end{array}$ \\
\hline $\begin{array}{c}10 c \\
40 h \\
150 \\
60 \\
9 c\end{array}$ & $\begin{array}{l}\text { 3383. } 302 \\
3382.44 \\
3380.934 \\
3379.300 \\
3377.375\end{array}$ & $\begin{array}{l}29548.47 \\
29556.0 \\
29569.17 \\
29583.46 \\
29600.32\end{array}$ & $\begin{array}{c}b^{3} \mathrm{D}_{3}-x^{3} \mathrm{D}_{3}^{2} \\
b^{3} \mathrm{G}_{5}-y^{3} \mathrm{G}_{4}^{3} \\
b^{1} \mathrm{G}_{1}-z^{1} \mathrm{~F}_{3}^{3} \\
b^{3} \mathrm{P}_{1}-z^{3} \mathrm{P}_{2}^{0}\end{array}$ & $\begin{array}{l}2 \\
1\end{array}$ & .140 & $\begin{array}{l}0 \\
0\end{array}$ & $\begin{array}{l}1.40 \\
.515\end{array}$ & $\begin{array}{r}(1.19) \\
.935\end{array}$ & $\begin{array}{l}1.14 \\
1.075\end{array}$ \\
\hline $\begin{array}{l}50 \\
20 h\end{array}$ & $\begin{array}{l}3374.252 \\
3374.087\end{array}$ & $\begin{array}{l}29627.72 \\
29629.17\end{array}$ & $a^{3} \mathrm{~F}_{3}-z{ }^{5} \mathrm{~F}_{4}$ & 2 & .253 & 0 & 2. 087 & 1.075 & 1. 328 \\
\hline $\begin{array}{l}120, \mathrm{~V} E \\
30 \\
50\end{array}$ & $\begin{array}{l}3372.565 \\
3370.609 \\
3370.154\end{array}$ & $\begin{array}{l}29642.54 \\
29659.74 \\
29663.74\end{array}$ & $\begin{array}{l}a^{3} \mathrm{G}_{5}-z^{3} \mathrm{~F}_{i} \\
b^{3} \mathrm{P}_{1}-z^{3} \mathrm{P}_{0}^{3} \\
d^{3} \mathrm{~F}_{4}-w^{3} \mathrm{G}_{3}^{3}\end{array}$ & $\begin{array}{l}1 \\
7 a\end{array}$ & & $\begin{array}{l}0 \\
0\end{array}$ & $\begin{array}{l}.86 \\
1.510\end{array}$ & $\begin{array}{r}(1.18) \\
1.510\end{array}$ & $\begin{array}{l}1.26 \\
0 / 0\end{array}$ \\
\hline $\begin{array}{l}100, \text { V E } \\
100 \\
10\end{array}$ & $\begin{array}{l}3369.155 \\
3365.94 \\
3365.883\end{array}$ & $\begin{array}{l}29672.58 \\
29700.9 \\
29701.38\end{array}$ & $\begin{array}{l}b^{3} \mathrm{G}_{4}-y^{3} \mathrm{G}_{4}^{2} \\
b^{3} \mathrm{D}_{1}-z{ }^{1} \mathrm{P}_{1} \\
a^{3} \mathrm{P}_{2}-z{ }^{3} \mathrm{~F}_{2}^{0}\end{array}$ & $\begin{array}{l}3 \\
3\end{array}$ & $\begin{array}{l}.10 \\
.244\end{array}$ & $0^{.40}$ & $1.07 \pm$ & $\begin{array}{l}1.02 \\
(.681)\end{array}$ & $\begin{array}{l}1.12 \\
.925\end{array}$ \\
\hline${ }_{20 h}^{100,}$ V $E$ & $\begin{array}{l}3365.594 \\
3362.17\end{array}$ & $\begin{array}{l}29703.93 \\
29734.2\end{array}$ & $\begin{array}{l}a^{3} \mathrm{~F}_{4}-2{ }^{5} \mathrm{~F}_{3}^{0} \\
c^{3} \mathrm{~F}_{3}-x^{3} \mathrm{G}_{4}\end{array}$ & $\begin{array}{l}2 \\
2\end{array}$ & .126 & $\begin{array}{l}0 \\
0\end{array}$ & $\begin{array}{l}1.864 \\
1.43\end{array}$ & $\begin{array}{c}1.234 \\
(1.083)\end{array}$ & $\begin{array}{l}1.360 \\
1.17\end{array}$ \\
\hline $\begin{array}{l}100 \\
2 \\
3 h \\
1 h \\
15, \mathrm{~V} E\end{array}$ & $\begin{array}{l}3360.904 \\
3357.156 \\
3355.92 \\
3355.67 \\
3353.509\end{array}$ & $\begin{array}{l}29745.38 \\
29778.60 \\
29789.6 \\
29791.8 \\
29810.97\end{array}$ & 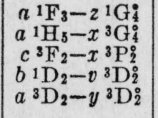 & 3 & .222 & .444 & 1. 293 & $\begin{array}{l}(1.007) \\
(1.002)\end{array}$ & 1. 224 \\
\hline $\begin{array}{c}5 h \\
3 h \\
3 h \\
100 \\
15\end{array}$ & $\begin{array}{l}3352.828 \\
3351.87 \\
3350.41 \\
3349.351 \\
3348.787\end{array}$ & $\begin{array}{l}29817.03 \\
29825.5 \\
29838.5 \\
29847.98 \\
29853.01\end{array}$ & $\begin{array}{l}c{ }^{3} \mathrm{~F}_{2}-x \\
a^{3} \mathrm{H}_{4}{ }^{3} \mathrm{P}_{1} \\
{ }^{3} \mathrm{G}_{4}^{8} \\
a^{1} \mathrm{G}_{4}-y^{3} \mathrm{D}_{3}^{3} \\
b^{3} \mathrm{G}_{1}-z^{1} \mathrm{~F}_{3}^{3}\end{array}$ & 2 & & 0 & 1.18 & $(1.083)$ & 1.05 \\
\hline $\begin{array}{l}20 \\
30 \\
20 c \\
8 h \\
80\end{array}$ & $\begin{array}{l}3348.28 \\
3346.760 \\
3346.286 \\
3344.25 \\
3343.967\end{array}$ & $\begin{array}{l}29857.5 \\
29871.09 \\
29875.32 \\
29893.5 \\
29896.03\end{array}$ & $\begin{array}{l}a{ }^{1} \mathrm{P}_{1}-x{ }^{3} \mathrm{P}_{0}^{0} \\
a^{3} \mathrm{~F}_{2}-2 \mathrm{~S}^{3} \mathrm{~F}_{3}^{3} \\
b^{3} \mathrm{H}_{4}-y^{3} \mathrm{H}_{4}^{4} \\
b^{3} \mathrm{D}_{2}-x^{3} \mathrm{D}_{2}^{2} \\
a^{3} \mathrm{~F}_{4}-z^{3} \mathrm{D}_{3}^{3}\end{array}$ & $\begin{array}{l}7 a \\
2\end{array}$ & .235 & $\begin{array}{l}0 \\
0\end{array}$ & $\begin{array}{l}1.120 \\
2.381\end{array}$ & $\begin{array}{r}1.120 \\
.716\end{array}$ & $\begin{array}{l}0 / 0 \\
1.271\end{array}$ \\
\hline $\begin{array}{c}6 \\
5 \\
150, \mathrm{~V} E \\
60 d \\
4 h\end{array}$ & $\begin{array}{l}3343.90 \\
3343.28 \\
3341.612 \\
3340.45 \\
3340.18\end{array}$ & $\begin{array}{l}29896.6 \\
29902.2 \\
29917.10 \\
29927.5 \\
29929.9\end{array}$ & $\begin{array}{c}a^{3} \mathrm{~F}_{3}-z{ }^{3} \mathrm{D}_{2}^{0} \\
b^{3} \mathrm{~F}_{4}-z^{3} \mathrm{H}_{5}^{0} \\
a^{3} \mathrm{H}_{0}-z^{3} \mathrm{G}_{5}^{0} \\
c^{3} \mathrm{~F}_{2}-x^{3} \mathrm{G}_{3}^{0}\end{array}$ & $\begin{array}{l}1 \\
2\end{array}$ & & $\begin{array}{l}0 \\
0\end{array}$ & $\begin{array}{r}.80 \\
1.05\end{array}$ & $\begin{array}{r}(1.157) \\
(.670)\end{array}$ & $\begin{array}{r}1.23 \\
.80\end{array}$ \\
\hline $\begin{array}{l}3 \\
2 h \\
5 c\end{array}$ & $\begin{array}{l}3338.38 \\
3337.20 \\
3335.669\end{array}$ & $\begin{array}{l}29946.1 \\
29956.7 \\
29970.40\end{array}$ & $\begin{array}{c}a{ }^{1} \mathrm{I}_{6}-z{ }^{1} \mathrm{H}^{3} \\
a^{3} \mathrm{G}_{4}-z{ }^{3} \mathrm{~F}_{4}^{8} \\
a^{3} \mathrm{~F}_{4}-z{ }^{5} \mathrm{D}_{4}^{4}\end{array}$ & & & & & & \\
\hline $\begin{array}{c}10 c \\
8\end{array}$ & $\begin{array}{l}3335.244 \\
3334.82\end{array}$ & $\begin{array}{l}29974.22 \\
29978.0\end{array}$ & $\begin{array}{l}a^{3} \mathrm{~s}_{2}-z{ }^{3} \mathrm{D}_{1}^{3} \\
b^{3} \mathrm{P}_{2}-y^{3} \mathrm{D}_{3}^{3}\end{array}$ & 1 & .73 & 0 & 0 & .73 & 1.46 \\
\hline $\begin{array}{c}8 c \\
20 h \\
3 h \\
7, E \\
20 h\end{array}$ & $\begin{array}{l}3334.529 \\
3329.16 \\
3327.246 \\
3326.54 \\
3325.436\end{array}$ & $\begin{array}{l}29980.65 \\
30029.0 \\
30046.28 \\
30052.7 \\
30062.63\end{array}$ & $\begin{array}{l}a^{1} \mathrm{G}_{4}-z{ }^{5} \mathrm{P}_{3}^{3} \\
d^{3} \mathrm{~F}_{2}-x{ }^{1} \mathrm{~F}_{3}^{3} \\
b{ }^{3} \mathrm{H}_{5}-y^{3} \mathrm{H}_{4}^{3}\end{array}$ & & & & & & \\
\hline $\begin{array}{c}4 h \\
50 \\
30 \\
30 \\
3 h\end{array}$ & $\begin{array}{l}3325.21 \\
3324.661 \\
3324.555 \\
3323.900 \\
3323.22\end{array}$ & $\begin{array}{l}30064.7 \\
30069.63 \\
30070.60 \\
30076.52 \\
30082.7\end{array}$ & 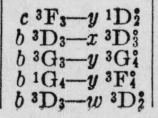 & $\begin{array}{l}3 \\
2 \\
3\end{array}$ & $\begin{array}{l}.358 \\
.155\end{array}$ & $\begin{array}{l}0 \\
.622\end{array}$ & $\begin{array}{l}\text { 1. } 300 \\
\text { 2. } 201\end{array}$ & $\begin{array}{r}(1.312) \\
(769 \\
(.950)\end{array}$ & $\begin{array}{l}1.288 \\
1.127 \\
1.105\end{array}$ \\
\hline
\end{tabular}


TABLE 1.-Second spectrum of columbium (Cb II)-Continued

\begin{tabular}{|c|c|c|c|c|c|c|c|c|c|}
\hline $\begin{array}{l}\text { Intensity } \\
\text { spark }\end{array}$ & $\lambda_{\text {sir }} \mathrm{A}$ & $\begin{array}{l}\text { Wav } \\
\text { No. } \\
\text { vac. } \\
\mathrm{cm}^{-1}\end{array}$ & $\begin{array}{l}\text { Term combi- } \\
\text { nation }\end{array}$ & $\begin{array}{c}\text { Zeeman } \\
\text { type }\end{array}$ & $\begin{array}{l}\text { Sepa- } \\
\text { ration }\end{array}$ & $\begin{array}{c}\text { Strong- } \\
\text { est } \\
p\end{array}$ & $\underset{n}{\text { Strongest }}$ & $g_{1}$ & $g_{2}$ \\
\hline 1 & 2 & 3 & 4 & 5 & 6 & 7 & 8 & 9 & 10 \\
\hline $\begin{array}{r}80 \\
2 \\
1 \\
100, \mathrm{~V} E \\
10 c, \mathrm{~V} E\end{array}$ & $\begin{array}{l}3320.808 \\
3320.56 \\
3320.124 \\
3319.590 \\
3319.22\end{array}$ & $\begin{array}{l}30104.52 \\
30106.8 \\
30110.72 \\
30115.58 \\
30118.9\end{array}$ & $\begin{array}{l}b^{8} \mathrm{~B}_{3}-y^{3} \mathrm{~F}_{2}^{0} \\
d^{3} \mathrm{~F}_{3}-x^{1} \mathrm{~F}_{3}^{3} \\
b^{3} \mathrm{P}_{2}-2{ }^{5} \mathrm{P}_{3}^{3} \\
a^{3} \mathrm{P}_{2}-z^{3} \mathrm{P}_{3}^{3} \\
a^{1} \mathrm{~F}_{3}-w^{3} \mathrm{~F}_{4}^{3}\end{array}$ & $7 b, 2$ & 0.183 & 0 & 0.786 & 1.444 & 1. 261 \\
\hline $\begin{array}{r}5 \\
1 \\
15 \\
2 \\
4\end{array}$ & $\begin{array}{l}3318.29 \\
3317.04 \\
3316.61 \\
3313.64 \\
3313.41\end{array}$ & $\begin{array}{l}30127.4 \\
30138.7 \\
30142.6 \\
30169.6 \\
30171.7\end{array}$ & $\begin{array}{l}c^{3} \mathrm{P}_{0}-x{ }^{3} \mathrm{P}_{1} \\
a^{3} \mathrm{D}_{2}-y \\
c^{3} \mathrm{D}_{2}-y \\
a^{3} \mathrm{P}_{1}-\mathrm{D}_{2} \\
a^{3} \mathrm{D}_{2}-y{ }^{3} \mathrm{D}_{1} \\
a^{3} \mathrm{D}_{1}-y \\
{ }^{3} \mathrm{D}_{2}\end{array}$ & 1 & .242 & 0 & .721 & 1.206 & .964 \\
\hline $\begin{array}{c}3 h \\
20 h \\
5 \\
70 \\
40\end{array}$ & $\begin{array}{l}3313.08 \\
3310.67 \\
3309.26 \\
3305.608 \\
3304.71\end{array}$ & $\begin{array}{l}30174.7 \\
30196.7 \\
30209.6 \\
30242.95 \\
30251.2\end{array}$ & $\begin{array}{l}b^{3}{ }^{3} \mathrm{D}_{3}-w^{3} \mathrm{D}_{3}^{3} \\
b^{3} \mathrm{~F}_{3}-y^{8} \mathrm{D}_{3}^{3} \\
b^{1} \mathrm{G}_{4}-y^{3} \mathrm{~F}_{3}^{3} \\
b^{3} \mathrm{G}_{3}-y^{3} \mathrm{~F}_{4}^{3} \\
b^{3} \mathrm{G}_{3}-z^{1} \mathrm{~F}_{3}^{3}\end{array}$ & $\begin{array}{l}2 \\
3\end{array}$ & .330 & ${ }^{0} .990$ & 1.45 & $\begin{array}{l}(1.19) \\
(.767)\end{array}$ & $\begin{array}{l}1.12 \\
1.097\end{array}$ \\
\hline $\begin{array}{r}50 \\
10 \\
3\end{array}$ & $\begin{array}{l}3303.323 \\
3302.619 \\
3301.96\end{array}$ & $\begin{array}{l}30263.86 \\
30270.32 \\
30276.4\end{array}$ & $\begin{array}{l}b^{3} \mathrm{~B}_{2}-2{ }^{3} \mathrm{P}_{2}^{0} \\
a^{3} \mathrm{H}_{1}-2{ }^{3} \mathrm{~F}_{3}^{0} \\
c^{3} \mathrm{P}_{2}-y \\
{ }^{1} \mathrm{P}^{3}\end{array}$ & $\begin{array}{l}3 \\
1\end{array}$ & $\begin{array}{l}.224 \\
.267\end{array}$ & $0^{.448}$ & 1. $261,1.482$ & $\begin{array}{l}1.482 \\
(.825)\end{array}$ & $\begin{array}{l}1.261 \\
1.092\end{array}$ \\
\hline 40 & $\begin{array}{l}3301.498 \\
3300.33\end{array}$ & $\begin{array}{l}30280.59 \\
30291.3\end{array}$ & & 2 & .216 & 0 & 2.050 & .970 & 1. 186 \\
\hline $\begin{array}{c}6 h \\
50, E \\
15 \\
20 \\
20 \\
150\end{array}$ & $\begin{array}{l}3300.33 \\
3299.57 \\
3297.673 \\
3297.055 \\
3295.506 \\
3294.367\end{array}$ & $\begin{array}{l}30291.3 \\
30298.3 \\
30315.71 \\
30321.40 \\
30335.65 \\
30346.14\end{array}$ & 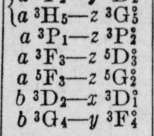 & $\begin{array}{l}2 \\
2 \\
3\end{array}$ & $\begin{array}{l}.899 \\
.34\end{array}$ & $\begin{array}{l}0 \\
0 \\
.32\end{array}$ & $\begin{array}{l}3.043 \\
1.07 \pm\end{array}$ & $\begin{array}{c}1.245 \\
(1.171) \\
(1.027)\end{array}$ & $\begin{array}{l}.346 \\
.83 \\
1.11\end{array}$ \\
\hline $\begin{array}{l}10 c \\
200, \mathrm{~V} E \\
30 \\
3 \\
10\end{array}$ & $\begin{array}{l}3292.365 \\
3292.020 \\
3291.055 \\
3290.55 \\
3289.551\end{array}$ & $\begin{array}{l}30364.58 \\
30367.77 \\
30376.68 \\
30381.3 \\
30390.56\end{array}$ & $\begin{array}{l}a^{3} \mathrm{~F}_{1}-z^{3} \mathrm{G}_{3}^{\circ} \\
b^{3} \mathrm{G}_{3}-y^{3} \mathrm{G}_{3}^{8} \\
a^{3} \mathrm{~F}_{1}-z^{3} \mathrm{G}_{3}^{\circ} \\
b^{3} \mathrm{D}_{2}-w^{3} \mathrm{D}_{1}^{\circ} \\
a^{3} \mathrm{~F}_{3}-z{ }^{3} \mathrm{D}_{4}^{0}\end{array}$ & $\begin{array}{l}2 \\
3 \\
2\end{array}$ & $\begin{array}{l}.46 \\
.254 \\
.433\end{array}$ & $\begin{array}{l}0 \\
0 \\
0\end{array}$ & $\begin{array}{r}2.59 \\
.760,1.014 \\
2.650\end{array}$ & $\begin{array}{c}1.21 \\
.765 \\
1.351 \\
(1.070)\end{array}$ & $\begin{array}{r}.75 \\
1.019 \\
.918 \\
1.477\end{array}$ \\
\hline 3 & 3287.75 & 30407.2 & 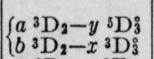 & & & & & & \\
\hline $\begin{array}{c}5 \\
40 \\
30 c \\
400, \mathrm{~V} E\end{array}$ & $\begin{array}{l}3286.81 \\
3286.340 \\
3285.70 \\
3283.463\end{array}$ & $\begin{array}{l}30415.9 \\
30420.25 \\
30426.2 \\
30446.91\end{array}$ & $\begin{array}{l}c^{3} \mathrm{P}_{2}-v^{3} \mathrm{~F}_{2} \\
b^{3} \mathrm{D}_{2}-w^{3} \mathrm{D}_{2}^{2} \\
b^{3} \mathrm{D}_{1}-x^{3} \mathrm{D}_{2}^{2} \\
b^{3} \mathrm{G}_{5}-y^{3} \mathrm{G}_{5}^{3}\end{array}$ & $\begin{array}{l}7 b \\
3\end{array}$ & & 0 & $\begin{array}{l}1.167 \\
1.175\end{array}$ & $\begin{array}{l}(1.171) \\
(1.19)\end{array}$ & $\begin{array}{l}1.163 \\
1.16\end{array}$ \\
\hline $\begin{array}{l}2 h \\
80 h \\
4 \\
20 c, V E \\
4\end{array}$ & $\begin{array}{l}3281.62 \\
3279.979 \\
3279.43 \\
3279.248 \\
3277.77\end{array}$ & $\begin{array}{l}30464.0 \\
30479.25 \\
30484.4 \\
30486.04 \\
30499.8\end{array}$ & $\begin{array}{l}c^{3} \mathrm{~F}_{1}-v^{3} \mathrm{~F}_{3}^{\circ} \\
b^{3} \mathrm{G}_{1}-y^{3} \mathrm{~F}_{3}^{8} \\
b^{3} \mathrm{~F}_{2}-y^{3} \mathrm{D}_{2}^{2} \\
a^{5} \mathrm{~F}_{3}-z{ }^{3} \mathrm{G}_{4}^{8} \\
a^{3} \mathrm{D}_{1}-y^{5} \mathrm{D}_{2}^{3}\end{array}$ & & & & & & 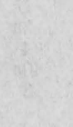 \\
\hline $\begin{array}{r}3 \\
6 \\
30\end{array}$ & $\begin{array}{l}3277.30 \\
3276.43 \\
3274.796\end{array}$ & $\begin{array}{l}30504.2 \\
30512.3 \\
30527.48\end{array}$ & $b^{3} \mathrm{D}_{2}-w^{3} \mathrm{D}_{3}^{2}$ & 2 & .144 & 0 & 14 & (1. 171) & 1. 315 \\
\hline $\begin{array}{l}15 \\
20\end{array}$ & $\begin{array}{l}3273.888 \\
3273.511\end{array}$ & $\begin{array}{l}30535.95 \\
30539.47\end{array}$ & $\begin{array}{l}a^{3} \mathrm{P}_{2}-z{ }^{6} \mathrm{D}_{2}^{2} \\
a^{3} \mathrm{P}_{1}-z{ }^{5} \mathrm{Fi}^{2}\end{array}$ & $\begin{array}{l}7 b \\
3\end{array}$ & & $\begin{array}{l}0 \\
1.38\end{array}$ & $\begin{array}{l}1.46 \\
1.48\end{array}$ & $\begin{array}{c}1.45 \\
(1.504)\end{array}$ & $\begin{array}{r}1.47 \\
.12\end{array}$ \\
\hline $\begin{array}{c}10 \\
100 \\
2 h\end{array}$ & $\begin{array}{l}3272.350 \\
3272.224 \\
3270.93\end{array}$ & $\begin{array}{l}30550.30 \\
30551.48 \\
30563.6\end{array}$ & $\begin{array}{l}b^{3} \mathrm{G}_{1}-y^{3} \mathrm{G}_{5}^{\circ} \\
a^{1} \mathrm{G}_{1}-z^{1} \mathrm{H}_{5}^{0} \\
a^{3} \mathrm{D}_{3}-y^{3} \mathrm{D}_{2}^{\circ}\end{array}$ & 1 & & 0 & .73 & $(1.083)$ & 1. 012 \\
\hline $\begin{array}{l}20 \\
30\end{array}$ & $\begin{array}{l}3269.125 \\
3267.684\end{array}$ & $\begin{array}{l}30580.44 \\
30593.92\end{array}$ & $\begin{array}{l}b^{3} \mathrm{P}_{1}-y^{3} \mathrm{P}_{0}^{0} \\
a^{3} \mathrm{H}_{4}-z^{3} \mathrm{G}_{5}^{0}\end{array}$ & $\begin{array}{l}7 a \\
2\end{array}$ & .403 & $\begin{array}{l}0 \\
0\end{array}$ & $\begin{array}{l}1.504 \\
2.854\end{array}$ & $\begin{array}{r}1.504 \\
.839\end{array}$ & $\begin{array}{l}0 / 0 \\
1.242\end{array}$ \\
\hline $\begin{array}{l}80 c \\
300, \mathrm{~V} E \\
6\end{array}$ & $\begin{array}{l}3266.11 \\
3263.365 \\
3262.56\end{array}$ & $\begin{array}{l}30608.7 \\
30634.41 \\
30642.0\end{array}$ & $a{ }^{1} \mathrm{H}_{0}-z{ }^{1} \mathrm{I}_{6}$ & $7 b, 2$ & & 0 & 1. 030 & $(.992)$ & 1.00 \\
\hline $\begin{array}{c}60 \\
350 c \text { V } E\end{array}$ & $\begin{array}{l}3261.702 \\
3260.564\end{array}$ & $\begin{array}{l}30650.03 \\
30660.73\end{array}$ & $\begin{array}{l}a^{1} \mathrm{~F}_{3}-y^{1} \mathrm{G}_{4} \\
b^{3} \mathrm{H}_{4}-z^{3} \mathrm{I}_{5}^{\circ}\end{array}$ & $\begin{array}{l}1 \\
2\end{array}$ & & $\begin{array}{l}0 \\
0\end{array}$ & $\begin{array}{l}.79 \\
.86\end{array}$ & $\begin{array}{r}(1.007) \\
(.880)\end{array}$ & $\begin{array}{l}.95 \\
.877\end{array}$ \\
\hline $\begin{array}{l}2 \\
1 h \\
5 h\end{array}$ & $\begin{array}{l}3256.75 \\
3255.80 \\
3255.27\end{array}$ & $\begin{array}{l}30696.6 \\
30705.6 \\
30710.6\end{array}$ & $\begin{array}{l}b^{3} \mathrm{P}_{0}^{0}-y^{3} \mathrm{P}_{1}^{0} \\
b^{3} \mathrm{H}_{0}-z^{3} \mathrm{I}_{5}^{\circ} \\
a^{3} \mathrm{~F}_{2}-z^{5} \mathrm{D}_{3}^{\circ}\end{array}$ & & & & & & \\
\hline$\stackrel{25}{200 r, \mathrm{~V} E}$ & $\begin{array}{l}3254.888 \\
3254.070\end{array}$ & $\begin{array}{l}30714.19 \\
30721.91\end{array}$ & $\begin{array}{c}b^{3} \mathrm{P}_{2}-y^{3} \mathrm{P}_{1}^{0} \\
a^{5} \mathrm{~F}_{2}-z^{5} \mathrm{G}_{2}^{0}\end{array}$ & $\begin{array}{l}1 \\
3\end{array}$ & 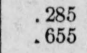 & $\begin{array}{l}0 \\
1.306\end{array}$ & $\begin{array}{c}1.18 \\
335, .995\end{array}$ & $\begin{array}{r}1.485 \\
.993\end{array}$ & $\begin{array}{r}1.745 \\
.338\end{array}$ \\
\hline $\begin{array}{l}1 \\
10 \\
50 \\
40 \\
80, \mathrm{~V} E\end{array}$ & $\begin{array}{l}3253.16 \\
3252.430 \\
3251.260 \\
3250.27 \\
3248.941\end{array}$ & $\begin{array}{l}30730.5 \\
30737.41 \\
30748.47 \\
30757.8 \\
30770.41\end{array}$ & $\begin{array}{l}b^{1} \mathrm{G}_{1}-x^{3} \mathrm{~F}_{3}^{2} \\
a^{1} \mathrm{~F}_{3}-x^{3} \mathrm{D}_{2}^{2} \\
b^{3} \mathrm{P}_{1}-y^{3} \mathrm{P}_{1} \\
b^{3} \mathrm{D}_{3}-x^{3} \mathrm{P}_{2}^{0} \\
a^{3} \mathrm{P}_{1}-z^{3} \mathrm{~F}_{2}\end{array}$ & $\begin{array}{l}1 \\
1 \\
1\end{array}$ & .458 & $\begin{array}{l}0 \\
0 \\
0\end{array}$ & $\begin{array}{l}1.23 \\
.565\end{array}$ & $\begin{array}{c}(1.312) \\
1.481\end{array}$ & $\begin{array}{l}1.35 \\
1.023\end{array}$ \\
\hline
\end{tabular}


TABLE 1.-Second spectrum of columbium (Cb II)-Continued

\begin{tabular}{|c|c|c|c|c|c|c|c|c|c|}
\hline $\begin{array}{l}\text { Intensity } \\
\text { spark }\end{array}$ & $\lambda_{\text {sir }} \mathrm{A}$ & $\begin{array}{l}\text { Wave } \\
\text { No. } \\
\text { vac. } \\
\mathrm{cm}^{-1}\end{array}$ & $\begin{array}{l}\text { Term combi- } \\
\text { nation }\end{array}$ & $\begin{array}{c}\text { Zeeman } \\
\text { type }\end{array}$ & $\begin{array}{l}\text { Sepa- } \\
\text { ration }\end{array}$ & $\begin{array}{c}\text { Strong- } \\
\text { est } \\
p\end{array}$ & $\underset{n}{\text { Strongest }}$ & $g_{1}$ & $g_{2}$ \\
\hline 1 & 2 & 3 & 4 & 5 & 6 & 7 & 8 & 8 & 10 \\
\hline $\begin{array}{c}150 c, V E \\
6 \\
5 \\
30 \\
6\end{array}$ & $\begin{array}{l}3247.478 \\
3246.69 \\
3245.07 \\
3244.515 \\
3243.83\end{array}$ & $\begin{array}{l}30784.28 \\
30791.7 \\
30807.1 \\
30812.39 \\
30818.9\end{array}$ & $\begin{array}{l}a^{3} \mathrm{~F}_{3}-z^{3} \mathrm{G}_{3}^{3} \\
b^{3} \mathrm{G}_{3}-x^{3} \mathrm{~F}_{2}^{0} \\
c^{3} \mathrm{~F}_{4}-v^{3} \mathrm{~F}_{4}^{0} \\
b^{3} \mathrm{~F}_{2}-y^{3} \mathrm{D}_{2}^{3} \\
c^{3} \mathrm{~F}_{3}-v^{3} \mathrm{~F}_{3}^{\circ}\end{array}$ & 3 & 0.494 & 0.996 & $0.850,1.338$ & 0.846 & 1. 340 \\
\hline $\begin{array}{c}40 \\
8 \\
40 \\
3 \\
2 h\end{array}$ & $\begin{array}{l}3242.532 \\
3242.423 \\
3241.818 \\
3241.29 \\
3239.23\end{array}$ & $\begin{array}{l}30831.23 \\
30832.27 \\
30838.02 \\
30843.0 \\
30862.7\end{array}$ & $\begin{array}{l}a{ }^{1} \mathrm{G}_{4}-y^{3} \mathrm{G}_{4}^{\circ} \\
a^{3} \mathrm{D}_{3}-y^{3} \mathrm{D}^{3} \\
b^{3} \mathrm{H}_{5}-z^{3} \mathrm{I}_{5}^{\circ} \\
b^{3} \mathrm{~F}_{2}-y^{3} \mathrm{D}^{3} \mathrm{i} \\
b^{3} \mathrm{D}_{3}-x^{3} \mathrm{~F}_{4}^{8}\end{array}$ & 3 & .188 & $\begin{array}{l}.16 \\
.94\end{array}$ & $1.10 \pm$ & $\begin{array}{c}1.08 \\
(1.052)\end{array}$ & $\begin{array}{r}1.12 \\
.864\end{array}$ \\
\hline $\begin{array}{l}80 c, \mathrm{~V} E \\
60 \\
300 r, \mathrm{~V} E \\
2 h \\
5 h\end{array}$ & $\begin{array}{l}3238.020 \\
3237.690 \\
3236.403 \\
3235.78 \\
3232.79\end{array}$ & $\begin{array}{l}30874.19 \\
30877.34 \\
30889.62 \\
30895.6 \\
30924.1\end{array}$ & $\begin{array}{l}a{ }^{5} \mathrm{P}_{2}-z^{1} \mathrm{D}_{2}^{0} \\
b^{3} \mathrm{G}_{3}-y^{3} \mathrm{~F}_{3}^{0} \\
a^{5} \mathrm{~F}_{3}-z^{3} \mathrm{G}_{3}^{0} \\
b^{3} \mathrm{D}_{3}-x^{3} \mathrm{G}_{3}^{0}\end{array}$ & $\begin{array}{l}3 \\
3\end{array}$ & $\begin{array}{l}.36 \\
.328\end{array}$ & $\begin{array}{l}1.10 \\
.984\end{array}$ & $.921,1.246$ & $\begin{array}{l}(.767) \\
1.248\end{array}$ & $\begin{array}{c}1.13 \\
.920\end{array}$ \\
\hline $\begin{array}{c}40 \\
100, \mathrm{~V} E \\
6 \\
6 \\
6\end{array}$ & $\begin{array}{l}3230.243 \\
3229.567 \\
3228.953 \\
3228.47 \\
3227.69\end{array}$ & $\begin{array}{l}30948.52 \\
30955.00 \\
30960.88 \\
30965.5 \\
30973.0\end{array}$ & $\begin{array}{l}b^{3}{ }^{3} \mathrm{~F}_{3}-y^{3} \mathrm{D}_{3}^{0} \\
a{ }^{3} \mathrm{P}_{2}-z^{5} \mathrm{D}_{3}^{3} \\
b^{3} \mathrm{H}_{5}-z^{1} \mathrm{G}_{4}^{\circ} \\
b^{3} \mathrm{~F}_{4}-y^{3} \mathrm{D}_{3}^{\circ}\end{array}$ & $\begin{array}{l}3 \\
7 b \\
1\end{array}$ & .107 & $\begin{array}{l}.34 \\
0\end{array}$ & $\begin{array}{r}1.033,1.138 \\
1.465 \\
.956\end{array}$ & $\begin{array}{c}1.140 \\
1.465 \\
(1.052)\end{array}$ & $\begin{array}{l}1.033 \\
1.465 \\
1.076\end{array}$ \\
\hline $\begin{array}{l}500 c R, \mathrm{~V} E \\
100, \mathrm{~V} E \\
50 \\
8 c \\
4 h\end{array}$ & $\begin{array}{l}3225.478 . \\
3223.332 \\
3222.065 \\
3221.655 \\
3220.48\end{array}$ & $\begin{array}{l}\text { 30994. } 24 \\
31014.87 \\
31027.07 \\
31031.02 \\
31042.3\end{array}$ & $\begin{array}{l}a{ }^{3} \mathrm{~F}_{1}-z^{5} \mathrm{G}_{2}^{\circ} \\
a^{3} \mathrm{~B}_{1}-z^{3} \mathrm{G}_{4}^{0} \\
a^{1} \mathrm{D}_{2}-y^{3} \mathrm{D}_{2}^{\circ} \\
b^{3} \mathrm{H}_{4}-y{ }^{3} \mathrm{H}^{3} \\
c^{3} \mathrm{P}_{2}-y^{3}{ }^{3} \mathrm{~S}_{1}^{\circ}\end{array}$ & $\begin{array}{l}2 \\
3 \\
3\end{array}$ & $\begin{array}{l}.36 \\
.156 \\
.220\end{array}$ & $\begin{array}{l}0 \\
.627 \\
.432\end{array}$ & $\begin{array}{r}.72 \\
\text { 1. } 076.1 .234 \\
1.002 .1 .226\end{array}$ & $\begin{array}{l}0.000 \\
1.233 \\
1.004\end{array}$ & $\begin{array}{l}.36 \\
1.077 \\
1.224\end{array}$ \\
\hline $\begin{array}{c}2 \\
3 h \\
60 c \\
2 \\
6\end{array}$ & $\begin{array}{l}3219.55 \\
3218.98 \\
3217.00 \\
3216.47 \\
3216.193\end{array}$ & $\begin{array}{l}31051.3 \\
31056.8 \\
31075.9 \\
31081.0 \\
31083.71\end{array}$ & $\begin{array}{l}a^{3} \mathrm{H}_{4}-z^{3} \mathrm{~F}_{4}^{\circ} \\
a^{3} \mathrm{P}_{1}-z{ }^{1} \mathrm{D}_{2}^{\circ} \\
b^{3} \mathrm{H}_{6}-y^{3} \mathrm{H}_{5}^{\circ} \\
b^{3} \mathrm{~F}_{2}-y{ }^{3} \mathrm{D}_{3}^{\circ} \\
a^{3} \mathrm{~F}_{3}-z^{3} \mathrm{~F}_{2}^{0}\end{array}$ & 2 & .38 & 0 & 1.84 & 1.08 & .70 \\
\hline $\begin{array}{l}300 c r, V E \\
5 h \\
3 \\
10 \\
20\end{array}$ & $\begin{array}{l}3215.595 \\
3215.00 \\
3213.91 \\
3212.14 \\
3211.814\end{array}$ & $\begin{array}{l}31089.49 \\
31095.2 \\
31105.8 \\
31122.9 \\
31126.09\end{array}$ & $\begin{array}{l}a^{3} F_{4}-z^{3} \mathrm{G}_{4} \\
b^{3} \mathrm{D}_{2}-x^{3} \mathrm{P}_{2}^{0} \\
a^{3} \mathrm{P}_{1}-z^{3} \mathrm{D}_{0}^{0} \\
b^{3} \mathrm{D}_{2}-x x^{3} \mathrm{Pi}^{3} \\
b^{1} \mathrm{G}_{4}-y^{3} \mathrm{H}_{4}^{8}\end{array}$ & $\begin{array}{l}7 b, 1 \\
7 b, 3\end{array}$ & .199 & .792 & $\begin{array}{r}1.151 \\
.954\end{array}$ & $\begin{array}{r}(1.171) \\
(.950)\end{array}$ & $\begin{array}{r}1.191 \\
.958\end{array}$ \\
\hline $\begin{array}{c}2 h \\
3 h \\
4 \\
40 c \\
3\end{array}$ & $\begin{array}{l}3211.63 \\
3211.40 \\
3210.20 \\
3208.585 \\
3208.409\end{array}$ & $\begin{array}{l}31127.9 \\
31130.1 \\
31141.7 \\
31157.41 \\
31159.12\end{array}$ & $\begin{array}{l}a^{1} \mathrm{G}_{4}-y{ }^{3} \mathrm{G}_{3}^{\circ} \\
c^{3} \mathrm{~F}_{2}-y{ }^{1} \mathrm{P}_{1}^{0} \\
b^{3} \mathrm{P}_{2}-z{ }^{1} \mathrm{~F}_{3}^{\circ} \\
b^{3} \mathrm{H}_{4}-w^{3} \mathrm{~F}^{\circ} \\
a^{3} \mathrm{D}_{2}-y^{3} \mathrm{D}_{3}^{\circ}\end{array}$ & $\begin{array}{l}1 \\
3\end{array}$ & $\begin{array}{l}.383 \\
.23\end{array}$ & ${ }^{0} .91$ & $.87,1.10$ & $\begin{array}{c}1.479 \\
.87\end{array}$ & $\begin{array}{l}\text { 1. } 096 \\
1.10\end{array}$ \\
\hline $\begin{array}{l}8 \\
2 \\
20 \\
300 \mathrm{rs}, \mathrm{V} E \\
100 \mathrm{c}\end{array}$ & $\begin{array}{l}3208.10 \\
3207.56 \\
3207.341 \\
3206.350 \\
3204.973\end{array}$ & $\begin{array}{l}31162.1 \\
31167.4 \\
31169.50 \\
31179.14 \\
31192.53\end{array}$ & $\begin{array}{l}c^{3} \mathrm{~F}_{3}-v^{3} \mathrm{~F}_{4}^{0} \\
a^{3} \mathrm{D}_{1}-z z^{3} \mathrm{P}_{0}^{0} \\
a^{3} \mathrm{P}_{0}-z{ }^{3} \mathrm{~F}_{1}^{0} \\
a^{3} \mathrm{~F}_{2}-z z^{3} \mathrm{G}_{3}^{\circ} \\
b^{3} \mathrm{H}_{0}-z{ }^{3} \mathrm{I}_{6}^{0}\end{array}$ & $\begin{array}{l}7 a \\
2 \\
3\end{array}$ & .11 & $\begin{array}{l}0 \\
0 \\
.66\end{array}$ & $\begin{array}{l}.135 \\
.87 \\
1.10 \pm\end{array}$ & $\begin{array}{l}0 / 0 \\
(.712) \\
1.155\end{array}$ & $\begin{array}{r}.135 \\
.765 \\
1.045\end{array}$ \\
\hline $\begin{array}{c}150 \\
10 h \\
3 \\
1 \\
1\end{array}$ & $\begin{array}{l}3203.357 \\
3203.148 \\
3201.66 \\
3200.78 \\
3200.69\end{array}$ & $\begin{array}{l}31208.26 \\
31210.30 \\
31224.8 \\
31233.4 \\
31234.3\end{array}$ & $\begin{array}{l}b^{{ }^{3} \mathrm{H}_{5}-y}{ }^{3} \mathrm{H}_{5}^{\circ} \\
b^{3} \mathrm{~B}_{2}-x{ }^{3} \mathrm{G}_{3}^{\circ} \\
b^{3} \mathrm{~B}_{3}-z{ }^{3} \mathrm{P}_{2}^{\circ}\end{array}$ & 3 & & .06 & $1.04 \pm$ & 1.05 & 1.03 \\
\hline $\begin{array}{c}3 h \\
4 h \\
20 \\
20 h \\
3\end{array}$ & $\begin{array}{l}3200.24 \\
3199.88 \\
3198.227 \\
3197.28 \\
3196.17\end{array}$ & $\begin{array}{l}31238.7 \\
31242.2 \\
31258.32 \\
31267.6 \\
31278.4\end{array}$ & $\begin{array}{l}c^{3} \mathrm{~F}_{4}-527_{3}^{\circ} \\
b^{3} \mathrm{P}_{2}-y^{3} \mathrm{G}_{3}^{\circ} \\
c^{3} \mathrm{~F}_{2}-v^{3} \mathrm{~F}_{3}^{\circ}\end{array}$ & 1 & .459 & 0 & .101 & 1.478 & 1. 019 \\
\hline $\begin{array}{c}2 \\
5 \\
700 R, \mathrm{~V} E \\
30 \mathrm{c} \\
3\end{array}$ & $\begin{array}{l}\text { 3195. } 96 \\
3195.216 \\
3194.983 \\
3194.27 \\
3193.47\end{array}$ & $\begin{array}{l}31280.5 \\
31287.77 \\
31290.06 \\
31297.0 \\
31304.9\end{array}$ & $\begin{array}{l}b^{3}{ }^{3} \mathrm{~F}_{3}-y^{5} \mathrm{D}_{4}^{0} \\
a^{3} \mathrm{P}_{1}-z^{5} \mathrm{D}_{1}^{0} \\
a^{5} \mathrm{~F}_{2}-z^{3} \mathrm{G}_{3}^{0} \\
a^{5} \mathrm{P}_{2}-y^{3} \mathrm{D}_{1}^{0} \\
b^{3} \mathrm{~F}_{4}-y^{5} \mathrm{D}_{4}^{0}\end{array}$ & $\begin{array}{l}1 \\
2\end{array}$ & $\begin{array}{l}.083 \\
.435\end{array}$ & $\begin{array}{l}0 \\
0\end{array}$ & $\begin{array}{r}.742 \\
2.258\end{array}$ & $\begin{array}{r}.991 \\
1.823\end{array}$ & $\begin{array}{r}.908 \\
1.388\end{array}$ \\
\hline $\begin{array}{c}2 \\
3 \\
250, \mathrm{~V} \\
200 \mathrm{c} \\
6\end{array}$ & $\begin{array}{l}3192.39 \\
3192.16 \\
3191.427 \\
3191.096 \\
3190.44\end{array}$ & $\begin{array}{l}31315.5 \\
31317.7 \\
31325.45 \\
31328.17 \\
31334.6\end{array}$ & $\begin{array}{l}c^{3} \mathrm{~F}_{4}-y{ }^{1} \mathrm{H}_{5} \\
a^{1}{ }^{1} \mathrm{~S}_{0}-z{ }^{1} \mathrm{P}^{0} \\
b^{3} \mathrm{H}_{5}-z \mathrm{I}_{6}^{8} \\
a^{5} \mathrm{H}_{5}-z^{5} \mathrm{G}_{5}^{\circ} \\
b^{3} \mathrm{H}_{5}-w^{3} \mathrm{~F}_{4}^{\circ}\end{array}$ & $\begin{array}{l}7 b, 2 \\
3\end{array}$ & .16 & ${ }^{0} .80$ & $\begin{array}{l}1.057 \\
1.31 \pm\end{array}$ & $\begin{array}{c}(1.052) \\
1.39\end{array}$ & $\begin{array}{l}1.062 \\
1.23\end{array}$ \\
\hline
\end{tabular}


TABLE 1.-Second spectrum of columbium (Cb II)-Continued

\begin{tabular}{|c|c|c|c|c|c|c|c|c|c|}
\hline $\begin{array}{l}\text { Intensity } \\
\text { spark }\end{array}$ & $\lambda_{\text {sir }} \mathrm{A}$ & $\begin{array}{l}\text { Wave } \\
\text { No. } \\
\text { vac. } \\
\mathrm{cm}^{-1}\end{array}$ & $\begin{array}{l}\text { Term combi- } \\
\text { nation }\end{array}$ & $\begin{array}{c}\text { Zeeman } \\
\text { type }\end{array}$ & $\begin{array}{l}\text { Sepa- } \\
\text { ration }\end{array}$ & $\begin{array}{l}\text { Strong- } \\
\text { est } \\
p\end{array}$ & $\underset{n}{\text { Strongest }}$ & $g_{1}$ & $g_{2}$ \\
\hline 1 & 2 & 3 & 4 & 5 & 6 & 7 & 8 & 9 & 10 \\
\hline $\begin{array}{c}180 c, \mathrm{~V} E \\
4 \\
4 \\
2 \\
2\end{array}$ & $\begin{array}{l}\text { 3189. } 288 \\
3188.85 \\
3188.36 \\
3185.23 \\
3184.94\end{array}$ & $\begin{array}{l}31345.93 \\
31350.2 \\
31355.0 \\
31385.9 \\
31388.7\end{array}$ & $\begin{array}{l}b^{3}{ }^{3} \mathrm{H}_{6}-y^{3} \mathrm{H}_{6}^{\circ} \\
a^{1} \mathrm{P}_{1}-y^{1} \mathrm{P}^{\circ} \\
a^{1} \mathrm{P}_{2}-y^{5} \mathrm{D}_{2}^{8} \\
a^{1} \mathrm{D}_{2}-y^{3} \mathrm{D}_{1} \\
b^{1} \mathrm{D}_{2}-x \\
{ }^{1} \mathrm{D}_{2}^{8}\end{array}$ & $\begin{array}{l}3 \\
7 b \\
3\end{array}$ & 0.333 & $\begin{array}{l}0.18 \\
0 \\
.666\end{array}$ & $\begin{array}{l}1.146 \pm \\
1.11\end{array}$ & $\begin{array}{l}1.16 \\
1.11 \\
(1.003)\end{array}$ & $\begin{array}{l}\text { 1. } 13 \\
\text { 1. } 11 \\
\text { 1. } 336\end{array}$ \\
\hline $\begin{array}{c}150 \\
40 \\
400, \mathrm{~V} E \\
8 \\
6\end{array}$ & $\begin{array}{l}3184.229 \\
3181.403 \\
3180.290 \\
3179.242 \\
3177.766\end{array}$ & $\begin{array}{l}31395.72 \\
31423.61 \\
31434.61 \\
31444.97 \\
31459.58\end{array}$ & $\begin{array}{l}b^{3} \mathrm{G}_{4}-y^{3} \mathrm{H}_{4}^{0} \\
a^{3} \mathrm{P}_{2}-z 3^{3} \mathrm{G}_{3}^{3} \\
a^{3} \mathrm{~F}_{3}-z^{3} \mathrm{G}_{4}^{4} \\
a^{3} \mathrm{D}_{2}-z^{3} \mathrm{P}_{2} \\
a^{3} \mathrm{~F}_{4}-z^{3} \mathrm{~F}_{3}^{2}\end{array}$ & $\begin{array}{l}3 \\
1 \\
7 b, 2 \\
3 \\
2\end{array}$ & $\begin{array}{l}.69 \\
.25 \\
.140\end{array}$ & $\begin{array}{l}.24 \\
0 \\
0 \\
0.50\end{array}$ & $\begin{array}{r}.99 \pm \\
.63 \\
1.085 \\
1.01,1.26 \\
1.650\end{array}$ & $\begin{array}{l}1.02 \\
1.44 \\
(1.070) \\
1.01 \\
1.230\end{array}$ & $\begin{array}{l}.96 \\
.75 \\
1.074 \\
1.26 \\
1.090\end{array}$ \\
\hline $\begin{array}{l}2 \\
4\end{array}$ & $\begin{array}{l}3177.41 \\
3176.14\end{array}$ & $\begin{array}{l}31463.1 \\
31475.7\end{array}$ & $\begin{array}{l}a^{3} \mathrm{a}_{3}-z{ }^{1} \mathrm{D}_{2}^{2} \\
b^{3} \mathrm{D}_{1}-x x^{3} \mathrm{P}_{0}^{2}\end{array}$ & & & & & & \\
\hline $150, \mathrm{~V} E$ & 3175.86 & 31478.5 & $\left\{\begin{array}{l}a^{3} \mathrm{r}_{2}-z^{3} \mathrm{~F}_{2}^{0} \\
b^{3} \mathrm{H}_{5}-y^{3} \mathrm{H}^{0}\end{array}\right.$ & & & & & & \\
\hline${ }_{1}^{50,} \mathrm{~V} E$ & $\begin{array}{l}3175.76 \\
3174.73\end{array}$ & $\begin{array}{l}31479.4 \\
31489.7\end{array}$ & $\begin{array}{l}a^{5} \mathrm{P}_{1}-y^{6} \mathrm{D}^{1} \\
a^{1} \mathrm{P}_{1}-v \\
{ }^{3} \mathrm{~F}_{2}^{0}\end{array}$ & 3 & 1. 105 & 1. 10 & 1. $37,2.48$ & 2. 48 & 1. 37 \\
\hline $\begin{array}{c}6 h \\
150 \\
1 \\
3 h \\
1\end{array}$ & $\begin{array}{l}3174.44 \\
3173.205 \\
3167.96 \\
3167.32 \\
3165.24\end{array}$ & $\begin{array}{l}31492.5 \\
31504.79 \\
31557.0 \\
31563.3 \\
31584.1\end{array}$ & 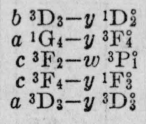 & 3 & & .110 & $1.094 \pm$ & 1.080 & 1. 108 \\
\hline $\begin{array}{c}1000 R, \mathrm{~V} E \\
10 \\
4 \\
4 c \\
15\end{array}$ & $\begin{array}{l}3163.403 \\
3163.149 \\
3161.56 \\
3161.244 \\
3159.855\end{array}$ & $\begin{array}{l}31602.41 \\
31604.95 \\
31620.8 \\
31624.00 \\
31637.89\end{array}$ & $\begin{array}{l}a^{5} \mathrm{~S}_{3}-z^{5} \mathrm{G}_{4}^{\circ} \\
a^{3} \mathrm{~S}_{1}-z^{5} \mathrm{D}_{2} \\
d^{3} \mathrm{D}_{0}-u^{3} \mathrm{D}_{1}^{0} \\
a^{1} \mathrm{D}_{2}-y^{5} \mathrm{D}^{3} \\
a^{1}{ }^{1} \mathrm{G}_{4}-y^{3} \mathrm{~F}_{3}^{3}\end{array}$ & $\begin{array}{l}1 \\
7 b, 1 \\
7 a \\
1\end{array}$ & .107 & $\begin{array}{l}0 \\
0 \\
0 \\
0\end{array}$ & $\begin{array}{l}.834 \\
1.46 \\
.645 \\
.99\end{array}$ & $\begin{array}{c}1.262 \\
1.49 \\
0 / 0 \\
(1.083)\end{array}$ & $\begin{array}{r}1.155 \\
1.48 \\
.645 \\
1.113\end{array}$ \\
\hline $\begin{array}{c}10 \\
3 \\
30 \\
150 c \\
10\end{array}$ & $\begin{array}{l}3158.104 \\
3156.00 \\
3155.599 \\
3154.820 \\
3153.851\end{array}$ & $\begin{array}{l}31655.43 \\
31676.5 \\
31680.56 \\
31688.38 \\
31698.12\end{array}$ & $\begin{array}{l}b^{3} \mathrm{D}_{1}-x x^{3} \mathrm{P}_{1}^{0} \\
b^{3} \mathrm{~F}_{4}-z 1^{1} \mathrm{H}^{\circ} \\
b^{3} \mathrm{P}_{0}-z^{3} \mathrm{~S}_{1}^{\circ} \\
b^{3} \mathrm{H}_{4}-y^{1} \mathrm{G}_{4} \\
b^{3} \mathrm{P}_{2}-z^{3} \mathrm{~S}_{1}\end{array}$ & $\begin{array}{l}3 \\
7 a \\
3 \\
1\end{array}$ & .525 & $\begin{array}{l}.526 \\
0.23 \\
0^{.23}\end{array}$ & $\begin{array}{r}.675,1.198 \\
1.824 \\
.92 \\
1.13\end{array}$ & $\begin{array}{c}.675 \\
0 / 0 \\
.89 \\
1.47\end{array}$ & $\begin{array}{l}1.200 \\
1.824 \\
.95 \\
1.81\end{array}$ \\
\hline $\begin{array}{c}4 h \\
70 \\
200 \\
50 \\
30\end{array}$ & $\begin{array}{l}3153.38 \\
3152.785 \\
3152.160 \\
3150.409 \\
3146.92\end{array}$ & $\begin{array}{l}31702.9 \\
31708.84 \\
31715.12 \\
31732.75 \\
31767.9\end{array}$ & 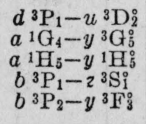 & $\begin{array}{l}2 \\
3 \\
3 \\
1 \\
1\end{array}$ & $\begin{array}{l}.319 \\
.355\end{array}$ & $\begin{array}{l}0 \\
.15 \\
0.319\end{array}$ & $\begin{array}{c}1.51 \\
1.003 \pm \\
1.508,1.827 \\
.415\end{array}$ & $\begin{array}{r}(1.083) \\
(.992) \\
1.508 \\
1.480\end{array}$ & $\begin{array}{l}\text { 1. } 17 \\
\text { 1. } 014 \\
1.827 \\
1.125\end{array}$ \\
\hline $\begin{array}{c}500 r s, \text { V } E \\
60 \\
4 \\
3 \\
100 c\end{array}$ & $\begin{array}{l}3145.405 \\
3144.353 \\
3143.41 \\
3143.17 \\
3142.26\end{array}$ & $\begin{array}{l}31783.23 \\
31793.87 \\
31803.4 \\
31805.8 \\
31815.0\end{array}$ & $\begin{array}{l}a^{3} \mathrm{~F}_{4}-z^{3} \mathrm{G}_{3}^{8} \\
b^{3} \mathrm{G}_{3}-y^{3} \mathrm{H}_{4}^{4} \\
a^{1} \mathrm{D}_{2}-z^{3} \mathrm{P}_{1}^{1} \\
a^{3} \mathrm{D}_{1}-z^{3} \mathrm{P}_{2}^{4}\end{array}$ & $\frac{7 b}{2}$ & .194 & $\begin{array}{l}0 \\
0\end{array}$ & $\begin{array}{l}\text { 1. } 23 \\
1.554\end{array}$ & $\begin{array}{c}(1.230) \\
.778\end{array}$ & $\begin{array}{r}1.230 \\
.972\end{array}$ \\
\hline $\begin{array}{c}4 \\
100 \\
2 h \\
3 \\
30\end{array}$ & $\begin{array}{l}3140.97 \\
3140.506 \\
3137.98 \\
3137.27 \\
3135.920\end{array}$ & $\begin{array}{l}31828.1 \\
31832.81 \\
31858.4 \\
31865.6 \\
31879.36\end{array}$ & $\begin{array}{l}d^{3} \mathrm{P}_{1}-u^{3} \mathrm{D}_{1}^{0} \\
b^{3} \mathrm{~F}_{2}-y^{3} \mathrm{D}_{3}^{8} \\
b^{1} \mathrm{G}_{4}-w^{3} \mathrm{~F}_{3}^{8} \\
b^{3} \mathrm{H}_{3}-y^{1} \mathrm{G}_{4}^{3} \\
a^{3} \mathrm{~F}_{3}-z^{3} \mathrm{~F}_{3}^{8}\end{array}$ & 2 & .181 & $\begin{array}{l}0 \\
.055\end{array}$ & 1.082 & 1. 073 & $\begin{array}{l}1.038 \\
1.091\end{array}$ \\
\hline $\begin{array}{r}25 \\
40 \\
60 \\
4 \\
10\end{array}$ & $\begin{array}{l}3135.409 \\
3134.342 \\
3132.767 \\
3132.140 \\
3132.015\end{array}$ & $\begin{array}{l}31884.55 \\
3189.41 \\
31911.44 \\
31917.83 \\
31919.11\end{array}$ & $\begin{array}{l}b^{3} \mathrm{P}_{2}-y^{3} \mathrm{P}_{2}^{0} \\
a^{3} \mathrm{P}_{2}-y^{3} \mathrm{P}_{1}^{0} \\
b^{1} \mathrm{G}_{4}-z^{3} \mathrm{I}_{5}^{\circ} \\
a^{3} \mathrm{P}_{0}-z^{3} \mathrm{D}_{1} \\
b^{3} \mathrm{P}_{1}-y^{3} \mathrm{P}_{2}^{\circ}\end{array}$ & $\begin{array}{c}3 \\
1 \\
1 \\
7 a \\
7 b, 2\end{array}$ & .770 & $\begin{array}{l}.045 \\
0 \\
0 \\
0 \\
0\end{array}$ & $\begin{array}{l}1.496 \\
.239 \\
.53 \\
1.45 \\
1.52\end{array}$ & $\begin{array}{c}1.485 \\
1.009 \\
(.950) \\
0 / 0 \\
(1.504)\end{array}$ & $\begin{array}{l}1.507 \\
1.779 \\
.87 \\
1.45 \\
1.51\end{array}$ \\
\hline $\begin{array}{c}1500 \mathrm{c} R, \mathrm{~V} E \\
60 \\
10 \mathrm{c} \\
20 \\
500, \mathrm{~V} E\end{array}$ & $\begin{array}{l}3130.780 \\
3129.65 \\
3128.92 \\
3128.372 \\
3127.526\end{array}$ & $\begin{array}{l}31931.70 \\
31943.2 \\
31950.7 \\
31956.27 \\
31964.92\end{array}$ & $\begin{array}{l}a{ }^{5} \mathrm{~F}_{4}-z^{5} \mathrm{G}_{5}^{\circ} \\
a^{5} \mathrm{P}_{1}-y^{5} \mathrm{D}_{0}^{0} \\
a^{5} \mathrm{P}_{8}-y^{3} \mathrm{D}_{2}^{8} \\
b^{3} \mathrm{~F}_{4}-y^{3} \mathrm{G}_{4}^{8} \\
b^{3} \mathrm{H}_{8}-z^{3} \mathrm{I}_{7}^{\circ}\end{array}$ & $\begin{array}{l}1 \\
7 a\end{array}$ & & $\begin{array}{l}0 \\
0 \\
.109\end{array}$ & $\begin{array}{l}1.13 \pm \\
1.124\end{array}$ & $\begin{array}{l}(1.350) \\
2.470\end{array}$ & $\begin{array}{l}1.25 \\
0 / 0\end{array}$ \\
\hline $\begin{array}{c}10 \\
6 c \\
10 c \\
20 \\
15\end{array}$ & $\begin{array}{l}3125.892 \\
3119.48 \\
3116.57 \\
3115.533 \\
3115.16\end{array}$ & $\begin{array}{l}31981.62 \\
32047.4 \\
32077.3 \\
32087.96 \\
32091.8\end{array}$ & $\begin{array}{c}a{ }^{1} \mathrm{~F}_{3}-x^{3} \mathrm{G}_{3}^{8} \\
a^{2} \mathrm{D}_{1}-y^{3} \mathrm{P}_{6}^{8}\end{array}$ & $7 a$ & & 0 & .506 & .506 & $0 / 0$ \\
\hline
\end{tabular}




\section{Journal of Research of the National Bureau of Standards}

TABLE 1.-Second spectrum of columbium (Cb II)-Continued

\begin{tabular}{|c|c|c|c|c|c|c|c|c|c|}
\hline $\begin{array}{l}\text { Intensity } \\
\text { spark }\end{array}$ & $\lambda_{\text {sir }} \mathrm{A}$ & $\begin{array}{l}\text { Wave } \\
\text { No. } \\
\text { vac. } \\
\mathrm{cm}^{-1}\end{array}$ & $\begin{array}{l}\text { Term combi- } \\
\text { nation }\end{array}$ & $\begin{array}{c}\text { Zeeman } \\
\text { type }\end{array}$ & $\begin{array}{l}\text { Sepa- } \\
\text { ration }\end{array}$ & $\begin{array}{c}\text { Strong- } \\
\text { est } \\
p\end{array}$ & $\underset{n}{\text { Strongest }}$ & $g_{1}$ & $g_{2}$ \\
\hline 1 & 2 & 3 & 4 & 5 & 6 & 7 & 8 & 9 & 10 \\
\hline $\begin{array}{r}30 \\
5 \\
20 \\
80 \\
10\end{array}$ & $\begin{array}{l}3113.17 \\
3111.63 \\
3110.800 \\
3106.980 \\
3106.520\end{array}$ & $\begin{array}{l}32112.3 \\
32128.2 \\
32136.78 \\
32176.29 \\
32181.05\end{array}$ & $\begin{array}{c}b^{3} \mathrm{~F}_{3}-z^{1} \mathrm{~F}_{3}^{0} \\
b^{3} \mathrm{G}_{4}-w^{3} \mathrm{~F}_{3}^{3} \\
b^{3} \mathrm{~F}_{1}-z^{1} \mathrm{~F}_{3}^{3} \\
a^{3} \mathrm{D}_{2}-y^{3} \mathrm{~F}_{2}^{0} \\
b^{3} \mathrm{G}_{4}-z^{3} \mathrm{I}_{5}^{0}\end{array}$ & $\begin{array}{l}3 \\
2 \\
3\end{array}$ & 0.268 & $\begin{array}{l}0.16 \\
0 \\
.540\end{array}$ & $\begin{array}{ll} & 1.29 \\
.738, & 1.003\end{array}$ & $\begin{array}{l}1.146 \\
(1.15) \\
1.004\end{array}$ & $\begin{array}{l}1.10 \\
.735\end{array}$ \\
\hline $\begin{array}{r}2 \\
8 \\
4 \\
20 \\
20\end{array}$ & $\begin{array}{l}3104.62 \\
3104.27 \\
3103.26 \\
3101.918 \\
3100.79\end{array}$ & $\begin{array}{l}32200.7 \\
32204.4 \\
32214.9 \\
32228.80 \\
32240.5\end{array}$ & $\begin{array}{l}b^{3} \mathrm{G}_{3}-z^{1} \mathrm{G}_{4} \\
b^{3} \mathrm{G}_{3}-w^{3} \mathrm{~F}_{2}^{\circ} \\
d^{3} \mathrm{P}_{2}-u^{3} \mathrm{D}_{2}^{\circ} \\
b^{3} \mathrm{~F}_{3}-y^{3} \mathrm{G}_{3}^{\circ} \\
a^{3} \mathrm{~F}_{4}-z^{3} \mathrm{~F}_{4}^{\circ}\end{array}$ & $\begin{array}{l}3 \\
3\end{array}$ & .13 & $\begin{array}{l}0 \\
.39 \\
.09\end{array}$ & 1. 246 & $\begin{array}{r}(.767) \\
(1.150) \\
1.233\end{array}$ & $\begin{array}{l}.64 \\
1.02 \\
1.259\end{array}$ \\
\hline $\begin{array}{c}50 \\
100, \mathrm{~V} E \\
10 \\
60 \mathrm{c} \\
2000 \mathrm{c} R, \mathrm{~V} E\end{array}$ & $\begin{array}{l}3100.25 \\
3099.180 \\
3098.47 \\
3097.115 \\
3094.172\end{array}$ & $\begin{array}{l}32246.1 \\
32257.27 \\
32264.7 \\
32278.76 \\
32309.47\end{array}$ & $\begin{array}{c}a^{5} \mathrm{~S}_{2}-z^{3} \mathrm{D}_{1} \\
a^{3} \mathrm{G}_{1}-z^{3} \mathrm{H}_{4} \\
a^{3} \mathrm{P}_{3}-y \\
a^{3} \mathrm{P}_{2} \mathrm{~F}_{8}-z^{5} \mathrm{G}_{6}^{\circ}\end{array}$ & $\begin{array}{l}2 \\
3 \\
2 \\
1\end{array}$ & $\begin{array}{l}.579 \\
.24 \\
.314\end{array}$ & $\begin{array}{l}0 \\
.96 \\
0\end{array}$ & $\begin{array}{l}2.290 \\
.90\end{array}$ & $\begin{array}{l}.999 \\
(1.052) \\
1.662 \\
(1.39)\end{array}$ & $\begin{array}{r}.420 \\
.81 \\
1.348 \\
(1.31)\end{array}$ \\
\hline $\begin{array}{c}6 \\
3 \\
90 \\
20 h \\
2\end{array}$ & $\begin{array}{l}3092.89 \\
3089.110 \\
3087.860 \\
3086.09 \\
\text { 3084. } 760\end{array}$ & $\begin{array}{l}32322.9 \\
32362.42 \\
32375.52 \\
32394.1 \\
32408.05\end{array}$ & 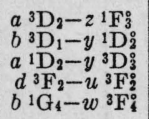 & $\begin{array}{l}2 \\
7 b, 2 \\
3\end{array}$ & $\begin{array}{l}.290 \\
.29\end{array}$ & $\begin{array}{l}0 \\
0 \\
0 \\
.58\end{array}$ & 1.053 & $\begin{array}{c}(1.002) \\
(.681) \\
(1.003) \\
(.70)\end{array}$ & $\begin{array}{l}1.08 \\
.971 \\
1.028 \\
.99\end{array}$ \\
\hline $\begin{array}{c}50 \\
30 c \\
40 \\
8 \\
100\end{array}$ & $\begin{array}{l}3084.369 \\
3083.32 \\
3081.77 \\
3081.09 \\
3080.345\end{array}$ & $\begin{array}{l}32412.16 \\
32423.2 \\
32439.5 \\
32446.7 \\
32454.50\end{array}$ & $\begin{array}{l}b^{3}{ }^{3} \mathrm{P}_{2}-z{ }^{3} \mathrm{~S}_{2}^{\circ} \\
d^{3} \mathrm{P}_{2}-u^{3} \mathrm{D}_{3}^{3} \\
a^{3} \mathrm{D}_{2}-y^{3} \mathrm{G}_{3} \\
b^{3} \mathrm{P}_{1}-z z^{5} \mathrm{~S}_{2}^{3} \\
a^{5} \mathrm{P}_{2}-y^{3} \mathrm{D}_{2}\end{array}$ & $\begin{array}{l}3 \\
2\end{array}$ & .465 & $\begin{array}{l}.936 \\
0 \\
1.176\end{array}$ & $\begin{array}{r}1.480,1.943 \\
1.034\end{array}$ & $\begin{array}{c}1.480 \\
(1.002) \\
1.810\end{array}$ & $\begin{array}{l}1.945 \\
1.013 \\
1.223\end{array}$ \\
\hline $\begin{array}{c}10 \\
200 \\
1 h\end{array}$ & $\begin{array}{l}3077.44 \\
3076.864 \\
3076.23\end{array}$ & $\begin{array}{l}32485.1 \\
32491.21 \\
32497.9\end{array}$ & $\begin{array}{l}a{ }^{1} \mathrm{~S}_{0}-x{ }^{3} \mathrm{D}_{1} \\
a^{5} \mathrm{~F}_{3}-z{ }^{3} \mathrm{D}_{2}^{\circ} \\
c^{3} \mathrm{P}_{2}-v \\
{ }^{3} \mathrm{D}_{3}^{\circ}\end{array}$ & $\begin{array}{l}7 a \\
1\end{array}$ & & $\begin{array}{l}0 \\
0\end{array}$ & $\begin{array}{l}.83 \\
1.478\end{array}$ & $\begin{array}{c}0 / 0 \\
(1.248)\end{array}$ & $\begin{array}{l}.83 \\
1.133\end{array}$ \\
\hline $\begin{array}{r}10 \\
5\end{array}$ & $\begin{array}{l}3075.250 \\
3074.27\end{array}$ & $\begin{array}{l}32508.27 \\
32518.6\end{array}$ & $\begin{array}{c}a{ }^{1} \mathrm{D}_{2}-z{ }^{5} \mathrm{P}_{3}^{3} \\
a^{3} \mathrm{P}_{2}-z^{3} \mathrm{~F}_{3}^{\circ}\end{array}$ & $\begin{array}{l}2 \\
1\end{array}$ & $\begin{array}{l}.562 \\
.360\end{array}$ & $\begin{array}{l}0 \\
0\end{array}$ & $\begin{array}{l}2.690 \\
.36\end{array}$ & $\begin{array}{l}1.004 \\
1.44\end{array}$ & $\begin{array}{l}1.566 \\
1.08\end{array}$ \\
\hline $\begin{array}{l}50 \\
60 \\
10 \\
90 c \\
40\end{array}$ & $\begin{array}{l}3073.232 \\
3072.502 \\
3072.18 \\
3071.55 \\
3071.18\end{array}$ & $\begin{array}{l}32529.61 \\
32537.34 \\
32540.7 \\
32547.4 \\
32551.3\end{array}$ & $\begin{array}{l}a{ }^{5} \mathrm{~F}_{1}-z{ }^{3} \mathrm{D}_{1}^{\circ} \\
a^{3} \mathrm{D}_{1}-y^{3} \mathrm{~F}_{2}^{\circ} \\
a^{5} \mathrm{P}_{3}-y{ }^{5} \mathrm{D}_{3}^{\circ} \\
b^{3} \mathrm{G}_{4}-y^{3} \mathrm{H}_{3}^{\circ}\end{array}$ & $\begin{array}{l}3 \\
2\end{array}$ & $\begin{array}{l}.418 \\
.23\end{array}$ & $0^{.418}$ & $\begin{array}{c}0.00, .417 \\
.96\end{array}$ & $\begin{array}{l}0.000 \\
.50\end{array}$ & $\begin{array}{l}.418 \\
.73\end{array}$ \\
\hline $\begin{array}{r}80 \\
100 \\
5 \\
5 \\
20\end{array}$ & $\begin{array}{l}3070.893 \\
3069.68 \\
3069.51 \\
3068.93 \\
3068.06\end{array}$ & $\begin{array}{l}32554.39 \\
32567.2 \\
32569.0 \\
32575.2 \\
32584.4\end{array}$ & $\begin{array}{c}a{ }^{1} \mathrm{G}_{4}-y{ }^{3} \mathrm{H}_{4} \\
a^{3} \mathrm{D}_{3}-y \\
b^{3} \mathrm{~B}_{4} \mathrm{G}_{4}-y \\
b^{3} \mathrm{P}^{3} \\
b^{3} \mathrm{D}_{2}-v^{3} \mathrm{~F}_{2}^{0} \\
b^{3} \mathrm{D}_{2}-v^{3} \mathrm{~F}_{3}^{0}\end{array}$ & 1 & .132 & $\begin{array}{l}0 \\
0\end{array}$ & .730 & 1. 258 & $\begin{array}{l}1.126 \\
1.10\end{array}$ \\
\hline $\begin{array}{r}20 \\
4\end{array}$ & $\begin{array}{l}3067.523 \\
3066.49\end{array}$ & $\begin{array}{l}32590.15 \\
32601.1\end{array}$ & $\begin{array}{l}b^{3}{ }^{3} \mathrm{D}_{3}-v^{3} \mathrm{~F}_{4} \\
a^{3} \mathrm{D}_{3}-y^{3} \mathrm{~F}_{2}^{2}\end{array}$ & 1 & & 0 & 1.14 & (1. 312) & 1. 28 \\
\hline $\begin{array}{c}60 \\
100 c \\
250 r\end{array}$ & $\begin{array}{l}3066.09 \\
3065.26 \\
3064.530\end{array}$ & $\begin{array}{l}32605.4 \\
32614.2 \\
32621.98\end{array}$ & $\begin{array}{r}b^{3}{ }^{3} \mathrm{~F}_{3}-y^{3}{ }^{3} \mathrm{~F}_{4}^{0} \\
a^{5} \mathrm{P}_{2}-z \\
a^{5} \mathrm{P}_{1}^{0} \mathrm{G}_{3}-z \\
a^{3} \mathrm{H}_{4}^{0}\end{array}$ & $\begin{array}{l}1 \\
1 \\
2\end{array}$ & .44 & $\begin{array}{l}0 \\
0 \\
0\end{array}$ & $\begin{array}{r}1.04 \\
1.38 \\
.87\end{array}$ & $\begin{array}{l}(1.150) \\
1.82 \\
(.765)\end{array}$ & $\begin{array}{r}1.12 \\
2.26 \\
.80\end{array}$ \\
\hline $\begin{array}{r}120 \\
40 \\
10 \\
5\end{array}$ & $\begin{array}{l}3063.782 \\
3063.126 \\
3061.95 \\
3061.408\end{array}$ & $\begin{array}{l}32629.94 \\
32636.93 \\
32649.5 \\
32655.25\end{array}$ & $\begin{array}{l}b^{3} \mathrm{~F}_{4}-y{ }^{3} \mathrm{~F}_{4}^{0} \\
a^{5} \mathrm{P}_{1}-y \\
a^{3} \mathrm{G}_{5}-z{ }^{3} \mathrm{D}_{5}^{\circ}\end{array}$ & $\begin{array}{l}3 \\
1 \\
3\end{array}$ & $\begin{array}{r}1.238 \\
.136\end{array}$ & ${ }_{0}^{.19}$ & ${ }_{0}^{1.07 \pm}$ & $\begin{array}{l}1.05 \\
2.476 \\
(1.180)\end{array}$ & $\begin{array}{l}1.10 \\
1.238 \\
1.044\end{array}$ \\
\hline 4 & 3060.844 & 32661.26 & $\left\{\begin{array}{c}c{ }^{3} \mathrm{P}_{2}-v \\
a^{1}{ }^{3} \mathrm{D}_{2}-z^{3} \mathrm{D}^{3} \\
{ }^{3} \mathrm{P}_{2}^{0}\end{array}\right.$ & & & & & & \\
\hline $\begin{array}{r}20 \\
3\end{array}$ & $\begin{array}{l}3059.294 \\
3058.62\end{array}$ & $\begin{array}{l}32677.81 \\
32685.0\end{array}$ & $b^{3} \mathrm{G}_{4}-w^{3} \mathrm{~F}_{4}^{\circ}$ & 3 & & .26 & $1.06 \pm$ & 1.03 & 1.09 \\
\hline $\begin{array}{r}12 \\
200 \\
90\end{array}$ & $\begin{array}{l}3057.03 \\
3055.520 \\
3053.631\end{array}$ & $\begin{array}{l}32702.0 \\
32718.17 \\
32738.41\end{array}$ & $\begin{array}{l}b^{3} \mathrm{G}_{3}-z{ }^{1} \mathrm{G}_{4}^{\circ} \\
b^{3} \mathrm{G}_{5}-y^{3} \mathrm{H}_{6}^{\circ} \\
b^{3} \mathrm{~F}_{3}-y^{3} \mathrm{H}_{3}^{\circ}\end{array}$ & $\begin{array}{l}2 \\
1 \\
3\end{array}$ & .325 & $\begin{array}{l}{ }^{0} \\
0 \\
.08\end{array}$ & $\begin{array}{c}.90 \\
1.138\end{array}$ & $\begin{array}{c}(.767) \\
(1.19) \\
1.152\end{array}$ & $\begin{array}{l}1.092 \\
1.14 \\
1.124\end{array}$ \\
\hline $\begin{array}{l}15 \\
40 c \\
5 \\
80 c \\
6 h\end{array}$ & $\begin{array}{l}3051.34 \\
3049.528 \\
3048.63 \\
3048.21 \\
3046.67\end{array}$ & $\begin{array}{l}32763.0 \\
32782.45 \\
32792.1 \\
32796.6 \\
32813.2\end{array}$ & $\begin{array}{l}b^{3}{ }^{3} \mathrm{~F}_{4}-y{ }^{3} \mathrm{~F}_{3}^{\circ} \\
a^{5} \mathrm{P}_{2}-y \\
a^{3} \mathrm{D}_{2} \mathrm{D}_{2}-z \\
{ }^{3} \mathrm{~F}_{2}^{\circ} \\
a^{5} \mathrm{P}_{1}-z{ }^{5} \mathrm{P}_{1}^{0} \\
a^{5} \mathrm{P}_{2}-y^{3} \mathrm{D}_{1}^{\circ}\end{array}$ & $\begin{array}{l}3 \\
3\end{array}$ & $\begin{array}{r}.47 \\
.20\end{array}$ & $\begin{array}{l}0.93 \\
.93 \\
.20\end{array}$ & $\begin{array}{r}1.22 \\
1.37,1.84 \\
2.26,2.46\end{array}$ & $\begin{array}{l}(1.152) \\
1.83\end{array}$ & $\begin{array}{l}1.13 \\
1.36\end{array}$ \\
\hline
\end{tabular}


TABLE 1.-Second spectrum of columbium (Cb II)-Continued

\begin{tabular}{|c|c|c|c|c|c|c|c|c|c|}
\hline $\begin{array}{l}\text { Intensity } \\
\text { spark }\end{array}$ & $\lambda_{\text {sir }} \mathbf{A}$ & $\begin{array}{l}\text { Wave } \\
\text { No. } \\
\text { vac. } \\
\mathrm{cm}^{-1}\end{array}$ & $\begin{array}{l}\text { Term combi- } \\
\text { nation }\end{array}$ & $\begin{array}{c}\text { Zeeman } \\
\text { type }\end{array}$ & $\begin{array}{l}\text { Sepa- } \\
\text { ration }\end{array}$ & $\begin{array}{c}\text { Strong- } \\
\text { est } \\
p\end{array}$ & $\underset{n}{\text { Strongest }}$ & $g_{1}$ & $g_{2}$ \\
\hline 1 & 2 & 3 & 4 & 5 & 6 & 7 & 8 & 8 & 10 \\
\hline $\begin{array}{c}150 \\
10 \\
15 \\
10 c \\
4\end{array}$ & $\begin{array}{l}3044.749 \\
3043.272 \\
3042.790 \\
3041.98 \\
3040.54\end{array}$ & $\begin{array}{l}32833.90 \\
32849.84 \\
32855.04 \\
32863.8 \\
32879.4\end{array}$ & $\begin{array}{l}b^{3} \mathrm{~F}_{4}-y^{3} \mathrm{G}^{0} \\
b^{3} \mathrm{~F}_{2}-y^{3} \mathrm{~F}_{2}^{0} \\
b^{3} \mathrm{~F}_{3}-y^{3} \mathrm{P}_{2}^{0} \\
a^{3} \mathrm{D}_{2}-x \\
a^{3} \mathrm{D}_{2} \mathrm{~F}_{2}^{0} \\
{ }^{3} \mathrm{~S}_{1}^{0}\end{array}$ & $\begin{array}{l}2 \\
3 \\
1\end{array}$ & $\begin{array}{l}0.11 \\
.362\end{array}$ & $\begin{array}{l}0 \\
0.22 \\
0\end{array}$ & $\begin{array}{l}1.193 \\
.78 \\
.430\end{array}$ & $\begin{array}{c}(1.152) \\
.84 \\
1.154\end{array}$ & $\begin{array}{l}1.160 \\
.73 \\
1.516\end{array}$ \\
\hline $\begin{array}{l}150 c \\
10 \\
200 c R \\
4 \\
400 r s\end{array}$ & $\begin{array}{l}3039.818 \\
3039.398 \\
3034.95 \\
3033.47 \\
3032.767\end{array}$ & $\begin{array}{l}32887.16 \\
32891.71 \\
32939.9 \\
32956.0 \\
32963.62\end{array}$ & 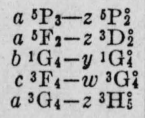 & $\begin{array}{l}1 \\
3 \\
7 b\end{array}$ & .141 & $\begin{array}{l}0 \\
0^{284} \\
0\end{array}$ & $\begin{array}{r}1.60 \\
.992,1.133 \\
.950\end{array}$ & $\begin{array}{r}(1.663) \\
.992 \\
.950 \\
(1.052)\end{array}$ & $\begin{array}{r}1.70 \\
1.133 \\
.950 \\
1.043\end{array}$ \\
\hline $\begin{array}{c}20 \\
60 \\
10 \\
300 c \\
2 h\end{array}$ & $\begin{array}{l}3029.86 \\
3029.76 \\
3028.76 \\
3028.436 \\
3026.80\end{array}$ & $\begin{array}{l}32995.2 \\
32996.3 \\
33007.2 \\
33010.76 \\
33028.6\end{array}$ & $\begin{array}{l}a{ }^{5} \mathrm{P}_{1}-y^{3} \mathrm{D}^{\mathrm{i}} \\
b^{3} \mathrm{~F}_{2}-z \\
c^{3} \mathrm{~F}_{3}^{3} \\
c^{3} \mathrm{~F}_{3}-v \\
a^{3} \mathrm{D}_{2} \mathrm{D}_{2}-z \\
b^{3} \mathrm{D}_{3}^{3} \mathrm{H}_{4}-x^{3} \mathrm{G}_{5}^{0}\end{array}$ & $\begin{array}{l}3 \\
2\end{array}$ & $\begin{array}{l}1.490 \\
.24\end{array}$ & $\begin{array}{l}1.490 \\
0 \\
0\end{array}$ & $\begin{array}{l}.983 \\
1.57 \\
1.37\end{array}$ & $\begin{array}{c}2.473 \\
.85 \\
(1.350)\end{array}$ & $\begin{array}{l}.983 \\
1.09 \\
1.34\end{array}$ \\
\hline $\begin{array}{r}40 \\
250 \\
10 \\
200 \\
5\end{array}$ & $\begin{array}{l}3025.372 \\
3024.735 \\
3024.258 \\
3022.738 \\
3022.48\end{array}$ & $\begin{array}{l}33044.19 \\
33051.15 \\
33056.36 \\
33072.99 \\
33075.8\end{array}$ & $\begin{array}{l}c^{3} \mathrm{~F}_{2}-w^{3} \mathrm{G}_{3}^{3} \\
a^{3} \mathrm{P}_{2}-y^{3} \mathrm{D}_{3}^{3} \\
a^{3} \mathrm{H}_{5}-z^{3} \mathrm{H}_{4} \\
b^{3} \mathrm{H}_{0}-x^{3} \mathrm{H}_{5}^{0} \\
b^{3} \mathrm{G}_{3}-w^{3} \mathrm{~F}_{4}^{0}\end{array}$ & $\begin{array}{l}2 \\
1\end{array}$ & $\begin{array}{l}.205 \\
.368\end{array}$ & $\begin{array}{l}0 \\
0\end{array}$ & $\begin{array}{r}1.282 \\
.706\end{array}$ & $\begin{array}{r}.667 \\
1.810 \\
(1.154)\end{array}$ & $\begin{array}{r}.872 \\
1.442\end{array}$ \\
\hline $\begin{array}{l}8 \\
3 \\
8 \\
3\end{array}$ & $\begin{array}{l}3021.885 \\
3019.780 \\
3019.57 \\
3019.20\end{array}$ & $\begin{array}{l}33082.31 \\
33105.38 \\
33107.7 \\
33111.7\end{array}$ & $\begin{array}{l}b^{3}{ }^{3} \mathrm{H}_{4}-x{ }^{3} \mathrm{~F}_{4} \\
b^{3} \mathrm{G}_{5}-y \\
b^{3} \mathrm{G}_{1} \\
a^{3} \mathrm{D}_{1}-v^{3} \mathrm{~F}_{2} \\
{ }^{1} \mathrm{D}_{2}-y^{3} \mathrm{P}^{3}\end{array}$ & & & & & & \\
\hline $\begin{array}{r}6 \\
100 \\
7 \\
2 \\
20\end{array}$ & $\begin{array}{l}3019.07 \\
3018.853 \\
3018.31 \\
3016.19 \\
3015.82\end{array}$ & $\begin{array}{l}33113.2 \\
33115.54 \\
33121.5 \\
33144.8 \\
33148.8\end{array}$ & $\begin{array}{l}b^{3} \mathrm{~F}_{2}-y^{3} \mathrm{G}_{3}^{0} \\
b^{3} \mathrm{H}_{4}-x^{3} \mathrm{G}_{3}^{3} \\
d^{3} \mathrm{~F}_{4}-u^{3} \mathrm{~F}_{3}^{\circ} \\
d^{3} \mathrm{~F}_{3}-u^{3} \mathrm{~F}_{3}^{\circ}\end{array}$ & 2 & & 0 & 1.09 & $(.880)$ & .810 \\
\hline $\begin{array}{r}30 \\
15 \\
3\end{array}$ & $\begin{array}{l}3015.02 \\
3014.438 \\
3013.62\end{array}$ & $\begin{array}{l}33157.6 \\
33164.04 \\
33173.1\end{array}$ & $a^{5} \mathrm{~F}_{1}-z^{3} \mathrm{D}_{2}^{2}$ & 2 & 1.140 & 0 & 2. 276 & 0.000 & 1. 136 \\
\hline $\begin{array}{l}25 \\
20\end{array}$ & $\begin{array}{l}3010.685 \\
3010.38\end{array}$ & $\begin{array}{l}33205.38 \\
33208.7\end{array}$ & $\begin{array}{l}b{ }^{3} \mathrm{H}_{5}-x^{3} \mathrm{G}_{5}^{0} \\
b^{3} \mathrm{G}_{4}-y^{1} \mathrm{G}_{4}^{0}\end{array}$ & $\begin{array}{l}3 \\
3\end{array}$ & .14 & $\begin{array}{l}.70 \\
.28\end{array}$ & $\begin{array}{c}1.12 \pm \\
.990 \pm\end{array}$ & $\begin{array}{l}1.05 \\
1.025\end{array}$ & $\begin{array}{l}1.19 \\
.955\end{array}$ \\
\hline $\begin{array}{c}7 \\
20 c \\
4\end{array}$ & $\begin{array}{l}3008.97 \\
3008.39 \\
3007.488\end{array}$ & $\begin{array}{l}33224.3 \\
33230.7 \\
33240.68\end{array}$ & $\begin{array}{l}a^{3} \mathrm{D}_{1}-x^{3} \mathrm{~F}_{2}^{0} \\
a{ }^{3} \mathrm{P}_{2}-z z^{3} \mathrm{P}_{1}^{0} \\
a^{3} \mathrm{D}_{1}-z{ }^{3} \mathrm{~S}_{1}^{0}\end{array}$ & 2 & .59 & 0 & 2. 39 & 1.80 & 1. 21 \\
\hline $\begin{array}{l}60 \\
10\end{array}$ & $\begin{array}{l}3005.764 \\
3004.65\end{array}$ & $\begin{array}{l}33259.75 \\
33272.1\end{array}$ & $\begin{array}{c}b^{3} \mathrm{H}_{5}-x^{3} \mathrm{~F}_{i}^{i} \\
a{ }^{1} \mathrm{~S}_{0}-x^{3} \mathrm{P}_{1}^{i}\end{array}$ & $\begin{array}{l}1 \\
7 a\end{array}$ & & $\begin{array}{l}0 \\
0\end{array}$ & $\begin{array}{l}.40 \\
1.202\end{array}$ & $\begin{array}{c}(1.052) \\
0 / 0\end{array}$ & $\begin{array}{l}\text { 1. } 215 \\
\text { 1. } 202\end{array}$ \\
\hline $\begin{array}{r}3 h \\
2 h\end{array}$ & $\begin{array}{l}3003.75 \\
3003.16\end{array}$ & $\begin{array}{l}33282.0 \\
33288.6\end{array}$ & $a^{3} \mathrm{D}_{3}-x^{3} \mathrm{~F}_{2}$ & & & & & & \\
\hline $\begin{array}{r}40 \\
150 \\
50\end{array}$ & $\begin{array}{l}3002.204 \\
3001.85 \\
3001.125\end{array}$ & $\begin{array}{l}33299.18 \\
33303.1 \\
33311.16\end{array}$ & $\begin{array}{l}a^{3} \mathrm{P}_{3}-y^{3} \mathrm{D}_{3}-w^{3} \mathrm{G}_{4} \\
c^{3}\end{array}$ & $\begin{array}{l}3 \\
2\end{array}$ & .630 & $\begin{array}{l}1.886 \\
0\end{array}$ & $\begin{array}{r}1.035,1.666 \\
1.025\end{array}$ & $\begin{array}{c}1.666 \\
(1.080)\end{array}$ & $\begin{array}{l}1.035 \\
1.066\end{array}$ \\
\hline $\begin{array}{l}4 h \\
1 \\
1 \\
8 \\
1\end{array}$ & $\begin{array}{l}2998.87 \\
2998.56 \\
2997.947 \\
2997.48 \\
2997.14\end{array}$ & $\begin{array}{l}33336.2 \\
33339.6 \\
33346.46 \\
33351.7 \\
33355.4\end{array}$ & $\begin{array}{l}a{ }^{1} \mathrm{G}_{4}-z^{3} \mathrm{I}_{5}^{\circ} \\
b^{3} \mathrm{D}_{3}-y^{1} \mathrm{~F}_{3}^{3} \\
c^{3} \mathrm{~F}_{2}-v^{3} \mathrm{D}_{3}^{8} \\
a^{1}{ }^{1} \mathrm{H}_{5}-w^{3} \mathrm{G}_{4}^{\circ}\end{array}$ & 2 & .52 & 0 & & $(.670)$ & 1.19 \\
\hline $\begin{array}{c}7 \\
4 \\
300 c \\
20 c \\
20\end{array}$ & $\begin{array}{l}2996.79 \\
2995.49 \\
2994.725 \\
2993.97 \\
2993.806\end{array}$ & $\begin{array}{l}33359.3 \\
33373.8 \\
33382.34 \\
33390.8 \\
33392.59\end{array}$ & 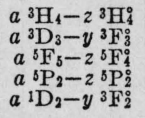 & $\begin{array}{l}7 b, 3 \\
2\end{array}$ & & 0 & $\begin{array}{c}.818 \\
1.60\end{array}$ & $\begin{array}{c}(.825) \\
(1.39)\end{array}$ & $\begin{array}{l}.811 \\
1.34\end{array}$ \\
\hline $\begin{array}{c}80 \\
6 \\
200 c \\
40 h \\
9\end{array}$ & $\begin{array}{l}2991.956 \\
2991.43 \\
2990.28 \\
2989.944 \\
2987.55\end{array}$ & $\begin{array}{l}33413.23 \\
33419.1 \\
33432.0 \\
33435.72 \\
33462.5\end{array}$ & $\begin{array}{l}a{ }^{5} \mathrm{P}_{1}-z^{3} \mathrm{P}_{1}^{0} \\
a^{1}{ }^{1} \mathrm{~F}_{3}-v^{3}{ }^{3} \mathrm{~F}_{2}^{0} \\
a^{5} \mathrm{P}_{3}-z^{5} \mathrm{P}_{3}^{\circ} \\
a^{1} \mathrm{G}_{4}-z^{1} \mathrm{G}_{4}\end{array}$ & $\begin{array}{l}3 \\
3 \\
7 b\end{array}$ & 1. 261 & $\begin{array}{l}1.260 \\
.24 \\
0\end{array}$ & $\begin{array}{r}1.225,2.487 \\
1.62 \pm \\
1.083\end{array}$ & $\begin{array}{l}2.486 \\
1.66 \\
1.083\end{array}$ & $\begin{array}{l}1.225 \\
1.58 \\
1.083\end{array}$ \\
\hline $\begin{array}{c}1 \\
3 \\
5 h \\
50 \\
3\end{array}$ & $\begin{array}{l}2987.16 \\
2986.90 \\
2986.40 \\
2985.04 \\
2982.90\end{array}$ & $\begin{array}{l}33466.9 \\
33469.8 \\
33475.4 \\
33490.6 \\
33514.7\end{array}$ & $\begin{array}{c}c{ }^{3} \mathrm{~F}_{2}-v^{3} \mathrm{D}_{2}^{\circ} \\
a^{3} \mathrm{D}_{2}-x^{3} \mathrm{~F}_{3}^{3} \\
b{ }^{1} \mathrm{D}_{2}-u^{3} \mathrm{~F}_{2}^{2} \\
a^{3} \mathrm{D}_{3}-y^{3} \mathrm{P}_{2}^{8} \\
c^{3} \mathrm{~F}_{2}-v^{3} \mathrm{D}_{1}^{0}\end{array}$ & 1 & .253 & 0 & .731 & 1. 237 & 1.490 \\
\hline
\end{tabular}


TABLE 1.-Second spectrum of columbium (Cb Ir)-Continued

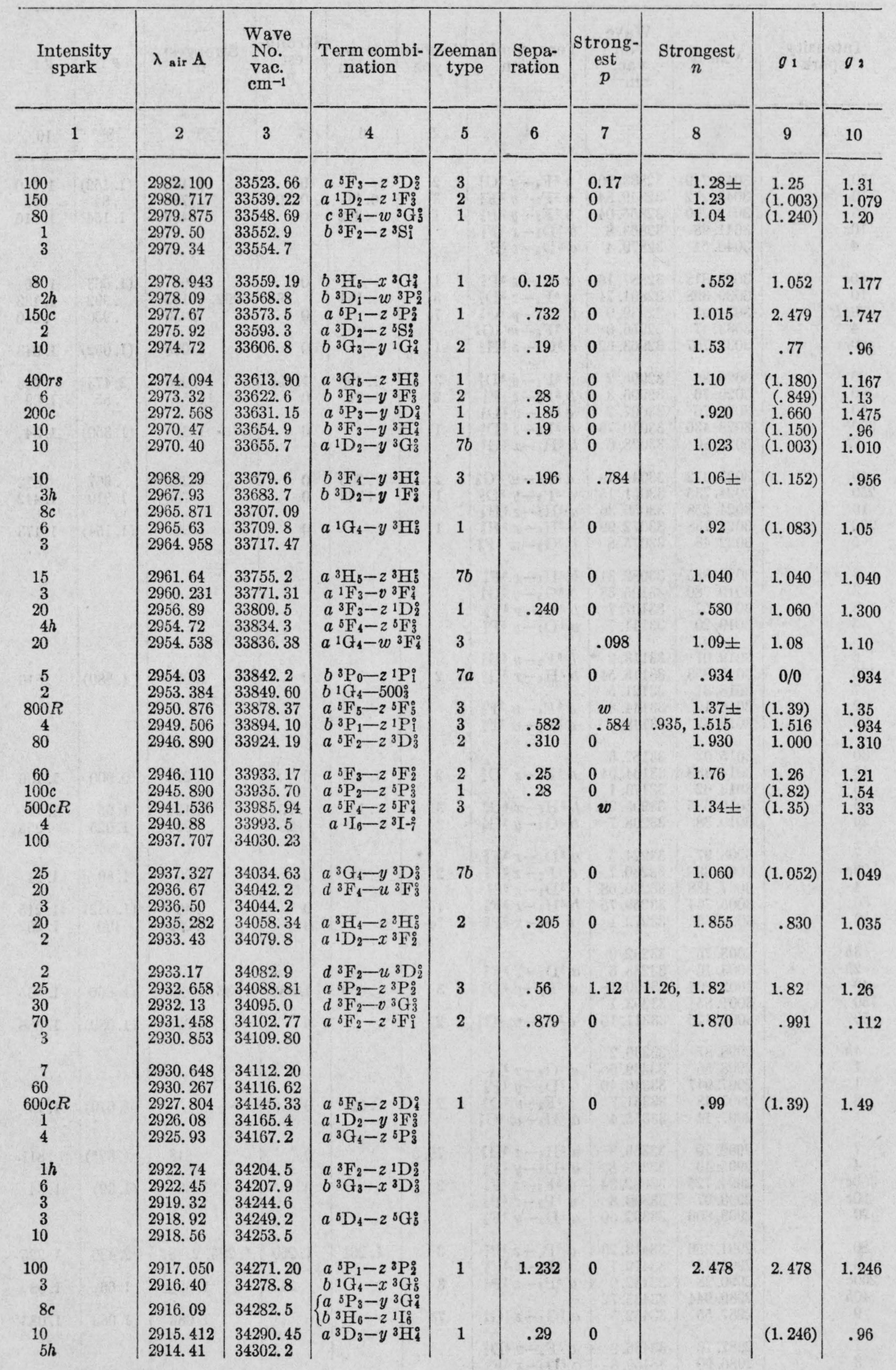


$\mathrm{T}_{\mathrm{ABLE}}$ 1.-Second spectrum of columbium (Cb II)-Continued

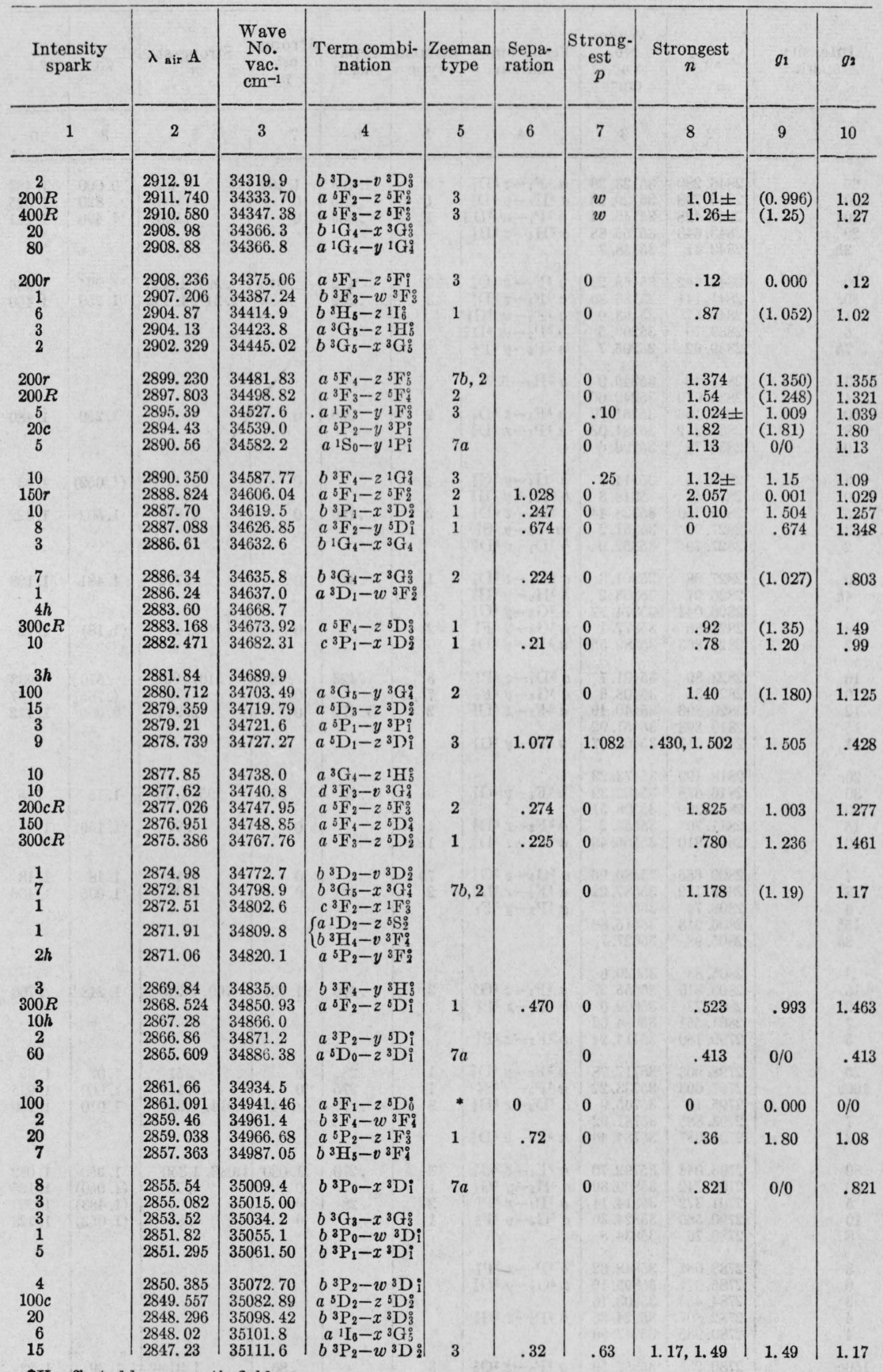

-Unaffected by magnetic field. 
498 Journal of Research of the National Bureau of Standards

TABLE 1.-Second spectrum of columbium (Cb II)-Continued

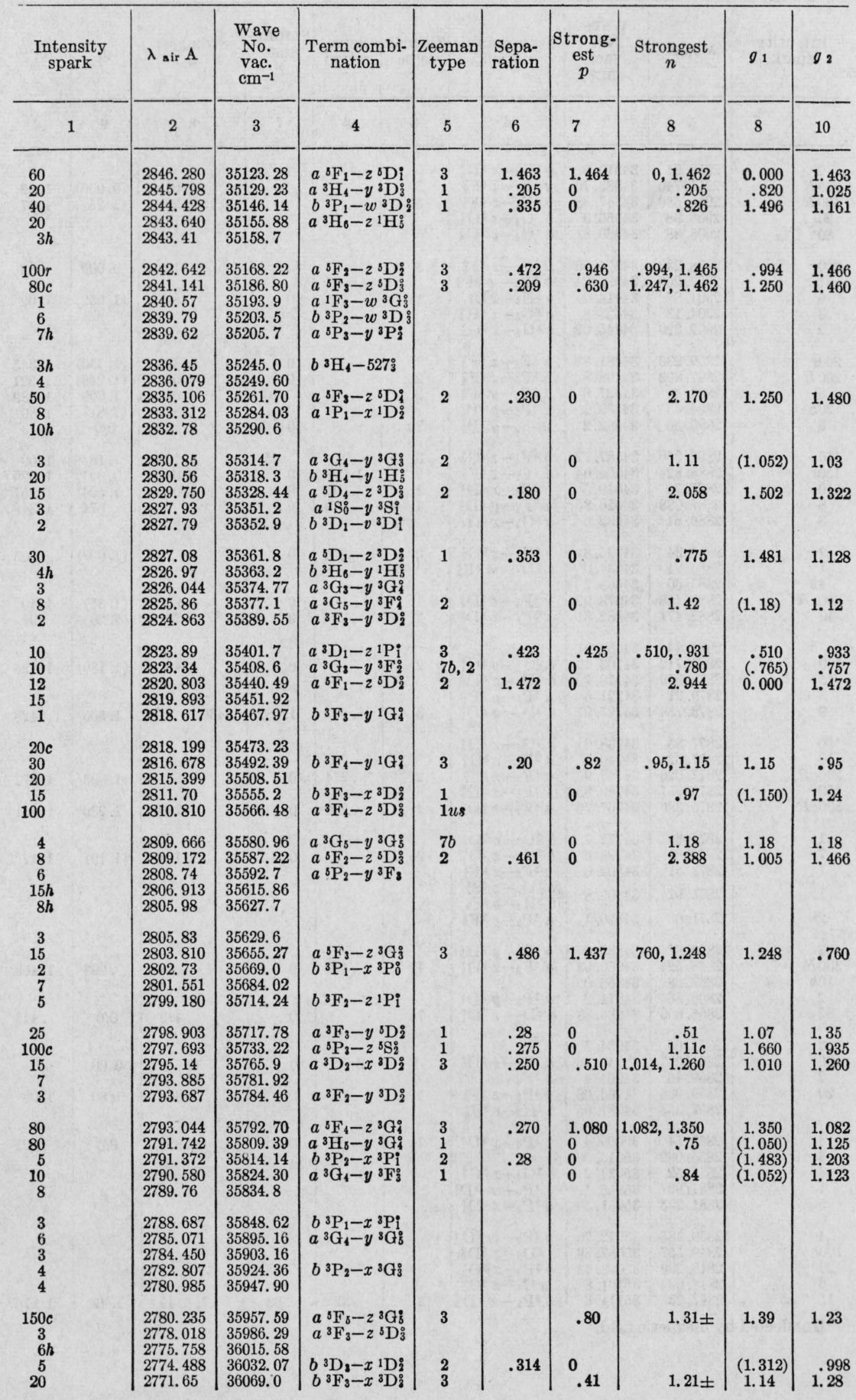


TABLE 1.-Second spectrum of columbium (Cb II) - Continued

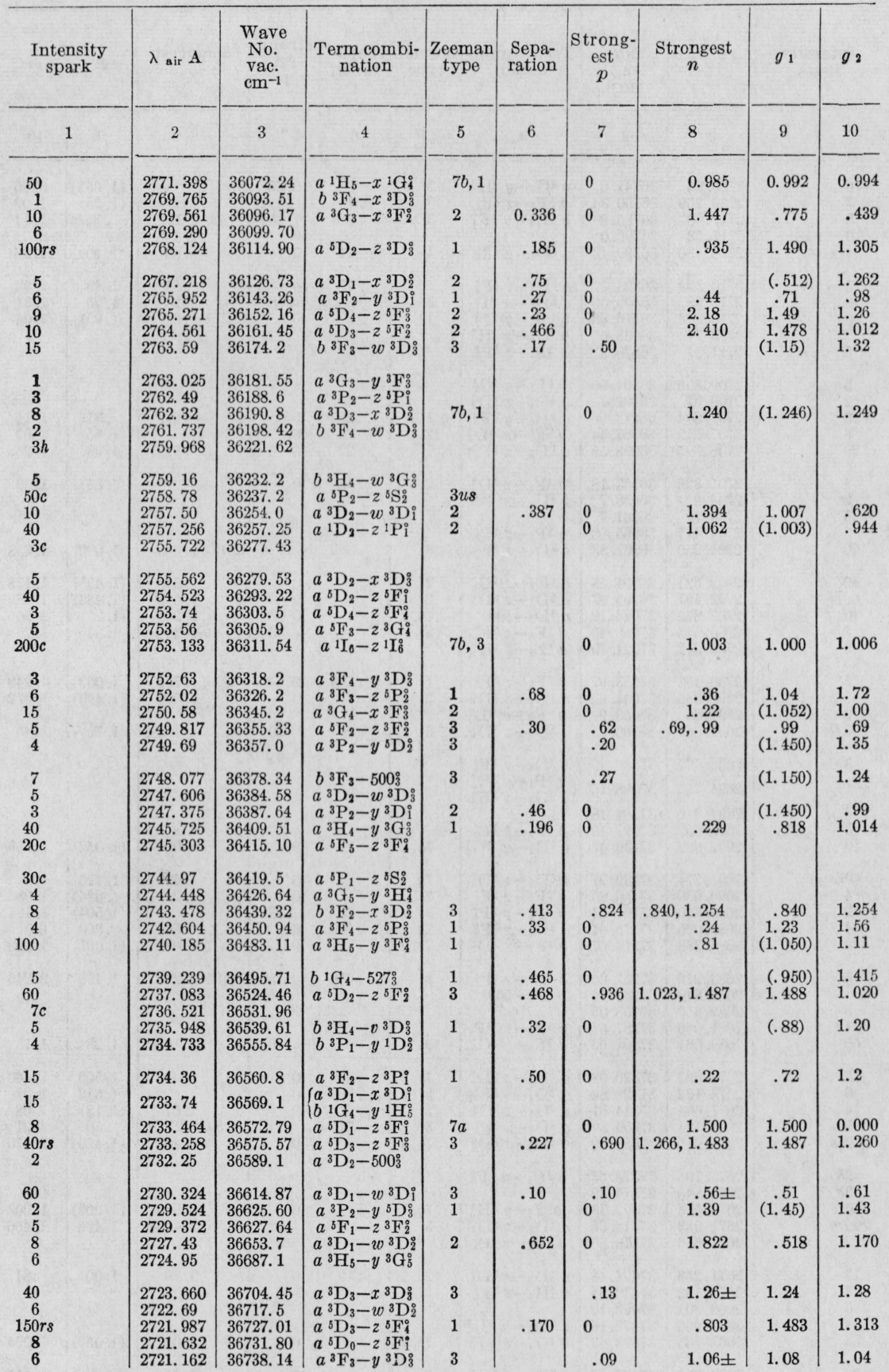


TABLE 1.-Second spectrum of columbium (Cb II)-Continued

\begin{tabular}{|c|c|c|c|c|c|c|c|c|c|}
\hline $\begin{array}{l}\text { Intensity } \\
\text { spark }\end{array}$ & $\lambda=$ ir $\mathbf{A}$ & $\begin{array}{l}\text { Wave } \\
\text { No. } \\
\text { vac. } \\
\mathrm{cm}^{-1}\end{array}$ & $\begin{array}{l}\text { Term combi- } \\
\text { nation }\end{array}$ & $\begin{array}{c}\text { Zeeman } \\
\text { type }\end{array}$ & $\begin{array}{l}\text { Sepa- } \\
\text { ration }\end{array}$ & $\begin{array}{c}\text { Strong- } \\
\text { est } \\
p\end{array}$ & $\underset{n}{\text { Strongest }}$ & $g_{1}$ & $g_{2}$ \\
\hline 1 & 2 & 3 & 4 & 5 & 6 & 7 & 8 & 9 & 10 \\
\hline $\begin{array}{c}6 \\
7 \\
8 \\
10 \\
150 r s\end{array}$ & $\begin{array}{l}2720.95 \\
2720.259 \\
2717.63 \\
2717.33 \\
2716.630\end{array}$ & $\begin{array}{l}36741.0 \\
36750.34 \\
36785.9 \\
36790.0 \\
36799.43\end{array}$ & $\begin{array}{l}a{ }^{3} \mathrm{G}_{4}-y^{3} \mathrm{H}^{\circ} \\
a{ }^{5} \mathrm{~F}_{3}-z^{3} \mathrm{~F}_{3}^{3} \\
a^{3} \mathrm{H}_{4}-y^{3} \mathrm{~F}_{4}^{\circ} \\
a^{5} \mathrm{D}_{4}-z^{5} \mathrm{~F}_{5}^{\circ}\end{array}$ & $\begin{array}{l}3 \\
3 \\
3 \\
1\end{array}$ & $\begin{array}{c}0.16 \\
.288 \\
.14\end{array}$ & $\begin{array}{l}0.36 \\
.48 \\
1.154 \\
0 \\
0\end{array}$ & $\begin{array}{c}82,1.11 \\
1.193 \\
.80\end{array}$ & $\begin{array}{c}(1.052) \\
(1.25) \\
.82 \\
1.50\end{array}$ & $\begin{array}{l}0.96 \\
109 \\
1.11 \\
1.36\end{array}$ \\
\hline $\begin{array}{r}15 \\
40 \\
50 \\
2 \\
5\end{array}$ & $\begin{array}{l}2716.309 \\
2715.882 \\
2715.344 \\
2713.74 \\
2711.37\end{array}$ & $\begin{array}{l}36803.77 \\
36809.56 \\
36816.85 \\
36838.6 \\
36870.8\end{array}$ & $\begin{array}{l}a{ }^{5} \mathrm{D}_{1}-z^{5} \mathrm{~F}_{2}^{\circ} \\
a^{3} \mathrm{D}_{3}-w^{3} \mathrm{D}^{3} \mathrm{D}_{3}^{\circ} \\
b^{1} \mathrm{G}_{4}-y \\
b^{3}{ }^{3} \mathrm{~F}_{4}-y \\
a^{3}{ }^{3} \mathrm{H}_{3}-z^{5} \mathrm{H}_{3}^{\circ}\end{array}$ & $\begin{array}{l}1 \\
3 \\
1\end{array}$ & .47 & $0^{0} .18$ & $\begin{array}{r}.55 \\
1.28 \\
.67\end{array}$ & $\begin{array}{l}1.49 \\
1.25 \\
(.95)\end{array}$ & $\begin{array}{l}1.02 \\
1.31 \\
1.04\end{array}$ \\
\hline $\begin{array}{r}3 \\
2 \\
20 \\
3 \\
20\end{array}$ & $\begin{array}{l}2709.595 \\
2709.07 \\
2707.834 \\
2707.212 \\
2706.395\end{array}$ & $\begin{array}{l}36894.96 \\
36902.1 \\
36918.96 \\
36927.44 \\
36938.58\end{array}$ & $\begin{array}{c}b^{3} \mathrm{~S}_{3}-x^{3} \mathrm{G}_{3}^{\circ} \\
b^{3} \mathrm{D}_{1}-x^{1} \mathrm{D}_{2}^{\circ} \\
a^{3} \mathrm{H}_{4}-y^{3} \mathrm{~F}_{3}^{0} \\
b^{3} \mathrm{~F}_{2}-w^{3} \mathrm{D}^{\circ} \\
a^{5} \mathrm{D}_{2}-z^{5} \mathrm{~F}_{3}^{\circ}\end{array}$ & $\begin{array}{l}1 \\
2 \\
1\end{array}$ & $\begin{array}{l}.295 \\
.23 \\
.22\end{array}$ & $\begin{array}{l}0 \\
0 \\
0\end{array}$ & $\begin{array}{r}-.07 \\
.82\end{array}$ & $\begin{array}{l}.815 \\
(.85) \\
1.48\end{array}$ & $\begin{array}{r}1.11 \\
.62 \\
1.26\end{array}$ \\
\hline $\begin{array}{r}4 \\
2 \\
5 \\
5 \\
20\end{array}$ & $\begin{array}{l}2705.326 \\
2704.92 \\
2704.70 \\
2704.417 \\
2704.250\end{array}$ & $\begin{array}{l}36953.18 \\
36958.7 \\
36961.7 \\
36965.60 \\
36967.88\end{array}$ & $\begin{array}{c}b{ }^{3} \mathrm{~F}_{2}-x{ }^{3} \mathrm{D}_{3}^{0} \\
b{ }^{3} \mathrm{H}_{4}-w^{3} \mathrm{G}_{4}^{\circ} \\
a^{3} \mathrm{P}_{2}-z{ }^{3} \mathrm{P}_{2}^{2} \\
a^{3} \mathrm{D}_{2}-x{ }^{3} \mathrm{P}_{2}^{0}\end{array}$ & 2 & .352 & .714 & $1.010,1.355$ & 1.006 & 1. 358 \\
\hline $\begin{array}{l}40 \\
60 r s \\
10 \\
7 \\
3\end{array}$ & $\begin{array}{l}2702.521 \\
2702.197 \\
2700.872 \\
2700.555 \\
2700.312\end{array}$ & $\begin{array}{l}36991.53 \\
36995.97 \\
37014.12 \\
37018.46 \\
37021.79\end{array}$ & $\begin{array}{l}a{ }^{5} \mathrm{D}_{4}-z{ }^{5} \mathrm{D}_{3}^{\circ} \\
a^{5} \mathrm{D}_{3}-z^{5} \mathrm{D}_{2}^{\circ} \\
a^{3} \mathrm{D}_{3}-500_{3}^{\circ} \\
a^{5} \mathrm{~F}_{4}-z^{3} \mathrm{~F}_{4}^{\circ} \\
a^{3} \mathrm{~F}_{4}-z^{1} \mathrm{H}_{5}^{\circ}\end{array}$ & $\begin{array}{l}2 \\
2 \\
7 b, 3 \\
3\end{array}$ & & $\begin{array}{l}0 \\
0 \\
0 \\
.30\end{array}$ & $\begin{array}{l}1.545 \\
1.519 \\
1.255 \\
1.30 \pm\end{array}$ & $\begin{array}{l}(1.495) \\
(1.486) \\
(1.25) \\
1.34\end{array}$ & $\begin{array}{l}\text { 1. } 478 \\
1.469 \\
1.26 \\
1.26\end{array}$ \\
\hline $\begin{array}{c}15 \\
100 \mathrm{rs} \\
2 \\
200 R s\end{array}$ & $\begin{array}{l}2700.153 \\
2698.866 \\
2697.66 \\
2697.067\end{array}$ & $\begin{array}{l}37023.97 \\
37041.62 \\
37058.2 \\
37066.33\end{array}$ & $\begin{array}{c}a^{3} \mathrm{~F}_{3}-z^{3} \mathrm{P}_{2}^{0} \\
a^{3} \mathrm{D}_{2}-z^{5} \mathrm{D}_{1}^{\circ} \\
b^{3} \mathrm{~F}_{2}-w^{3} \mathrm{D}_{3}^{\circ} \\
a^{5} \mathrm{D}_{4}-z^{5} \mathrm{D}_{4}^{0}\end{array}$ & $\begin{array}{l}1 \\
2 \\
3\end{array}$ & .180 & $\begin{array}{l}\begin{array}{l}0 \\
0 \\
.20\end{array} \\
\end{array}$ & $\begin{array}{r}.702 \\
\text { 1. } 508\end{array}$ & $\begin{array}{c}1.062 \\
(1.490) \\
1.50\end{array}$ & $\begin{array}{l}1.242 \\
1.472 \\
1.45\end{array}$ \\
\hline $\begin{array}{l}3 \\
4\end{array}$ & 2695. 601 & 37086.49 & $\begin{array}{l}b^{3} \mathrm{G}_{4}-y{ }^{1} \mathrm{~F}_{3}^{0} \\
\int^{a^{3} \mathrm{P}_{1}-y}{ }^{3} \mathrm{D}_{2}^{2}\end{array}$ & & & & & & \\
\hline $\begin{array}{l}4 \\
4\end{array}$ & $\begin{array}{l}2694.753 \\
2694.316\end{array}$ & $\begin{array}{l}37098.16 \\
37104.18\end{array}$ & $\left\{\begin{array}{l}a^{3} \mathrm{G}_{3}-y^{3} \mathrm{H}_{4}^{0} \\
{ }^{3} \mathrm{H}^{0}\end{array}\right.$ & & & & & & \\
\hline $\begin{array}{r}3 \\
10\end{array}$ & $\begin{array}{l}2692.652 \\
2692.002\end{array}$ & $\begin{array}{l}37127.10 \\
37136.07\end{array}$ & $\begin{array}{l}b{ }^{3} \mathrm{P}_{2}-y \\
{ }^{1} \mathrm{P}^{3} \mathrm{i}_{5}-w^{3} \mathrm{G}_{1}\end{array}$ & $7 b, 1$ & & 0 & 1. 024 & (1. 052) & 1.059 \\
\hline $\begin{array}{c}60 r s \\
4 \\
2 \\
4 \\
100\end{array}$ & $\begin{array}{l}2691.774 \\
2690.930 \\
2690.150 \\
2689.066 \\
2686.388\end{array}$ & $\begin{array}{l}37139.22 \\
37150.86 \\
37161.63 \\
37176.61 \\
37213.67\end{array}$ & 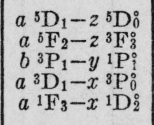 & $\begin{array}{l}7 a \\
2 \\
3 \\
7 a \\
7 b, 2\end{array}$ & $\begin{array}{l}.38 \\
.500\end{array}$ & $\begin{array}{l}0 \\
0 \\
0 \\
0 \\
0\end{array}$ & $\begin{array}{l}1.496 \\
1.24 \\
.500 \\
1.020\end{array}$ & $\begin{array}{c}1.496 \\
(.996) \\
(1.504) \\
.500 \\
(1.007)\end{array}$ & $\begin{array}{l}0 / 0 \\
1.08 \\
1.124 \\
0 / 0 \\
1.000\end{array}$ \\
\hline $\begin{array}{r}6 \\
1 \\
8 \\
1 \\
50\end{array}$ & $\begin{array}{l}2683.216 \\
2682.849 \\
2682.469 \\
2681.066 \\
2680.061\end{array}$ & $\begin{array}{l}37257.66 \\
37262.76 \\
37268.03 \\
37287.54 \\
37301.52\end{array}$ & $\begin{array}{l}a^{3} \mathrm{P}_{1}-z{ }^{5} \mathrm{P}_{1}^{\circ} \\
b^{3} \mathrm{~F}_{2}-500_{3}^{\circ} \\
a^{5} \mathrm{P}_{3}-w^{3} \mathrm{~F}_{4}^{0} \\
a^{3} \mathrm{~F}_{4}-y^{3} \mathrm{G}_{4}^{\circ}\end{array}$ & 3 & .756 & .756 & 1. $490,2.245$ & 1.490 & 1.13 \\
\hline $\begin{array}{c}15 \\
6 \\
15 \\
5 \\
80 r 8\end{array}$ & $\begin{array}{l}2678.663 \\
2678.102 \\
2677.664 \\
2676.124 \\
2675.945\end{array}$ & $\begin{array}{l}37320.98 \\
37328.80 \\
37334.91 \\
37356.39 \\
37358.89\end{array}$ & $\begin{array}{l}a{ }^{5} \mathrm{D}_{1}-z{ }^{5} \mathrm{D}_{1}^{\circ} \\
a^{3} \mathrm{D}_{1}-x \\
a^{3} \mathrm{~S}_{2}^{\circ} \mathrm{G}_{5}-z \mathrm{G}_{4}^{\circ} \\
a^{3} \mathrm{D}_{1}-x^{3} \mathrm{P}_{1}^{\circ} \\
a^{5} \mathrm{D}_{2}-z z^{5} \mathrm{D}_{2}^{\circ}\end{array}$ & $\begin{array}{l}7 b, 3 \\
2 \\
2 \\
3 \\
7 b, 3\end{array}$ & $\begin{array}{l}.86 \\
.71\end{array}$ & $\begin{array}{l}0 \\
0 \\
0 \\
0^{\cdot 71}\end{array}$ & $\begin{array}{c}1.470 \\
1.57 \\
.50,1.21 \\
1.480\end{array}$ & $\begin{array}{c}1.500 \\
(.512) \\
(1.18) \\
.50 \\
(1.490)\end{array}$ & $\begin{array}{l}1.440 \\
1.37 \\
1.08 \\
1.21 \\
1.470\end{array}$ \\
\hline $\begin{array}{c}2 h \\
3 h \\
250 r s \\
200 r s \\
2\end{array}$ & $\begin{array}{l}2675.104 \\
2674.840 \\
2673.566 \\
2671.933 \\
2671.656\end{array}$ & $\begin{array}{l}37370.63 \\
37374.32 \\
37392.13 \\
37414.98 \\
37418.86\end{array}$ & $\begin{array}{r}a^{1} \mathrm{P}_{1}-u^{3} \mathrm{~F}_{2}^{0} \\
a{ }^{1} \mathrm{I}_{6}-y \\
a^{3}{ }^{1} \mathrm{H}_{5}^{\circ} \\
a^{3} \mathrm{D}_{3}-z \\
{ }^{3} \mathrm{D}_{3}-z \\
{ }^{3} \mathrm{D}_{2}^{\circ}\end{array}$ & $\begin{array}{l}7 b, 1 \\
3\end{array}$ & & ${ }^{0} .12$ & $\begin{array}{l}.985 \\
1.476 \pm\end{array}$ & $\begin{array}{c}(1.000) \\
1.496\end{array}$ & $\begin{array}{l}1.003 \\
1.456\end{array}$ \\
\hline $\begin{array}{r}12 \\
3 \\
5 \\
4 \\
35\end{array}$ & $\begin{array}{l}2671.255 \\
2670.152 \\
2668.501 \\
2667.996 \\
2667.765\end{array}$ & $\begin{array}{l}37424.48 \\
37439.94 \\
37463.10 \\
37470.19 \\
37473.43\end{array}$ & $\begin{array}{l}a^{1} \mathrm{D}_{2}-x^{3} \mathrm{D}_{1} \\
a^{3} \mathrm{H}_{4}-x^{3} \mathrm{~F}_{3}^{3} \\
a^{1} \mathrm{D}_{2}-w^{3} \mathrm{D}_{1} \\
a^{3} \mathrm{G}_{4}-w^{3} \mathrm{~F}_{3}\end{array}$ & $7 b, 1$ & .19 & 0 & 1.017 & (1.052) & 1.064 \\
\hline $\begin{array}{r}30 \\
6 \\
5 \\
50 \\
2\end{array}$ & $\begin{array}{l}2667.300 \\
2667.146 \\
2667.072 \\
2666.595 \\
2666.164\end{array}$ & $\begin{array}{l}37479.97 \\
37482.13 \\
37483.17 \\
37489.87 \\
37495.94\end{array}$ & $\begin{array}{c}a^{5} \mathrm{D}_{0}-z{ }^{5} \mathrm{D}_{1}^{\circ} \\
a^{3} \mathrm{~F}_{4}-z^{1} \mathrm{~F}_{3}^{\circ} \\
b^{1} \mathrm{G}_{4}-w^{3} \mathrm{G}_{3}^{\circ} \\
a^{5} \mathrm{D}_{3}-z^{5} \mathrm{D}_{4}^{\circ} \\
a^{1} \mathrm{D}_{2}-x^{3} \mathrm{D}_{3}^{\circ}\end{array}$ & $7 b, 1$ & & 0 & 1. 457 & 1.486 & $\begin{array}{l}1.457 \\
1.474\end{array}$ \\
\hline
\end{tabular}


TABLE 1.-Second spectrum of columbium (Cb II)-Continued

\begin{tabular}{|c|c|c|c|c|c|c|c|c|c|}
\hline $\begin{array}{l}\text { Intensity } \\
\text { spark }\end{array}$ & $\lambda_{\text {air }} \mathbf{A}$ & $\begin{array}{l}\text { Wave } \\
\text { No. } \\
\text { vac. } \\
\mathrm{cm}^{-1}\end{array}$ & $\begin{array}{c}\text { Term combi- } \\
\text { nation }\end{array}$ & $\begin{array}{c}\text { Zeeman } \\
\text { type }\end{array}$ & $\begin{array}{l}\text { Sepa- } \\
\text { ration }\end{array}$ & $\begin{array}{c}\text { Strong- } \\
\text { est } \\
p\end{array}$ & $\begin{array}{c}\text { Strongest } \\
n\end{array}$ & $g_{1}$ & $\mathrm{O}_{2}$ \\
\hline 1 & 2 & 3 & 4 & 5 & 6 & 7 & 8 & 8 & 10 \\
\hline $\begin{array}{r}2 \\
80 \\
3 \\
2 \\
10\end{array}$ & $\begin{array}{l}2666.056 \\
2665.247 \\
2664.010 \\
2663.705 \\
2663.052\end{array}$ & $\begin{array}{l}37497.45 \\
37508.83 \\
37526.25 \\
37530.55 \\
37532.70\end{array}$ & $\begin{array}{l}a^{3} \mathrm{D}_{3}-x^{3} \mathrm{~F}_{4}^{0} \\
a^{3} \mathrm{G}_{3}-w^{3} \mathrm{~F}_{2}^{\circ} \\
a^{3} \mathrm{G}_{4}-z^{3} \mathrm{I}_{3}^{\circ} \\
a^{3} \mathrm{D}_{3}-x^{3} \mathrm{G}_{3}^{2} \\
a^{3} \mathrm{H}_{5}-y^{3} \mathrm{H}_{4}^{0}\end{array}$ & 2 & 0.142 & 0 & 1.34 & $(1.050)$ & .98 \\
\hline $\begin{array}{c}15 \\
15 \\
5 \\
1 \\
80 r 8\end{array}$ & $\begin{array}{l}2660.036 \\
2669.049 \\
2658.875 \\
2658.711 \\
2656.076\end{array}$ & $\begin{array}{l}37582.31 \\
37596.26 \\
37598.72 \\
37601.04 \\
37638.34\end{array}$ & $\begin{array}{l}a^{3} \mathrm{G}_{5}-y^{3} \mathrm{H}_{5}^{\circ} \\
b^{3} \mathrm{H}^{6}-w^{3} \mathrm{G}_{5}^{3} \\
a^{3} \mathrm{~F}_{4}-y^{3} \mathrm{G}_{3}^{3} \\
a^{1} \mathrm{D}_{2}-w^{3} \mathrm{D}_{3}^{3} \\
a^{5} \mathrm{D}_{1}-z^{3} \mathrm{D}_{2}^{3}\end{array}$ & $\begin{array}{l}3 \\
1 \\
2 \\
7 b, 1\end{array}$ & .22 & $\begin{array}{l}.70 \\
0 \\
0 \\
0\end{array}$ & $\begin{array}{l}\text { 1. } 11 \pm \\
\text { 1. } 10\end{array}$ & $\begin{array}{l}1.18 \\
(1.154) \\
(1.230) \\
(1.500)\end{array}$ & $\begin{array}{l}1.04 \\
1.165 \\
1.01\end{array}$ \\
\hline $\begin{array}{r}8 \\
2 \\
10 \\
80 \\
3\end{array}$ & $\begin{array}{l}2655.865 \\
2655.314 \\
2651.810 \\
2651.122 \\
2649.714\end{array}$ & $\begin{array}{l}37641.33 \\
37649.14 \\
37698.88 \\
37708.67 \\
37728.70\end{array}$ & $\begin{array}{l}b^{3} \mathrm{~F}_{2}-x^{3} \mathrm{P}_{2}^{\circ} \\
a^{3} \mathrm{G}_{4}-z \mathrm{C}_{4}^{2} \\
a^{3} \mathrm{G}_{5}-z^{3} \mathrm{I}_{6}^{6} \\
a^{3} \mathrm{G}_{5}-w^{3} \mathrm{~F}^{2} \\
b^{3} \mathrm{H}_{5}-w^{3} \mathrm{G}_{5}^{6}\end{array}$ & $\begin{array}{l}1 \\
2\end{array}$ & .50 & $\begin{array}{l}0 \\
0\end{array}$ & $\begin{array}{r}.86,1.36 \\
.44 \\
1.46\end{array}$ & $\begin{array}{c}.86 \\
(1.180) \\
(1.180)\end{array}$ & $\begin{array}{l}1.06 \\
1.11\end{array}$ \\
\hline $\begin{array}{c}3 \\
200 r s \\
3 \\
5 \\
150 r s\end{array}$ & $\begin{array}{l}2648.034 \\
2646.258 \\
2644.932 \\
2642.566 \\
2642.233\end{array}$ & $\begin{array}{l}37752.64 \\
37777.97 \\
37796.91 \\
37830.75 \\
37835.52\end{array}$ & \begin{tabular}{|l}
$b^{3} \mathrm{G}_{4}-w^{3} \mathrm{G}_{3}^{\circ}$ \\
$a^{3} \mathrm{D}_{2}-22^{5} \mathrm{D}_{3}^{3}$ \\
$a^{3} \mathrm{D}_{3}-x^{3} \mathrm{G}_{4}^{8}$ \\
$a^{3} \mathrm{G}_{3}-w^{3} \mathrm{~F}_{3}^{3}$ \\
$a^{3} \mathrm{H}_{4}-y^{3} \mathrm{H}_{4}^{8}$
\end{tabular} & $7 b, 1$ & .130 & .53 & $.824, .950$ & (1. 490) & .952 \\
\hline $\begin{array}{r}30 \\
5 \\
2 \\
5 \\
7\end{array}$ & $\begin{array}{l}2641.060 \\
2639.883 \\
2639.420 \\
2638.877 \\
2638.591\end{array}$ & $\begin{array}{l}37852.32 \\
37869.20 \\
37875.84 \\
37883.63 \\
37887.74\end{array}$ & $\begin{array}{l}a^{3} \mathrm{a}_{5}-y^{3} \mathrm{H}_{6}^{8} \\
a^{3} \mathrm{H}_{2}-y^{3} \mathrm{P}_{1}^{0} \\
b^{3} \mathrm{P}_{0}-y^{3} \mathrm{~S}_{1}^{1} \\
a^{5} \mathrm{D}_{3}-z^{3} \mathrm{G}_{3}^{3} \\
a^{3} \mathrm{P}_{0}-z^{5} \mathrm{P}_{1}^{8}\end{array}$ & $7 a$ & & 0 & 2. 256 & (1. 180) & 2. 256 \\
\hline $\begin{array}{c}10 \\
3 h \\
60 \\
3 \\
20\end{array}$ & $\begin{array}{l}2637.976 \\
2635.837 \\
2632.510 \\
2631.442 \\
2630.983\end{array}$ & $\begin{array}{l}37896.57 \\
37927.32 \\
37975.25 \\
37990.67 \\
37997.29\end{array}$ & $\begin{array}{l}a^{3} \mathrm{G}_{4}-y^{3} \mathrm{H}_{5}^{\circ} \\
c^{3} \mathrm{H}_{4}-u^{3} \mathrm{~F}_{4}^{4} \\
a^{3} \mathrm{~F}_{4}-y^{3} \mathrm{~F}^{4} \\
b^{3} \mathrm{H}_{4}-x^{1} \mathrm{~F}_{3}^{8} \\
a^{1} \mathrm{G}_{4}-y^{1} \mathrm{H}^{3}\end{array}$ & $7 b, 1$ & & $\begin{array}{l}0 \\
.47\end{array}$ & 1. $17 \pm$ & $\begin{array}{c}(1.052) \\
1.23\end{array}$ & $\begin{array}{l}1.045 \\
1.11\end{array}$ \\
\hline $\begin{array}{l}1 \\
3 \\
3 \\
3 c \\
4\end{array}$ & $\begin{array}{l}2630.354 \\
2629.209 \\
2628.408 \\
2627.055 \\
2626.636\end{array}$ & $\begin{array}{l}38006.37 \\
38022.93 \\
38034.51 \\
38054.10 \\
38060.17\end{array}$ & $\begin{array}{l}a^{3} \mathrm{G}_{3}-z^{1} \mathrm{G}_{4}^{0} \\
a^{3} \mathrm{G}_{4}-w^{3} \mathrm{~F}^{3} \mathrm{~F}^{\circ} \\
a^{3} \mathrm{P}_{1}-z^{5} \mathrm{P}^{2} \\
b^{3} \mathrm{P}_{2}-527_{3}^{2} \\
b^{3} \mathrm{G}_{4}-v^{3} \mathrm{D}_{3}^{3}\end{array}$ & 3 & & .20 & & $(1.052)$ & 1.10 \\
\hline $\begin{array}{r}4 \\
2 \\
6 \\
5 \\
20\end{array}$ & $\begin{array}{l}2626.401 \\
2624.330 \\
2623.321 \\
2623.170 \\
2622.952\end{array}$ & $\begin{array}{l}38063.58 \\
38093.61 \\
38108.26 \\
38110.46 \\
38113.62\end{array}$ & $\begin{array}{l}a^{3} \mathrm{D}_{1}-y^{1} \mathrm{D}_{2}^{0} \\
a^{3} \mathrm{P}_{1}-z^{3} \mathrm{P}_{0}^{2} \\
a^{3} \mathrm{~F}_{4}-y^{3} \mathrm{~F}_{3}^{3} \\
a^{5} \mathrm{D}_{4}-z^{3} \mathrm{G}_{4}^{4} \\
a^{3} \mathrm{P}_{2}-y^{3} \mathrm{P}_{1}^{0}\end{array}$ & 2 & .45 & 0 & 1.14 & 1. 44 & 1.74 \\
\hline $\begin{array}{c}80 \\
3 \\
10 \\
3 h \\
15\end{array}$ & $\begin{array}{l}2620.440 \\
2618.444 \\
2617.427 \\
2617.102 \\
2616.219\end{array}$ & $\begin{array}{l}38150.16 \\
38179.24 \\
38194.07 \\
38198.81 \\
38211.70\end{array}$ & $\begin{array}{l}a^{3} \mathrm{~F}_{2}-y^{3} \mathrm{~F}_{2}^{0} \\
a^{3} \mathrm{~F}_{4}-y^{3} \mathrm{G}_{5}^{2} \\
c^{3} \mathrm{P}_{2}-u^{3} \mathrm{D}_{3}^{3} \\
a^{1} \mathrm{D}_{2}-x^{3} \mathrm{P}_{1}^{\circ}\end{array}$ & $7 b, 3$ & .18 & 0 & .726 & $(.712)$ & 1. 19 \\
\hline $\begin{array}{r}10 \\
8 \\
6 \\
8 \\
2\end{array}$ & $\begin{array}{l}2614.759 \\
2614.306 \\
2613.927 \\
2613.854 \\
2609.622\end{array}$ & $\begin{array}{l}38233.04 \\
38239.66 \\
38245.21 \\
38246.27 \\
38308.30\end{array}$ & 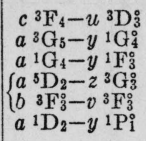 & 2 & .232 & 0 & & (1. 180) & .948 \\
\hline $\begin{array}{r}1 \\
10 \\
2 \\
5 \\
10\end{array}$ & $\begin{array}{l}2609.199 \\
2608.958 \\
2608.690 \\
2605.064 \\
2605.013\end{array}$ & $\begin{array}{l}38314.51 \\
38318.04 \\
38321.98 \\
38375.32 \\
38376.07\end{array}$ & $\begin{array}{l}a^{3} \mathrm{H}_{6}-y^{3} \mathrm{H}^{\circ} \\
a^{3} \mathrm{H}_{5}-z^{3} \mathrm{I}_{5}^{\circ} \\
a^{1} \mathrm{D}_{2}-x^{3} \mathrm{G}_{3}^{\circ} \\
b^{3} \mathrm{P}_{2}-y^{1} \mathrm{~F}_{3}^{3} \\
b^{3} \mathrm{~F}_{2}-y^{1} \mathrm{D}_{2}^{\circ}\end{array}$ & 3 & .18 & .90 & & (1. 050) & .87 \\
\hline $\begin{array}{r}10 c \\
10 \\
4 \\
100 \\
2\end{array}$ & $\begin{array}{l}2604.753 \\
2603.731 \\
2602.485 \\
2601.285 \\
2600.615\end{array}$ & $\begin{array}{l}38379.90 \\
38394.96 \\
38413.34 \\
38431.06 \\
38440.96\end{array}$ & $\begin{array}{l}a^{3} \mathrm{G}_{3}-w^{3} \mathrm{~F}^{9} \\
a^{3} \mathrm{~F}_{3}-y^{3} \mathrm{~F}_{4}^{4} \\
a^{3} \mathrm{~F}_{2}-y^{3} \mathrm{G}^{3} \\
a^{3} \mathrm{H}_{6}-z^{3} \mathrm{I}^{6} \\
a^{3} \mathrm{H}_{5}-z^{1} \mathrm{G}_{4}^{4}\end{array}$ & $\begin{array}{l}2 \\
2 \\
3\end{array}$ & .305 & $\begin{array}{l}0 \\
0 \\
.62\end{array}$ & $\begin{array}{l}1.18 \\
1.10 \pm\end{array}$ & $\begin{array}{c}(1.070) \\
(.712) \\
1.15\end{array}$ & $\begin{array}{l}\text { 1. } 10 \\
\text { 1. } 017 \\
1.05\end{array}$ \\
\hline $\begin{array}{c}10 \\
3 h \\
6 \\
4 c \\
50\end{array}$ & $\begin{array}{l}2600.156 \\
2599.522 \\
2598.037 \\
2594.964 \\
2594.736\end{array}$ & $\begin{array}{l}38447.75 \\
38457.13 \\
38479.11 \\
38524.67 \\
38528.06\end{array}$ & $\begin{array}{l}a^{3} \mathrm{D}_{2}-v^{3} \mathrm{~F}_{2}^{\circ} \\
a^{3} \mathrm{D}_{2}-v^{3} \mathrm{~F}_{3}^{3} \\
b^{3} \mathrm{G}_{4}-w^{3} \mathrm{G}_{4}^{8} \\
a^{3} \mathrm{P}_{3}-w^{8} \mathrm{D}^{8} \\
a^{3} \mathrm{~F}_{3}-y^{3} \mathrm{~F}_{3}^{3}\end{array}$ & $\begin{array}{l}3 \\
3\end{array}$ & .20 & $\begin{array}{l}.13 \\
.15\end{array}$ & $\begin{array}{l}1.043 \pm \\
1.094 \pm\end{array}$ & $\begin{array}{c}(1.027) \\
1.069\end{array}$ & 1.059 \\
\hline
\end{tabular}


TABLE 1.-Second spectrum of columbium (Cb II)-Continued

\begin{tabular}{|c|c|c|c|c|c|c|c|c|c|}
\hline $\begin{array}{l}\text { Intensity } \\
\text { spark }\end{array}$ & $\lambda_{\text {air }} \mathrm{A}$ & $\begin{array}{l}\text { Wave } \\
\text { No. } \\
\text { vac. } \\
\mathrm{cm}^{-1}\end{array}$ & $\begin{array}{c}\text { Term eombi- } \\
\text { nation }\end{array}$ & $\begin{array}{c}\text { Zeeman } \\
\text { type }\end{array}$ & $\begin{array}{l}\text { Sepa- } \\
\text { ration }\end{array}$ & $\begin{array}{c}\text { Strong- } \\
\text { est } \\
p\end{array}$ & $\underset{n}{\text { Strongest }}$ & $g_{1}$ & $g_{2}$ \\
\hline 1 & 2 & 3 & 4 & 5 & 6 & 7 & 8 & 9 & 10 \\
\hline $\begin{array}{c}10 \\
4 \\
200 R \\
8 \\
4\end{array}$ & $\begin{array}{l}2594.337 \\
2592.035 \\
2590.940 \\
2588.966 \\
2587.952\end{array}$ & $\begin{array}{l}38533.98 \\
38568.20 \\
38584.50 \\
38613.92 \\
38629.04\end{array}$ & $\begin{array}{c}a{ }^{5} \mathrm{D}_{3}-z^{3} \mathrm{G}_{4}^{0} \\
a 3^{3} \mathrm{H}_{4}-w^{3} \mathrm{~F}^{3} \\
a^{3} \mathrm{H}_{6}-y^{3} \mathrm{H}^{6} \\
b^{3} \mathrm{~F}_{4}-v^{3} \mathrm{~F}_{4}^{6} \\
a^{3} \mathrm{~F}_{4}-x^{3} \mathrm{~F}_{3}^{6}\end{array}$ & 3 & 0.22 & 0.18 & $1.14 \pm$ & $\begin{array}{c}1.155 \\
(1.230)\end{array}$ & $\begin{array}{l}1.125 \\
1.01\end{array}$ \\
\hline $\begin{array}{c}8 \\
4 \\
250 \\
30 \\
6\end{array}$ & $\begin{array}{l}2587.409 \\
2586.911 \\
2583.982 \\
2580.284 \\
2575.968\end{array}$ & $\begin{array}{l}38637.15 \\
38644.59 \\
38688.39 \\
38743.83 \\
38808.74\end{array}$ & 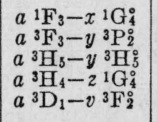 & $\begin{array}{l}7 b, 1 \\
1 \\
7 b, 3 \\
3 \\
2\end{array}$ & $\begin{array}{l}.26 \\
.30\end{array}$ & $\begin{array}{l}0 \\
0 \\
0 \\
1.04 \\
0\end{array}$ & $\begin{array}{r}.995 \\
1.037 \\
0.83,1.08 \\
1.10\end{array}$ & $\begin{array}{c}(1.007) \\
(1.070) \\
(1.050) \\
.83 \\
.50\end{array}$ & $\begin{array}{l}\text { 1. } 004 \\
1.515 \\
1.024 \\
1.08 \\
.80\end{array}$ \\
\hline $\begin{array}{r}2 \\
40 \\
5 \\
15 \\
5\end{array}$ & $\begin{array}{l}2575.570 \\
2574.843 \\
2574.074 \\
2573.136 \\
2573.022\end{array}$ & $\begin{array}{l}38814.74 \\
3882.70 \\
38837.30 \\
38851.45 \\
38853.18\end{array}$ & $\begin{array}{l}a^{3} \mathrm{H}_{5}-w^{3} \mathrm{~F}_{2}^{2} \\
a^{3} \mathrm{D}_{1}-z 3^{3} \mathrm{~F}_{2}^{2} \\
a^{3} \mathrm{~F}_{2}-x 3^{3} \mathrm{~F}_{2}^{2} \\
c^{3} \mathrm{~F}_{2}-v^{3} G_{3}^{2} \\
a^{3} \mathrm{~F}_{2}-z^{3} \mathrm{~S}_{1}^{3}\end{array}$ & 3 & .29 & .58 & & $(.712)$ & .42 \\
\hline 60 & $\begin{array}{l}2571.74 \\
2571.324\end{array}$ & $\begin{array}{l}38872.5 \\
38878.83\end{array}$ & $\begin{array}{l}a^{3} \mathrm{D}_{3}-v^{3} \mathrm{~F}_{2}^{0} \\
a^{5} \mathrm{D}_{4}-z^{3} G_{5}^{2}\end{array}$ & 1 & .264 & 0 & .176 & 1.496 & 1. 232 \\
\hline 3 & 2569. 187 & 38911. 17 & $\left\{\begin{array}{l}a a_{1} \mathrm{G}_{4}-w^{3} \mathrm{G}_{3}^{3} \\
a^{3} \mathrm{G}_{3}-y^{1} \mathrm{G}_{4}^{3}\end{array}\right.$ & & & & & & \\
\hline$\frac{2}{10}$ & $\begin{array}{l}2568.677 \\
2568.409\end{array}$ & $\begin{array}{l}38918.89 \\
38922.95\end{array}$ & $\begin{array}{l}a{ }^{1}{ }^{1} \mathrm{D}_{2}-y \\
a^{3} \mathrm{~F}_{2}-y_{2} \mathrm{D}_{2}^{0} \\
{ }^{3} \mathrm{~F}_{3}^{3}\end{array}$ & & & & & & \\
\hline $\begin{array}{r}5 \\
4 \\
20 \\
15 \\
1\end{array}$ & $\begin{array}{l}2566.075 \\
2565.675 \\
2565.504 \\
2564.846 \\
2564.526\end{array}$ & $\begin{array}{l}38958.35 \\
38964.42 \\
38967.02 \\
38977.02 \\
38981.89\end{array}$ & $\begin{array}{r}a^{3} \mathrm{H}_{5}-z^{3} \mathrm{H}_{6}^{0} \\
c^{3} \mathrm{~F}_{2}-u^{3} \mathrm{D}_{1}^{0} \\
c^{3} \mathrm{~F}_{4}-v^{3} \mathrm{G}_{0}^{1} \\
-c^{3} \mathrm{~F}_{3}-v^{3} \mathrm{G}_{4}^{1} \\
b^{3} \mathrm{~F}_{2}-y^{1} \mathrm{P}_{1}^{0}\end{array}$ & $7 b, 2$ & & 0 & 1.13 & (1.08) & 1.09 \\
\hline $\begin{array}{l}15 \\
10\end{array}$ & $\begin{array}{l}2564.070 \\
2563.913\end{array}$ & $\begin{array}{l}38988.81 \\
38991.20\end{array}$ & $\begin{array}{l}b^{3} \mathrm{D}_{1}-u^{3} \mathrm{~F}_{2}^{\circ} \\
a^{3} \mathrm{H}_{4}-y^{3} \mathrm{H}^{2}\end{array}$ & 2 & .29 & 0 & & (.681) & .97 \\
\hline $\begin{array}{r}120 \\
1 \\
6\end{array}$ & $\begin{array}{l}2562.402 \\
2561.926 \\
2561.708\end{array}$ & $\begin{array}{l}39014.19 \\
39021.45 \\
39024.76\end{array}$ & $\begin{array}{l}a^{3}{ }^{3} \mathrm{P}_{1}-y^{3} \mathrm{P}_{0}^{0} \\
a^{1} \mathrm{H}_{5}-v^{3} \mathrm{G}_{4}^{4} \\
a^{3} \mathrm{~F}_{4}-y^{3} \mathrm{H}_{4}^{4}\end{array}$ & $7 a$ & & 0 & 1. 500 & 1.500 & $0 / 0$ \\
\hline $\begin{array}{c}2 c \\
3 \\
20 \\
60 \\
3\end{array}$ & $\begin{array}{l}2561.465 \\
2560.741 \\
2560.622 \\
2560.112 \\
2558.628\end{array}$ & $\begin{array}{l}39028.46 \\
39039.50 \\
39041.31 \\
39049.09 \\
39071.74\end{array}$ & $\begin{array}{l}a^{3} \mathrm{P}_{2}-w^{3} \mathrm{D}^{3} \\
a^{3} \mathrm{~F}_{2}-y^{3} \mathrm{P}^{3} \\
b^{3} \mathrm{P}_{2}-w^{3} \mathrm{G}_{3}^{3} \\
a^{3} \mathrm{~F}_{3}-x^{3} \mathrm{~F}^{3} \\
b^{3} \mathrm{G}_{4}-w^{3} \mathrm{G}_{5}^{3}\end{array}$ & 1 & .592 & 0 & .300 & 1.484 & .892 \\
\hline $\begin{array}{r}120 \\
60\end{array}$ & $\begin{array}{l}2556.933 \\
2555.626\end{array}$ & $\begin{array}{l}39097.63 \\
39117.63\end{array}$ & $\begin{array}{l}a^{3} \mathrm{P}_{2}-z^{3} \mathrm{~S} \\
a^{3} \mathrm{~S}_{4} \\
{ }^{3} \mathrm{H}^{3} \mathrm{~F}\end{array}$ & $\begin{array}{l}1 \\
3\end{array}$ & $\begin{array}{l}.368 \\
.277\end{array}$ & $\begin{array}{l}0 \\
1.114\end{array}$ & $\begin{array}{c}1.079 \\
.828,1.099\end{array}$ & $\begin{array}{r}1.447 \\
.825\end{array}$ & $\begin{array}{l}1.815 \\
1.102\end{array}$ \\
\hline $\begin{array}{r}8 \\
8 \\
15\end{array}$ & $\begin{array}{l}2555.314 \\
2554.793 \\
2553.490\end{array}$ & $\begin{array}{l}39122.40 \\
39130.38 \\
39150.35\end{array}$ & $\begin{array}{l}b^{3} \mathrm{~F}_{4}-y y^{1} \mathrm{H}^{5} \\
b^{3} \mathrm{~F}_{2}-v^{3} \mathrm{~F}_{3}^{3} \\
b^{3} \mathrm{D}_{2}-u^{3} \mathrm{~F}_{3}^{\circ}\end{array}$ & $\begin{array}{l}2 \\
2\end{array}$ & .24 & $\begin{array}{l}0 \\
0\end{array}$ & $\begin{array}{l}\text { 1. } 57 \\
1.411\end{array}$ & $\begin{array}{c}.85 \\
(1.171)\end{array}$ & $\begin{array}{l}\text { 1. } 09 \\
1.251\end{array}$ \\
\hline $\begin{array}{c}120 \\
2 \\
2 h \\
1\end{array}$ & $\begin{array}{l}2551.382 \\
2550.037 \\
2549.435 \\
2549.039 \\
2510\end{array}$ & $\begin{array}{l}39182.69 \\
39203.36 \\
39212.62 \\
39218.70\end{array}$ & $\begin{array}{l}a^{3} \mathrm{P}_{1}-y^{3} \mathrm{P}^{\circ} \\
a^{3} \mathrm{H}_{6}-z^{3} \mathrm{I}_{7}^{0} \\
a^{5} \mathrm{P}_{3}-x^{3} \mathrm{~F}_{4}^{8} \\
a^{1} \mathrm{G}_{4}-v^{3} \mathrm{D}_{3}^{8}\end{array}$ & 3 & .264 & .266 & 1. $498,1.760$ & 1. 497 & 1. 761 \\
\hline & 2548.634 & 39224.94 & $a{ }^{3} \mathrm{D}_{3}-v{ }^{3} \mathrm{~F}_{4}^{8}$ & $7 b, 2$ & & 0 & 1. 282 & $(1.246)$ & 1.25 \\
\hline $\begin{array}{c}2 \\
2 \\
2 \\
200 R \\
1\end{array}$ & $\begin{array}{l}2547.556 \\
2546.353 \\
2545.404 \\
2544.802 \\
2544.435\end{array}$ & $\begin{array}{l}39241.53 \\
39260.07 \\
39274.71 \\
39284.00 \\
39289.66\end{array}$ & $\begin{array}{l}b^{1} \mathrm{C}_{4}-x{ }^{1} \mathrm{~F}_{3}^{0} \\
a^{3} \mathrm{G}_{4}-w^{3} \mathrm{D}^{3} \\
c^{3} \mathrm{P}_{0}-u^{3} \mathrm{D}^{3} \\
a^{3} \mathrm{P}_{2}-y^{3} \mathrm{P}_{2} \\
a^{3} \mathrm{G}_{3}-495_{2}^{\circ}\end{array}$ & 3 & & .102 & 1. $480 \pm$ & 1.454 & 1.505 \\
\hline $\begin{array}{r}50 \\
2\end{array}$ & $\begin{array}{l}2541.424 \\
2541.074\end{array}$ & $\begin{array}{l}39336.21 \\
39341.63\end{array}$ & $\begin{array}{l}a^{5} \mathrm{D}_{4}-z^{3} \mathrm{~F}_{4} \\
a^{3} \mathrm{D}_{2}-2^{3} \mathrm{~F}^{3}\end{array}$ & 3 & .235 & .94 & 1. $260,1.495$ & 1. 495 & 1. 260 \\
\hline 3 & 2540.811 & 39345.70 & 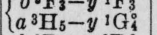 & & & & & & \\
\hline $\begin{array}{l}80 \\
10\end{array}$ & $\begin{array}{l}2540.611 \\
2539.224\end{array}$ & $\begin{array}{l}39348.80 \\
39370.29\end{array}$ & $\begin{array}{l}b^{3} \mathrm{P}_{\mathbf{2}}-v^{3} \mathrm{D}_{\mathbf{3}}^{6} \\
b^{3} \mathrm{~F}_{4}-y^{1} \mathrm{~F}_{3}^{6}\end{array}$ & 1 & .273 & 0 & .66 & 1.479 & 1. 206 \\
\hline $\begin{array}{l}10 h \\
30 \\
30 \\
80 \\
3\end{array}$ & $\begin{array}{l}2534.445 \\
2533.188 \\
2531.252 \\
2530.968 \\
2530.168\end{array}$ & $\begin{array}{l}39444.52 \\
39464.08 \\
39494.27 \\
39498.70 \\
39511.19\end{array}$ & 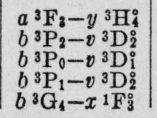 & $\begin{array}{l}3 \\
7 a \\
1\end{array}$ & $\begin{array}{l}.30 \\
.32\end{array}$ & $\begin{array}{l}.60 \\
0^{.60}\end{array}$ & $\begin{array}{l}.515 \\
.840\end{array}$ & $\begin{array}{c}(1.483) \\
0 / 0 \\
1.48\end{array}$ & $\begin{array}{l}1.18 \\
1.515\end{array}$ \\
\hline
\end{tabular}


TABLE 1. -Second spectrum of columbium (Cb II)-Continued

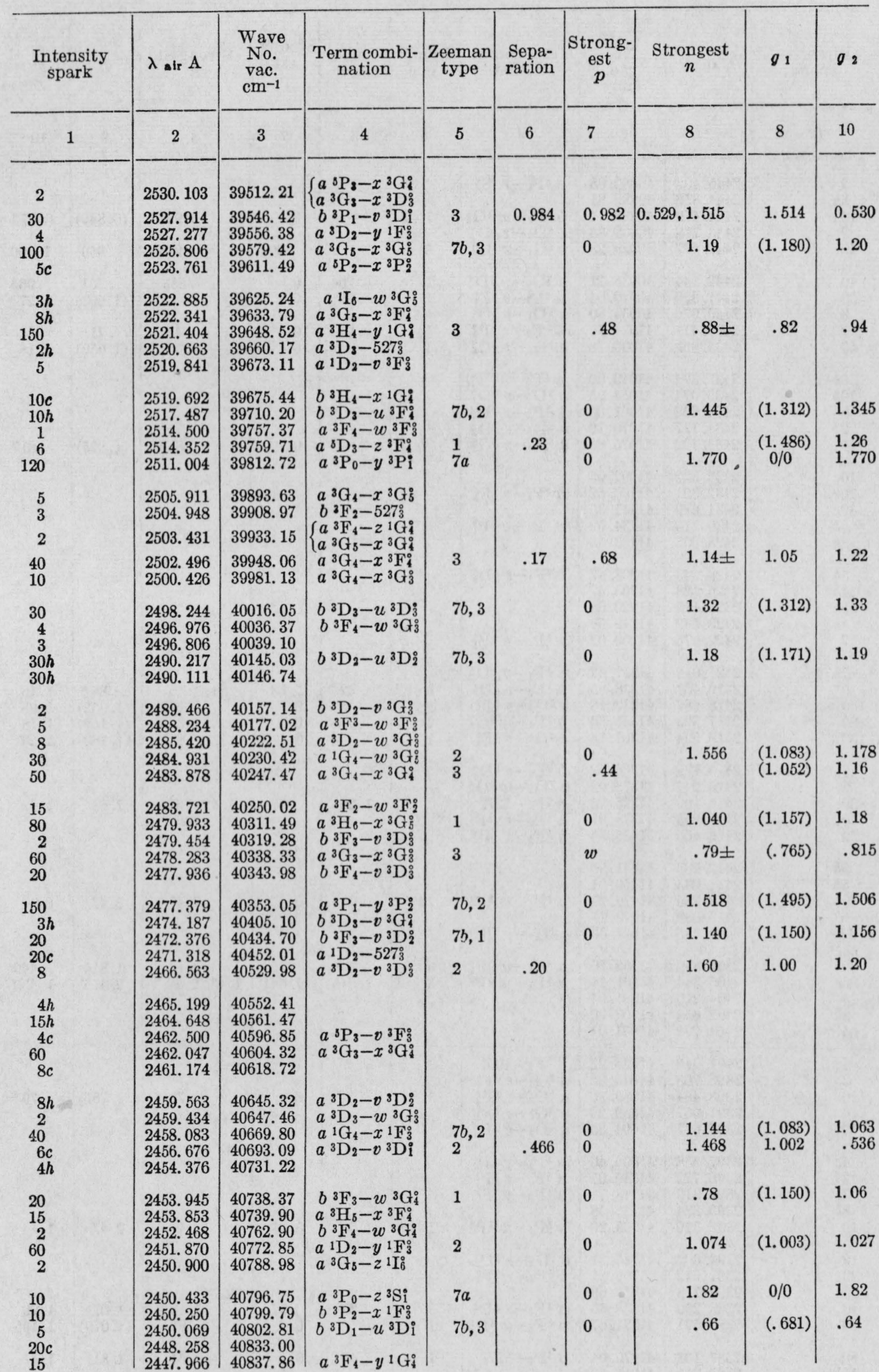


504 Journal of Research of the National Bureau of Standards

TABLE 1.-Second spectrum of columbium (Cb II)-Continued

\begin{tabular}{|c|c|c|c|c|c|c|c|c|c|}
\hline $\begin{array}{l}\text { Intensity } \\
\text { spark }\end{array}$ & $\lambda_{\text {sir } A}$ & $\begin{array}{l}\text { Wave } \\
\text { No. } \\
\text { vac. } \\
\mathrm{cm}^{-1}\end{array}$ & $\begin{array}{l}\text { Term combi- } \\
\text { nation }\end{array}$ & $\begin{array}{c}\text { Zeeman } \\
\text { type }\end{array}$ & $\begin{array}{l}\text { Sepa- } \\
\text { ration }\end{array}$ & $\begin{array}{c}\text { Strong- } \\
\text { est } \\
p\end{array}$ & $\underset{n}{\text { Strongest }}$ & $g_{1}$ & $g_{2}$ \\
\hline 1 & 2 & 3 & 4 & 5 & 6 & 7 & 8 & 9 & 10 \\
\hline $\begin{array}{c}2 \\
3 h \\
10 \\
2 \\
40\end{array}$ & $\begin{array}{l}2445.402 \\
2444.856 \\
2444.479 \\
2443.718 \\
2442.677\end{array}$ & $\begin{array}{l}40880.68 \\
40889.81 \\
40896.11 \\
40908.85 \\
40926.28\end{array}$ & $\begin{array}{l}a^{3} \mathrm{P}_{1}-z^{5} \mathrm{~S}_{2} \\
b^{3} \mathrm{~B}_{2}-w^{3} \mathrm{G}_{3} \\
a^{3} \mathrm{D}_{3}-z^{1} \mathrm{D}_{2} \\
b^{1} \mathrm{G}_{1}-x^{1} \mathrm{G}_{4}^{8}\end{array}$ & $7 b, 2$ & & $\begin{array}{l}0 \\
.20\end{array}$ & $\begin{array}{r}0.921 \\
.975 \pm\end{array}$ & $\begin{array}{c}(0.849) \\
.950\end{array}$ & $\begin{array}{l}0.873 \\
1.000\end{array}$ \\
\hline $\begin{array}{r}40 \\
30 \\
8 \\
50 \\
50\end{array}$ & $\begin{array}{l}2442.144 \\
2441.856 \\
2440.976 \\
2437.411 \\
2435.952\end{array}$ & $\begin{array}{l}40935.21 \\
40940.04 \\
40954.80 \\
41014.70 \\
41039.26\end{array}$ & $\begin{array}{c}a^{3} \mathrm{G}_{3}-y{ }^{1} \mathrm{D}_{2}^{\circ} \\
a^{3} \mathrm{P}_{3}-v{ }^{3} \mathrm{~F}_{4}^{0} \\
a^{3} \mathrm{D}_{3}-v{ }^{3} \mathrm{D}_{3}^{3} \\
a^{3} \mathrm{~F}_{2}-z \\
a^{3} \mathrm{P}_{1}^{0} \mathrm{H}_{3}-x \\
{ }^{3} \mathrm{G}_{4}^{\circ}\end{array}$ & $\begin{array}{l}1 \\
1\end{array}$ & $\begin{array}{c}0.204 \\
.39 \\
.24\end{array}$ & $\begin{array}{l}0 \\
0 \\
0 \\
0\end{array}$ & $\begin{array}{l}.47 \\
.53\end{array}$ & $\begin{array}{c}.761 \\
(1.663) \\
.71 \\
(1.050)\end{array}$ & $\begin{array}{r}.965 \\
1.27 \\
.95 \\
1.18\end{array}$ \\
\hline $\begin{array}{c}5 c \\
10 h \\
3 \\
2 \\
60\end{array}$ & $\begin{array}{l}2435.374 \\
2435.074 \\
2434.662 \\
2434.117 \\
2433.792\end{array}$ & $\begin{array}{l}41049.00 \\
41054.05 \\
41061.00 \\
41070.19 \\
41075.68\end{array}$ & 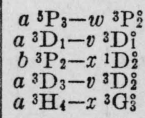 & $7 b, 2$ & & 0 & .850 & $(.825)$ & .817 \\
\hline $\begin{array}{r}10 c \\
10 c \\
30 c \\
4 h \\
8 h\end{array}$ & $\begin{array}{l}2432.822 \\
2432.321 \\
2431.679 \\
2430.310 \\
2428.603\end{array}$ & $\begin{array}{l}41092.05 \\
41100.52 \\
41111.36 \\
41134.52 \\
41163.43\end{array}$ & $\begin{array}{l}a^{5} \mathrm{P}_{2}-v^{3} \mathrm{~F}_{3}^{0} \\
a^{5} \mathrm{P}_{1}-y{ }^{1} \mathrm{P}_{1}^{\circ}\end{array}$ & & & & & & \\
\hline $\begin{array}{l}5 h \\
4 h \\
5 h \\
4 h \\
2\end{array}$ & $\begin{array}{l}2426.243 \\
2426.128 \\
2425.112 \\
2423.532 \\
2422.976\end{array}$ & $\begin{array}{l}41203.47 \\
41205.42 \\
41222.68 \\
41249.56 \\
41259.02\end{array}$ & $b{ }^{3} \mathrm{~F}_{2}-v{ }^{3} \mathrm{D}_{3}^{\circ}$ & & & & & & \\
\hline $\begin{array}{r}2 \\
10 \\
150 \\
20 \\
150\end{array}$ & $\begin{array}{l}2421.016 \\
2419.467 \\
2418.687 \\
2417.323 \\
2416.994\end{array}$ & $\begin{array}{l}41292.42 \\
41318.85 \\
41332.18 \\
41355.50 \\
41361.13\end{array}$ & $\begin{array}{c}a{ }^{3} \mathrm{~F}_{1}-y^{3} \mathrm{D}_{1}^{\mathrm{i}} \\
b^{3} \mathrm{~F}_{2}-v^{3} \mathrm{D}_{2}^{2} \\
a^{3} \mathrm{G}_{4}-v^{3} \mathrm{~F}_{3}^{0} \\
b^{3} \mathrm{~F}_{4}-v^{3} \mathrm{G}^{3} \\
a^{3} \mathrm{G}_{5}-v^{3} \mathrm{~F}_{4}^{0}\end{array}$ & $\begin{array}{l}3 \\
1 \\
2 \\
1\end{array}$ & .32 & $\begin{array}{l}0^{.63} \\
0 \\
0\end{array}$ & $\begin{array}{r}.84,1.16 \\
.97 \\
1.31 \\
.88\end{array}$ & $\begin{array}{c}.84 \\
(1.052) \\
(1.152) \\
(1.180)\end{array}$ & $\begin{array}{l}1.16 \\
1.08 \\
1.18 \\
1.26\end{array}$ \\
\hline $\begin{array}{c}2 \\
3 \\
30 \\
10 c \\
150\end{array}$ & $\begin{array}{l}2416.679 \\
2416.247 \\
2416.169 \\
2415.955 \\
2412.460\end{array}$ & $\begin{array}{l}41366.52 \\
41373.92 \\
41375.25 \\
41378.91 \\
41438.86\end{array}$ & $\begin{array}{c}b^{3} \mathrm{SF}_{2}-v^{3} \mathrm{Di}_{1}^{\circ} \\
a^{3} \mathrm{D}_{3}-w^{3} \mathrm{G}_{4}^{\circ} \\
a^{5} \mathrm{P}_{3}-527_{3}^{\circ} \\
a^{5} \mathrm{P}_{2}-w^{3} \mathrm{P}_{1} \\
a^{3} \mathrm{~F}_{4}-x^{3} \mathrm{D}_{3}^{\circ}\end{array}$ & 3 & 1. 26 & .77 & $1.42, .68$ & 1.68 & 1.42 \\
\hline $\begin{array}{c}5 h \\
5 h \\
20 c \\
6 \\
20 h\end{array}$ & $\begin{array}{l}\text { 2412. } 283 \\
2411.235 \\
2410.285 \\
2410.080 \\
2407.685\end{array}$ & $\begin{array}{l}41441.90 \\
41459.91 \\
41476.25 \\
41479.77 \\
41521.03\end{array}$ & $\begin{array}{l}a{ }^{5} \mathrm{P}_{1}-w^{3} \mathrm{P}_{0}^{0} \\
a^{3} \mathrm{H}_{6}-2{ }^{1} \mathrm{I}_{6}^{8}\end{array}$ & $7 a$ & & 0 & 2.47 & 2.47 & $0 / 0$ \\
\hline $\begin{array}{c}60 c \\
50 c \\
20 \\
8 h \\
4\end{array}$ & $\begin{array}{l}2405.850 \\
2405.344 \\
2404.210 \\
2402.658 \\
2401.272\end{array}$ & $\begin{array}{l}41552.70 \\
41561.44 \\
41581.04 \\
41607.90 \\
41631.91\end{array}$ & $\begin{array}{l}a^{5} \mathrm{P}_{2}-w^{8} \mathrm{P}_{2}^{0} \\
a^{5} \mathrm{P}_{1}-w^{3} \mathrm{P}_{1}^{0}\end{array}$ & $\begin{array}{l}3 \\
3\end{array}$ & $\begin{array}{r}.375 \\
1.045\end{array}$ & $\begin{array}{l}.75 \\
1.041\end{array}$ & $\begin{array}{l}1.44,1.81 \\
1.43,2.48\end{array}$ & $\begin{array}{l}1.815 \\
2.475\end{array}$ & $\begin{array}{l}1.440 \\
1.430\end{array}$ \\
\hline $\begin{array}{r}4 \\
2 \\
120 \\
6 \\
2\end{array}$ & $\begin{array}{l}2401.040 \\
2398.768 \\
2398.484 \\
2397.967 \\
2397.677\end{array}$ & $\begin{array}{l}41635.93 \\
41675.36 \\
41680.30 \\
41689.29 \\
41694.33\end{array}$ & $\begin{array}{l}a^{3} \mathrm{~F}_{3}-495_{2}^{\circ} \\
a^{3} \mathrm{G}_{4}-v^{3} \mathrm{~F}_{4}^{\circ} \\
a^{3} \mathrm{G}_{3}-v^{3} \mathrm{~F}_{2}^{0} \\
a^{3} \mathrm{G}_{3}-v^{3} \mathrm{~F}_{3}^{0} \\
a^{5} \mathrm{D}_{2}-y^{5} \mathrm{D}_{1}^{\circ}\end{array}$ & 1 & & 0 & .72 & $(.765)$ & .79 \\
\hline $\begin{array}{l}4 c \\
2 \\
3 c \\
8 h \\
40\end{array}$ & $\begin{array}{l}2397.558 \\
2396.772 \\
2396.310 \\
2395.824 \\
2395.329\end{array}$ & $\begin{array}{l}41696.40 \\
41710.07 \\
41718.11 \\
41726.58 \\
41735.20\end{array}$ & $\begin{array}{l}a^{5} \mathrm{P}_{3}-y^{1} \mathrm{~F}_{3}^{0} \\
a^{5} \mathrm{~F}_{1}-z{ }^{3} \mathrm{P}_{1}^{0} \\
a^{5} \mathrm{P}_{2}-y^{3} \mathrm{~S}_{1}^{\circ} \\
a^{5} \mathrm{P}_{1}-w^{3} \mathrm{P}_{2}^{0}\end{array}$ & 1 & 1.03 & 0 & .41 & 2.47 & 1.44 \\
\hline $\begin{array}{c}2 \\
20 \\
3 h \\
40 \\
80\end{array}$ & $\begin{array}{l}2394.697 \\
2391.912 \\
2390.718 \\
2388.269 \\
2387.521\end{array}$ & $\begin{array}{l}41746.21 \\
41794.81 \\
41815.69 \\
41858.56 \\
41871.67\end{array}$ & $\begin{array}{l}a{ }^{1} \mathrm{D}_{2}-v{ }^{3} \mathrm{D}_{3}^{\circ} \\
b{ }^{3} \mathrm{~F}_{4}-x \\
{ }^{1} \mathrm{~F}_{3}^{\circ} \\
a^{3} \mathrm{~F}_{3}-x \\
a^{3} \mathrm{D}_{3}^{\circ} \mathrm{D}_{3}-w^{3} \mathrm{D}_{2}^{\circ}\end{array}$ & $\begin{array}{l}3 \\
1\end{array}$ & .22 & $0^{.67}$ & $\begin{array}{l}1.18 \pm \\
.88\end{array}$ & $\begin{array}{l}1.07 \\
(1.070)\end{array}$ & $\begin{array}{l}1.29 \\
1.165\end{array}$ \\
\hline $\begin{array}{c}80 \\
10 h \\
5 h \\
3 \\
2\end{array}$ & $\begin{array}{l}2387.101 \\
2385.251 \\
2384.852 \\
2381.717 \\
2381.122\end{array}$ & $\begin{array}{l}41879.04 \\
41911.52 \\
41918.52 \\
41973.80 \\
41984.19\end{array}$ & $\begin{array}{l}a^{5} \mathrm{P}_{2}-527^{\circ} \\
a^{5} \mathrm{D}_{1}-y^{5} \mathrm{D}_{1}^{\circ} \\
a^{3} \mathrm{P}_{2}-x^{8} \mathrm{D}_{2}^{\circ}\end{array}$ & 1 & .40 & 0 & .61 & 1.81 & 1.41 \\
\hline
\end{tabular}


TABLE 1.-Second spectrum of columbium (Cb II)-Continued

\begin{tabular}{|c|c|c|c|c|c|c|c|c|c|}
\hline $\begin{array}{l}\text { Intensity } \\
\text { spark }\end{array}$ & $\lambda_{\mathrm{sir}} \mathrm{A}$ & $\begin{array}{l}\text { Wave } \\
\text { No. } \\
\text { vac. } \\
\mathrm{cm}^{-1}\end{array}$ & $\begin{array}{l}\text { Term combi- } \\
\text { nation }\end{array}$ & $\begin{array}{c}\text { Zeeman } \\
\text { type }\end{array}$ & $\begin{array}{l}\text { Sepa- } \\
\text { ration }\end{array}$ & $\begin{array}{c}\text { Strong- } \\
\text { est } \\
p\end{array}$ & $\underset{n}{\text { Strongest }}$ & $g_{1}$ & $g_{3}$ \\
\hline 1 & 2 & 3 & 4 & 5 & 6 & 7 & 8 & 9 & 10 \\
\hline $\begin{array}{c}10 h \\
5 h \\
4 \\
10 \\
100\end{array}$ & $\begin{array}{l}2380.148 \\
2379.122 \\
2378.476 \\
2377.989 \\
2376.398\end{array}$ & $\begin{array}{l}42001.37 \\
42019.48 \\
42030.89 \\
42039.50 \\
42067.64\end{array}$ & $\begin{array}{l}a^{3} \mathrm{~F}_{2}-495_{2} \\
b{ }^{3} \mathrm{H}_{4}-v^{3} \mathrm{G}_{3} \\
a^{5} \mathrm{D}_{3}-2{ }^{3} \mathrm{H}_{4}\end{array}$ & & & & & & \\
\hline $\begin{array}{c}8 \\
10 c \\
60 \\
8 c \\
100\end{array}$ & $\begin{array}{l}2374.167 \\
2373.967 \\
2372.730 \\
2372.227 \\
2369.954\end{array}$ & $\begin{array}{l}42107.16 \\
42110.71 \\
42132.66 \\
42141.60 \\
42182.01\end{array}$ & $\begin{array}{l}b{ }^{3} \mathrm{H}_{5}-u^{3} \mathrm{~F}_{4}^{0} \\
a{ }^{3} \mathrm{G}_{4}-527_{3}^{\circ} \\
a{ }^{5} \mathrm{D}_{0}-y{ }^{3} \mathrm{D}_{1}^{\circ} \\
a^{3} \mathrm{G}_{3}-w^{3} \mathrm{P}_{2}^{\circ} \\
a^{3} \mathrm{~F}_{2}-x{ }^{3} \mathrm{D}_{1}^{\circ}\end{array}$ & 1 & & 0 & 0.62 & $(0.712)$ & 0.81 \\
\hline $\begin{array}{c}8 c \\
20 h \\
10 h \\
20 h \\
3 h\end{array}$ & $\begin{array}{l}2368.941 \\
2366.198 \\
2365.745 \\
2365.624 \\
2365.305\end{array}$ & $\begin{array}{l}42200.05 \\
42248.96 \\
42257.05 \\
42259.21 \\
42264.91\end{array}$ & $\begin{array}{l}a{ }^{5} \mathrm{P}_{2}-y{ }^{1} \mathrm{~F}_{3}^{0} \\
b^{3} \mathrm{G}_{3}-u^{3} \mathrm{~F}_{2}^{0} \\
a^{3} \mathrm{~F}_{4}-x^{3} \mathrm{G}_{3}\end{array}$ & & & & t & & \\
\hline $\begin{array}{l}70 \\
2 h \\
3 \\
80 \\
3\end{array}$ & $\begin{array}{l}2365.215 \\
2364.270 \\
2363.587 \\
2360.302 \\
2357.437\end{array}$ & $\begin{array}{l}42266.52 \\
42283.41 \\
42295.63 \\
42354.49 \\
42405.96\end{array}$ & $\begin{array}{l}a^{3} \mathrm{~F}_{2}-w^{3} \mathrm{D}_{2}^{0} \\
b^{1} \mathrm{G}_{4}-u^{3} \mathrm{~F}_{3}^{0} \\
a^{3} \mathrm{~F}_{2}-z^{3} \mathrm{P}_{2}^{0} \\
a^{1} \mathrm{G}_{4}-x{ }^{1} \mathrm{G}_{4}^{0} \\
a^{3}{ }^{3} \mathrm{D}_{3}-x{ }^{1} \mathrm{~F}_{3}^{0}\end{array}$ & 3 & 0.46 & .27 & $1.05 \pm$ & $\begin{array}{r}.70 \\
1.09\end{array}$ & $\begin{array}{l}1.16 \\
1.01\end{array}$ \\
\hline 50 & 2356. 290 & 42426.60 & $\left\{\begin{array}{l}a^{3} \mathrm{P}_{2}-x{ }^{3} \mathrm{D} \\
a^{3} \mathrm{~B}_{4}-v^{3} \mathrm{~F}_{3}\end{array}\right.$ & & & & & & \\
\hline $\begin{array}{r}40 \\
8 \\
50 \\
3\end{array}$ & $\begin{array}{l}2356.005 \\
2355.680 \\
2354.040 \\
2353.765\end{array}$ & $\begin{array}{l}42431.73 \\
42437.59 \\
42467.15 \\
42472.11\end{array}$ & 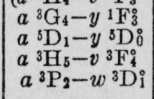 & $7 b, 2$ & & 0 & 1.07 & $(1.052)$ & 1. 04 \\
\hline $\begin{array}{c}60 \\
60 \\
4 \\
20 h \\
5 h\end{array}$ & $\begin{array}{l}2352.837 \\
2352.338 \\
2351.613 \\
2350.488 \\
2349.411\end{array}$ & $\begin{array}{l}42488.86 \\
42497.87 \\
42510.98 \\
42531.32 \\
42550.81\end{array}$ & $\begin{array}{l}a{ }^{5} \mathrm{D}_{3}-y{ }^{3} \mathrm{D}_{2} \\
a^{3} \mathrm{P}_{2}-x{ }^{3} \mathrm{D}_{3} \\
a^{3} \mathrm{P}_{2}-w^{3} \mathrm{D}_{2} \\
a^{3} \mathrm{~F}_{1}-x^{3} \mathrm{G}_{4}^{\circ}\end{array}$ & & & & & & . \\
\hline $\begin{array}{l}2 \\
3 \\
50 \\
15 h \\
10\end{array}$ & $\begin{array}{l}2347.111 \\
2346.961 \\
2346.532 \\
2345.333 \\
2343.271\end{array}$ & $\begin{array}{l}42592.50 \\
42595.22 \\
42603.01 \\
42624.79 \\
42662.30\end{array}$ & $\begin{array}{l}a^{5} \mathrm{~F}_{3}-y^{3} \mathrm{G}_{4} \\
a^{3} \mathrm{P}_{2}-w^{3} \mathrm{D}^{3} \\
b^{3} \mathrm{H}_{4}-v^{3} \mathrm{G}_{4} \\
a^{5} \mathrm{D}_{4}-y^{5} \mathrm{D}_{3}^{8}\end{array}$ & 1 & & 0 & 1. 10 & (1. 450$)$ & 1. 33 \\
\hline $\begin{array}{l}3 h \\
4 h \\
20 h \\
30 \\
20\end{array}$ & $\begin{array}{l}2343.103 \\
2342.852 \\
2340.025 \\
2335.620 \\
2335.322\end{array}$ & $\begin{array}{l}42665.36 \\
42669.93 \\
42721.47 \\
42802.04 \\
42807.68\end{array}$ & $\begin{array}{l}a^{5} \mathrm{P}_{3}-v^{3} \mathrm{D}_{3}^{\circ} \\
b^{3} \mathrm{H}_{5}-v^{3} \mathrm{G}_{i}^{\circ} \\
a^{3} \mathrm{P}_{2}-500_{3}^{\circ}\end{array}$ & & & & & & \\
\hline $\begin{array}{c}100 \\
10 \\
3 h \\
4 \\
3 h\end{array}$ & $\begin{array}{l}2334.802 \\
2332.896 \\
2332.295 \\
2328.028 \\
2327.521\end{array}$ & $\begin{array}{l}42817.03 \\
42852.01 \\
42863.05 \\
42941.61 \\
42950.96\end{array}$ & $\begin{array}{l}a{ }^{5} \mathrm{D}_{3}-y \\
a{ }^{5} \mathrm{D}_{2}-y \\
{ }^{3} \mathrm{D}_{2} \\
a^{3} \mathrm{~F}_{2}-x \\
a^{3} \mathrm{P}_{2}^{\circ} \\
{ }^{3} \mathrm{~F}_{3}-x \\
{ }^{3} \mathrm{G}_{4}\end{array}$ & & & 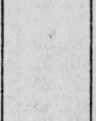 & & & \\
\hline $\begin{array}{c}10 \\
20 \\
6 h \\
3 h \\
60\end{array}$ & $\begin{array}{l}2327.131 \\
2326.221 \\
2325.504 \\
2324.409 \\
2324.237\end{array}$ & $\begin{array}{l}42958.16 \\
42974.96 \\
42988.21 \\
43008.46 \\
43011.75\end{array}$ & $\begin{array}{c}a^{3} \mathrm{P}_{0}-y{ }^{1} \mathrm{P}_{i}^{\circ} \\
a^{3} \mathrm{H}_{5}-y{ }^{1} \mathrm{H}_{5}^{\circ} ? \\
a^{5} \mathrm{D}_{2}-2{ }^{5} \mathrm{P}^{\circ}\end{array}$ & 1 & .75 & 0 & .75 & 1. 50 & 2.25 \\
\hline $\begin{array}{l}40 \\
10 h \\
30 \\
3 h \\
7 h\end{array}$ & $\begin{array}{l}2324.063 \\
2323.512 \\
2321.996 \\
2321.271 \\
2320.659\end{array}$ & $\begin{array}{l}43014.86 \\
43025.06 \\
43053.15 \\
43066.60 \\
43077.95\end{array}$ & $\begin{array}{l}b^{3} \mathrm{H}_{6}-v^{3} \mathrm{G}_{5}^{0} \\
a^{3} \mathrm{P}_{1}-x{ }^{3} \mathrm{D}_{2}^{2}\end{array}$ & & & & & & \\
\hline $\begin{array}{c}25 \\
5 \\
25 \\
3 h \\
10\end{array}$ & $\begin{array}{l}2320.238 \\
2319.847 \\
2319.589 \\
2318.180 \\
2317.784\end{array}$ & $\begin{array}{l}43085.75 \\
43093.03 \\
43097.82 \\
43124.01 \\
43131.38\end{array}$ & $\begin{array}{l}a{ }^{5} \mathrm{D}_{3}-y{ }^{5} \mathrm{D}_{3}^{\circ} \\
a^{3} \mathrm{G}_{4}-w{ }^{3} \mathrm{G}_{3} \\
a^{5} \mathrm{D}_{1}-y{ }^{3} \mathrm{D}_{2}\end{array}$ & & & & & & \\
\hline
\end{tabular}


TABLE 1.-Second spectrum of columbium (Cb II)-Continued

\begin{tabular}{|c|c|c|c|c|c|c|c|c|c|}
\hline $\begin{array}{l}\text { Intensity } \\
\text { spark }\end{array}$ & $\lambda_{\text {air }} \mathrm{A}$ & $\begin{array}{l}\text { Wave } \\
\text { No. } \\
\text { vac. } \\
\mathrm{cm}^{-1}\end{array}$ & $\begin{array}{c}\text { Term combi- } \\
\text { nation }\end{array}$ & $\begin{array}{c}\text { Zeeman } \\
\text { type }\end{array}$ & $\begin{array}{l}\text { Sepa- } \\
\text { ration }\end{array}$ & $\begin{array}{c}\text { Strong- } \\
\text { est } \\
p\end{array}$ & $\underset{n}{\text { Strongest }}$ & $g_{1}$ & $g_{2}$ \\
\hline 1 & 2 & 3 & 4 & 5 & 6 & 7 & 8 & 8 & 10 \\
\hline $15 h$ & 2316.929 & 43147.49 & $\left\{\begin{array}{l}b{ }^{3} \mathrm{H}_{5}-v^{3} \mathrm{G}_{5}^{0} \\
b^{3} \mathrm{P}_{2}-u^{3} \mathrm{~B}_{2}^{0}\end{array}\right.$ & & & & & & \\
\hline $\begin{array}{r}20 \\
50 \\
3 \\
15\end{array}$ & $\begin{array}{l}2315.173 \\
2314.850 \\
2314.240 \\
2313.524\end{array}$ & $\begin{array}{l}43180.02 \\
43186.04 \\
43197.43 \\
43210.79\end{array}$ & $\begin{array}{c}a^{5} \mathrm{D}_{2}-y{ }^{5} \mathrm{D}_{2}^{0} \\
a^{3} \mathrm{P}_{2}-x \\
a^{3} \mathrm{P}_{2}^{0} \\
a^{1} \mathrm{D}_{2}-x \\
a^{5} \mathrm{~F}_{3}^{0} \\
{ }^{5} \mathrm{D}_{2}-y \\
{ }^{3} \mathrm{D}_{1}^{0}\end{array}$ & 3 & & 0.20 & $1.41 \pm$ & 1.46 & 1.36 \\
\hline $\begin{array}{c}20 h \\
2 \\
8\end{array}$ & $\begin{array}{l}2311.456 \\
2310.570 \\
2310.313\end{array}$ & $\begin{array}{l}43249.45 \\
43266.03 \\
43270.84\end{array}$ & $a^{5} \mathrm{~F}_{3}-y{ }^{3} \mathrm{~F}_{4}^{\circ}$ & & & & & & 6 \\
\hline $\begin{array}{r}3 \\
100\end{array}$ & $\begin{array}{l}2309.742 \\
2309.239\end{array}$ & $\begin{array}{l}43281.54 \\
43290.97\end{array}$ & 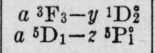 & 3 & 0.76 & .76 & $1.50,2.26$ & 1.50 & 2. 26 \\
\hline $\begin{array}{l}6 \\
3 \\
4 h \\
4 \\
40\end{array}$ & $\begin{array}{l}2308.807 \\
2307.477 \\
2305.426 \\
2303.154 \\
2302.695\end{array}$ & $\begin{array}{l}43299.07 \\
43324.02 \\
43362.56 \\
43405.33 \\
43413.98\end{array}$ & $\begin{array}{l}a{ }^{5} \mathrm{~F}_{1}-y{ }^{3} \mathrm{~F}_{2}^{\circ} \\
a^{3} \mathrm{P}_{2}-x \\
{ }^{3} \mathrm{G}_{3}^{\circ} \\
a^{3} \mathrm{G}_{4}-v{ }^{3} \mathrm{D}_{3}^{\circ} \\
a^{5} \mathrm{D}_{4}-y^{3} \mathrm{D}_{3}^{0}\end{array}$ & & & & & & \\
\hline $\begin{array}{r}200 \\
50\end{array}$ & $\begin{array}{l}2302.086 \\
2300.785\end{array}$ & $\begin{array}{l}43425.47 \\
43450.02\end{array}$ & 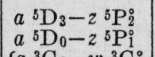 & $\begin{array}{l}1 \\
7 a\end{array}$ & .25 & $\begin{array}{l}0 \\
0\end{array}$ & $\begin{array}{l}1.00 \\
2.255\end{array}$ & $\begin{array}{l}1.50 \\
0 / 0\end{array}$ & $\begin{array}{l}1.75 \\
2.255\end{array}$ \\
\hline 5 & 2300.519 & 43455.04 & $\mid \begin{array}{l}a a^{3} \mathrm{G}_{3}-w^{3} \mathrm{G}_{3}^{0} \\
b^{3} \mathrm{~F}_{3}-x^{1} \mathrm{G}_{4}^{0}\end{array}$ & & & & & & \\
\hline $\begin{array}{l}30 \\
20\end{array}$ & $\begin{array}{l}2300.339 \\
2299.226\end{array}$ & $\begin{array}{l}43458.44 \\
43479.48\end{array}$ & $\mid \begin{array}{cccc}a & 1 & \mathrm{D}_{2}-x & { }^{1} \mathrm{D}_{2}^{0} \\
b^{3} & 3 \mathrm{~F}_{4}-x & { }^{1} \mathrm{G}_{4}^{2}\end{array}$ & $7 b$ & & 0 & 1.002 & 1.002 & 1. 002 \\
\hline $\begin{array}{l}5 \\
10 \\
40 h \\
50 \\
3\end{array}$ & $\begin{array}{l}2298.662 \\
2298.385 \\
2297.853 \\
2297.611 \\
2296.748\end{array}$ & $\begin{array}{l}43490.14 \\
43495.38 \\
43505.45 \\
43510.04 \\
43526.38\end{array}$ & $\begin{array}{l}a{ }^{5} \mathrm{D}_{1}-y^{3} \mathrm{D}_{1}^{\circ} \\
a^{1} \mathrm{P}_{1}-x^{3} \mathrm{D}_{1}^{\circ} \\
\\
a^{5}{ }^{5} \mathrm{G}_{3}-w^{3}{ }^{3} \mathrm{G}_{4}^{\circ} \\
a^{3} \mathrm{H}_{4}-y^{1} \mathrm{~F}_{3}^{8}\end{array}$ & & & & . & & \\
\hline $\begin{array}{c}8 \\
300 \\
60 \\
40 \\
8 h\end{array}$ & $\begin{array}{l}2295.972 \\
2295.681 \\
2294.983 \\
2293.926 \\
2292.325\end{array}$ & $\begin{array}{l}43541.10 \\
43546.61 \\
43559.85 \\
43579.93 \\
43610.36\end{array}$ & $\begin{array}{c}a^{3} \mathrm{P}_{1}-w^{3} \mathrm{D}_{1} \\
a^{5} \mathrm{D}_{4}-z^{5} \mathrm{P}_{3}^{\circ} \\
b^{3} \mathrm{G}_{4}-v^{3} \mathrm{G}_{3}^{3} \\
a^{3} \mathrm{P}_{1}-w^{3} \mathrm{D}_{2}^{\circ}\end{array}$ & & & & & & \\
\hline $\begin{array}{c}20 h \\
8 \\
6 \\
30 \\
7\end{array}$ & $\begin{array}{l}2291.644 \\
2291.383 \\
2290.289 \\
2288.861 \\
2286.892\end{array}$ & $\begin{array}{l}43623.32 \\
43628.29 \\
43649.12 \\
43676.35 \\
43713.95\end{array}$ & $\begin{array}{c}a{ }^{5} \mathrm{D}_{2}-z{ }^{3} \mathrm{P}_{1}^{0} \\
a^{5} \mathrm{D}_{0}-y \\
a^{3} \mathrm{D}_{1} \\
a^{3} \mathrm{~F}_{2}-y \\
a^{5} \mathrm{D}_{2}^{0} \\
{ }^{5} \mathrm{~F}_{2}-x \\
{ }^{3} \mathrm{~F}_{2}^{0}\end{array}$ & & & & & & \\
\hline $\begin{array}{l}20 \\
10 h \\
5 h \\
60 \\
10\end{array}$ & $\begin{array}{l}2286.749 \\
2286.352 \\
2285.673 \\
2285.223 \\
2284.356\end{array}$ & $\begin{array}{l}43716.69 \\
43724.28 \\
43737.26 \\
43745.88 \\
43762.47\end{array}$ & $\begin{array}{l}a{ }^{5} \mathrm{D}_{4}-y{ }^{5} \mathrm{D}_{4}^{\circ} \\
a^{3} \mathrm{G}_{3}-v^{3} \mathrm{D}_{3}^{\circ}\end{array}$ & $7 b, 3$ & & 0 & 1.490 & (1.495) & 1.485 \\
\hline $\begin{array}{l}300 \\
80 h \\
30 \\
50 \\
4 h\end{array}$ & $\begin{array}{l}2283.004 \\
2281.830 \\
2281.136 \\
2280.450 \\
2278.477\end{array}$ & $\begin{array}{l}43788.39 \\
43810.92 \\
43824.25 \\
43837.43 \\
43875.38\end{array}$ & $\begin{array}{l}a^{5} \mathrm{D}_{2}-2{ }^{5} \mathrm{P}_{2}^{\circ} \\
a^{3} \mathrm{G}_{4}-w^{3} \mathrm{~B}_{4}^{\circ} \\
a^{5} \mathrm{D}_{3}-y^{3} \mathrm{D}_{3}^{0} \\
b^{1} \mathrm{G}_{4}-v^{3} \mathrm{G}_{4}^{\circ}\end{array}$ & 3 & .25 & .50 & 1. $49,1.74$ & 1.49 & 1.74 \\
\hline $\begin{array}{c}4 h \\
3 \\
6 \\
60 \\
100\end{array}$ & $\begin{array}{l}2278.320 \\
2276.361 \\
2276.170 \\
2274.198 \\
2274.128\end{array}$ & $\begin{array}{l}43878.41 \\
43916.16 \\
43919.85 \\
43957.93 \\
43959.28\end{array}$ & $\begin{array}{l}a{ }^{5} \mathrm{~F}_{2}-y^{3} \mathrm{P}_{2}^{0} \\
a^{3} \mathrm{~F}_{3}-x \\
b^{3} \mathrm{~F}_{3}^{0} \mathrm{G}_{3}-v^{3} \mathrm{G}_{3}^{0} \\
a^{3} \mathrm{~F}_{4}-v^{3} \mathrm{~F}_{4}^{\circ}\end{array}$ & $7 b, 3$ & & $w$ & 1. 246 & (1. 230) & 1.260 \\
\hline $\begin{array}{c}150 \\
6 h \\
100 \\
2 \\
150\end{array}$ & $\begin{array}{l}2273.566 \\
2273.120 \\
2272.730 \\
2270.65 \\
2270.180\end{array}$ & $\begin{array}{l}43970.15 \\
43978.77 \\
43986.32 \\
44026.6 \\
44035.72\end{array}$ & $\begin{array}{l}a{ }^{5} \mathrm{D}_{3}-2{ }^{5} \mathrm{P}_{3}^{\circ} \\
a{ }^{5} \mathrm{~F}_{1}-x{ }^{3} \mathrm{~F}_{2}^{\circ} \\
a^{3} \mathrm{~F}_{3}-v{ }^{3} \mathrm{~F}_{2}^{2} \\
a^{3} \mathrm{~F}_{3}-v^{3} \mathrm{~F}_{3}^{0}\end{array}$ & $7 b$ & & .25 & $1.52 \pm$ & 1.48 & 1.07 \\
\hline $\begin{array}{l}100 \\
10 h \\
150 \\
100 \\
100\end{array}$ & $\begin{array}{l}2269.865 \\
2269.202 \\
2268.527 \\
2266.732 \\
2265.676\end{array}$ & $\begin{array}{l}44041.83 \\
44054.70 \\
44067.81 \\
44102.70 \\
44123.26\end{array}$ & $\begin{array}{l}b^{3} \mathrm{G}_{5}-v^{3} \mathrm{G}_{4}^{\circ} \\
a^{5} \mathrm{D}_{1}-z^{5} \mathrm{P}_{2}^{2} \\
a^{3} \mathrm{G}_{5}-w^{3} \mathrm{G}_{5}^{\circ} \\
a^{5} \mathrm{D}_{3}-z^{3} \mathrm{P}_{2}^{\circ}\end{array}$ & & & & & & \\
\hline
\end{tabular}


TABLE 1.-Second spectrum of columbium (Cb II)-Continued

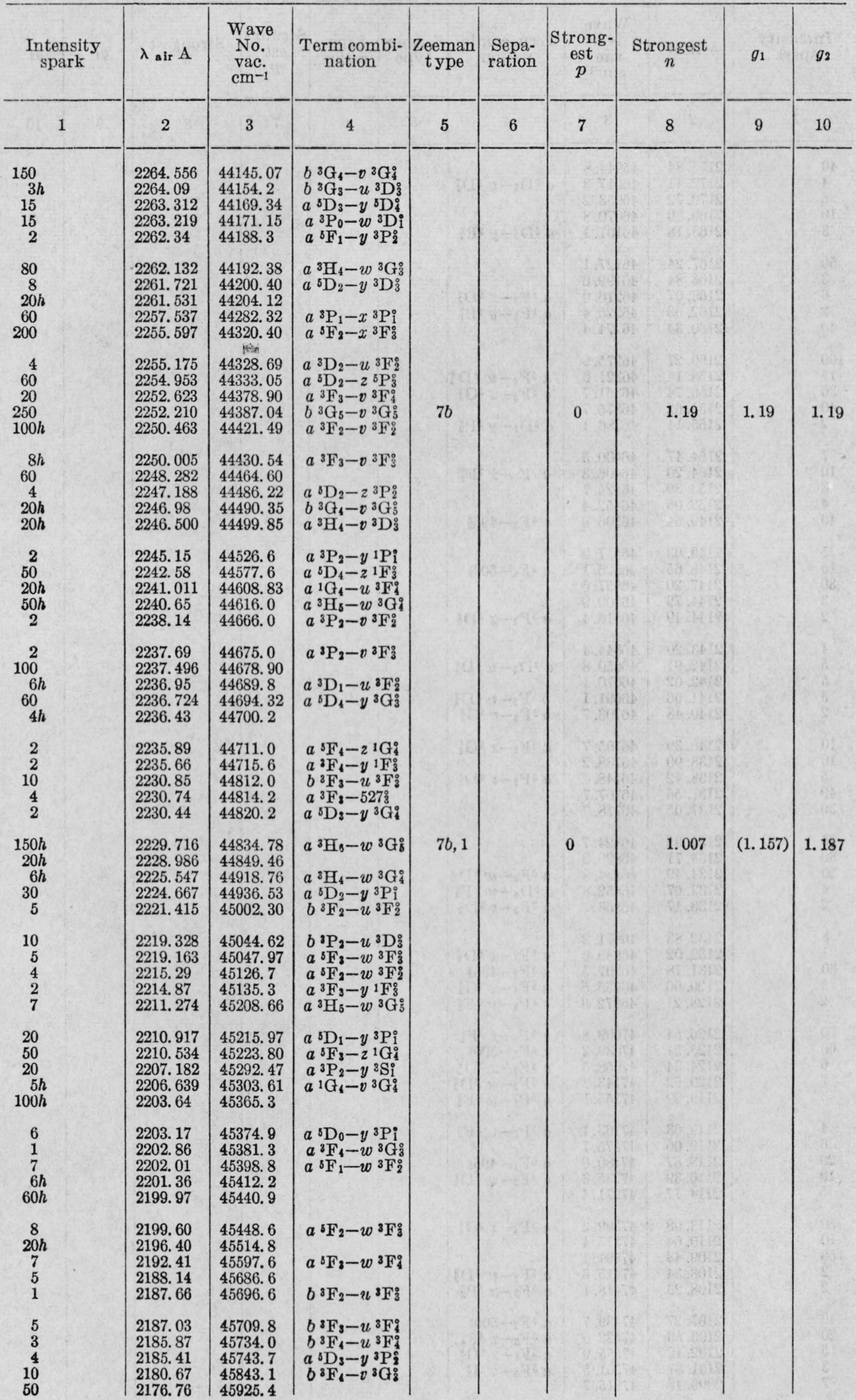


508 Journal of Research of the National Bureau of Standards

TABLE 1.-Second spectrum of columbium (Cb II)-Continued

\begin{tabular}{|c|c|c|c|c|c|c|c|c|c|}
\hline $\begin{array}{c}\text { Intensity } \\
\text { spark }\end{array}$ & $\lambda_{\text {air }} \mathrm{A}$ & $\begin{array}{l}\text { Wave } \\
\text { No. } \\
\text { vac. } \\
\mathrm{cm}^{-1}\end{array}$ & $\begin{array}{c}\text { Term combi- } \\
\text { nation }\end{array}$ & $\begin{array}{c}\text { Zeeman } \\
\text { type }\end{array}$ & $\begin{array}{l}\text { Sepa- } \\
\text { ration }\end{array}$ & $\begin{array}{l}\text { Strong- } \\
\text { est } \\
p\end{array}$ & $\underset{n}{\text { Strongest }}$ & $g_{1}$ & $g_{2}$ \\
\hline 1 & 2 & 3 & 4 & 5 & 6 & 7 & 8 & 9 & 10 \\
\hline $\begin{array}{r}40 \\
4 \\
5 \\
10 \\
3\end{array}$ & $\begin{array}{l}2175.84 \\
2172.41 \\
2170.72 \\
2169.89 \\
2168.18\end{array}$ & $\begin{array}{l}45944.8 \\
46017.3 \\
46053.2 \\
46070.8 \\
46107.1\end{array}$ & $\begin{array}{l}a^{3} \mathrm{D}_{2}-u^{3} \mathrm{D}_{2}^{\circ} \\
a^{5} \mathrm{D}_{2}-y^{3} \mathrm{P}_{2}^{\circ}\end{array}$ & & & & & & 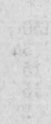 \\
\hline $\begin{array}{r}50 \\
3 \\
6 \\
2 \\
40\end{array}$ & $\begin{array}{l}2167.24 \\
2163.84 \\
2163.07 \\
2162.63 \\
2160.34\end{array}$ & $\begin{array}{l}46127.1 \\
46199.6 \\
46216.0 \\
46225.4 \\
46274.4\end{array}$ & 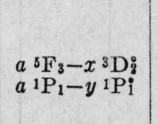 & & & & & & \\
\hline $\begin{array}{r}100 \\
15 \\
30 \\
40 \\
2\end{array}$ & $\begin{array}{l}2160.27 \\
2158.14 \\
2156.74 \\
2155.62 \\
2155.14\end{array}$ & $\begin{array}{l}46275.9 \\
46321.6 \\
46351.7 \\
46375.7 \\
46386.1\end{array}$ & $\begin{array}{l}a{ }^{5} \mathrm{~F}_{4}-w^{3} \mathrm{D}^{0} \\
a^{5} \mathrm{D}_{5}-x^{3} \mathrm{G}_{5}^{3} \\
a^{5} \mathrm{D}_{1}-y^{3} \mathrm{P}_{2}^{\mathrm{o}}\end{array}$ & & & & & & 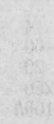 \\
\hline $\begin{array}{r}7 \\
10 \\
3 \\
4 \\
40\end{array}$ & $\begin{array}{l}2154.47 \\
2154.20 \\
2153.30 \\
2152.06 \\
2149.54\end{array}$ & $\begin{array}{l}46400.5 \\
46406.3 \\
46425.7 \\
46452.4 \\
46506.9\end{array}$ & $\begin{array}{l}a{ }^{5} \mathrm{~F}_{5}-x{ }^{3} \mathrm{~F}_{4} \\
a^{3} \mathrm{~F}_{3}-4952\end{array}$ & & & & & & s. \\
\hline $\begin{array}{r}5 \\
15 \\
30 \\
3 \\
2\end{array}$ & $\begin{array}{l}2149.03 \\
2148.65 \\
2147.20 \\
2144.79 \\
2144.49\end{array}$ & $\begin{array}{l}46517.9 \\
46526.1 \\
46557.6 \\
46609.9 \\
46616.4\end{array}$ & $\begin{array}{l}a{ }^{5} \mathrm{~F}_{4}-5002 \\
a^{5} \mathrm{~F}_{2}-x{ }^{3} \mathrm{D}_{2}^{:}\end{array}$ & & & & 2 & & s. \\
\hline $\begin{array}{l}4 \\
5 \\
5 \\
3 \\
2 \\
2\end{array}$ & $\begin{array}{l}2143.20 \\
2142.91 \\
2142.02 \\
2141.06 \\
2140.48\end{array}$ & $\begin{array}{l}46644.4 \\
46650.8 \\
46670.1 \\
46691.1 \\
46703.7\end{array}$ & $\begin{array}{l}a^{3} \mathrm{D}_{3}-u^{3} \mathrm{D}_{3}^{3} \\
b^{3} \mathrm{~F}_{2}-u^{3} \mathrm{D}^{3} \\
b^{3} \mathrm{~F}_{2}-v^{3} \mathrm{G}_{3}^{3}\end{array}$ & & & & & & 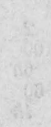 \\
\hline $\begin{array}{r}10 \\
10 \\
5 \\
40 \\
30\end{array}$ & $\begin{array}{l}2140.39 \\
2138.90 \\
2138.42 \\
2137.55 \\
2137.05\end{array}$ & $\begin{array}{l}46705.7 \\
46738.2 \\
46748.7 \\
46767.7 \\
46778.7\end{array}$ & $\begin{array}{l}a{ }^{5} \mathrm{~F}_{5}-x^{3} \mathrm{G}_{4} \\
a^{3} \mathrm{P}_{2}-v^{3} \mathrm{D}_{3}^{\circ}\end{array}$ & & & & & & \\
\hline $\begin{array}{r}25 \\
30 \\
20 \\
4 \\
2\end{array}$ & $\begin{array}{l}2134.95 \\
2134.71 \\
2134.49 \\
2133.67 \\
2133.17\end{array}$ & $\begin{array}{l}46824.7 \\
46829.9 \\
46834.8 \\
46852.8 \\
46863.7\end{array}$ & $\begin{array}{c}a^{5} \mathrm{~F}_{3}-w^{3} \mathrm{D}^{3} \\
a^{3} \mathrm{D}_{4}-w^{3} \mathrm{~F}_{3}^{3} \\
a^{3} \mathrm{P}_{2}-v^{3} \mathrm{D}_{2}^{3}\end{array}$ & & & & & & \\
\hline $\begin{array}{r}8 \\
1 \\
60 \\
3 \\
2\end{array}$ & $\begin{array}{l}2132.83 \\
2132.02 \\
2131.18 \\
2129.00 \\
2128.21\end{array}$ & $\begin{array}{l}46871.2 \\
46889.0 \\
46907.5 \\
46955.5 \\
46972.9\end{array}$ & $\begin{array}{l}a^{5} \mathrm{~F}_{1}-x^{3} \mathrm{D}^{2} \\
a^{5} \mathrm{~F}_{2}-495_{2}^{\circ} \\
a^{5} \mathrm{~F}_{4}-x^{3} \mathrm{G}^{2} \\
a^{5} \mathrm{P}_{2}-u^{3} \mathrm{~F}_{2}^{2}\end{array}$ & & & & & & \\
\hline $\begin{array}{r}60 \\
60 \\
6 \\
7 \\
3\end{array}$ & $\begin{array}{l}2126.54 \\
2125.21 \\
2124.34 \\
2120.52 \\
2119.99\end{array}$ & $\begin{array}{l}47009.8 \\
47039.2 \\
47058.5 \\
47143.3 \\
47155.1\end{array}$ & $\begin{array}{l}a^{5} \mathrm{~F}_{4}-x^{3} \mathrm{~F}_{4}^{\circ} \\
a^{5} \mathrm{~F}_{3}-500_{3}^{\circ} \\
a^{5} \mathrm{~F}_{2}-x^{3} \mathrm{D}_{1}^{\circ} \\
a^{5} \mathrm{~F}_{2}-w^{3} \mathrm{D}_{2}^{2} \\
a^{5} \mathrm{P}_{1}-u^{3} \mathrm{~F}_{2}^{\circ}\end{array}$ & & & & & & his \\
\hline $\begin{array}{r}4 \\
6 \\
20 \\
10 \\
5\end{array}$ & $\begin{array}{l}2119.63 \\
2119.06 \\
2118.87 \\
2116.39 \\
2114.77\end{array}$ & $\begin{array}{l}47163.1 \\
47175.7 \\
47180.0 \\
47235.3 \\
47271.4\end{array}$ & $\begin{array}{l}a^{5} \mathrm{P}_{3}-u^{3} \mathrm{~F}_{3}^{0} \\
a^{5} \mathrm{~F}_{1}-495_{2}^{\circ} \\
a^{5} \mathrm{~F}_{2}-w^{3} \mathrm{D}_{3}^{\circ}\end{array}$ & & & & & & \\
\hline $\begin{array}{r}50 \\
10 \\
150 \\
2 \\
2\end{array}$ & $\begin{array}{l}2113.08 \\
2110.04 \\
2109.43 \\
2108.34 \\
2108.23\end{array}$ & $\begin{array}{l}47309.2 \\
47377.4 \\
47391.1 \\
47415.6 \\
47418.1\end{array}$ & $\begin{array}{l}a^{5} \mathrm{~F}_{4}-x{ }^{3} \mathrm{G}_{4} \\
a^{5} \mathrm{~F}_{1}-w^{3} \mathrm{D}^{2} \\
a^{5} \mathrm{~F}_{3}-x^{3} \mathrm{P}_{2}^{2}\end{array}$ & & & & a. & & , \\
\hline $\begin{array}{r}40 \\
20 \\
5 \\
3 \\
7\end{array}$ & $\begin{array}{l}2107.27 \\
2103.59 \\
2102.12 \\
2101.87 \\
2099.48\end{array}$ & $\begin{array}{l}47439.7 \\
47522.6 \\
47555.9 \\
47561.5 \\
47615.7\end{array}$ & 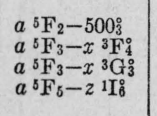 & & & & & & \\
\hline
\end{tabular}


TABLE 1.-Second spectrum of columbium (Cb II)-Continued

\begin{tabular}{|c|c|c|c|c|c|c|c|c|c|}
\hline $\begin{array}{l}\text { Intensity } \\
\text { spark }\end{array}$ & $\lambda_{\text {sir } A}$ & $\begin{array}{l}\text { Wave } \\
\text { No. } \\
\text { vac. } \\
\mathrm{cm}^{-1}\end{array}$ & $\begin{array}{l}\text { Term combi- } \\
\text { nation }\end{array}$ & $\begin{array}{c}\text { Zeeman } \\
\text { type }\end{array}$ & $\begin{array}{l}\text { Sepa- } \\
\text { ration }\end{array}$ & $\begin{array}{l}\text { Strong. } \\
\text { est } \\
p\end{array}$ & $\begin{array}{c}\text { Strongest } \\
n\end{array}$ & $g_{1}$ & $g_{2}$ \\
\hline 1 & 2 & 3 & 4 & 5 & 6 & 7 & 8 & 8 & 10 \\
\hline $\begin{array}{r}15 \\
5 \\
10 \\
20 \\
2\end{array}$ & $\begin{array}{l}2098.05 \\
2097.23 \\
2094.95 \\
2090.41 \\
2089.36\end{array}$ & $\begin{array}{l}47648.1 \\
47666.7 \\
47718.6 \\
47822.2 \\
47846.3\end{array}$ & $\begin{array}{l}a{ }^{5} \mathrm{P}_{2}-u^{3} \mathrm{~F}_{3}^{0} \\
a^{5} \mathrm{~S}_{3}-x^{3} \mathrm{C}_{4}^{8} \\
a^{5} \mathrm{~F}_{3}-x^{3} \mathrm{P}_{1}^{6}\end{array}$ & & & & & & \\
\hline $\begin{array}{r}1 \\
10 \\
15 \\
1 \\
3\end{array}$ & $\begin{array}{l}2085.56 \\
2085.37 \\
2084.30 \\
2083.51 \\
2082.89\end{array}$ & $\begin{array}{l}47933.4 \\
47937.8 \\
47962.4 \\
47980.6 \\
47994.9\end{array}$ & $\begin{array}{l}a^{5}{ }^{5} \mathrm{D}_{4}-y{ }^{1} \mathrm{G}_{4}^{0} \\
a^{3} \mathrm{~F}_{1}-x{ }^{3} \mathrm{P}_{0}^{0} \\
a^{3} \mathrm{P}_{1}-v^{3} \mathrm{D}^{\circ}\end{array}$ & & & & & & \\
\hline $\begin{array}{r}6 \\
5 \\
10 \\
15 \\
30\end{array}$ & $\begin{array}{l}2081.37 \\
2080.33 \\
2080.05 \\
2079.71 \\
2076.88\end{array}$ & $\begin{array}{l}48029.9 \\
48053.9 \\
48060.4 \\
48068.2 \\
48133.7\end{array}$ & $\begin{array}{l}a{ }^{5} \mathrm{P}_{3}-u{ }^{3} \mathrm{~F}_{4} \\
a \\
a{ }^{5} \mathrm{~F}_{3}-v{ }^{3}{ }^{3} \mathrm{~F}_{4}\end{array}$ & & & & & & \\
\hline $\begin{array}{r}4 \\
2 \\
5 \\
10 \\
15\end{array}$ & $\begin{array}{l}2074.32 \\
2073.33 \\
2072.07 \\
2065.71 \\
2064.21\end{array}$ & $\begin{array}{l}48193.1 \\
48216.1 \\
48245.5 \\
48394.0 \\
48429.1\end{array}$ & $\begin{array}{l}a^{3} \mathrm{~F}_{2}-x{ }^{1} \mathrm{D}_{2}^{2} \\
a^{5} \mathrm{~F}_{4}-v^{3} \mathrm{~F}_{3}^{0}\end{array}$ & & & & & & \\
\hline $\begin{array}{r}2 \\
15 \\
20 \\
4 \\
5\end{array}$ & $\begin{array}{l}2061.97 \\
2061.45 \\
2057.05 \\
2054.19 \\
2051.16\end{array}$ & $\begin{array}{l}48481.7 \\
48494.0 \\
48597.7 \\
48665.3 \\
48737.2\end{array}$ & $\begin{array}{l}a^{3} \mathrm{G}_{5}-u^{3} \mathrm{~F}_{4} \\
a^{5} \mathrm{~F}_{4}-v^{3} \mathrm{~F}_{4}^{\circ}\end{array}$ & & & & & & \\
\hline $\begin{array}{r}10 \\
10 \\
1 \\
1 \\
1\end{array}$ & $\begin{array}{l}2050.93 \\
2049.89 \\
2048.70 \\
2047.44 \\
2044.41\end{array}$ & $\begin{array}{l}48742.7 \\
48767.4 \\
48795.7 \\
48825.7 \\
48898.1\end{array}$ & $\begin{array}{l}a^{3} \mathrm{G}_{4}-u^{3} \mathrm{~F}_{4}^{0} \\
a^{5} \mathrm{~F}_{1}-y^{1} \mathrm{D}_{2}^{2} \\
a^{5} \mathrm{~F}_{3}-v^{3} \mathrm{D}_{2}^{2}\end{array}$ & & & & & & \\
\hline $\begin{array}{r}5 \\
1 \\
4 \\
4 \\
10\end{array}$ & $\begin{array}{l}2044.22 \\
2044.02 \\
2043.53 \\
2043.18 \\
2037.95\end{array}$ & $\begin{array}{l}48902.6 \\
48907.4 \\
48919.2 \\
48927.5 \\
49053.1\end{array}$ & $a^{5} \mathrm{~F}_{3}-v^{3} \mathrm{~F}_{3}^{0}$ & & & & & & \\
\hline $\begin{array}{r}20 \\
30 \\
10 \\
3 \\
3\end{array}$ & $\begin{array}{l}2032.99 \\
2029.33 \\
2025.31 \\
2019.79 \\
2018.67\end{array}$ & $\begin{array}{l}49172.7 \\
49261.4 \\
49359.2 \\
49494.1 \\
49521.5\end{array}$ & $\begin{array}{l}a^{5} \mathrm{~F}_{4}-527_{3}^{\circ} \\
a^{5} \mathrm{~F}_{3}-w^{3} \mathrm{P}_{2}^{0} \\
a^{5} \mathrm{~F}_{4}-y^{1} \mathrm{~F}_{3}^{3} \\
a^{3} \mathrm{G}_{5}-v^{3} \mathrm{G}_{5}^{\circ}\end{array}$ & & & & & & \\
\hline $\begin{array}{r}5 \\
1 \\
10 \\
3 \\
6\end{array}$ & $\begin{array}{l}2017.28 \\
2016.67 \\
2016.05 \\
2015.69 \\
2011.99\end{array}$ & $\begin{array}{l}49555.6 \\
49570.6 \\
49585.9 \\
49594.7 \\
49685.9\end{array}$ & $\begin{array}{l}a^{5} \mathrm{~F}_{1}-v^{3} \mathrm{~F}_{2}^{\circ} \\
a^{5} \mathrm{~F}_{2}-w^{3} \mathrm{P}_{1}^{\circ} \\
a^{5} \mathrm{~F}_{3}-527_{3}^{\circ}\end{array}$ & & & & & & \\
\hline $\begin{array}{l}7 \\
7 \\
5 \\
4 \\
3\end{array}$ & $\begin{array}{l}2008.99 \\
2008.46 \\
2005.02 \\
2004.76 \\
2002.41\end{array}$ & $\begin{array}{l}49760.1 \\
49773.2 \\
49858.6 \\
49865.1 \\
49923.6\end{array}$ & $a^{5} \mathrm{~F}_{1}-w{ }^{3} \mathrm{P}_{0}^{\circ}$ & & & & & & \\
\hline
\end{tabular}

\section{TERMS OF THE CB II SPECTRUM}

The established terms (atomic energy states) of the $\mathrm{Cb}$ II spectrum are described in table 2 , in which successive columns show (1) the electron configuration responsible for the term, (2) term symbols, (3) level values, (4) level separations in polyfold terms, (5) $g$-values derived from the Zeeman effect, and (6) observed combinations. All level values are relative to $\left(4 d^{4}\right) a^{5} \mathrm{D}_{0}=0.00$. The total number of levels is 183 , of which 27 represent singlet terms, 116 comprise 40 triplet terms, 37 belong to 9 quintet terms, and 3 are fragments of 
$d^{2} s p$ terms. Negative signs in column 4 show that 2 polyfold terms are inverted and 14 partially inverted. The remainder are regular, but many violations of the rule that intervals are proportional to the larger $j$-values occur. Of special interest is the term $z^{5} \mathrm{D}^{\circ}$, whose fourth interval is abnormally small. Attention was called to the same abnormality in the analogous V II spectrum [7]. The term $y^{5} \mathrm{D}^{\circ}$ is also extremely irregular in both cases.

TABLE 2.-Terms of the $\mathrm{Cb}$ II spectrum

\begin{tabular}{|c|c|c|c|c|c|}
\hline $\begin{array}{l}\text { Electron } \\
\text { configura- } \\
\text { tion }\end{array}$ & $\begin{array}{c}\text { Term } \\
\text { symbol }\end{array}$ & Level & $\begin{array}{l}\text { Differ- } \\
\text { ence }\end{array}$ & $\underset{g}{\text { Observed }}$ & Combinations \\
\hline 1 & 2 & 3 & 4 & 5 & 6 \\
\hline$d^{4}$ & $\begin{array}{l}a{ }^{5} \mathrm{D}_{0} \\
{ }^{5} \mathrm{D}_{1} \\
a^{5} \mathrm{D}_{2} \\
{ }^{5} \mathrm{D}_{3} \\
a^{5} \mathrm{D}_{4}\end{array}$ & $\begin{array}{r}0.00 \\
158.99 \\
438.38 \\
801.38 \\
1224.87\end{array}$ & $\begin{array}{l}158.99 \\
279.39 \\
363.00 \\
423.49\end{array}$ & $\begin{array}{l}0 / 0 \\
1.500 \\
1.490 \\
1.486 \\
1.495\end{array}$ & 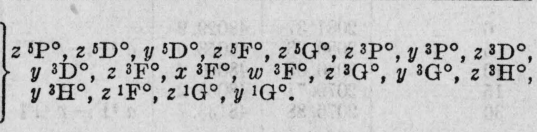 \\
\hline$d^{3} s$ & $\begin{array}{l}a{ }^{5} \mathrm{~F}_{1} \\
a^{5} \mathrm{~F}_{2} \\
a^{5} \mathrm{~F}_{3} \\
a^{5} \mathrm{~F}_{4} \\
a^{5} \mathrm{~F}_{3}\end{array}$ & $\begin{array}{l}2356.76 \\
2629.07 \\
3029.57 \\
3542.50 \\
4146.00\end{array}$ & $\begin{array}{l}272.31 \\
400.50 \\
512.93 \\
603.50\end{array}$ & $\begin{array}{l}0.000 \\
.996 \\
1.248 \\
1.350 \\
1.390\end{array}$ & $\left\{\begin{array}{l}z^{\circ} \mathrm{D}^{\circ}, z{ }^{3} \mathrm{~F}^{\circ}, z^{3} \mathrm{G}^{\circ}, z^{3} \mathrm{P}^{\circ}, y^{3} \mathrm{P}^{\circ}, x{ }^{3} \mathrm{P}^{\circ}, w^{3} \mathrm{P}^{\circ}, z^{3} \mathrm{D}^{\circ}, \\
y{ }^{3} \mathrm{D}^{\circ}, x{ }^{3} \mathrm{D}^{\circ}, w^{3} \mathrm{D}^{\circ}, u^{3} \mathrm{D}^{\circ}, z^{3} \mathrm{~F}^{\circ}, y^{3} \mathrm{~F}^{\circ}, x^{3} \mathrm{~F}^{\circ} \\
w^{3} \mathrm{~F}^{\circ}, u^{3} \mathrm{~F}^{\circ}, z^{3} \mathrm{G}^{\circ}, y^{3} \mathrm{G}^{\circ}, x^{3} \mathrm{G}^{\circ}, w^{3} \mathrm{G}^{\circ}, y^{1} \mathrm{D}^{\circ}, \\
y^{1} \mathrm{I}^{\circ}, z^{1} \mathrm{G}^{\circ}, z^{1} \mathrm{I}^{\circ}, 495^{\circ}, 500^{\circ}, 527^{\circ} .\end{array}\right.$ \\
\hline$d^{4}$ & $\begin{array}{l}a^{3} \mathrm{P}_{0} \\
a^{3} \mathrm{P}_{1} \\
a^{3} \mathrm{P}_{2}\end{array}$ & $\begin{array}{l}5562.26 \\
6192.33 \\
7261.33\end{array}$ & $\begin{array}{r}630.07 \\
1069.00\end{array}$ & $\begin{array}{l}0 / 0 \\
1.495 \\
1.450\end{array}$ & $\left\{\begin{array}{c}z{ }^{\circ} \mathrm{S}^{\circ}, z{ }^{5} \mathrm{P}^{\circ}, z{ }^{5} \mathrm{D}^{\circ}, y{ }^{3} \mathrm{D}^{\circ}, z{ }^{5} \mathrm{~F}^{\circ}, z{ }^{3} \mathrm{~S}^{\circ}, y^{3} \mathrm{~S}^{\circ}, z{ }^{3} \mathrm{P}^{\circ}, \\
y{ }^{3} \mathrm{P}^{\circ}, x^{3} \mathrm{P}^{\circ}, z{ }^{3} \mathrm{D}^{\circ}, y y^{3} \mathrm{D}^{\circ}, x{ }^{3} \mathrm{D}^{\circ}, w^{3} \mathrm{D}^{\circ}, u^{3} \mathrm{D}^{\circ}, \\
z^{3} \mathrm{~F}^{\circ}, u^{3} \mathrm{~F}^{\circ}, z^{3} \mathrm{G}^{\circ}, x^{3} \mathrm{G}^{\circ}, z^{1} \mathrm{P}^{\circ}, y^{1} \mathrm{P}^{\circ}, 500^{\circ} .\end{array}\right.$ \\
\hline$d^{3} s$ & $\begin{array}{l}a \mathrm{~F}_{8} \\
a^{3} \mathrm{~F}_{3} \\
a^{3} \mathrm{~F}_{4}\end{array}$ & $\begin{array}{l}7505.78 \\
7900.65 \\
8320.40\end{array}$ & $\begin{array}{l}394.87 \\
419.75\end{array}$ & $\begin{array}{r}.712 \\
1.070 \\
1.230\end{array}$ & 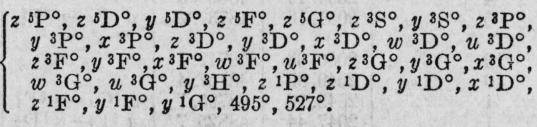 \\
\hline$d^{4}$ & $\begin{array}{l}a{ }^{3} \mathrm{H}_{4} \\
a{ }^{3} \mathrm{H}_{3} \\
a^{3} \mathrm{H}_{6}\end{array}$ & $\begin{array}{r}9509.67 \\
9812.56 \\
10186.41\end{array}$ & $\begin{array}{l}302.89 \\
373.85\end{array}$ & $\begin{array}{r}.825 \\
1.050 \\
1.157\end{array}$ & $\left\{\begin{array}{l}z \mathrm{D}^{\circ}, z \mathrm{~F}^{\circ}, z^{5} \mathrm{G}^{\circ}, z^{3} \mathrm{D}^{\circ}, y^{3} \mathrm{D}^{\circ}, v^{3} \mathrm{D}^{\circ}, u^{3} \mathrm{D}^{\circ}, z^{3} \mathrm{~F}^{\circ}, \\
y^{3} \mathrm{~F}^{\circ}, x^{3} \mathrm{~F}^{\circ}, w^{3} \mathrm{~F}^{\circ}, v^{3} \mathrm{~F}^{\circ}, z^{3} \mathrm{G}^{\circ}, y^{3} \mathrm{G}^{\circ}, x^{3} \mathrm{G}^{\circ}, w^{3} \mathrm{G}^{\circ}, \\
u^{3} \mathrm{G}^{\circ}, z^{3} \mathrm{H}^{\circ}, y^{3} \mathrm{H}^{\circ}, z^{3} \mathrm{I}^{\circ}, z^{1} \mathrm{~F}^{\circ}, y^{1} \mathrm{~F}^{\circ}, z^{1} \mathrm{G}^{\circ}, y^{1} \mathrm{G}^{\circ}, \\
z^{1} \mathrm{I}^{\circ} .\end{array}\right.$ \\
\hline$d^{4}$ & $\begin{array}{l}a^{3} \mathrm{G}_{3} \\
a^{3} \mathrm{G}_{1} \\
a^{3} \mathrm{G}_{5}\end{array}$ & $\begin{array}{l}10247.04 \\
10604.28 \\
10918.52\end{array}$ & $\begin{array}{l}357.24 \\
314.24\end{array}$ & $\begin{array}{r}.765 \\
1.052 \\
1.180\end{array}$ & 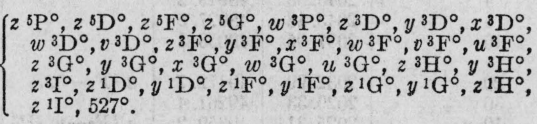 \\
\hline$d^{3} s$ & $\begin{array}{l}a{ }^{5} \mathrm{P}_{1} \\
a^{5} \mathrm{P}_{2} \\
a^{5} \mathrm{P}_{3}\end{array}$ & $\begin{array}{l}10653.40 \\
10835.85 \\
11339.56\end{array}$ & $\begin{array}{l}182.45 \\
503.71\end{array}$ & $\begin{array}{l}2.477 \\
1.815 \\
1.663\end{array}$ & 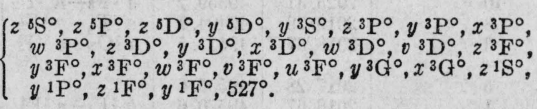 \\
\hline$d^{4}$ & $a 1 \mathrm{D}_{2}$ & 12263.26 & & 1.003 & $\left\{\begin{array}{l}z^{5 \mathrm{~S}^{\circ}, z}{ }^{5} \mathrm{P}^{\circ}, y^{5} \mathrm{D}^{\circ}, z^{3} \mathrm{P}^{\circ}, y^{3} \mathrm{P}^{\circ}, x^{3} \mathrm{P}^{\circ}, y^{3} \mathrm{D}^{\circ}, x^{3} \mathrm{D}^{\circ}, \\
w^{3} \mathrm{D}^{\circ}, v^{3} \mathrm{D}^{\circ}, z^{3} \mathrm{~F}^{\circ}, y^{3} \mathrm{~F}^{\circ}, x^{3} \mathrm{~F}^{\circ}, v^{3} \mathrm{~F}^{\circ}, y^{3} \mathrm{G}^{\circ}, x^{3} \mathrm{G}^{\circ}, \\
z^{1} \mathrm{P}^{\circ}, y^{1} \mathrm{P}^{\circ}, z^{1} \mathrm{D}^{\circ}, y^{1} \mathrm{D}^{\circ}, x^{1} \mathrm{D}^{\circ}, z^{1} \mathrm{~F}^{\circ}, y^{1} \mathrm{~F}^{\circ}, x^{1} \mathrm{~F}^{\circ}, \\
527^{\circ} .\end{array}\right.$ \\
\hline$d^{4}$ & $\begin{array}{l}b^{3} \mathrm{~F}_{2} \\
b{ }^{3} \mathrm{~F}_{3} \\
b^{3} \mathrm{~F}_{4}\end{array}$ & $\begin{array}{l}12805.98 \\
13690.20 \\
13665.68\end{array}$ & $\begin{array}{r}884.22 \\
-24.52\end{array}$ & $\begin{array}{l}.849 \\
1.150 \\
1.152\end{array}$ & 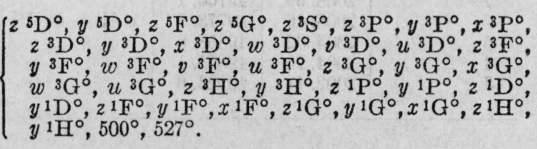 \\
\hline$d^{4}$ & $\begin{array}{l}a{ }^{3} \mathrm{D}_{1} \\
a^{3} \mathrm{D}_{2} \\
a^{3} \mathrm{D}_{3}\end{array}$ & $\begin{array}{l}13118.62 \\
13479.50 \\
13054.69\end{array}$ & $\begin{array}{r}360.88 \\
-424.81\end{array}$ & $\begin{array}{l}.512 \\
1.002 \\
1.246\end{array}$ & $\left\{\begin{array}{l}z^{5} \mathrm{~S}^{\circ}, y{ }^{5} \mathrm{D}^{\circ}, z{ }^{5} \mathrm{~F}^{\circ}, z z^{5} \mathrm{G}^{\circ}, z^{3} \mathrm{~S}^{\circ}, z^{3} \mathrm{P}^{\circ}, y^{3} \mathrm{P}^{\circ}, x^{3} \mathrm{P}^{\circ}, \\
y^{3} \mathrm{D}^{\circ}, y^{3} \mathrm{D}^{\circ}, x{ }^{3} \mathrm{D}^{\circ}, w^{3} \mathrm{D}^{\circ}, v^{3} \mathrm{D}^{\circ}, u^{3} \mathrm{D}^{\circ}, z^{3} \mathrm{~F}^{\circ}, \\
y^{3} \mathrm{~F}^{\circ}, x^{\circ}, w^{3} \mathrm{~F}^{\circ}, v^{3} \mathrm{~F}^{\circ}, u^{3} \mathrm{~F}^{\circ}, z^{3} \mathrm{G}^{\circ}, y^{3} \mathrm{G}^{\circ}, x^{3} \mathrm{G}^{\circ}, \\
w^{3} \mathrm{G}^{\circ}, y^{3} \mathrm{H}^{\circ}, z z^{1} \mathrm{~S}^{\circ}, z^{1} \mathrm{P}^{\circ}, y^{1} \mathrm{P}^{\circ}, z^{1} \mathrm{D}^{\circ}, z^{1} \mathrm{~F}^{\circ}, y^{1} \mathrm{~F}^{\circ}, \\
x^{1} \mathrm{~F}^{\circ}, 500^{\circ}, 527^{\circ} .\end{array}\right.$ \\
\hline$d^{3} s$ & $\begin{array}{l}b{ }^{3} \mathrm{P}_{0} \\
b^{3} \mathrm{P}_{1} \\
b^{3} \mathrm{P}_{2}\end{array}$ & $\begin{array}{l}14678.40 \\
14626.26 \\
14660.77\end{array}$ & $\begin{array}{r}-52.14 \\
34.51\end{array}$ & $\begin{array}{l}0 / 0 \\
1.504 \\
1.483\end{array}$ & 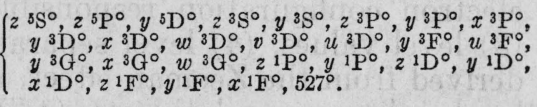 \\
\hline$d^{s}$ & $a{ }^{1} \mathrm{G}_{4}$ & 14790.79 & & 1.083 & $\left\{\begin{array}{l}z^{5} \mathrm{P}^{\circ}, z{ }^{3} \mathrm{D}^{\circ}, y{ }^{3} \mathrm{D}^{\circ}, z \mathrm{~F}^{\circ}, z^{3} \mathrm{D}^{\circ}, y^{3} \mathrm{D}^{\circ}, v^{3} \mathrm{D}^{\circ}, z^{3} \mathrm{~F}^{\circ}, \\
y^{3} \mathrm{~F}^{\circ}, w^{3} \mathrm{~F}^{\circ}, u^{3} \mathrm{~F}^{\circ}, z^{3} \mathrm{G}^{\circ}, y^{3} \mathrm{G}^{\circ}, w^{3} \mathrm{G}^{\circ}, u^{3} \mathrm{G}^{\circ}, \\
z^{3} \mathrm{H}^{\circ}, y^{3} \mathrm{H}^{\circ}, z^{3} \mathrm{O}^{\circ}, y^{1} \mathrm{~F}^{\circ}, x \mathrm{I}^{\circ}, z^{1} \mathrm{G}^{\circ}, y^{1} \mathrm{G}^{\circ}, x^{1} \mathrm{G}^{\circ}, \\
z^{1} \mathrm{H}^{\circ}, y^{1} \mathrm{H}^{\circ} .\end{array}\right.$ \\
\hline
\end{tabular}


TABLE 2.-Terms of the $\mathrm{Cb}$ II spectrum-Continued

\begin{tabular}{|c|c|c|c|c|c|}
\hline $\begin{array}{l}\text { Electron } \\
\text { configura- } \\
\text { tion }\end{array}$ & $\begin{array}{l}\text { Term } \\
\text { symbol }\end{array}$ & Level & $\begin{array}{l}\text { Differ- } \\
\text { ence }\end{array}$ & Observed & Combinations \\
\hline 1 & 2 & 3 & 4 & 5 & 6 \\
\hline$d i$ & $a^{1} \mathrm{I}_{0}$ & 15396.10 & & 1. 000 & $x^{8} \mathrm{G}^{\circ}, w^{3} \mathrm{G}^{\circ}, u^{3} \mathrm{G}^{\circ}, z^{3} \mathrm{H}^{\circ}, z^{3} \mathrm{I}^{\circ}, z^{1} \mathrm{H}^{\circ}, y^{1} \mathrm{H}^{\circ}, z^{1} \mathrm{I}^{\circ}$. \\
\hline$d^{3} \approx$ & $\begin{array}{l}b^{3} \mathrm{G}_{8} \\
b^{3} \mathrm{G}_{4} \\
b^{3} \mathrm{G}_{5}\end{array}$ & $\begin{array}{l}15551.30 \\
15949.40 \\
16052.72\end{array}$ & $\begin{array}{l}398.10 \\
103.32\end{array}$ & $\begin{array}{r}.767 \\
1.027 \\
1.190\end{array}$ & 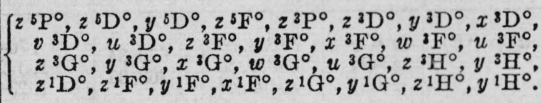 \\
\hline$d^{3} s$ & $0: G_{4}$ & 16219.04 & & .950 & $\left\{\begin{array}{c}z^{\circ} \mathrm{P}^{\circ}, y^{\circ} \mathrm{D}^{\circ}, y^{3} \mathrm{D}^{\circ}, z^{3} \mathrm{~F}^{\circ}, y^{3} \mathrm{~F}^{\circ}, x^{3} \mathrm{~F}^{\circ}, w^{3} \mathrm{~F}^{\circ}, u^{3} \mathrm{~F}^{\circ}, \\
y^{3} \mathrm{G}^{\circ}, x^{3} \mathrm{G}^{\circ}, w^{3} \mathrm{G}^{\circ}, u^{3} \mathrm{G}^{\circ}, y^{3} \mathrm{H}^{\circ}, z^{3} \mathrm{I}^{\circ}, z^{1} \mathrm{~F}^{\circ}, y^{1} \mathrm{~F}^{\circ}, \\
x{ }^{1} \mathrm{~F}^{\circ}, y^{1} \mathrm{G}^{\circ}, x{ }^{1} \mathrm{G}^{\circ}, z^{1} \mathrm{H}^{\circ}, y^{1} \mathrm{H}^{\circ}, 500^{\circ}, 527^{\circ} .\end{array}\right.$ \\
\hline$d 4$ & $a 18_{0}$ & 17202. 72 & & $0 / 0$ & $y^{5} \mathrm{D}^{\circ}, y^{8} \mathrm{~S}^{\circ}, x^{8} \mathrm{P}^{\circ}, x^{8} \mathrm{D}^{\circ}, z^{1} \mathrm{P}^{\circ}, y^{1} \mathrm{P}^{\circ}$. \\
\hline$d^{3} s$ & $\begin{array}{l}b^{3} \mathrm{H}_{4} \\
b^{8} \mathrm{H}_{5} \\
b_{6} \mathrm{H}_{6}\end{array}$ & $\begin{array}{l}17469.77 \\
17292.49 \\
17424.88\end{array}$ & $\begin{array}{r}-177.28 \\
132.39\end{array}$ & $\begin{array}{r}.880 \\
1.052 \\
1.154\end{array}$ & $\left\{\begin{array}{c}y^{3} \mathrm{D}^{\circ}, v^{3} \mathrm{D}^{\circ}, z^{3} \mathrm{~F}^{\circ}, y^{3} \mathrm{~F}^{\circ}, x^{3} \mathrm{~F}^{\circ}, w^{3} \mathrm{~F}^{\circ}, v^{3} \mathrm{~F}^{\circ}, u^{3} \mathrm{~F}^{\circ}, \\
y^{3} \mathrm{G}^{\circ}, x^{3} \mathrm{G}^{\circ}, w{ }^{3} \mathrm{G}^{\circ}, u^{3} \mathrm{G}^{\circ}, z^{8} \mathrm{H}^{\circ}, y^{3} \mathrm{H}^{\circ}, z{ }^{8} \mathrm{I}^{\circ}, \\
z^{1} \mathrm{~F}^{\circ}, x^{1} \mathrm{~F}^{\circ}, z^{1} \mathrm{G}^{\circ}, y^{1} \mathrm{G}^{\circ}, x \mathrm{i}^{\circ}, y^{1} \mathrm{H}^{\circ}, z \mathrm{I}^{\circ}, 527^{\circ} .\end{array}\right.$ \\
\hline & $a^{1} F_{3}$ & 18508.15 & & 1. 007 & $\left\{\begin{array}{c}y^{\circ} \mathrm{D}^{\circ}, y^{3} \mathrm{P}^{\circ}, x^{3} \mathrm{D}^{\circ}, z^{2} \mathrm{~F}^{\circ}, w^{3} \mathrm{~F}^{\circ}, v^{3} \mathrm{~F}^{\circ}, z^{3} \mathrm{G}^{\circ} \\
x^{3} \mathrm{G}^{\circ}, w^{3} \mathrm{G}^{\circ}, x \mathrm{D}^{\circ}, y^{1} \mathrm{~F}^{\circ}, z^{1} \mathrm{G}^{\circ}, y^{1} \mathrm{G}^{\circ}, x^{1} \mathrm{G}^{\circ} .\end{array}\right.$ \\
\hline$d^{3} s$ & $\begin{array}{l}b^{3} \mathrm{D}_{1} \\
b^{3} \mathrm{D}_{2} \\
b^{3} \mathrm{D}_{3}\end{array}$ & $\begin{array}{l}18819.57 \\
19351.98 \\
19689.54\end{array}$ & $\begin{array}{l}532.41 \\
337.56\end{array}$ & $\begin{array}{l}.681 \\
\text { 1. } 171 \\
1.312\end{array}$ & 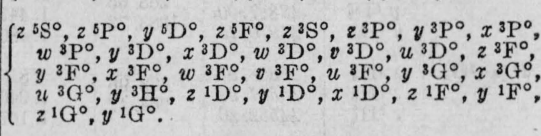 \\
\hline$d^{3} s$ & $\begin{array}{l}c^{3} \mathrm{Po}_{0} \\
c^{3} \mathrm{P}_{1} \\
c^{3} \mathrm{P}_{2}\end{array}$ & $\begin{array}{l}20347.55 \\
21039.56 \\
21511.46\end{array}$ & $\begin{array}{l}692.01 \\
471.90\end{array}$ & $\begin{array}{l}0 / 0 \\
\text { 1. } 218 \\
\text { 1. } 468\end{array}$ & $\left\{\begin{array}{l}{ }^{8} \mathrm{~S}^{\circ}, y{ }^{5} \mathrm{D}^{\circ}, z{ }^{3} \mathrm{~S}^{\circ}, y^{3} \mathrm{~S}^{\circ}, z^{3} \mathrm{P}^{\circ}, y^{3} \mathrm{P}^{\circ}, x{ }^{3} \mathrm{P}^{\circ}, y^{3} \mathrm{D}^{\circ}, \\
x^{3} \mathrm{D}^{\circ}, w^{3} \mathrm{D}^{\circ}, v^{3} \mathrm{D}^{\circ}, u^{3} \mathrm{D}^{\circ}, y^{3} \mathrm{~F}^{\circ}, x^{3} \mathrm{~F}^{\circ}, w^{3} \mathrm{~F}^{\circ}, \\
{ }^{3} \mathrm{~F}^{\circ}, y^{3} \mathrm{G}^{\circ}, z \mathrm{~S}^{\circ}, z \mathrm{P}^{\circ}, y \mathrm{P}^{\circ}, z \mathrm{D}^{\circ}, x \mathrm{D}^{\circ} .\end{array}\right.$ \\
\hline$d^{3} s$ & $a \mathbf{P}_{1}$ & 20437.58 & & 1. 115 & $\left\{\begin{array}{l}y{ }^{3} \mathrm{D}^{\circ}, z{ }^{3} \mathrm{~S}^{\circ}, z{ }^{3} \mathrm{P}^{\circ}, y^{3} \mathrm{P}^{\circ}, x^{3} \mathrm{P}^{\circ}, y{ }^{3} \mathrm{D}^{\circ}, x^{3} \mathrm{D}^{\circ}, w^{3} \mathrm{D}^{\circ}, \\
y \mathrm{~F}^{\circ}, v{ }^{3} \mathrm{~F}^{\circ}, u^{3} \mathrm{~F}^{\circ}, z \mathrm{~S}^{\circ}, z \mathrm{P}^{\circ}, y \mathrm{P}^{\circ}, z \mathrm{D}^{\circ}, \\
x^{1} \mathrm{D}^{\circ}, 495^{\circ} .\end{array}\right.$ \\
\hline$d^{4}$ & $\begin{array}{l}c^{3} \mathrm{~F}_{2} \\
c^{3} \mathrm{~F}_{3} \\
c^{3} \mathrm{~F}_{4}\end{array}$ & $\begin{array}{l}20657.82 \\
21117.47 \\
21472.52\end{array}$ & $\begin{array}{l}459.55 \\
355.05\end{array}$ & $\begin{array}{r}.670 \\
1.080 \\
1.240\end{array}$ & 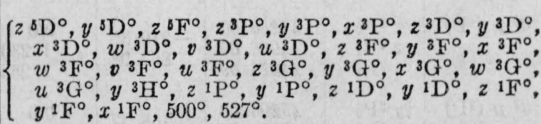 \\
\hline$d^{3} 8$ & $a^{1} \mathrm{H}_{5}$ & 21073.05 & & .992 & $\left\{\begin{array}{l}y \mathrm{D}^{\circ}, y{ }^{3} \mathrm{~F}^{\circ}, w{ }^{3} \mathrm{~F}^{\circ}, y^{8} \mathrm{G}^{\circ}, x^{3} \mathrm{G}^{\circ}, w^{3} \mathrm{G}^{\circ} \cdot y^{3} \mathrm{G}^{\circ}, \\
z^{3} \mathrm{H}^{\circ}, y^{3} \mathrm{H}^{\circ}, z^{3} \mathrm{I}^{\circ}, z^{1} \mathrm{G}^{\circ}, y^{1} \mathrm{G}^{\circ}, x \mathrm{i}^{\circ}, y^{1} \mathrm{H}^{\circ}, z^{1 \mathrm{I}^{\circ}} .\end{array}\right.$ \\
\hline$d^{3} 8$ & $b 1 \mathrm{D}_{2}$ & 24332.87 & & .98 & $\left\{\begin{array}{l}{ }^{3} \mathrm{P}^{\circ}, x^{3} \mathrm{P}^{\circ}, y^{3} \mathrm{D}^{\circ}, x^{3} \mathrm{D}^{\circ}, w^{3} \mathrm{D}^{\circ}, v^{3} \mathrm{D}^{\circ}, v^{3} \mathrm{~F}^{\circ}, u^{3} \mathrm{~F}^{\circ}, \\
x^{3} \mathrm{G}^{\circ}, y{ }^{1} \mathrm{P}^{\circ}, y 1 \mathrm{D}^{\circ}, y \mathrm{~F}^{\circ}, 527^{\circ} .\end{array}\right.$ \\
\hline$d^{3} 8$ & $\begin{array}{l}d^{3} \mathrm{~F}_{2} \\
d^{3} \mathrm{~F}_{3} \\
d^{3} \mathrm{~F}_{4}\end{array}$ & $\begin{array}{l}25414.24 \\
25353.66 \\
25357.50\end{array}$ & $\begin{array}{r}-60.58 \\
3.84\end{array}$ & .71 & $\left\{\begin{array}{l}y{ }^{3} \mathrm{P}^{\circ}, x^{3} \mathrm{P}^{\circ}, x^{3} \mathrm{D}^{\circ}, w^{3} \mathrm{D}^{\circ}, v{ }^{3} \mathrm{D}^{\circ}, u^{3} \mathrm{D}^{\circ}, y{ }^{3} \mathrm{~F}^{\circ}, \\
x^{3} \mathrm{~F}^{\circ}, w^{3} \mathrm{~F}^{\circ}, v^{3} \mathrm{~F}^{\circ}, u^{3} \mathrm{~F}^{\circ}, x^{3} \mathrm{G}^{\circ}, w^{3} \mathrm{G}^{\circ}, u^{3} \mathrm{G}^{\circ}, \\
y^{1} \mathrm{~F}^{\circ}, x^{1} \mathrm{~F}^{\circ}, 500^{\circ}, 527^{\circ} .\end{array}\right.$ \\
\hline$d^{4}$ & $\begin{array}{l}d^{3} \mathrm{P}_{0} \\
d^{3} \mathrm{P}_{1} \\
d^{3} \mathrm{P}_{2}\end{array}$ & $\begin{array}{l}28001.37 \\
27794.15 \\
27282.18\end{array}$ & $\begin{array}{l}-207.22 \\
-511.97\end{array}$ & $\begin{array}{l}0 / 0 \\
1.49 \\
1.49\end{array}$ & $y^{3} \mathrm{D}^{\circ}, y^{3} \mathrm{~S}^{\circ}, x^{3} \mathrm{P}^{\circ}, x^{3} \mathrm{D}^{\circ}, w^{3} \mathrm{D}^{\circ}, u^{3} \mathrm{D}^{\circ}, 495^{\circ}$ \\
\hline$d^{4}$ & $c \mathrm{G}_{4}$ & 29634. 24 & & 1. 087 & ${ }^{0}{ }^{3} \mathrm{D}^{\circ}, y^{3} \mathrm{~F}^{\circ}, y^{3} \mathrm{H}^{\circ}, z^{1} \mathrm{~F}^{\circ}, x^{1} \mathrm{~F}^{\circ}, y^{1} \mathrm{G}^{\circ}, x^{1} \mathrm{G}^{\circ}, y^{1} \mathrm{H}^{\circ}$. \\
\hline$d^{4}$ & $c^{1} \mathrm{D}_{2}$ & 31064.80 & & 1.01 & $y{ }^{1} \mathrm{D}^{\circ}, y^{1} \mathrm{P}^{\circ}, y^{3} \mathrm{~S}^{\circ}, 527^{\circ}, x{ }^{1} \mathrm{~F}^{\circ}, x^{1} \mathrm{D}^{\circ}$ \\
\hline$d^{3} s$ & $6 \mathrm{~F}_{8}$ & 31762.31 & & 1.01 & $v^{3} \mathrm{~F}^{\circ}, w^{3} \mathrm{G}^{\circ}, y^{1} \mathrm{D}^{\circ}, x^{1} \mathrm{D}^{\circ}, x^{1} \mathrm{~F}^{\circ}, z^{1} \mathrm{G}^{\circ}, y^{1} \mathrm{G}^{\circ}, x^{1} \mathrm{G}^{\circ}$. \\
\hline$d^{3} p\left({ }^{4} \mathrm{~F}\right)$ & $\begin{array}{l}z^{5} \mathrm{G}_{2}^{\circ} \\
z^{5} \mathrm{G}_{3}^{\circ} \\
z^{5} \mathrm{G}_{4}^{\circ} \\
z^{5} \mathrm{G}_{4} \\
z^{\circ} \mathrm{G}_{8}^{\circ}\end{array}$ & $\begin{array}{l}39351.00 \\
39919.20 \\
34632.00 \\
3547.17 \\
36455.47\end{array}$ & $\begin{array}{l}568.20 \\
712.80 \\
842.17 \\
981.30\end{array}$ & $\begin{array}{l}.345 \\
.913 \\
\text { 1. } 152 \\
\text { 1. } 250 \\
\text { 1. } 31\end{array}$ & $\int a^{5} \mathrm{D}, a^{5} \mathrm{~F}, a^{3} \mathrm{D}, a^{3}$ \\
\hline$d^{3} p\left({ }^{4} \mathrm{~F}\right)$ & $\begin{array}{l}z^{3} \mathrm{D}_{1}^{\circ} \\
z^{3} \mathrm{D}_{2}^{2} \\
2^{3} \mathrm{D}_{3}^{\circ}\end{array}$ & $\begin{array}{l}34886.39 \\
35520.83 \\
36553.27\end{array}$ & $\begin{array}{r}634.50 \\
1032.44\end{array}$ & $\begin{array}{r}.420 \\
1.138 \\
1.311\end{array}$ & $\left\{\begin{array}{l}a^{5} \mathrm{P}, a^{5} \mathrm{D}, a^{5} \mathrm{~F}, a^{3} \mathrm{P}, a^{3} \mathrm{D}, a^{3} \mathrm{~F}, b^{3} \mathrm{~F}, c^{3} \mathrm{~F}, a^{3} \mathrm{G}, b^{3} \mathrm{G} \\
a^{3} \mathrm{H}, a^{1} \mathrm{G} .\end{array}\right.$ \\
\hline$d^{3} p(4 \mathrm{~F})$ & $\begin{array}{ll}z & 5 \mathrm{~F}_{1}^{\circ} \\
z & 6 \mathrm{~F}_{2}^{2} \\
z & 5 \mathrm{~F}_{3}^{0} \\
2 & 5 \mathrm{~F}^{\circ} \\
z & 5 \mathrm{~F}_{6}^{0}\end{array}$ & $\begin{array}{l}\$ 6781.79 \\
36968.76 \\
37376.91 \\
37528.39 \\
\text { 38024. } 92\end{array}$ & $\begin{array}{l}230.97 \\
414.15 \\
151.48 \\
495.93\end{array}$ & $\begin{array}{l}.122 \\
\text { 1. } 023 \\
\text { 1. } 266 \\
\text { 1. } 325 \\
\text { 1. } 357\end{array}$ & $\left\{\begin{array}{l}a^{5} \mathrm{D}, a^{5} \mathrm{~F}, a^{3} \mathrm{P}, c^{3} \mathrm{P}, a^{3} \mathrm{D}, b^{3} \mathrm{D}, a^{3} \mathrm{~F}, b^{3} \mathrm{~F}, c^{3} \mathrm{~F}, a^{3} \mathrm{G}, \\
b^{3} \mathrm{G}, a^{3} \mathrm{H}, a^{1} \mathrm{G} .\end{array}\right.$ \\
\hline$d^{3} p\left({ }^{4} \mathrm{~F}\right)$ & 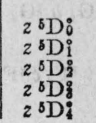 & $\begin{array}{l}37298.20 \\
37480.03 \\
37797.29 \\
38816.37 \\
38291.25\end{array}$ & $\begin{array}{r}181.83 \\
317.26 \\
419.08 \\
74.88\end{array}$ & $\begin{array}{l}0 / 0 \\
1.460 \\
1.469 \\
1.466 \\
1.473\end{array}$ & $\begin{array}{l}a^{5} \mathrm{P}, a^{5} \mathrm{D}, a^{5} \mathrm{~F}, a^{3} \mathrm{P}, a^{3} \mathrm{~F}, b^{3} \mathrm{~F}, c^{3} \mathrm{~F}, a^{3} \mathrm{G}, b^{3} \mathrm{G}, a^{3} \mathrm{H} \\
a \mathrm{i}^{\mathrm{G}} \mathrm{C}\end{array}$ \\
\hline
\end{tabular}


TABLE 2.-Terms of the $\mathrm{Cb}$ II spectrum-Continued

\begin{tabular}{|c|c|c|c|c|c|}
\hline $\begin{array}{l}\text { Electron } \\
\text { configura- } \\
\text { tion }\end{array}$ & $\begin{array}{c}\text { Term } \\
\text { symbol }\end{array}$ & Level & $\begin{array}{l}\text { Differ- } \\
\text { ence }\end{array}$ & $\underset{g}{\text { Observed }}$ & Combinations \\
\hline 1 & 2 & 3 & 4 & 5 & 6 \\
\hline$d^{3} p\left({ }^{4} \mathrm{~F}\right)$ & $\begin{array}{l}z^{3} \mathrm{BG}_{3}^{2} \\
z^{3} \mathrm{G}_{4}^{4} \\
z^{3} \mathrm{G}_{3}^{3}\end{array}$ & $\begin{array}{l}38684.96 \\
39385.90 \\
40103.61\end{array}$ & $\begin{array}{l}650.34 \\
768.31\end{array}$ & $\begin{array}{r}.764 \\
1.072 \\
1.234\end{array}$ & $\left\{\begin{array}{l}a^{3} \mathrm{D}, a{ }^{3} \mathrm{~F}, a^{3} \mathrm{P}, a^{3} \mathrm{D}, a^{3} \mathrm{~F}, b^{3} \mathrm{~F}, c^{3} \mathrm{~F}, a^{3} \mathrm{G}, b^{3} \mathrm{G}, a^{3} \mathrm{H}, \\
a^{1} \mathrm{~F}, a{ }^{\mathrm{G}} \mathrm{G} .\end{array}\right.$ \\
\hline$d^{3} p\left({ }^{4} \mathrm{~F}\right)$ & 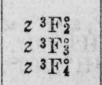 & $\begin{array}{l}38984.40 \\
39779.95 \\
40561.00\end{array}$ & $\begin{array}{l}795.55 \\
781.05\end{array}$ & $\begin{array}{r}.691 \\
1.088 \\
1.260\end{array}$ & $\left\{\begin{array}{l}a^{5} \mathrm{P}, a^{5} \mathrm{D}, a^{3} \mathrm{~F}, a^{3} \mathrm{P}, a^{3} \mathrm{D}, b^{3} \mathrm{D}, a^{3} \mathrm{~F}, b^{3} \mathrm{~F}, c^{3} \mathrm{~F}, a^{3} \mathrm{G}, \\
b^{3} \mathrm{G}, a^{3} \mathrm{H}, b^{3} \mathrm{H}, a^{1} \mathrm{D}, a^{1} \mathrm{~F}, a^{1} \mathrm{G}, b^{1} \mathrm{G} .\end{array}\right.$ \\
\hline$d^{3} p\left({ }^{2} \mathrm{P}\right)$ & $2{ }^{1} \mathrm{~S}_{0}^{0}$ & 39791.90 & 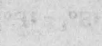 & $0 / 0$ & $a^{5} \mathrm{P}, c^{3} \mathrm{P}, a^{3} \mathrm{D}, a^{1} \mathrm{P}$ \\
\hline$d^{3} p\left({ }^{2} \mathrm{P}\right)$ & $z^{1} \mathrm{D}_{2}^{\circ}$ & 41710.15 & & 1.312 & $\left\{\begin{aligned} a^{3} \mathrm{P}, a^{3} \mathrm{D}, b^{3} \mathrm{P}, c^{3} \mathrm{P}, a^{3} \mathrm{D}, b^{3} \mathrm{D}, a^{3} \mathrm{~F}, b^{3} \mathrm{~F}, c^{3} \mathrm{~F}, a^{3} \mathrm{G}, \\
b^{3} \mathrm{G}, a^{1} \mathrm{P}, a^{1} \mathrm{D}, b^{1} \mathrm{D} .\end{aligned}\right.$ \\
\hline$d^{3} p\left({ }^{4} \mathrm{P}\right)$ & 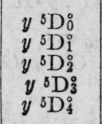 & $\begin{array}{l}42596.58 \\
42132.70 \\
43618.35 \\
49887.00 \\
44970.73\end{array}$ & $\begin{array}{r}-463.88 \\
1485.65 \\
268.65 \\
1083.73\end{array}$ & $\begin{array}{l}0 / 0 \\
1.385 \\
1.347 \\
1.447 \\
1.480\end{array}$ & $\left\{\begin{array}{l}a^{5} \mathrm{P}, a^{3} \mathrm{D}, a^{3} \mathrm{P}, b^{3} \mathrm{P}, c^{3} \mathrm{P}, d^{3} \mathrm{P}, a^{3} \mathrm{D}, b^{3} \mathrm{D}, a^{3} \mathrm{~F}, b^{3} \mathrm{~F}, \\
c^{3} \mathrm{~F}, b^{3} \mathrm{G}, a^{1} \mathrm{~S}, a^{1} \mathrm{P}, a^{1} \mathrm{D}, a^{1} \mathrm{~F}, a^{1} \mathrm{G}, b^{1} \mathrm{G}, a^{1} \mathrm{H}\end{array}\right.$ \\
\hline$d^{3} p\left({ }^{2} G\right)$ & $\begin{array}{l}z^{3} \mathrm{H}_{i}^{0} \\
z^{3} \mathrm{H}_{5}^{3} \\
z^{3} \mathrm{H}_{6}\end{array}$ & $\begin{array}{l}42868.97 \\
43567.93 \\
44538.40\end{array}$ & $\begin{array}{l}698.96 \\
964.47\end{array}$ & $\begin{array}{r}.810 \\
1.040 \\
1.167\end{array}$ & $a^{5} \mathrm{D}, b^{3} \mathrm{~F}, a^{3} \mathrm{G}, b^{3} \mathrm{G}, a^{3} \mathrm{H}, b^{3} \mathrm{H}, a^{1} \mathrm{G}, b^{1} \mathrm{G}, a^{1} \mathrm{H}, a^{1} \mathrm{I}$. \\
\hline$d^{3} p\left({ }^{4} \mathrm{P}\right)$ & $\begin{array}{l}y^{3} \mathrm{D}_{1}^{\circ} \\
y^{3} \mathrm{D}_{2}^{2} \\
y^{3} \mathrm{D}_{3}^{3}\end{array}$ & $\begin{array}{l}49649.19 \\
43290.34 \\
44638.77\end{array}$ & $\begin{array}{r}-358.85 \\
1348.43\end{array}$ & $\begin{array}{l}.980 \\
1.227 \\
1.035\end{array}$ & $\left\{\begin{array}{l}a{ }^{3} \mathrm{P}, a^{5} \mathrm{D}, a^{5} \mathrm{~F}, b^{3} \mathrm{P}, c^{3} \mathrm{P}, a^{3} \mathrm{D}, b^{3} \mathrm{D}, a^{3} \mathrm{~F}, b^{3} \mathrm{~F}, c^{3} \mathrm{~F}, \\
a^{3} \mathrm{G}, b^{3} \mathrm{G}, a^{3} \mathrm{H}, b^{3} \mathrm{H}, a^{1} \mathrm{P}, a^{1} \mathrm{D}, b^{1} \mathrm{D}, a^{1} \mathrm{G}, b^{1} \mathrm{G}\end{array}\right.$ \\
\hline$d^{2} p\left({ }^{4} \mathrm{P}\right)$ & 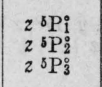 & $\begin{array}{l}43450.00 \\
44226.81 \\
44771.47\end{array}$ & $\begin{array}{l}776.81 \\
544.66\end{array}$ & $\begin{array}{l}2.255 \\
1.745 \\
1.562\end{array}$ & $\left\{\begin{array}{l}a{ }^{5} \mathrm{P}, a^{5} \mathrm{D}, a^{3} \mathrm{P}, b{ }^{3} \mathrm{P}, b^{3} \mathrm{D}, a^{3} \mathrm{~F}, a^{3} \mathrm{G}, b^{3} \mathrm{G}, a^{3} \mathrm{H}, \\
a{ }^{1} \mathrm{D}, a^{1} \mathrm{G}, b^{1} \mathrm{G} .\end{array}\right.$ \\
\hline$d^{3} p\left({ }^{4} \mathrm{P}\right)$ & $\begin{array}{l}2^{3} \mathrm{P}_{0}^{0} \\
2^{3} \mathrm{P}_{1}^{0} \\
z^{3} \mathrm{P}_{2}^{2}\end{array}$ & $\begin{array}{r}44286.00 \\
44066.65 \\
44924.59\end{array}$ & $\begin{array}{r}-219.35 \\
857.94\end{array}$ & $\begin{array}{l}0 / 0 \\
1.230 \\
1.253\end{array}$ & $\left\{\begin{array}{l}a^{5} \mathrm{P}, a^{5} \mathrm{D}, a^{5} \mathrm{~F}, a^{3} \mathrm{P}, b^{3} \mathrm{P}, c^{3} \mathrm{P}, a^{3} \mathrm{D}, b^{3} \mathrm{D}, a^{3} \mathrm{~F}, b^{3} \mathrm{~F}, \\
c^{3} \mathrm{~F}, b^{3} \mathrm{G}, a^{1} \mathrm{P}, a^{1} \mathrm{D}, b^{1} \mathrm{D} .\end{array}\right.$ \\
\hline$d^{3} p\left({ }^{2} \mathrm{P}\right)$ & $\begin{array}{l}y^{3} \mathrm{P}_{0}^{0} \\
y^{3} \mathrm{P}_{1}^{0} \\
y^{3} \mathrm{P}_{2}^{0}\end{array}$ & $\begin{array}{l}45206.59 \\
45374.96 \\
46545.28\end{array}$ & $\begin{array}{r}168.37 \\
1170.32\end{array}$ & \begin{tabular}{l|l}
$0 / 0$ \\
1.765 \\
1.506
\end{tabular} & $\left\{\begin{array}{l}a^{5} \mathrm{P}, a^{3} \mathrm{D}, a^{5} \mathrm{~F}, a^{3} \mathrm{P}, b^{3} \mathrm{P}, c^{3} \mathrm{P}, a^{3} \mathrm{D}, b^{3} \mathrm{D}, a^{3} \mathrm{~F}, b^{3} \mathrm{~F} \\
c^{3} \mathrm{~F}, d^{3} \mathrm{~F}, a^{1} \mathrm{P}, a^{1} \mathrm{D}, a^{1} \mathrm{~F} .\end{array}\right.$ \\
\hline$d^{3} p\left({ }^{2} G\right)$ & $2{ }^{1} \mathrm{H}_{5}^{\circ}$ & 45342.25 & & 1.018 & $a^{3} \mathrm{~F}, b^{3} \mathrm{~F}, a^{3} \mathrm{G}, b^{3} \mathrm{G}, a^{3} \mathrm{H}, b^{3} \mathrm{H}, a^{1} \mathrm{G}, b^{1} \mathrm{G}, a^{1} \mathrm{H}, a^{1} \mathrm{I}$. \\
\hline$d^{3} p\left({ }^{2} G\right)$ & $\begin{array}{l}y^{3} \mathrm{~F}_{2}^{0} \\
y^{3} \mathrm{~F}_{3}^{2} \\
y^{3} \mathrm{~F}_{4}^{2}\end{array}$ & $\begin{array}{l}45655.84 \\
46428.63 \\
46295.61\end{array}$ & $\begin{array}{r}772.79 \\
-133.02\end{array}$ & $\begin{array}{r}.740 \\
1.123 \\
1.111\end{array}$ & $\left\{\begin{array}{l}a^{3 \mathrm{P}}, a^{3} \mathrm{~F}, b^{3} \mathrm{P}, c^{3} \mathrm{P}, a^{3} \mathrm{D}, b^{3} \mathrm{D}, a^{3} \mathrm{~F}, b^{3} \mathrm{~F}, c^{3} \mathrm{~F}, d^{3} \mathrm{~F}, \\
a^{3} \mathrm{G}, b^{3} \mathrm{G}, a^{3} \mathrm{H}, b^{3} \mathrm{H}, a^{1 \mathrm{P}}, a^{1} \mathrm{D}, a^{1} \mathrm{G}, b^{1} \mathrm{G}, c^{1} \mathrm{G}, \\
a^{1} \mathrm{H} .\end{array}\right.$ \\
\hline$d^{3} p\left({ }^{2} G\right)$ & $\begin{array}{l}y^{3} \mathrm{G}_{3}^{8} \\
y^{3} \mathrm{G}_{4} \\
y^{3} \mathrm{G}_{5}^{4}\end{array}$ & $\begin{array}{l}45919.08 \\
45621.97 \\
46499.62\end{array}$ & -297.11 & $\begin{array}{l}1.014 \\
1.126 \\
1.171\end{array}$ & $\left\{\begin{array}{l}a^{5} \mathrm{P}, a^{5} \mathrm{D}, a^{5} \mathrm{~F}, b^{3} \mathrm{P}, c^{3} \mathrm{P}, a^{3} \mathrm{D}, b^{3} \mathrm{D}, a^{3} \mathrm{~F}, b^{3} \mathrm{~F}, c^{3} \mathrm{~F}, \\
a^{3} \mathrm{G}, b^{3} \mathrm{G}, a^{3} \mathrm{H}, b^{3} \mathrm{H}, a^{1} \mathrm{D}, a^{1} \mathrm{G}, b^{1} \mathrm{G}, a^{1} \mathrm{H} .\end{array}\right.$ \\
\hline$d^{3} p\left({ }^{2} \mathrm{G}\right)$ & $21 \mathrm{~F}_{3}^{2}$ & 45802.49 & & 1.085 & $\left\{\begin{array}{c}a^{5} \mathrm{P}, a^{3} \mathrm{D}, b^{3} \mathrm{P}, c^{3} \mathrm{P}, a^{3} \mathrm{D}, b^{3} \mathrm{D}, a^{3} \mathrm{~F}, b^{3} \mathrm{~F}, c^{3} \mathrm{~F}, a^{3} \mathrm{G}, \\
b^{3} \mathrm{G}, a^{3} \mathrm{H}, b^{3} \mathrm{H}, a^{1} \mathrm{D}, a^{1} \mathrm{~F}, b^{1} \mathrm{~F}, b^{1} \mathrm{G}, c^{1} \mathrm{G} .\end{array}\right.$ \\
\hline$d^{3} p\left({ }^{2} \mathrm{D}\right)$ & $\begin{array}{l}x^{3} \mathrm{~F}_{2}^{2} \\
x^{3} \mathrm{~F}_{3}^{2} \\
x^{3} \mathrm{~F}_{4}^{2}\end{array}$ & $\begin{array}{l}46843.10 \\
46949.47 \\
50552.26\end{array}$ & $\begin{array}{r}606.37 \\
3602.79\end{array}$ & $\begin{array}{l}.43 \\
1.00 \\
1.22\end{array}$ & $\left\{\begin{array}{l}a^{5} \mathrm{P}, a^{3} \mathrm{~F}, c^{8} \mathrm{P}, a^{3} \mathrm{D}, b^{3} \mathrm{D}, a^{3} \mathrm{~F}, c^{3} \mathrm{~F}, d^{3} \mathrm{~F}, a^{3} \mathrm{G}, a^{3} \mathrm{H}, \\
b^{3} \mathrm{H}, a^{1} \mathrm{D}, b^{1} \mathrm{G} .\end{array}\right.$ \\
\hline$d^{3} p\left({ }^{2} \mathrm{P}\right)$ & $2{ }^{3} \mathrm{~S}_{\mathrm{i}}$ & 46858.94 & & 1.821 & $a^{3} \mathrm{P}, b^{3} \mathrm{P}, c^{3} \mathrm{P}, a^{3} \mathrm{D}, b^{3} \mathrm{D}, a^{3} \mathrm{~F}, b^{3} \mathrm{~F}, a^{1} \mathrm{P}$. \\
\hline$d^{3} p\left({ }^{4} \mathrm{P}\right)$ & ${ }^{3}{ }^{5} \mathrm{~S}_{2}$ & 47072.92 & & 1.940 & $a{ }^{5} \mathrm{P}, a^{3} \mathrm{P}, b^{3} \mathrm{P}, c^{3} \mathrm{P}, a^{3} \mathrm{D}, b^{3} \mathrm{D}, a^{1} \mathrm{D}$ \\
\hline$d^{3} p\left({ }^{2} \mathrm{H}\right)$ & $\begin{array}{l}y^{3}{ }^{3} \mathrm{H}_{4}^{8} \\
y^{3} \mathrm{H}_{5}^{6} \\
y^{3} \mathrm{H}_{6}^{6}\end{array}$ & $\begin{array}{r}47345.18 \\
48500.80 \\
48770.90\end{array}$ & $\begin{array}{r}1155.62 \\
270.10\end{array}$ & $\begin{array}{r}.963 \\
1.038 \\
1.132\end{array}$ & $\left\{\begin{array}{l}a{ }^{5} \mathrm{D}, a^{3} \mathrm{D}, b{ }^{3} \mathrm{D}, a^{3} \mathrm{~F}, b^{3} \mathrm{~F}, c^{3} \mathrm{~F}, a^{3} \mathrm{G}, b^{3} \mathrm{G}, a^{3} \mathrm{H}, \\
b^{3} \mathrm{H}, a^{1} \mathrm{G}, b^{1} \mathrm{G}, c^{1} \mathrm{G}, a^{1} \mathrm{H} .\end{array}\right.$ \\
\hline$d^{3} p\left({ }^{2} \mathrm{D}\right)$ & $\begin{array}{l}w^{3} \mathrm{~F}_{2}^{0} \\
w^{3} \mathrm{~F}_{3}^{3} \\
w^{3} \mathrm{~F}_{4}^{3}\end{array}$ & $\begin{array}{r}47755.76 \\
48077.67 \\
48627.20\end{array}$ & $\begin{array}{l}321.91 \\
549.53\end{array}$ & $\begin{array}{l}.63 \\
1.065 \\
1.100\end{array}$ & $\left\{\begin{array}{l}a{ }^{5} \mathrm{P}, a{ }^{5} \mathrm{D}, a^{3} \mathrm{~F}, c^{3} \mathrm{P}, d^{3} \mathrm{P}, a^{3} \mathrm{D}, b^{3} \mathrm{D}, a^{3} \mathrm{~F}, b^{3} \mathrm{~F}, \\
c^{3} \mathrm{~F}, d^{3} \mathrm{~F}, a^{3} \mathrm{G}, b^{3} \mathrm{G}, a^{3} \mathrm{H}, b^{3} \mathrm{H}, b^{1} \mathrm{D}, a^{1} \mathrm{~F}, a^{1} \mathrm{G}, \\
b^{1} \mathrm{G}, a^{11} \mathrm{H} .\end{array}\right.$ \\
\hline$d^{3} p\left({ }^{2} \mathrm{H}\right)$ & $\begin{array}{l}z^{3} I_{5}^{\circ} \\
z^{3} I_{6}^{\circ} \\
z^{3} I_{7}^{\circ}\end{array}$ & $\begin{array}{r}48150.50 \\
48617.49 \\
49389.73\end{array}$ & $\begin{array}{l}486.99 \\
772.24\end{array}$ & $\begin{array}{l}.870 \\
1.051 \\
1.15\end{array}$ & $a^{3} \mathrm{G}, b^{3} \mathrm{G}, a^{3} \mathrm{H}, b^{3} \mathrm{H}, a^{1} \mathrm{G}, b^{1} \mathrm{G}, a^{1} \mathrm{H}, a^{1} \mathrm{I}$ \\
\hline$d^{3} p\left({ }^{2} G\right)$ & ${ }^{1}{ }^{1} \mathrm{G}:$ & 48258. 44 & & 1.083 & $\left\{\begin{array}{c}a{ }^{5} \mathrm{D}, a^{3} \mathrm{~F}, b^{3} \mathrm{D}, a^{3} \mathrm{~F}, b^{3} \mathrm{~F}, a^{3} \mathrm{G}, b^{3} \mathrm{G}, a^{3} \mathrm{H}, b^{3} \mathrm{H}, \\
a^{1} \mathrm{~F}, b^{1} \mathrm{~F}, a^{1} \mathrm{G}, a^{1} \mathrm{H} .\end{array}\right.$ \\
\hline$d^{3} p\left({ }^{2} \mathrm{P}\right)$ & ${ }^{2}{ }^{1} \mathrm{Pi}$ & 48520.41 & & .936 & $\left\{\begin{array}{l}a^{3} \mathrm{P}, b^{3} \mathrm{P}, c^{3} \mathrm{P}, a^{3} \mathrm{D}, b^{3} \mathrm{D}, a^{3} \mathrm{~F}, b^{3} \mathrm{~F}, c^{3} \mathrm{~F}, a^{1} \mathrm{~S}, a^{1} \mathrm{P}, \\
a^{1} \mathrm{D} .\end{array}\right.$ \\
\hline
\end{tabular}


TABLE 2.-Terms of the $\mathrm{Cb}$ II spectrum-Continued

\begin{tabular}{|c|c|c|c|c|c|}
\hline $\begin{array}{l}\text { Electron } \\
\text { configura- } \\
\text { tion }\end{array}$ & $\begin{array}{l}\text { Term } \\
\text { symbol }\end{array}$ & Level & $\begin{array}{l}\text { Differ- } \\
\text { ence }\end{array}$ & $\underset{\rho}{\text { Observed }}$ & Combinations \\
\hline 1 & 2 & 3 & 4 & 5 & 6 \\
\hline$d^{3} p\left({ }^{2} \mathrm{H}\right)$ & $y^{1} G_{i}$ & 49158.16 & & .950 & $\left\{\begin{array}{c}a^{3} \mathrm{D}, b^{3} \mathrm{D}, a^{3} \mathrm{~F}, b^{3} \mathrm{~F}, c^{3} \mathrm{~F}, a^{3} \mathrm{G}, b^{3} \mathrm{G}, a^{3} \mathrm{H}, b^{3} \mathrm{H}, \\
a^{1} \mathrm{~F}, b^{1} \mathrm{~F}, a^{1} \mathrm{G}, b^{1} \mathrm{G}, c^{1} \mathrm{G}, a^{1} \mathrm{H} .\end{array}\right.$ \\
\hline$d^{2} s p$ & $495_{2}^{\circ}$ & 49536.62 & & & $a{ }^{5} \mathrm{~F}, d^{3} \mathrm{P}, a^{3} \mathrm{~F}, a^{3} \mathrm{G}, a^{1} \mathrm{P}$ \\
\hline$d^{2} s p$ & $\begin{array}{l}x^{3} \mathrm{D}_{1}^{\mathrm{i}} \\
x^{3} \mathrm{D}_{2}^{0} \\
x^{3} \mathrm{D}_{3}^{2}\end{array}$ & $\begin{array}{l}49687.72 \\
49245.55 \\
49759.19\end{array}$ & $\begin{array}{r}-442.17 \\
513.64\end{array}$ & $\begin{array}{l}.820 \\
1.257 \\
1.288\end{array}$ & $\left\{\begin{array}{l}a^{8} \mathrm{~F}, a^{3} \mathrm{P}, b{ }^{3} \mathrm{P}, c^{3} \mathrm{P}, d^{3} \mathrm{P}, a^{3} \mathrm{D}, b^{3} \mathrm{D}, a^{3} \mathrm{~F}, b^{3} \mathrm{~F}, \\
c^{3} \mathrm{~F}, d^{3} \mathrm{~F}, a^{3} \mathrm{G}, b^{3} \mathrm{G}, a^{1} \mathrm{~S}, a^{1} \mathrm{P}, a^{1} \mathrm{D}, b^{1} \mathrm{D}, a^{1} \mathrm{~F} .\end{array}\right.$ \\
\hline$d^{3} p\left({ }^{2} \mathrm{D}\right)$ & $\begin{array}{l}w^{3} \mathrm{Di}_{1} \\
w^{3} \mathrm{D}_{2}^{2} \\
w^{3} \mathrm{D}_{3}^{3}\end{array}$ & $\begin{array}{l}49738.45 \\
49772.30 \\
49864.25\end{array}$ & $\begin{array}{l}38.85 \\
91.95\end{array}$ & $\begin{array}{l}.616 \\
1.167 \\
1.308\end{array}$ & $\left\{\begin{array}{l}a^{5} \mathrm{P}, a{ }^{5} \mathrm{~F}, a^{3} \mathrm{P}, b^{3} \mathrm{P}, c^{3} \mathrm{P}, d^{3} \mathrm{P}, a^{3} \mathrm{D}, b^{3} \mathrm{D}, a^{3} \mathrm{~F}, \\
b^{3} \mathrm{~F}, c^{3} \mathrm{~F}, d^{3} \mathrm{~F}, a^{3} \mathrm{G}, a^{1} \mathrm{P}, a^{1} \mathrm{D} .\end{array}\right.$ \\
\hline$d^{2} s p$ & $503_{3}^{\circ}$ & 50068.70 & & 1. 245 & $a^{3} \mathrm{~F}, a^{3} \mathrm{P}, c^{3} \mathrm{P}, a^{3} \mathrm{D}, b^{3} \mathrm{~F}, c^{3} \mathrm{~F}, d^{3} \mathrm{~F}, b^{1} \mathrm{G}$. \\
\hline$d^{3} p\left({ }^{2} \mathrm{D}\right)$ & $\begin{array}{l}x^{3} \mathrm{P}_{0}^{0} \\
x^{3} \mathrm{Pi}_{1}^{1} \\
x^{3} \mathrm{P}_{2}^{0}\end{array}$ & $\begin{array}{l}50295.20 \\
5047.92 \\
50447.36\end{array}$ & $\begin{array}{r}179.72 \\
-27.56\end{array}$ & $\begin{array}{l}0 / 0 \\
1.202 \\
1.360\end{array}$ & $\left\{\begin{array}{l}a^{5} \mathrm{P}, a{ }^{3} \mathrm{~F}, a^{3} \mathrm{P}, b^{3} \mathrm{P}, c^{3} \mathrm{P}, a^{3} \mathrm{D}, b^{3} \mathrm{D}, a^{3} \mathrm{~F}, b^{3} \mathrm{~F}, \\
c^{3} \mathrm{~F}, a^{1} \mathrm{P}, a^{1} \mathrm{D} .\end{array}\right.$ \\
\hline$d^{3} p\left({ }^{2} \mathrm{H}\right)$ & $\begin{array}{l}x^{3} \mathrm{G}_{3}^{2} \\
x^{3} \mathrm{G}: \\
x^{3} \mathrm{G} ;\end{array}$ & $\begin{array}{l}50585.81 \\
50851.70 \\
50497.90\end{array}$ & $\begin{array}{r}266.39 \\
-353.80\end{array}$ & $\begin{array}{l}.811 \\
1.171 \\
1.188\end{array}$ & $\left\{\begin{array}{l}a^{\mathrm{s}} \mathrm{P}, a^{3} \mathrm{~F}, a^{3} \mathrm{P}, b^{3} \mathrm{P}, a^{3} \mathrm{D}, b^{3} \mathrm{D}, a^{3} \mathrm{~F}, b^{3} \mathrm{~F}, c^{3} \mathrm{~F}, \\
d^{3} \mathrm{~F}, a^{3} \mathrm{G}, b^{3} \mathrm{G}, a^{3} \mathrm{H}, b^{3} \mathrm{H}, a^{1} \mathrm{D}, b^{1} \mathrm{D}, a^{1} \mathrm{~F}, b^{1} \mathrm{G}, \\
a^{1} \mathrm{H}, a^{1} \mathrm{I} .\end{array}\right.$ \\
\hline$d^{3} p\left({ }^{2} \mathrm{D}\right)$ & $y 1 \mathrm{D} 2$ & 51182.16 & & .965 & $\left\{\begin{array}{c}a^{5 \mathrm{~F},}, b^{3} \mathrm{P}, c^{3} \mathrm{P}, a^{3} \mathrm{D}, b{ }^{3} \mathrm{D}, a^{3} \mathrm{~F}, b^{3} \mathrm{~F}, c^{3} \mathrm{~F}, d^{3} \mathrm{~F}, \\
a^{3} \mathrm{G}, a^{1} \mathrm{D}, b^{1} \mathrm{D}, b^{1} \mathrm{~F} .\end{array}\right.$ \\
\hline$d^{3} p\left({ }^{2} \mathrm{H}\right)$ & $21 \mathrm{I}_{0}$ & 51707.49 & & 1.010 & $a^{5} \mathrm{~F}, a^{3} \mathrm{G}, a^{3} \mathrm{H}, b^{3} \mathrm{H}, a^{1} \mathrm{H}, a^{1} \mathrm{I}$. \\
\hline$d^{3} p\left({ }^{2} \mathrm{D}\right)$ & $y 1 \mathrm{Pi}$ & 51787.87 & & 1.13 & $\left\{\begin{array}{c}a^{5} \mathrm{P}, a{ }^{3} \mathrm{P}, b \\
a \\
a\end{array}\right.$ \\
\hline$d^{3} p\left({ }^{2} \mathrm{~F}\right)$ & $\begin{array}{l}v^{2} \mathrm{~F}: \\
v^{3} \mathrm{~F}_{3}^{2} \\
v^{3} \mathrm{Fi}\end{array}$ & $\begin{array}{l}51927.28 \\
51936.39 \\
52279.64\end{array}$ & $\begin{array}{r}9.11 \\
343.25\end{array}$ & $\begin{array}{l}.80 \\
1.085 \\
1.261\end{array}$ & $\left\{\begin{array}{l}a^{\mathrm{s}} \mathrm{P}, a^{b} \mathrm{~F}, a^{3} \mathrm{P}, c^{3} \mathrm{P}, a^{3} \mathrm{D}, b^{3} \mathrm{D}, a^{3} \mathrm{~F}, b^{3} \mathrm{~F}, c^{3} \mathrm{~F}, \\
d^{3} \mathrm{~F}, a^{3} \mathrm{G}, a^{3} \mathrm{H}, b^{3} \mathrm{H}, a^{1} \mathrm{P}, a^{1} \mathrm{D}, b^{1} \mathrm{D}, a^{1} \mathrm{~F}, b^{1} \mathrm{~F}\end{array}\right.$ \\
\hline$d^{2} s p$ & $\begin{array}{l}w^{3} \mathrm{P}_{0}^{0} \\
w^{3} \mathrm{P}_{1}^{0} \\
w^{3} \mathrm{P}_{2}^{\circ}\end{array}$ & $\begin{array}{l}52129.80 \\
52214.85 \\
52388.59\end{array}$ & $\begin{array}{r}85.05 \\
173.74\end{array}$ & $\begin{array}{l}0 / 0 \\
1.43 \\
1.44\end{array}$ & $a^{5} \mathrm{P}, a^{3} \mathrm{~F}, b^{3} \mathrm{D}, c^{3} \mathrm{~F}, a^{3} \mathrm{G}$ \\
\hline$d^{3} p\left({ }^{4} \mathrm{P}\right)$ & $y^{3} \mathrm{Si}$ & 52553.90 & & 1.820 & $a^{5} \mathrm{P}, a^{3} \mathrm{P}, b^{3} \mathrm{P}, c^{3} \mathrm{P}, d^{3} \mathrm{P}, a^{3} \mathrm{~F}, a^{1} \mathrm{~S}$ \\
\hline$d^{2} s p$ & $527_{3}^{\circ}$ & 52714.88 & & 1.415 & $\begin{array}{l}a^{5} \mathrm{P}, a^{3} \mathrm{~F}, b{ }^{3} \mathrm{P}, a^{3} \mathrm{D}, a^{3} \mathrm{~F}, b^{3} \mathrm{~F}, c{ }^{3} \mathrm{~F}, d^{3} \mathrm{~F}, a^{3} \mathrm{G}, \\
{ }^{3} \mathrm{H},{ }^{1} \mathrm{D}, b{ }^{1} \mathrm{G} .\end{array}$ \\
\hline$d^{3} p\left({ }^{2} \mathrm{H}\right)$ & $y{ }^{1} \mathrm{H}_{5}^{\circ}$ & 52788.10 & & 1.006 & $b^{3} \mathrm{~F}, c^{3} \mathrm{~F}, b^{3} \mathrm{G}, b^{3} \mathrm{H}, a^{1} \mathrm{G}, b^{1} \mathrm{G}, c^{1} \mathrm{G}, a^{1} \mathrm{H}, a^{11}$. \\
\hline$d^{2} p\left({ }^{2} \mathrm{D}\right)$ & $y^{1} \mathrm{~F}_{3}^{\circ}$ & $530 \$ 5.95$ & & 1.036 & $\begin{array}{c}a^{5} \mathrm{P}, a^{5} \mathrm{~F}, b^{3} \mathrm{P}, a^{3} \mathrm{D}, b^{3} \mathrm{D}, a^{3} \mathrm{~F}, b^{3} \mathrm{~F}, c^{3} \mathrm{~F}, d^{3} \mathrm{~F}, a^{3} \mathrm{G}, \\
\quad b^{3} \mathrm{G}, a^{3} \mathrm{H}, a^{1} \mathrm{D}, b^{1} \mathrm{D}, a^{1} \mathrm{~F}, a^{1} \mathrm{G}, b^{1} \mathrm{G} .\end{array}$ \\
\hline$d^{3} p\left({ }^{2} \mathrm{~F}\right)$ & $\begin{array}{l}w^{3} \mathrm{G}_{3}^{\circ} \\
w^{3} \mathrm{G}_{4}^{\circ} \\
w^{3} \mathrm{G}_{5}^{6}\end{array}$ & $\begin{array}{l}53702.02 \\
54428.57 \\
55021.20\end{array}$ & $\begin{array}{l}726.55 \\
592.63\end{array}$ & $\begin{array}{l}.88 \\
1.062 \\
1.180\end{array}$ & $\left\{\begin{array}{l}a^{5} \mathrm{~F}, b^{3} \mathrm{P}, a^{3} \mathrm{D}, a^{3} \mathrm{~F}, b^{3} \mathrm{~F}, c^{3} \mathrm{~F}, d^{3} \mathrm{~F}, a^{3} \mathrm{G}, b^{3} \mathrm{G}, a^{3} \mathrm{H}, \\
b^{3} \mathrm{H}, a^{1} \mathrm{D}, a^{1} \mathrm{~F}, b^{1} \mathrm{~F}, a^{1} \mathrm{G}, b^{1} \mathrm{G}, a^{1} \mathrm{H}, a^{1} \mathrm{I} .\end{array}\right.$ \\
\hline$d^{3} p$ ('F) & $\begin{array}{l}{ }^{3} \mathrm{Di}_{1}^{\mathrm{i}} \\
v^{3} \mathrm{D}_{2}^{\mathbf{3}} \\
v^{3} \mathrm{D}_{3}^{\circ}\end{array}$ & $\begin{array}{l}54172.60 \\
54124.80 \\
54009.50\end{array}$ & $\begin{array}{r}-47.80 \\
-115.30\end{array}$ & $\begin{array}{l}.527 \\
1.164 \\
1.200\end{array}$ & $\left\{\begin{array}{c}a^{5} \mathrm{P}, a^{3} \mathrm{~F}, a^{3} \mathrm{P}, b^{3} \mathrm{P}, c^{3} \mathrm{P}, a^{3} \mathrm{D}, b^{3} \mathrm{D}, b^{3} \mathrm{~F}, c^{3} \mathrm{~F}, d^{3} \mathrm{~F}, \\
a^{3} \mathrm{G}, b^{3} \mathrm{G}, a^{3} \mathrm{H}, b^{3} \mathrm{H}, a^{1} \mathrm{D}, b^{1} \mathrm{D}, a^{1} \mathrm{G}, c^{1} \mathrm{G} .\end{array}\right.$ \\
\hline$d^{3} p\left({ }^{2} F\right)$ & $x^{1} \mathrm{~F}_{3}^{\circ}$ & 55460.54 & & 1.06 & $\begin{array}{l}b^{3} \mathrm{P}, a^{3} \mathrm{D}, b^{3} \mathrm{~F}, c^{3} \mathrm{~F}, d^{3} \mathrm{~F}, b^{3} \mathrm{G}, b^{3} \mathrm{H}, a^{1} \mathrm{D}, b^{1} \mathrm{~F}, b^{1} \mathrm{G}, \\
\quad c^{1} \mathrm{G} .\end{array}$ \\
\hline$d^{3} p\left({ }^{2} \mathrm{~F}\right)$ & $x{ }^{1} \mathrm{D}_{2}$ & 55721.74 & & 1.000 & $b^{3} \mathrm{P}, c^{3} \mathrm{P}, b^{3} \mathrm{D}, a^{3} \mathrm{~F}, a^{1} \mathrm{P}, a^{1} \mathrm{D}, b^{1} \mathrm{D}, a^{1} \mathrm{~F}, b^{1} \mathrm{~F}$. \\
\hline$d^{3} p\left({ }^{2} \mathrm{~F}\right)$ & $x^{1} \mathrm{G}_{4}$ & 57145.25 & & 1.000 & $b^{3} \mathrm{~F}, b^{3} \mathrm{H}, a^{1} \mathrm{~F}, b^{1} \mathrm{~F}, a^{1} \mathrm{G}, b^{1} \mathrm{G}, a^{1} \mathrm{H}$ \\
\hline$d^{2} s p\left({ }^{4} F\right)$ & $\begin{array}{l}u^{3} \mathrm{~F}_{2}^{\circ} \\
u^{3} \mathrm{~F}_{3}^{\circ} \\
u^{3} \mathrm{~F}_{4}^{\circ}\end{array}$ & $\begin{array}{l}57808.85 \\
58502.48 \\
59399.80\end{array}$ & $\begin{array}{l}694.08 \\
897.37\end{array}$ & $\begin{array}{r}.98 \\
1.25 \\
1.34\end{array}$ & $\left\{\begin{array}{l}a^{5 \mathrm{P}}, b^{3} \mathrm{P}, a^{3} \mathrm{D}, b^{3} \mathrm{D}, a^{3} \mathrm{~F}, b^{3} \mathrm{~F}, c^{3} \mathrm{~F}, d^{3} \mathrm{~F}, a^{3} \mathrm{G}, b^{3} \mathrm{G}, \\
b^{3} \mathrm{H}, a^{1} \mathrm{P}, b{ }^{1} \mathrm{D}, a^{1} \mathrm{G}, b{ }^{1} \mathrm{G} .\end{array}\right.$ \\
\hline$d^{2} s p\left({ }^{4} \mathrm{~F}\right)$ & $\begin{array}{l}u{ }^{3} \mathrm{D}_{1}^{\mathbf{i}} \\
u^{3} \mathrm{D}_{2}^{\mathbf{2}} \\
u^{3} \mathrm{D}_{3}^{3}\end{array}$ & $\begin{array}{l}59622.26 \\
59497.02 \\
59705.50\end{array}$ & $\begin{array}{r}-125.24 \\
208.48\end{array}$ & $\begin{array}{r}.64 \\
1.19 \\
1.33\end{array}$ & $\left\{\begin{array}{l}b^{3} \mathrm{P}, c^{3} \mathrm{P}, d^{3} \mathrm{P}, a^{3} \mathrm{D}, b^{3} \mathrm{D}, a^{3} \mathrm{~F}, b^{3} \mathrm{~F}, c^{3} \mathrm{~F}, d^{3} \mathrm{~F}, b^{3} \mathrm{G}, \\
a^{3} \mathrm{H} .\end{array}\right.$ \\
\hline$d^{2} s p\left({ }^{4} \mathrm{~F}\right)$ & $\begin{array}{l}v^{3} \mathrm{G}_{3}^{\circ} \\
v^{3} \mathrm{G} \\
v^{3} \mathrm{G}_{5}^{\circ}\end{array}$ & $\begin{array}{l}59509.23 \\
60094.50 \\
60439.75\end{array}$ & $\begin{array}{l}585.27 \\
345.25\end{array}$ & 1. 19 & $\left\{\begin{array}{l}b^{3} \mathrm{D}, a^{3} \mathrm{~F}, b^{3} \mathrm{~F}, c^{3} \mathrm{~F}, d^{3} \mathrm{~F}, a^{3} \mathrm{G}, b^{3} \mathrm{G}, a^{3} \mathrm{H}, b^{3} \mathrm{H}, a^{1} \mathrm{G}, \\
b^{1} \mathrm{G}, a^{1} \mathrm{H} .\end{array}\right.$ \\
\hline
\end{tabular}




\section{Journal of Research of the National Bureau of Standards}

The observed $g$-values (magnetic splitting factors) entered in column 5 of table 2 may be compared with Landé values for $L S$-coupling by referring to published tables [17]. Many departures from theoretical $L S$-values will be noticed, but the larger deviations apparently result from $g$-sharing rather than from $j j$-coupling. An interesting example is $z^{5} \mathrm{~F}_{1}^{\circ}$ the $g$-value of which is observed to be 0.122 instead of 0.000 as required by theory. There are two other levels, $z^{3} D_{1}^{\circ}$ and $z^{5} D_{1}^{\circ}$, associated with $z^{5} \mathrm{~F}_{1}^{\circ}$, and their deficiency in theoretical $g$-value amounts to 0.120 . Summing all $g$-values for levels ascribed to given electron configurations leads to the following results. The $g$-sum for 38 levels originating with $d^{4}$ is 33.016 , compared with the Landé sum 33.000 . The sum for 75 levels ascribed to $d^{3} p$ is 104.23 , compared with the Landé sum 105.00.

The combining properties of the terms are displayed in the last column of table 2. All combinations obey the selection rules $\Delta L=0$, $\pm 1, \pm 2, \pm 3$, and $\Delta J=0$ (except 0 to 0 ), \pm 1 , but, as usual, not all permitted combinations have been observed, either because of low intensity or masking by stronger lines. The total number of observed combinations of terms is 619 , of which 60 are singlet-singlet, 238 triplet-triplet, 12 quintet-quintet, 174 singlet-triplet, 24 singletquintet, 89 triplet-quintet, and 22 with 3 unidentified odd levels.

Since the number of levels and combinations was greatly increased in this analysis as compared with the earlier one [5], it was necessary to adjust some of the older values. Three of the old levels (11047.15, 40231.97, 44936.01) were not confirmed by the Zeeman effect-they have been replaced by genuine levels.

\section{ELECTION CONFIGURATIONS, THEORETICAL AND OBSERVED TERMS}

Columbium has atomic number 41 and the extra-nuclear structure of neutral columbium atoms is represented by $1 s^{2} 2 s^{2} 2 p^{6} 3 s^{2} 3 p^{6} 3 d^{10}$ $4 s^{2} 4 p^{6} 4 d^{4} 5 s$ (see below). Chemical valence and the first optical spectrum $(\mathrm{Cb} \mathrm{I})$ are associated with the last five electrons, and if one of these is removed by ionization, the ion may emit the second spectrum ( $\mathrm{Cb}$ II) characteristic of four valence electrons. These four may be distributed in various ways, and each configuration produces a different family of (even) spectral terms (atomic energy states) as follows:

$$
\begin{aligned}
& 4 d^{4},{ }^{1}(\mathrm{SDG}),{ }^{3}(\mathrm{PF}),{ }^{1}(\mathrm{SDFGI}),{ }^{3}(\mathrm{PDFGH}),{ }^{5}(\mathrm{D}) . \\
& 4 d^{3} 5 s, 1,3(\mathrm{D}),{ }^{1,3}(\mathrm{PDFGH}),{ }^{3,5}(\mathrm{PF}) . \\
& 4 d^{2} 5 s^{2},{ }^{1} \text { (SDG), }{ }^{3}(\mathrm{PF}) .
\end{aligned}
$$

In this analysis of the $\mathrm{Cb}$ II spectrum, all the $4 d^{4}$ terms have been found except the highest ${ }^{1} \mathrm{~S}$, all the $4 d^{3} 5 s$ terms except the highest 1,3(D), but no trace of $4 d^{2} 5 s^{2}$ terms that yield the ground state of the $\mathrm{Zr}$ I spectrum, which $\mathrm{Cb}$ II should resemble, according to the displacement law. Similar results were reported and explained for the V II spectrum [7].

Almost all the observed $\mathrm{Cb}$ II lines arise from combinations of the levels from (even) $4 d^{4}$ or $4 d^{3} 5 s$ configurations with excited states associated with (odd) configurations $4 d^{3} 5 p$ or $4 d^{2} 5 s 5 p$. The predicted and observed terms from these configurations are displayed in table 3. 
The lines remaining unclassified are too scattered and few to fix further terms with certainty. Efforts to use them for this purpose, in particular to find series-forming terms from the configuration $4 d^{3} 6 s$, proved futile, and it appears that the spectroscopic ionization potential of $\mathrm{Cb}^{+}$cannot be determined without study of the $\mathrm{Cb}$ II spectrum from a source that favors the development of series terms. The evidence from other spectra indicates that the principal ionization potential of $\mathrm{Cb}^{+}$will be about 14 volts.

TABLE 3.- $\mathrm{Cb}$ II predicted and observed terms

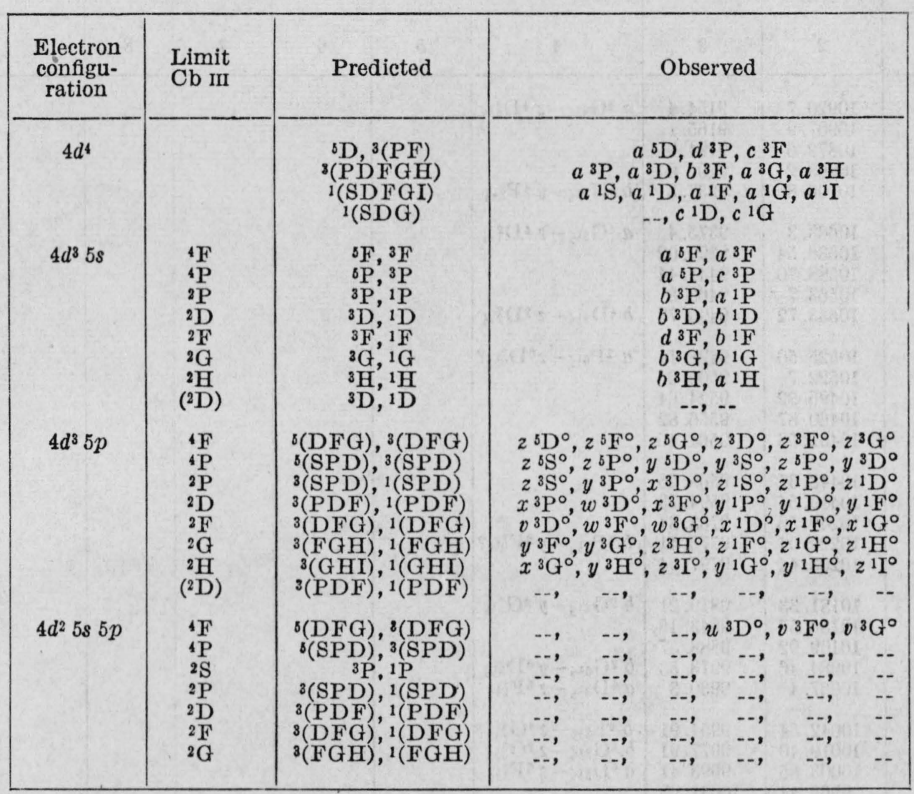

\section{TERM ANALYSIS OF $\mathrm{Cb}$ I}

\section{LINES OF THE $\mathrm{Cb}$ I SPECTRUM}

The data for the lines of $\mathrm{Cb}$, characteristic of neutral $\mathrm{Cb}$ atoms, are displayed in table 4, which is prepared according to the same plan as table 1 for $\mathrm{Cb}$ II. Column 1 shows the estimated intensity and character, column 2 the measured wavelength, column 3 the vacuum wave number, column 4 the term combinations, and the last six columns contain data on the Zeeman effect. There are 3,313 entries in the table, comprising all classified lines and nearly all unclassified lines, only those of doubtful origin and very weak lines of estimated intensity 2 or less being omitted. Where a line is classified in two or more ways, the more probable transition is written first. In many instances these double classifications are unresolved blends, the measured position falling between the calculated positions of the respective components. Statistical information as to classification and distribution of the classified lines into various categories is given in table 5 . 


\section{Journal of Research of the National Bureau of Standards}

\section{TABLE 4.-First spectrum of columbium ( $\mathrm{Cb}$ I)}

$?=$ Observed and calculated wave numbers disagree by $0.2 \mathrm{~cm}^{-1}$ or more.

* = Leads to value of observed $g$ showing excessive variation from values derived from other patterns. Components of opposite polarization only used in reduction if present.

$t=$ Classification not in agreement with observed Zeeman effect. Possibly fortuitous.

$t=$ Zeeman components show resolved hyperfine structure.

** $=$ Unafiected by magnetic field.

ur = Unresolved.

$\mathrm{L}=$ Landé $g$ assumed.

\begin{tabular}{|c|c|c|c|c|c|c|c|c|c|}
\hline $\begin{array}{l}\text { Intensity } \\
\text { Arc }\end{array}$ & $\lambda_{\text {air }} \mathrm{A}$ & $\begin{array}{l}\text { Wave } \\
\text { No. } \\
\text { vac. } \\
\mathrm{cm}^{-1}\end{array}$ & $\begin{array}{l}\text { Term combi- } \\
\text { nation }\end{array}$ & $\begin{array}{c}\text { Zeeman } \\
\text { type }\end{array}$ & $\begin{array}{l}\text { Separa- } \\
\text { tion }\end{array}$ & $\begin{array}{c}\text { Strong- } \\
\text { est } \\
p\end{array}$ & $\begin{array}{c}\text { Strong- } \\
\text { est } \\
n\end{array}$ & $g_{1}$ & $g_{2}$ \\
\hline 1 & 2 & 3 & 4 & 5 & 6 & 7 & 8 & 9 & 10 \\
\hline $\begin{array}{l}5 c \\
5 c \\
3 \\
5 \\
3\end{array}$ & $\begin{array}{l}10920.7 \\
10907.9 \\
10872.6 \\
10863.2 \\
10706.8\end{array}$ & $\begin{array}{l}9154.4 \\
9165.1 \\
9194.9 \\
9202.9 \\
9337.3\end{array}$ & $\begin{array}{l}a^{4} \mathrm{G}_{43 / 2}-2{ }^{4} \mathrm{D}_{31 / 2}^{0} \\
b^{2} \mathrm{H}_{41 / 2}-y^{4} \mathrm{~F}_{31 / 2}^{0}\end{array}$ & & & & & & \\
\hline $\begin{array}{c}2 \\
4 \\
7 \\
10 c \\
4\end{array}$ & $\begin{array}{l}10663.3 \\
10636.54 \\
10588.70 \\
10563.7 \\
10533.72\end{array}$ & $\begin{array}{l}9375.4 \\
9399.02 \\
9441.44 \\
9463.8 \\
9490.73\end{array}$ & $\begin{array}{l}a^{4} \mathrm{G}_{31 / 2}-z{ }^{4} \mathrm{D}_{31 / 2}^{\circ} \\
b^{4} \mathrm{D}_{31 / 2}-z^{2} \mathrm{D}_{21 / 3}^{0}\end{array}$ & & & & & $6 x$ & \\
\hline $\begin{array}{l}5 c \\
3 \\
5 c \\
3 \\
3\end{array}$ & $\begin{array}{l}10525.50 \\
10522.7 \\
10496.22 \\
10460.87 \\
10452.53\end{array}$ & $\begin{array}{l}9498.14 \\
9500.6 \\
9524.64 \\
9556.82 \\
9564.46\end{array}$ & $a^{2} \mathrm{P}_{01 / 2}-z^{6} \mathrm{D}_{03 / 2}^{\circ} ?$ & & & & & $1:$ & \\
\hline $\begin{array}{c}3 \\
10 c \\
5 c \\
2 \\
8 c\end{array}$ & $\begin{array}{l}10448.37 \\
10419.54 \\
10249.56 \\
10220.04 \\
10203.44\end{array}$ & $\begin{array}{l}9568.26 \\
9594.73 \\
9753.84 \\
9773.41 \\
9797.94\end{array}$ & $b^{4} \mathrm{P}_{13 / 2}-y^{6} \mathrm{~F}_{01 / 2}^{\circ} ?$ & & & & & & \\
\hline $\begin{array}{c}10 c \\
3 \\
4 \\
5 c \\
20 c\end{array}$ & $\begin{array}{l}\text { 10181. } 33 \\
10156.57 \\
10109.92 \\
10084.46 \\
10067.4\end{array}$ & $\begin{array}{l}9819.21 \\
9843.15 \\
9888.57 \\
9913.53 \\
9930.3\end{array}$ & 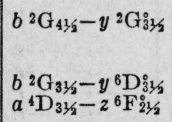 & & & & & & \\
\hline $\begin{array}{c}10 c \\
2 \\
30 c \\
4 c \\
15\end{array}$ & $\begin{array}{r}10042.54 \\
10019.40 \\
10003.85 \\
9965.44 \\
9957.29\end{array}$ & $\begin{array}{r}9954.91 \\
9977.91 \\
9993.41 \\
10031.93 \\
10040.14\end{array}$ & 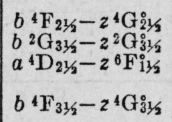 & & & & & & \\
\hline $\begin{array}{l}25 \\
20 \\
5 \\
4 c \\
2\end{array}$ & $\begin{array}{l}9912.26 \\
9910.35 \\
9896.6 \\
9890.09 \\
9815.56\end{array}$ & $\begin{array}{l}10085.75 \\
10087.70 \\
10101.7 \\
10108.36 \\
10185.11\end{array}$ & $\begin{array}{c}a{ }^{4} \mathrm{D}_{11 / 2}-z^{6} \mathrm{~F}_{01 / 2}^{0} \\
183_{51 / 2}-y^{2} \mathrm{G}_{1 / 2}^{0} \\
a^{2} \mathrm{D}_{13 / 2}-z^{6} \mathrm{D}_{03 / 2}^{\circ} ?\end{array}$ & & & & & & \\
\hline $\begin{array}{c}4 \\
50 \\
3 \\
12 \\
50 c\end{array}$ & $\begin{array}{l}9677.5 \\
9676.75 \\
9669.8 \\
9650.97 \\
9631.11\end{array}$ & $\begin{array}{l}10330.4 \\
10331.22 \\
10338.6 \\
10358.81 \\
10380.18\end{array}$ & 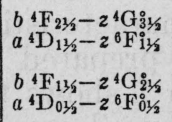 & & & & & & \\
\hline $\begin{array}{c}100 c \\
10 \\
2 \\
6 c \\
60 c\end{array}$ & $\begin{array}{l}9626.88 \\
9620.96 \\
9614.7 \\
9598.72 \\
9595.06\end{array}$ & $\begin{array}{l}10384.74 \\
10391.12 \\
10397.87 \\
10415.20 \\
10419.17\end{array}$ & 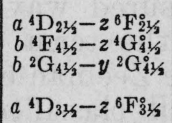 & & & & & & \\
\hline $\begin{array}{l}8 c \\
3 \\
5 \\
4 \\
6\end{array}$ & $\begin{array}{l}9549.13 \\
9518.30 \\
9474.57 \\
9472.02 \\
9470.93\end{array}$ & $\begin{array}{l}10469.29 \\
10503.20 \\
10551.67 \\
10554.52 \\
10555.73\end{array}$ & 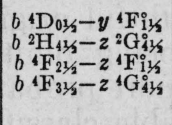 & & & & & & \\
\hline $\begin{array}{c}8 h \\
8 c \\
4 \\
20 \\
4\end{array}$ & $\begin{array}{l}9438.7 \\
9435.48 \\
9412.39 \\
9408.60 \\
9393.56\end{array}$ & $\begin{array}{l}10591.8 \\
10595.39 \\
10621.38 \\
10625.66 \\
10642.67\end{array}$ & $\begin{array}{l}b^{4} \mathrm{~F}_{31 / 2}-z^{4} \mathrm{~F}_{21 / 3}^{0} \\
b^{4} \mathrm{D}_{11 / 2}-y^{4} \mathrm{~F}_{21 / 2}^{2} \\
a^{4} \mathrm{D}_{01 / 2}-z^{6} \mathrm{~F}_{11 / 2}^{6}\end{array}$ & & & & & & \\
\hline
\end{tabular}


TABLE 4.-First spectrum of columbium (Cb I)-Continued

\begin{tabular}{|c|c|c|c|c|c|c|c|c|c|}
\hline $\begin{array}{c}\text { Intensity } \\
\text { Arc }\end{array}$ & $\lambda=\operatorname{sir} A$ & $\begin{array}{l}\text { Wave } \\
\text { No. } \\
\text { vac. } \\
\text { cm-1 }^{-1}\end{array}$ & $\begin{array}{l}\text { Term combi- } \\
\text { nation }\end{array}$ & $\begin{array}{c}\text { Zeeman } \\
\text { type }\end{array}$ & $\begin{array}{l}\text { Separa- } \\
\text { tion }\end{array}$ & $\begin{array}{c}\text { Strong- } \\
\text { est } \\
p\end{array}$ & $\begin{array}{c}\text { Strong- } \\
\text { est } \\
n\end{array}$ & $g_{1}$ & $g_{2}$ \\
\hline 1 & 2 & 3 & 4 & 5 & 6 & 7 & 8 & 9 & 10 \\
\hline $\begin{array}{c}10 \\
4 \\
3 \\
40 c \\
10\end{array}$ & $\begin{array}{l}9353.17 \\
9344.4 \\
9341.52 \\
9323.54 \\
9299.2\end{array}$ & $\begin{array}{l}10688.63 \\
10688.7 \\
10701.96 \\
10722.60 \\
10750.7\end{array}$ & $\begin{array}{l}a^{2} \mathrm{P}_{11 / 2}-z{ }^{4} \mathrm{P}_{01 / 2}^{0} b^{4} \mathrm{D}_{21 / 2}-y^{4} \mathrm{~F}_{51 / 2}^{0} \\
b^{4} \mathrm{D}^{4} \mathrm{~F}_{31 / 2}-z \\
a^{4} \mathrm{P}_{21 / 2}^{0} \\
a^{4} \mathrm{D}_{13 / 2}-z^{6} \mathrm{~F}_{21 / 2}^{0}\end{array}$ & & & 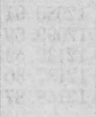 & & & 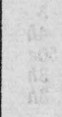 \\
\hline $\begin{array}{l}10 c \\
15 \\
20 \\
50 c \\
10 c\end{array}$ & $\begin{array}{l}9240.9 \\
9197.60 \\
9186.96 \\
9141.31 \\
9129.44\end{array}$ & $\begin{array}{l}10818.5 \\
10869.42 \\
10882.01 \\
10936.35 \\
10950.57\end{array}$ & $\begin{array}{l}b^{4} \mathrm{~F}_{41 / 2}-z^{4} \mathrm{~F}_{31 / 2}^{0} \\
b^{4} \mathrm{~F}_{21 / 2}-z^{4} \mathrm{~F}_{21 / 2}^{\circ} \\
b^{2} \mathrm{G}_{31 / 2}-y^{2} \mathrm{G}_{31 / 2}^{\circ} \\
a^{4} \mathrm{D}_{21 / 2}-z^{6} \mathrm{D}_{21 / 2}^{0}\end{array}$ & & & & tis & 18 & \\
\hline $\begin{array}{r}10 \\
3 \\
7 \\
7 \\
4 c\end{array}$ & $\begin{array}{l}9125.25 \\
9123.60 \\
9117.68 \\
9084.91 \\
9066.54\end{array}$ & $\begin{array}{l}10955.60 \\
10957.58 \\
10964.70 \\
11004.25 \\
11026.54\end{array}$ & $\begin{array}{l}b^{4} \mathrm{~F}_{11 / 2}-z^{4} \mathrm{~F}_{1 / 2}^{\circ} \\
b^{2} \mathrm{H}_{51 / 2}-y^{2} \mathrm{G}_{415}^{\circ} \\
a^{4} \mathrm{G}_{21 / 2}-z^{4} \mathrm{G}_{31 / 2}^{\circ} \\
b^{2} \mathrm{H}_{41 / 2}-y^{2} \mathrm{G}_{31 / 2}^{\circ}\end{array}$ & & & & & & \\
\hline $\begin{array}{c}20 c \\
5 \\
2 \\
8 \\
7\end{array}$ & $\begin{array}{l}9061.43 \\
9042.23 \\
9041.27 \\
9039.18 \\
9011.74\end{array}$ & $\begin{array}{l}11032.76 \\
11056.19 \\
11057.36 \\
11059.92 \\
11093.59\end{array}$ & $\begin{array}{c}b^{4} \mathrm{~F}_{31 / 2}-z^{4} \mathrm{~F}_{31 / 2}^{0} \\
b^{2} \mathrm{G}_{31 / 2}-x^{6} \mathrm{D}_{13 / 2}^{0} \\
b^{4} \mathrm{~F}_{41 / 2}-z^{4} \mathrm{G}_{51 / 2}^{0} \\
a^{4} \mathrm{D}_{11 / 2}-z^{6} \mathrm{D}_{11 / 2}^{0}\end{array}$ & & & & & & \\
\hline $\begin{array}{c}4 \\
20 c \\
20 c \\
6 \\
3\end{array}$ & $\begin{array}{l}8983.15 \\
8967.76 \\
8959.75 \\
8933.43 \\
8930.70\end{array}$ & $\begin{array}{l}11128.90 \\
11148.00 \\
11157.96 \\
11190.84 \\
11194.26\end{array}$ & $\begin{array}{l}b^{4} \mathrm{D}_{31 / 2}-y{ }^{4} \mathrm{~F}_{41 / 2}^{0} \\
a^{4} \mathrm{G}_{51 / 2}-2{ }^{4} \mathrm{G}_{51 / 2}^{0}\end{array}$ & & & & & & \\
\hline $\begin{array}{c}4 c \\
20 \\
6 \\
4\end{array}$ & $\begin{array}{l}8915.76 \\
8905.78 \\
8897.5 \\
8896.4\end{array}$ & $\begin{array}{l}11213.02 \\
11225.58 \\
11236.0 \\
11237.4\end{array}$ & 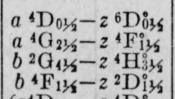 & & & & & & \\
\hline $100 c$ & 8815.56 & 11340.47 & $\left\{\begin{array}{l}a^{4} \mathrm{D}_{31 / 4}-2 \mathrm{D}_{21 / 2}^{2} \\
a^{4} \mathrm{D}_{21 / 4}-2 \mathrm{D}_{11 / 2}^{0}\end{array}\right.$ & & & & & & \\
\hline $\begin{array}{l}7 \\
3 \\
7 c \\
12 \\
20\end{array}$ & $\begin{array}{l}8799.76 \\
8798.22 \\
8769.57 \\
8767.97 \\
8740.96\end{array}$ & $\begin{array}{l}11360.83 \\
11362.82 \\
11399.94 \\
11402.02 \\
11437.25\end{array}$ & $\begin{array}{l}b^{4} \mathrm{~F}_{41 / 2}-z^{4} \mathrm{~F}_{115} \\
a^{4} \mathrm{G}_{31 / 5}-z^{4} \mathrm{G}_{41 / 5}^{0} \\
a^{4} \mathrm{D}_{11 / 5}-z^{4} \mathrm{D}_{01 / 5}^{0} \\
a^{4} \mathrm{G}_{31 / 5}-z^{4} \mathrm{~F}_{21 / 5}^{0}\end{array}$ & & & & & & $\frac{x}{45}$ \\
\hline $\begin{array}{c}4 \\
40 c \\
2 \\
2 h\end{array}$ & $\begin{array}{l}8717.09 \\
8697.55 \\
8695.10 \\
8681.94\end{array}$ & $\begin{array}{l}11468.57 \\
11494.34 \\
11497.58 \\
11515.00\end{array}$ & $\begin{array}{l}a^{4} \mathrm{G}_{51 / 5}-z^{4} \mathrm{~F}_{41 / 3} \\
b^{4} \mathrm{D}_{11 / 2}-y^{4} \mathrm{D}_{11 / 2}^{0} \\
b^{2} \mathrm{G}_{31 / 5}-y^{2} \mathrm{G}_{41 / 2}^{0}\end{array}$ & & & & & & \\
\hline $20 c$ & 8614.45 & 11605.22 & $\left\{\begin{array}{l}b^{2} \mathrm{H}_{415}-v^{2} \mathrm{G}_{41 / 2} \\
a^{2} \mathrm{G}_{31 / 5}-z^{6} \mathrm{~F}_{41 / 5}^{0}\end{array}\right.$ & 6 & & 0.27 & 1. 11 & 1.09 & $(1.12)$ \\
\hline $\begin{array}{c}30 c \\
30 c \\
20 c \\
50 \\
150 c\end{array}$ & $\begin{array}{l}8575.86 \\
8560.54 \\
8547.25 \\
8526.99 \\
8475.98\end{array}$ & $\begin{array}{l}11657.44 \\
11678.30 \\
11696.46 \\
11724.25 \\
11794.81\end{array}$ & 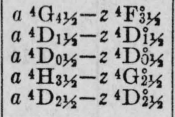 & $\begin{array}{c}4 \\
6 \\
* * \\
5 \\
6\end{array}$ & & $\begin{array}{c}0 \\
0 \\
0 \\
w \\
w\end{array}$ & $\begin{array}{l}1.20 \\
1.22 \\
0 \\
0.90 \\
1.38\end{array}$ & $\begin{array}{c}1.23 \\
(1.20) \\
0 \\
(0.69) \\
(1.36)\end{array}$ & $\begin{array}{l}(1.24) \\
1.24 \\
0 \\
0.61 \\
1.40\end{array}$ \\
\hline $\begin{array}{c}25 c \\
3 h \\
4 h \\
4 \\
5\end{array}$ & $\begin{array}{l}8439.76 \\
8434.31 \\
8433.90 \\
8417.07 \\
8414.72\end{array}$ & $\begin{array}{l}11845.43 \\
11853.08 \\
11853.66 \\
11877.36 \\
11880.67\end{array}$ & $a^{2} \mathrm{P}_{01 / 2}-2{ }^{4} \mathrm{P}_{01 / 2}^{0}$ & 5 & 1.78 & $\begin{array}{l}0 \\
0 \\
0.89\end{array}$ & $\begin{array}{l}1.41 \\
1.40 \\
1.55\end{array}$ & $\begin{array}{l}(1.23) \\
0.66\end{array}$ & 1. 29 \\
\hline $\begin{array}{c}15 c \\
3 h \\
4 h \\
4 h \\
20\end{array}$ & $\begin{array}{l}8406.23 \\
8389.36 \\
8387.88 \\
8371.39 \\
8350.03\end{array}$ & $\begin{array}{l}11892.67 \\
11916.59 \\
11918.69 \\
11942.17 \\
11972.72\end{array}$ & $a^{4} \mathrm{D}_{01 / 2}-2{ }^{4} \mathrm{Di}_{1 / 5}$ & 5 & 1.20 & 0.60 & 1.87 & 0.07 & 1.27 \\
\hline $\begin{array}{c}60 \\
500 c \\
3 H \\
1 h \\
1 h\end{array}$ & $\begin{array}{l}8346.07 \\
8320.93 \\
8293.64 \\
8277.98 \\
8277.39\end{array}$ & $\begin{array}{l}11978.40 \\
12014.59 \\
12054.12 \\
12076.93 \\
12077.79\end{array}$ & 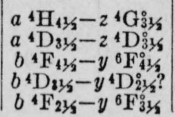 & $\begin{array}{r}7 b \\
6\end{array}$ & 0 & $\begin{array}{r}0 \\
w\end{array}$ & $\begin{array}{l}0.98 \\
1.43\end{array}$ & $\begin{array}{l}(0.98) \\
(1.42)\end{array}$ & $\begin{array}{l}0.98 \\
1.44\end{array}$ \\
\hline
\end{tabular}


518 Journal of Research of the National Bureau of Standards

TABLE 4.-First spectrum of columbium (Cb I)-Continued

\begin{tabular}{|c|c|c|c|c|c|c|c|c|c|}
\hline $\begin{array}{l}\text { Intensity } \\
\text { Arc }\end{array}$ & $\lambda_{\text {sir }} A$ & $\begin{array}{l}\text { Wave } \\
\text { No. } \\
\text { vac. } \\
\mathrm{cm}^{-1}\end{array}$ & $\begin{array}{l}\text { Term combi- } \\
\text { nation }\end{array}$ & $\begin{array}{c}\text { Zeeman } \\
\text { type }\end{array}$ & $\begin{array}{l}\text { Separa- } \\
\text { tion }\end{array}$ & $\begin{array}{c}\text { Strong- } \\
\text { est } \\
p\end{array}$ & $\begin{array}{c}\text { Strong- } \\
\text { est } \\
n\end{array}$ & $g_{1}$ & $\theta_{2}$ \\
\hline 1 & 2 & 3 & 4 & 5 & 6 & 7 & 8 & 9 & 10 \\
\hline $\begin{array}{c}3 \\
4 h \\
50 c \\
3 h \\
3 h\end{array}$ & $\begin{array}{l}8275.25 \\
8262.41 \\
8240.00 \\
8236.80 \\
8228.96\end{array}$ & $\begin{array}{l}12080.91 \\
12099.69 \\
12132.59 \\
12137.30 \\
12148.87\end{array}$ & 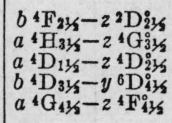 & 5 & & $w h$ & 1.55 & $(1.20)$ & 1.34 \\
\hline $\begin{array}{l}3 h \\
3 h \\
5 \\
2 H l \\
4\end{array}$ & $\begin{array}{l}8226.08 \\
8211.23 \\
8205.38 \\
8202.67 \\
8190.64\end{array}$ & $\begin{array}{l}12153.12 \\
12175.10 \\
12183.78 \\
12187.80 \\
12205.72\end{array}$ & 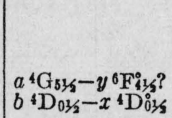 & & & & & & \\
\hline $\begin{array}{l}3 h \\
5 \\
6 \\
3 \\
3 h\end{array}$ & $\begin{array}{l}8189.33 \\
8176.22 \\
8173.03 \\
8168.48 \\
8157.00\end{array}$ & $\begin{array}{l}12207.66 \\
12227.23 \\
12232.01 \\
12238.82 \\
12256.04\end{array}$ & 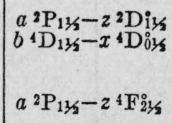 & & & 0 & 0.83 & & \\
\hline $\begin{array}{c}2 h \\
80 \\
6 h\end{array}$ & $\begin{array}{l}8154.42 \\
8135.20 \\
8118.67\end{array}$ & $\begin{array}{l}12259.92 \\
12288.89 \\
12313.91\end{array}$ & $\begin{array}{l}a^{4} \mathrm{G}_{31 / 2}-y^{6}{ }^{6} \mathrm{~F}_{21 / 2}^{2} \\
a^{4} \mathrm{H}_{53 / 2}-z^{4} \mathrm{G}_{41 / 2}^{4}\end{array}$ & 4 & & 0 & 1.06 & (1.12) & 1.14 \\
\hline $\begin{array}{l}7 w \\
2 h\end{array}$ & $\begin{array}{l}8118.17 \\
8107.85\end{array}$ & $\begin{array}{l}12314.66 \\
12330.34\end{array}$ & $\begin{array}{l}b{ }^{4} \mathrm{D}_{31 / 5}-y{ }^{4} \mathrm{D}_{31 / 2}^{0} \\
b^{4} \mathrm{D}_{21 / 2}-z^{2} \mathrm{~F}_{21 / 5}^{2}\end{array}$ & 6 & & $w$ & 1.41 & 1.41 & (1.42) \\
\hline $\begin{array}{c}4 \\
2 \\
4 h \\
5 \\
10\end{array}$ & $\begin{array}{l}8085.17 \\
8084.27 \\
8037.74 \\
8036.08 \\
8028.74\end{array}$ & $\begin{array}{l}12364.93 \\
12366.30 \\
12437.89 \\
12440.46 \\
12451.83\end{array}$ & $\begin{array}{l}a^{2} \mathrm{P}_{11 / 5}-z{ }^{4} \mathrm{P}_{21 / 5}^{0} \\
b^{4} \mathrm{P}_{03 / 2}-y \\
{ }^{6} \mathrm{D}_{1 / 2}^{0} \\
b^{4} \mathrm{D}_{23 / 2}-z^{2} \mathrm{P}_{11 / 2}^{0}\end{array}$ & & & & & & . \\
\hline $\begin{array}{r}4 h \\
50 c \\
5 h \\
3 h \\
3 h\end{array}$ & $\begin{array}{l}8024.74 \\
8017.67 \\
8017.23 \\
8010.85 \\
8007.50\end{array}$ & $\begin{array}{l}12458.04 \\
12469.03 \\
12469.71 \\
12479.64 \\
12484.86\end{array}$ & 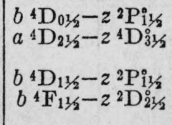 & 5 & & 0 & 1. $45^{*}$ & (1.36) & 1.40 \\
\hline $\begin{array}{l}8 \\
3 h\end{array}$ & $\begin{array}{l}8002.46 \\
7997.81\end{array}$ & $\begin{array}{l}12492.73 \\
12499.99\end{array}$ & $a^{4} \mathrm{H}_{41 / 2}-z^{4} \mathrm{G}_{41 / 3}^{0}$ & & & & & & \\
\hline $\begin{array}{l}3 h \\
4\end{array}$ & $\begin{array}{l}7993.74 \\
7979.99\end{array}$ & $\begin{array}{l}12506.35 \\
12527.90\end{array}$ & $\begin{array}{l}b^{4} \mathrm{P}_{11 / 2}-y^{4} \mathrm{D}_{01 / 2}^{0}{ }^{4} \mathrm{P}^{4} \mathrm{P}_{21 / 2}-x \\
0\end{array}$ & & & & & & 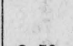 \\
\hline 10 & 7954.76 & 12567.64 & $a^{2} \mathrm{D}_{13 / 2}-z^{4} \mathrm{P}_{01 / 2}^{\circ}$ & 4 & 1.53 & .77 & 0.20 & 0.97 & 2.50 \\
\hline $\begin{array}{l}20 \\
3 h \\
3 \\
4 h \\
10 H w\end{array}$ & $\begin{array}{l}7938.90 \\
7922.78 \\
7909.36 \\
7902.14 \\
7898.88\end{array}$ & $\begin{array}{l}12592.74 \\
12618.37 \\
12639.78 \\
12651.32 \\
12656.55\end{array}$ & 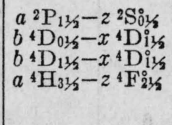 & 4 & 1.00 & 0.51 & 0.68 & 1.18 & 2.18 \\
\hline $\begin{array}{c}1 \\
60 \\
2 h\end{array}$ & $\begin{array}{l}7892.56 \\
7885.30 \\
7881.05\end{array}$ & $\begin{array}{l}12666.68 \\
12678.34 \\
12685.18\end{array}$ & 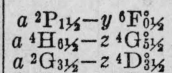 & 4 & & 0 & 1.04 & $(1.22)$ & 1.25 \\
\hline $\begin{array}{l}50 c \\
10\end{array}$ & $\begin{array}{l}7873.40 \\
7862.66\end{array}$ & $\begin{array}{l}12697.51 \\
12714.85\end{array}$ & $\begin{array}{l}b^{4} \mathrm{P}_{21 / 2}-y \mathrm{~A}^{4} \mathrm{D}^{\circ} 1 / 2 \\
b^{4} \mathrm{P}_{21 / 2}-27621 / 2\end{array}$ & $\begin{array}{l}4 \\
6\end{array}$ & 0.14 & $\begin{array}{l}0 \\
0.34\end{array}$ & $\begin{array}{l}1.30 \\
1.36 *\end{array}$ & $\begin{array}{l}1.52 \\
1.51\end{array}$ & $\begin{array}{l}(1.42) \\
(1.37)\end{array}$ \\
\hline $\begin{array}{r}15 \\
5\end{array}$ & $\begin{array}{l}7856.05 \\
7838.01\end{array}$ & $\begin{array}{l}12725.55 \\
12754.84\end{array}$ & $\begin{array}{l}b^{4} \mathrm{P}_{136}-y{ }^{4} \mathrm{D}_{11 / 2}^{\circ} a^{\circ} \\
a^{4} \mathrm{G}_{21 / 5}-z^{2} \mathrm{D}_{21 / 6}^{0}\end{array}$ & & & & & & 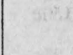 \\
\hline 8 & 7829.12 & 12769.32 & $\left\{\begin{array}{l}a^{2} \mathrm{D}_{21 / 6}-z^{4} \mathrm{P}_{136}^{0} \\
b^{4} \mathrm{D}_{11 / 6}-2^{4} \mathrm{~S}_{11 / 6}\end{array}\right.$ & & & & & & \\
\hline 10 & $\begin{array}{l}7787.08 \\
7772.11\end{array}$ & $\begin{array}{l}12838.26 \\
12862.98\end{array}$ & & & & 0.26 & 1.14 & & \\
\hline $\begin{array}{r}8 \\
8 \\
30 \\
10 \\
3\end{array}$ & $\begin{array}{l}7761.37 \\
7758.33 \\
7757.29 \\
7750.92 \\
7748.53\end{array}$ & $\begin{array}{l}12880.78 \\
12885.83 \\
12887.56 \\
12898.15 \\
12902.13\end{array}$ & $\begin{array}{l}a^{2} \mathrm{P}_{01 / 2}-z{ }^{4} \mathrm{P}_{1 / 2}^{0} \\
y^{6} \mathrm{~F}^{0}{ }^{0} 1 / 5-e^{6} \mathrm{D}_{41 / 5} \\
b^{4} \mathrm{P}_{21 / 5}-z^{2} \mathrm{~F}_{21 / 2}^{0}\end{array}$ & 4 & & $w$ & 1.09 & (1. 45) & 1.53 \\
\hline $\begin{array}{c}10 w \\
6 c \\
3 \\
100 \\
3\end{array}$ & $\begin{array}{l}7746.92 \\
7735.85 \\
7730.25 \\
7726.67 \\
7724.01\end{array}$ & $\begin{array}{l}12904.81 \\
12923.27 \\
12932.64 \\
12938.63 \\
12943.09\end{array}$ & $\begin{array}{l}b^{4} \mathrm{P}_{03 / 2}-x{ }^{6} \mathrm{D}_{01 / 2}^{0} \\
a^{6} \mathrm{~S}_{23 / 2}-z^{6} \mathrm{P}^{\circ} \mathrm{P}_{1 / 2}^{\circ}\end{array}$ & 4 & 0.38 & 0.19 & 1.41 & 1.98 & 2.36 \\
\hline
\end{tabular}


TABLE 4.-First spectrum of columbium (Cb $\mathrm{r}$ )-Continued

\begin{tabular}{|c|c|c|c|c|c|c|c|c|c|}
\hline $\begin{array}{c}\text { Intensity } \\
\text { Are }\end{array}$ & $\lambda_{\text {sir }} \mathrm{A}$ & $\begin{array}{l}\text { Wave } \\
\text { No. } \\
\text { vac. } \\
\mathrm{cm}^{-1}\end{array}$ & $\begin{array}{c}\text { Term combi- } \\
\text { nation }\end{array}$ & $\begin{array}{c}\text { Zeeman } \\
\text { type }\end{array}$ & $\begin{array}{l}\text { Separa- } \\
\text { tion }\end{array}$ & $\begin{array}{l}\text { Strong- } \\
\text { est } \\
p\end{array}$ & $\begin{array}{c}\text { Strong- } \\
\text { est } \\
n\end{array}$ & $g_{1}$ & $g_{2}$ \\
\hline 1 & 2 & 3 & 4 & 5 & 6 & 7 & 8 & 9 & 10 \\
\hline $\begin{array}{l}4 h \\
5 \\
50 w \\
3 h\end{array}$ & $\begin{array}{l}7716.83 \\
7707.39 \\
7703.29 \\
7694.37\end{array}$ & $\begin{array}{l}12955.13 \\
12971.00 \\
12977.90 \\
12992.94\end{array}$ & $\begin{array}{l}a^{4} \mathrm{H}_{51 / 2}-z^{4} \mathrm{G}_{51 / 2}^{0} \\
a^{4} \mathrm{H}_{41 / 2}-z^{4} \mathrm{~F}_{31 / 2}^{0} \\
y^{6} \mathrm{~F}_{41 / 2}^{0}-e^{6} \mathrm{D}_{31 / 2}^{0}\end{array}$ & 4 & & $H w$ & 0.92 & (1. 43) & 1.58 \\
\hline $3 h$ & 7686.50 & 13006.25 & $\left\{\begin{array}{l}a^{2} \mathrm{D}_{21 / 2}-z^{4} \mathrm{~F}_{11 / 2}^{0} \\
b^{4} \mathrm{D}_{11 / 2}-y^{4} \mathrm{P}_{21 / 2}^{0}\end{array}\right.$ & & & & & & \\
\hline $\begin{array}{l}4 h \\
3 h \\
10 H w \\
10 \\
5 h\end{array}$ & $\begin{array}{l}7678.34 \\
7669.13 \\
7662.74 \\
7661.62 \\
7660.32\end{array}$ & $\begin{array}{l}13020.07 \\
13035.71 \\
13046.58 \\
13048.49 \\
13050.70\end{array}$ & $\begin{array}{l}b^{4} \mathrm{~F}_{41 / 5}-y^{4} \mathrm{~F}_{31 / 2}^{0} \\
{ }^{6}{ }^{6} \mathrm{P}_{21 / 5}^{0}-e^{6} \mathrm{D}_{13 / 5}\end{array}$ & & & & t & & \\
\hline $\begin{array}{l}12 \\
5 H w \\
3 H \\
20 w \\
10\end{array}$ & $\begin{array}{l}7659.02 \\
7649.69 \\
7648.46 \\
7647.60 \\
7644.15\end{array}$ & $\begin{array}{l}13052.91 \\
13068.83 \\
13070.93 \\
13072.40 \\
13078.31\end{array}$ & $\begin{array}{l}b^{2} \mathrm{G}_{31 / 2}-x^{4} \mathrm{~F}_{21 / 2}^{0} \\
a^{4} \mathrm{P}_{21 / 2}-z^{6} \mathrm{~F}_{11 / 2}^{\circ} \\
y^{6} \mathrm{~F}_{31 / 3}^{0}-e^{6} \mathrm{D}_{21 / 2} \\
b^{4} \mathrm{~F}_{31 / 3}-y^{4} \mathrm{~F}_{21 / 2}^{0}\end{array}$ & 4 & & $H w$ & 0.68 & (1.38) & 1.66 \\
\hline $\begin{array}{l}1 h \\
5 c \\
12 \\
15 h c \\
10\end{array}$ & $\begin{array}{l}7641.77 \\
7640.72 \\
7639.82 \\
7638.15 \\
7628.16\end{array}$ & $\begin{array}{l}13082.38 \\
13084.17 \\
13085.72 \\
13088.58 \\
13105.72\end{array}$ & 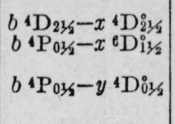 & & & 0.25 & 0.89 & & . \\
\hline $\begin{array}{l}4 \\
8 \\
4 h \\
5 c \\
1 h\end{array}$ & $\begin{array}{l}7625.57 \\
7621.14 \\
7615.88 \\
7603.40 \\
7595.06\end{array}$ & $\begin{array}{l}13110.17 \\
13117.79 \\
13126.85 \\
13148.40 \\
13162.83\end{array}$ & 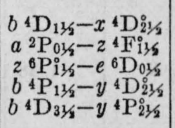 & 4 & 0.36 & 0.18 & $0.56^{*}$ & 1.68 & $(1.32)$ \\
\hline $\begin{array}{c}2 h \\
20 h l \\
8 \\
20 \\
500\end{array}$ & $\begin{array}{l}7593.63 \\
7585.29 \\
7584.07 \\
7583.22 \\
7574.57\end{array}$ & $\begin{array}{l}13165.31 \\
13179.79 \\
13181.91 \\
13183.39 \\
13198.44\end{array}$ & $\begin{array}{l}b^{4} \mathrm{P}_{2132}-x^{4} \mathrm{D}_{11 / 2}^{0} \\
y^{6} \mathrm{~F}_{21 / 5}-e^{6} \mathrm{D}_{11 / 2} \\
b^{4} \mathrm{~F}_{31 / 2}-y^{4} \mathrm{~F}_{31 / 2}^{0} \\
a^{6} \mathrm{~S}_{21 / 2}-z^{6} \mathrm{P}_{21 / 2}^{0}\end{array}$ & $\begin{array}{l}4 \\
6 \\
6\end{array}$ & $\begin{array}{l}0.12 \\
0.11\end{array}$ & $\begin{array}{l}0 \\
0.43 \\
0.27\end{array}$ & $\begin{array}{l}1.66 \\
1.29 * \\
1.93\end{array}$ & $\begin{array}{l}1.53 \\
(1.12) \\
1.99\end{array}$ & $\begin{array}{l}(1.44) \\
1.24 \\
1.88\end{array}$ \\
\hline $\begin{array}{c}10 \\
12 h \\
4 h \\
4 \\
10\end{array}$ & $\begin{array}{l}7551.96 \\
7547.71 \\
7543.38 \\
7540.18 \\
7535.36\end{array}$ & $\begin{array}{l}13237.96 \\
13245.41 \\
13253.01 \\
13258.64 \\
13267.12\end{array}$ & $\begin{array}{l}b^{4} \mathrm{~F}_{21 / 5}-y^{4} \mathrm{~F}_{11 / 2}^{0} \\
y^{6} \mathrm{~F}_{135}^{0}-e^{6} \mathrm{D}_{01 / 2} \\
b^{4} \mathrm{D}_{315}-z^{2} \mathrm{~F}_{31 / 2}^{0} \\
a^{4} \mathrm{H}_{31 / 2}-z^{4} \mathrm{~F}_{11 / 2}^{0} \\
b^{4} \mathrm{D}_{315}-x^{4} \mathrm{D}_{21 / 2}^{\circ}\end{array}$ & 4 & 2. 10 & 1.05 & -. & (1.06) & 3.16 \\
\hline $12 h$ & 7532.08 & 13272.90 & $z^{6} \mathrm{P}_{31 / 2}^{0}-e^{6} \mathrm{D}_{31 / 2}$ & 6 & 0.12 & 0.41 & 1. 61 & 1. 68 & 1.56 \\
\hline $\begin{array}{l}6 \\
50 w c \\
4 \\
8\end{array}$ & $\begin{array}{l}7523.34 \\
7519.77 \\
7517.51 \\
7517.14\end{array}$ & $\begin{array}{l}13288.31 \\
13294.62 \\
13298.62 \\
13299.28\end{array}$ & $\begin{array}{l}b^{4} \mathrm{~F}_{41 / 2}-y^{4} \mathrm{~F}_{41 / 2}^{0} \\
z^{6} \mathrm{P}_{21 / 2}^{0}-e^{6} \mathrm{D}_{21 / 2}\end{array}$ & 6 & 0.08 & 0.35 & 1. 29 & 1.25 & 1. 33 \\
\hline $\begin{array}{c}100 \\
15 w \\
8 w \\
20 \\
4 h\end{array}$ & $\begin{array}{l}7515.92 \\
7512.37 \\
7492.76 \\
7478.20 \\
7460.82\end{array}$ & $\begin{array}{l}13301.43 \\
13307.72 \\
13342.55 \\
13368.52 \\
13399.67\end{array}$ & $\begin{array}{l}a^{4} \mathrm{~A}_{11 / 2}-z \mathrm{P}_{01 / 2} \\
b \mathrm{P}^{4} \mathrm{P}_{01 / 2}-y \\
\end{array}$ & 6 & 1. 25 & 0.57 & $1.13^{*}$ & $\begin{array}{l}1.20 \\
(0.85)\end{array}$ & 1.08 \\
\hline $\begin{array}{r}10 h \\
2 h\end{array}$ & $\begin{array}{l}7459.08 \\
7458.29\end{array}$ & $\begin{array}{l}13402.79 \\
13404.21\end{array}$ & $b 4 \mathrm{P}_{11 / 2}-276_{21 / 5}^{\circ}$ & 4 & & $H w$ & 0.87 & 1. 70 & (1.37) \\
\hline $\begin{array}{l}12 h \\
12 h\end{array}$ & $\begin{array}{l}7456.31 \\
7452.88\end{array}$ & $\begin{array}{l}13404.21 \\
13407.77 \\
13413.94\end{array}$ & $\begin{array}{l}y^{6} \mathrm{~F}_{31 / 4}^{0}-e^{6} \mathrm{D}_{31 / 5} \\
y^{6} \mathrm{~F}_{135}^{\circ}-e^{6} \mathrm{D}_{135}\end{array}$ & $\begin{array}{l}6 \\
6\end{array}$ & $\begin{array}{l}0.18 \\
0.82\end{array}$ & $\begin{array}{l}0.63 \\
1.23\end{array}$ & $\begin{array}{l}\text { 1. } 59^{*} \\
1.43\end{array}$ & $\begin{array}{c}(1.38) \\
1.02\end{array}$ & $\begin{array}{l}1.56 \\
1.84\end{array}$ \\
\hline $10 h$ & 7446.60 & 13425.26 & $\left\{\begin{array}{l}y^{6}{ }^{6} \mathrm{~F}_{015}^{0}-e^{6} \mathrm{D}_{01 / 2} \\
a^{6} \mathrm{~S}_{21 / 5}-y^{6} \mathrm{~F}_{315}^{0}\end{array}\right.$ & & & & & & \\
\hline $\begin{array}{l}8 h \\
7 \\
7 \\
7 \\
7\end{array}$ & $\begin{array}{l}7445.26 \\
7436.99 \\
7436.02 \\
7435.37 \\
7434.62\end{array}$ & $\begin{array}{l}13427.67 \\
13442.60 \\
13444.36 \\
13445.53 \\
13446.89\end{array}$ & $\begin{array}{l}a^{2} \mathrm{~F}_{21 / 5}-y^{6} \mathrm{D}_{31 / 5}^{\circ} \\
y{ }^{6} \mathrm{~F}_{21 / 5}^{0}-e^{6} \mathrm{D}_{21 / 5} \\
a^{2} \mathrm{D}_{21 / 5}-z^{6} \mathrm{P}_{21 / 2}^{2}\end{array}$ & & & $H w$ & 0.69 & & \\
\hline 10 & 7430.12 & 13455.03 & $\left\{\begin{array}{l}b^{4} \mathrm{P}_{13,5}-x^{4} \mathrm{D}_{014} \\
a^{2} \mathrm{P}_{11,5}-z^{2} \mathrm{D}_{21 / 3}^{\circ}\end{array}\right.$ & & & & & & \\
\hline 12 & 7428.50 & 13457.97 & $b{ }^{4} \mathrm{~F}_{31 / 2}-y{ }^{4} \mathrm{~F}_{41 / 2}^{0}$ & & & & & & \\
\hline 8 & 7426.06 & 13462.39 & $\left\{\begin{array}{l}a^{6} \mathrm{P}_{21 / 5}-z^{6} \mathrm{~F}_{21 / 2}^{2} \\
a^{4} \mathrm{H}_{135}-z^{4} \mathrm{~F}_{1 / 5}\end{array}\right.$ & & & & & & \\
\hline 12 & $\begin{array}{l}7425.26 \\
7419.83\end{array}$ & $\begin{array}{l}13463.84 \\
13473.69\end{array}$ & $b \cdot \mathrm{F}_{21 / 2}-y-\mathrm{F}_{31 / 2}$ & & & & & & \\
\hline
\end{tabular}


TABLE 4.-First spectrum of columbium (Cb I)-Continued

\begin{tabular}{|c|c|c|c|c|c|c|c|c|c|}
\hline $\begin{array}{c}\text { Intensity } \\
\text { Arc }\end{array}$ & $\lambda_{\text {sir }} \mathbf{A}$ & $\begin{array}{l}\text { Wave } \\
\text { No. } \\
\text { vac. } \\
\mathrm{cm}^{-1}\end{array}$ & $\begin{array}{l}\text { Term combi- } \\
\text { nation }\end{array}$ & $\begin{array}{c}\text { Zeeman } \\
\text { type }\end{array}$ & $\begin{array}{l}\text { Separa- } \\
\text { tion }\end{array}$ & $\begin{array}{l}\text { Strong- } \\
\text { est } \\
p\end{array}$ & $\begin{array}{l}\text { Strong- } \\
\text { est } \\
n\end{array}$ & $g_{1}$ & $g_{2}$ \\
\hline 1 & 2 & 3 & 4 & 5 & 6 & 7 & 7 & 8 & 10 \\
\hline $\begin{array}{c}8 \\
500 \\
6 h \\
4 \\
100 c\end{array}$ & $\begin{array}{l}7409.81 \\
7372.51 \\
7366.48 \\
7362.10 \\
7353.17\end{array}$ & $\begin{array}{l}13491.91 \\
13560.17 \\
13571.27 \\
13579.35 \\
13595.84\end{array}$ & 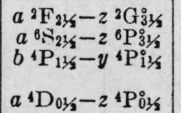 & $\ddagger 6$ & 0.31 & 0.14 & 1.22 & 1.97 & 2.45 \\
\hline $\begin{array}{c}4 h \\
2 h \\
4 \\
10 \\
3\end{array}$ & $\begin{array}{l}7350.58 \\
7348.86 \\
7340.26 \\
7332.30 \\
7331.44\end{array}$ & $\begin{array}{l}13600.63 \\
13603.81 \\
13619.75 \\
13634.54 \\
13636.13\end{array}$ & 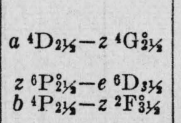 & & & & & & \\
\hline $\begin{array}{l}30 \\
25 h \\
20 w \\
8 h \\
3\end{array}$ & $\begin{array}{l}7328.38 \\
7323.91 \\
7317.03 \\
7311.93 \\
7300.69\end{array}$ & $\begin{array}{l}13641.83 \\
13650.14 \\
13662.99 \\
13672.52 \\
13693.57\end{array}$ & 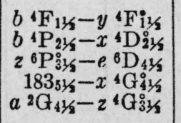 & & & & & & \\
\hline $\begin{array}{l}3 \mathrm{H} \\
10 \\
10 \\
8 h \\
2 h\end{array}$ & $\begin{array}{l}7293.22 \\
7276.76 \\
7274.78 \\
7268.92 \\
7262.80\end{array}$ & $\begin{array}{l}13707.59 \\
13738.60 \\
13742.34 \\
13753.42 \\
13765.01\end{array}$ & 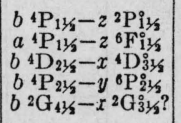 & & & & & & \\
\hline $\begin{array}{l}15 \\
10 \\
12 w\end{array}$ & $\begin{array}{l}7258.90 \\
7256.19 \\
7253.19\end{array}$ & $\begin{array}{l}13772.40 \\
13777.55 \\
13783.24\end{array}$ & $\begin{array}{l}b{ }^{4} \mathrm{~F}_{1315}-y^{4} \mathrm{~F}_{21 / 5}^{0} \\
a^{2} \mathrm{D}_{21 / 5}-z^{4} \mathrm{~F}_{31 / 6}^{2}\end{array}$ & & & & & & \\
\hline $\begin{array}{c}40 \\
8\end{array}$ & $\begin{array}{l}7252.35 \\
7248.12\end{array}$ & $\begin{array}{l}13784.81 \\
13792.89\end{array}$ & $\begin{array}{l}a^{2}{ }^{2} \mathrm{P}_{01 / 2}-z^{2} \mathrm{~S}_{013}^{0} \\
a^{4} \mathrm{P}_{01 / 2}-z^{6} \mathrm{~F}^{0} \mathrm{~F}_{01 / 2}\end{array}$ & 6 & 1.48 & 0.74 & 1.39 & 0.65 & 2.13 \\
\hline 7 & 7244.55 & 13799.68 & $a^{4} \mathrm{P}_{2165}-2^{6} \mathrm{D}_{136}$ & & & & & & \\
\hline 7 & 7241.81 & 13804.90 & $\left\{\begin{array}{l}a^{2} \mathrm{D}_{13 / 6}-z^{4} \mathrm{~F}_{1} 1 / 6 \\
b^{4} \mathrm{D}_{23,6}-z^{4} \mathrm{H}^{3} 1 / 6\end{array}\right.$ & & & & & & \\
\hline $\begin{array}{l}10 h \\
8 h \\
8 H\end{array}$ & $\begin{array}{l}7240.11 \\
7235.68 \\
7233.87\end{array}$ & $\begin{array}{l}13808.14 \\
13816.60 \\
13820.06\end{array}$ & 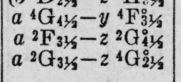 & & & & & & \\
\hline $\begin{array}{l}2 \\
2 \\
15 c \\
10 c \\
15\end{array}$ & $\begin{array}{l}7221.07 \\
7213.58 \\
7208.95 \\
7207.92 \\
7191.38\end{array}$ & $\begin{array}{l}13844.55 \\
13858.93 \\
13867.83 \\
13869.80 \\
13901.71\end{array}$ & 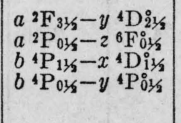 & & & & & & \\
\hline 8 & 7186.17 & 13911.79 & $\left\{\begin{array}{l}a^{4} G_{21 / 5}-y{ }^{4} F_{115}^{0} \\
a^{2} F_{316}-x^{6} D_{315}^{3}\end{array}\right.$ & & & & & & \\
\hline $\begin{array}{c}8 h \\
8 h \\
10 w \\
15 w\end{array}$ & $\begin{array}{l}7185.57 \\
7184.89 \\
7180.00 \\
7178.27\end{array}$ & $\begin{array}{l}13912.95 \\
13914.27 \\
13923.74 \\
13927.10\end{array}$ & 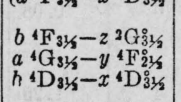 & & & & & & \\
\hline 4 & 7172.98 & 13937.37 & $\left\{\begin{array}{l}a^{2} \mathrm{H}_{31 / 6}-y^{4} \mathrm{~F}^{13 / 5} \\
b^{2} \mathrm{G}_{415}-v^{4} \mathrm{~F}_{31 / 6}^{3}\end{array}\right.$ & & & & & & \\
\hline $\begin{array}{c}4 \\
10 w \\
3 \\
100\end{array}$ & $\begin{array}{l}7170.75 \\
7165.84 \\
7163.92 \\
7159.44\end{array}$ & $\begin{array}{l}13941.71 \\
13951.26 \\
13955.00 \\
13963.73\end{array}$ & 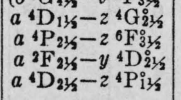 & 4 & 0.23 & 0.12 & 1.00 & 1.35 & 1.59 \\
\hline $\begin{array}{l}10 w \\
8 w \\
4 \\
12 \\
40\end{array}$ & $\begin{array}{l}7151.45 \\
7146.19 \\
7136.09 \\
7130.06 \\
7126.18\end{array}$ & $\begin{array}{l}13979.33 \\
13989.62 \\
14009.42 \\
14021.27 \\
14028.90\end{array}$ & 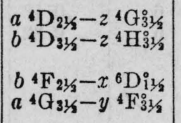 & & & & & & \\
\hline $\begin{array}{l}10 \\
10\end{array}$ & $\begin{array}{l}7122.96 \\
7121.91\end{array}$ & $\begin{array}{l}14035.24 \\
14037.31\end{array}$ & $b^{4} \mathrm{P}_{01 / 2}-x^{4} \mathrm{D}_{01 / 5}^{\circ}$ & & & 0 & 1.06 & & \\
\hline 10 & 7121.25 & 14038.62 & 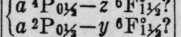 & & & & & & \\
\hline $\begin{array}{l}10 \\
15\end{array}$ & $\begin{array}{l}7120.66 \\
7119.32\end{array}$ & $\begin{array}{l}14039.78 \\
14042.42\end{array}$ & $a^{4} \mathrm{G}_{21 / 5}-y^{4} \mathrm{~F}_{23 / 5}^{\circ}$ & & & & & & \\
\hline $\begin{array}{l}3 h \\
30 w \\
50 c \\
3 \\
4\end{array}$ & $\begin{array}{l}7108.58 \\
7102.02 \\
7098.94 \\
7091.30 \\
7090.73\end{array}$ & $\begin{array}{l}14063.63 \\
14076.62 \\
14082.73 \\
14097.91 \\
14099.04\end{array}$ & 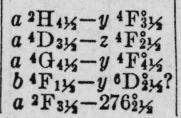 & & & & & & \\
\hline
\end{tabular}


TABLE 4.-First spectrum of columbium (Cb I) - Continued

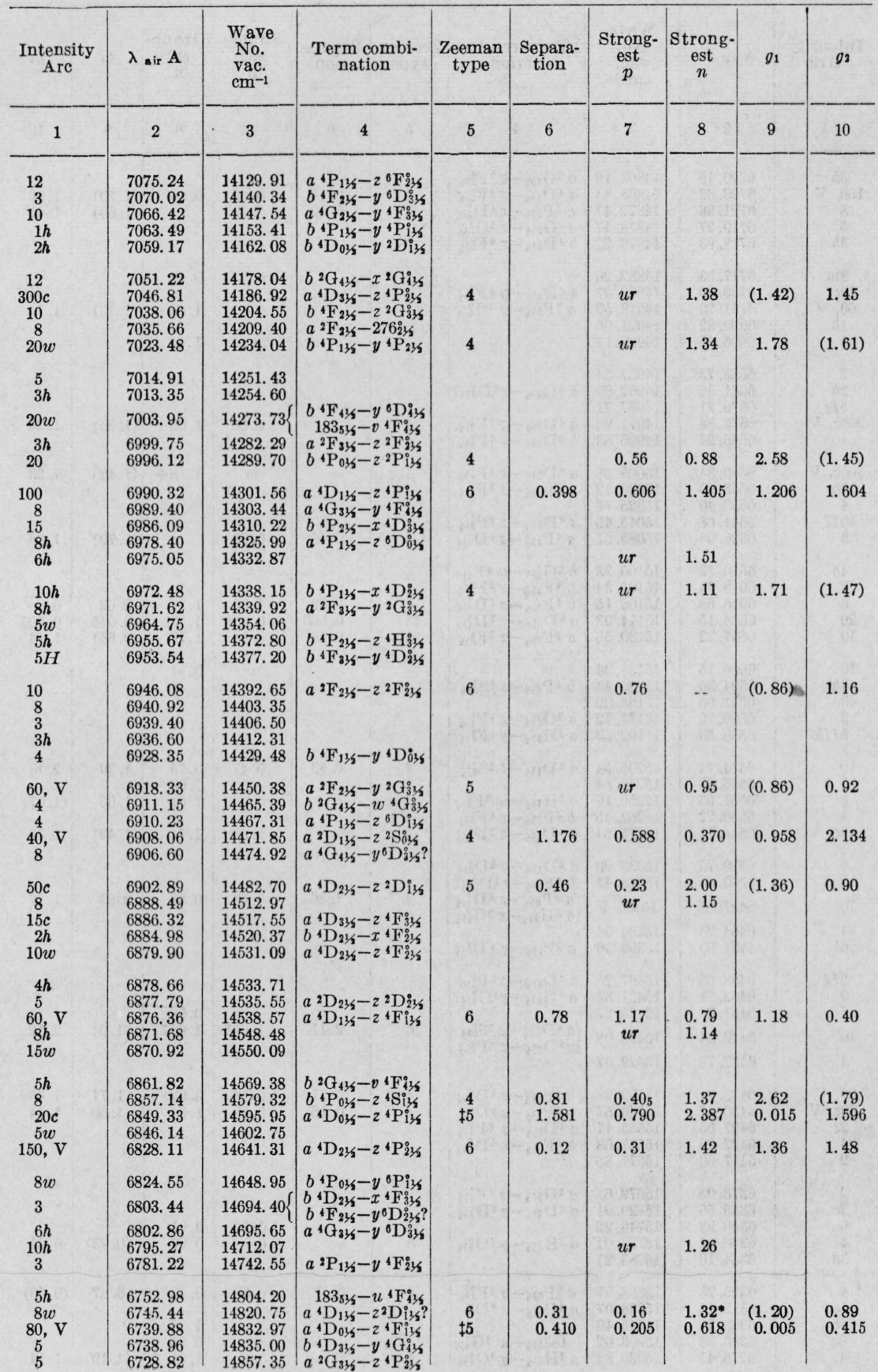


TaBle 4.-First spectrum of columbium (Cb I)-Continued

\begin{tabular}{|c|c|c|c|c|c|c|c|c|c|}
\hline $\begin{array}{c}\text { Intensity } \\
\text { Arc }\end{array}$ & $\lambda_{\text {air }} A$ & $\begin{array}{l}\text { Wave } \\
\text { No. } \\
\text { vac. } \\
\mathrm{cm}^{-1}\end{array}$ & $\begin{array}{l}\text { Term combi- } \\
\text { nation }\end{array}$ & $\begin{array}{c}\text { Zeeman } \\
\text { type }\end{array}$ & $\begin{array}{l}\text { Separa- } \\
\text { tion }\end{array}$ & $\begin{array}{l}\text { Strong- } \\
\text { est } \\
p\end{array}$ & $\begin{array}{c}\text { Strong- } \\
\text { est } \\
n\end{array}$ & $g_{1}$ & $g_{2}$ \\
\hline 1 & 2 & 3 & 4 & 5 & 6 & 7 & 8 & 9 & 10 \\
\hline $\begin{array}{c}5 h \\
150, \mathrm{~V} \\
8 \\
5 \\
3 h\end{array}$ & $\begin{array}{l}6726.19 \\
6723.62 \\
6721.98 \\
6719.27 \\
6718.93\end{array}$ & $\begin{array}{l}14863.16 \\
14868.84 \\
14872.47 \\
14878.47 \\
14879.22\end{array}$ & 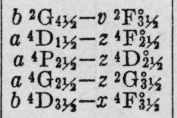 & $\begin{array}{l}4 \\
6\end{array}$ & $\begin{array}{l}0.14 \\
0.19\end{array}$ & $\begin{array}{l}0.07 \\
0.48\end{array}$ & 0.86 & $\begin{array}{l}(1.20) \\
(1.60)\end{array}$ & $\begin{array}{l}1.06 \\
1.41\end{array}$ \\
\hline $\begin{array}{l}8 w \\
10 c \\
60, \mathrm{~V} \\
4 h \\
5\end{array}$ & $\begin{array}{l}6717.56 \\
6709.89 \\
6701.20 \\
6699.82 \\
6696.45\end{array}$ & $\begin{array}{l}14882.26 \\
14899.27 \\
14918.59 \\
14921.66 \\
14929.17\end{array}$ & $\begin{array}{l}a^{4} \mathrm{D}_{31 / 5}-y^{6}{ }^{6} \mathrm{~F}_{21 / 2}^{0} \\
a^{2} \mathrm{~F}_{31 / 2}-y^{2} \mathrm{G}_{41 / 5}^{0}\end{array}$ & 4 & & $\begin{array}{l}0.25 \\
0 \\
u r\end{array}$ & $\begin{array}{l}0.73 \\
1.08 \\
1.03\end{array}$ & $(1.13)$ & 1.11 \\
\hline $\begin{array}{c}2 \\
2 h \\
5 H \\
200 c, \mathrm{v} \\
4\end{array}$ & $\begin{array}{l}6683.75 \\
6681.36 \\
6679.21 \\
6677.34 \\
6666.24\end{array}$ & $\begin{array}{l}14957.54 \\
14962.89 \\
14967.71 \\
14971.90 \\
14996.83\end{array}$ & $\begin{array}{l}a^{4} \mathrm{G}_{51 / 2}-x^{6} \mathrm{D}_{41 / 2}^{0} ? \\
a^{4} \mathrm{D}_{21 / 2}-z^{4} \mathrm{~F}_{31 / 2}^{3} \\
b^{4} \mathrm{D}_{31 / 2}-x^{4} \mathrm{~F}_{41 / 2}^{\circ}\end{array}$ & 4 & & 0.06 & $1.00+$ & $(1.36)$ & 1.24 \\
\hline $\begin{array}{l}300 c, \mathrm{~V} \\
10 h \\
4 \\
10 H \\
8\end{array}$ & $\begin{array}{l}6660.84 \\
6655.90 \\
6653.40 \\
6644.68 \\
6626.98\end{array}$ & $\begin{array}{l}15008.98 \\
15020.12 \\
15025.77 \\
15045.49 \\
15085.67\end{array}$ & 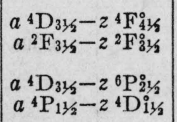 & 4 & 0.46 & 0.68 & $1.18+$ & (1. 72) & 1.33 \\
\hline $\begin{array}{l}4 h \\
2 \\
6 \\
20 \\
10\end{array}$ & $\begin{array}{l}6620.59 \\
6618.35 \\
6616.68 \\
6614.15 \\
6607.32\end{array}$ & $\begin{array}{l}15100.23 \\
15105.34 \\
15109.15 \\
15114.93 \\
15130.56\end{array}$ & $\begin{array}{l}b^{2} \mathrm{G}_{41 / 1}-u^{4} \mathrm{~F}^{0} i 1 / 2 \\
b^{4} \mathrm{~F}_{21 / 2}-z^{2} \mathrm{~F}_{21 / 5}^{0} \\
a^{4} \mathrm{P}_{01 / 2}-z^{4} \mathrm{D}_{01 / 2}^{0} \\
a^{4} \mathrm{D}_{01 / 2}-z^{2} \mathrm{D}_{11 / 2}^{1} \\
a^{2} \mathrm{~F}_{21 / 2}-z^{2} \mathrm{~F}_{31 / 2}^{0}\end{array}$ & $\begin{array}{r}6 \\
\ddagger 5 \\
5\end{array}$ & $\begin{array}{l}2.62 \\
0.860 \\
0.28\end{array}$ & $\begin{array}{l}1.31 \\
0.431 \\
0.14\end{array}$ & $\begin{array}{l}\text { 1. } 31 \\
\text { 1. } 336 \\
1.77\end{array}$ & $\begin{array}{l}2.62 \\
0.045 \\
(0.86)\end{array}$ & $\begin{array}{l}0 \\
0.905 \\
1.14\end{array}$ \\
\hline $\begin{array}{l}10 \\
4 h \\
40 c \\
3 \\
5 \text { Hld }\end{array}$ & $\begin{array}{l}6606.15 \\
6594.30 \\
6591.00 \\
6586.79 \\
6580.50\end{array}$ & $\begin{array}{l}15133.24 \\
15160.43 \\
15168.02 \\
15177.72 \\
15192.23\end{array}$ & $\begin{array}{l}b^{4} \mathrm{P}_{215}-z^{6} \mathrm{~S}_{21 / 2}^{0} \\
a^{2} \mathrm{G}_{41 / 2}-z^{4} \mathrm{~F}^{2} \mathrm{~F}^{4} / 1 \\
a^{4} \mathrm{H}_{51 / 2}-y^{4} \mathrm{~F}^{0} 1 / 2\end{array}$ & & & $u r$ & 0.91 & & 89: \\
\hline $\begin{array}{l}10 \\
6 \\
8 \\
4 \\
80 c, V\end{array}$ & $\begin{array}{l}6574.74 \\
6556.93 \\
6551.63 \\
6550.22 \\
6544.61\end{array}$ & $\begin{array}{l}15205.54 \\
15246.84 \\
15259.17 \\
15262.45 \\
15275.54\end{array}$ & $\begin{array}{l}a^{4} \mathrm{D}_{11 / 2}-z^{2} \mathrm{~S}_{01 / 5}^{\circ} \\
b^{2} \mathrm{H}_{41 / 5}-w^{2} \mathrm{~F}_{33 / 5}^{0} \\
b^{4} \mathrm{P}_{21 / 3}-x^{4} \mathrm{~F}_{31 / 5}^{0} \\
a^{4} \mathrm{D}_{31 / 3}-z^{2} \mathrm{D}_{21 / 2}^{0}\end{array}$ & $\begin{array}{l}4 \\
5\end{array}$ & 0.93 & $\begin{array}{l}0.47 \\
0 \\
0.09\end{array}$ & $\begin{array}{l}0.74 \\
1.08 \\
1.01 \\
1.71\end{array}$ & $\begin{array}{l}1.20 \\
1.05 \\
(1.42)\end{array}$ & $\begin{array}{c}2.13 \\
(1.07) \\
1.30\end{array}$ \\
\hline $\begin{array}{l}6 \\
2\end{array}$ & $\begin{array}{l}6519.70 \\
6500.83\end{array}$ & $\begin{array}{l}15333.90 \\
15378.41\end{array}$ & $\begin{array}{l}a^{2} \mathrm{D}_{11 / 2}-z^{2} \mathrm{D}_{21 / 6}^{0} \\
b^{4} \mathrm{~F}_{11 / 2}-x^{4} \mathrm{D}_{01 / 2}^{0}\end{array}$ & & & & & & \\
\hline 10 & 6497.85 & 15385.46 & $\left\{\begin{array}{l}a a^{4} \mathrm{P}_{01 / 2}-z^{4} \mathrm{D}_{1 / 1} \\
b^{2} \mathrm{P}_{41 / 2}-x^{2} \mathrm{G}_{41 / 2}\end{array}\right.$ & 4 & 1.36 & 0.68 & 0.58 & 2. 63 & 1.27 \\
\hline $\begin{array}{l}4 \\
6 h\end{array}$ & $\begin{array}{l}6494.23 \\
6491.90\end{array}$ & $\begin{array}{l}15394.04 \\
15399.56\end{array}$ & $a{ }^{2} \mathrm{P}_{1 / 3}-y^{4} \mathrm{D}_{013 / 2}^{0}$ & & & & & & atif \\
\hline $\begin{array}{l}2 H \\
2 \\
8\end{array}$ & $\begin{array}{l}6488.66 \\
6482.73 \\
6464.32\end{array}$ & $\begin{array}{l}15407.25 \\
15421.35 \\
15465.27\end{array}$ & $\begin{array}{l}a^{4} \mathrm{D}_{31 / 2}-z^{6} \mathrm{P}_{3}^{\circ} \mathrm{P}_{3 / 2}^{\circ} \\
a^{4} \mathrm{G}_{51 / 2}-y^{2} \mathrm{G}_{41 / 2}^{0} ?\end{array}$ & & & $u r$ & 0.98 & & \\
\hline $\begin{array}{r}10 \\
4\end{array}$ & $\begin{array}{l}6449.84 \\
6437.76\end{array}$ & $\begin{array}{l}15499.98 \\
15529.07\end{array}$ & 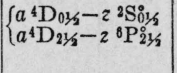 & $\ddagger 6$ & 2.11 & 1.055 & 1067 & 1.01 & 2.12 \\
\hline $\begin{array}{l}30, \mathrm{~V} \\
80 c, \mathrm{~V} \\
2 c \\
4 \\
2\end{array}$ & $\begin{array}{l}6433.24 \\
6430.47 \\
6427.66 \\
6422.06 \\
6417.60\end{array}$ & $\begin{array}{l}15539.98 \\
15546.67 \\
15553.47 \\
15567.03 \\
15577.85\end{array}$ & $\begin{array}{l}a^{4} \mathrm{P}_{11 / 2}-z^{4} \mathrm{D}_{21 / 6}^{0} \\
a^{4} \mathrm{P}_{21 / 2}-z^{4} \mathrm{D}_{31 / 2}^{0} \\
a^{4} \mathrm{D}_{11 / 2}-z^{6} \mathrm{P}_{13 / 2}^{13} \\
b^{4} \mathrm{~F}_{31 / 2}-x^{4} \mathrm{D}_{21 / 2}^{2}\end{array}$ & $\begin{array}{l}4 \\
4\end{array}$ & $\begin{array}{l}0.31 \\
0.16\end{array}$ & $\begin{array}{l}0.16 \\
0.08\end{array}$ & $\begin{array}{l}0.93 \\
1.04\end{array}$ & $\begin{array}{l}1.71 \\
1.60\end{array}$ & $\begin{array}{l}1.40 \\
1.44\end{array}$ \\
\hline $\begin{array}{l}2 \\
2 c \\
6 \\
4 \\
3 h\end{array}$ & $\begin{array}{l}6375.95 \\
6355.56 \\
6348.98 \\
6346.24 \\
6334.10\end{array}$ & $\begin{array}{l}15679.61 \\
15729.91 \\
15746.22 \\
15753.01 \\
15783.21\end{array}$ & $\begin{array}{l}a^{2} \mathrm{G}_{31 / 5}-z^{4} \mathrm{~F}_{41 / 2}^{\circ} \\
a^{4} \mathrm{D}_{21 / 2}-z^{2} \mathrm{D}_{21 / 2}^{2} \\
a^{2} \mathrm{H}_{43 / 2}-y^{2} \mathrm{G}_{31 / 2}^{\circ}\end{array}$ & 5 & & $\begin{array}{l}0.00 \\
0\end{array}$ & $\begin{array}{l}0.91 \\
0.97\end{array}$ & $(0.93)$ & 0.91 \\
\hline $\begin{array}{l}4 \\
5 c \\
4 c \\
6 c \\
4\end{array}$ & $\begin{array}{l}6325.78 \\
6312.16 \\
6309.24 \\
6286.38 \\
6275.41\end{array}$ & $\begin{array}{l}15803.97 \\
15838.07 \\
15845.40 \\
15903.02 \\
15930.82\end{array}$ & 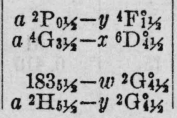 & 4 & 0.17 & $\begin{array}{l}0.09 \\
u r \\
u r \\
u r\end{array}$ & $\begin{array}{l}0.42 \\
1.13 \\
0.85 \\
1.01\end{array}$ & $(1.10)$ & $(0.50)$ \\
\hline
\end{tabular}


TABLE 4.-First spectrum of columbium (Cb I)-Continued

\begin{tabular}{|c|c|c|c|c|c|c|c|c|c|}
\hline $\begin{array}{l}\text { Intensity } \\
\text { Arc }\end{array}$ & $\lambda_{\text {sir }} \mathrm{A}$ & $\begin{array}{l}\text { Wave } \\
\text { No. } \\
\text { vac. } \\
\mathrm{cm}^{-1}\end{array}$ & $\begin{array}{l}\text { Term combi- } \\
\text { nation }\end{array}$ & $\begin{array}{c}\text { Zeeman } \\
\text { type }\end{array}$ & $\begin{array}{l}\text { Separa- } \\
\text { tion }\end{array}$ & $\begin{array}{c}\text { Strong- } \\
\text { est } \\
p\end{array}$ & $\begin{array}{c}\text { Strong- } \\
\text { est } \\
n\end{array}$ & $g_{1}$ & $g_{2}$ \\
\hline 1 & 2 & 3 & 4 & 5 & 6 & 7 & 7 & 8 & 10 \\
\hline $\begin{array}{c}8 c \\
4 h \\
20 c, \mathrm{~V} \\
5 h \\
20 \mathrm{c}, \mathrm{V}\end{array}$ & $\begin{array}{l}6260.77 \\
6257.38 \\
6251.76 \\
6228.25 \\
6221.95\end{array}$ & $\begin{array}{l}15968.07 \\
15976.72 \\
15991.08 \\
16051.44 \\
16067.70\end{array}$ & $\begin{array}{l}b^{2} \mathrm{G}_{41 / 2}-z^{2} \mathrm{I}_{51 / 2}^{\circ} \\
{ }^{1} 3_{51 / 2}-z^{2} \mathrm{I}_{61 / 4}^{\circ} \\
a^{4} \mathrm{D}_{11 / 2}-z^{2} \mathrm{D}_{21 / 2}^{\circ}\end{array}$ & 5 & & $\begin{array}{l}u r \\
u r \\
u r\end{array}$ & $\begin{array}{l}0.82 \\
1.18 \\
1.33\end{array}$ & $(1.20)$ & $(0.95)$ \\
\hline $\begin{array}{l}5 h \\
2 h \\
8, \mathrm{~V} \\
6 \\
12, \mathrm{~V}\end{array}$ & $\begin{array}{l}6219.59 \\
6218.44 \\
6213.06 \\
6204.75 \\
6164.30\end{array}$ & $\begin{array}{l}16073.79 \\
16076.06 \\
16090.69 \\
16112.24 \\
16217.96\end{array}$ & $\begin{array}{l}a^{4} \mathrm{G}_{41 / 5}-y^{2} \mathrm{G}_{41 / 5} \\
b^{4} \mathrm{D}_{01 / 2}-w^{4} \mathrm{~F}_{1 / 5} \\
b^{4} \mathrm{D}_{13 / 5}-w^{4} \mathrm{~F}_{11 / 2} \\
a^{2} \mathrm{~F}_{21 / 2}-y^{2} \mathrm{D}_{11 / 2}^{\circ}\end{array}$ & $\begin{array}{l}5 \\
6 \\
5\end{array}$ & $\begin{array}{l}0.47 \\
0.70\end{array}$ & $\begin{array}{l}0.24 \\
1.05 \\
u r\end{array}$ & $\begin{array}{l}1.32 \\
0.73 \\
0.88 \\
0.90\end{array}$ & $\begin{array}{l}0.03 \\
1.20 \\
(0.86)\end{array}$ & $\begin{array}{c}0.50 \\
(0.50) \\
0.81\end{array}$ \\
\hline $\begin{array}{l}10 c, \mathrm{~V} \\
7 c \\
7 c \\
4 c \\
6\end{array}$ & $\begin{array}{l}6148.11 \\
6142.53 \\
6128.68 \\
6118.10 \\
6110.94\end{array}$ & $\begin{array}{l}16260.67 \\
16275.44 \\
16312.22 \\
16340.43 \\
16359.58\end{array}$ & $\begin{array}{l}a^{2} \mathrm{~F}_{31 / 5}-y^{2} \mathrm{D}_{21 / 5}^{\circ} b^{4} \mathrm{P}_{21 / 2}-y^{4} \mathrm{D}_{1 / 5}^{0} \\
b^{4} \mathrm{D}_{21 / 2}^{\circ}-v^{4} \mathrm{~F}_{21 / 5}^{\circ}\end{array}$ & $\begin{array}{l}4 \\
4\end{array}$ & $\begin{array}{l}0.20 \\
0.41\end{array}$ & $\begin{array}{l}0.13 \\
0.20 \\
u r \\
0.56\end{array}$ & $\begin{array}{l}0.62 \\
0.93 \\
1.04 \\
1.04\end{array}$ & $\begin{array}{l}1.12 \\
1.55\end{array}$ & $\begin{array}{l}\text { (1. } 32) \\
1.96\end{array}$ \\
\hline $\begin{array}{l}9 \\
2 \\
4 c \\
6 \\
4\end{array}$ & $\begin{array}{l}6107.69 \\
6105.30 \\
6103.49 \\
6083.55 \\
6067.81\end{array}$ & $\begin{array}{l}16368.28 \\
16374.69 \\
16379.54 \\
16433.23 \\
16475.86\end{array}$ & 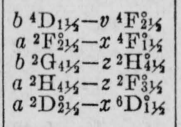 & $\begin{array}{l}4 \\
4\end{array}$ & & $\begin{array}{l}u r \\
0\end{array}$ & $\begin{array}{l}0.26 \\
0.81\end{array}$ & $\begin{array}{l}1.22 \\
(0.93) \\
(1.21)\end{array}$ & $\begin{array}{l}(1.05) \\
1.12 \\
1.47\end{array}$ \\
\hline $12 c$ & 6056.64 & 16506.25 & $\left\{\begin{array}{r}b^{4} \mathrm{D}_{23 / 5}-v^{4} \mathrm{~F}_{31 / 5}^{0} \\
18351 / 5-z^{2} \mathrm{H}_{51 / 5}^{6}\end{array}\right.$ & 4 & & $u r$ & 1.04 & 1.46 & (1.34) \\
\hline $\begin{array}{c}1 \\
10 c \\
2 h \\
20, \mathrm{~V}\end{array}$ & $\begin{array}{l}6052.59 \\
6048.71 \\
6047.46 \\
6045.49\end{array}$ & $\begin{array}{l}16517.29 \\
16527.88 \\
16531.30 \\
16536.69\end{array}$ & 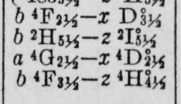 & 6 & & $\begin{array}{l}0.22 \\
u r\end{array}$ & $\begin{array}{l}0.97 \\
0.60\end{array}$ & $\begin{array}{l}0.99 \\
(1.12)\end{array}$ & $\begin{array}{l}0.95 \\
1.00\end{array}$ \\
\hline $\begin{array}{c}4 \\
10, \mathrm{~V}\end{array}$ & $\begin{array}{l}6041.96 \\
6031.83\end{array}$ & $\begin{array}{l}16546.35 \\
16574.14\end{array}$ & 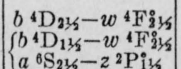 & 4 & & & 0.71 & 1.19 & $(1.00)$ \\
\hline $\begin{array}{l}12, \mathrm{~V} \\
5 c \\
1\end{array}$ & $\begin{array}{l}6029.74 \\
6028.73 \\
6022.50\end{array}$ & $\begin{array}{l}16579.88 \\
16582.66 \\
16599.81\end{array}$ & 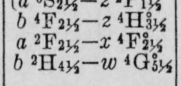 & 5 & & 0 & 0.93 & $(0.85)$ & 0.89 \\
\hline $\begin{array}{c}50 c, \mathrm{~V} \\
5 \\
30, \mathrm{~V} \\
100, \mathrm{IV} ? \\
5 c\end{array}$ & $\begin{array}{l}5997.86 \\
5986.61 \\
5986.08 \\
5983.21 \\
5971.25\end{array}$ & $\begin{array}{l}16668.01 \\
16699.33 \\
16700.81 \\
16708.82 \\
16742.28\end{array}$ & 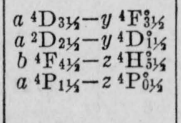 & $\begin{array}{l}6 \\
4 \\
4 \\
4\end{array}$ & 0.75 & $\begin{array}{l}0.79 \\
u r \\
0.21_{5} \\
0.38\end{array}$ & $\begin{array}{l}1.33 \\
1.12 \\
0.76+ \\
1.35 \\
1.35\end{array}$ & $\begin{array}{l}1.44 \\
(1.21) \\
(1.22) \\
1.72\end{array}$ & $\begin{array}{l}1.21 \\
\text { 1. } 27 \\
\text { 1. } 14 \\
\text { 2. } 47\end{array}$ \\
\hline $\begin{array}{l}4 c \\
1\end{array}$ & $\begin{array}{l}5965.36 \\
5964.56\end{array}$ & $\begin{array}{l}16758.82 \\
16761.06\end{array}$ & $\begin{array}{l}b^{2} \mathrm{H}_{51 / 5}-w^{2} \mathrm{G}_{i 1 / 2} \\
a^{2} \mathrm{P}_{13 / 2}-x^{4} \mathrm{D}_{11 / 3}\end{array}$ & 4 & & $u r$ & 0.70 & 1.03 & $(1.10)$ \\
\hline $\begin{array}{c}15, \mathrm{~V} \\
4 \\
2\end{array}$ & $\begin{array}{l}5957.69 \\
5949.90 \\
5938.62\end{array}$ & $\begin{array}{l}16780.39 \\
16802.36 \\
16834.28\end{array}$ & $\mid \begin{array}{l}b^{4} \mathrm{~F}_{31 / 5}-y \\
b^{2} \mathrm{G}_{41 / 5} \mathrm{G}_{31 / 5}^{1} z^{2} \mathrm{H}_{51 / 5}^{5} \\
a^{4} \mathrm{G}_{51 / 3}-z^{4} \mathrm{H}_{51 / 5}^{5}\end{array}$ & $\begin{array}{l}6 \\
5\end{array}$ & 0.11 & $\begin{array}{l}0.34 \\
u r\end{array}$ & $\begin{array}{l}1.07 \\
1.10\end{array}$ & $\begin{array}{l}(1.12) \\
0.99\end{array}$ & $\begin{array}{c}1.01 \\
(1.01)\end{array}$ \\
\hline $\begin{array}{l}15 c, \mathrm{~V} \\
12 c, \mathrm{~V}\end{array}$ & $\begin{array}{l}5934.15 \\
5928.234\end{array}$ & $\begin{array}{l}16846.96 \\
16863.77\end{array}$ & $\begin{array}{c}b^{2} \mathrm{H}_{51 / 2}-z^{2} \mathrm{I}_{615}^{\circ} \\
a^{6} \mathrm{~S}_{216}-z^{4} \mathrm{~S}_{115}\end{array}$ & 5 & & $u r$ & 1.52 & 1.00 & $(1.08)$ \\
\hline $\begin{array}{l}10 \\
2 h \\
1 h\end{array}$ & $\begin{array}{l}5927.40 \\
5924.27 \\
5920.16\end{array}$ & $\begin{array}{l}16866.14 \\
16875.05 \\
16886.76\end{array}$ & $\begin{array}{l}b^{4} \mathrm{D}_{215}-w^{4} \mathrm{~F}_{31 / 5} \\
a^{4} \mathrm{H}_{315}-z^{2} \mathrm{~F}_{21 / 2}^{2} ? \\
a^{4} \mathrm{D}_{21 / 5}-y^{4} \mathrm{~F}^{1} \mathrm{~F}_{1 / 5}\end{array}$ & 4 & 0.23 & 0.12 & $0.72^{*}$ & 1.43 & $(1.20)$ \\
\hline $\begin{array}{c}2 h \\
5 \\
20 c, \mathrm{~V} \\
200 c, \mathrm{~V} \\
3\end{array}$ & $\begin{array}{l}5910.55 \\
5904.48 \\
5903.80 \\
5900.59 \\
5894.61\end{array}$ & $\begin{array}{l}16914.22 \\
16931.61 \\
16933.56 \\
16942.77 \\
16959.96\end{array}$ & 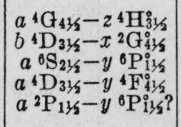 & $\begin{array}{l}5 \\
4 \\
4\end{array}$ & $\begin{array}{l}0.34 \\
0.32\end{array}$ & $\begin{array}{c}0.17 \\
0.16 \\
w\end{array}$ & $\begin{array}{l}1.55^{*} \\
1.46^{*} \\
1.09+\end{array}$ & $\begin{array}{l}(1.23) \\
1.41 \\
(1.42)\end{array}$ & $\begin{array}{c}0.89 \\
(1.09) \\
1.35\end{array}$ \\
\hline $\begin{array}{l}15 c, \mathrm{~V} \\
4 \\
5\end{array}$ & $\begin{array}{l}5893.43 \\
5885.94 \\
5878.97\end{array}$ & $\begin{array}{l}16963.36 \\
16984.94 \\
17005.08\end{array}$ & $\begin{array}{l}b^{4} \mathrm{P}_{11 / 6}-y^{4} \mathrm{~S}_{11 / 6}^{0} \\
b^{4} \mathrm{D}_{21 / 5}-u^{4} \mathrm{~F}_{31 / 6}^{3} \\
b^{4} \mathrm{~F}_{31 / 5}-x^{4} \mathrm{~F}_{21 / 6}^{5}\end{array}$ & $\begin{array}{l}6 \\
4\end{array}$ & $\begin{array}{l}0.26 \\
0.27\end{array}$ & $\begin{array}{l}0.39 \\
0.13\end{array}$ & $\begin{array}{l}1.88 \\
0.29\end{array}$ & $\begin{array}{l}\text { 1. } 70 \\
1.385\end{array}$ & $\begin{array}{l}(1.96) \\
\left(1.11_{5}\right)\end{array}$ \\
\hline $\begin{array}{l}20, \mathrm{~V} \\
8 \mathrm{c}\end{array}$ & $\begin{array}{l}5877.78 \\
5876.30\end{array}$ & $\begin{array}{l}17008.52 \\
17012.81\end{array}$ & 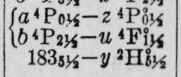 & $\begin{array}{l}6 \\
6\end{array}$ & 0.18 & $\begin{array}{l}0.09 \\
0.30\end{array}$ & $1.22-$ & 2. 64 & 2. 46 \\
\hline $\begin{array}{c}7 c \\
40 c, \mathrm{~V} \\
4 d \\
6 \\
80, \mathrm{IV} ?\end{array}$ & $\begin{array}{l}5875.24 \\
5874.68 \\
5872.46 \\
5868.89 \\
5866.45\end{array}$ & $\begin{array}{l}17015.88 \\
17017.50 \\
17023.93 \\
17034.29 \\
17041.37\end{array}$ & 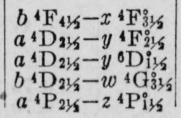 & $\begin{array}{l}5 \\
6 \\
4 \\
7 b, 4\end{array}$ & 0.36 & $\begin{array}{l}0.20 \\
0\end{array}$ & $\begin{array}{l}1.35 \\
1.21 \\
0.19 \\
1.58\end{array}$ & $\begin{array}{l}(1.22) \\
1.35 \\
1.45 \\
(1.60)\end{array}$ & $\begin{array}{l}1.19 \\
1.08 \\
1.09 \\
1.60\end{array}$ \\
\hline
\end{tabular}


TABLE 4.-First spectrum of columbium (Cb I)-Continued

\begin{tabular}{|c|c|c|c|c|c|c|c|c|c|}
\hline $\begin{array}{l}\text { Intensity } \\
\text { Arc }\end{array}$ & $\lambda_{\text {air }} \mathrm{A}$ & $\begin{array}{l}\text { Wave } \\
\text { No. } \\
\text { vac. } \\
\mathrm{cm}^{-1}\end{array}$ & $\begin{array}{l}\text { Term combi- } \\
\text { nation }\end{array}$ & $\underset{\text { type }}{\text { Zeeman }}$ & $\begin{array}{c}\text { Separa- } \\
\text { tion }\end{array}$ & $\begin{array}{l}\text { Strong- } \\
\text { est } \\
p\end{array}$ & $\begin{array}{c}\text { Strong- } \\
\text { est } \\
n\end{array}$ & $g_{1}$ & gs \\
\hline 1 & 2 & 3 & 4 & 5 & 6 & 7 & 8 & 9 & 10 \\
\hline $\begin{array}{l}6 \\
2 c \\
4 \\
5 \\
6\end{array}$ & $\begin{array}{l}5864.94 \\
5863.21 \\
5862.12 \\
5856.70 \\
5855.72\end{array}$ & $\begin{array}{l}17045.76 \\
17050.79 \\
17053.96 \\
17069.74 \\
17072.60\end{array}$ & $\begin{array}{l}b^{4} \mathrm{D}_{31 / 3}-w^{4} \mathrm{~F}_{3 / 2}^{\circ} \\
a^{4} \mathrm{G}_{33 / 2}-x^{4} \mathrm{D}_{31 / 2}^{\circ}\end{array}$ & 6 & 0.15 & $\begin{array}{c}u r \\
0.54\end{array}$ & $\begin{array}{l}0.90 \\
1.15\end{array}$ & 1. 07 & 1. 23 \\
\hline $\begin{array}{l}6 \\
1 \\
2 \\
20 c, \mathrm{~V} \\
30, \mathrm{~V}\end{array}$ & $\begin{array}{l}5852.79 \\
5851.94 \\
5850.67 \\
5846.10 \\
5842.47\end{array}$ & $\begin{array}{l}17081.14 \\
17083.63 \\
17087.33 \\
17100.69 \\
17111.32\end{array}$ & 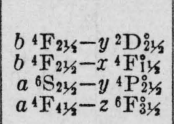 & $\begin{array}{l}6 \\
4\end{array}$ & 0.38 & $u r$ & $\begin{array}{l}1.78 \\
1.10\end{array}$ & $\begin{array}{l}1.97 \\
(1.33)\end{array}$ & $\begin{array}{l}1.59 \\
1.39\end{array}$ \\
\hline $\begin{array}{l}2 \\
100 c, \text { IV } \\
15 c\end{array}$ & & & & $\begin{array}{l}4 \\
5\end{array}$ & 0.39 & & & $\begin{array}{l}(1.36) \\
1.16\end{array}$ & $\begin{array}{l}1.26 \\
0.76\end{array}$ \\
\hline $40, \mathrm{~V}$ & $\begin{array}{l}5838.13 \\
5834.88\end{array}$ & $\begin{array}{l}17132.04 \\
17133.57\end{array}$ & $b^{4} \mathrm{~F}_{43 / 2}-x^{4} \mathrm{~F}_{41 / 5}$ & 6 & 0.05 & 0.12 & 1. 23 & 1.24 & 1. 21 \\
\hline 2 & 5834.40 & 17134. 98\{ & 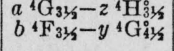 & & & & & & \\
\hline $\begin{array}{c}3 \\
20 c\end{array}$ & $\begin{array}{l}5825.41 \\
5820.61\end{array}$ & $\begin{array}{l}17161.43 \\
17175.58\end{array}$ & 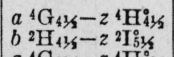 & $\begin{array}{l}6 \\
4\end{array}$ & 0.22 & $w^{0.98}$ & $0 .-64$ & (1. 23) & $\begin{array}{l}1.01 \\
(0.95)\end{array}$ \\
\hline $80, \mathrm{~V} ?$ & 5819.415 & 17179.11\{ & 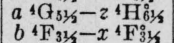 & 4 & & & $1.09+$ & $(1.26)$ & 1. 23 \\
\hline $\begin{array}{c}15 c \\
3\end{array}$ & $\begin{array}{l}5815.316 \\
5811.89\end{array}$ & $\begin{array}{l}\text { 17191.21 } \\
17201.35\end{array}$ & 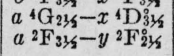 & $\begin{array}{l}5 \\
5\end{array}$ & 0.49 & ${ }_{u r}^{0.25}$ & $\begin{array}{l}2.51 \\
1.58\end{array}$ & $\begin{array}{l}0.79 \\
(1.13)\end{array}$ & $\begin{array}{l}1.28 \\
0.95\end{array}$ \\
\hline $\begin{array}{c}5 c \\
1 \\
4 c \\
30 d, \mathrm{~V} \\
1 h\end{array}$ & $\begin{array}{l}5810.68 \\
5805.91 \\
5804.65 \\
5804.02 \\
5801.78\end{array}$ & $\begin{array}{l}17204.93 \\
17219.07 \\
17222.80 \\
17224.67 \\
17231.32\end{array}$ & 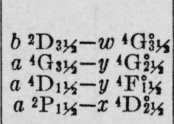 & 6 & 0.74 & 1.08 & 0.83 & 1. 20 & 0.46 \\
\hline $\begin{array}{l}2 c \\
20, \mathrm{~V} \\
7 c \\
80, \mathrm{III} \\
8\end{array}$ & & & & $\begin{array}{l}5 \\
5 \\
4 \\
6\end{array}$ & & $\begin{array}{l}w \\
w r \\
w \\
0.38\end{array}$ & & $\begin{array}{l}(0.74) \\
(1.26) \\
(1.24 \\
(0.85)\end{array}$ & $\begin{array}{l}0.87 \\
1.20 \\
1.34 \\
1.00\end{array}$ \\
\hline $\begin{array}{c}2 \\
30 c, \mathrm{~V} \\
4 \\
10 c \\
40, \mathrm{~V}\end{array}$ & & & 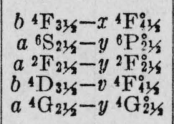 & $\begin{array}{l}5 \\
6 \\
6 \\
4 \\
6 \\
6\end{array}$ & 0.24 & $\begin{array}{l}u r \\
0.81^{*} \\
0 \\
0.12 \\
0.11\end{array}$ & & $\begin{array}{c}(1.12) \\
1.99 \\
(0.86) \\
1.45 \\
0.75\end{array}$ & $\begin{array}{l}1.23 \\
(1.77) \\
0.80 \\
(1.21) \\
0.71\end{array}$ \\
\hline $\begin{array}{l}80, \text { IV } \\
2 c \\
4 h \\
4 c \\
3\end{array}$ & $\begin{array}{l}5760.33 \\
5759.00 \\
5758.11 \\
5754.44 \\
5753.26\end{array}$ & $\begin{array}{l}17355.31 \\
17359.32 \\
17362.01 \\
17373.08 \\
17376.64\end{array}$ & 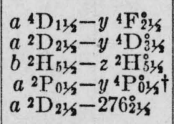 & $\begin{array}{l}4 \\
6\end{array}$ & & $\begin{array}{l}w \\
u r \\
--\end{array}$ & $\begin{array}{l}0.91+ \\
1.01 \\
1.23\end{array}$ & $(1.20)$ & $\begin{array}{l}1.08 \\
(1.01)\end{array}$ \\
\hline $\begin{array}{l}40, \mathrm{~V} \\
3 c \\
3 \\
6 \\
7\end{array}$ & $\begin{array}{l}5751.43 \\
5743.847 \\
5743.384 \\
5738.19 \\
5737.35\end{array}$ & $\begin{array}{l}17382.17 \\
17405.12 \\
17406.52 \\
17422.28 \\
17424.83\end{array}$ & 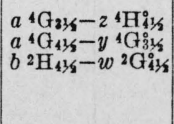 & $\begin{array}{l}4 \\
6\end{array}$ & & $\begin{array}{l}w \\
0.18 \\
0 \\
0.12\end{array}$ & $\begin{array}{l}0.76 \\
1.02 \\
1.07 \\
--\end{array}$ & $\begin{array}{l}(1.08) \\
1.00\end{array}$ & 1.01 \\
\hline $\begin{array}{c}1 \mathrm{c} \\
80, \mathrm{III} \\
9 \\
4 \\
30, \mathrm{~V}\end{array}$ & $\begin{array}{l}5734.35 \\
5729.185 \\
5725.66 \\
5722.70 \\
5716.34\end{array}$ & $\begin{array}{l}17433.94 \\
17449.66 \\
17460.40 \\
17469.44 \\
17488.87\end{array}$ & 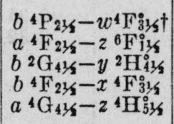 & $\begin{array}{l}4 \\
6 \\
5 \\
4\end{array}$ & 0.32 & $\begin{array}{l}0.34 \\
w \\
d \\
0.16 \\
w\end{array}$ & $\begin{array}{l}1.24 \\
0.85 \\
0.96 \\
2.03^{*} \\
0.76+\end{array}$ & $\begin{array}{l}(1.03) \\
0.97 \\
(0.85) \\
(1.23)\end{array}$ & $\begin{array}{l}1.15 \\
0.95 \\
1.17 \\
1.15\end{array}$ \\
\hline 6 & 5715. 58 & 17491. 20\{ & 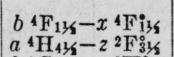 & $7 b$ & & 0 & 0.39 & 0.39 & 0.39 \\
\hline $5 c$ & 5713. 79 & 17496.68\{ & $\begin{array}{l}b^{2} \mathrm{G}_{31 / 6}-z^{2} \mathrm{H}_{1 / 6} \\
b^{2} \mathrm{P}_{135}-v^{4} \mathrm{~F}_{1 / 6}\end{array}$ & 4 & & 0 & 0.68 & 0.86 & $(0.82)$ \\
\hline $\begin{array}{r}2 \\
15\end{array}$ & $\begin{array}{l}5713.44 \\
5709.33\end{array}$ & $\begin{array}{l}17497.75 \\
17510.34\end{array}$ & $\begin{array}{l}a^{2} \mathrm{D}_{11 / 4}-y^{4} \mathrm{D}_{1 / 5}^{0} \\
a^{2} \mathrm{~F}_{31 / 4}-y^{2} \mathrm{~F}_{31 / 4}^{\circ}\end{array}$ & $7 b, 6$ & & 0 & 1. 14 & (1.13) & 1.15 \\
\hline 50, IV? & 5706.47 & 17519.12 & 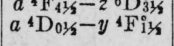 & 5 & 0.41 & 0.21 & 0.68 & 0.06 & 0.47 \\
\hline $\begin{array}{r}15 \\
4 \\
5 \\
8 \\
5 c\end{array}$ & $\begin{array}{l}5706.16 \\
5698.01 \\
5697.89 \\
5693.07 \\
5684.45\end{array}$ & \begin{tabular}{l|}
17520.07 \\
17545.13 \\
17545.50 \\
17560.35 \\
17586.98
\end{tabular} & 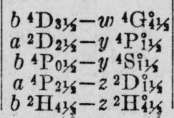 & $\begin{array}{l}4 \\
4 \\
4 \\
5 \\
6 \\
6\end{array}$ & $\begin{array}{l}0.70 \\
0.70 \\
0.68 \\
0.22\end{array}$ & $\begin{array}{l}0.11 \\
0.35 \\
0.35 \\
0.34 \\
1,01-\end{array}$ & $\begin{array}{l}1.63 \\
1.63 \\
2.62 \\
-.\end{array}$ & $\begin{array}{l}(1.43) \\
2.68 \\
2.68 \\
1.60 \\
1.04\end{array}$ & $\begin{array}{l}1.21 \\
1.98 \\
1.98 \\
0.92 \\
(0.82)\end{array}$ \\
\hline
\end{tabular}


TABLE 4.-First spectrum of columbium (Cb I)-Continued

\begin{tabular}{|c|c|c|c|c|c|c|c|c|c|}
\hline $\begin{array}{l}\text { Intensity } \\
\text { Are }\end{array}$ & $\lambda_{\text {air }} \mathrm{A}$ & $\begin{array}{l}\text { Wave } \\
\text { No. } \\
\text { vac. } \\
\mathrm{cm}^{-1}\end{array}$ & $\begin{array}{l}\text { Term combi- } \\
\text { nation }\end{array}$ & $\begin{array}{c}\text { Zeeman } \\
\text { type }\end{array}$ & $\begin{array}{c}\text { Separa- } \\
\text { tion }\end{array}$ & $\begin{array}{c}\text { Strong- } \\
\text { est } \\
p\end{array}$ & $\begin{array}{c}\text { Strong- } \\
\text { est } \\
n\end{array}$ & $g_{1}$ & $g_{2}$ \\
\hline 1 & 2 & 3 & 4 & 5 & 6 & 7 & 8 & 9 & 10 \\
\hline $\begin{array}{l}10 \\
3 \\
50, \text { IV } \\
60, \mathrm{~V} \\
9\end{array}$ & $\begin{array}{l}5677.453 \\
5675.942 \\
5671.90 \\
5671.09 \\
5666.86\end{array}$ & $\begin{array}{l}17608.66 \\
17613.34 \\
17625.90 \\
17628.41 \\
17641.57\end{array}$ & 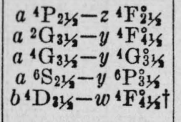 & $\begin{array}{l}6 \\
4\end{array}$ & $\begin{array}{l}0.07 \\
0.32\end{array}$ & $\begin{array}{l}0.24 \\
0.16 \\
0.14\end{array}$ & $\begin{array}{l}1.04 \\
1.83^{*} \\
0.42\end{array}$ & $\begin{array}{l}1.07 \\
2.02\end{array}$ & $\begin{array}{l}1.06 \\
1.00 \\
(1.70)\end{array}$ \\
\hline $\begin{array}{l}120, \text { III } \\
100, \text { III } \\
1 \\
5 \\
3 c\end{array}$ & $\begin{array}{l}5665.63 \\
5664.70 \\
5662.04 \\
5654.13 \\
5648.40\end{array}$ & $\begin{array}{l}17645.40 \\
17648.30 \\
17656.59 \\
17681.29 \\
17699.23\end{array}$ & 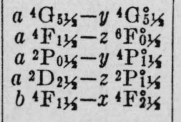 & $\begin{array}{l}7 b, 6 \\
5 \\
4 \\
5\end{array}$ & $\begin{array}{l}0.772 \\
0.23 \\
0.605\end{array}$ & $\begin{array}{l}0.12 \\
0.303\end{array}$ & $\begin{array}{l}1.26 \\
0.785 \\
0.76^{*} \\
2.038^{*}\end{array}$ & $\begin{array}{l}(1.26) \\
0.399 \\
(1.21) \\
(0.402)\end{array}$ & $\begin{array}{c}1.26 \\
-0.373 \\
1.44 \\
1.007\end{array}$ \\
\hline $\begin{array}{l}10 \\
80, \mathrm{III} \\
20 c \\
2 c \\
6 c\end{array}$ & $\begin{array}{l}5645.30 \\
5642.10 \\
5635.42 \\
5633.64 \\
5629.81\end{array}$ & $\begin{array}{l}17708.95 \\
17718.99 \\
17739.99 \\
17745.60 \\
17757.67\end{array}$ & 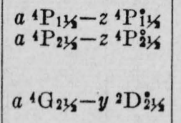 & $\begin{array}{l}6 \\
6\end{array}$ & $\begin{array}{l}0.09 \\
0.109\end{array}$ & $\begin{array}{l}0.13 \\
0.273 \\
u r\end{array}$ & $\begin{array}{l}\text { 1. } 65 \\
\text { 1. } 547 \\
\text { 1. } 08+\end{array}$ & $\begin{array}{l}1.70 \\
1.601\end{array}$ & $\begin{array}{l}\text { 1. } 61 \\
\text { 1. } 492\end{array}$ \\
\hline $\begin{array}{c}40, \mathrm{~V} \\
10 c \\
1 h \\
3\end{array}$ & $\begin{array}{l}5629.17 \\
5628.25 \\
5619.78 \\
5618.69\end{array}$ & $\begin{array}{l}17759.69 \\
17762.59 \\
17789.36 \\
17792.82\end{array}$ & 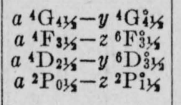 & $\begin{array}{l}6 \\
6 \\
4 \\
5\end{array}$ & 0.77 & $\begin{array}{l}u \\
0.53\end{array}$ & $\begin{array}{l}1.24 \\
1.63^{*} \\
1.10 h \\
1.85\end{array}$ & $\begin{array}{l}1.21 \\
(1.24) \\
(1.36) \\
0.70\end{array}$ & $\begin{array}{l}\text { (1. } 28) \\
1.39 \\
1.24 \\
1.47\end{array}$ \\
\hline $\begin{array}{l}7 \\
30, \mathrm{~V} \\
10 c \\
5 h \\
6 c\end{array}$ & $\begin{array}{l}5603.93 \\
5603.51 \\
5599.57 \\
5596.87 \\
5594.87\end{array}$ & $\begin{array}{l}17839.68 \\
17841.02 \\
17853.57 \\
17862.18 \\
17868.57\end{array}$ & 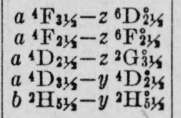 & $\begin{array}{l}6 \\
4 \\
5\end{array}$ & $\begin{array}{l}0.326 \\
0.37\end{array}$ & $\begin{array}{l}0.817 \\
0.20 \\
\text { ur }\end{array}$ & $\begin{array}{l}1.191 \\
1 . \overline{5} 2\end{array}$ & $\begin{array}{l}1.028 \\
(1.36) \\
(1.42)\end{array}$ & $\begin{array}{l}1.354 \\
0.99 \\
1.34\end{array}$ \\
\hline $\begin{array}{l}15 c \\
30, I V\end{array}$ & $\begin{array}{l}5590.95 \\
5586.99\end{array}$ & $\begin{array}{l}17881.10 \\
17893.77\end{array}$ & 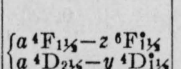 & 6 & 0.75 & $\begin{array}{l}u r \\
1.11\end{array}$ & $\begin{array}{l}1.15 \\
0.77\end{array}$ & 0.40 & 1.15 \\
\hline${ }_{4 c}^{15, \mathrm{~V}}$ & $\begin{array}{l}5578.28 \\
5578.07\end{array}$ & $\begin{array}{l}17921.71 \\
17922.38\end{array}$ & 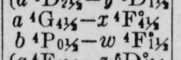 & 6 & & 0.010 & 1.22 & 1. 23 & 1.21 \\
\hline $15 c, \mathrm{~V}$ & 5576.16 & 17928.52 & 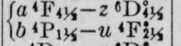 & & & & & & \\
\hline $\begin{array}{l}2 \\
10 c \\
2 \\
10, \mathrm{~V} \\
4\end{array}$ & $\begin{array}{l}5572.52 \\
5571.41 \\
5566.12 \\
5562.99 \\
5553.115\end{array}$ & $\begin{array}{l}17940.23 \\
17943.81 \\
17960.86 \\
17970.97 \\
18002.92\end{array}$ & 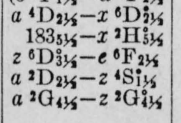 & 4 & & -- & $\begin{array}{l}1.08 \pm \\
0.31\end{array}$ & 1. 20 & (1.79) \\
\hline $\begin{array}{l}5 \\
60, \mathrm{~V} \\
2 \\
8 c \\
2 c\end{array}$ & $\begin{array}{l}5552.33 \\
5551.34 \\
5550.18 \\
5549.60 \\
5545.52\end{array}$ & $\begin{array}{l}18005.47 \\
18008.68 \\
18012.44 \\
18014.33 \\
18027.58\end{array}$ & 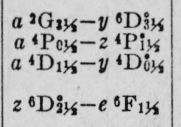 & 4 & & 0.52 & 1.08 & 2. 64 & 1.60 \\
\hline $\begin{array}{c}9 \\
3 \\
4 \\
30, I V ? \\
3\end{array}$ & $\begin{array}{l}5541.461 \\
5538.736 \\
5532.59 \\
5523.57 \\
5521.89\end{array}$ & $\begin{array}{l}18040.78 \\
18049.66 \\
18069.71 \\
18099.22 \\
18104.72\end{array}$ & 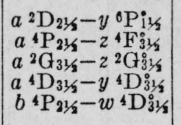 & 6 & & 0 & 1.41 & $(1.42)$ & 1.40 \\
\hline $\begin{array}{l}5 \\
15 c, \mathrm{~V} \\
4 c \\
2 h \\
7\end{array}$ & $\begin{array}{l}5517.39 \\
5512.81 \\
5511.22 \\
5510.69 \\
5509.13\end{array}$ & $\begin{array}{l}\text { 18119. } 49 \\
18134.54 \\
18139.78 \\
18141.52 \\
18146.66\end{array}$ & 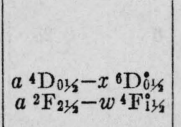 & 5 & & 0 & 1.11 & $(0.86)$ & 0.50 \\
\hline $1 c$ & 5506.74 & 18154.53 & $\left\{\begin{array}{l}a^{2} \mathrm{H}_{51 / 4}-y^{4} \mathrm{G}_{51 / 5}^{0} \\
b^{2} \mathrm{H}_{31 / 2}-v^{4} \mathrm{G}_{51 / 5}\end{array}\right.$ & & & & & & \\
\hline $\begin{array}{l}30 c, \text { III } \\
5 \\
1 \\
1\end{array}$ & $\begin{array}{l}5504.58 \\
5501.759 \\
5500.53 \\
5499.87\end{array}$ & $\begin{array}{l}18161.66 \\
18170.97 \\
18175.03 \\
18177.21\end{array}$ & $\begin{array}{l}a^{4} \mathrm{~F}_{31 / 2}-z^{6} \mathrm{D}_{31 / 5}^{\circ} \\
a^{2} \mathrm{D}_{11 / 5}-276_{21 / 6}^{\circ} \\
a^{2} \mathrm{H}_{435}-x^{4} \mathrm{~F}_{41 / 5}^{6}\end{array}$ & & & -- & 1. $09 h$ & & \\
\hline $\begin{array}{l}7 \\
5 h \\
4\end{array}$ & $\begin{array}{l}5499.53 \\
5494.09 \\
5491.062\end{array}$ & $\begin{array}{l}\text { 18178. } 33 \\
18196.33 \\
18206.37\end{array}$ & 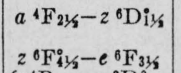 & & & & & & \\
\hline 1 & 5484.58 & 18227.88 & $\left\{\begin{array}{l}a^{4} \mathrm{P}_{11 / 2}-z^{2} \mathrm{D}_{13 / 5}^{\circ} \\
a^{4} \mathrm{H}_{413 / 2}-z^{4} \mathrm{H}_{31 / 6}^{\circ}\end{array}\right.$ & & & & & & \\
\hline 8 & 5483.48 & 18231.54 & $\left|a^{4} \mathrm{D}_{1 / 3,5}-y^{4} \mathrm{D}_{1 / 6}\right|$ & 6 & & -- & 1. 25 & (1. 20) & 1. 30 \\
\hline
\end{tabular}


TABLE 4.-First spectrum of columbium (Cb I)-Continued

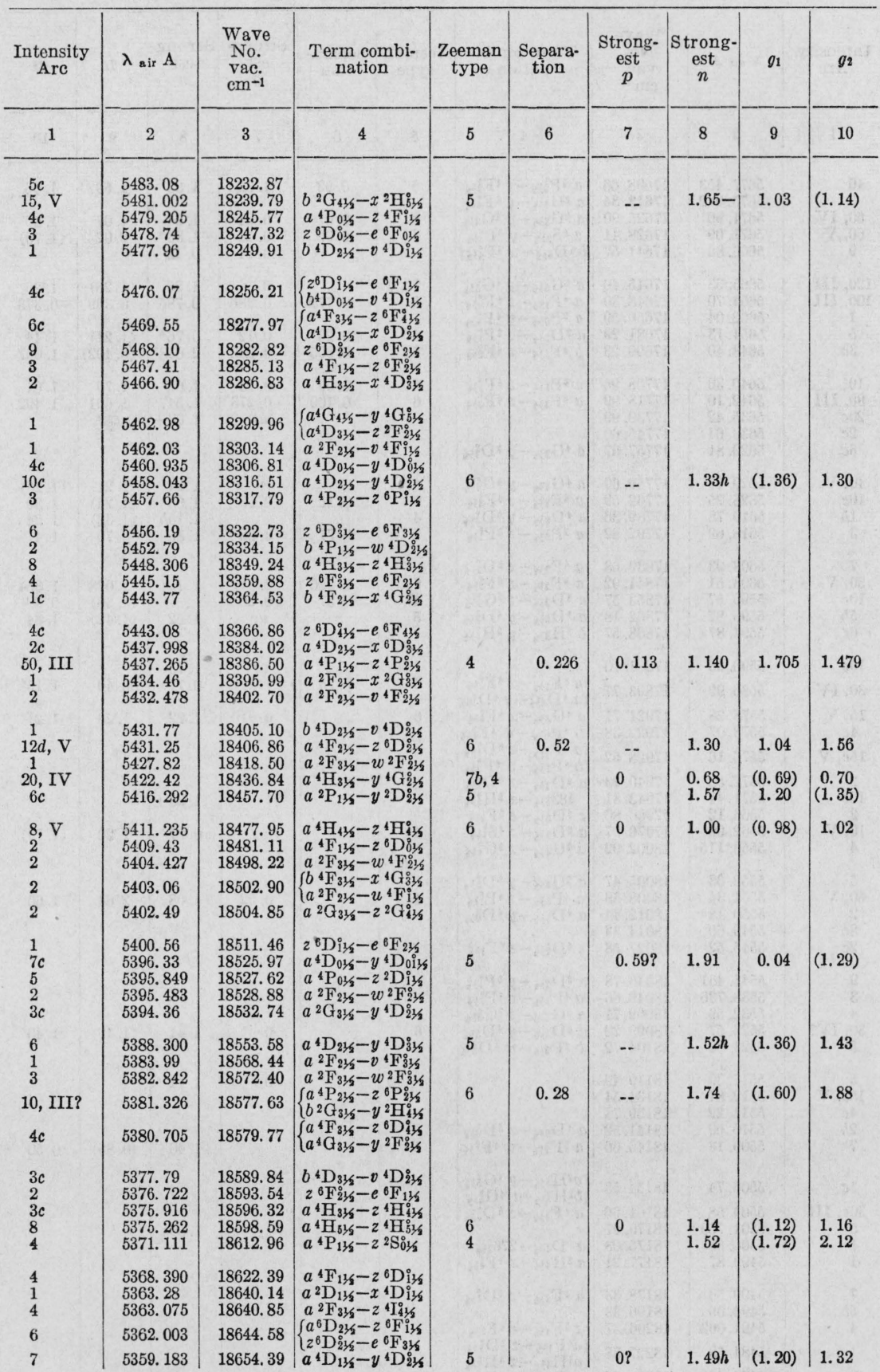


TABLE 4.-First spectrum of columbium (Cb I) - Continued

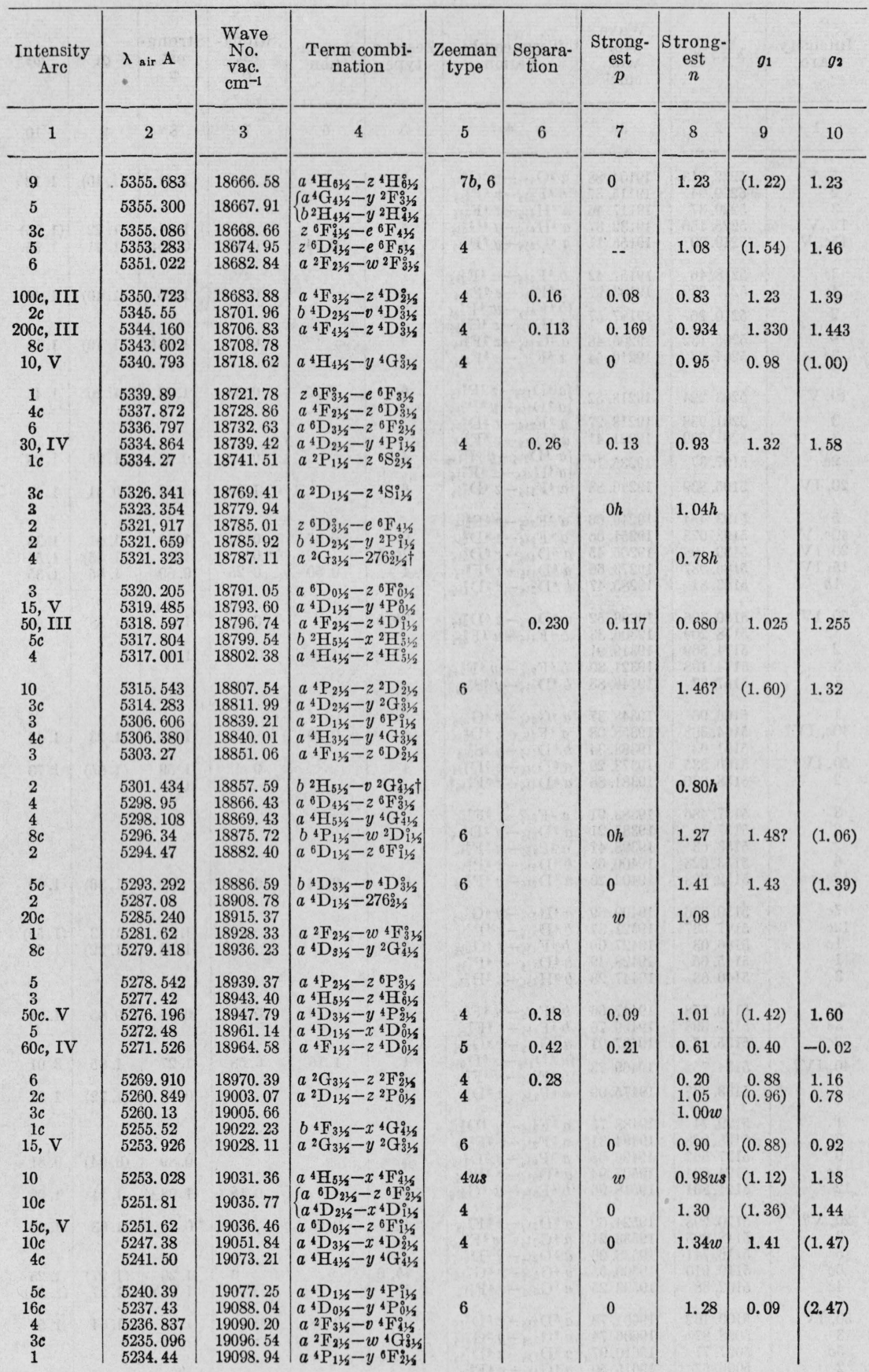


TABLE 4.-First spectrum of columbium (Cb I)-Continued

\begin{tabular}{|c|c|c|c|c|c|c|c|c|c|}
\hline $\begin{array}{c}\text { Intensity } \\
\text { Arc }\end{array}$ & $\lambda_{\text {air }} \mathrm{A}$ & $\begin{array}{l}\text { Wave } \\
\text { No. } \\
\text { vac. } \\
\mathrm{cm}^{-1}\end{array}$ & $\begin{array}{l}\text { Term combi- } \\
\text { nation }\end{array}$ & $\begin{array}{c}\text { Zeeman } \\
\text { type }\end{array}$ & $\begin{array}{c}\text { Separa- } \\
\text { tion }\end{array}$ & $\begin{array}{l}\text { Strong- } \\
\text { est } \\
p\end{array}$ & $\begin{array}{c}\text { Strong- } \\
\text { est } \\
n\end{array}$ & $g_{1}$ & $g_{2}$ \\
\hline 1 & 2 & 3 & 4 & 5 & 6 & 7 & 8 & 9 & 10 \\
\hline $\begin{array}{l}25, \mathrm{~V} \\
2 \\
3 \\
12, \mathrm{~V} \\
15 c, \mathrm{~V}\end{array}$ & $\begin{array}{l}5232.813 \\
5229.94 \\
5229.37 \\
5225.156 \\
5219.09\end{array}$ & $\begin{array}{l}19104.88 \\
19115.37 \\
19117.46 \\
19132.87 \\
19155.11\end{array}$ & 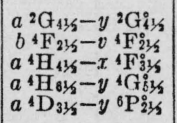 & $\begin{array}{l}4 \\
4\end{array}$ & 0.34 & $\begin{array}{l}w \\
0.17\end{array}$ & $\begin{array}{l}1.11 \\
1.14 w \\
0.56 w\end{array}$ & $\begin{array}{l}1.22 \\
1.41\end{array}$ & $\begin{array}{l}1.12 \\
(1.25) \\
1.75\end{array}$ \\
\hline $\begin{array}{l}3 c \\
4 c\end{array}$ & $\begin{array}{l}\text { 5218. } 46 \\
\text { 5211. } 239\end{array}$ & $\begin{array}{l}19157.42 \\
19183.97\end{array}$ & $\begin{array}{l}b^{4} \mathrm{~F}_{33 / 3}-u^{4} \mathrm{~F}_{23 / 5}^{2} \\
2^{6} \mathrm{~F}_{33 / 3}-e^{6} \mathrm{~F}_{13 / 5}\end{array}$ & 5 & & 0 & 1.66 & $(1.40)$ & 1.46 \\
\hline 2 & 5210.26 & 19187.57 & 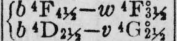 & & & & & & \\
\hline $\begin{array}{l}8 \\
3 c\end{array}$ & $\begin{array}{l}5205.132 \\
5204.03\end{array}$ & $\begin{array}{l}19206.48 \\
19210.54\end{array}$ & $\begin{array}{c}a^{2} \mathrm{G}_{1 / 3}-z^{2} \mathrm{~F}_{31 / 2}^{2} \\
z^{6} \mathrm{~F}_{21 / 2}^{2}-e^{6} \mathrm{~F}_{31 / 2}\end{array}$ & 4 & & $0 ?$ & 1. $03 h$ & $(1.10)$ & 1.14 \\
\hline $10, \mathrm{~V}$ & 5203. 224 & 19213. 52 & $\left\{\begin{array}{l}a^{4}{ }^{4} \mathrm{D}_{113 /}-z^{2} \mathrm{P}_{11 / 3} \\
a^{2} \mathrm{D}_{13 / 5}-y^{6} \mathrm{P}_{21 / 6}^{2}\end{array}\right.$ & 6 & 0.25 & 0.39 & 1.33 & 1.20 & 1.45 \\
\hline & $\begin{array}{l}5201.938 \\
5201.089\end{array}$ & $\begin{array}{l}19218.27 \\
19221.41\end{array}$ & 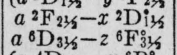 & & & & & & \\
\hline $2 c$ & 5197.37 & 19235.16 & 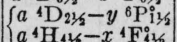 & 4 & & 0 & 0.43 & $(1.36)$ & 1.98 \\
\hline $20, \mathrm{IV}$ & 5195.839 & 19240.83 & $a^{4} \mathrm{~F}_{11 / 2}-z^{4} \mathrm{D}_{11 / 2}$ & 6 & & 1.29 & 0.84 & 0.41 & 1.27 \\
\hline $\begin{array}{c}5 \\
40 c, \mathrm{~V} \\
20, \mathrm{IV} \\
15, \mathrm{IV} \\
1 h\end{array}$ & $\begin{array}{l}5193.454 \\
5193.078 \\
5189.198 \\
5186.987 \\
5183.81\end{array}$ & $\begin{array}{l}19249.66 \\
19251.06 \\
19265.45 \\
19273.66 \\
19285.47\end{array}$ & 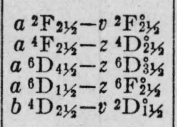 & $\begin{array}{l}6 \\
7 b, 5 \\
4\end{array}$ & $\begin{array}{l}0.37 \\
0.50\end{array}$ & $\begin{array}{l}0.94 \\
0 \\
0.25\end{array}$ & $\begin{array}{l}1.19 \\
1.57 \\
0.60\end{array}$ & $\begin{array}{c}1.01 \\
(1.55) \\
1.85\end{array}$ & $\begin{array}{l}1.38 \\
1.54 \\
1.35\end{array}$ \\
\hline $\begin{array}{l}50, \text { IV } \\
3 c \\
2 \\
3 \\
1\end{array}$ & $\begin{array}{l}5180.306 \\
5178.209 \\
5174.569 \\
5174.198 \\
5167.37\end{array}$ & $\begin{array}{l}19298.52 \\
19306.33 \\
19319.91 \\
19321.30 \\
19346.83\end{array}$ & $\begin{array}{c}a^{6} \mathrm{D}_{31 / 5}-z^{6} \mathrm{D}_{21 / 5}^{2} \\
b^{4} \mathrm{~F}_{43 / 5}-u^{4} \mathrm{~F}_{31 / 5}^{0} \\
b^{4} \mathrm{~F}_{21 / 5}-w^{4} \mathrm{~F}^{4} 21 / 2 \\
b^{4} \mathrm{D}_{01 / 2}-y^{2} \mathrm{~S}_{01 / 2}^{2}\end{array}$ & $7 b, 5$ & & $\begin{array}{l}0 \\
0\end{array}$ & $\begin{array}{l}1.61 \\
1.15\end{array}$ & $(1.58)$ & 1.56 \\
\hline $\begin{array}{c}1 \\
40 c, \text { IV? } \\
1\end{array}$ & $\begin{array}{l}\text { 5166. } 96 \\
5164.368 \\
5161.63\end{array}$ & $\begin{array}{l}19348.37 \\
19358.08 \\
19368.34\end{array}$ & $\begin{array}{l}a^{4} \mathrm{G}_{31 / 5}-x^{4} \mathrm{G}_{31 / 2}^{0} \\
a^{4} \mathrm{~F}_{31 / 5}-z^{4} \mathrm{D}_{31 / 2} \\
b^{4} \mathrm{D}_{13 / 5}-y^{2} \mathrm{~S}_{031 / 5}^{3}\end{array}$ & 6 & 0.20 & -. & 1.33 & 1.23 & 1.43 \\
\hline${ }_{2}^{50, \text { IV? }}$ & $\begin{array}{l}5160.335 \\
5158.030\end{array}$ & $\begin{array}{l}19373.20 \\
19381.86\end{array}$ & $\begin{array}{l}a^{6} \mathrm{D}_{21 / 2}-z \\
a^{6} \mathrm{D}_{41 / 2} \mathrm{D}^{11 / 2} \\
{ }^{6} \mathrm{~F}_{11 / 3}{ }^{4}\end{array}$ & 4 & & $\begin{array}{l}0 \\
-.\end{array}$ & $\begin{array}{l}1.59 \\
1.80\end{array}$ & $(1.65)$ & 1.73 \\
\hline $\begin{array}{c}3 \\
2 \\
2 \\
6 \\
12 c\end{array}$ & $\begin{array}{l}\text { 5157. } 486 \\
5157.14 \\
5153.63 \\
5153.028 \\
5152.623\end{array}$ & $\begin{array}{l}19383.91 \\
19385.21 \\
19398.41 \\
19400.68 \\
19402.20\end{array}$ & 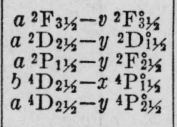 & 6 & 0.24 & 0.60 & $?$ & $(1.36)$ & 1.60 \\
\hline $\begin{array}{r}7 c \\
12 c \\
1 c \\
1 \\
3\end{array}$ & $\begin{array}{l}5150.636 \\
5147.537 \\
5146.03 \\
5145.65 \\
5140.68\end{array}$ & $\begin{array}{l}19409.69 \\
19421.37 \\
19427.06 \\
19428.49 \\
19447.28\end{array}$ & 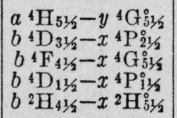 & $\begin{array}{l}4 \\
5\end{array}$ & & $\begin{array}{l}w \\
w\end{array}$ & $\begin{array}{l}1.13 \\
1.30\end{array}$ & $\begin{array}{c}1.43 \\
(1.22)\end{array}$ & $\begin{array}{l}(1.55) \\
1.24\end{array}$ \\
\hline $\begin{array}{l}8 \\
3 c \\
2 c\end{array}$ & $\begin{array}{l}5140.578 \\
5137.398 \\
5135.47\end{array}$ & $\begin{array}{l}19447.66 \\
19459.70 \\
19467.01\end{array}$ & $\begin{array}{l}b^{4} \mathrm{~F}_{21 / 2}-u^{4} \mathrm{~F}_{21 / 2}^{0} \\
b^{4} \mathrm{~F}_{41 / 2}-v^{4} \mathrm{~F}_{41 / 2}^{0} \\
a^{4} \mathrm{G}_{21 / 2}-x^{4} \mathrm{G}_{31 / 2}^{1} 15\end{array}$ & 6 & 0.19 & 0.47 & 0.94 & 0.85 & 1.04 \\
\hline 40, IV? & 5134.752 & 19469. 73 & $\left\{\begin{array}{l}a{ }^{6} \mathrm{D}_{11 / 1}-z^{6} \mathrm{C}^{6} \mathrm{D}_{01 / 2} \\
b^{4} \mathrm{~F}_{313}-u^{4} \mathrm{~F}^{0} \mathrm{~F}_{3136}\end{array}\right.$ & 4 & 1.16 & 0.58 & 1.27 & 1.85 & 3. 01 \\
\hline 7 & 5133.338 & 19475.09 & $a^{4} \mathrm{P}_{11 / 2}-z^{2} \mathrm{D}_{21 / 2}^{3}$ & 4 & & -. & 0.70 & (1. 72) & 1. 31 \\
\hline $\begin{array}{c}1 \\
2 \\
9 \\
4 c \\
12\end{array}$ & $\begin{array}{l}5129.74 \\
5128.278 \\
5127.662 \\
5124.694 \\
5121.801\end{array}$ & $\begin{array}{l}19488.75 \\
19494.31 \\
19496.65 \\
19507.94 \\
19518.96\end{array}$ & 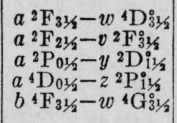 & $\begin{array}{l}5 \\
6\end{array}$ & & 0.18 & $1.08 \pm$ & $(0.64)$ & $\begin{array}{l}0.81 \\
1.06\end{array}$ \\
\hline $\begin{array}{l}20, \mathrm{~V} ? \\
3 \\
5 \\
6 c \\
4 c\end{array}$ & $\begin{array}{l}5120.298 \\
5118.065 \\
5116.741 \\
5110.910 \\
5102.38\end{array}$ & $\begin{array}{l}19524.69 \\
19533.21 \\
19538.26 \\
19560.55 \\
19593.25\end{array}$ & $\begin{array}{l}a^{6} \mathrm{D}_{21 / 2}-z^{6} \mathrm{~F}_{31 / 2}^{0} \\
a^{4} \mathrm{G}_{21 / 2}-w^{4} \mathrm{~F}_{11 / 2} \\
a^{2} \mathrm{D}_{21 / 2}-y^{2} \mathrm{D}_{21 / 2}^{2} \\
a^{4} \mathrm{G}_{51 / 2}-x^{4} \mathrm{G}_{51 / 2}^{1} \\
a^{4} \mathrm{G}_{51 / 2}-v^{4} \mathrm{~F}_{41 / 2}^{0}\end{array}$ & $\begin{array}{l}4 \\
7 b, 6 \\
5\end{array}$ & 0.23 & $w^{0}$ & $\begin{array}{l}0.82 \\
\\
1.26 \\
1.37 h\end{array}$ & $\begin{array}{l}1.63 \\
\\
(1.27) \\
1.27\end{array}$ & $\begin{array}{c}1.40 \\
\\
1.25 \\
(1.22)\end{array}$ \\
\hline $\begin{array}{l}30, \text { IV } \\
3 \\
5 c \\
2 \\
7 c\end{array}$ & $\begin{array}{l}5100.162 \\
5098.87 \\
5097.77 \\
5096.57 \\
5095.535\end{array}$ & $\begin{array}{l}19601.78 \\
19606.74 \\
19610.97 \\
19615.59 \\
19619.57\end{array}$ & $\begin{array}{l}a^{6} \mathrm{D}_{21 / 2}-z^{6} \mathrm{D}_{21 / 1} \\
a^{2} \mathrm{G}_{31 / 2}-y^{2} \mathrm{G}_{41 / 2} \\
a^{6} \mathrm{D}_{11 / 2}-z^{6} \mathrm{D}_{11 / 2} \\
a^{4} \mathrm{G}_{41 / 2}-v^{4} \mathrm{~F}_{31 / 2}^{0} \\
b^{4} \mathrm{~F}_{11 / 2}-u^{4} \mathrm{~F}_{11 / 2}\end{array}$ & 6 & 0.08 & 0.19 & 1.60 & 1.64 & 1.56 \\
\hline
\end{tabular}


TABLE 4.-First spectrum of columbium (Cb I) - Continued

\begin{tabular}{|c|c|c|c|c|c|c|c|c|c|}
\hline $\begin{array}{l}\text { Intensity } \\
\text { Arc }\end{array}$ & $\lambda_{\text {sir }} \mathbf{A}$ & $\begin{array}{l}\text { Wave } \\
\text { No. } \\
\text { vac. } \\
\mathrm{cm}^{-1}\end{array}$ & $\begin{array}{l}\text { Term combi- } \\
\text { nation }\end{array}$ & $\begin{array}{c}\text { Zeeman } \\
\text { type }\end{array}$ & $\begin{array}{l}\text { Separa- } \\
\text { tion }\end{array}$ & $\begin{array}{c}\text { Strong- } \\
\text { est } \\
p\end{array}$ & $\begin{array}{c}\text { Strong- } \\
\text { est } \\
n\end{array}$ & $g_{1}$ & $g_{2}$ \\
\hline 1 & 2 & 3 & 4 & 5 & 6 & 7 & 7 & 8 & 10 \\
\hline $\begin{array}{l}80 c, \text { IV? } \\
3 \\
9 c \\
9 c \\
3 h\end{array}$ & $\begin{array}{l}5095.298 \\
5095.14 \\
5094.41 \\
5088.82 \\
5086.83\end{array}$ & $\begin{array}{l}19620.48 \\
19621.09 \\
19623.90 \\
19645.46 \\
19653.14\end{array}$ & $\begin{array}{l}a^{6} \mathrm{D}_{31 / 2}-z^{6} \mathrm{D}_{31 / 2}^{0} \\
a^{2} \mathrm{~F}_{31 / 2}-u^{4} \mathrm{~F}_{41 / 2}^{0} \\
a^{6} \mathrm{D}_{01 / 2}-z^{6} \mathrm{D}_{01 / 2}^{0} \\
b^{4} \mathrm{~F}_{11 / 2}-w^{2} \mathrm{~F}_{21 / 2}^{15} \\
a^{2} \mathrm{H}_{51 / 2}-z^{4} \mathrm{I}_{41 / 2}^{2}\end{array}$ & 6 & 0.58 & 0.25 & 1.86 & $\begin{array}{l}1.59 \\
0.41\end{array}$ & 0.99 \\
\hline $\begin{array}{c}2 \\
3 \\
2 \\
3 \\
150, \text { IV }\end{array}$ & $\begin{array}{l}5085.00 \\
5084.85 \\
5082.31 \\
5079.314 \\
5078.959\end{array}$ & $\begin{array}{l}19660.22 \\
19660.80 \\
19670.62 \\
19682.23 \\
19683.60\end{array}$ & $\begin{array}{l}a^{4} \mathrm{G}_{51 / 2}-z^{4} \mathrm{I}_{61 / 2}^{\circ} \\
b^{4} \mathrm{P}_{11 / 2}-v^{4} \mathrm{D}_{21 / 2}^{2} \\
a^{4} \mathrm{G}_{31 / 2}-v^{4} \mathrm{~F}_{21 / 2}^{2} \\
a^{2} \mathrm{~F}_{21 / 2}-w^{2} \mathrm{D}_{115}^{\circ} \\
a^{6} \mathrm{D}_{41 / 2}-z^{6} \mathrm{D}_{415 / 2}\end{array}$ & $7 b$ & & 0 & 1.55 & $(1.55)$ & 1.55 \\
\hline $\begin{array}{l}7 c \\
5 c \\
3 c\end{array}$ & $\begin{array}{l}5077.391 \\
5075.97 \\
5072.56\end{array}$ & $\begin{array}{l}19689.68 \\
19695.19 \\
19708.43\end{array}$ & $\begin{array}{l}a^{4} \mathrm{G}_{23 / 2}-v^{4} \mathrm{~F}_{11 / 2}^{\circ} \\
a^{4} \mathrm{~F}_{13 / 2}-z^{4} \mathrm{D}_{21 / 2} \\
a^{2} \mathrm{G}_{31 / 2}-z^{2} \mathrm{~F}_{31 / 2}\end{array}$ & 4 & & & 0.64 & $(0.74)$ & 0.81 \\
\hline $4 c$ & 5071.66 & 19711.93 & 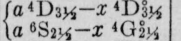 & & & & & & \\
\hline 20, IV? & 5065.256 & 19736.85 & $a^{6} \mathrm{D}_{31 / 2}-z^{6} \mathrm{~F}_{41 / 2}^{0}$ & 4 & & $w$ & $0.95+$ & $(1.58)$ & 1.44 \\
\hline $\begin{array}{l}3 c \\
1 c \\
10 \\
40, \text { IV? } \\
2 c\end{array}$ & $\begin{array}{l}5064.45 \\
5060.99 \\
5059.353 \\
5057.999 \\
5055.62\end{array}$ & $\begin{array}{l}19739.99 \\
19753.49 \\
19759.88 \\
19765.17 \\
19774.47\end{array}$ & $\begin{array}{l}a^{4} \mathrm{D}_{13 / 2}-y^{4} \mathrm{P}_{2135}^{0} \\
b^{4} \mathrm{~F}_{413 / 2}-v^{2} \mathrm{~F}_{31 / 2}^{3} \\
b^{4} \mathrm{~F}_{21 / 2}-u^{4} \mathrm{~F}_{31 / 2}^{3} \\
a^{6} \mathrm{D}_{015}-z^{6} \mathrm{D}^{1} 13 / 2 \\
a^{4} \mathrm{D}_{31 / 2}-z^{4} \mathrm{H}_{31 / 2}^{1}\end{array}$ & $\begin{array}{l}5 \\
4\end{array}$ & & 0.82 & $\begin{array}{l}1.80- \\
0.90\end{array}$ & $\begin{array}{l}(0.85) \\
3.34\end{array}$ & $\begin{array}{l}1.12 \\
1.71\end{array}$ \\
\hline 8 & 5054.671 & 19778.18 & $\begin{cases}b^{4} \mathrm{f}_{4315}-w^{4} \mathrm{~F}_{41 / 3}^{0} \\
b^{2} \mathrm{H}_{51 / 2}-y^{4} \mathrm{H}_{613}^{6}\end{cases}$ & 6 & & 0.15 & 1. $23 \pm$ & 1. 21 & 1.25 \\
\hline $\begin{array}{c}2 \\
12 c \\
3 \\
5\end{array}$ & $\begin{array}{l}5049.91 \\
5047.956 \\
5046.756 \\
5043.985\end{array}$ & $\begin{array}{l}19796.83 \\
19804.49 \\
19809.20 \\
19820.08\end{array}$ & $\begin{array}{l}a^{4} \mathrm{G}_{31 / 2}-w^{2} \mathrm{~F}_{21 / 2}^{2} \\
b^{4} \mathrm{P}_{21 / 2}-x^{4} \mathrm{P}_{2132} \\
b^{4} \mathrm{~F}_{21 / 2}-w^{4} \mathrm{G}_{31 / 2}{ }^{3} \\
b^{4} \mathrm{~F}_{31 / 2}-w^{4} \mathrm{G}_{41 / 2}^{0}\end{array}$ & $7 b$ & & 0 & 1.56 & $(1.56)$ & 1.56 \\
\hline $\begin{array}{l}1 h \\
3 c\end{array}$ & $\begin{array}{l}5043.47 \\
5043.030\end{array}$ & $\begin{array}{l}19822.11 \\
19823.84\end{array}$ & $a^{2} \mathrm{D}_{21 / 2}-z^{6} \mathrm{~S}_{21 / 2}^{0}$ & & & & & & \\
\hline $\begin{array}{l}40, I V \\
1 c \\
6 c\end{array}$ & $\begin{array}{l}5039.032 \\
5037.89 \\
5035.99\end{array}$ & $\begin{array}{l}19839.56 \\
19844.06 \\
19851.55\end{array}$ & $\begin{array}{l}a^{6} \mathrm{D}_{11 / 2}-z^{6} \mathrm{D}_{21 / 5}^{\circ} \\
a^{4} \mathrm{D}_{11 / 2}-x^{4} \mathrm{D}_{21 / 3} \\
b^{4} \mathrm{~F}_{11 / 2}-u^{4} \mathrm{~F}_{2135}^{0}\end{array}$ & 4 & 0.298 & 0.149 & 1. 123 & 1. 868 & 1. 570 \\
\hline $5 c$ & 5031.884 & 19867. 75 & $\begin{array}{l}a^{4} \mathrm{G}_{31 / 2}-x^{4} \mathrm{G}_{41 / 2}^{0} \\
\int^{2}{ }^{2} \mathrm{~F}_{31 / 3}-w^{2} \mathrm{D}_{21 / 2}^{2}\end{array}$ & 4 & & .. & 1.05 & 1.14 & $(1.21)$ \\
\hline 7 & 5030.130 & 19874.67 & $\left\{\begin{array}{l}a \\
b^{4} \mathrm{D}_{31 / 5}-v^{4} \mathrm{G}_{43 / 5}^{21 / 2}\end{array}\right.$ & 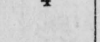 & & & & & \\
\hline $\begin{array}{l}3 \\
2 \\
20, \mathrm{~V}\end{array}$ & $\begin{array}{l}5029.67 \\
5028.65 \\
5026.362\end{array}$ & $\begin{array}{l}19876.49 \\
19880.52 \\
19889.57\end{array}$ & $\begin{array}{l}a^{4} \mathrm{G}_{33 / 5}-w^{4} \mathrm{~F}_{21 / 5} \\
a^{2} \mathrm{G}_{43 / 5}-x^{4} \mathrm{D}_{31 / 5} \\
a^{4} \mathrm{G}_{23 / 5}-u^{4} \mathrm{~F}_{1 / 5}\end{array}$ & 4 & & -- & 0.72 & $(0.74)$ & 0.77 \\
\hline $\begin{array}{l}8 c \\
40, \text { IV? } \\
3 c \\
9 c\end{array}$ & $\begin{array}{l}5019.512 \\
5017.743 \\
5017.363 \\
5013.275\end{array}$ & $\begin{array}{l}\text { 19916. } 72 \\
19923.74 \\
19925.25 \\
19941.49\end{array}$ & 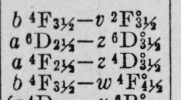 & $\begin{array}{l}6 \\
4 \\
5\end{array}$ & & $\bar{w}$ & $\begin{array}{l}1.16 \\
1.32 \\
1.40-\end{array}$ & $\begin{array}{l}(1.12) \\
(1.65) \\
(1.12)\end{array}$ & $\begin{array}{l}1.20 \\
1.55 \\
1.22\end{array}$ \\
\hline 4 & 5011.750 & 19947.56 & 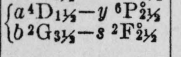 & & & & & & \\
\hline $\begin{array}{l}6 \\
12 \\
20 \mathrm{c}, \mathrm{V} \\
4 \\
15, \mathrm{~V}\end{array}$ & $\begin{array}{l}5008.039 \\
5002.247 \\
5000.958 \\
5000.712 \\
4997.880\end{array}$ & $\begin{array}{l}19962.34 \\
19985.46 \\
19990.61 \\
19991.59 \\
20002.92\end{array}$ & 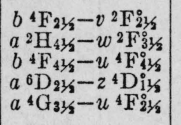 & $\begin{array}{l}6 \\
6 \\
5\end{array}$ & & $\begin{array}{l}0 \\
0 \\
0\end{array}$ & $\begin{array}{l}0.84 \\
1.24 \\
1.12\end{array}$ & $\begin{array}{c}(0.85) \\
1.23 \\
(1.08)\end{array}$ & $\begin{array}{l}0.83 \\
(1.25) \\
1.05\end{array}$ \\
\hline $\begin{array}{r}12 \\
1\end{array}$ & $\begin{array}{l}4994.303 \\
4993.83\end{array}$ & $\begin{array}{l}20017.25 \\
20019.14\end{array}$ & $a^{4} \mathrm{G}_{31 / 2}-z^{4} \mathrm{I}_{413}^{\circ}$ & & & & $1.55-$ & & \\
\hline 3 & 4993. 232 & 20021.54 & $\left\{\begin{array}{l}b^{4} \mathrm{~F}_{31 / 5}-w^{4} \mathrm{D}^{8} 3_{3 / 6} \\
a^{4} \mathrm{D}_{31 / 6}-z^{4} \mathrm{H}_{11 / 2}^{0}\end{array}\right.$ & & & & & & \\
\hline $\begin{array}{l}6 c \\
40, \mathrm{IV}\end{array}$ & $\begin{array}{l}4992.466 \\
4988.972\end{array}$ & $\begin{array}{l}20024.61 \\
20038.64\end{array}$ & $\mid \begin{array}{l}a^{4} \mathrm{G}_{13 / 5}-z^{4} \mathrm{I}_{53 / 5}^{\circ} \\
a^{0} \mathrm{D}_{31 / 3}-z^{6} \mathrm{D}_{41 / 5}\end{array}$ & 4 & & 0 & 1.45 & (1. 58) & 1.53 \\
\hline $\begin{array}{c}1 \\
1 \\
10 c \\
15, \mathrm{~V} \\
10 c\end{array}$ & $\begin{array}{l}4985.18 \\
4976.75 \\
4975.135 \\
4973.138 \\
4971.917\end{array}$ & $\begin{array}{l}20053.88 \\
20087.85 \\
20094.37 \\
20102.44 \\
20107.37\end{array}$ & 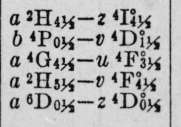 & $\begin{array}{l}5 \\
4\end{array}$ & & $w$ & $\begin{array}{l}\text { 1. } 56- \\
\text { 1. } 20^{*}\end{array}$ & $\begin{array}{l}\text { 1. } 21 \\
1.22 ?\end{array}$ & $\begin{array}{l}(1.11) \\
(1.22)\end{array}$ \\
\hline $\begin{array}{l}30 c, \text { IV } \\
25 c, \mathrm{~V} \\
7 \\
5 h \\
8 c\end{array}$ & $\begin{array}{l}4967.777 \\
4965.375 \\
4963.189 \\
4962.950 \\
4957.393\end{array}$ & $\begin{array}{l}20124.13 \\
20133.86 \\
20142.73 \\
20143.70 \\
20166.28\end{array}$ & 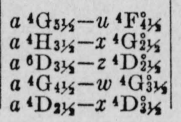 & 5 & & 0 & 0.81 & $(0.69)$ & 0.64 \\
\hline
\end{tabular}


TABLE 4.-First spectrum of columbium (Cb I)-Continued

\begin{tabular}{|c|c|c|c|c|c|c|c|c|c|}
\hline $\begin{array}{l}\text { Intensity } \\
\text { Arc }\end{array}$ & $\lambda_{\text {sir }} A$ & $\begin{array}{l}\text { Wave } \\
\text { No. } \\
\text { vac. } \\
\mathrm{cm}^{-1}\end{array}$ & $\begin{array}{l}\text { Term combi- } \\
\text { nation }\end{array}$ & $\begin{array}{c}\text { Zeeman } \\
\text { type }\end{array}$ & $\begin{array}{l}\text { Separa- } \\
\text { tion }\end{array}$ & $\begin{array}{l}\text { Strong- } \\
\text { est } \\
p\end{array}$ & $\begin{array}{l}\text { Strong. } \\
\text { est } \\
n\end{array}$ & $g_{1}$ & $g_{2}$ \\
\hline 1 & 2 & 3 & 4 & 5 & 6 & 7 & 7 & 8 & 10 \\
\hline $\begin{array}{l}3 c \\
7 \\
1\end{array}$ & $\begin{array}{l}4956.60 \\
4953.124 \\
4951.54\end{array}$ & $\begin{array}{l}20169.51 \\
20183.66 \\
20190.12\end{array}$ & $\begin{array}{l}a^{2} \mathrm{H}_{51 / 2}-z^{4} \mathrm{I}_{11 / 5}^{\circ} \\
a^{2} \mathrm{D}_{11 / 2}-y^{2} \mathrm{D}_{11 / 5}^{1} \\
a^{2} \mathrm{G}_{41 / 2}-z^{4} \mathrm{H}_{415}^{0}\end{array}$ & 6 & & & $0.88 w$ & $(0.95)$ & 0.81 \\
\hline $\begin{array}{r}12 c \\
2 h\end{array}$ & $\begin{array}{l}4945.432 \\
4941.905\end{array}$ & $\begin{array}{l}20215.05 \\
20229.48\end{array}$ & 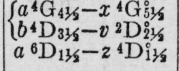 & 5 & & 0 & 1.34 & (1.23) & 1.26 \\
\hline $\begin{array}{l}9 \\
1 h \\
2 \\
2 \\
4\end{array}$ & $\begin{array}{l}4941.513 \\
4937.45 \\
4933.20 \\
4932.140 \\
4929.556\end{array}$ & $\begin{array}{l}20231.09 \\
20247.73 \\
20265.18 \\
20269.53 \\
20280.16\end{array}$ & 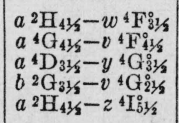 & & & & & & $n^{2}$ \\
\hline $\begin{array}{c}15, \text { IV? } \\
6 c \\
1 \\
3 h \\
15 c\end{array}$ & $\begin{array}{l}4928.969 \\
4924.862 \\
4921.07 \\
4920.75 \\
4916.392\end{array}$ & $\begin{array}{l}20282.57 \\
20299.49 \\
20315.13 \\
20316.45 \\
20334.46\end{array}$ & 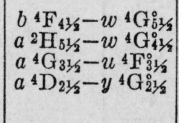 & 5 & & 0 & 1.32 & (1. 22) & 1.25 \\
\hline $\begin{array}{l}7 \\
30, \mathrm{IV} \\
7 \mathrm{c} \\
3 \\
25, \mathrm{IV}\end{array}$ & $\begin{array}{l}4915.842 \\
4910.948 \\
4908.716 \\
4908.331 \\
4904.534\end{array}$ & $\begin{array}{l}20336.74 \\
20357.00 \\
20366.26 \\
20367.85 \\
20383.62\end{array}$ & 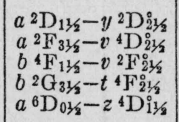 & $\dagger$ & & 1.03 & $\begin{array}{l}1.10+ \\
0.24\end{array}$ & (3. 32) & 1.27 \\
\hline $1 h$ & 4903.02 & 20389.92 & $\left\{\begin{array}{l}a a^{2} \mathrm{P}_{11 / 2}-v{ }^{4} \mathrm{~F}_{1 / 5}^{\circ} \\
b^{2} \mathrm{H}_{51 / 5}-x^{4} \mathrm{H}_{11 / 3}^{6}\end{array}\right.$ & & & & & & \\
\hline $\begin{array}{l}20, \text { III } \\
\frac{4}{2}\end{array}$ & $\begin{array}{l}4900.786 \\
4898.491 \\
4897.51\end{array}$ & $\begin{array}{l}20399.21 \\
20408.77 \\
20412.86\end{array}$ & $\begin{array}{l}a^{2} \mathrm{H}_{43 / 5}-w^{4} \mathrm{G}_{31 / 2}^{4 / 2} \\
a^{4} \mathrm{G}_{31 / 2}-w^{4} \mathrm{D}_{21 / 2}^{\circ} \\
b^{2} \mathrm{G}_{43 / 2}-w^{2} \mathrm{H}_{41 / 2}^{2}\end{array}$ & 4 & & $w$ & $0.51+$ & $(0.93)$ & 1.05 \\
\hline $10 c$ & 4895.574 & 20420.93 & 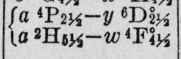 & & & & & & 2 \\
\hline $12 c$ & 4892.50 & 20433. 76 & $\left\{\begin{array}{l}a{ }^{4} \mathrm{G}_{21 / 1}-u^{4} \mathrm{~F}_{3}^{0} \\
a^{2} \mathrm{G}_{41 / 2}-y^{4} \mathrm{G}_{31 / 2}^{3}\end{array}\right.$ & & & & & & \\
\hline $\begin{array}{l}25, \mathrm{IV} \\
3 \\
7 \mathrm{c} \\
5\end{array}$ & $\begin{array}{l}4890.739 \\
4889.809 \\
4889.551 \\
4885.765\end{array}$ & $\begin{array}{l}20441.12 \\
20445.00 \\
20446.08 \\
20461.93\end{array}$ & $\begin{array}{l}a^{4} \mathrm{H}_{413 / 2}-x^{4} \mathrm{G}_{31 / 2} \\
a^{6} \mathrm{D}_{21 / 2}-z^{4} \mathrm{C}_{21 / 2}^{2} \\
a^{6} \mathrm{D}_{43 / 2}-z^{4} \mathrm{D}_{31 / 2}^{23}\end{array}$ & 4 & & $\begin{array}{l}0 \\
w\end{array}$ & $\begin{array}{l}0.96 \\
1.20^{*}\end{array}$ & $(0.98)$ & 0.99 \\
\hline $\begin{array}{l}2 \\
8 c \\
1 \\
5\end{array}$ & $\begin{array}{l}4881.68 \\
4880.714 \\
4879.20 \\
4878.496\end{array}$ & $\begin{array}{l}20479.05 \\
20483.10 \\
20489.46 \\
20492.41\end{array}$ & 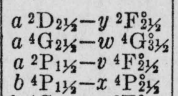 & & & & & & \\
\hline 5 & 4872.505 & 20517.61 & $\begin{cases}\left.a^{4} \mathrm{G}_{31 / 2}-1\right)^{2} & \mathrm{~F}_{215}^{2} \\
a^{2} \mathrm{G}_{43 / 2}-z^{4} \mathrm{H}_{53 / 5}\end{cases}$ & & & & & & \\
\hline $20, \mathrm{~V}$ & 4868.99 & 20532.42 & $\left\{\begin{array}{l}z^{6} \mathrm{G}_{631 / 2}^{\circ}-e^{6} \mathrm{~F}_{51 / 2} \\
a^{2} \mathrm{G}_{31 / 2}-y^{4} \mathrm{G}_{23 / 2}^{0} ?\end{array}\right.$ & & & & & & \\
\hline $12 c$ & 4866.842 & 20541.49 & $\left\{\begin{array}{l}b^{4} \mathrm{P}_{11 / 2}-v{ }^{2} \mathrm{D}_{11 / 5} \\
a^{4} \mathrm{G}_{41 / 2}-v^{2} \mathrm{~F}_{31 / 6}^{1}\end{array}\right.$ & & & $w$ & $1.00+*$ & & 3 \\
\hline $\begin{array}{l}2 \\
1 h \\
1\end{array}$ & $\begin{array}{l}4860.994 \\
4857.31 \\
4853.89\end{array}$ & $\begin{array}{l}20566.20 \\
20581.79 \\
20596.30\end{array}$ & $\begin{array}{l}a^{4} \mathrm{G}_{41 / 2}-w^{4} \mathrm{~F}_{41 / 2} \\
a^{4} \mathrm{P}_{13 / 2}-y^{6} \mathrm{D}_{015 / 2}^{0} \\
b^{4} \mathrm{P}_{13 / 2}-y^{2} \mathrm{~S}_{03 / 2}^{0}\end{array}$ & & & & & & \\
\hline 6 & 4852.273 & 20603.16 & & & & & & & \\
\hline $7 c$ & 4851.881 & 20604.83 & $\left\{\begin{array}{l}a^{4} \mathrm{G}_{21 / 3}-x^{2} \mathrm{D}_{115 / 5} \\
b^{2} \mathrm{G}_{3135}-u^{4} \mathrm{G}_{41 / 5}\end{array}\right.$ & & & & & & \\
\hline$\underset{3 h}{2}$ & $\begin{array}{l}4849.35 \\
4848.359 \\
4847.39\end{array}$ & $\begin{array}{l}20615.58 \\
20619.79 \\
20623.91\end{array}$ & $\begin{array}{l}a^{2} \mathrm{P}_{11 / 3}-w^{2} \mathrm{~F}_{21 / 3}^{2} \\
a^{4} \mathrm{D}_{31 / 3}-y^{4} \mathrm{G}_{41 / 6}^{2} \\
b^{4} \mathrm{P}_{01 / 3}-y^{2} \mathrm{P}_{13 / 6}^{0}\end{array}$ & 4 & & $w$ & $0.77+$ & $(1.42)$ & 1.28 \\
\hline $\begin{array}{l}2 \\
20, \mathrm{~V} \\
20 \mathrm{c} \\
1 \\
1\end{array}$ & $\begin{array}{l}4845.46 \\
4845.170 \\
4842.139 \\
4840.39 \\
4840.28\end{array}$ & $\begin{array}{l}20632.13 \\
20633.36 \\
20646.28 \\
20653.74 \\
20654.21\end{array}$ & 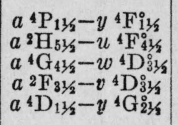 & $\begin{array}{l}4 \\
4\end{array}$ & & $\begin{array}{l}w \\
w\end{array}$ & $\begin{array}{l}0.45+ \\
0.94\end{array}$ & $\begin{array}{l}1.10 \\
1.25\end{array}$ & $\begin{array}{l}(1.25) \\
(1.34)\end{array}$ \\
\hline $\begin{array}{l}20 c, \mathrm{~V} \\
15\end{array}$ & $\begin{array}{l}4837.98 \\
4837.615\end{array}$ & $\begin{array}{l}20664.03 \\
20665.59\end{array}$ & 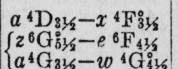 & & & & & at & \\
\hline $\begin{array}{l}40, \mathrm{III} ? \\
3 \\
2\end{array}$ & $\begin{array}{l}4833.362 \\
4832.21 \\
4830.69\end{array}$ & $\begin{array}{l}20683.77 \\
20688.70 \\
20695.21\end{array}$ & 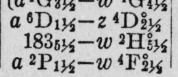 & 4 & 0.46 & 0.23 & 0.72 & 1.86 & 1.40 \\
\hline
\end{tabular}


TABLE 4.-First spectrum of columbium (Cb I)-Continued

\begin{tabular}{|c|c|c|c|c|c|c|c|c|c|}
\hline $\begin{array}{l}\text { Intensity } \\
\text { Arc }\end{array}$ & $\lambda_{\text {air }} \mathrm{A}$ & $\begin{array}{l}\text { Wave } \\
\text { No. } \\
\text { vac. } \\
\mathrm{cm}^{-1}\end{array}$ & $\begin{array}{l}\text { Term combi- } \\
\text { nation }\end{array}$ & $\begin{array}{c}\text { Zeeman } \\
\text { type }\end{array}$ & $\begin{array}{l}\text { Separa- } \\
\text { tion }\end{array}$ & $\begin{array}{l}\text { Strong- } \\
\text { est } \\
p\end{array}$ & $\begin{array}{c}\text { Strong- } \\
\text { est } \\
n\end{array}$ & $g_{1}$ & $g_{2}$ \\
\hline 1 & 2 & 3 & 4 & 5 & 6 & 7 & 8 & 9 & 10 \\
\hline $\begin{array}{c}30, \mathrm{~V} \\
1 \\
1 \\
6 c \\
3\end{array}$ & $\begin{array}{l}4829.302 \\
4826.97 \\
4826.50 \\
4824.954 \\
4821.11\end{array}$ & $\begin{array}{l}20701.16 \\
20711.16 \\
20713.18 \\
20719.81 \\
20736.33\end{array}$ & 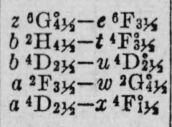 & & & $w$ & $0.89+$ & 1.29 & $(1.40)$ \\
\hline 1 & 4820.07 & 20740.81 & $\begin{array}{l}b^{4} \mathrm{D}_{13 / 1}-u^{4} \mathrm{D}_{21 / 5}^{\circ} \\
\int^{4} \mathrm{H}_{51 / 1}-x^{4} \mathrm{G}_{11 / 6}\end{array}$ & 4 & & 0 & 1.10 & (1.12) & 1.12 \\
\hline $80 c$, IV & 4816.375 & 20756. 72 & $\left\{a^{4} \mathrm{H}_{43 / 5}-x^{2} \mathrm{G}_{31 / 5}\right.$ & & & & & & \\
\hline $\begin{array}{c}2 \\
2 \\
12 c\end{array}$ & $\begin{array}{l}4814.98 \\
4812.12 \\
4811.287\end{array}$ & $\begin{array}{l}20762.73 \\
20775.07 \\
20778.67\end{array}$ & $\begin{array}{l}a^{4} \mathrm{P}_{13 / 1}-y^{4} \mathrm{~F}_{21 / 5}^{2} \\
b^{2} \mathrm{H}_{5,3}-y^{2} \mathrm{I}_{b / 5}^{0} \\
a^{4} \mathrm{G}_{43 / 5}-u^{4} \mathrm{~F}_{43 / 5}^{6}\end{array}$ & 6 & & 0 & 1.23 & 1.21 & (1.25) \\
\hline $\begin{array}{l}100 c, \mathrm{~V} \\
30, \mathrm{~V} \\
4 \\
4 c \\
10\end{array}$ & $\begin{array}{l}4810.584 \\
4809.357 \\
4809.12 \\
4809.03 \\
4807.052\end{array}$ & $\begin{array}{l}20781.70 \\
20787.01 \\
20788.03 \\
20788.42 \\
20796.98\end{array}$ & 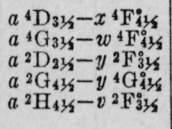 & & & & $1+$ & & \\
\hline $\begin{array}{l}3 \\
9 \\
2 \\
5 c\end{array}$ & $\begin{array}{l}4805.61 \\
4802.442 \\
4798.818 \\
4793.07\end{array}$ & $\begin{array}{l}20803.22 \\
20816.94 \\
20832.66 \\
20857.64\end{array}$ & 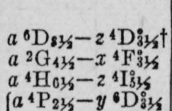 & & & 2. 56 & $1.44+$ & & \\
\hline 9 & 4790.902 & 20867.08 & $\left\{\begin{array}{l}a^{4} \mathrm{G}_{31 / 3}-w^{4} \mathrm{D}_{31 / 3} \\
b^{4} \mathrm{D}_{23 / 5}-u^{4} \mathrm{D}_{31 / 4}\end{array}\right.$ & & & & & & \\
\hline $\begin{array}{l}6 d \\
3 \\
1\end{array}$ & $\begin{array}{l}4790.58 \\
4787.73 \\
4787.56\end{array}$ & $\begin{array}{l}20868.48 \\
20880.91 \\
20881.65\end{array}$ & $\begin{array}{l}a^{4} \mathrm{~F}_{31 / 5}-z^{4} \mathrm{G}_{33 / 5}^{3} \\
a^{4} \mathrm{G}_{21 / 5}-v^{2} \mathrm{~F}_{31 / 5}^{0} \\
a^{4} \mathrm{P}_{01 / 5}-y^{6} \mathrm{D}_{015}^{0}\end{array}$ & & & & & & \\
\hline $\begin{array}{l}7 \\
5 c\end{array}$ & $\begin{array}{l}4785.697 \\
4784.30\end{array}$ & $\begin{array}{l}20889.78 \\
20895.88\end{array}$ & $\begin{array}{l}b{ }^{4} \mathrm{~F}_{313}-t^{4} \mathrm{D}_{215}^{2} \\
b^{2} \mathrm{G}_{3135}-r^{2} \mathrm{~F}_{2136}^{2}\end{array}$ & 5 & & 0 & 0.91 & 0.89 & $(0.88)$ \\
\hline 4 & 4782.948 & 20901. 78 & $a^{2} \mathrm{H}_{435}-w{ }^{4} \mathrm{D}^{3} 3 / 6$ & & & & 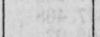 & & \\
\hline 1 & 4782.44 & 20904. 00 & 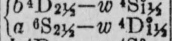 & & & & & & \\
\hline $\begin{array}{l}5 c \\
1 \\
6 c\end{array}$ & $\begin{array}{l}4780.98 \\
4780.07 \\
4777.62\end{array}$ & $\begin{array}{l}20910.38 \\
20914.37 \\
20925.09\end{array}$ & 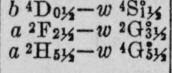 & $t$ & & $w$ & $1.46-$ & & \\
\hline $\begin{array}{l}2 \\
2\end{array}$ & $\begin{array}{l}4776.224 \\
4776.07\end{array}$ & $\begin{array}{l}20931.21 \\
20931.88\end{array}$ & 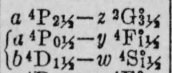 & & & & 3. & & \\
\hline $\begin{array}{c}20 c \\
5 c \\
1\end{array}$ & $\begin{array}{l}4773.24 \\
4772.79 \\
4772.20\end{array}$ & $\begin{array}{l}20944.29 \\
20946.27 \\
20948.86\end{array}$ & $\begin{array}{l}a{ }^{4} \mathrm{D}_{235}-x^{4} \mathrm{~F}_{215}^{\circ} \\
b^{2} \mathrm{G}_{435}-u^{2} \mathrm{G}_{313}^{6} \\
a^{2}{ }^{2} \mathrm{G}_{315}-y^{2} \mathrm{D}_{235}^{\circ}\end{array}$ & & & & & \}) & \\
\hline $\begin{array}{l}7 \\
5\end{array}$ & $\begin{array}{l}4771.852 \\
4767.07\end{array}$ & $\begin{array}{l}20950.38 \\
20971.40\end{array}$ & $\begin{array}{l}a^{2} \mathrm{G}_{415}-x^{4} \mathrm{~F}^{2} 1 / 6 \\
a^{4} \mathrm{P}_{21 / 2}-y^{4} \mathrm{D}_{1 / 6}\end{array}$ & & & & & & \\
\hline $30 c, \mathrm{~V}$ & 4766.80 & 20972. 59 & 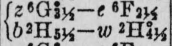 & $\begin{array}{l}4 \\
5\end{array}$ & & $\begin{array}{l}0 \\
0\end{array}$ & $\begin{array}{l}0.68 \\
1.12\end{array}$ & $\begin{array}{l}1.13 \\
0.99\end{array}$ & $\begin{array}{l}(1.31) \mathrm{L} \\
(0.93)\end{array}$ \\
\hline $\begin{array}{l}6 \\
2 c\end{array}$ & $\begin{array}{l}4766.53 \\
4758.12\end{array}$ & $\begin{array}{l}20973.78 \\
21010.85\end{array}$ & 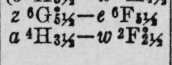 & & & & & & \\
\hline $\begin{array}{c}4 \\
10 c \\
5 \\
3 d \\
1\end{array}$ & $\begin{array}{l}4756.538 \\
4755.318 \\
4754.957 \\
4753.48 \\
4752.86\end{array}$ & $\begin{array}{l}21017.84 \\
21023.23 \\
21024.82 \\
21031.36 \\
21034.10\end{array}$ & 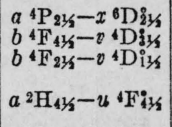 & & & & & & \\
\hline $\begin{array}{c}5 h \\
20 \\
5\end{array}$ & $\begin{array}{l}4752.10 \\
4751.44 \\
4750.74\end{array}$ & $\begin{array}{l}21037.46 \\
21040.39 \\
21043.49\end{array}$ & 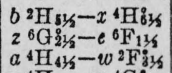 & & & & & & \\
\hline $\begin{array}{l}200 c, \mathrm{~V} \\
3 c\end{array}$ & $\begin{array}{l}4749.706 \\
4748.84\end{array}$ & $\begin{array}{l}21048.07 \\
21051.91\end{array}$ & $\begin{array}{l}a{ }^{4} \mathrm{H}_{63 / 2}-x^{4} \mathrm{G} \\
b^{4} \mathrm{D}_{31 / 2}-u^{4} \mathrm{D}_{31 / 6}^{3}\end{array}$ & 4 & & $w$ & $1.14 w$ & 1.21 & $(1.24)$ \\
\hline $\begin{array}{l}3 h \\
9 c\end{array}$ & $\begin{array}{l}4748.04 \\
4746.987\end{array}$ & $\begin{array}{l}21055.45 \\
21060.12\end{array}$ & $a^{4} \mathrm{~F}_{23,5}-2^{4} \mathrm{G}_{24,4}$ & & & & & & \\
\hline $10 c$ & 4745.020 & 21068.85 & 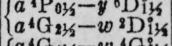 & & & & & & \\
\hline $\begin{array}{l}30, \mathrm{~V} \\
15, \mathrm{~V}\end{array}$ & $\begin{array}{l}4744.622 \\
4743.838\end{array}$ & $\begin{array}{l}21070.62 \\
21074.10\end{array}$ & $\left\{\begin{array}{l}a^{4} a_{436}-w^{4} \\
a^{4} \mathrm{D}_{115}-y^{2} \mathrm{D}_{213} \\
a^{4} \mathrm{D}_{15-x^{4}} \mathrm{~F}^{2}\end{array}\right.$ & 5 & & 0 & 1.41 & (1. 23) & 1.26 \\
\hline
\end{tabular}


TABLE 4.-First spectrum of columbium (Cb I)-Continued

\begin{tabular}{|c|c|c|c|c|c|c|c|c|c|}
\hline $\begin{array}{l}\text { Intensity } \\
\text { Arc }\end{array}$ & $\lambda_{\text {air }} \mathbf{A}$ & $\begin{array}{l}\text { Wave } \\
\text { No. } \\
\text { vac. } \\
\mathrm{cm}^{-1}\end{array}$ & $\begin{array}{l}\text { Term combi- } \\
\text { nation }\end{array}$ & $\begin{array}{c}\text { Zeeman } \\
\text { type }\end{array}$ & $\begin{array}{l}\text { Separa- } \\
\text { tion }\end{array}$ & $\begin{array}{c}\text { Strong- } \\
\text { est } \\
p\end{array}$ & $\begin{array}{c}\text { Strong- } \\
\text { est } \\
n\end{array}$ & $g_{1}$ & $g_{2}$ \\
\hline 1 & 2 & 3 & 4 & 5 & 6 & 7 & 8 & 9 & 10 \\
\hline $\begin{array}{c}4 c \\
3 \\
15 c \\
2 \\
20 c\end{array}$ & $\begin{array}{l}4742.43 \\
4742.072 \\
4740.61 \\
4740.426 \\
4736.49\end{array}$ & $\begin{array}{l}21080.36 \\
21081.95 \\
21088.45 \\
21089.27 \\
21106.80\end{array}$ & 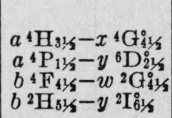 & 5 & & $\begin{array}{l}0 \\
w\end{array}$ & $\begin{array}{l}1.50 \\
1.55-\end{array}$ & $\begin{array}{l}(1.72) \\
1.01\end{array}$ & $\begin{array}{c}1.63 \\
(1.09)\end{array}$ \\
\hline $\begin{array}{l}10 \\
60 c, \mathrm{~V} \\
30 c, \mathrm{~V} \\
20 \\
10 c\end{array}$ & $\begin{array}{l}4735.339 \\
4733.885 \\
4733.483 \\
4730.312 \\
4727.320\end{array}$ & $\begin{array}{l}21111.93 \\
21118.41 \\
21120.20 \\
21134.36 \\
21147.74\end{array}$ & 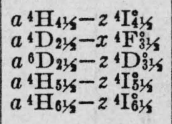 & 6 & 0.17 & $\begin{array}{l}0.09 \\
0.79\end{array}$ & $\begin{array}{c}0.77+ \\
\ldots\end{array}$ & $\begin{array}{c}1.37 \\
(1.12)\end{array}$ & 0.98 \\
\hline $\begin{array}{c}4 d \\
2 \\
15 c \\
3 \\
2 \\
2\end{array}$ & $\begin{array}{l}4726.77 \\
4724.48 \\
4723.795 \\
4723.146 \\
4720.10\end{array}$ & $\begin{array}{l}21150.20 \\
21160.45 \\
21163.52 \\
21166.43 \\
21180.08\end{array}$ & $\begin{array}{l}a^{2} \mathrm{G}_{31 / 5}-x^{4} \mathrm{~F}_{21 / 5}^{0} \\
z^{6} \mathrm{G}_{415}^{0}-e^{6} \mathrm{~F}_{41 / 2} \\
b^{4} \mathrm{~F}_{21 / 5}-v^{4} \mathrm{D}_{21 / 6}^{\circ}\end{array}$ & & & 0 & 0.96 & & के \\
\hline $\begin{array}{c}8 \\
20 \\
80, \mathrm{~V} \\
9 \mathrm{c} \\
6 d\end{array}$ & $\begin{array}{l}4718.024 \\
4715.819 \\
4713.495 \\
4713.038 \\
4711.872\end{array}$ & $\begin{array}{l}21189.40 \\
21199.31 \\
.21209 .76 \\
21211.82 \\
21217.07\end{array}$ & $\left\{\begin{array}{l}z^{6} \mathrm{G}^{\mathrm{i} 1 / 2}-e^{0} \mathrm{~F}_{01 / 2} \\
a^{4} \mathrm{~F}_{41 / 2}-z^{4} \mathrm{~F}_{31 / 2}^{0} \\
a^{4} \mathrm{D}_{01 / 2}-y^{2} \mathrm{D}_{11 / 2}^{10} \\
a^{4} \mathrm{H}_{31 / 2}-u^{4} \mathrm{~F}_{1 / 2}^{2} \\
a^{2} \mathrm{H}_{41 / 2}-w^{2} \mathrm{G}_{31 / 6}^{0}\end{array}\right.$ & $\begin{array}{l}4 \\
5\end{array}$ & & $\begin{array}{l}0.34 \\
w\end{array}$ & $\begin{array}{l}0.34 h \\
1.635\end{array}$ & $\begin{array}{l}0.00 \\
(1.330)\end{array}$ & $\begin{array}{l}-0.68 \\
1.243\end{array}$ \\
\hline $\begin{array}{l}150, \mathrm{IV} ? \\
5 d \\
100 c, \mathrm{~V} \\
4 \\
4\end{array}$ & $\begin{array}{l}4708.284 \\
4707.103 \\
4706.132 \\
4705.09 \\
4703.92\end{array}$ & $\begin{array}{l}21233.24 \\
21238.57 \\
21242.95 \\
21247.65 \\
21252.94\end{array}$ & 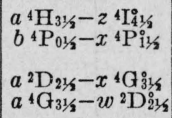 & 5 & 0.146 & $\begin{array}{l}0.216 \\
0\end{array}$ & $\begin{array}{l}1.273 \\
1.082\end{array}$ & $(0.690)$ & 0.836 \\
\hline $\begin{array}{c}5 \\
4 \\
30, \mathrm{~V} \\
2 \\
20 c, \mathrm{~V}\end{array}$ & $\begin{array}{l}4702.02 \\
4697.752 \\
4697.468 \\
4695.93 \\
4695.46\end{array}$ & $\begin{array}{l}21261.53 \\
21280.84 \\
21282.13 \\
21289.10 \\
21291.23\end{array}$ & 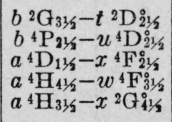 & $\begin{array}{l}4 \\
5\end{array}$ & $\begin{array}{l}0.19 \\
0.40\end{array}$ & 0.09 & $0.72+$ & $\begin{array}{l}1.20 \\
(0.690)\end{array}$ & $\begin{array}{l}1.01 \\
1.09\end{array}$ \\
\hline $\begin{array}{l}15 c, \mathrm{~V} \\
3 \\
10 \\
5 c \\
4\end{array}$ & $\begin{array}{l}4694.50 \\
4692.255 \\
4689.162 \\
4688.83 \\
4688.49\end{array}$ & $\begin{array}{l}21295.58 \\
21305.77 \\
21319.82 \\
21321.33 \\
21322.88\end{array}$ & $\begin{array}{r}z^{6} \mathrm{G}_{21 / 5}^{0}-e^{6} \mathrm{~F}_{21 / 5} \\
183_{51 / 3}-t^{2} \mathrm{G}_{41 / 5}^{2}\end{array}$ & & & 0 & 0.96 & & I \\
\hline $\begin{array}{l}3 \\
1 h\end{array}$ & $\begin{array}{l}4687.790 \\
4687.19\end{array}$ & $\begin{array}{l}21326.06 \\
21328.79\end{array}$ & 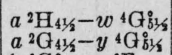 & & & & & & \\
\hline 9 & 4685.925 & 21334.55 & $\left\{\begin{array}{l}z^{6} \mathrm{G}_{33 / 1}^{0}-e^{6} \mathrm{~F}_{33 / 4} \\
a^{2} \mathrm{G}_{31 / 5}-x^{4} \mathrm{~F}_{31 / 6}^{3}\end{array}\right.$ & & & & & & \\
\hline $\begin{array}{c}9 \\
100, \mathrm{~V} ?\end{array}$ & $\begin{array}{l}4685.518 \\
4685.133\end{array}$ & $\begin{array}{l}21336.41 \\
21338.16\end{array}$ & $\begin{array}{l}a^{2} \mathrm{P}_{11 / 5}-v^{2} \mathrm{~F}^{21 / 1} \\
a^{4} \mathrm{H}_{41 / 2}-z^{4} \mathrm{I}_{51 / 2}^{2}\end{array}$ & $7 b, 5$ & & 0 & 0.986 & $(0.984)$ & 0.984 \\
\hline $\begin{array}{c}1 \\
7 \\
10 c \\
2 \\
3\end{array}$ & $\begin{array}{l}4683.68 \\
4682.984 \\
4682.664 \\
4681.997 \\
4681.66\end{array}$ & $\begin{array}{l}21344.78 \\
21347.95 \\
21349.41 \\
21352.45 \\
21353.99\end{array}$ & $\begin{array}{l}b^{2} \mathrm{G}_{413 / 5}-u^{2} \mathrm{G}_{41 / 5}^{0} \\
a^{2} \mathrm{~F}_{21 / 5}-v^{2} \mathrm{D}_{11 / 3} \\
z^{6} \mathrm{G}_{11 / 3}{ }^{1}-e^{6} \mathrm{~F}_{11 / 5}\end{array}$ & & & & & $y$ & \\
\hline $\begin{array}{c}1 \\
4 c \\
22 d, \mathrm{~V} \\
150, \mathrm{~V} ? \\
12 c\end{array}$ & $\begin{array}{l}4681.53 \\
4680.890 \\
4678.48 \\
4675.371 \\
4673.589\end{array}$ & $\begin{array}{l}21354.58 \\
21357.50 \\
21368.50 \\
21382.71 \\
21390.86\end{array}$ & $\begin{array}{l}a^{4} \mathrm{H}_{31 / 5}-v^{4} \mathrm{~F}_{41 / 2}^{0} \\
a^{4} \mathrm{D}_{01 / 2}-x^{4} \mathrm{~F}_{11 / 3} \\
a^{4} \mathrm{~F}_{31 / 2}-z^{4} \mathrm{G}_{41 / 5}^{0} \\
b^{2} \mathrm{G}_{41 / 2}-t^{2} \mathrm{G}^{3} 31 / 2\end{array}$ & $\begin{array}{l}5 \\
4\end{array}$ & 0.38 & $\underset{w}{0.19}$ & $\begin{array}{l}0.62 \\
0.91\end{array}$ & $\begin{array}{c}0.05 \\
(1.24)\end{array}$ & $\begin{array}{l}0.43 \\
1.17\end{array}$ \\
\hline $\begin{array}{c}200 c, \mathrm{~V} ? \\
3 c\end{array}$ & $\begin{array}{l}4672.097 \\
4670.87\end{array}$ & $\begin{array}{l}21397.69 \\
21403.32\end{array}$ & 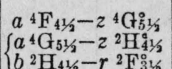 & 4 & & $w$ & 0.96 & (1. 33) & 1. 26 \\
\hline $\begin{array}{c}10 \\
2 c \\
3 c\end{array}$ & $\begin{array}{l}4669.868 \\
4669.32 \\
4668.966\end{array}$ & $\begin{array}{l}21407.91 \\
21410.42 \\
21412.04\end{array}$ & $\begin{array}{l}a^{4} \mathrm{H}_{41 / 2}-u^{4} \mathrm{~F}_{31 / 2}^{0} \\
a^{4} \mathrm{H}_{31 / 2}-w^{4} \mathrm{~F}_{31 / 2}^{0}\end{array}$ & & & & & & 18 \\
\hline $\begin{array}{c}6 \\
50, \mathrm{~V}\end{array}$ & $\begin{array}{l}4668.232 \\
4667.224\end{array}$ & $\begin{array}{l}21415.41 \\
21420.03\end{array}$ & $\left\{\begin{array}{l}a^{4} \mathrm{P}_{11 / 2}-x{ }^{6} \mathrm{D}_{11 / 2} \mathrm{i} \\
a^{4} \mathrm{H}^{4} \mathrm{~F}_{31 / 2}-z \\
a^{4} \mathrm{P}_{21 / 2}\end{array}\right.$ & 5 & 0.179 & 0.093 & 1. 673 & (1.235) & 1. 056 \\
\hline $\begin{array}{c}6 c \\
100, \text { IV? } \\
10\end{array}$ & $\begin{array}{l}4666.630 \\
4666.251 \\
4665.333\end{array}$ & $\begin{array}{l}21422.76 \\
21424.50 \\
21428.72\end{array}$ & 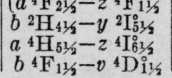 & 4 & & $w$ & 0.92 & (1.12) & 1.09 \\
\hline
\end{tabular}


TABLE 4.-First spectrum of columbium (Cb I)-Continued

\begin{tabular}{|c|c|c|c|c|c|c|c|c|c|}
\hline $\begin{array}{l}\text { Intensity } \\
\text { Are }\end{array}$ & $\lambda_{\text {air }} A$ & $\begin{array}{l}\text { Wave } \\
\text { No. } \\
\text { vac. } \\
\mathrm{cm}^{-1}\end{array}$ & $\begin{array}{l}\text { Term combi- } \\
\text { nation }\end{array}$ & $\begin{array}{c}\text { Zeeman } \\
\text { type }\end{array}$ & $\begin{array}{c}\text { Separa- } \\
\text { tion }\end{array}$ & $\begin{array}{l}\text { Strong- } \\
\text { est } \\
p\end{array}$ & $\begin{array}{c}\text { Strong- } \\
\text { est } \\
n\end{array}$ & $g_{1}$ & $g_{2}$ \\
\hline 1 & 2 & 3 & 4 & 5 & 6 & 7 & 7 & 8 & 10 \\
\hline 5 & 4664. 366 & 21433.16 & $b^{4} \mathrm{~F}_{31 / 5}-z^{2} \mathrm{H}_{41 / 6}^{\circ}$ & & & & & & \\
\hline 4 & 4663. 97 & 21434. 98 & & & & & & & \\
\hline $\begin{array}{c}100, \text { IV? } \\
4 c \\
5 h\end{array}$ & $\begin{array}{l}4663.831 \\
4662.89 \\
4660.98\end{array}$ & $\begin{array}{l}21435.62 \\
21439.94 \\
21448.73\end{array}$ & 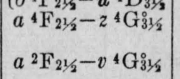 & 4 & & & 0.862 & $(1.029)$ & 0.981 \\
\hline $\begin{array}{c}3 h \\
12 \\
1 \\
1 \\
3\end{array}$ & $\begin{array}{l}4660.70 \\
4658.186 \\
4655.953 \\
4654.89 \\
4652.08\end{array}$ & \begin{tabular}{l|}
21450.02 \\
21461.59 \\
21471.89 \\
21477.79 \\
21489.77
\end{tabular} & 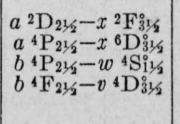 & & & & & & \\
\hline $\begin{array}{l}2 \\
40, \mathrm{~V} \\
100, \text { IV? }\end{array}$ & $\begin{array}{l}4649.622 \\
4649.255 \\
4648.949\end{array}$ & $\begin{array}{l}21501.12 \\
21502.82 \\
21504.24\end{array}$ & $\begin{array}{l}a{ }^{2} \mathrm{H}_{51 / 5}-z^{2} \mathrm{I}_{51 / 5}^{2} \\
a^{4} \mathrm{~F}_{11 / 3}-z{ }^{4} \mathrm{G}_{21 / 3}^{2}\end{array}$ & 5 & 0.175 & 0.087 & 0.841 & 0.403 & 0.578 \\
\hline $\begin{array}{l}20 c \\
4 c\end{array}$ & $\begin{array}{l}4646.952 \\
4645.84\end{array}$ & $\begin{array}{l}21513.48 \\
21518.63\end{array}$ & $183_{51 / 2}-v^{2} \mathrm{H}_{41 / 2}$ & 0 & 0.170 & $w$ & $0.79+$ & 0.700 & 0.070 \\
\hline $\begin{array}{c}5 c \\
10 c \\
10 \\
3\end{array}$ & $\begin{array}{l}4643.86 \\
4643.682 \\
4643.315 \\
4642.46\end{array}$ & $\begin{array}{l}21527.80 \\
21528.63 \\
21530.33 \\
21534.29\end{array}$ & 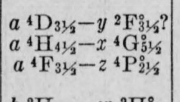 & & & & & & \\
\hline & 4640. 248 & 21544.56 & $b^{2} \mathrm{H}_{51 / 5}-w^{2} \mathrm{H}_{51 / 5}^{0}$ & & & & & & \\
\hline 4 & 4638. 17 & 21554.21 & 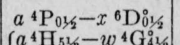 & 6 & & $d ?$ & 2. $54 \pm$ & $(2.65)$ & 2.43 \\
\hline 10 & 4638. 105 & 21554.51 & 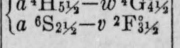 & & & & & & \\
\hline $\begin{array}{l}5 c \\
1 \\
3\end{array}$ & $\begin{array}{l}4633.80 \\
4632.25 \\
4631.976\end{array}$ & $\begin{array}{l}21574.54 \\
21581.76 \\
21583.03\end{array}$ & $a^{2} \mathrm{P}_{03 / 2}-v{ }^{4} \mathrm{~F}_{13 / 5}^{0}$ & & & & & & \\
\hline $\begin{array}{l}3 h \\
100, \mathrm{IV} ? \\
7 d \\
1 \\
4 c\end{array}$ & $\begin{array}{l}4630.30 \\
4630.115 \\
4627.477 \\
4625.335 \\
4624.576\end{array}$ & $\begin{array}{l}21590.85 \\
21591.71 \\
21604.02 \\
21614.02 \\
21617.57\end{array}$ & 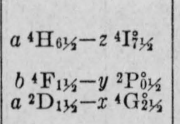 & 4 & & $w$ & 0.994 & 1. 22 & 1.19 \\
\hline $\begin{array}{c}6 \\
2 \\
3 \\
50, \mathrm{~V} \\
5 \mathrm{c}\end{array}$ & $\begin{array}{l}4622.338 \\
4620.02 \\
4619.42 \\
4616.162 \\
4614.740\end{array}$ & $\begin{array}{l}21628.04 \\
21638.89 \\
21641.70 \\
21656.97 \\
21663.64\end{array}$ & 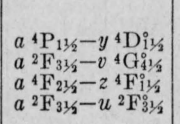 & 5 & 0.607 & 0.301 & 1. $12 \pm$ & 1.030 & 0.416 \\
\hline $\begin{array}{l}4 \\
8 \\
2 \\
7 c \\
4\end{array}$ & $\begin{array}{l}4612.465 \\
4612.108 \\
4612.02 \\
4610.698 \\
4610.106\end{array}$ & $\begin{array}{l}21674.33 \\
21676.01 \\
21676.42 \\
21682.64 \\
21685.43\end{array}$ & 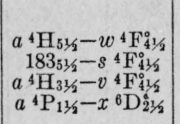 & & & & & & \\
\hline $\begin{array}{c}9 c \\
1 \\
200, \text { III } \\
4 \\
5 c\end{array}$ & $\begin{array}{l}4608.567 \\
4607.28 \\
4606.760 \\
4605.649 \\
4603.797\end{array}$ & $\begin{array}{l}21692.66 \\
21698.72 \\
21701.17 \\
21706.41 \\
21715.14\end{array}$ & 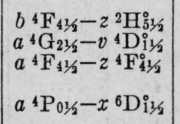 & $7 b, 6$ & & 9 & 1.334 & $(1.332)$ & 1.336 \\
\hline $\begin{array}{c}7 \\
2 \\
30, \mathrm{~V} \\
10 c \\
4\end{array}$ & $\begin{array}{l}4602.864 \\
4602.48 \\
4600.22 \\
4599.475 \\
4592.42\end{array}$ & $\begin{array}{l}21719.54 \\
21721.35 \\
21732.02 \\
21735.54 \\
21768.93\end{array}$ & 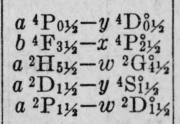 & $7 b$ & & 0 & $1.10-$ & $(1.10)$ & 1.10 \\
\hline $\begin{array}{l}6 c \\
7 c \\
4\end{array}$ & $\begin{array}{l}4584.849 \\
4583.49 \\
4583.132\end{array}$ & $\begin{array}{l}21804.88 \\
2181.34 \\
21813.05\end{array}$ & $\begin{array}{l}b{ }^{4} \mathrm{P}_{113}-u{ }^{4} \mathrm{D}_{11 / 2}^{11} \\
a^{4} \mathrm{C}_{443 / 2}-v{ }^{4} \mathrm{D}_{31 / 2}\end{array}$ & & & & & & \\
\hline $\begin{array}{r}{ }^{4}, \mathrm{~V} \\
100, \mathrm{~V}\end{array}$ & $\begin{array}{l}45833.132 \\
4582.283 \\
4581.623\end{array}$ & 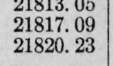 & 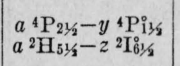 & $\begin{array}{l}4 \\
4\end{array}$ & & $w$ & $\begin{array}{l}1.521 \\
1.03\end{array}$ & $\begin{array}{l}(1.596) \\
(1.10)\end{array}$ & $\begin{array}{l}1.646 \\
1.08\end{array}$ \\
\hline $\begin{array}{l}12 \\
25 c, \mathrm{~V} \\
2 \\
7 \\
200 c, \mathrm{III}\end{array}$ & $\begin{array}{l}4575.372 \\
4574.848 \\
4574.572 \\
4574.338 \\
4573.077\end{array}$ & $\begin{array}{l}21850.04 \\
21852.54 \\
21853.86 \\
21854.98 \\
21861.01\end{array}$ & 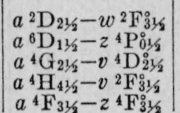 & 4 & & 0.298 & 1.23 & $(1.863)$ & 2. 459 \\
\hline
\end{tabular}




\section{Journal of Research of the National Bureau of Standards}

TABLE 4.-First spectrum of columbium (Cb I)-Continued

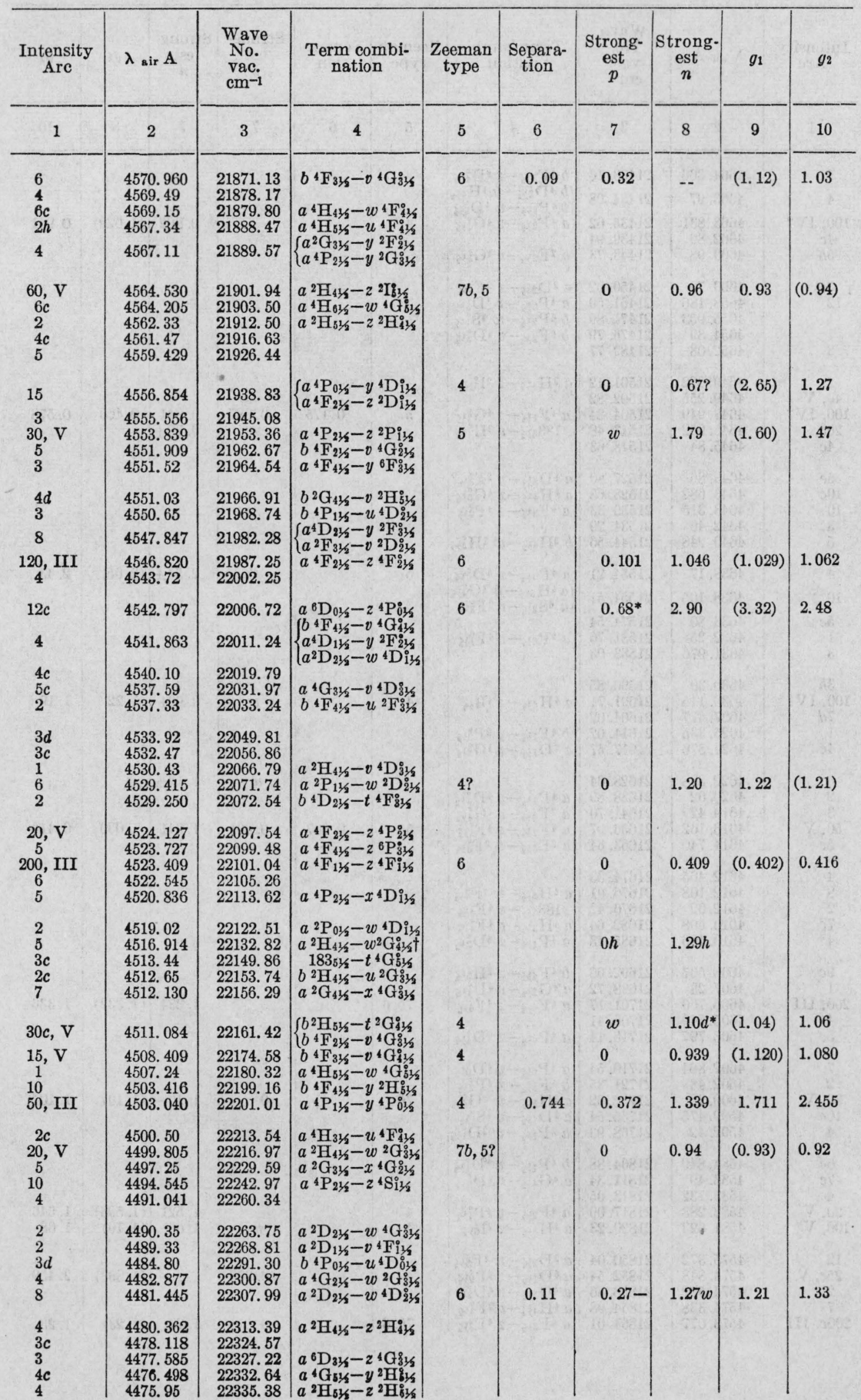


TABLE 4.-First spectrum of columbium (Cb I) - Continued

\begin{tabular}{|c|c|c|c|c|c|c|c|c|c|}
\hline $\begin{array}{c}\text { Intensity } \\
\text { Arc }\end{array}$ & $\lambda_{\text {air }} \mathbf{A}$ & $\begin{array}{l}\text { Wave } \\
\text { No. } \\
\text { vac. } \\
\mathrm{cm}^{-1}\end{array}$ & $\begin{array}{l}\text { Term combi- } \\
\text { nation }\end{array}$ & $\begin{array}{l}\text { Zeeman } \\
\text { type }\end{array}$ & $\begin{array}{l}\text { Separa- } \\
\text { tion }\end{array}$ & $\begin{array}{l}\text { Strong- } \\
\text { est } \\
p\end{array}$ & $\begin{array}{c}\text { Strong- } \\
\text { est } \\
n\end{array}$ & $g_{1}$ & $g_{2}$ \\
\hline 1 & 2 & 3 & at & 5 & 6 & 7 & 8 & 9 & 10 \\
\hline $\begin{array}{l}6 \\
2 \\
40 c, \text { IV } \\
50 c, \text { V } \\
40 c, \text { IV }\end{array}$ & $\begin{array}{l}4475.278 \\
4472.874 \\
4472.536 \\
4471.292 \\
4469.714\end{array}$ & $\begin{array}{l}22338.73 \\
22350.74 \\
22352.43 \\
22358.65 \\
22366.54\end{array}$ & $\begin{array}{c}b^{4} \mathrm{~F}_{41 / 2}-y^{2} \mathrm{H}_{41 / 2}^{0} \\
a^{4} \mathrm{~F}_{31 / 2}-z^{4} \mathrm{~F}_{41 / 2}^{0} \\
a^{2} \mathrm{G}_{41 / 2}-x^{2} \mathrm{~F}_{31 / 2}^{0} \\
b^{4} \mathrm{~F}_{11 / 2}-v^{4} \mathrm{G}_{21 / 2}^{\circ}\end{array}$ & $\begin{array}{l}5 \\
4 \\
5\end{array}$ & 0.228 & $\begin{array}{l}0 \\
w \\
w \\
0.114\end{array}$ & $\begin{array}{l}1.03 \\
1.56- \\
0.76 \\
\ldots\end{array}$ & $\begin{array}{l}(1.24) \\
(1.10) \\
(0.402)\end{array}$ & $\begin{array}{l}1.31 \\
1.20 \\
0.630\end{array}$ \\
\hline 10 & 4469.322 & 22368.50 & $\left\{\begin{array}{l}a^{4} \mathrm{P}_{13 / 3}-x^{4} \mathrm{D}_{031 / 3}^{\circ} \\
a^{2} \mathrm{D}_{11 / 2}-v^{4} \mathrm{~F}_{21 / 3}^{\circ}\end{array}\right.$ & & & & & & \\
\hline $\begin{array}{l}1 \\
5 \\
2 \\
7\end{array}$ & $\begin{array}{l}4468.54 \\
4466.421 \\
4466.198 \\
4465.926\end{array}$ & $\begin{array}{l}22372.42 \\
22383.03 \\
22384.15 \\
22385.51\end{array}$ & $\mid \begin{array}{c}a \\
a \\
a^{6} \mathrm{~S}_{21 / 2}-v^{4} \mathrm{D}_{11 / 2}-z^{2} \mathrm{D}_{11 / 2} \\
a^{4} \mathrm{H}_{11 / 2}-w^{4} \mathrm{G}_{51 / 2} \\
a^{2} \mathrm{H}_{21 / 2}-x^{2} \mathrm{D}_{11 / 2}^{0}\end{array}$ & & & & & & \\
\hline 4 & 4465.232 & 22388.99 & $a^{4} \mathrm{~F}_{31 / 2}-z{ }^{6} \mathrm{P}_{21 / 2}^{0}$ & & & & & & \\
\hline 20, IV & 4464.151 & 22394.41 & $\left\{\begin{array}{l}a^{4} \mathrm{~F}_{41 / 2}-y{ }^{6} \mathrm{~F}_{41 / 5} \\
a^{2} \mathrm{H}_{41 / 2}-y^{2} \mathrm{H}_{4152}^{0}\end{array}\right.$ & 6 & 0.09 & 0.39 & $1.35 \pm$ & 1.31 & 1.40 \\
\hline $\begin{array}{l}1 \\
20 c, \mathrm{~V} \\
15 c, \mathrm{~V}\end{array}$ & $\begin{array}{l}4461.49 \\
4460.423 \\
4460.203\end{array}$ & $\begin{array}{l}22407.77 \\
22413.13 \\
22414.23\end{array}$ & $\begin{array}{l}b^{4} \mathrm{~F}_{21 / 2}-u^{2} \mathrm{~F}_{21 / 2}^{0} \\
a^{2} \mathrm{~F}_{31 / 2}-u^{2} \mathrm{D}_{21 / 2}^{\circ}\end{array}$ & 5 & & 0 & 1.09 & (1.13) & 1.16 \\
\hline$\stackrel{2}{15}, \mathrm{~V}$ & $\begin{array}{l}4459.671 \\
4458.117\end{array}$ & $\begin{array}{l}22416.91 \\
22424.72\end{array}$ & $\begin{array}{l}a^{2} \mathrm{D}_{21 / 5}-v^{2} \mathrm{~F}_{21 / 4}^{2} \\
a^{2} \mathrm{~F}_{21 / 3}-u^{2} \mathrm{D}_{11 / 6}\end{array}$ & & & & & & \\
\hline 50, IV? & 4457.424 & 22428. 21 & $a^{4} \mathrm{~F}_{21 / 2}-z^{4} \mathrm{~F}_{31 / 2}^{0}$ & 5 & & & 1.776 & (1.029) & 1. 242 \\
\hline $4 c$ & $\begin{array}{l}4456.800 \\
4456.331\end{array}$ & $\begin{array}{l}22431.35 \\
22433.71\end{array}$ & $\begin{array}{l}a^{4} \mathrm{~F}_{11 / 2}-z^{4} \mathrm{~F}_{21 / 5}^{0} \\
b^{2} \mathrm{G}_{41 / 2}-q^{2} \mathrm{~F}_{31 / 2}^{\circ}\end{array}$ & 5 & 0.718 & 0.363 & -- & $(0.402)$ & 1.120 \\
\hline $\begin{array}{l}3 c \\
6 \\
2\end{array}$ & $\begin{array}{l}4454.68 \\
4448.769 \\
4448.06\end{array}$ & $\begin{array}{l}22442.02 \\
22471.84 \\
22475.42\end{array}$ & $\begin{array}{l}a^{4} \mathrm{D}_{21 / 2}-x^{4} \mathrm{G}_{31 / 2}^{1} \\
a^{2} \mathrm{G}_{41 / 2}-x^{2} \mathrm{G}_{31 / 5}^{3}\end{array}$ & & & & & & \\
\hline$\frac{100, \text { IV }}{4}$ & $\begin{array}{l}4447.184 \\
4446.232\end{array}$ & $\begin{array}{l}22479.85 \\
22484.66\end{array}$ & $\begin{array}{l}a^{4} \mathrm{P}_{21 / 2}-y{ }^{4} \mathrm{P}_{21 / 2}^{0} \\
a^{4} \mathrm{P}_{11 / 2}-y{ }^{4} \mathrm{P}_{11 / 2}^{0}\end{array}$ & $7 b, 6$ & & 0 & 1. 597 & 1. 596 & 1. 598 \\
\hline $12, \mathrm{~V}$ & 4446.181 & 22484.92 & $b^{4} \mathrm{~F}_{41 / 2}-v^{4} \mathrm{G}_{53 / 5}^{0}$ & & & & & & \\
\hline $15 c$ & 4445.843 & 22486.63 & $\left\{\begin{array}{l}a^{6} \mathrm{D}_{41 / 3}-z^{4} \mathrm{G}_{41 / 5}^{0} \\
b^{4} \mathrm{~F}_{21 / 2}-u^{2} \mathrm{~F}_{31 / 2}^{0}\end{array}\right.$ & & & & & & \\
\hline $\begin{array}{l}1 \\
2 \\
2\end{array}$ & $\begin{array}{l}4444.26 \\
4443.80 \\
4443.302\end{array}$ & $\begin{array}{l}22494.64 \\
22496.97 \\
22499.49\end{array}$ & $\begin{array}{l}a^{2} \mathrm{D}_{11 / 2}-w^{2} \mathrm{~F}_{21 / 2}^{\circ} \\
a^{2} \mathrm{P}_{01 / 2}-x^{2} \mathrm{D}_{11 / 2}^{2} \\
a^{4} \mathrm{P}_{11 / 5}-z^{2} \mathrm{~F}_{21 / 2}^{0}\end{array}$ & & & & & & \\
\hline $\begin{array}{c}1 \\
10 c, \mathrm{~V} \\
3 c \\
5\end{array}$ & $\begin{array}{l}4443.05 \\
4441.802 \\
4441.61 \\
4440.435\end{array}$ & $\begin{array}{l}22500.77 \\
22507.09 \\
22508.06 \\
22514.02\end{array}$ & $\begin{array}{l}a^{4} \mathrm{P}_{01 / 2}-y^{4} \mathrm{P}_{01 / 5}^{0} \\
a^{4} \mathrm{D}_{31 / 2}-x^{4} \mathrm{G}_{41 / 2}^{0} \\
b^{2} \mathrm{G}_{31 / 2}-t^{2} \mathrm{G}_{31 / 2}^{0} \\
b^{4} \mathrm{~F}_{31 / 2}-y^{2} \mathrm{H}_{41 / 2}^{0}\end{array}$ & & & & & & \\
\hline $1 c$ & 4439.38 & 22519.37 & $\left\{\begin{array}{l}b^{4} \mathrm{~F}_{11 / 2}-y{ }^{2} \mathrm{~S}_{01 / 5}^{1} \\
b^{2} \mathrm{G}_{41 / 2}-8^{4} \mathrm{G}_{31 / 5}^{1}\end{array}\right.$ & & & & & & \\
\hline $\begin{array}{c}7 c \\
100 c, V \\
5 \\
3 \\
3\end{array}$ & $\begin{array}{l}4437.918 \\
4437.218 \\
4436.712 \\
4435.919 \\
4434.997\end{array}$ & $\begin{array}{l}22526.79 \\
22530.34 \\
22532.91 \\
22536.94 \\
22541.62\end{array}$ & $\begin{array}{l}b^{2} \mathrm{H}_{51 / 2}-v^{2} \mathrm{H}_{51 / 4}^{\circ} \\
a^{2} \mathrm{~F}_{31 / 2}-v^{2} \mathrm{G}_{31 / 4} \\
a^{4} \mathrm{~F}_{11 / 5}-z^{4} \mathrm{P}_{21 / 5}{ }^{2}\end{array}$ & & & 0 & 1.04 & & \\
\hline $\begin{array}{l}1 \\
3 c \\
2 c \\
1 \\
20 c, \mathrm{~V}\end{array}$ & $\begin{array}{l}4433.895 \\
4433.496 \\
4432.912 \\
4430.029 \\
4429.446\end{array}$ & $\begin{array}{l}22547.22 \\
22549.25 \\
22552.23 \\
22566.90 \\
22569.87\end{array}$ & $\begin{array}{l}b{ }^{4} \mathrm{D}_{31 / 2}-t{ }^{4} \mathrm{~F}_{41 / 2}^{0} \\
b^{2} \mathrm{H}_{41 / 2}-u^{2} \mathrm{G}_{41 / 2} \\
a^{4} \mathrm{G}_{31 / 2}-x{ }^{4} \mathrm{P}_{21 / 2}^{0} \\
a^{4} \mathrm{P}_{21 / 2}-z^{2} \mathrm{~F}_{31 / 2}^{0}\end{array}$ & 4 & 0.48 & 0.23 & & 1. 60 & 1.12 \\
\hline $\begin{array}{l}2 \\
1 \\
20, \mathrm{~V} \\
6 c \\
2\end{array}$ & $\begin{array}{l}4428.576 \\
4427.866 \\
4426.690 \\
4423.868 \\
4421.305\end{array}$ & $\begin{array}{l}22574.30 \\
22577.92 \\
22583.92 \\
22598.33 \\
22611.43\end{array}$ & $\begin{array}{l}a^{2} \mathrm{D}_{13 / 2}-w^{4} \mathrm{~F}_{21 / 2}^{2} \\
a^{4} \mathrm{~F}_{21 / 2}-y^{6} \mathrm{~F}_{11 / 2}^{\circ} \\
a^{4} \mathrm{P}_{21 / 2}-x^{4} \mathrm{D}_{21 / 2}^{\circ} \\
b^{2} \mathrm{H}_{41 / 2}-t^{2} \mathrm{G}_{31 / 2}^{\circ} \\
a^{2} \mathrm{~F}_{21 / 2}-u^{4} \mathrm{D}_{11 / 2}^{\circ}\end{array}$ & 6 & 0.117 & 0.293 & 1. $726^{*}$ & (1.596) & 1. 479 \\
\hline $\begin{array}{l}30 c, \mathrm{~V} \\
10, \mathrm{~V} \\
10, \mathrm{~V} \\
40, \mathrm{IV} \\
4\end{array}$ & $\begin{array}{l}4420.637 \\
4420.455 \\
4419.839 \\
4419.448 \\
4418.166\end{array}$ & $\begin{array}{l}22614.84 \\
22615.78 \\
22618.91 \\
22620.93 \\
22627.49\end{array}$ & $\begin{array}{l}a^{6} \mathrm{D}_{21 / 2}-z^{4} \mathrm{P}_{11 / 2}^{0} \\
a^{4} \mathrm{~F}_{31 / 2}-y^{6} \mathrm{~F}_{31 / 5}^{0} \\
a^{4} \mathrm{~F}_{31 / 2}-z^{2} \mathrm{D}_{21 / 6}^{0} \\
a^{4} \mathrm{P}_{11 / 2}-z^{2} \mathrm{P}_{11 / 2}^{0}\end{array}$ & 6 & 0.26 & 0.39 & 1. 59 & 1. 72 & 1.46 \\
\hline $\begin{array}{l}6 \\
2\end{array}$ & $\begin{array}{l}4416.406 \\
4415.080\end{array}$ & $\begin{array}{l}22636.51 \\
22643.31\end{array}$ & $\begin{array}{l}a^{4} \mathrm{G}_{21 / 5}-v^{4} \mathrm{G}_{21 / 4} \\
a^{2} \mathrm{~F}_{21 / 5}-v^{2} \mathrm{G}_{31 / 5}^{0}\end{array}$ & & & & & & \\
\hline $8 c$ & 4414.879 & 22644.34 & $\left\{\begin{array}{l}a^{4} \mathrm{D}_{21 / 5}-x^{2} \mathrm{~F}_{31 / 5}^{0} \\
a^{2} \mathrm{G}_{436}-v \mathrm{v}_{31 / 6}^{\circ}\end{array}\right.$ & & & & & & \\
\hline $\begin{array}{l}2 \\
5\end{array}$ & $\begin{array}{l}4413.000 \\
4412.184\end{array}$ & $\begin{array}{l}22653.98 \\
22658.17\end{array}$ & $\begin{array}{l}a^{2} \mathrm{H}_{51 / 2}-v^{4} \mathrm{G}_{41 / 5} \\
a^{2} \mathrm{G}_{315}-x^{4} \mathrm{G}_{31 / 5}^{3}\end{array}$ & & & & & & \\
\hline
\end{tabular}


536 Journal of Research of the National Bureau of Standards

TABLE 4.-First spectrum of columbium (Cb I)-Continued

\begin{tabular}{|c|c|c|c|c|c|c|c|c|c|}
\hline $\begin{array}{l}\text { Intensity } \\
\text { Arc }\end{array}$ & $\lambda$ air $A$ & $\begin{array}{l}\text { Wave } \\
\text { No. } \\
\text { vac. } \\
\mathrm{cm}^{-1}\end{array}$ & $\begin{array}{c}\text { Term combi- } \\
\text { nation }\end{array}$ & $\begin{array}{c}\text { Zeeman } \\
\text { type }\end{array}$ & $\begin{array}{l}\text { Separa- } \\
\text { tion }\end{array}$ & $\begin{array}{l}\text { Strong. } \\
\text { est } \\
p\end{array}$ & $\begin{array}{c}\text { Strong- } \\
\text { est } \\
n\end{array}$ & $g_{1}$ & $g_{3}$ \\
\hline 1 & 2 & 3 & 4 & 5 & 6 & 7 & 8 & 9 & 10 \\
\hline $15, \mathrm{~V}$ & 4411.526 & 22661.55 & $a^{2} \mathrm{D}_{21 / 2}-v^{2} \mathrm{~F}_{31 / 6}^{0}$ & 5 & & 0 & 1.11 & (1. 21) & 1.16 \\
\hline 2 & 4410.882 & 22664.86 & $\left\{\begin{array}{l}a^{4} \mathrm{D}_{21 / 5}-v^{4} \mathrm{~F}_{11 / 5}^{0} \\
a^{2} \mathrm{~F}_{31 / 5}-u^{4} \mathrm{D}_{21 / 6}\end{array}\right.$ & & & & & & \\
\hline $\begin{array}{l}60, \text { IV } \\
10 c \\
1\end{array}$ & $\begin{array}{l}4410.214 \\
4406.546 \\
4404.74\end{array}$ & $\begin{array}{l}22668.29 \\
22687.16 \\
22696.46\end{array}$ & 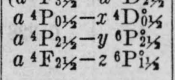 & 6 & 2. 42 & 1.21 & 1. 42 & 2. 63 & 0.21 \\
\hline $\begin{array}{l}2 \\
7 c\end{array}$ & $\begin{array}{l}4403.925 \\
4402.056\end{array}$ & $\begin{array}{l}22700.66 \\
22710.30\end{array}$ & $a^{2} \mathrm{D}_{13 / \Omega}-u^{4} \mathrm{~F}_{21 / 5}^{0}$ & & & & & & \\
\hline 10 & 4400.832 & 22716.62 & $a^{1} \mathrm{G}_{31 / 2}-v^{4} \mathrm{G}_{31 / 2}^{2}$ & & & & & & \\
\hline $\begin{array}{l}10 c \\
10\end{array}$ & $\begin{array}{l}4400.368 \\
4397.036\end{array}$ & $\begin{array}{l}22719.01 \\
22736.23\end{array}$ & $\begin{array}{l}b^{2} \mathrm{G}_{31 / 2}-t^{2} \mathrm{G}_{41 / 2}^{0} \\
a^{2} \mathrm{H}_{41 / 2}-z^{2} \mathrm{H}_{51 / 2}^{\circ}\end{array}$ & 5 & & $w$ & 1. $34-$ & $(0.93)$ & 1.00 \\
\hline $\begin{array}{l}1 \\
3 \\
30, \mathrm{~V} \\
4 c \\
7\end{array}$ & $\begin{array}{l}4395.61 \\
4394.229 \\
4392.692 \\
4391.59 \\
4391.214\end{array}$ & $\begin{array}{l}22743.60 \\
22750.75 \\
22758.71 \\
22764.42 \\
22766.37\end{array}$ & $\begin{array}{l}b^{4} \mathrm{D}_{11 / 2}-x^{2} \mathrm{P}_{01 / 3}^{0} \\
a^{4} \mathrm{~F}_{31 / 2}-z^{6} \mathrm{P}_{31 / 5}^{0} \\
a^{2} \mathrm{G}_{41 / 2}-w^{2} \mathrm{~F}_{31 / 2}^{0} \\
a^{4} \mathrm{D}_{21 / 5}-v^{4} \mathrm{~F}_{21 / 5}^{0} \\
a^{2} \mathrm{D}_{21 / 2}-w^{4} \mathrm{D}_{31 / 2}^{0}\end{array}$ & 5 & & 0 & 1. 14 & (1. 10) & 1.08 \\
\hline $2 \mathrm{~V}$ & 4389.496 & 22775. 28 & $a^{2} \mathrm{~F}_{21 / 3}-u{ }^{4} \mathrm{D}_{21 / 3}^{2}$ & & & & & & \\
\hline $\begin{array}{l}30, V \\
15\end{array}$ & $\begin{array}{l}4388.357 \\
4387.743\end{array}$ & $\begin{array}{l}22781.19 \\
22784.38\end{array}$ & $\begin{array}{l}a^{4} \mathrm{P}_{11 / 2}-x^{4} \mathrm{D}_{11 / 2} \\
a^{4} \mathrm{P}_{01 / 2}-y^{4} \mathrm{P}_{11 / 2}^{0}\end{array}$ & $\begin{array}{l}0 \\
4\end{array}$ & $\begin{array}{l}0.283 \\
1.012\end{array}$ & $\begin{array}{l}0.421 \\
0.506\end{array}$ & $\begin{array}{l}\text { 1. } 090 \\
\text { 1. } 162\end{array}$ & 2. 680 & $\begin{array}{l}\text { 1. } 448 \\
1.668\end{array}$ \\
\hline$\frac{4}{15 c}$ & $\begin{array}{l}4386.302 \\
4384.870\end{array}$ & $\begin{array}{l}22791.87 \\
22799.31\end{array}$ & $a^{4} \mathrm{G}_{41 / 2}-v^{4} \mathrm{G}_{435}$ & & & & & & \\
\hline 1 & 4383.70 & 22805.39 & $b^{4} \mathrm{~F}_{21 / 2}-v^{2} \mathrm{D}_{21 / 2}^{0}$ & & & & & & \\
\hline $9 c$ & 4382.856 & 22809. 79 & $\left\{\begin{array}{l}a^{4} \mathrm{~F}_{21 / 5}-y{ }^{6} \mathrm{~F}_{21 / 1} \\
a^{2} \mathrm{D}_{11 / 5}-w^{4} \mathrm{D}_{116}\end{array}\right.$ & & & & & & \\
\hline 7 & 4382.496 & 22811.66 & $b^{4} \mathrm{~F}_{11 / 2}-u^{2} \mathrm{~F}_{21 / 2}^{0}$ & & & & & & \\
\hline $10 c$ & 4381.114 & 22818.86 & $\left\{\begin{array}{l}a^{2} \mathrm{~F}_{31 / 2}-v^{2} \mathrm{G}_{41 / 3}^{0} \\
a^{2} \mathrm{~F}_{31 / 2}-u^{4} \mathrm{D}_{31 / 3}^{\circ}\end{array}\right.$ & & & & & 4 & \\
\hline $4 c$ & 4380.664 & 22821.20 & $a^{4} \mathrm{G}_{41 / 2}-u^{2} \mathrm{~F}_{31 / 4}^{0}$ & & & & & & \\
\hline $\begin{array}{l}12 \\
30 c, V\end{array}$ & $\begin{array}{l}4379.525 \\
4377.958\end{array}$ & $\begin{array}{l}22827.13 \\
22835.30\end{array}$ & $\begin{array}{l}a^{2} \mathrm{G}_{41 / 5}-z^{4} \mathrm{I}_{41 / 2}^{0} \\
a^{4} \mathrm{G}_{21 / 5}-v^{4} \mathrm{G}_{31 / 5}^{2}\end{array}$ & 5 & 0.264 & 0.135 & 1. 659 & 0.734 & 0.998 \\
\hline $\begin{array}{l}5 \\
4\end{array}$ & $\begin{array}{l}4377.823 \\
4376.707\end{array}$ & $\begin{array}{l}22836.01 \\
22841.83\end{array}$ & $a^{2} \mathrm{H}_{51 / 2}-y^{2} \mathrm{H}_{51 / 3}^{\circ}$ & & & & & & \\
\hline 6 & 4375.246 & 22849.46 & $\left\{\begin{array}{l}a^{2} \mathrm{D}_{21 / 2}-w^{2} \mathrm{D}_{11 / 2} \\
a^{4} \mathrm{G}_{21 / 2}-x^{4} \mathrm{P}_{11 / 2}^{0}\end{array}\right.$ & & & & & & \\
\hline $12 c$ & 4374. 789 & 22851.84 & $a^{6} \mathrm{D}_{21 / 2}-z^{4} \mathrm{~F}_{11 / 2}^{0}$ & & & & & & \\
\hline $\begin{array}{r}15 \\
8\end{array}$ & $\begin{array}{l}4370.361 \\
4369.618\end{array}$ & 22875.00 & $a^{4} \mathrm{~F}_{41 / 2}-y^{6} \mathrm{~F}_{51 / 2}$ & & & & & & \\
\hline 50, IV? & $\begin{array}{l}4309.018 \\
4368.434\end{array}$ & $\begin{array}{l}22878.88 \\
22885.09\end{array}$ & $a^{2} \mathrm{G}_{41 / 2}-x^{2} \mathrm{G}_{41 / 2}^{21 / 2}$ & 6ur & & 0.079 & 1. 096 & 1. 105 & 1. 087 \\
\hline 9 & 4361.656 & 22920.65 & $a^{4} \mathrm{P}_{01 / 2}-z{ }^{2} \mathrm{P}_{11 / 5}^{0}$ & & 1. 198 & 0.599 & 0.856 & 2. 653 & 1. 455 \\
\hline $5 c$ & 4360.504 & 22926. 71 & $b^{2} \mathrm{G}_{31 / 2}-v^{2} \mathrm{H}_{41 / 2}^{\circ}$ & & & & & & \\
\hline$\frac{40 c, V}{7}$ & $\begin{array}{l}4359.865 \\
4356.847\end{array}$ & $\begin{array}{l}22930.07 \\
22945.95\end{array}$ & $\begin{array}{l}a^{4} \mathrm{D}_{21 / 2}-v{ }^{4} \mathrm{~F}_{31 / 2}^{0} \\
b^{4} \mathrm{~F}_{31 / 2}-u^{2} \mathrm{D}_{21 / 2}^{0}\end{array}$ & $\begin{array}{l}7 b, 4 \\
4\end{array}$ & & $\begin{array}{l}0 \\
0\end{array}$ & $\begin{array}{l}1.330 \\
1.05\end{array}$ & $\begin{array}{l}(1.360) \\
(1.12)\end{array}$ & $\begin{array}{l}1.347 \\
1.18\end{array}$ \\
\hline $5 c$ & 4355.245 & 22954. 39 & $\left\{\begin{array}{l}a^{4} \mathrm{D}_{31 / 2}-u^{4} \mathrm{~F}^{0} 1 / 5 \\
b^{4} \mathrm{D}_{11 / 5}-t^{4} \mathrm{D}_{21 / 6}^{0}\end{array}\right.$ & & & & & & \\
\hline 4 & 4354. 784 & 22956.82 & & & & & & & \\
\hline 6 & 4354.188 & 22959.96 & $a{ }^{4} \mathrm{H}_{41 / 2}-z^{2} \mathrm{I}_{53 / 6}^{0}$ & & & & & & \\
\hline & $\begin{array}{l}4354.006 \\
4353.266\end{array}$ & $\begin{array}{l}22960.92 \\
22964.83\end{array}$ & $\begin{array}{l}a^{2} \mathrm{P}_{01 / 3}-w^{2} \mathrm{D}_{111 / 2}^{0} \\
a^{6} \mathrm{D}_{41 / 2}-z^{4} \mathrm{~F}_{31 / 2}^{0}\end{array}$ & 5 & 0.310 & & & (1. 549) & 1. 239 \\
\hline $2 c$ & 4352.243 & 22970.22 & $a^{4} \mathrm{D}_{21 / 2}-w^{4} \mathrm{~F}_{21 / 2}^{6}$ & & & & & & \\
\hline 40, IV? & 4351.573 & 22973.76 & $a^{2} \mathrm{G}_{31 / 2}-x^{2} \mathrm{G}_{31 / 2}^{0}$ & 6 & & 0 & 0.896 & $(0.885)$ & 0.906 \\
\hline 9 & 4350.302 & 22980.47 & $\left\{\begin{array}{l}a^{2} \mathrm{G}_{31 / 2}-v{ }^{4} \mathrm{~F}_{21 / 2}^{0} \\
a^{4} \mathrm{P}_{116}-y{ }^{6} \mathrm{P}_{116}^{0}\end{array}\right.$ & 4 & 0.163 & 0.082 & & $(0.885)$ & 1. 048 \\
\hline $30, \mathrm{~V}$ & 4349.026 & 22987.21 & $\left\{\begin{array}{l}a^{4} \mathrm{G}_{41 / 2}-y^{2} \mathrm{H}_{51 / 5}^{6 / 3} \\
a^{4} \mathrm{H}_{515}-w^{2} \mathrm{G}^{2} \mathrm{H}^{2}\end{array}\right.$ & 4 & 0.115 & 0.062 & 0.650 & 1. 283 & 1.168 \\
\hline $\begin{array}{c}40 c, V \\
8 c \\
10\end{array}$ & $\begin{array}{l}4348.652 \\
4347.312 \\
4346.120\end{array}$ & $\begin{array}{l}22989.19 \\
22996.28 \\
23002.58\end{array}$ & $\begin{array}{l}a^{6} \mathrm{D}_{31 / 2}-z^{4} \mathrm{P}_{21 / 2}^{0} \\
a^{2} \mathrm{~F}_{31 / 2}-t^{2} \mathrm{~F}_{31 / 2}^{0} \\
a^{4} \mathrm{D}_{11 / 2}-v^{4} \mathrm{~F}_{11 / 2}^{0}\end{array}$ & $5 u r$ & & $0 w$ & 1. 862 & (1. 582) & 1.470 \\
\hline $\begin{array}{c}6 c \\
20 d\end{array}$ & $\begin{array}{l}4345.518 \\
4345.315\end{array}$ & $\begin{array}{l}23005.77 \\
23006.84\end{array}$ & $\begin{array}{l}b^{2} \mathrm{H}_{53 / 2}-t^{4} \mathrm{G}_{51 / 2} \\
a^{6} \mathrm{D}_{016}-z^{4} \mathrm{P}_{11 / 6}^{5}\end{array}$ & & 1. 719 & 0.860 & 0.748 & 3.327 & 1. 608 \\
\hline $25, \mathrm{~V}$ & 4342.818 & 23020.07 & $a^{4} \mathrm{G}_{31 / 2}-v{ }^{4} \mathrm{G}_{41 / 2}^{1}$ & $7 b, 4$ & & & 1. 067 & (1.081) & 1.076 \\
\hline 6 & $\begin{array}{l}4342.400 \\
4338.698\end{array}$ & $\begin{array}{l}23021.97 \\
23041.93\end{array}$ & $\begin{array}{l}a^{4} \mathrm{~F}_{11 / 2}-y^{0} \mathrm{~F}_{11 / 2} \\
a^{4} \mathrm{G}_{31 / 2}-u^{2} \mathrm{~F}_{31 / 2}^{01}\end{array}$ & & & & & & \\
\hline
\end{tabular}


TABLE 4.-First spectrum of columbium (Cb I) - Continued

\begin{tabular}{|c|c|c|c|c|c|c|c|c|c|}
\hline $\begin{array}{c}\text { Intensity } \\
\text { Are }\end{array}$ & $\lambda_{\text {air }} \mathrm{A}$ & $\begin{array}{l}\text { Wave } \\
\text { No. } \\
\text { vac. } \\
\mathrm{cm}^{-1}\end{array}$ & $\begin{array}{l}\text { Term combi- } \\
\text { nation }\end{array}$ & $\begin{array}{c}\text { Zeeman } \\
\text { type }\end{array}$ & $\begin{array}{l}\text { Separa- } \\
\text { tion }\end{array}$ & $\begin{array}{l}\text { Strong- } \\
\text { est } \\
p\end{array}$ & $\begin{array}{c}\text { Strong- } \\
\text { est } \\
n\end{array}$ & $g_{1}$ & $g_{2}$ \\
\hline 1 & 2 & 3 & 4 & 5 & 6 & 7 & 7 & 8 & 10 \\
\hline $\begin{array}{c}12 c \\
6 \\
2 h \\
5 \\
1\end{array}$ & $\begin{array}{l}4337.561 \\
4336.548 \\
4334.23 \\
4332.428 \\
4332.17\end{array}$ & $\begin{array}{l}23047.97 \\
23053.36 \\
23065.68 \\
23075.28 \\
23076.65\end{array}$ & 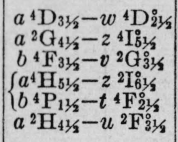 & 5 & & $w$ & 1. $60 h$ & (1. 42) & 1. 35 \\
\hline $\begin{array}{l}60, \mathrm{~V} \\
2 \\
20 c, \mathrm{~V} \\
4 c \\
30 c, \mathrm{~V}\end{array}$ & $\begin{array}{l}\text { 4331. } 371 \\
4331.252 \\
4329.732 \\
4329.47 \\
4328.428\end{array}$ & $\begin{array}{l}23080.91 \\
23081.55 \\
23089.65 \\
23091.04 \\
23096.61\end{array}$ & $\begin{array}{l}a^{4} \mathrm{P}_{01 / 2}-x{ }^{4} \mathrm{D}_{11 / 2}{ }^{0} \\
a^{4} \mathrm{G}_{21 / 2}-u^{2} \mathrm{~F}_{21 / 2}^{0} \\
a^{6} \mathrm{D}_{11 / 2}-z^{4} \mathrm{~F}_{11 / 2} \\
b^{2} \mathrm{G}_{31 / 2}-400_{21 / 2}^{0} \\
a^{4} \mathrm{D}_{21 / 2}-u^{4} \mathrm{~F}_{21 / 2}^{0}\end{array}$ & 4 & $\begin{array}{l}1.212 \\
0.323\end{array}$ & 0.797 & 0.831 & $\begin{array}{r}2.649 \\
(1.360)\end{array}$ & 1. 437 \\
\hline $\begin{array}{c}25 c, \mathrm{~V} \\
9\end{array}$ & $\begin{array}{l}4327.385 \\
4326.540\end{array}$ & $\begin{array}{l}23102.17 \\
23106.68\end{array}$ & $\begin{array}{l}a^{4} \mathrm{D}_{11 / 2}-v^{4} \mathrm{~F}_{21 / 2}^{\circ} \\
\left\{\begin{array}{l}a^{2} \mathrm{~F}_{21 / 2}-t^{2} \mathrm{~F}_{31 / 2}^{\circ} \\
a^{2} \mathrm{G}_{31 / 4}-w^{2} \mathrm{~F}^{\circ}\end{array}\right.\end{array}$ & 4 & 0.150 & 0.076 & 0.820 & 1.195 & 1.045 \\
\hline $\begin{array}{c}100 c, \mathrm{~V} \\
8 \\
1\end{array}$ & $\begin{array}{l}4326.320 \\
4323.466 \\
4322.148\end{array}$ & $\begin{array}{l}23107.86 \\
23123.11 \\
23130.16\end{array}$ & $\begin{array}{l}a^{4} \mathrm{D}_{31 / 2}-v^{4} \mathrm{~F}_{41 / 2}^{0} \\
a^{2} \mathrm{G}_{41 / 2}-u^{4} \mathrm{~F}_{31 / 2}^{0} \\
b^{4} \mathrm{~F}_{41 / 2}-x{ }^{2} \mathrm{H}_{51 / 2}^{0}\end{array}$ & $\begin{array}{l}4 \\
4\end{array}$ & 0.202 & $\begin{array}{l}0.102 \\
0\end{array}$ & $\begin{array}{l}0.495 \\
1.058\end{array}$ & $\begin{array}{l}1.419 \\
1.094\end{array}$ & $\begin{array}{l}1.217 \\
(1.115)\end{array}$ \\
\hline $\begin{array}{l}3 c \\
6 \\
1 \\
3 \\
10\end{array}$ & $\begin{array}{l}4321.88 \\
4320.800 \\
4319.52 \\
4319.147 \\
4318.010\end{array}$ & $\begin{array}{l}23131.60 \\
23137.38 \\
23144.23 \\
23146.23 \\
23152.33\end{array}$ & 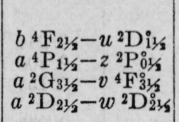 & $7 b, 6$ & & $\begin{array}{l}0 \\
0\end{array}$ & 1. 207 & $\begin{array}{l}(0.852) \\
(1.206)\end{array}$ & $\begin{array}{l}0.831 \\
1.208\end{array}$ \\
\hline $12 c$ & 4316.476 & 23160.55 & $\left\{\begin{array}{l}a^{4} \mathrm{G}_{21 / 2}-u^{2} \mathrm{~F}_{31 / 2}^{0} \\
b^{2} \mathrm{G}_{31 / 2}-s^{4} \mathrm{G}_{21 / 2}^{0}\end{array}\right.$ & & & & & & \\
\hline $\begin{array}{l}1 \\
3 \\
10 c \\
25, V\end{array}$ & $\begin{array}{l}4315.144 \\
4314.262 \\
4313.887 \\
4312.454\end{array}$ & $\begin{array}{l}23167.70 \\
23172.44 \\
23174.45 \\
23182.15\end{array}$ & $\begin{array}{l}a^{4} \mathrm{H}_{51 / 2}-z^{2} \mathrm{H}_{41 / 2}^{\circ} \\
a^{2} \mathrm{G}_{41 / 2}-w^{4} \mathrm{G}_{31 / 2} \\
b^{2} \mathrm{H}_{41 / 2}-v^{2} \mathrm{H}_{53 / 2}^{0} \\
a^{6} \mathrm{D}_{21 / 2}-z^{4} \mathrm{~F}_{21 / 2}^{0}\end{array}$ & 6 & 0.596 & 1. 483 & 1. 351 & 1. 649 & 1.053 \\
\hline $\begin{array}{l}4 \\
15 \\
20, \mathrm{~V} \\
50 c \\
4\end{array}$ & $\begin{array}{l}4312.121 \\
4311.695 \\
4311.37 \\
4311.27 \\
4310.826\end{array}$ & $\begin{array}{l}23183.95 \\
23186.23 \\
23187.98 \\
23188.52 \\
23190.91\end{array}$ & $\begin{array}{l}a^{2} \mathrm{D}_{11 / 2}-x^{2} \mathrm{D}_{11 / 2}^{0} \\
a^{4} \mathrm{~F}_{21 / 5}-z^{2} \mathrm{D}_{21 / 5}^{\circ} \\
b^{4} \mathrm{~F}_{41 / 2}-v^{2} \mathrm{G}_{41 / 2}^{0} \\
b^{4} \mathrm{~F}_{41 / 5}-u^{4} \mathrm{D}_{31 / 5}^{0} \\
a^{4} \mathrm{H}_{41 / 5}-w^{2} \mathrm{G}_{41 / 5}^{0}\end{array}$ & 6 & & $w$ & 1.077 & (1. 224) & $0.930 ?$ \\
\hline $\begin{array}{l}20, \mathrm{~V} \\
20 c \\
15 c \\
2 c \\
15\end{array}$ & $\begin{array}{l}4309.564 \\
4308.692 \\
4308.117 \\
4307.233 \\
4306.283\end{array}$ & $\begin{array}{l}23197.70 \\
23202.40 \\
23205.49 \\
23210.25 \\
23215.38\end{array}$ & $\begin{array}{c}b^{4} \mathrm{~F}_{31 / 2}-u{ }^{4} \mathrm{D}_{21 / 2}^{0} \\
a^{4} \mathrm{D}_{11 / 2}-u^{4} \mathrm{~F}_{11 / 2}^{11} \\
a^{4} \mathrm{D}_{21 / 2}-w^{4} \mathrm{D}_{11 / 2}^{0} \\
a^{4} \mathrm{P}_{01 / 2}-z^{4} \mathrm{~S}_{11 / 2}^{0} \\
a^{2} \mathrm{D}_{11 / 2}-v^{2} \mathrm{~F}_{21 / 2}^{0}\end{array}$ & $\begin{array}{l}4 \\
6 \\
5 \\
4\end{array}$ & $\begin{array}{l}0.197 \\
0.405\end{array}$ & $\begin{array}{l}0.100 \\
0.608 \\
0.092\end{array}$ & $\begin{array}{l}0.628 \\
0.991 \\
1.36^{*}\end{array}$ & $\begin{array}{c}1.120 \\
1.194 \\
(1.360)\end{array}$ & $\begin{array}{l}1.317 \\
0.789 \\
1.176 \\
0.828\end{array}$ \\
\hline $\begin{array}{l}4 c \\
9 c \\
3 \\
4\end{array}$ & $\begin{array}{l}4304.659 \\
4303.875 \\
4302.435 \\
4301.204\end{array}$ & $\begin{array}{l}23224.13 \\
23228.36 \\
23236.14 \\
23242.79\end{array}$ & $\begin{array}{c}a^{4} \mathrm{D}_{11 / 3}-w^{2} \mathrm{~F}_{21 / 2}^{0} \\
b^{4} \mathrm{~F}_{21 / 2}-u^{2} \mathrm{D}_{21 / 2}^{0} \\
a^{2} \mathrm{H}_{41 / 2}-y^{2} \mathrm{H}_{51 / 3}^{\circ}\end{array}$ & & & & & & . \\
\hline $100, \mathrm{~V}$ & 4300.989 & 23243.95 & $\left\{\begin{array}{l}a^{4} \mathrm{P}_{21 / 2}-x{ }^{4} \mathrm{D}_{31 / 2}^{0} \\
a^{6} \mathrm{D}_{01 / 2}-z^{4} \mathrm{~F}_{1 / 2}^{0}\end{array}\right.$ & 4 & 0.357 & 0.178 & 0.347 & 1. 598 & 1. 241 \\
\hline $\begin{array}{c}100, \mathrm{~V} \\
6\end{array}$ & $\begin{array}{l}4299.596 \\
4297.920\end{array}$ & $\begin{array}{l}23251.48 \\
23260.55\end{array}$ & $\begin{array}{l}a^{4} \mathrm{P}_{11 / 2}-x{ }^{4} \mathrm{D}_{21 / 2}{ }^{2} \\
a^{2} \mathrm{G}_{31 / 2}-w^{2} \mathrm{~F}_{31 / 2}\end{array}$ & 4 & 0.245 & 0.122 & 1. 106 & 1. 719 & 1.474 \\
\hline $\begin{array}{l}20 c, \mathrm{~V} \\
15, \mathrm{~V} \\
1\end{array}$ & $\begin{array}{l}4296.159 \\
4295.620 \\
4295.253\end{array}$ & $\begin{array}{l}23270.08 \\
23273.00 \\
23274.99\end{array}$ & $\begin{array}{l}a^{4} \mathrm{G}_{41 / 2}-v^{4} \mathrm{G}_{51 / 2}^{\circ} \\
a^{4} \mathrm{H}_{43 / 2}-w^{2} \mathrm{G}_{31 / 2}\end{array}$ & 4 & & $\begin{array}{l}0.06 \\
0 w\end{array}$ & $\begin{array}{l}0.98 \\
0.86\end{array}$ & (1. 23) & 1.16 \\
\hline $\begin{array}{l}1 \\
40 c, \mathrm{~V} \\
20 c, \mathrm{~V} \\
1 \\
25, \mathrm{~V}\end{array}$ & $\begin{array}{l}4294.33 \\
4292.480 \\
4292.035 \\
4291.344 \\
4291.195\end{array}$ & $\begin{array}{l}23279.99 \\
23290.03 \\
23292.44 \\
23296.19 \\
23297.00\end{array}$ & $\begin{array}{l}a^{4} \mathrm{P}_{01 / 2}-y^{6} \mathrm{P}_{13 / 2}^{0} \\
a^{4} \mathrm{D}_{21 / 2}-w^{4} \mathrm{~F}_{31 / 2}^{0} \\
a^{6} \mathrm{D}_{21 / 3}-z^{4} \mathrm{P}_{21 / 2}^{0} \\
b^{4} \mathrm{D}_{21 / 2}-w^{4} \mathrm{P}_{21 / 2}^{1} \\
a^{4} \mathrm{D}_{01 / 2}-v^{4} \mathrm{~F}_{11 / 2}^{0}\end{array}$ & 5 & 0.162 & 0.081 & 1. 19 & $\begin{array}{l}1.361 \\
(0.06)\end{array}$ & 0.81 \\
\hline $\begin{array}{c}4 \\
30, \mathrm{~V} \\
1 \\
6 \\
1\end{array}$ & $\begin{array}{l}4289.85 \\
4289.443 \\
4288.429 \\
4288.299 \\
4288.12\end{array}$ & $\begin{array}{l}23304.31 \\
23306.52 \\
23312.04 \\
23312.73 \\
23313.71\end{array}$ & $\begin{array}{l}a^{4} \mathrm{P}_{21 / 2}-z^{4} \mathrm{H}_{31 / 2}^{0} \\
a^{4} \mathrm{H}_{31 / 2}-w^{2} \mathrm{G}_{4135}^{0} ? \\
a^{2} \mathrm{G}_{31 / 2}-u^{4} \mathrm{~F}_{21 / 2}^{0} \\
a^{4} \mathrm{H}_{01 / 2}-z^{2} \mathrm{H}_{51 / 2}^{0}\end{array}$ & 4 & 0.716 & 0.359 & -0.901 & 1.606 & 0.890 \\
\hline $\begin{array}{l}60, \mathrm{IV} \\
15, \mathrm{~V} \\
2 \\
1 \\
1 h\end{array}$ & $\begin{array}{l}4286.987 \\
4286.216 \\
4285.842 \\
4285.31 \\
4284.683\end{array}$ & $\begin{array}{l}23319.87 \\
23324.06 \\
23326.10 \\
23328.99 \\
23332.41\end{array}$ & 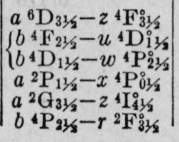 & $\begin{array}{l}6 \\
4\end{array}$ & $\begin{array}{l}0.342 \\
0.344\end{array}$ & $\begin{array}{l}1.197 \\
0.174\end{array}$ & $\begin{array}{l}1.414 \\
0.335\end{array}$ & $\begin{array}{l}1.584 \\
0.851\end{array}$ & $\begin{array}{l}\text { 1. } 243 \\
1.195\end{array}$ \\
\hline
\end{tabular}


538 Journal of Research of the National Bureau of Standards

TABLE 4.-First spectrum of columbium (Cb I)-Continued

\begin{tabular}{|c|c|c|c|c|c|c|c|c|c|}
\hline $\begin{array}{l}\text { Intensity } \\
\text { Arc }\end{array}$ & $\lambda_{\text {air }} A$ & $\begin{array}{l}\text { Wave } \\
\text { No. } \\
\text { vac. } \\
\mathrm{cm}^{-1}\end{array}$ & $\begin{array}{l}\text { Term combi- } \\
\text { nation }\end{array}$ & $\begin{array}{l}\text { Zeeman } \\
\text { type }\end{array}$ & $\begin{array}{l}\text { Separa- } \\
\text { tion }\end{array}$ & $\begin{array}{l}\text { Strong- } \\
\text { est } \\
p\end{array}$ & $\begin{array}{l}\text { Strong- } \\
\text { est } \\
n\end{array}$ & $g_{1}$ & $g_{2}$ \\
\hline 1 & 2 & 3 & 4 & 5 & 6 & 7 & 8 & 9 & 10 \\
\hline $\begin{array}{l}1 h \\
1 \\
3 \\
3 \\
2\end{array}$ & $\begin{array}{l}4284.14 \\
4283.906 \\
4283.055 \\
4282.968 \\
4281.120\end{array}$ & $\begin{array}{l}23335.36 \\
23336.64 \\
23341.27 \\
23341.75 \\
23351.82\end{array}$ & $\begin{array}{l}b^{2} \mathrm{G}_{43 / 3}-8^{4} \mathrm{G}_{51 / 2}^{0} \\
a^{2} \mathrm{P}_{13 / 2}-v^{4} \mathrm{G}_{21 / 5}^{8} \\
b^{4} \mathrm{~F}_{33 / 2}-u^{4} \mathrm{D}_{31 / 2}^{0}\end{array}$ & & & & C.5\% & & \\
\hline $\begin{array}{l}30, \mathrm{~V} \\
1 \\
8\end{array}$ & $\begin{array}{l}4280.586 \\
4280.38 \\
4279.707\end{array}$ & $\begin{array}{l}23354.74 \\
23355.86 \\
23359.54\end{array}$ & 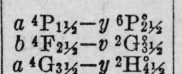 & 5 & 0.131 & $\begin{array}{l}0 \\
0.061\end{array}$ & 1.805 & $\begin{array}{l}(1.721) \\
(1.081)\end{array}$ & $\begin{array}{l}1.755 \\
0.950\end{array}$ \\
\hline $\begin{array}{r}5 \\
20\end{array}$ & $\begin{array}{l}4279.53 \\
4279.49\end{array}$ & $\begin{array}{l}23360.50 \\
23360.72\end{array}$ & 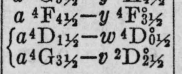 & 5 & 1.148 & 0.574 & 1.770 & 1.196 & 0.048 \\
\hline $20, \mathrm{~V}$ & 4277.500 & 23371.59 & 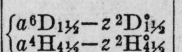 & 6 & 0.967 & 1.452 & 1.384 & 1.868 & 0.901 \\
\hline 20 & 4274.692 & 23386.94 & $a^{2} \mathrm{G}_{31 / 2}-x^{2} \mathrm{a}_{01 / 2}^{2}$ & 5 & 0.203 & 0.091 & 1.794 & 0.881 & 1.084 \\
\hline 5 & 4273.357 & 23394.25 & $\left\{a^{2} \mathrm{H}_{43 / 2}-y^{2} \mathrm{H}_{41 / 6}^{2}\right.$ & & & & & & \\
\hline${ }_{4 c}^{8}$ & $\begin{array}{l}4272.972 \\
4272.027\end{array}$ & $\begin{array}{l}23396.35 \\
23401.53\end{array}$ & $\begin{array}{l}a^{4} \mathrm{H}_{31 / 1}-w^{2} \mathrm{G}_{31 / 6} \\
a^{4} \mathrm{D}_{31 / 2}-v^{2} \mathrm{~F}_{31 / 2}\end{array}$ & & 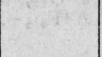 & & & & \\
\hline $\begin{array}{l}50 c, \mathrm{~V} \\
15, \mathrm{~V} \\
50, \mathrm{IV}\end{array}$ & $\begin{array}{l}4270.691 \\
4268.667 \\
4266.020 \\
4264.612\end{array}$ & $\begin{array}{l}23408.85 \\
23419.95 \\
23434.48 \\
2342.29\end{array}$ & 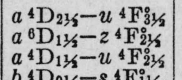 & $\begin{array}{l}4 \\
4 \\
4\end{array}$ & $\begin{array}{l}0.244 \\
0.806 \\
0.155\end{array}$ & $\begin{array}{l}0.122 \\
0.405 \\
0.078\end{array}$ & $\begin{array}{l}0.508 \\
0.140 \\
0.807\end{array}$ & $\begin{array}{l}\text { 1. } 358 \\
1.876 \\
1.195\end{array}$ & $\begin{array}{l}\text { 1. } 114 \\
1.070 \\
1.040\end{array}$ \\
\hline $100, \mathrm{IV}$ & $\begin{array}{l}4262.056 \\
4262.056\end{array}$ & $\begin{array}{l}23456.28 \\
23428\end{array}$ & $a^{0} \mathrm{D}_{41 / 2}-z^{4} \mathrm{~F}_{41 / 2}^{11 / 2}$ & 6 & 0.216 & 0.977 & 1.444 & 1. 552 & 1. 336 \\
\hline $\begin{array}{c}20 c, \mathrm{~V} \\
5 \\
12 c \\
1 \\
5\end{array}$ & $\begin{array}{l}4261.717 \\
4260.661 \\
4258.917 \\
4258.120 \\
4257.853\end{array}$ & $\begin{array}{l}23458.14 \\
23463.95 \\
23473.56 \\
23477.96 \\
23479.43\end{array}$ & 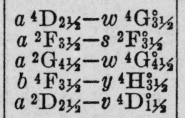 & 6 & & 0.50 & $\begin{array}{l}1.16 w \\
1.187\end{array}$ & 1.205 & $\begin{array}{l}1.21 \\
(1.230)\end{array}$ \\
\hline $\begin{array}{l}4 \\
20 c \\
60 c, \mathrm{~V} \\
30, \mathrm{~V} \\
50 c, \mathrm{v}\end{array}$ & $\begin{array}{l}4256.303 \\
4255.943 \\
4255.439 \\
4254.693 \\
4253.695\end{array}$ & $\begin{array}{l}23487.98 \\
23489.97 \\
23492.75 \\
23496.87 \\
23502.38\end{array}$ & $\begin{array}{l}b^{4} \mathrm{~F}_{21 / 2}-u^{4} \mathrm{D}_{21 / 5}^{0} \\
a^{4} \mathrm{H}_{31 / 2}-z^{2} \mathrm{H}_{41 / 2}^{0} \\
a^{4} \mathrm{D}_{01 / 2}-u^{4} \mathrm{~F}_{11 / 2}^{1} \\
a^{4} \mathrm{D}_{21 / 2}-w^{4} \mathrm{D}_{21 / 2}\end{array}$ & $\begin{array}{l}5 \\
5 \\
6\end{array}$ & $\begin{array}{l}0.134 \\
0.714\end{array}$ & $\begin{array}{l}0.063 \\
0.357 \\
0.14^{*}\end{array}$ & $\begin{array}{l}1.156 \\
1.345\end{array}$ & $\begin{array}{c}(0.690) \\
0.085 \\
(1.360)\end{array}$ & $\begin{array}{l}0.824 \\
0.799 \\
1.330\end{array}$ \\
\hline $\begin{array}{l}80 c, \mathrm{~V} \\
20, \mathrm{IV} \\
3 \\
8 \\
4 c\end{array}$ & $\begin{array}{l}4252.977 \\
4249.457 \\
4248.961 \\
4248.658 \\
4247.689\end{array}$ & $\begin{array}{l}23506.35 \\
23525.82 \\
23528.56 \\
23530.24 \\
23535.61\end{array}$ & 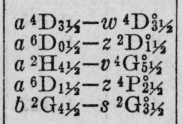 & $\begin{array}{l}6 \\
4\end{array}$ & 2.430 & $\begin{array}{l}0.31^{*} \\
1.214\end{array}$ & $\begin{array}{r}1.385 \\
-0.317\end{array}$ & $\begin{array}{l}(1.420) \\
3.328\end{array}$ & $\begin{array}{l}1.351 \\
0.898\end{array}$ \\
\hline $\begin{array}{l}20, \mathrm{~V} \\
1 \\
20 c, \mathrm{~V} \\
12 \\
4\end{array}$ & $\begin{array}{l}4246.293 \\
4245.15 \\
4242.637 \\
4241.453 \\
4241.077\end{array}$ & $\begin{array}{l}23543.35 \\
23549.69 \\
23563.63 \\
23570.21 \\
23572.30\end{array}$ & 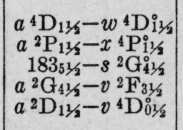 & $7 b, 6$ & & $\begin{array}{l}0 \\
0.26 \\
0 w\end{array}$ & $\begin{array}{l}1.196 \\
1.06 \\
0.89\end{array}$ & (1.197) & $\begin{array}{l}1.195 \\
1.16\end{array}$ \\
\hline $\begin{array}{l}12 c \\
4 c \\
25, \mathrm{IV} \\
1 \\
20, \mathrm{~V}\end{array}$ & $\begin{array}{l}4237.814 \\
4236.998 \\
4231.954 \\
4231.624 \\
4230.320\end{array}$ & $\begin{array}{l}23590.45 \\
23595.00 \\
23623.14 \\
23624.96 \\
23632.24\end{array}$ & $\begin{array}{l}a^{4} \mathrm{H}_{51 / 2}-z^{2} \mathrm{H}_{51 / 1}^{\circ} \\
a^{2} \mathrm{G}_{41 / 2}-w^{4} \mathrm{~F}_{41 / 2}^{\circ} \\
a^{6} \mathrm{D}_{21 / 1}-z^{4} \mathrm{~F}_{31 / 2}^{0} \\
a^{2} \mathrm{G}_{31 / 2}-u^{4} \mathrm{~F}_{31 / 2}^{0} \\
b^{4} \mathrm{~F}_{11 / 2}-u^{4} \mathrm{D}_{015}^{0}\end{array}$ & $\begin{array}{l}6 \\
4 \\
5\end{array}$ & $\begin{array}{l}0.12 \\
0.413\end{array}$ & $\begin{array}{l}0.65 \\
0.208 \\
0.188\end{array}$ & $\begin{array}{l}1.07 \\
0.211 \\
0.584\end{array}$ & $\begin{array}{l}1.13 \\
1.657 \\
0.396\end{array}$ & $\begin{array}{l}1.01 \\
1.244 \\
0.021\end{array}$ \\
\hline $\begin{array}{c}25, \mathrm{~V} \\
1 h \\
100 c, \mathrm{~V} \\
8 c \\
8\end{array}$ & $\begin{array}{l}4229.832 \\
4229.570 \\
4229.154 \\
4228.726 \\
4227.514\end{array}$ & $\begin{array}{l}23634.97 \\
23636.43 \\
23638.76 \\
23641.15 \\
23647.93\end{array}$ & 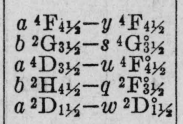 & $\begin{array}{l}7 b, 6 \\
4 \\
6\end{array}$ & $\begin{array}{l}0.172 \\
0.104\end{array}$ & $\begin{array}{l}0 \\
0.086 \\
0.156\end{array}$ & $\begin{array}{c}1.333 \\
\ldots \\
-.\end{array}$ & $\begin{array}{c}(1.332) \\
1.420 \\
(0.953)\end{array}$ & $\begin{array}{l}1.334 \\
1.248 \\
1.057\end{array}$ \\
\hline $\begin{array}{c}8 c \\
12 d \\
1 \\
5 c \\
9\end{array}$ & $\begin{array}{l}4226.528 \\
4226.218 \\
4225.85 \\
4224.690 \\
4222.676\end{array}$ & $\begin{array}{l}23653.44 \\
23655.18 \\
23657.24 \\
23663.73 \\
23675.02\end{array}$ & 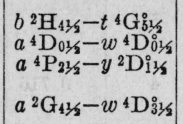 & 6 & & $0^{--}$ & $\begin{array}{l}0.080 \\
0.91\end{array}$ & 0.114 & $(0.046)$ \\
\hline $\begin{array}{l}150 c, \mathrm{IV} \\
100 \mathrm{c}, \mathrm{V} \\
10 \mathrm{c} \\
10 \mathrm{c} \\
15, \mathrm{~V}\end{array}$ & $\begin{array}{l}4217.946 \\
4214.732 \\
4213.463 \\
4213.256 \\
4212.535\end{array}$ & $\begin{array}{l}23701.57 \\
23719.64 \\
23726.79 \\
23727.95 \\
23732.01\end{array}$ & 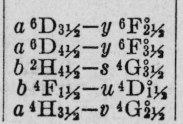 & $\begin{array}{l}5 \\
5\end{array}$ & $\begin{array}{l}0.279 \\
0.176\end{array}$ & 0.140 & 2. 284 & $\begin{array}{l}1.586 \\
(1.549)\end{array}$ & $\begin{array}{l}1.307 \\
1.373\end{array}$ \\
\hline
\end{tabular}


TABLE 4.-First spectrum of columbium (Cb I) - Continued

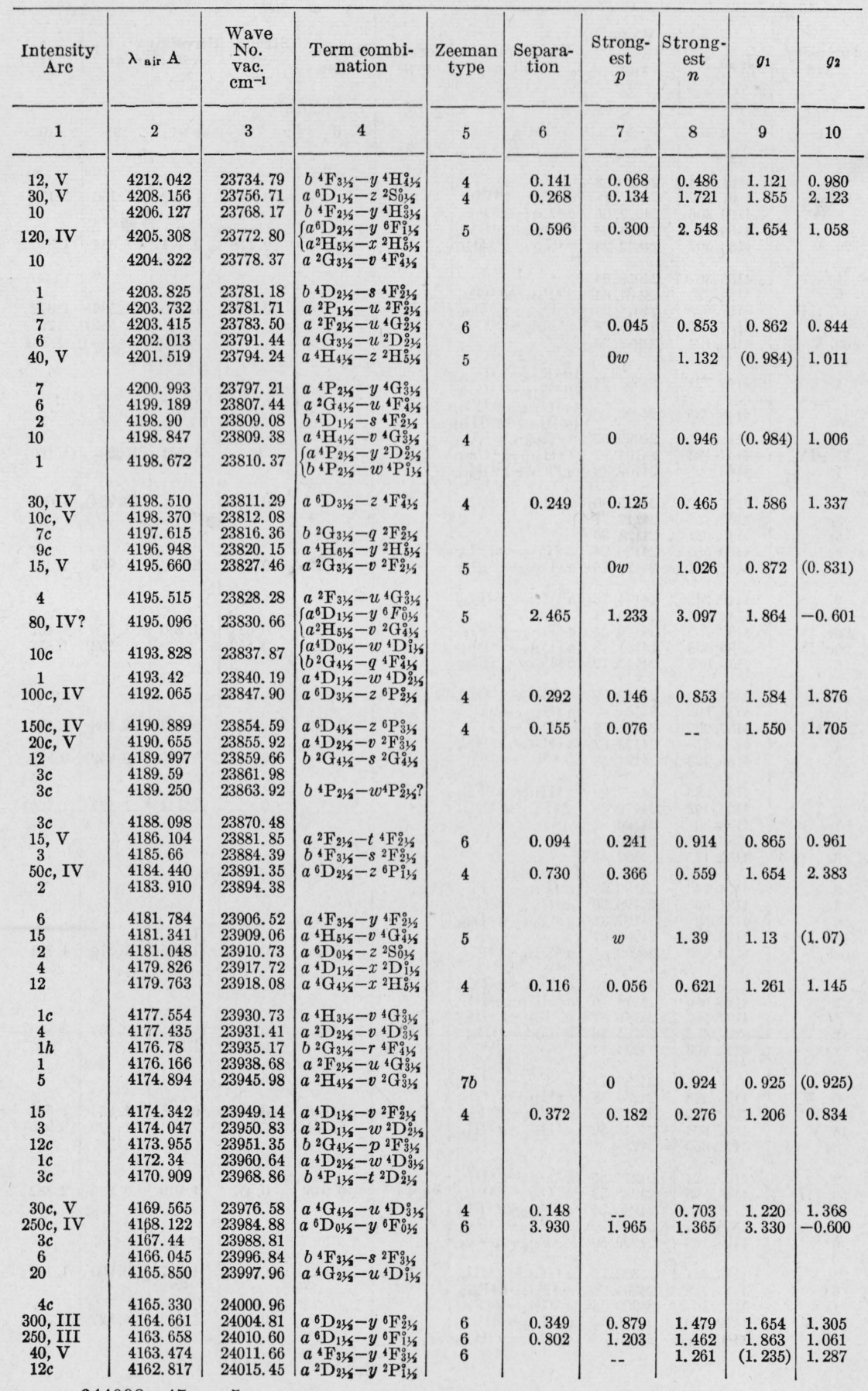

$644098-45-5$ 
TABLE 4.-First spectrum of columbium ( $\mathrm{Cb}$ I) - Continued

\begin{tabular}{|c|c|c|c|c|c|c|c|c|c|}
\hline $\begin{array}{c}\text { Intensity } \\
\text { Are }\end{array}$ & $\lambda_{\text {air }} \mathrm{A}$ & $\begin{array}{l}\text { Wave } \\
\text { No. } \\
\text { vac. } \\
\mathrm{cm}^{-1}\end{array}$ & $\begin{array}{l}\text { Term combi- } \\
\text { nation }\end{array}$ & $\begin{array}{c}\text { Zeeman } \\
\text { type }\end{array}$ & $\begin{array}{l}\text { Separa- } \\
\text { tion }\end{array}$ & $\begin{array}{l}\text { Strong- } \\
\text { est } \\
p\end{array}$ & $\begin{array}{c}\text { Strong- } \\
\text { est } \\
n\end{array}$ & $g_{1}$ & $g_{2}$ \\
\hline 1 & 2 & 3 & 4 & 5 & 6 & 7 & 8 & 9 & 10 \\
\hline $\begin{array}{c}3 c \\
20 c \\
8 \\
1 \\
20, \mathrm{~V}\end{array}$ & $\begin{array}{l}4161.884 \\
4161.252 \\
4160.806 \\
4159.49 \\
4158.007\end{array}$ & $\begin{array}{l}24020.83 \\
24024.48 \\
24027.05 \\
24034.66 \\
24043.23\end{array}$ & $\begin{array}{l}a^{2} \mathrm{~F}_{31 / 2}-t^{4} \mathrm{~F}_{31 / 2}^{0} \\
a^{4} \mathrm{~F}_{41 / 2}-y^{6} \mathrm{D}_{31 / 2}^{0} \\
b^{2} \mathrm{G}_{31 / 2}-s^{4} \mathrm{D}_{31 / 2}^{0} \\
a^{4} \mathrm{G}_{31 / 2}-u^{4} \mathrm{D}_{21 / 2}^{2}\end{array}$ & 6 & 0.235 & 0.118 & 1.162 & 1.081 & 1.173 \\
\hline $\begin{array}{c}5 c \\
6 \\
500, \text { III } \\
20 c, \mathrm{~V} \\
100, \mathrm{~V}\end{array}$ & $\begin{array}{l}4155.36 \\
4154.826 \\
4152.575 \\
4152.040 \\
4150.124\end{array}$ & $\begin{array}{l}24058.54 \\
24061.64 \\
24074.68 \\
24077.78 \\
24088.90\end{array}$ & 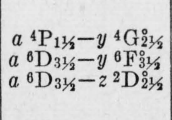 & $\begin{array}{l}6 \\
5\end{array}$ & $\begin{array}{l}0.214 \\
0.285\end{array}$ & $\begin{array}{l}0.680 \\
-- \\
--\end{array}$ & $\begin{array}{l}1.480 \\
0.97\end{array}$ & $\begin{array}{c}1.586 \\
(1.582)\end{array}$ & $\begin{array}{l}\text { 1. } 372 \\
1.297\end{array}$ \\
\hline 4 & 4149. 721 & 24091. 24 & $\left\{\begin{array}{l}a^{4} \mathrm{~F}_{413 / 2}-z^{2} \mathrm{G}_{31 / 2} \\
b^{4} \mathrm{D}_{11 / 2}-t^{4} \mathrm{G}_{21 / 2}\end{array}\right.$ & & & & & & \\
\hline 15 & 4148.735 & 24096. 96 & $\left\{\begin{array}{l}a^{2} \mathrm{G}_{31 / 2}-w^{4} \mathrm{~F}_{41 / 2} \\
a^{4} \mathrm{H}_{51 / 2}-y^{2} \mathrm{H}_{51 / 2}^{4}\end{array}\right.$ & 5 & & 0 & 1. $06^{*}$ & & \\
\hline$\underset{1}{25 c, \text { IV? }}$ & $\begin{array}{l}4148.321 \\
4147.184 \\
4146.598\end{array}$ & $\begin{array}{l}24099.37 \\
24105.97 \\
24109.38\end{array}$ & $\begin{array}{l}a^{2} \mathrm{G}_{41 / 2}-w^{4} \mathrm{G}_{51 / 2} \\
a^{4} \mathrm{H}_{31 / 2}-v^{4} \mathrm{G}_{5} 3 / 2 \\
a^{2} \mathrm{~F}_{31 / 2}-x^{4} \mathrm{H}_{31 / 2}^{3}\end{array}$ & 5 & & $h$ & 1. 571 & 1. 223 & (1. 160) \\
\hline $\begin{array}{l}10 \\
3 c \\
5 c \\
4 c \\
80 c, \mathrm{IV}\end{array}$ & $\begin{array}{l}4146.000 \\
4145.155 \\
4143.931 \\
4143.58 \\
4143.201\end{array}$ & $\begin{array}{l}24112.86 \\
24117.77 \\
24124.90 \\
24126.94 \\
24129.15\end{array}$ & $\begin{array}{l}a^{4} \mathrm{H}_{41 / 2}-v^{4} \mathrm{G}_{41 / 2}^{\circ} \\
a^{2} \mathrm{P}_{01 / 2}-y^{2} \mathrm{P}_{11 / 2}^{\circ} \\
a^{6} \mathrm{D}_{11 / 2}-z^{6} \mathrm{P}_{11 / 2}^{\circ}\end{array}$ & 6 & 0.519 & 0.779 & 2. 123 & $(0.984)$ & 2. 382 \\
\hline $\begin{array}{l}9 \\
4\end{array}$ & $\begin{array}{l}4142.243 \\
4141.32\end{array}$ & $\begin{array}{l}24134.73 \\
24140.11\end{array}$ & $a^{4} \mathrm{H}_{4 / 2}-u^{2} \mathrm{~F}_{31 / 2}^{0}$ & & & & & & \\
\hline $\begin{array}{c}400 c, \text { IV } \\
90 c, \text { IV } \\
6\end{array}$ & $\begin{array}{l}4139.702 \\
4139.430 \\
4139.088\end{array}$ & $\begin{array}{l}24149.54 \\
24151.13 \\
24153.12\end{array}$ & $\begin{array}{l}a^{6} \mathrm{D}_{41 / 2}-y^{6} \mathrm{~F}_{41 / 2}^{0} \\
a^{6} \mathrm{D}_{21 / 2}-z^{6} \mathrm{P}_{21 / 2}^{0} \\
b^{4} \mathrm{D}_{21 / 2}-s^{4} \mathrm{~F}_{31 / 2}^{0}\end{array}$ & $\begin{array}{l}6 \\
6\end{array}$ & $\begin{array}{l}0.139 \\
0.221\end{array}$ & $\begin{array}{l}0.681 \\
0.552\end{array}$ & $\begin{array}{l}1.506 \\
--\end{array}$ & $\begin{array}{c}1.576 \\
(1.652)\end{array}$ & $\begin{array}{l}1.437 \\
1.873\end{array}$ \\
\hline $\begin{array}{l}10 c \\
12 c\end{array}$ & $\begin{array}{l}4138.300 \\
4137.590\end{array}$ & $\begin{array}{l}24157.72 \\
24161.87\end{array}$ & $\begin{array}{l}b^{2} \mathrm{G}_{41 / 1}-r^{2} \mathrm{G}_{41 / 2}^{\circ} \\
a^{4} \mathrm{G}_{21 / 2}-u^{4} \mathrm{D}_{21 / 2}^{2}\end{array}$ & & & & & & \\
\hline $\begin{array}{c}200, \text { III } \\
4 \\
10\end{array}$ & $\begin{array}{l}4137.090 \\
4135.57 \\
4135.423\end{array}$ & $\begin{array}{l}24164.79 \\
24173.67 \\
24174.53\end{array}$ & $\begin{array}{l}a^{6} \mathrm{D}_{01 / 2}-y^{6} \mathrm{~F}_{11} \\
a^{2} \mathrm{H}_{41 / 2}-x^{2} \mathrm{H}_{51 / 2}^{5} \\
b^{4} \mathrm{~F}_{21 / 2}-s^{2} \mathrm{~F}_{21 / 2}^{2}\end{array}$ & 4 & 2. 197 & $\begin{array}{l}1.135 \\
0\end{array}$ & $\begin{array}{l}0.073 \\
0.846\end{array}$ & $\begin{array}{c}3.368 \\
(0.852)\end{array}$ & $\begin{array}{l}1.171 \\
0.840\end{array}$ \\
\hline $\begin{array}{l}6 \\
30, \mathrm{IV} \\
1 \\
5 h \\
3 c\end{array}$ & $\begin{array}{l}4134.985 \\
4134.592 \\
4134.33 \\
4133.40 \\
4132.175\end{array}$ & $\begin{array}{l}24177.09 \\
24179.39 \\
24180.92 \\
24186.36 \\
24193.53\end{array}$ & $\begin{array}{l}a^{4} \mathrm{H}_{31 / 2}-u^{2} \mathrm{~F}_{21 / 2}^{0} \\
a^{2} \mathrm{P}_{13 / 2}-v^{2} \mathrm{D}_{21 / 2}^{2}\end{array}$ & 4 & & 0 & 1. 144 & 1.171 & $(1.160)$ \\
\hline $\begin{array}{l}2 \\
3 \\
6\end{array}$ & $\begin{array}{l}4131.74 \\
4131.60 \\
4131.53\end{array}$ & $\begin{array}{l}24196.08 \\
24196.90 \\
24197.31\end{array}$ & $\begin{array}{l}a^{4} \mathrm{P}_{21 / 1}-x^{4} \mathrm{~F}_{31 / 2}^{0} \\
a^{4} \mathrm{G}_{33 / 1}-v^{2} \mathrm{G}_{41 / 3}^{1} \\
a^{4} \mathrm{G}_{31 / 2}-u^{4} \mathrm{D}_{31 / 2}^{0}\end{array}$ & - & & & & & \\
\hline $\begin{array}{l}150 c, \mathrm{~V} \\
100 c, \mathrm{~V}\end{array}$ & $\begin{array}{l}4129.931 \\
4129.430\end{array}$ & $\begin{array}{l}24206.67 \\
24209.61\end{array}$ & $a^{6} \mathrm{D}_{31 / 2}-z^{6} \mathrm{P}_{31 / 2}^{\circ}$ & 6 & 0.131 & 0.476 & $\begin{array}{l}\text { 1. } 08 \\
1.635\end{array}$ & 1.570 & 1. 701 \\
\hline $\begin{array}{c}4 \\
2 \\
1 \\
12 c \\
8 c\end{array}$ & $\begin{array}{l}4129.000 \\
4128.654 \\
4127.69 \\
4127.458 \\
4126.903\end{array}$ & $\begin{array}{l}24212.13 \\
24214.16 \\
24219.82 \\
24221.18 \\
24224.44\end{array}$ & $\begin{array}{l}a^{4} \mathrm{D}_{01 / 2}-x^{2} \mathrm{D}_{11 / 2}^{0} \\
a^{2} \mathrm{H}_{51 / 2}-y^{4} \mathrm{H}_{41 / 2}^{4} \\
a^{2} \mathrm{~F}_{21 / 2}-x^{4} \mathrm{H}^{3} 1 / 2 \\
b^{2} \mathrm{G}_{31 / 2}-411_{31 / 2}^{3}\end{array}$ & 6 & & $\begin{array}{l}0.06 \\
0\end{array}$ & $1 . \overline{12}$ & $(0.90)$ & 0.88 \\
\hline $\begin{array}{l}12 \\
20 c, \mathrm{~V}\end{array}$ & $\begin{array}{l}4125.573 \\
4125.243\end{array}$ & $\begin{array}{l}24232.24 \\
24234.18\end{array}$ & $\begin{array}{l}a^{4} \mathrm{~F}_{31 / 2}-y^{6} \mathrm{D}_{21 / 1}^{2} 2 \\
a^{4} \mathrm{H}_{31 / 2}-v^{4} \mathrm{G}_{41 / 2}^{2}\end{array}$ & & & & & & \\
\hline $\begin{array}{l}400, \text { III } \\
15, \mathrm{~V} \\
3 c\end{array}$ & $\begin{array}{l}4123.812 \\
4122.804 \\
4121.060\end{array}$ & $\begin{array}{l}24242.59 \\
24248.52 \\
24258.78\end{array}$ & $\begin{array}{l}a^{6} \mathrm{D}_{11 / 2}-y^{6} \mathrm{~F}_{21 / 2}^{5} \\
a^{4} \mathrm{H}_{51 / 2}-y^{2} \mathrm{H}_{41 / 2}^{0}\end{array}$ & $\begin{array}{l}4 \\
5\end{array}$ & $\begin{array}{l}0.558 \\
0.174\end{array}$ & $\begin{array}{l}0.282 \\
--\end{array}$ & $\begin{array}{c}0.468 \\
--\end{array}$ & $\begin{array}{l}1.863 \\
1.129\end{array}$ & $\begin{array}{l}1.305 \\
(0.955)\end{array}$ \\
\hline $\begin{array}{c}2 \\
50, \text { III } \\
5 \\
2 \\
6\end{array}$ & $\begin{array}{l}\text { 4117. } 824 \\
4116.895 \\
4116.401 \\
4116.274 \\
4114.155\end{array}$ & $\begin{array}{l}24277.85 \\
24283.32 \\
24286.24 \\
24286.99 \\
24299.50\end{array}$ & $\begin{array}{l}a^{2} \mathrm{D}_{11 / 2}-v^{4} \mathrm{D}_{11 / 2}^{0} \\
a^{6} \mathrm{D}_{01 / 2}-z^{6} \mathrm{P}_{11 / 2}^{0} \\
a^{4} \mathrm{~F}_{31 / 2}-y^{4} \mathrm{~F}^{0} 11 / 2 \\
b^{4} \mathrm{~F}_{21 / 2}-s^{2} \mathrm{~F}_{31 / 2}^{3} \\
a^{2} \mathrm{~F}_{33 / 2}-r^{2} \mathrm{~F}_{21 / 2}^{3}\end{array}$ & 4 & 0.943 & 0.473 & 1.910 & 3.325 & 2. 382 \\
\hline $\begin{array}{l}25, \mathrm{~V} \\
6 \\
1 \\
20 c, \mathrm{~V} \\
2\end{array}$ & $\begin{array}{l}4113.941 \\
4113.348 \\
4112.49 \\
4112.130 \\
4110.12\end{array}$ & $\begin{array}{l}24300.76 \\
24304.26 \\
24309.33 \\
24311.46 \\
24323.35\end{array}$ & $\begin{array}{l}a^{4} \mathrm{H}_{41 / 2}-y^{2} \mathrm{H}_{51 / 2}^{\circ} \\
b^{4} \mathrm{~F}_{31 / 2}-t^{4} \mathrm{~F}_{21 / 2}^{0} \\
a^{2} \mathrm{G}_{31 / 2}-u^{4} \mathrm{~F}_{41 / 2}^{6} \\
b^{2} \mathrm{H}_{41 / 2}-411_{31 / 3}^{0} \\
a^{4} \mathrm{G}_{31 / 2}-y^{4} \mathrm{H}_{31 / 2}^{0}\end{array}$ & 5 & 0.132 & 0.066 & 1.717 & $\begin{array}{l}0.990 \\
0.82 ?\end{array}$ & $\begin{array}{l}1.122 \\
(0.76)\end{array}$ \\
\hline
\end{tabular}


TABLE 4.-First spectrum of columbium (Cb I) - Continued

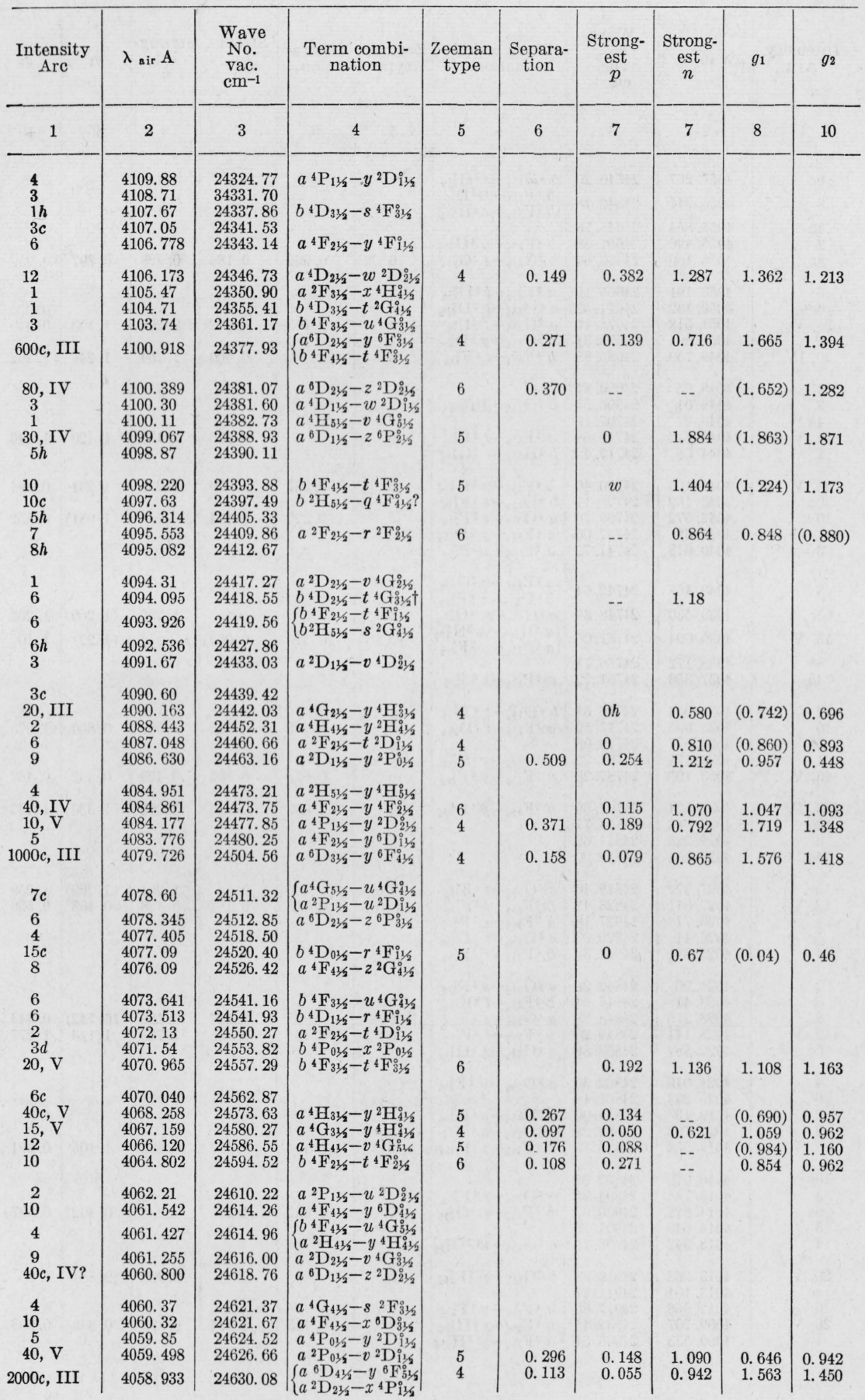


TABLE 4.-First spectrum of columbium (Cb I)-Continued

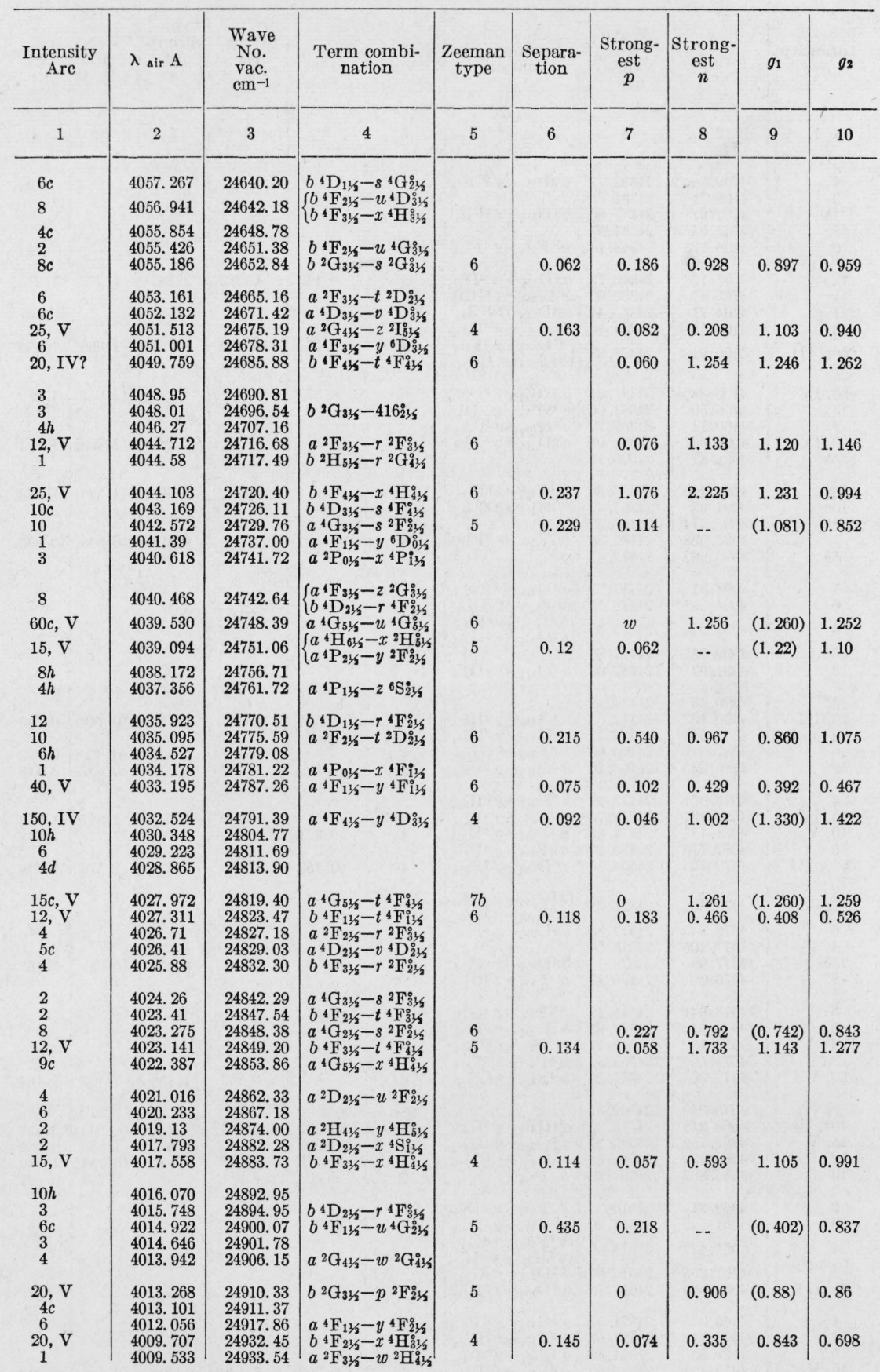


TABLE 4.-First spectrum of columbium (Cb I) - Continued

\begin{tabular}{|c|c|c|c|c|c|c|c|c|c|}
\hline $\begin{array}{c}\text { Intensity } \\
\text { Arc }\end{array}$ & $\lambda_{\text {air }} \mathrm{A}$ & $\begin{array}{l}\text { Wave } \\
\text { No. } \\
\text { vac. } \\
\mathrm{cm}^{-1}\end{array}$ & $\begin{array}{l}\text { Term combi- } \\
\text { nation }\end{array}$ & $\begin{array}{c}\text { Zeeman } \\
\text { type }\end{array}$ & $\begin{array}{l}\text { Separa- } \\
\text { tion }\end{array}$ & $\begin{array}{l}\text { Strong- } \\
\text { est } \\
p\end{array}$ & $\begin{array}{c}\text { Strong- } \\
\text { est } \\
n\end{array}$ & $g_{1}$ & $g_{2}$ \\
\hline 1 & 2 & 3 & 4 & 5 & 6 & 7 & 8 & 9 & 10 \\
\hline $\begin{array}{l}30, \mathrm{~V} \\
4 d \\
4 \\
2 \\
4 h\end{array}$ & $\begin{array}{l}4008.286 \\
4006.05 \\
4005.915 \\
4005.13 \\
4002.25\end{array}$ & $\begin{array}{l}24941.29 \\
24955.22 \\
24956.06 \\
24960.95 \\
24978.91\end{array}$ & $\begin{array}{l}a^{2} \mathrm{D}_{21 / 2}-u^{2} \mathrm{~F}_{31 / 2}^{0} \\
b^{2} \mathrm{G}_{31 / 2}-q^{4} \mathrm{~F}_{41 / 2}^{\circ} \\
a^{4} \mathrm{G}_{21 / 2}-s^{2} \mathrm{~F}_{31 / 2}^{0}\end{array}$ & 4 & 0.101 & 0.050 & 0.865 & 1. 218 & 1. 117 \\
\hline $\begin{array}{c}15 c, \mathrm{~V} \\
1 \\
1 \\
40, \mathrm{~V} \\
7\end{array}$ & $\begin{array}{l}4001.135 \\
4000.446 \\
3999.868 \\
3999.182 \\
3998.447\end{array}$ & $\begin{array}{l}24985.87 \\
24990.17 \\
24993.78 \\
24998.07 \\
25002.67\end{array}$ & $\begin{array}{l}a^{4} \mathrm{G}_{41 / 2}-u^{4} \mathrm{G}_{31 / 2}^{0} \\
a^{2} \mathrm{G}_{41 / 2}-w^{2} \mathrm{G}_{31 / 2}^{0} \\
a^{2} \mathrm{P}_{015}-x^{4} \mathrm{~S}_{11 / 2}^{0} \\
b^{4} \mathrm{~F}_{41 / 2}-x^{4} \mathrm{H}_{51 / 2}^{0} \\
b^{4} \mathrm{D}_{21 / 2}-q^{2} \mathrm{~F}_{31 / 2}^{0}\end{array}$ & 4 & 0.127 & 0.061 & 0.517 & 1. 216 & 1.089 \\
\hline $\begin{array}{l}4 \\
2 d \\
4 h \\
4 \\
1\end{array}$ & $\begin{array}{l}3998.23 \\
3997.780 \\
3997.377 \\
3997.157 \\
3996.95\end{array}$ & $\begin{array}{l}25004.02 \\
25006.83 \\
25009.36 \\
25010.74 \\
25012.03\end{array}$ & $\begin{array}{l}a^{4} \mathrm{H}_{41 / 2}-v^{2} \mathrm{G}_{31 / 2}^{0} \\
b^{4} \mathrm{D}_{21 / 2}-s^{4} \mathrm{D}_{21 / 2}^{0} \\
b^{2} \mathrm{G}_{31 / 2}-q^{4} \mathrm{~F}_{31 / 2}^{0}\end{array}$ & & & & & & \\
\hline $\begin{array}{c}3 \\
6 \\
1 \\
4 \\
40 c, V\end{array}$ & $\begin{array}{l}3995.588 \\
3994.420 \\
3993.36 \\
3992.165 \\
3991.677\end{array}$ & $\begin{array}{l}25020.55 \\
25027.87 \\
25034.52 \\
25042.01 \\
25045.07\end{array}$ & $\begin{array}{l}a^{2} \mathrm{H}_{51 / 2}-u^{4} \mathrm{G}_{41 / 2}^{0} \\
a^{4} \mathrm{H}_{51 / 2}-x^{2} \mathrm{H}_{51 / 2}^{0} \\
b^{4} \mathrm{D}_{11 / 2}-s^{4} \mathrm{D}_{21 / 2}^{2} \\
a^{2} \mathrm{~F}_{21 / 2}-x^{2} \mathrm{P}_{11 / 2}^{0}\end{array}$ & & 0.19 & 0.10 & & & \\
\hline $\begin{array}{c}1 \\
6 \\
20 c \\
4 c \\
6 c\end{array}$ & $\begin{array}{l}3990.926 \\
3990.665 \\
3988.158 \\
3986.17 \\
3985.18\end{array}$ & $\begin{array}{l}25049.78 \\
25051.42 \\
25067.17 \\
25079.67 \\
25085.90\end{array}$ & 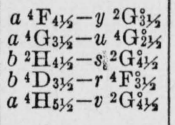 & & & $0 h$ & 1. 188 & (1. 04) & $1.34 ?$ \\
\hline $\begin{array}{l}4 \\
6\end{array}$ & $\begin{array}{l}3985.074 \\
3984.810\end{array}$ & $\begin{array}{l}25086.57 \\
25088.23\end{array}$ & $\begin{array}{l}a_{2}^{2} \mathrm{G}_{41 / 2}-z{ }^{2} \mathrm{H}_{41 / 3}^{\circ} \\
b^{4} \mathrm{D}_{21 / 2}-s^{4} \mathrm{G}_{31 / 2}^{\circ}\end{array}$ & & & & & & \\
\hline $10 c$ & 3982.055 & 25105.59 & $b^{4} \mathrm{~F}_{41 / 2}-y 2 \mathrm{I}_{51 / 2}^{\circ}$ & 4 & 0.269 & 0.137 & & (1. 224) & 0.955 \\
\hline $\begin{array}{l}60 c, \text { IV } \\
10, \mathrm{~V}\end{array}$ & $\begin{array}{l}3980.483 \\
3979.370\end{array}$ & $\begin{array}{l}25115.50 \\
25122.53\end{array}$ & $\begin{array}{c}a^{6} \mathrm{D}_{41 / 2}-y{ }^{4} \mathrm{~F}_{31 / 2}^{\circ} \\
b^{4} \mathrm{~F}_{21 / 2}-r \\
{ }^{2} \mathrm{~F}_{21 / 2}\end{array}$ & $\begin{array}{l}5 \\
6\end{array}$ & 0.304 & $\begin{array}{c}0.151 \\
w\end{array}$ & $\begin{array}{l}2.614 \\
0.864\end{array}$ & $\begin{array}{l}1.552 \\
(0.854)\end{array}$ & $\begin{array}{l}1.248 \\
0.874\end{array}$ \\
\hline $\begin{array}{l}4 \\
12, \mathrm{~V} \\
20 c, \mathrm{~V} \\
15 c, \mathrm{~V} \\
6 h\end{array}$ & $\begin{array}{l}3978.909 \\
3978.753 \\
3977.940 \\
3976.677 \\
3975.20\end{array}$ & $\begin{array}{l}25125.44 \\
25126.42 \\
25131.56 \\
25139.54 \\
25148.88\end{array}$ & 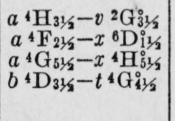 & & & & & & \\
\hline $\begin{array}{l}2 d \\
20 c \\
40 c,-\mathrm{V} \\
15, \mathrm{~V} \\
20, \mathrm{~V}\end{array}$ & $\begin{array}{l}3973.87 \\
3973.624 \\
3972.512 \\
3971.932 \\
3971.852\end{array}$ & $\begin{array}{l}25157.30 \\
25158.85 \\
25165.89 \\
25169.57 \\
25170.08\end{array}$ & $\begin{array}{l}a^{4} \mathrm{D}_{31 / 2}-v^{4} \mathrm{G}_{21 / 2} \\
b^{2} \mathrm{H}_{41 / 2}-p^{2} \mathrm{~F}_{31 / 2}^{0} \\
a^{4} \mathrm{G}_{11 / 2}-u^{4} \mathrm{G}_{41 / 2}^{0} \\
a^{4} \mathrm{~F}_{41 / 2}-x{ }^{6} \mathrm{D}_{41 / 2}^{0} \\
a^{4} \mathrm{G}_{21 / 2}-u^{4} \mathrm{G}_{21 / 2}^{0}\end{array}$ & & & $w$ & 1. 51 & & \\
\hline $\begin{array}{c}1 \\
12, \mathrm{~V} \\
4 h \\
5 c \\
7 c\end{array}$ & $\begin{array}{l}3971.35 \\
3970.650 \\
3970.10 \\
3968.465 \\
3968.32\end{array}$ & $\begin{array}{l}25173.26 \\
25177.70 \\
25181.18 \\
25191.55 \\
25192.48\end{array}$ & $\begin{array}{l}b^{4} \mathrm{~F}_{21 / 2}-t^{2} \mathrm{D}_{11 / 2}^{\circ} \\
a^{4} \mathrm{~F}_{31 / 5}-z^{2} \mathrm{G}_{11 / 2}^{\circ} \\
b^{4} \mathrm{D}_{31 / 5}-s^{4} \mathrm{D}_{21 / 2}^{\circ}\end{array}$ & 4 & 0.163 & 0.085 & -. & $(1.235)$ & 1. 072 \\
\hline $\begin{array}{c}1 \\
1 \\
150, \mathrm{~V} \\
25, \mathrm{~V} \\
40, \mathrm{IV}\end{array}$ & $\begin{array}{l}3967.61 \\
3967.441 \\
3966.246 \\
3966.094 \\
3965.692\end{array}$ & $\begin{array}{l}25196.99 \\
25198.06 \\
25205.65 \\
25206.61 \\
25209.17\end{array}$ & 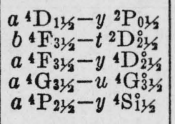 & $\begin{array}{l}4 \\
4\end{array}$ & 0.365 & 0.182 & $\begin{array}{l}1.016 \\
1.049\end{array}$ & $\begin{array}{c}(1.235) \\
1.596\end{array}$ & $\begin{array}{l}1.322 \\
1.961\end{array}$ \\
\hline $\begin{array}{l}7 \\
3 \\
3 h \\
4 \\
10 c, V\end{array}$ & $\begin{array}{l}3964.665 \\
3963.60 \\
3963.22 \\
3962.154 \\
3960.994\end{array}$ & $\begin{array}{l}25215.71 \\
25222.48 \\
25224.90 \\
25231.68 \\
25239.07\end{array}$ & $\begin{array}{l}a^{2} \mathrm{D}_{11 / 2}-v{ }^{4} \mathrm{G}_{21 / 2}^{\circ} \\
a^{4} \mathrm{H}_{41 / 2}-x{ }^{2} \mathrm{H}_{51 / 2}^{\circ} \\
a^{4} \mathrm{G}_{51 / 2}-y{ }^{2} \mathrm{I}_{51 / 2}^{0}\end{array}$ & & & . & & & \\
\hline $\begin{array}{c}4 \\
4 \\
2 \\
15 c, V \\
3\end{array}$ & $\begin{array}{l}3960.636 \\
3959.978 \\
3959.54 \\
3959.356 \\
3959.144\end{array}$ & $\begin{array}{l}25241.35 \\
25245.55 \\
25248.34 \\
25249.51 \\
25250.86\end{array}$ & 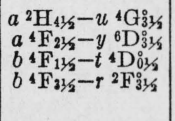 & 6 & & 0 & 1.116 & $(1.120)$ & 1. 112 \\
\hline $\begin{array}{l}7 c \\
1 \\
1 \\
7 \\
3 c\end{array}$ & $\begin{array}{l}3958.129 \\
3957.724 \\
3957.25 \\
3956.83 \\
3956.626\end{array}$ & $\begin{array}{l}25257.34 \\
25259.93 \\
25262.95 \\
25265.63 \\
25266.94\end{array}$ & 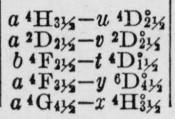 & & & & & & \\
\hline
\end{tabular}


544 Journal of Research of the National Bureau of Standards

TABLE 4.-First spectrum of columbium (Cb I)-Continued

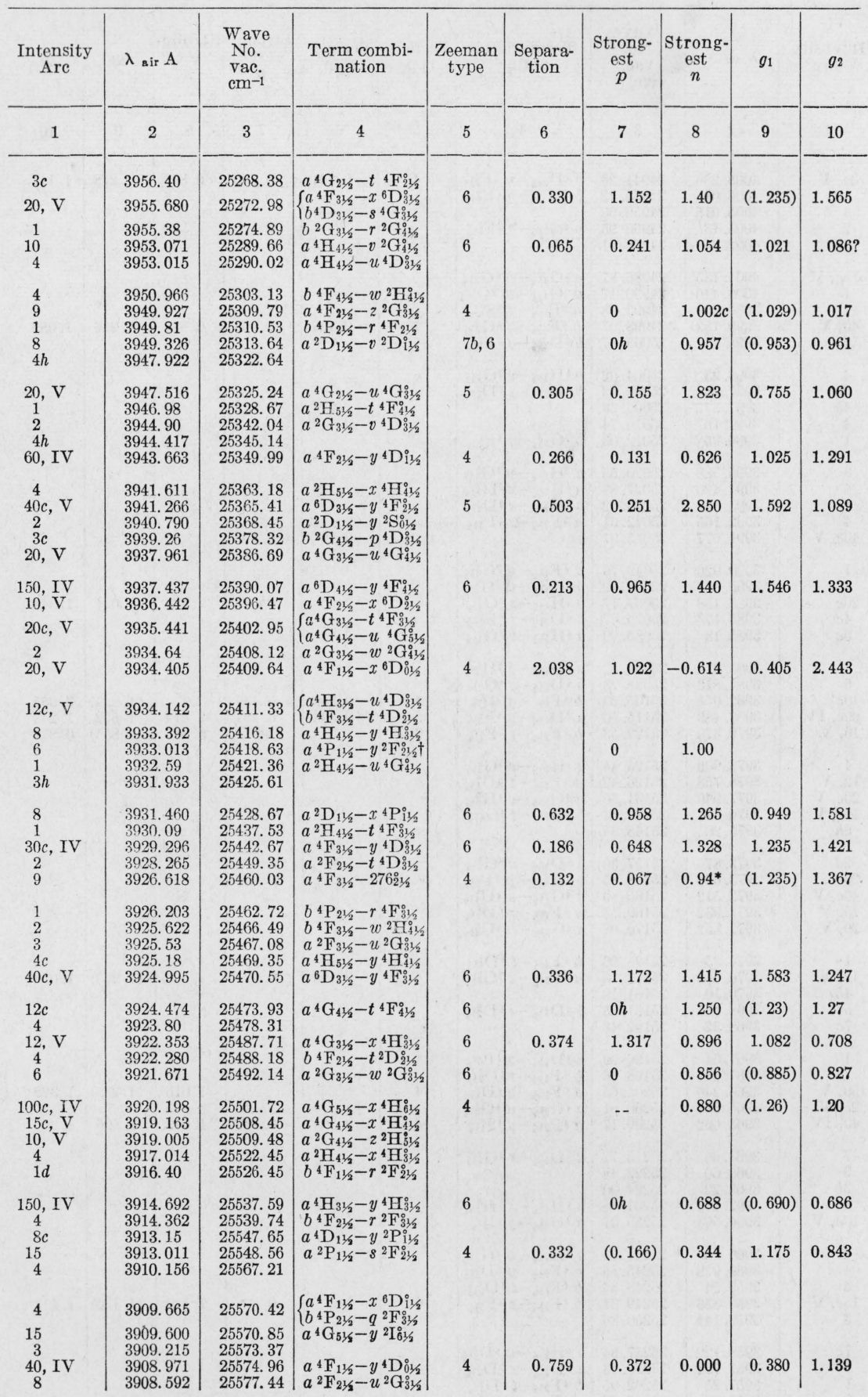


TABLE 4. -First spectrum of columbium (Cb I)-Continued

\begin{tabular}{|c|c|c|c|c|c|c|c|c|c|}
\hline $\begin{array}{l}\text { Intensity } \\
\text { Arc }\end{array}$ & $\lambda_{\text {air }} \mathrm{A}$ & $\begin{array}{l}\text { Wave } \\
\text { No. } \\
\text { vac. } \\
\text { cm-1 }^{-1}\end{array}$ & $\begin{array}{l}\text { Term combi- } \\
\text { nation }\end{array}$ & $\begin{array}{c}\text { Zeeman } \\
\text { type }\end{array}$ & $\begin{array}{c}\text { Separa- } \\
\text { tion }\end{array}$ & $\begin{array}{c}\text { Strong- } \\
\text { est } \\
p\end{array}$ & $\begin{array}{c}\text { Strong- } \\
\text { est } \\
n\end{array}$ & $g_{1}$ & $g_{2}$ \\
\hline 1 & 2 & 3 & 4 & 5 & 6 & 7 & 8 & 9 & 10 \\
\hline $\begin{array}{l}12, \mathrm{~V} \\
1 h\end{array}$ & $\begin{array}{l}3906.900 \\
3906.35\end{array}$ & $\begin{array}{l}25588.52 \\
35592.12\end{array}$ & $\begin{array}{l}a^{2} \mathrm{G}_{31 / 1}-z^{4} \mathrm{H}_{41 / 2}^{81} \\
a^{2} \mathrm{D}_{23 / 2}-u^{2} \mathrm{D}_{1 / 1 / 2}^{1}\end{array}$ & 4 & & $w$ & 0.566 & $(0.885)$ & 0.814 \\
\hline $\begin{array}{l}2 \\
30 c, \mathrm{~V}\end{array}$ & $\begin{array}{l}3904.83 \\
3904.188 \\
3902.829\end{array}$ & $\begin{array}{l}\begin{array}{l}25602.08 \\
25606.29 \\
25615.21\end{array}\end{array}$ & $\begin{array}{l}a{ }^{4} \mathrm{G}_{21 / 2}-x^{4} \mathrm{H}_{31 / 2}^{\circ} \\
b^{4} \mathrm{D}_{21 / 2}-w^{2} \mathrm{P}_{1 / 2}^{1}\end{array}$ & 4 & & $w$ & 0.634 & $(0.742)$ & 0.694 \\
\hline $\begin{array}{l}3 \\
5 \\
15 c, \mathrm{~V} \\
4 \\
20, \mathrm{~V}\end{array}$ & $\begin{array}{l}3901.88 \\
3900.83 \\
3899.24 \\
3898.94 \\
3898.563\end{array}$ & $\begin{array}{l}25621.44 \\
25628.34 \\
25638.79 \\
25640.76 \\
25643.24\end{array}$ & $\begin{array}{l}b^{4} \mathrm{D}_{01 / 2}-w^{2} \mathrm{P}_{11 / 2}^{0} \\
a^{4} \mathrm{~F}_{41 / 2}-y^{2} \mathrm{G}_{41 / 2}^{0} \\
b^{4} \mathrm{D}_{31 / 2}-s^{4} \mathrm{G}_{41 / 2}^{41} \\
a^{2} \mathrm{H}_{51 / 2}-x^{4} \mathrm{H}_{51 / 2} \\
a^{4} \mathrm{~F}_{31 / 2}-z^{2} \mathrm{~F}_{21 / 2}^{5}\end{array}$ & & & & & & \\
\hline & $\begin{array}{l}3898.01 \\
3896.621 \\
3896.00\end{array}$ & $\begin{array}{l}25646.88 \\
25656.02\end{array}$ & $b^{4} \mathrm{P}_{21 / 2}-s^{4} \mathrm{G}_{3 / 2}^{0}$ & & & & & & \\
\hline$\stackrel{4}{20,}, \mathrm{~V}$ & $\begin{array}{l}3896.00 \\
3895.895 \\
3894.99\end{array}$ & $\begin{array}{l}25660.11 \\
25660.80 \\
25666.76\end{array}$ & $\begin{array}{c}a^{2} \mathrm{D}_{1 / 1 / 2}-u{ }^{2} \mathrm{~F}_{21 / 2}^{8} \\
b^{4} \mathrm{~F}_{1 / 2}-t^{4} \mathrm{D}_{113 / 2}\end{array}$ & 4 & 0.094 & 0.047 & 0.727 & 0.962 & 0.868 \\
\hline 10 & $\begin{array}{l}3894.70 \\
3894.33\end{array}$ & $\begin{array}{l}25668.67 \\
25671.11\end{array}$ & 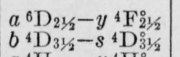 & & & & & & \\
\hline $\begin{array}{l}50, \mathrm{IV} \\
40, \mathrm{IV} \\
6\end{array}$ & $\begin{array}{l}3894.039 \\
3893.733 \\
3893.325\end{array}$ & $\begin{array}{l}25673.03 \\
25675.05 \\
25677.74\end{array}$ & 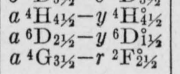 & $\begin{array}{l}6 \\
4\end{array}$ & 0.164 & $\begin{array}{l}0 h \\
0.080\end{array}$ & $\begin{array}{l}0.977 \\
1.413\end{array}$ & $\begin{array}{c}(0.984) \\
1.660\end{array}$ & $\begin{array}{l}0.970 \\
1.824\end{array}$ \\
\hline $\begin{array}{l}4 \\
4 \\
5\end{array}$ & $\begin{array}{l}3892.87 \\
3892.76 \\
3892.33\end{array}$ & $\begin{array}{l}25680.74 \\
25681.46 \\
25684.30\end{array}$ & $\begin{array}{l}a^{2} \mathrm{D}_{113 / 4}-x^{4} \mathrm{~S}_{1113}^{\circ} ? \\
a^{4} \mathrm{D}_{31 / 5}-u^{2} \mathrm{~F}_{31 / 2}^{1} ?\end{array}$ & & & & & & \\
\hline $\begin{array}{c}60, \mathrm{IV} \\
6\end{array}$ & $\begin{array}{l}389.756 \\
3891.302 \\
3890.75\end{array}$ & 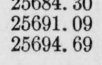 & $\begin{array}{l}a^{6} \mathrm{C}_{313}-y^{6} \mathrm{D}_{21 / 2}^{2} \\
a^{4} \mathrm{G}_{31 / 2}-t^{4} \mathrm{~F}_{41 / 2}^{2}\end{array}$ & 4 & & $w$ & 1. 519 & (1. 582) & 1.632 \\
\hline $\begin{array}{l}6 \\
8 c \\
1 \\
2 \\
1\end{array}$ & $\begin{array}{l}3889.800 \\
3889.64 \\
3889.440 \\
3888.68 \\
3888.49\end{array}$ & $\begin{array}{l}25701.01 \\
25702.06 \\
25703.39 \\
25708.41 \\
25709.67\end{array}$ & $\begin{array}{l}a^{4} \mathrm{~F}_{31 / 2}-y^{2} \mathrm{G}_{31 / 2}^{0} \\
a^{2} \mathrm{P}_{01 / 2}-u^{2} \mathrm{D}_{11 / 2}^{\circ} \\
b^{4} \mathrm{~F}_{41 / 2}-t^{4} \mathrm{D}_{31 / 2}^{31} \\
a^{4} \mathrm{D}_{21 / 2}-v^{2} \mathrm{D}_{11 / 2}\end{array}$ & & & & & & \\
\hline $\begin{array}{c}3 \\
6 h \\
20, \mathrm{~V} \\
100, \mathrm{IV} \\
150 \mathrm{c}, \mathrm{IV}\end{array}$ & $\begin{array}{l}3888.29 \\
3886.664 \\
3886.074 \\
3885.686 \\
3885.453\end{array}$ & $\begin{array}{l}25710.99 \\
25721.74 \\
25725.65 \\
25728.22 \\
25729.76\end{array}$ & 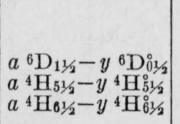 & $\begin{array}{l}4 \\
6 \\
6\end{array}$ & & $\begin{array}{c}0.718 \\
0 h \\
0 h\end{array}$ & $\begin{array}{l}\text { 1. } 184 \\
\text { 1. } 13 \\
1.225\end{array}$ & $\begin{array}{l}(1.863) \\
(1.12) \\
(1.22)\end{array}$ & $\begin{array}{l}3.220 \\
1.15 \\
1.23\end{array}$ \\
\hline $\begin{array}{l}3 \\
80, \mathrm{~V} ? \\
6 \\
8 \\
2\end{array}$ & $\begin{array}{l}3884.519 \\
3883.141 \\
3882.894 \\
3882.660 \\
3880.543\end{array}$ & $\begin{array}{l}25735.95 \\
25745.08 \\
25746.72 \\
25748.27 \\
25762.31\end{array}$ & $\begin{array}{r}a^{6} \mathrm{D}_{31 / 2}-y^{4} \mathrm{~F}_{41 / 2}^{\circ} \\
a^{2} \mathrm{H}_{51 / 2}-y^{2}{ }^{2} \mathrm{I}_{5 / 2}^{\circ}\end{array}$ & 4 & 0.244 & 0.123 & 0.480 & 1. 580 & 1.335 \\
\hline $\begin{array}{l}7 \\
20, \mathrm{~V} \\
40, \mathrm{~V} ?\end{array}$ & $\begin{array}{l}3879.644 \\
3878.965 \\
3878.817\end{array}$ & $\begin{array}{l}25768.29 \\
25772.79 \\
25773.78\end{array}$ & 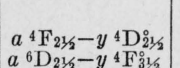 & 4 & 0.404 & 0.191 & 0.231 & 1645 & 1. 241 \\
\hline $60 c, \mathrm{~V}$ & $\begin{array}{l}3878.082 \\
3877.557\end{array}$ & $\begin{array}{l}25778.66 \\
25782.15\end{array}$ & $\begin{array}{l}a^{2}{ }^{2} \mathrm{D}_{21 / 1}-u^{4} \mathrm{D}_{11 / 2} \\
a^{6} \mathrm{D}_{14 / 2}-y \\
{ }^{6} \mathrm{D}_{3136}\end{array}$ & 5 & 0.292 & 0.150 & 0.201 & $(1.549)$ & 1.257 \\
\hline $20 c, \mathrm{~V}$ & $\begin{array}{l}3876.964 \\
3876.810\end{array}$ & $\begin{array}{l}25786.10 \\
25787.12\end{array}$ & $a^{4} \mathrm{G}_{41 / 2}-x^{4} \mathrm{H}_{51 / 2}^{0}$ & & & & & & \\
\hline $\begin{array}{l}20, \mathrm{~V} \\
5\end{array}$ & $\begin{array}{l}3875.77 \\
3875.697\end{array}$ & $\begin{array}{l}25794.04 \\
25794.52\end{array}$ & $\begin{array}{l}a^{4} \mathrm{H}_{11 / 2}-y^{4} \mathrm{D} \\
\left\{\begin{array}{l}{ }^{4} \mathrm{H}_{31 / 2}-y^{4} \mathrm{H} \\
a^{2} \mathrm{P}_{011}^{3}-u^{4} \mathrm{D}\end{array}\right.\end{array}$ & & & & & & \\
\hline $10 d$ & 3875.421 & 25796.36 & $a^{4} \mathrm{G}_{21 / 2}-r^{2} \mathrm{~F}_{21 / 2}^{01 / 2}$ & & & & & & \\
\hline $\begin{array}{l}1 \\
5 \\
5\end{array}$ & $\begin{array}{l}3875.10 \\
3873.288 \\
3871.76\end{array}$ & $\begin{array}{l}25798.50 \\
25810.57 \\
25820\end{array}$ & 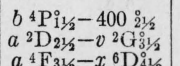 & 4 & 0.291 & -- & 0.198 & 1.216 & 0.925 \\
\hline $20 \mathrm{c}, \mathrm{V}$ & $\begin{array}{l}3870.665 \\
3871.188 \\
3870\end{array}$ & $\begin{array}{l}25824.57 \\
25828.06\end{array}$ & $\begin{array}{l}a^{4} \mathrm{D}_{21 / 2}-x^{4} 4 \mathrm{P}_{1 / 2} \\
a^{2} \mathrm{G}_{41 / 2}-v_{4}^{4} \mathrm{G}_{41 / 2}^{4}\end{array}$ & 4 & 0.232 & 0.114 & $1.026 w$ & 1. 363 & 1. 595 \\
\hline 1 & 3869.586 & 25835.26 & $a^{4} \mathrm{P}_{21 / 2}-x^{2} \mathrm{G}_{31 / 2}^{0}$ & & & & & & \\
\hline $9 d$ & 3868.829 & 25840.32 & & & & & & & \\
\hline 8 & 3868.570 & 25842.05 & $\left\{\begin{array}{l}a^{4} \mathrm{~A}_{21 / 2}-v^{4} \mathrm{~F}_{21 / 2}^{2} \\
a^{4} \mathrm{D}_{01 / 2}-y^{2} \mathrm{P}_{11 / 2}^{0}\end{array}\right.$ & & & & & & \\
\hline $\begin{array}{l}50 c, \mathrm{~V} ? \\
1\end{array}$ & $\begin{array}{l}3867.918 \\
3867.393\end{array}$ & $\begin{array}{l}25846.40 \\
25849.91\end{array}$ & 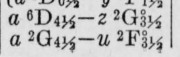 & 5us & 0.532 & 0.267 & 3. 409 & 1. 549 & 1.017 \\
\hline
\end{tabular}


TABLE 4.-First spectrum of columbium (Cb I)-Continued

\begin{tabular}{|c|c|c|c|c|c|c|c|c|c|}
\hline $\begin{array}{l}\text { Intensity } \\
\text { Arc }\end{array}$ & $\lambda_{\text {sir }} \mathbf{A}$ & $\begin{array}{l}\text { Wave } \\
\text { No. } \\
\text { vac. } \\
\text { cm-1 }^{-1}\end{array}$ & $\begin{array}{l}\text { Term combi- } \\
\text { nation }\end{array}$ & $\begin{array}{c}\text { Zeeman } \\
\text { type }\end{array}$ & $\begin{array}{c}\text { Separa- } \\
\text { tion }\end{array}$ & $\begin{array}{c}\text { Strong- } \\
\text { est } \\
p\end{array}$ & $\begin{array}{c}\text { Strong- } \\
\text { est } \\
n\end{array}$ & $g_{1}$ & $g_{2}$ \\
\hline 1 & 2 & 3 & 4 & 5 & 6 & 7 & 8 & 9 & 10 \\
\hline $\begin{array}{c}4 \\
10 d \\
1 \\
8 \\
1\end{array}$ & $\begin{array}{l}3866.24 \\
3865.041 \\
3864.698 \\
3864.364 \\
3864.111\end{array}$ & $\begin{array}{l}25857.62 \\
25865.64 \\
25867.94 \\
25870.17 \\
25871.87\end{array}$ & 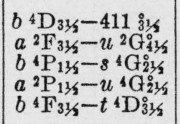 & & & & & & \\
\hline $\begin{array}{c}3 c \\
3 c \\
50, \mathrm{~V} ? \\
20 c, \mathrm{~V} \\
2\end{array}$ & $\begin{array}{l}3863.78 \\
3863.61 \\
3863.383 \\
3862.926 \\
3861.384\end{array}$ & $\begin{array}{l}25874.08 \\
25875.22 \\
25876.74 \\
25879.80 \\
25890.14\end{array}$ & 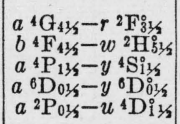 & 6 & 0.239 & 0.358 & 1.839 & 1.720 & 1.959 \\
\hline $\begin{array}{c}3 c \\
12 c \\
1\end{array}$ & $\begin{array}{l}3861.081 \\
3860.860 \\
3860.694\end{array}$ & $\begin{array}{l}25892.17 \\
25893.65 \\
25894.76\end{array}$ & 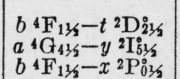 & & & & & & \\
\hline $\begin{array}{l}4 c \\
40, \mathrm{~V} ?\end{array}$ & $\begin{array}{l}3859.237 \\
3858.953\end{array}$ & $\begin{array}{l}25904.54 \\
25906.45\end{array}$ & $a 0_{1135}-y^{4} \mathrm{~F}_{21 / 5}^{\circ}$ & 4 & 0.784 & 0.392 & -0.099 & 1.861 & 1.077 \\
\hline $\begin{array}{r}1 \\
1 \\
6 \\
15 \\
12\end{array}$ & $\begin{array}{l}3858.162 \\
3858.00 \\
3856.683 \\
3855.456 \\
3855.146\end{array}$ & $\begin{array}{l}25911.76 \\
25912.84 \\
25921.70 \\
25929.94 \\
25932.03\end{array}$ & 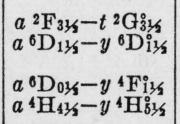 & 4 & & -. & 0.948 & (3. 323) & 0.475 \\
\hline $\begin{array}{l}9 \\
6 \\
4 c\end{array}$ & $\begin{array}{l}3854.698 \\
3854.127 \\
3853.59\end{array}$ & $\begin{array}{l}25935.04 \\
25938.88 \\
25942.50\end{array}$ & 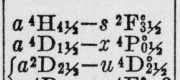 & & & & & & \\
\hline $\begin{array}{c}4 c \\
20 \\
4\end{array}$ & $\begin{array}{l}3853.59 \\
3853.388 \\
3853.096\end{array}$ & $\begin{array}{l}25942.50 \\
25943.86 \\
25945.83\end{array}$ & 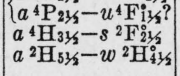 & 4 & 0.156 & 0.078 & 0.299 & 0.689 & 0.845 \\
\hline $6 c$ & 3849.744 & 25968.41 & $\left\{\begin{array}{l}a^{2} \mathrm{P}_{11 / 6}-t t^{4} \mathrm{~F}_{13 / 6}^{0} \\
a^{4} \mathrm{P}_{23 / 3}-w^{2} \mathrm{~F}_{213}^{2}\end{array}\right.$ & & & & & & \\
\hline $\begin{array}{l}1 \\
40, \mathrm{~V}\end{array}$ & $\begin{array}{l}3846.00 \\
3845.900\end{array}$ & $\begin{array}{l}25993.69 \\
25994.37\end{array}$ & 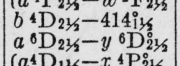 & 6 & & 0.080 & 1. 627 & 1. 643 & 1.611 \\
\hline $\begin{array}{l}4 c \\
2 c\end{array}$ & $\begin{array}{l}3845.307 \\
3845.079\end{array}$ & $\begin{array}{l}25998.38 \\
25999.92\end{array}$ & 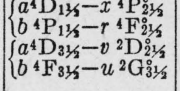 & & & & & & \\
\hline $\begin{array}{c}12 \\
10 c \\
4 \\
4 \\
20, \mathrm{~V}\end{array}$ & $\begin{array}{l}3844.090 \\
3843.927 \\
3843.615 \\
3843.453 \\
3842.709\end{array}$ & $\begin{array}{l}26006.61 \\
26007.71 \\
26009.82 \\
26010.92 \\
26015.96\end{array}$ & 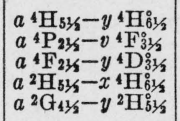 & 5 & 0.248 & 0.115 & 1. 200 & $\begin{array}{l}(1.596) \\
(1.103)\end{array}$ & 1.348 \\
\hline 30 & 3841.820 & 26021.97 & 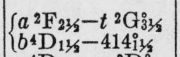 & 5 & & $0 h$ & 1.004 & $(0.860)$ & 0.924 \\
\hline $\begin{array}{c}2 \\
4 \\
12 c \\
5 c\end{array}$ & $\begin{array}{l}3838.072 \\
3837.995 \\
3837.077 \\
3836.738\end{array}$ & $\begin{array}{l}26047.39 \\
26047.91 \\
26054.14 \\
26056.44\end{array}$ & 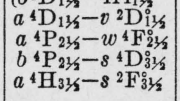 & 4 & 0.191 & 0.094 & 0.884 & 1. 558 & 1.367 \\
\hline $20, \mathrm{~V}$ & $\begin{array}{l}3836.452 \\
3836.260\end{array}$ & $\begin{array}{l}26058.38 \\
26059.69\end{array}$ & $a^{2} \mathrm{D}_{13 / 3}-v^{2} \mathrm{D}_{21 / 2}^{2}$ & 5 & 0.198 & 0. 098 & 1.456 & 0.952 & 1. 160 \\
\hline $\begin{array}{l}40, \mathrm{IV} ? \\
2 \\
6\end{array}$ & $\begin{array}{l}3835.176 \\
3834.575 \\
3833.940\end{array}$ & $\begin{array}{l}26067.05 \\
26071.14 \\
26075.46\end{array}$ & 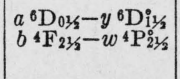 & 4 & 1. 508 & 0.754 & 1.061 & 3. 322 & 1.815 \\
\hline $\begin{array}{c}2 \\
10 c \\
1 \\
6 d \\
2\end{array}$ & $\begin{array}{l}3833.769 \\
3833.257 \\
3832.134 \\
3831.198 \\
3831.074\end{array}$ & $\begin{array}{l}26076.62 \\
26080.10 \\
26087.75 \\
26094.12 \\
26094.96\end{array}$ & 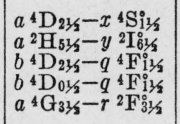 & $7 b, 4 ?$ & & 0 & 1.11 & (1.10) & 1.10 \\
\hline $\begin{array}{r}1 \\
10\end{array}$ & $\begin{array}{l}3830.820 \\
3830.006\end{array}$ & $\begin{array}{l}26096.69 \\
26102.24\end{array}$ & 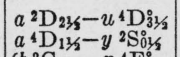 & 4 & 0.880 & 0.440 & 0.761 & 1. 201 & 2. 081 \\
\hline 4 & 3829.659 & 26104. 61 & $\left\{\begin{array}{l}b^{2} \mathrm{G}_{313}-p{ }^{4} \mathrm{~F}_{21 / 1}^{0} \\
b^{4} \mathrm{D}_{23}-8^{2} \mathrm{G}_{316}^{2}\end{array}\right.$ & & & & & & \\
\hline $\begin{array}{l}4 \\
6\end{array}$ & $\begin{array}{l}3828.045 \\
3827.090\end{array}$ & $\begin{array}{l}26115.61 \\
26122.13\end{array}$ & $\begin{array}{l}b^{4} \mathrm{D}_{131}-q^{4} \mathrm{~F}_{11 / 3} \\
a^{4} \mathrm{P}_{21 / 2}-w^{2} \mathrm{~F}_{31 / 2}^{6}\end{array}$ & & & & & & \\
\hline $\begin{array}{r}12 \\
3 \\
5\end{array}$ & $\begin{array}{l}3827.015 \\
3826.574\end{array}$ & $\begin{array}{l}26122.64 \\
26125.65\end{array}$ & 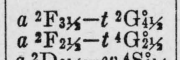 & 4 & 0.104 & $w$ & 0.648 & (1.130) & 1.044 \\
\hline $\begin{array}{c}5 \\
100, \mathrm{IV} \\
3\end{array}$ & $\begin{array}{l}3825.410 \\
3824.882 \\
3823.255\end{array}$ & $\begin{array}{l}26133.60 \\
26137.21 \\
26148.33\end{array}$ & 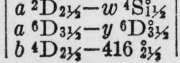 & 6 & 0.333 & 1. 171 & 1.415 & 1. 581 & 1. 248 \\
\hline
\end{tabular}


TABLE 4.-First spectrum of columbium (Cb I)-Continued

\begin{tabular}{|c|c|c|c|c|c|c|c|c|c|}
\hline $\begin{array}{c}\text { Intensity } \\
\text { Are }\end{array}$ & $\lambda_{\text {air }} \mathbf{A}$ & $\begin{array}{l}\text { Wave } \\
\text { No. } \\
\text { vac. } \\
\mathrm{cm}^{-1}\end{array}$ & $\begin{array}{l}\text { Term combi- } \\
\text { nation }\end{array}$ & $\begin{array}{c}\text { Zeeman } \\
\text { type }\end{array}$ & $\begin{array}{l}\text { Separa- } \\
\text { tion }\end{array}$ & $\begin{array}{c}\text { Strong- } \\
\text { est } \\
p\end{array}$ & $\begin{array}{c}\text { Strong. } \\
\text { est } \\
n\end{array}$ & $g_{1}$ & $g_{2}$ \\
\hline 1 & 2 & 3 & 4 & 5 & 6 & 7 & 7 & 8 & 10 \\
\hline $\begin{array}{c}6 \\
4 \\
12 c \\
40, \mathrm{~V} \\
6 c\end{array}$ & $\begin{array}{l}3823.135 \\
3822.956 \\
3821.201 \\
3819.148 \\
3818.190\end{array}$ & $\begin{array}{l}26149.15 \\
26150.38 \\
26162.38 \\
26176.45 \\
26183.01\end{array}$ & 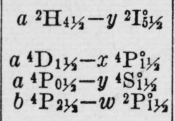 & $\begin{array}{l}6 \\
4\end{array}$ & $\begin{array}{l}0.390 \\
0.692\end{array}$ & $\begin{array}{l}0.585 \\
--\end{array}$ & $\begin{array}{l}1.393 \\
1.604\end{array}$ & $\begin{array}{l}1.198 \\
2.642\end{array}$ & $\begin{array}{l}\text { 1. } 588 \\
\text { 1. } 950\end{array}$ \\
\hline $\begin{array}{l}3 c \\
15 \\
60 c, \text { IV? } \\
4 \\
5\end{array}$ & $\begin{array}{l}3816.645 \\
3816.342 \\
3815.507 \\
3814.188 \\
3813.470\end{array}$ & $\begin{array}{l}26193.62 \\
26195.69 \\
26201.43 \\
26210.49 \\
26215.42\end{array}$ & $\begin{array}{l}a^{4} \mathrm{~F}_{21 / 2}-y^{4} \mathrm{P}_{11 / 2}^{0} \\
a^{6} \mathrm{D}_{31 / 2}-z^{2} \mathrm{G}_{31 / 2}^{0} \\
a^{4} \mathrm{~F}_{21 / 2}-z^{2} \mathrm{~F}_{21 / 3}^{3}\end{array}$ & $\begin{array}{l}4 \\
6\end{array}$ & $\begin{array}{l}0.624 \\
0.567\end{array}$ & $\begin{array}{l}0.314 \\
1.989\end{array}$ & $\begin{array}{l}0.090 \\
1.296\end{array}$ & $\begin{array}{l}1.026 \\
1.580\end{array}$ & $\begin{array}{l}1.650 \\
1.013\end{array}$ \\
\hline $\begin{array}{l}5 c \\
50, \text { III? } \\
3 c \\
80 c, \mathrm{~V} \\
2 c\end{array}$ & $\begin{array}{l}3813.01 \\
3811.035 \\
3810.88 \\
3810.50 \\
3807.95\end{array}$ & $\begin{array}{l}26218.59 \\
26232.17 \\
26233.24 \\
26235.85 \\
26253.43\end{array}$ & 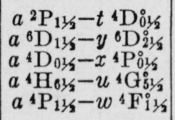 & $\begin{array}{l}4 \\
4 ?\end{array}$ & 0.242 & $\begin{array}{l}0.125 \\
w\end{array}$ & $\begin{array}{l}1.248 \\
1.18\end{array}$ & $\begin{array}{l}1.852 \\
(1.22)\end{array}$ & $\begin{array}{l}1.610 \\
1.23\end{array}$ \\
\hline $\begin{array}{l}1 \\
10 \\
20, \mathrm{~V} \\
40, \mathrm{Cb} \text { II } \\
10 c\end{array}$ & $\begin{array}{l}3807.485 \\
3806.631 \\
3806.196 \\
3804.733 \\
3804.204\end{array}$ & $\begin{array}{l}26256.63 \\
26262.52 \\
26265.52 \\
26275.62 \\
26279.27\end{array}$ & $\begin{array}{l}a^{4} \mathrm{G}_{31 / 2}-t^{4} \mathrm{D}_{21 / 5}^{\circ} \\
b{ }^{4} \mathrm{P}_{11 / 5}-s^{4} \mathrm{D}_{21 / 5}^{\circ} \\
a^{4} \mathrm{H}_{31 / 5}-u^{4} \mathrm{G}_{21 / 5}^{\circ} \\
a^{4} \mathrm{H}_{51 / 5}-u^{4} \mathrm{G}_{41 / 2}^{0} \\
b^{4} \mathrm{D}_{21 / 5}-q^{4} \mathrm{~F}_{21 / 2}^{0}\end{array}$ & $\begin{array}{l}4 \\
4 \\
4\end{array}$ & $\begin{array}{l}0.376 \\
0.147\end{array}$ & $\begin{array}{l}0.19 ? \\
0.074 \\
w\end{array}$ & $\begin{array}{l}0.725 \\
0.324 \\
1.01\end{array}$ & $\begin{array}{r}1.665 \\
0.691 \\
(1.12)\end{array}$ & $\begin{array}{l}1.289 \\
0.838 \\
1.17\end{array}$ \\
\hline $\begin{array}{c}100, \text { IV? } \\
1 \\
400 r, \text { IV? } \\
6 \\
5\end{array}$ & $\begin{array}{l}3803.879 \\
3803.65 \\
3802.928 \\
3802.636 \\
3802.555\end{array}$ & $\begin{array}{l}26281.52 \\
26283.10 \\
26288.09 \\
26290.11 \\
26290.67\end{array}$ & $\begin{array}{c}a^{6} \mathrm{D}_{41 / 5}-z^{2} \mathrm{G}_{41 / 5}^{\circ} \\
a^{4} \mathrm{P}_{21 / 5}-w^{4} \mathrm{D}_{11 / 2}^{\circ} \\
a^{6} \mathrm{D}_{31 / 5}-x^{6} \mathrm{D}_{21 / 5}^{\circ} \\
b^{6} \mathrm{~F}_{21 / 5}-u^{2} \mathrm{G}_{31 / 5}^{\circ}\end{array}$ & $\begin{array}{l}6 \\
4\end{array}$ & 0.477 & $\begin{array}{l}2.135 \\
w\end{array}$ & $\begin{array}{l}1.313 \\
1.514\end{array}$ & $\begin{array}{c}1.551 \\
(1.582)\end{array}$ & $\begin{array}{l}1.074 \\
1.636\end{array}$ \\
\hline $\begin{array}{l}4 \\
20, \mathrm{~V} \\
20, \mathrm{~V} \\
5 \\
5\end{array}$ & $\begin{array}{l}3802.480 \\
3801.302 \\
3800.941 \\
3800.197 \\
3799.486\end{array}$ & $\begin{array}{l}26291.19 \\
26299.34 \\
26301.84 \\
26306.98 \\
26311.91\end{array}$ & $\begin{array}{l}a^{4} \mathrm{~F}_{31 / 2}-y{ }^{4} \mathrm{P}_{21 / 2}^{0} \\
a^{4} \mathrm{H}_{41 / 2}-u^{4} \mathrm{G}_{31 / 2}^{0} \\
a^{2} \mathrm{G}_{41 / 2}-v^{4} \mathrm{G}_{51 / 2}^{0} \\
b^{4} \mathrm{D}_{11 / 2}-q^{4} \mathrm{~F}_{21 / 2}^{0} \\
a^{4} \mathrm{G}_{31 / 2}-w^{2} \mathrm{H}^{0} 11 / 2\end{array}$ & & & & & & . \\
\hline $\begin{array}{l}300 r, \mathrm{III} \\
15 c \\
12 \\
20 c, \mathrm{~V} \\
30 h, \mathrm{~V}\end{array}$ & $\begin{array}{l}3798.127 \\
3796.850 \\
3796.599 \\
3796.440 \\
3795.543\end{array}$ & $\begin{array}{l}26321.32 \\
26330.17 \\
26331.92 \\
26333.02 \\
26339.24\end{array}$ & $\begin{array}{l}a^{0} \mathrm{D}_{21 / 5}-x{ }^{6} \mathrm{D}_{11 / 2}^{0} \\
a^{2} \mathrm{~F}_{31 / 2}-v^{2} \mathrm{H}_{41 / 2}^{0} \\
a^{4} \mathrm{~F}_{21 / 2}-z^{2} \mathrm{P}_{11 / 2}^{1} \\
b^{4} \mathrm{D}_{31 / 2}-416_{21 / 2}^{0}\end{array}$ & & & & & & \\
\hline $\begin{array}{c}2 \\
15 \\
5 c \\
7 c \\
8\end{array}$ & $\begin{array}{l}3795.182 \\
3794.476 \\
3793.727 \\
3791.999 \\
3791.446\end{array}$ & $\begin{array}{l}26341.75 \\
26346.65 \\
26351.85 \\
26363.86 \\
26367.70\end{array}$ & $\begin{array}{l}a^{4} \mathrm{D}_{01 / 2}-v^{2} \mathrm{D}_{11 / 2}^{0} \\
a^{2} \mathrm{H}_{43 / 2}-w^{2} \mathrm{H}_{41 / 2}^{0} \\
a^{2} \mathrm{G}_{31 / 2}-u^{2} \mathrm{~F}_{31 / 2}^{0} \\
a^{4} \mathrm{H}_{31 / 2}-t^{4} \mathrm{~F}_{21 / 2}^{0} \\
a^{4} \mathrm{P}_{21 / 2}-w^{4} \mathrm{~F}_{31 / 2}^{0}\end{array}$ & 6 & & 0.049 & 0.929 & 0.923 & 0.936 \\
\hline $\begin{array}{l}300 r, \text { III } \\
200 r, \text { III } \\
20 \\
3 c \\
4\end{array}$ & $\begin{array}{l}3791.209 \\
3790.138 \\
3789.502 \\
3788.69 \\
3788.188\end{array}$ & $\begin{array}{l}26369.35 \\
26376.80 \\
26381.23 \\
26386.88 \\
26390.38\end{array}$ & $\begin{array}{l}a^{6} \mathrm{D}_{41 / 2}-y{ }^{6} \mathrm{D}_{11 / 2}^{0} \\
a^{6} \mathrm{D}_{41 / 2}-x{ }^{6} \mathrm{D}_{31 / 2}^{0} \\
a^{4} \mathrm{~F}_{31 / 2}-z^{2} \mathrm{~F}_{31 / 2}^{3} \\
a^{2} \mathrm{D}_{11 / 2}-u^{2} \mathrm{D}_{11 / 2}^{0}\end{array}$ & $\begin{array}{l}6 \\
4\end{array}$ & 0.120 & $\begin{array}{l}0.552 \\
0 h \\
0\end{array}$ & $\begin{array}{l}1.483 \\
1.517 \\
0.682\end{array}$ & $\begin{array}{c}1.543 \\
(1.549)\end{array}$ & $\begin{array}{l}1.422 \\
1.567\end{array}$ \\
\hline $\begin{array}{c}15 \\
8 c \\
150, \text { IV? } \\
10, \mathrm{~V} \\
6 c\end{array}$ & $\begin{array}{l}3787.480 \\
3787.280 \\
3787.064 \\
3786.227 \\
3786.10\end{array}$ & $\begin{array}{l}26395.31 \\
26396.70 \\
26398.21 \\
26404.05 \\
26404.93\end{array}$ & 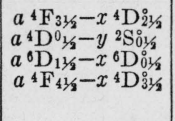 & 4 & 0.577 & 0.288 & 1. 572 & 1. 860 & 2. 437 \\
\hline $\begin{array}{c}3 \\
1 \\
20 c \\
1 \\
5 c\end{array}$ & $\begin{array}{l}3785.384 \\
3784.73 \\
3783.844 \\
3782.71 \\
3782.40\end{array}$ & $\begin{array}{l}26409.92 \\
26414.49 \\
26420.67 \\
26428.59 \\
26430.76\end{array}$ & $\begin{array}{l}a^{4} \mathrm{P}_{13 / 2}-v{ }^{4} \mathrm{~F}_{11 / 2}^{0} \\
a^{4} \mathrm{D}_{11 / 2}-x{ }^{4} \mathrm{~S}_{11 / 2}^{0} \\
a^{4} \mathrm{H}_{31 / 2}-u^{4} \mathrm{G}_{31 / 2} \\
a^{4} \mathrm{G}_{21 / 2}-x^{2} \mathrm{P}_{11 / 2}^{0} \\
a^{4} \mathrm{D}_{31 / 2}-u^{2} \mathrm{D}_{21 / 2}^{0}\end{array}$ & 6 & 0.367 & 1.285 & 0.872 & 1.055 & 0.688 \\
\hline $\begin{array}{l}80, \text { III } \\
4 \\
1 \\
5 \\
15 c\end{array}$ & $\begin{array}{l}3781.017 \\
3779.22 \\
3779.03 \\
3778.675 \\
3777.670\end{array}$ & $\begin{array}{l}26440.43 \\
26453.00 \\
26454.33 \\
26456.82 \\
26463.85\end{array}$ & $\begin{array}{l}a^{6} \mathrm{D}_{21 / 2}-y^{6} \mathrm{D}_{31 / 2}^{\circ} \\
a^{4} \mathrm{D}_{21 / 2}-v^{2} \mathrm{D}_{21 / 2}^{\circ} \\
a^{4} \mathrm{D}_{01 / 2}-x^{4} \mathrm{P}_{11 / 2}^{0} \\
b^{4} \mathrm{D}_{31 / 2}-q^{4} \mathrm{~F}_{21 / 2}^{0}\end{array}$ & $4 u s$ & 0.403 & 0.201 & 0.235 & 1. 647 & 1. 243 \\
\hline $\begin{array}{l}8 \\
7 \\
6 c \\
20, V \\
2\end{array}$ & $\begin{array}{l}3777.277 \\
3776.605 \\
3776.157 \\
3775.449 \\
3775.16\end{array}$ & $\begin{array}{l}26466.61 \\
26471.32 \\
26474.46 \\
26479.42 \\
26481.45\end{array}$ & $\begin{array}{l}a^{4} \mathrm{~F}_{41 / 2}-z^{4} \mathrm{H}_{31 / 2}^{0} \\
a^{4} \mathrm{~F}_{11 / 2}-276_{21 / 2}^{0} \\
b^{1} \mathrm{~F}_{41 / 2}-s^{4} \mathrm{~F}_{31 / 2}^{0} \\
a^{4} \mathrm{H}_{41 / 2}-u^{4} \mathrm{G}_{41 / 2}^{\circ} \\
a^{2} \mathrm{D}_{13 / 2}-u^{4} \mathrm{D}_{01 / 5}^{0}\end{array}$ & 6 & 0.204 & 0.914 & 1.088 & 0.986 & 1. 190 \\
\hline
\end{tabular}


548 Journal of Research of the National Bureau of Standards

TABLE 4.-First spectrum of columbium (Cb I) - Continued

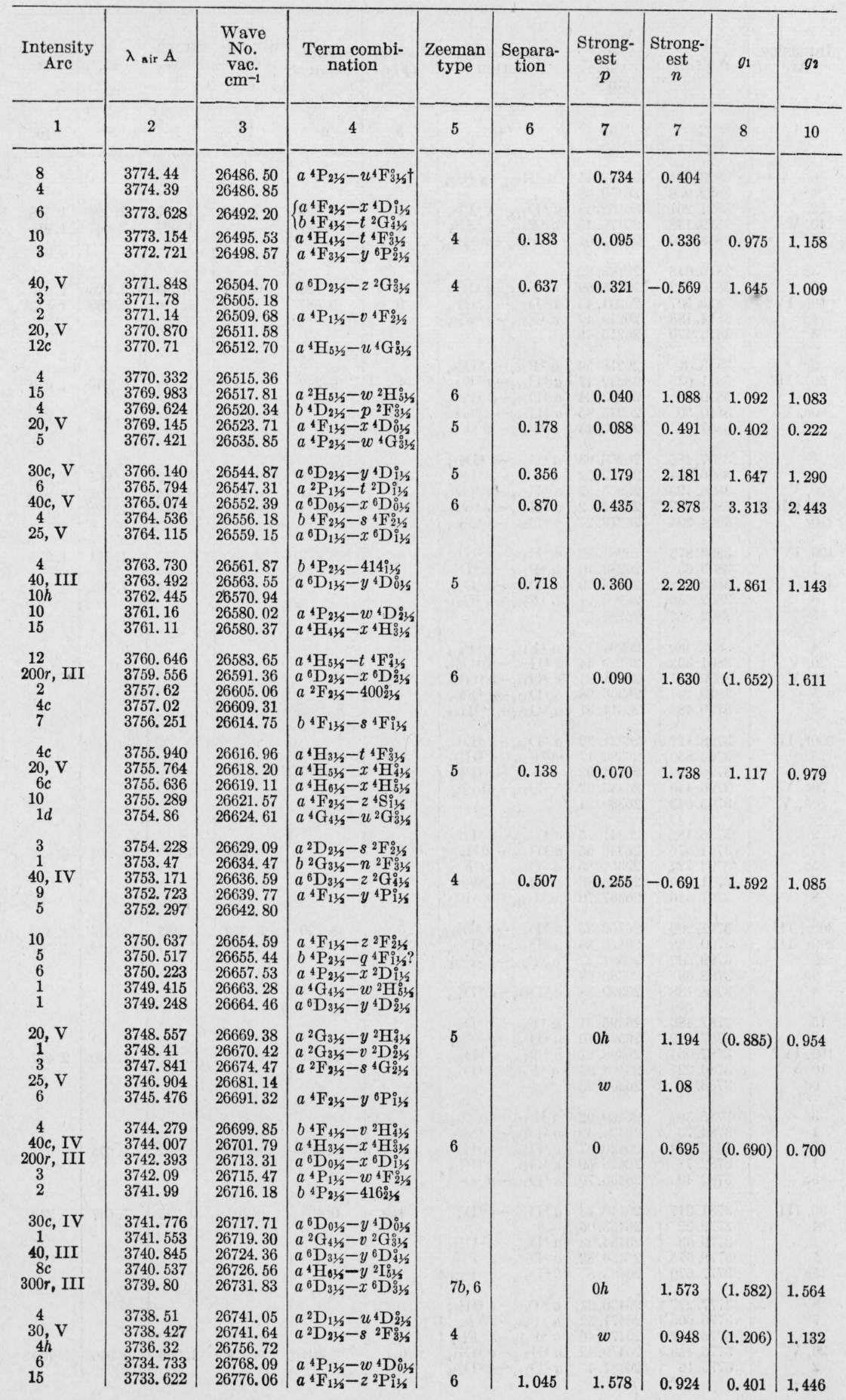


TABLE 4.-First spectrum of columbium (Cb I)-Continued

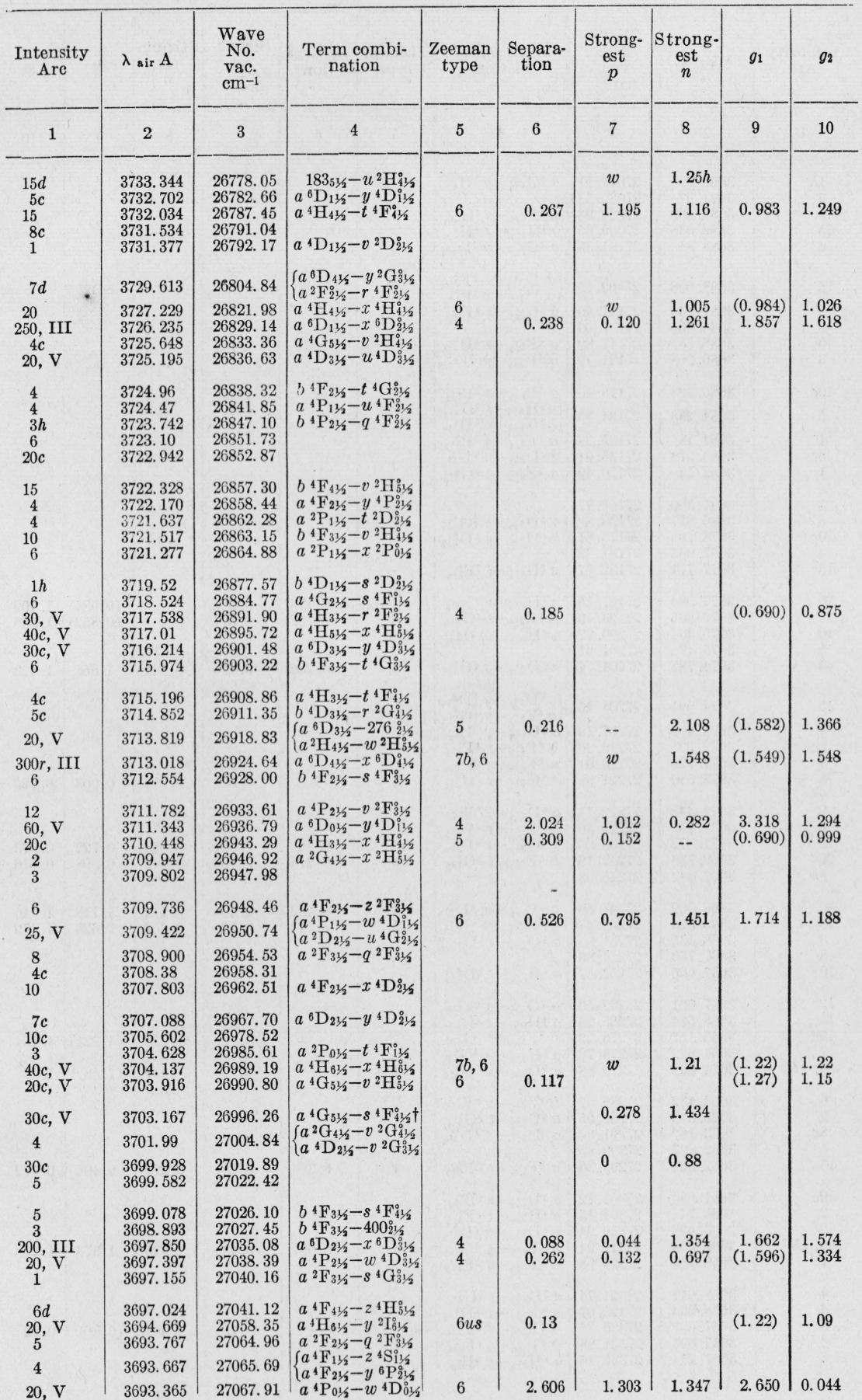


TABLE 4.-First spectrum of columbium (Cb I)-Continued

\begin{tabular}{|c|c|c|c|c|c|c|c|c|c|}
\hline $\begin{array}{l}\text { Intensity } \\
\text { Arc }\end{array}$ & $\lambda_{\text {air }} \mathbf{A}$ & $\begin{array}{l}\text { Wave } \\
\text { No. } \\
\text { vac. } \\
\mathrm{cm}^{-1}\end{array}$ & $\begin{array}{l}\text { Term combi- } \\
\text { nation }\end{array}$ & $\begin{array}{c}\text { Zeeman } \\
\text { type }\end{array}$ & $\begin{array}{l}\text { Separa- } \\
\text { tion }\end{array}$ & $\begin{array}{l}\text { Strong- } \\
\text { est } \\
p\end{array}$ & $\begin{array}{c}\text { Strong. } \\
\text { est } \\
n\end{array}$ & $g_{1}$ & $g_{2}$ \\
\hline 1 & 2 & 3 & 4 & 5 & 6 & 7 & 8 & 9 & 10 \\
\hline $\begin{array}{r}1 h \\
3 \\
5 \\
15 \\
4\end{array}$ & $\begin{array}{l}3692.518 \\
3691.37 \\
3689.409 \\
3689.038 \\
3688.81\end{array}$ & $\begin{array}{l}27074.11 \\
27082.53 \\
27096.93 \\
27099.65 \\
27101.33\end{array}$ & 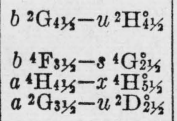 & & & & & & \\
\hline 15 & 3688.698 & 27102.15 & $\left\{\begin{array}{l}a^{6} \mathrm{D}_{31 / 2}-z^{2} \mathrm{~F}_{21 / 3}^{\circ} \\
a^{6} \mathrm{BS}_{23 / 3}-x^{2} \mathrm{P}^{0}\end{array}\right.$ & & & & & . & \\
\hline $\begin{array}{r}8 \\
3 \\
10 \\
3\end{array}$ & $\begin{array}{l}3687.440 \\
3686.665 \\
3686.557 \\
3686.068\end{array}$ & $\begin{array}{l}27111.40 \\
27117.09 \\
27117.89 \\
27121.49\end{array}$ & $\begin{array}{l}a^{4} \mathrm{G}_{33 / 5}-s^{4} \mathrm{~F}_{21 / 5}^{0} \\
a^{4} \mathrm{~F}_{33 / 5}-z^{4} \mathrm{H}_{31 / 2}^{0} \\
a^{4} \mathrm{P}_{21 / 5}-w^{2} \mathrm{D}_{135}^{0}\end{array}$ & & & & & & \\
\hline $10 c$ & 3685.128 & 27128.40 & $a^{2} \mathrm{P}_{11 / 2}-x^{2} \mathrm{P}_{1 / 43}^{0} ?$ & & & & & & \\
\hline 5 & 3684.253 & 27134.85 & $\left\{\begin{array}{l}a^{2} \mathrm{H}_{53 / 2}-t^{2} \mathrm{G}_{41 / 2}^{0} \\
b^{2} \mathrm{G}\end{array}\right.$ & & & & & & \\
\hline $\begin{array}{l}1 \\
5 c \\
1\end{array}$ & $\begin{array}{l}3684.18 \\
3683.973 \\
3682.13\end{array}$ & $\begin{array}{l}27135.39 \\
27136.91 \\
27150.49\end{array}$ & 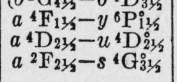 & & & & & & \\
\hline $\begin{array}{c}3 \\
5 \\
10 c \\
4 \\
15\end{array}$ & $\begin{array}{l}3681.360 \\
3680.857 \\
3678.70 \\
3677.905 \\
3677.771\end{array}$ & $\begin{array}{l}27156.17 \\
27159.88 \\
27175.81 \\
27181.68 \\
27182.67\end{array}$ & $\begin{array}{l}a^{6} \mathrm{D}_{31 / 2}-y^{2} \mathrm{G}_{31 / 5}^{\circ} \\
b^{4} \mathrm{D}_{21 / 2}-q^{4} \mathrm{D}_{21 / 2}^{0} \\
a^{2} \mathrm{G}_{43 / 2}-t^{2} \mathrm{~F}_{31 / 2}^{\circ}\end{array}$ & & & & & & \\
\hline $\begin{array}{r}20 \\
20 \\
10 \\
5\end{array}$ & $\begin{array}{l}3677.084 \\
3676.307 \\
3675.304 \\
3675.17\end{array}$ & $\begin{array}{l}27187.75 \\
27193.50 \\
27200.92 \\
27201.91\end{array}$ & $\begin{array}{l}a^{4} \mathrm{H}_{43 / 4}-r^{2} \mathrm{~F}_{31 / 5}^{0} \\
b^{4} \mathrm{~F}_{21 / 5}-t^{4} \mathrm{G}_{31 / 2}^{0} \\
a^{4} \mathrm{H}_{51 / 3}-w^{2} \mathrm{H}_{41 / 2}^{4}\end{array}$ & $\begin{array}{l}4 \\
5\end{array}$ & $\begin{array}{l}0.125 \\
0.252\end{array}$ & $\begin{array}{l}0.065 \\
0.377\end{array}$ & $\begin{array}{l}-- \\
--\end{array}$ & $\begin{array}{l}(0.984) \\
(0.852)\end{array}$ & $\begin{array}{l}1.109 \\
1.106\end{array}$ \\
\hline $40 c, \mathrm{~V}$ & 3674.787 & 27204. 74 & $a^{6} \mathrm{D}_{23 / 2}-y^{4} \mathrm{D}_{31 / 2}^{0}$ & 4 & 0.232 & 0.116 & 0.841 & 1.655 & 1. 423 \\
\hline 15 & 3674.691 & 27205.45 & $\left\{\begin{array}{l}a^{6} \mathrm{D}_{113}-y^{4} \mathrm{D}_{21 / 2}^{0} \\
a^{4} \mathrm{~F}_{313}-y^{4} \mathrm{G}^{2}\end{array}\right.$ & & & & & & \\
\hline $\begin{array}{l}4 \\
8 c \\
4 \\
8\end{array}$ & $\begin{array}{l}3674.469 \\
3673.227 \\
3672.726 \\
3672.580\end{array}$ & $\begin{array}{l}27207.10 \\
27216.30 \\
27220.01 \\
27221.09\end{array}$ & 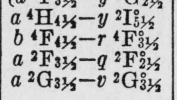 & 6 & & 0.065 & 0.914 & 0.903 & 0.925 \\
\hline $\begin{array}{c}9 \\
6 \\
12 \\
20 d \\
8 h\end{array}$ & $\begin{array}{l}3672.443 \\
3671.735 \\
3671.372 \\
3669.736 \\
3669.347\end{array}$ & $\begin{array}{l}27222.11 \\
27227.36 \\
27230.05 \\
27242.19 \\
27245.08\end{array}$ & 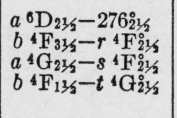 & $\begin{array}{l}6 \\
5\end{array}$ & $\begin{array}{l}0.251 \\
0.218\end{array}$ & $\begin{array}{l}0.602 \\
0.109\end{array}$ & $\begin{array}{l}0.856 \\
0.943\end{array}$ & $\begin{array}{l}0.722 \\
0.398\end{array}$ & $\begin{array}{l}0.973 \\
0.616\end{array}$ \\
\hline $\begin{array}{l}40, \mathrm{~V} \\
20, \mathrm{~V} \\
3 \\
8 \\
10 c\end{array}$ & $\begin{array}{l}3669.009 \\
3668.626 \\
3668.435 \\
3667.760 \\
3667.665\end{array}$ & $\begin{array}{l}27247.58 \\
27250.43 \\
27251.85 \\
27256.86 \\
27257.57\end{array}$ & 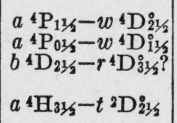 & $\begin{array}{l}4 \\
5\end{array}$ & $\begin{array}{l}0.402 \\
1.444\end{array}$ & $\begin{array}{l}0.196 \\
0.722\end{array}$ & $\begin{array}{l}0.712 \\
0.457\end{array}$ & $\begin{array}{l}1.718 \\
2.623\end{array}$ & $\begin{array}{l}1.316 \\
1.179\end{array}$ \\
\hline $\begin{array}{l}12 c \\
15 \\
10 d \\
7 \\
80, \text { III }\end{array}$ & $\begin{array}{l}3667.001 \\
3666.534 \\
3665.157 \\
3664.822 \\
3664.692\end{array}$ & $\begin{array}{l}27262.50 \\
27265.98 \\
27276.22 \\
27278.72 \\
27279.68\end{array}$ & 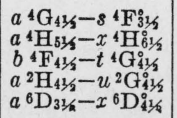 & $\begin{array}{l}7 b, 6 \\
4\end{array}$ & & $\begin{array}{l}0 \\
w\end{array}$ & $\begin{array}{l}1.229 \\
1.437\end{array}$ & $\begin{array}{l}(1.224) \\
(1.582)\end{array}$ & $\begin{array}{l}1.234 \\
1.528\end{array}$ \\
\hline $\begin{array}{c}6 \\
2 \\
8 c \\
5 \\
15\end{array}$ & $\begin{array}{l}3663.432 \\
3663.313 \\
3663.167 \\
3662.927 \\
3662.051\end{array}$ & $\begin{array}{l}27289.07 \\
27289.95 \\
27291.04 \\
27292.83 \\
27299.36\end{array}$ & $\begin{array}{l}b{ }^{4} \mathrm{~F}_{21 / 3}-r \\
a^{4} \mathrm{G}_{31 / 2} \mathrm{~F}_{13 / 2}^{0}-t^{2} \mathrm{G}_{31 / 2}^{0} \\
a^{4} \mathrm{D}_{21 / 2}-u^{4} \mathrm{D}_{31 / 2}^{\circ} \\
a^{4} \mathrm{~F}_{11 / 2}-z^{2} \mathrm{P}_{01 / 2}^{0}\end{array}$ & 4 & 0.366 & 0.181 & 0.223 & 0.406 & 0.772 \\
\hline $\begin{array}{c}10 c \\
2 \\
2 \\
100, \mathrm{IV} \\
7 d\end{array}$ & $\begin{array}{l}3661.680 \\
3660.74 \\
3660.498 \\
3660.364 \\
3658.750\end{array}$ & $\begin{array}{l}27302.12 \\
27309.13 \\
27310.94 \\
27311.94 \\
27323.98\end{array}$ & 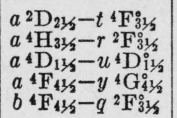 & 6 & 0.097 & 0.436 & 1.325 & 1. 373 & 1. 276 \\
\hline $\begin{array}{l}4 \\
4 \\
8 \\
4 \\
4\end{array}$ & $\begin{array}{l}3658.648 \\
3658.604 \\
3657.897 \\
3657.693 \\
3657.254\end{array}$ & $\begin{array}{l}27324.74 \\
27325.07 \\
27330.36 \\
27331.88 \\
27335.16\end{array}$ & 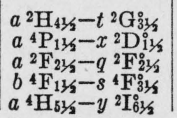 & 6 & & 0.15 & $0.69^{*}$ & $(0.86)$ & 0.92 \\
\hline
\end{tabular}


TABLE 4.-First spectrum of columbium (Cb I) - Continued

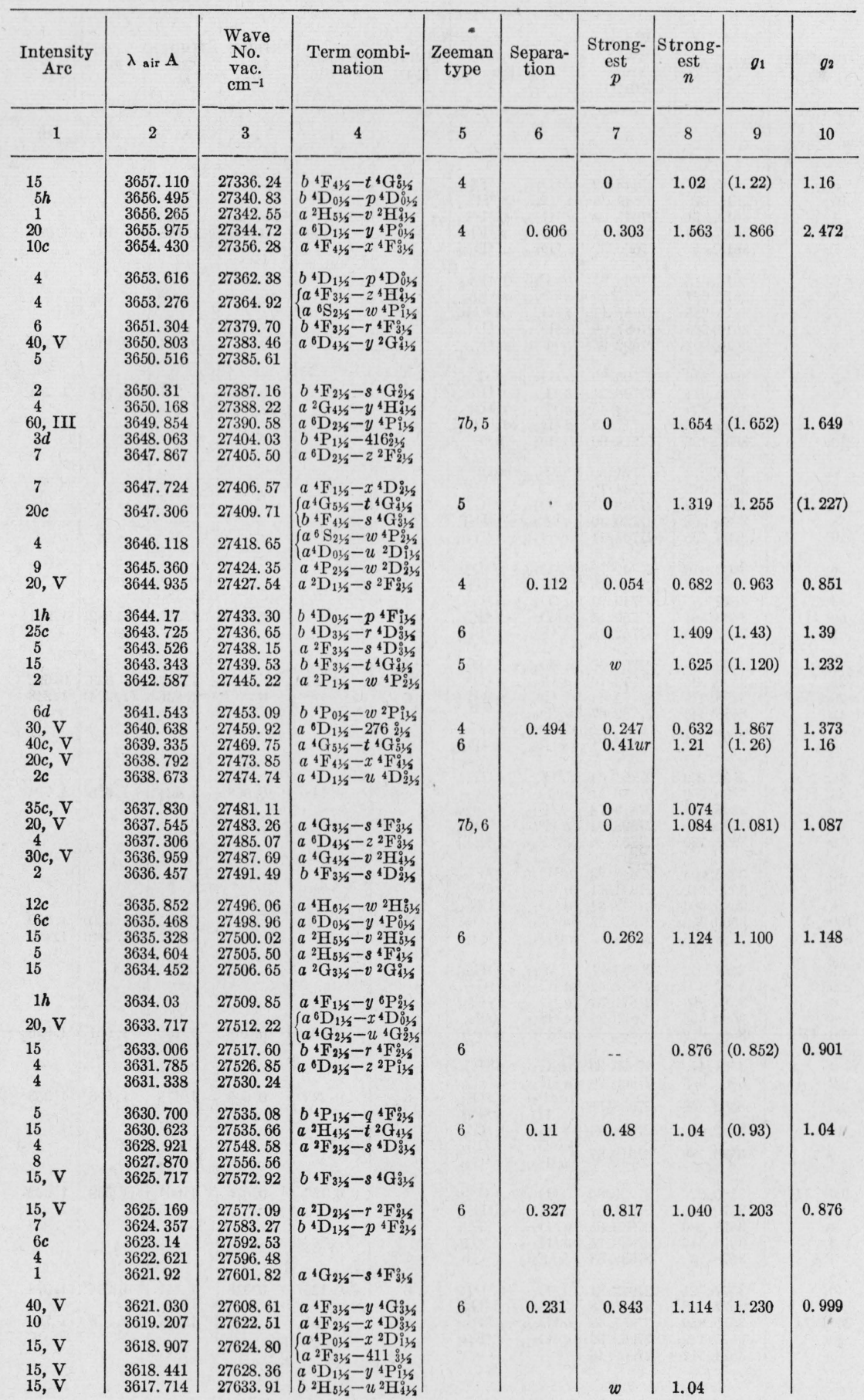


TABLE 4.-First spectrum of columbium (Cb I) - Continued

\begin{tabular}{|c|c|c|c|c|c|c|c|c|c|}
\hline $\begin{array}{c}\text { Intensity } \\
\text { Arc }\end{array}$ & $\lambda_{\text {air }} \mathrm{A}$ & $\begin{array}{l}\text { Wave } \\
\text { No. } \\
\text { vac. } \\
\mathrm{cm}^{-1}\end{array}$ & $\underset{\text { nation }}{\text { Term combi- }}$ & $\begin{array}{c}\text { Zeeman } \\
\text { type }\end{array}$ & $\begin{array}{c}\text { Separa- } \\
\text { tion }\end{array}$ & $\begin{array}{l}\text { Strong- } \\
\text { est } \\
p\end{array}$ & $\begin{array}{c}\text { Strong- } \\
\text { est } \\
n\end{array}$ & $g_{1}$ & $g_{2}$ \\
\hline 1 & 2 & 3 & 4 & 5 & 6 & 7 & 8 & 9 & 10 \\
\hline $\begin{array}{c}6 \\
10 \\
4 \\
30 c, \mathrm{~V} \\
7 c\end{array}$ & $\begin{array}{l}3616.497 \\
3616.216 \\
3615.971 \\
3615.500 \\
3613.45\end{array}$ & $\begin{array}{l}27643.22 \\
27645.36 \\
27647.24 \\
27650.84 \\
27666.52\end{array}$ & 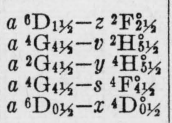 & 6 & 0.100 & 0.450 & 1. 148 & 1. 198 & 1.098 \\
\hline $\begin{array}{l}9 \\
9 \\
7 c \\
7 \\
8 c\end{array}$ & $\begin{array}{l}3613.013 \\
3612.657 \\
3611.285 \\
3610.765 \\
3610.002\end{array}$ & $\begin{array}{l}27669.87 \\
27672.59 \\
27683.11 \\
27687.09 \\
27692.95\end{array}$ & 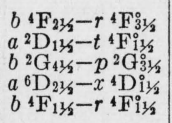 & 6 & & 0 & 0.424 & $(0.402)$ & 0.446 \\
\hline $\begin{array}{c}9 \\
10 d \\
7 \\
8 \\
10 d\end{array}$ & $\begin{array}{l}3608.316 \\
3608.013 \\
3607.33 \\
3606.806 \\
3606.492\end{array}$ & $\begin{array}{l}27705.89 \\
27708.21 \\
27713.46 \\
27717.48 \\
27719.90\end{array}$ & 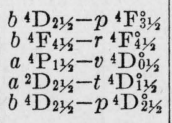 & 6 & & 0.099 & 1. 231 & 1. 217 & 1. 244 \\
\hline $\begin{array}{c}8 c \\
7 c \\
8 c \\
8 \\
10\end{array}$ & $\begin{array}{l}3606.272 \\
3604.643 \\
3604.072 \\
3603.962 \\
3603.435\end{array}$ & $\begin{array}{l}27721.59 \\
27734.12 \\
27738.51 \\
27739.36 \\
27743.41\end{array}$ & $\begin{array}{l}b^{4} \mathrm{~F}_{11 / 2}-400_{21 / 2}^{\circ} \\
a^{6} \mathrm{D}_{31 / 2}-y^{2} \mathrm{G}_{41 / 2}^{0} \\
a^{2} \mathrm{P}_{01 / 2}-t^{2} \mathrm{D}_{11 / 2}^{11} \\
a^{2} \mathrm{H}_{41 / 2}-v^{2} \mathrm{H}_{41 / 2}^{11}\end{array}$ & & & & & & \\
\hline $\begin{array}{c}6 \\
2 \\
3 \\
60, \mathrm{III} \\
2\end{array}$ & $\begin{array}{l}3602.876 \\
3602.74 \\
3602.68 \\
3602.561 \\
3602.219\end{array}$ & $\begin{array}{l}27747.72 \\
27748.76 \\
27749.23 \\
27750.14 \\
27752.78\end{array}$ & $\begin{array}{l}b^{4} \mathrm{D}_{11 / 2}-p^{4} \mathrm{D}_{21 / 2}^{2} \\
a^{4} \mathrm{G}_{331 / 2}-t^{4} \mathrm{G}_{31 / 2}^{\circ} \\
a^{2} \mathrm{D}_{11 / 1}-u^{4} \mathrm{G}_{21 / 2}^{2} \\
a^{6} \mathrm{D}_{31 / 2}-y^{4} \mathrm{P}_{21 / 2}^{2} \\
b^{4} \mathrm{~F}_{31 / 2}-q^{2} \mathrm{~F}_{21 / 2}^{2}\end{array}$ & 4 & & 0 & 1. 558 & (1. 582) & 1. 601 \\
\hline $\begin{array}{l}3 \\
20, \mathrm{~V} \\
20 c, \mathrm{~V} \\
12 d\end{array}$ & $\begin{array}{l}3600.678 \\
3599.635 \\
3599.279 \\
3598.343\end{array}$ & $\begin{array}{l}27764.66 \\
27772.70 \\
27775.45 \\
27782.67\end{array}$ & $\begin{array}{l}a^{6} \mathrm{D}_{113 / 2}-z^{2} \mathrm{P}_{11 / 2}^{0} \\
a^{4} \mathrm{~F}_{21 / 2}-y^{4} \mathrm{G}_{21 / 2} \\
b^{4} \mathrm{~F}_{41 / 2}-s^{4} \mathrm{G}_{41 / 2}^{2} \\
a^{6} \mathrm{D}_{01 / 2}-y^{4} \mathrm{P}_{11 / 2}^{0}\end{array}$ & 6 & 0.331 & $\begin{array}{l}0.829 \\
0\end{array}$ & $\begin{array}{l}\text { 1. } 715 \\
1.236\end{array}$ & $\begin{array}{c}1.023 \\
(1.224)\end{array}$ & $\begin{array}{l}0.692 \\
1.248\end{array}$ \\
\hline 8 & 3597.514 & 27789.07 & $\left\{\begin{array}{l}a^{4} \mathrm{D}_{31 / 2}-t^{4} \mathrm{~F}_{21 / 2}^{0} \\
a^{4} \mathrm{P}_{11 / 2}-w^{2} \mathrm{D}_{11 / 2}\end{array}\right.$ & & & & & & \\
\hline $\begin{array}{l}16 c \\
80, \text { III } \\
2 \\
3 \\
9\end{array}$ & $\begin{array}{l}3597.260 \\
3593.966 \\
3593.543 \\
3592.360 \\
3591.790\end{array}$ & $\begin{array}{l}27791.04 \\
27816.51 \\
27819.78 \\
27828.94 \\
27833.36\end{array}$ & $\begin{array}{l}b^{4} \mathrm{~F}_{11 / 2}-s^{4} \mathrm{G}_{21 / 2}^{0} \\
a^{6} \mathrm{D}_{21 / 2}-z^{4} \mathrm{~S}_{11 / 2}^{0} \\
b^{4} \mathrm{P}_{21 / 2}-r{ }^{4} \mathrm{D}_{31 / 2}^{1} \\
a^{2} \mathrm{P}_{013}-t t^{4} \mathrm{D}_{11 / 2}^{1} \\
a^{4} \mathrm{~F}_{31 / 2}-x^{4} \mathrm{~F}_{21 / 2}^{2}\end{array}$ & $4 u s$ & 0.144 & 0.068 & 1. 434 & 1.650 & 1. 794 \\
\hline $\begin{array}{l}10 \\
10 c \\
8 \\
100 c, \mathrm{~V} \\
100, \mathrm{IV} ?\end{array}$ & $\begin{array}{l}3590.904 \\
3590.712 \\
3589.960 \\
3589.356 \\
3589.106\end{array}$ & $\begin{array}{l}27840.23 \\
27841.71 \\
27847.54 \\
27852.23 \\
27854.17\end{array}$ & $\begin{array}{l}a^{6} \mathrm{D}_{31 / 2}-z^{2} \mathrm{~F}_{31 / 2}^{0} \\
a^{4} \mathrm{G}_{531 / 2}-r^{4} \mathrm{~F}_{41 / 2}^{0} \\
a^{2} \mathrm{D}_{11 / 2}-t^{4} \mathrm{~F}_{21 / 2}^{0} \\
a^{4} \mathrm{~F}_{413 / 2}-y^{4} \mathrm{G}_{51 / 2}^{5} \\
a^{6} \mathrm{D}_{31 / 2}-x^{4} \mathrm{D}_{21 / 2}{ }^{2}\end{array}$ & $\begin{array}{l}4 \\
5\end{array}$ & & $\begin{array}{l}w \\
--\end{array}$ & $\begin{array}{l}0.835 \\
1.882\end{array}$ & $\begin{array}{l}(1.330) \\
(1.582)\end{array}$ & $\begin{array}{l}1.240 \\
1.462\end{array}$ \\
\hline $\begin{array}{c}2 \\
9 d \\
6 \\
4 \\
100, \text { IV? }\end{array}$ & $\begin{array}{l}3587.942 \\
3587.400 \\
3586.869 \\
3586.695 \\
3584.972\end{array}$ & $\begin{array}{l}27863.21 \\
27867.42 \\
27871.54 \\
27872.89 \\
27886.29\end{array}$ & 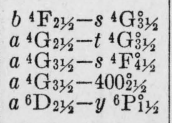 & 4 & 0.325 & 0.163 & 1.162 & 1. 651 & 1. 976 \\
\hline $\begin{array}{l}4 \\
2\end{array}$ & $\begin{array}{l}3584.47 \\
3583.095\end{array}$ & $\begin{array}{l}27890.20 \\
27900.90\end{array}$ & $\begin{array}{l}a^{2} \mathrm{G}_{31 / 2}-y^{4} \mathrm{H}_{41 / 2}^{\circ} \\
a^{2} \mathrm{H}_{41 / 2}-v^{2} \mathrm{H}_{51 / 2}^{10}\end{array}$ & & & & & & \\
\hline 25 & 3582.369 & 27906.55 & $\left\{\begin{array}{l}a^{4} \mathrm{P}_{21 / 3}-v^{4} \mathrm{D}_{21 / 2}^{2} \\
a^{2} \mathrm{H}_{42}-s^{4} \mathrm{~F}_{4112}^{0}\end{array}\right.$ & 6 & 0.243 & 0.609 & 1.475 & 1. 596 & 1. 353 \\
\hline $\begin{array}{c}10 c \\
9\end{array}$ & $\begin{array}{l}3582.062 \\
3580.788\end{array}$ & $\begin{array}{l}27908.94 \\
27918.87\end{array}$ & $\left\{\begin{array}{l}a^{4} \mathrm{G}_{51 / 2}-s^{4} \mathrm{G}_{41 / 2}^{2} \\
a^{6} \mathrm{D}_{01 / 2}-z^{2} \mathrm{P}_{11 / 2}^{10} \\
a^{2} \mathrm{H}_{51 / 2}-s^{4} \mathrm{G}_{41 / 2}^{0}\end{array}\right.$ & & & & & & \\
\hline $\begin{array}{l}400 r, \text { III? } \\
3 \\
4 c \\
3 \\
4\end{array}$ & $\begin{array}{l}3580.277 \\
3579.186 \\
3578.586 \\
3578.317 \\
3578.23\end{array}$ & $\begin{array}{l}27922.86 \\
27931.37 \\
27936.05 \\
27938.15 \\
27938.83\end{array}$ & $\begin{array}{l}a^{6} \mathrm{D}_{41 / 3}-y^{6} \mathrm{P}_{31 / 2}^{0} \\
a^{4} \mathrm{H}_{31 / 2}-t^{4} \mathrm{D}_{31 / 2}^{0} \\
a^{4} \mathrm{D}_{21 / 2}-s^{2} \mathrm{~F}_{31 / 2}^{0} \\
a^{4} \mathrm{H}_{41 / 2}-u^{2} \mathrm{G}_{3}^{3} 3 \\
b^{4} \mathrm{~F}_{31 / 2}-s^{4} \mathrm{G}^{4} 11 / 2\end{array}$ & 4 & 0.153 & 0.075 & 1.001 & 1.539 & 1. 692 \\
\hline $\begin{array}{c}40, \mathrm{~V} \\
8 c \\
200, \mathrm{III} \\
20 c \\
3\end{array}$ & $\begin{array}{l}3577.721 \\
3577.238 \\
3575.850 \\
3575.139 \\
3574.312\end{array}$ & $\begin{array}{l}27942.81 \\
27946.58 \\
27957.43 \\
27962.99 \\
27969.46\end{array}$ & $\begin{array}{l}a^{2} \mathrm{D}_{21 / 2}-t^{2} \mathrm{D}_{21 / 2}^{0} \\
b^{2} \mathrm{G}_{41 / 2}-p^{2} \mathrm{G}_{41 / 2}^{0} \\
a^{6} \mathrm{D}_{31 / 3}-y^{6} \mathrm{P}_{21 / 2}^{0} \\
a^{4} \mathrm{G}_{21 / 2}-r^{4} \mathrm{~F}^{\circ} 11 / 2\end{array}$ & $\begin{array}{l}6 \\
4\end{array}$ & $\begin{array}{l}0.125 \\
0.181\end{array}$ & $\begin{array}{l}0.313 \\
0.092\end{array}$ & $\begin{array}{l}1.141 \\
1.121\end{array}$ & $\begin{array}{l}1.204 \\
1.573\end{array}$ & $\begin{array}{l}1.078 \\
1.764\end{array}$ \\
\hline
\end{tabular}


TABLE 4.-First spectrum of columbium (Cb I) - Continued

\begin{tabular}{|c|c|c|c|c|c|c|c|c|c|}
\hline $\begin{array}{l}\text { Intensity } \\
\text { Arc }\end{array}$ & $\lambda_{\text {sir }} \mathbf{A}$ & $\begin{array}{l}\text { Wave } \\
\text { No. } \\
\text { vac. } \\
\mathrm{cm}^{-1}\end{array}$ & $\begin{array}{l}\text { Term combi- } \\
\text { nation }\end{array}$ & $\begin{array}{c}\text { Zeeman } \\
\text { type }\end{array}$ & $\begin{array}{l}\text { Separa- } \\
\text { tion }\end{array}$ & $\begin{array}{l}\text { Strong- } \\
\text { est } \\
p\end{array}$ & $\begin{array}{l}\text { Strong- } \\
\text { est } \\
n\end{array}$ & $g_{1}$ & $g_{2}$ \\
\hline 1 & 2 & 3 & 4 & 5 & 6 & 7 & 7 & 8 & 10 \\
\hline $\begin{array}{l}5 \\
1 \\
6 c \\
3 \\
7 c\end{array}$ & $\begin{array}{l}3573.096 \\
3572.10 \\
3571.485 \\
3571.124 \\
3569.854\end{array}$ & $\begin{array}{l}27978.98 \\
27986.78 \\
27991.60 \\
27994.42 \\
28004.39\end{array}$ & $\begin{array}{l}a^{2} \mathrm{H}_{51 / 2}-t^{4} \mathrm{G}_{51 / 2}^{\circ} \\
b^{2} \mathrm{G}_{31 / 5}-0^{4} \mathrm{D}_{21 / 2}^{\circ} \\
a^{4} \mathrm{G}_{21 / 5}-400_{21 / 2}^{\circ} \\
a^{2} \mathrm{D}_{21 / 2}-r^{2} \mathrm{~F}_{31 / 2}^{\circ} \\
a^{4} \mathrm{G}_{431 / 2}-r^{4} \mathrm{~F}_{31 / 2}^{0}\end{array}$ & & & & & & \\
\hline $\begin{array}{l}5 \\
40, \mathrm{~V} \\
20, \mathrm{~V} \\
5 c \\
6\end{array}$ & $\begin{array}{l}3569.692 \\
3569.464 \\
3568.727 \\
3567.099 \\
3565.855\end{array}$ & $\begin{array}{l}28005.66 \\
28007.45 \\
28013.23 \\
28026.01 \\
28035.79\end{array}$ & $\begin{array}{l}a^{4} \mathrm{~F}_{31 / 2}-x^{4} \mathrm{~F}_{31 / 2}^{0} \\
a^{4} \mathrm{P}_{01 / 2}-v^{4} \mathrm{D}_{01 / 2}^{0} \\
a^{4} \mathrm{D}_{31 / 2}-u^{4} \mathrm{G}_{41 / 2}^{0} \\
a^{4} \mathrm{~F}_{21 / 2}-y^{2} \mathrm{D}_{1 / 2}^{1}\end{array}$ & $\begin{array}{l}6 \\
6\end{array}$ & 0.056 & $\begin{array}{l}0.166 \\
0^{*}\end{array}$ & $\begin{array}{l}1.204 \\
1.564\end{array}$ & $\begin{array}{c}1.232 \\
(2.650)\end{array}$ & $\begin{array}{l}1.176 \\
0.477\end{array}$ \\
\hline $\begin{array}{l}10 c \\
6 c\end{array}$ & $\begin{array}{l}3565.052 \\
3564.028 \\
3563\end{array}$ & 28042.10 & $\begin{array}{l}a{ }^{4} \mathrm{D}_{31 / 2}-t^{4} \mathrm{~F}_{31 / 2}^{\circ} \\
b^{4} \mathrm{D}_{31 / 3}-q^{4} \mathrm{D}_{31 / 2}^{3}\end{array}$ & & & & & & \\
\hline $\begin{array}{c}80, \text { IV } \\
100, \text { IV } \\
6\end{array}$ & $\begin{array}{l}3563.624 \\
3563.501 \\
3563.232\end{array}$ & $\begin{array}{l}28053.34 \\
28054.31 \\
28056.43\end{array}$ & $\begin{array}{l}a^{6} \mathrm{D}_{21 / 2}-y{ }^{4} \mathrm{P}_{21 / 2} \\
a^{6} \mathrm{D}_{11 / 2}-z^{4} \mathrm{~S}_{11 / 2} \\
a^{2} \mathrm{~F}_{31 / 2}-s^{2} \mathrm{G}_{31 / 2} 1\end{array}$ & $\begin{array}{l}6 \\
6\end{array}$ & 0.074 & $\begin{array}{l}0.11^{*} \\
0.111\end{array}$ & $\begin{array}{l}1.618 \\
1.835\end{array}$ & $\begin{array}{c}(1.652) \\
1.872\end{array}$ & $\begin{array}{l}1.585 \\
1.798\end{array}$ \\
\hline $\begin{array}{c}3 \\
6 c \\
6 \\
12 \\
7\end{array}$ & $\begin{array}{l}3562.846 \\
3562.649 \\
3561.697 \\
3561.142 \\
3560.357\end{array}$ & $\begin{array}{l}28059.47 \\
28061.02 \\
28068.52 \\
28072.89 \\
28079.08\end{array}$ & $\begin{array}{l}a^{4} \mathrm{H}_{31 / 2}-u^{2} \mathrm{G}_{31 / 2}^{0} \\
a^{4} \mathrm{G}_{21 / 2}-s^{4} \mathrm{G}_{21 / 2}^{2} \\
a^{4} \mathrm{D}_{21 / 2}-t^{4} \mathrm{~F}_{11 / 2}^{0} \\
a^{4} \mathrm{G}_{31 / 2}-r^{4} \mathrm{~F}_{21 / 2}^{21} \\
a^{6} \mathrm{D}_{01 / 2}-x^{4} \mathrm{D}_{11 / 2}^{1}\end{array}$ & & & & & & \\
\hline $\begin{array}{c}1 \\
12, \mathrm{~V} \\
9 \\
1 \\
2\end{array}$ & $\begin{array}{l}3559.470 \\
3559.128 \\
3558.015 \\
3557.684 \\
3557.023\end{array}$ & $\begin{array}{l}28086.08 \\
28088.78 \\
28097.56 \\
28100.18 \\
28105.40\end{array}$ & 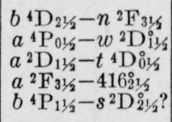 & 4 & 1. 592 & 0.796 & 0.262 & 2. 640 & 1.058 \\
\hline $\begin{array}{l}10 \\
80, \text { III } \\
60, \text { IV? } \\
10 c \\
6\end{array}$ & $\begin{array}{l}3556.022 \\
3554.666 \\
3554.524 \\
3553.613 \\
3552.227\end{array}$ & $\begin{array}{l}28113.31 \\
28124.03 \\
28125.16 \\
28132.37 \\
28143.34\end{array}$ & 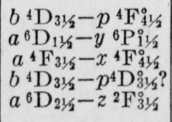 & $\begin{array}{l}6 \\
4\end{array}$ & 0.120 & 0.180 & $\begin{array}{l}1.919 \\
1.144\end{array}$ & $\begin{array}{c}1.861 \\
(1.235)\end{array}$ & $\begin{array}{l}1.981 \\
1.202\end{array}$ \\
\hline $\begin{array}{c}5 \\
1 \\
8 \\
6 \\
50, \text { IV? }\end{array}$ & $\begin{array}{l}3552.000 \\
3551.37 \\
3551.102 \\
3550.624 \\
3550.448\end{array}$ & $\begin{array}{l}28145.14 \\
28150.14 \\
28152.26 \\
28156.05 \\
28157.45\end{array}$ & $\begin{array}{l}a^{4} \mathrm{D}_{21 / 5}-u^{4} \mathrm{G}_{21 / 1}^{2} \\
a^{2} \mathrm{~F}_{21 / 2}-q^{4} \mathrm{~F}_{13 / 2} \\
a^{2} \mathrm{G}_{31 / 5}-s^{2} \mathrm{~F}^{3} 1 / 2 \\
a^{2} \mathrm{D}_{21 / 2}-t^{4} \mathrm{D}_{21 / 2}^{21} \\
a^{6} \mathrm{D}_{21 / 2}-x^{4} \mathrm{D}_{21 / 2}^{21}\end{array}$ & 6 & 0.179 & 0.448 & 1. 562 & 1. 652 & 1.472 \\
\hline $\begin{array}{l}10 \\
1 \\
12 \\
15, \mathrm{~V} \\
3\end{array}$ & $\begin{array}{l}3550.237 \\
3549.96 \\
3549.263 \\
3548.130 \\
3547.426\end{array}$ & $\begin{array}{l}28159.12 \\
28161.31 \\
28166.84 \\
28175.84 \\
28181.43\end{array}$ & 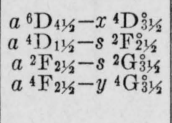 & $\begin{array}{l}5 \\
4\end{array}$ & & $\begin{array}{l}0.21 \\
--\end{array}$ & $\begin{array}{l}1.10^{*} \\
0.962\end{array}$ & $\begin{array}{l}(0.86) \\
(1.029)\end{array}$ & $\begin{array}{l}0.94 \\
0.999\end{array}$ \\
\hline $\begin{array}{r}2 \\
8 \\
5 \\
12 \\
6 c\end{array}$ & $\begin{array}{l}3546.910 \\
3546.489 \\
3546.160 \\
3546.031 \\
3545.381\end{array}$ & $\begin{array}{l}28185.53 \\
28188.88 \\
28191.49 \\
28192.52 \\
28197.68\end{array}$ & $\begin{array}{l}b^{4} \mathrm{~F}_{11 / 2}-s^{4} \mathrm{D}_{21 / 2}^{2} \\
a^{4} \mathrm{~F}_{21 / 2}-y^{2} \mathrm{D}_{21 / 2}^{2} \\
a^{4} \mathrm{G}_{21 / 2}-r^{4} \mathrm{~F}_{21 / 2}^{2} \\
a^{4} \mathrm{~F}_{21 / 2}-x^{4} \mathrm{~F}_{11 / 2}^{11} \\
a^{4} \mathrm{G}_{41 / 2}-s^{4} \mathrm{G}_{31 / 5}\end{array}$ & & & & & & \\
\hline $\begin{array}{l}50, \text { IV } \\
50, \text { IV? } \\
12, \mathrm{~V} \\
5 \\
1\end{array}$ & $\begin{array}{l}3544.656 \\
3544.031 \\
3543.936 \\
3543.745 \\
3543.55\end{array}$ & $\begin{array}{l}28203.45 \\
28208.43 \\
28209.18 \\
28210.70 \\
28212.25\end{array}$ & 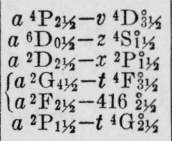 & $\begin{array}{l}4 \\
4\end{array}$ & $\begin{array}{l}0.197 \\
1.523\end{array}$ & $\begin{array}{l}0.104 \\
0.763\end{array}$ & $\begin{array}{l}0.901 \\
1.030\end{array}$ & $\begin{array}{l}\text { 1. } 590 \\
\text { 3. } 314\end{array}$ & $\begin{array}{l}\text { 1. } 394 \\
1.791\end{array}$ \\
\hline $\begin{array}{l}15, \mathrm{~V} \\
10 \\
20 c \\
15 d, \mathrm{~V} \\
2\end{array}$ & $\begin{array}{l}3542.983 \\
3542.560 \\
3541.898 \\
3539.650 \\
3538.076\end{array}$ & $\begin{array}{l}28216.77 \\
28220.14 \\
28225.41 \\
28243.34 \\
28255.90\end{array}$ & 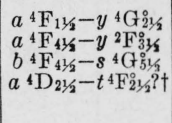 & $\begin{array}{l}5 \\
5\end{array}$ & $\begin{array}{l}0.292 \\
0.200\end{array}$ & $\begin{array}{l}0.146 \\
0.105 \\
0.685\end{array}$ & 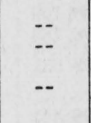 & $\begin{array}{l}(0.402) \\
(1.330)\end{array}$ & $\begin{array}{l}0.694 \\
1.130\end{array}$ \\
\hline $\begin{array}{c}150, \mathrm{III} \\
4 c \\
1\end{array}$ & $\begin{array}{l}3537.475 \\
3536.212 \\
3535.845\end{array}$ & $\begin{array}{l}28260.70 \\
28270.80 \\
28273.73\end{array}$ & $\begin{array}{l}a^{6}{ }^{6} \mathrm{D}_{21 / 1}-y{ }^{6} \mathrm{P}_{21 / 2}^{0} \\
b^{4} \mathrm{D}_{31 / 2}-u^{2} \mathrm{~F}_{31 / 2}^{8} \\
b^{4} \mathrm{P}_{21 / 2}-p^{4} \mathrm{~F}_{31 / 2}^{0}\end{array}$ & 6 & 0.122 & 0.306 & 1. 739 & 1. 678 & 1.800 \\
\hline $\begin{array}{c}400 c, \text { III } \\
1 h\end{array}$ & $\begin{array}{l}3535.304 \\
3534,85\end{array}$ & $\begin{array}{l}28278.06 \\
28281,69\end{array}$ & $\left\{\begin{array}{l}a^{6} \mathrm{D}_{31 / 2}-y{ }^{6} \mathrm{P}_{31 / 2}^{0} \\
a^{6} \mathrm{D}_{01 / 2}^{0}-y{ }^{6} \mathrm{P}_{1 / 2}^{0} \\
b^{2} \mathrm{H}_{41 / 2}-u^{2} \mathrm{H}_{41 / 2}^{0}\end{array}\right.$ & $\begin{array}{l}6 \\
4\end{array}$ & 0.124 & $\begin{array}{l}0.425 \\
0.674\end{array}$ & $\begin{array}{l}\text { 1. } 642 \\
2.616\end{array}$ & $\begin{array}{l}\text { 1. } 580 \\
\text { 3. } 326\end{array}$ & $\begin{array}{l}1.704 \\
1.986\end{array}$ \\
\hline
\end{tabular}


TABLE 4.-First spectrum of columbium (Cb I)-Continued

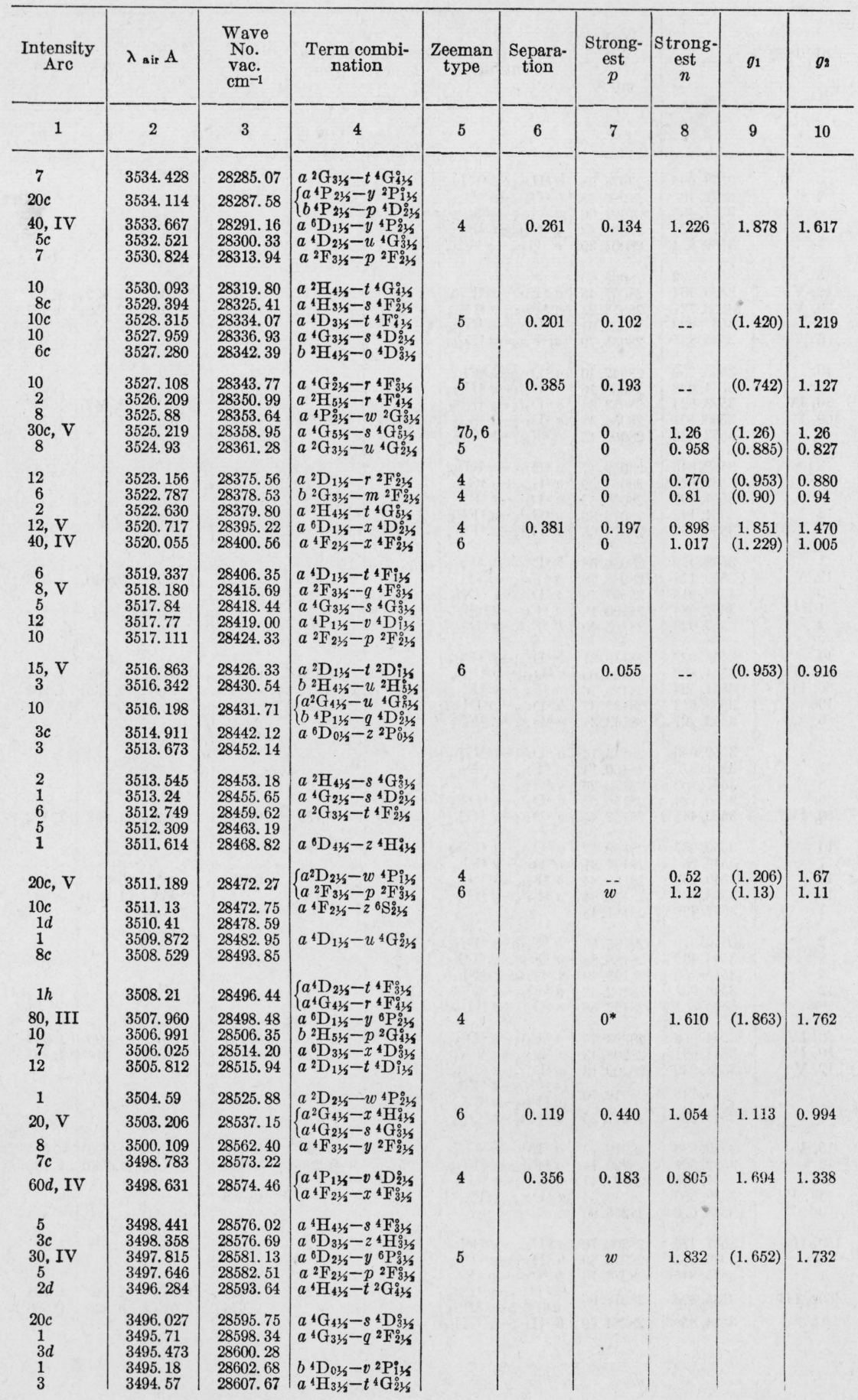


TABLE 4.-First spectrum of columbium (Cb I)-Continued

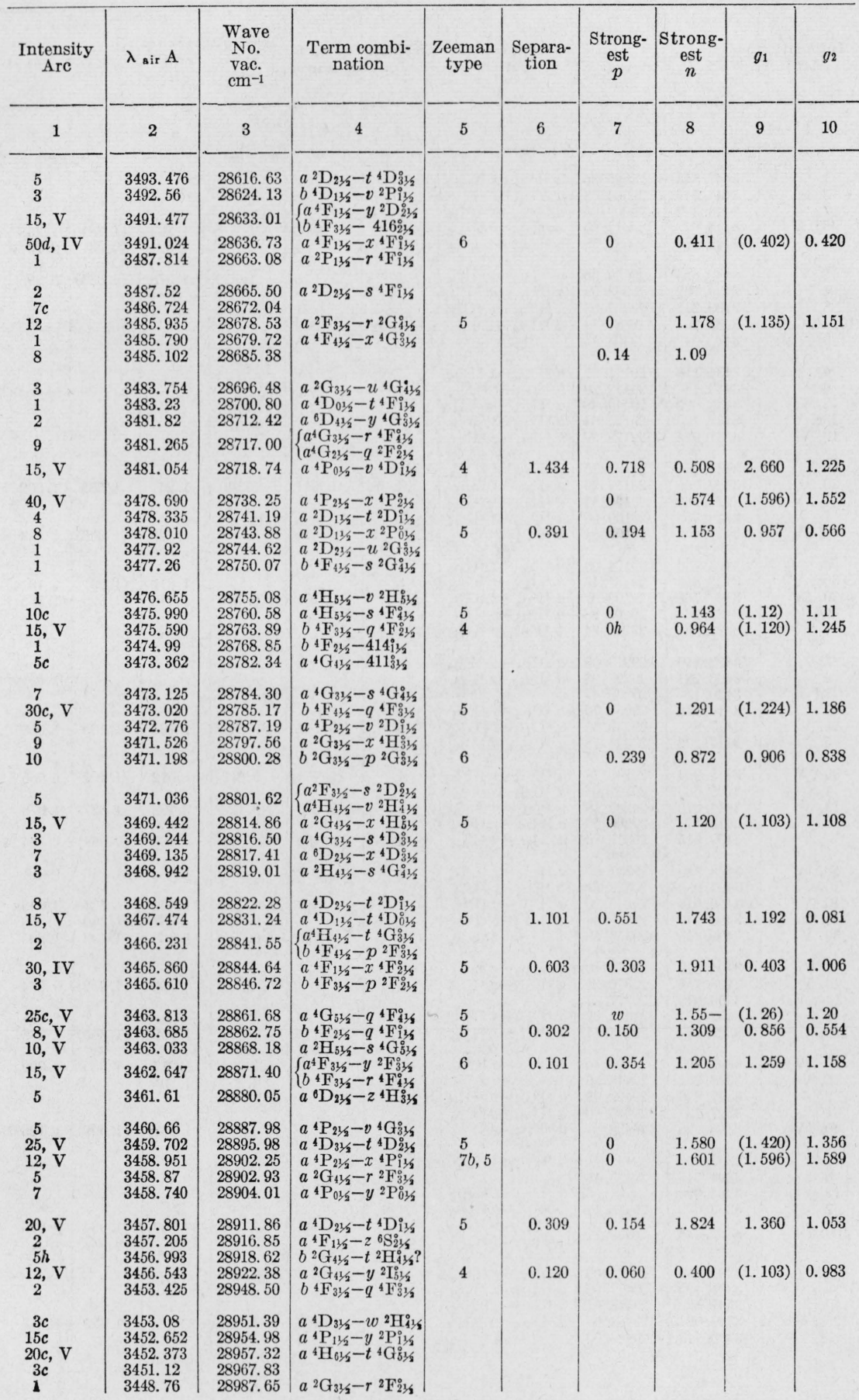


TABLE 4. -First spectrum of columbium (Cb I) - Continued

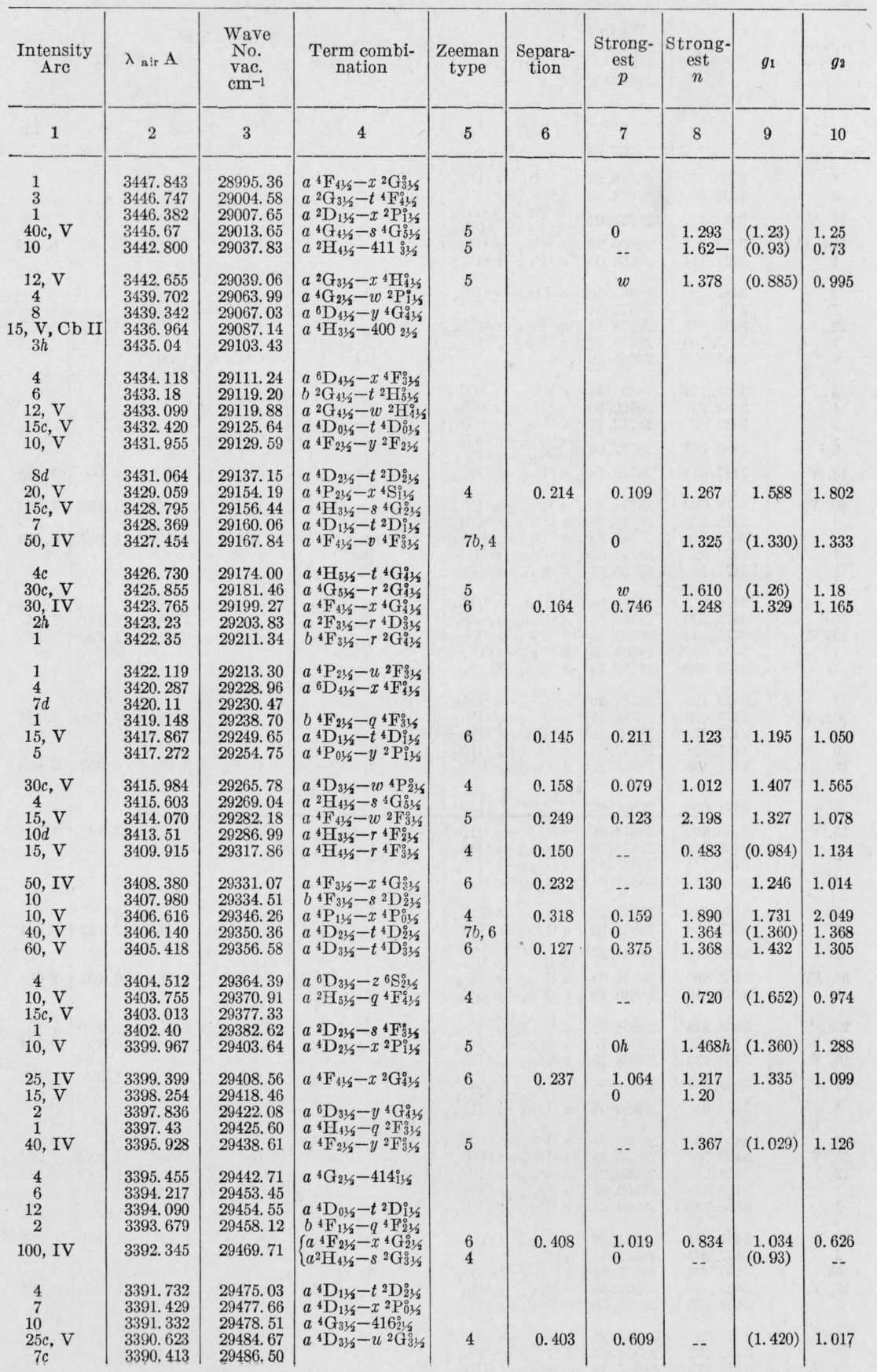


TABLE 4.-First spectrum of columbium (Cb I)—Continued

\begin{tabular}{|c|c|c|c|c|c|c|c|c|c|}
\hline $\begin{array}{l}\text { Intensity } \\
\text { Arc }\end{array}$ & $\lambda$ air $\mathrm{A}$ & $\begin{array}{l}\text { Wave } \\
\text { No. } \\
\text { vac. } \\
\mathrm{cm}^{-1}\end{array}$ & $\begin{array}{c}\text { Term combi- } \\
\text { nation }\end{array}$ & $\begin{array}{c}\text { Zeeman } \\
\text { type }\end{array}$ & $\begin{array}{l}\text { Separa- } \\
\text { tion }\end{array}$ & $\begin{array}{l}\text { Strong- } \\
\text { est } \\
p\end{array}$ & $\begin{array}{c}\text { Strong- } \\
\text { est } \\
n\end{array}$ & $g_{1}$ & $g_{2}$ \\
\hline 1 & 2 & 3 & 4 & 5 & 6 & 7 & 8 & 9 & 10 \\
\hline $\begin{array}{l}1 c \\
1 \\
15, \mathrm{~V} \\
10, \mathrm{~V} \\
20\end{array}$ & $\begin{array}{l}3390.08 \\
3389.73 \\
3387.754 \\
3387.579 \\
3386.995\end{array}$ & $\begin{array}{l}29489.39 \\
29492.44 \\
29509.64 \\
29511.16 \\
29516.25\end{array}$ & $\begin{array}{l}a^{2} \mathrm{~F}_{21 / 2}-p{ }^{4} \mathrm{~F}_{11 / 2}^{0} \\
b^{2} \mathrm{G}_{41 / 2}-0^{2} \mathrm{G}_{31 / 2}^{0} \\
a^{4} \mathrm{P}_{11 / 2}-y^{2} \mathrm{~S}_{01 / 3}^{0} \\
a^{4} \mathrm{H}_{41 / 2}-s^{4} \mathrm{G}_{31 / 2}^{0} \\
a^{4} \mathrm{G}_{41 / 2}-q^{4} \mathrm{~F}_{41 / 2}^{0}\end{array}$ & $\begin{array}{l}4 \\
4\end{array}$ & 0.356 & 0.178 & $\begin{array}{l}1.587 \\
0.646\end{array}$ & $\begin{array}{c}1.724 \\
(0.984)\end{array}$ & $\begin{array}{l}2.080 \\
1.080\end{array}$ \\
\hline $\begin{array}{l}10 \\
12, \mathrm{~V} \\
4 \\
20, \mathrm{~V} \\
1 h\end{array}$ & $\begin{array}{l}3385.815 \\
3385.665 \\
3385.192 \\
3384.662 \\
3384.50\end{array}$ & $\begin{array}{l}29526.54 \\
29527.85 \\
29531.97 \\
29536.60 \\
29538.01\end{array}$ & $\begin{array}{l}a^{4} \mathrm{~F}_{41 / 2}-w^{4} \mathrm{~F}_{31 / 2}^{0} \\
a^{4} \mathrm{P}_{21 / 2}-v^{2} \mathrm{D}_{21 / 2}^{0} \\
a^{4} \mathrm{G}_{21 / 2}-q^{4} \mathrm{~F}_{11 / 2}^{0} \\
a^{4} \mathrm{G}_{41 / 2}-s^{2} \mathrm{G}_{41 / 2}^{0}\end{array}$ & 5 & 0.200 & 0.098 & 1. 058 & 0.758 & 0.558 \\
\hline $\begin{array}{l}15, \mathrm{~V} \\
2 \\
20 \\
7 \\
40, \mathrm{III}\end{array}$ & $\begin{array}{l}3383.802 \\
3382.91 \\
3380.862 \\
3380.490 \\
3380.420\end{array}$ & $\begin{array}{l}29544.10 \\
29551.89 \\
29569.80 \\
29573.04 \\
29573.66\end{array}$ & $\begin{array}{l}a^{4} \mathrm{D}_{01 / 2}-t{ }^{4} \mathrm{D}_{11 / 2} \\
b^{2} \mathrm{G}_{31 / 2}-r^{4} \mathrm{G}_{31 / 2}^{0} \\
a^{4} \mathrm{P}_{11 / 2}-x^{4} \mathrm{P}_{11 / 2}^{0} \\
a^{4} \mathrm{G}_{41 / 2}-q^{4} \mathrm{~F}_{31 / 2}^{0} \\
a^{4} \mathrm{~F}_{11 / 2}-y^{2} \mathrm{~F}_{21 / 2}^{0}\end{array}$ & 5 & 0.475 & 0.234 & 1. 604 & 0.416 & 0.891 \\
\hline $\begin{array}{l}20, \mathrm{~V} \\
6 \\
15, \mathrm{IV} \\
1 \\
12, \mathrm{IV}\end{array}$ & $\begin{array}{l}3380.055 \\
3377.743 \\
3376.732 \\
3376.59 \\
3376.341\end{array}$ & $\begin{array}{l}29576.86 \\
29597.10 \\
29605.96 \\
29607.20 \\
29609.39\end{array}$ & $\begin{array}{l}a^{4} \mathrm{~F}_{41 / 2}-z^{4} \mathrm{I}_{51 / 2}^{0} \\
a^{4} \mathrm{G}_{21 / 2}-416_{21 / 2}^{\circ} \\
a^{4} \mathrm{H}_{51 / 2}-r{ }^{4} \mathrm{~F}_{41 / 2}^{0} \\
a^{6} \mathrm{D}_{41 / 2}-y^{4} \mathrm{G}_{51 / 2}^{0} \\
a^{4} \mathrm{G}_{31 / 2}-q^{4} \mathrm{~F}_{21 / 2}^{0}\end{array}$ & 4 & 0.330 & 0.165 & -- & $(1.330)$ & 1.000 \\
\hline $\begin{array}{l}60, \text { IV } \\
5 \\
1 \\
6\end{array}$ & $\begin{array}{l}3374.928 \\
3374.029 \\
3373.71 \\
3372.164\end{array}$ & $\begin{array}{l}29621.78 \\
29629.68 \\
29632.48 \\
29646.06\end{array}$ & $\begin{array}{l}a^{2} \mathrm{G}_{31 / 2}-w^{2} \mathrm{H}^{\circ}{ }^{\circ} / 2 \\
a^{4} \mathrm{G}_{41 / 2}-p^{2} \mathrm{~F}_{31 / 2}^{5} \\
a^{4} \mathrm{H}_{31 / 2}-s^{4} \mathrm{G}_{31 / 2}^{\circ} \\
a^{4} \mathrm{P}_{01 / 2}-x^{4} \mathrm{P}_{01 / 2}^{\circ}\end{array}$ & 5 & & $w$ & 1.111 & $(0.885)$ & 0.935 \\
\hline $10 \mathrm{~V}$ & 3372.101 & 29646.62 & $\left\{\begin{array}{l}a^{4} \mathrm{~F}_{41 / 2}-u^{4} \mathrm{~F}_{31 / 2}^{0} \\
a^{4} \mathrm{~F}_{31 / 2}-x^{2} \mathrm{G}_{31 / 2}^{0}\end{array}\right.$ & & & & & & \\
\hline 40, IV & 3371.331 & 29653.39 & $\left\{\begin{array}{l}a{ }^{4} \mathrm{~F}_{31 / 2}-v^{4} \mathrm{~F}_{21 / 2}^{0} \\
a^{2} \mathrm{G}_{41 / 2}-u^{2} \mathrm{G}_{31 / 3}\end{array}\right.$ & 5 & 0.184 & 0.092 & 1. 700 & 1. 240 & 1.056 \\
\hline $\begin{array}{l}20 d \\
4 \\
20 c \\
10 d\end{array}$ & $\begin{array}{l}3369.840 \\
3369.719 \\
3369.081 \\
3368.426\end{array}$ & $\begin{array}{l}29666.51 \\
29667.57 \\
29673.19 \\
29678.96\end{array}$ & $\begin{array}{l}a^{4} \mathrm{D}_{21 / 2}-w^{4} \mathrm{P}^{1} 1 / 3 \\
a^{6} \mathrm{D}_{21 / 2}-z^{6} \mathrm{~S}_{21 / 2}^{0} \\
a^{4} \mathrm{H}_{51 / 2}-s^{4} \mathrm{G}_{11 / 3}^{0} \\
b^{2}{ }^{2} \mathrm{H}_{51 / 2}-t^{2} \mathrm{H}_{51 / 2}^{1}\end{array}$ & & & & & & \\
\hline $\begin{array}{l}25, \mathrm{~V} \\
8 \\
50, \mathrm{IV} \\
6 \\
15 d\end{array}$ & $\begin{array}{l}3367.382 \\
3367.085 \\
3366.956 \\
3365.883 \\
3363.750\end{array}$ & $\begin{array}{l}29688.16 \\
29690.78 \\
29691.92 \\
29701.38 \\
29720.22\end{array}$ & $\begin{array}{l}a^{4} \mathrm{D}_{11 / 2}-t^{4} \mathrm{D}_{21 / 2}^{\circ} \\
a^{2} \mathrm{H}_{51 / 2}-r^{2} \mathrm{G}_{41 / 2}{ }^{2} \\
a^{2} \mathrm{G}_{41 / 2}-w^{2} \mathrm{H}_{51 / 2}^{\circ} \\
a^{4} \mathrm{D}_{21 / 2}-w^{4} \mathrm{P}_{21 / 2}^{\circ}\end{array}$ & $\begin{array}{l}5 \\
4 \\
6\end{array}$ & & $\begin{array}{l}0 h \\
0 \\
0.606\end{array}$ & $\begin{array}{l}1.602 \\
1.041 \\
--\end{array}$ & $\begin{array}{l}(1.197) \\
(1.103) \\
(1.360)\end{array}$ & $\begin{array}{l}\text { 1. } 359 \\
\text { 1. } 084 \\
\text { 1. } 602\end{array}$ \\
\hline $\begin{array}{c}10 \\
2 d \\
2 \\
7 \\
4 \\
250 r, \text { III } \\
4 d\end{array}$ & $\begin{array}{l}3362.866 \\
3361.86 \\
3361.35 \\
3359.874 \\
3358.77 \\
3358.422 \\
3357.899\end{array}$ & $\begin{array}{l}29728.03 \\
29736.92 \\
29741.44 \\
29754.50 \\
29764.28 \\
29767.37 \\
29772.00\end{array}$ & $\begin{array}{l}a^{4} \mathrm{G}_{21 / 3}-q^{4} \mathrm{~F}_{21 / 2}^{0} \\
b^{4} \mathrm{~F}_{31 / 2}-r^{4} \mathrm{D}_{31 / 2}^{0} \\
a^{4} \mathrm{D}_{11 / 3}-x^{2} \mathrm{P}_{11 / 2}^{0} \\
a^{4} \mathrm{P}_{01 / 2}-v^{2} \mathrm{D}_{11 / 2}^{0} \\
a^{2} \mathrm{P}_{11 / 2}-w^{2} \mathrm{P}_{11 / 2}^{0} \\
a^{4} \mathrm{~F}_{41 / 2}-x^{4} \mathrm{G}_{51 / 2}^{0} \\
\left\{\begin{array}{l}a^{4} \mathrm{D}_{01 / 2}-x^{2} \mathrm{P}_{01 / 2}^{0} \\
a^{2} \mathrm{H}_{41 / 2}-q^{4} \mathrm{~F}_{41 / 2}^{0}\end{array}\right.\end{array}$ & 4 & & $w$ & 0.826 & (1. 330) & 1. 238 \\
\hline $\begin{array}{l}25, \mathrm{~V} \\
1 \\
8 \mathrm{c}\end{array}$ & $\begin{array}{l}3357.043 \\
3356.76 \\
3356.465\end{array}$ & $\begin{array}{l}29779.59 \\
29782.10 \\
29784.72\end{array}$ & $\begin{array}{l}a^{4} \mathrm{~F}_{31 / 3}-w^{2} \mathrm{~F}_{21 / 2}^{0} \\
a^{2} \mathrm{~F}_{21 / 2}-p{ }^{4} \mathrm{D}_{21 / 2}^{0}\end{array}$ & 5 & 0.256 & 0.128 & 1. 878 & 1. 238 & 0.982 \\
\hline $\begin{array}{l}12, \text { V } \\
80, \text { III }\end{array}$ & $\begin{array}{l}3355.423 \\
3354.743\end{array}$ & $\begin{array}{l}29793.97 \\
29800.01\end{array}$ & $\begin{array}{l}a^{4} \mathrm{G}_{31 / 2}-q^{4} \mathrm{~F}_{31 / 2}^{0} \\
a^{4} \mathrm{~F}_{41 / 2}-v^{4} \mathrm{~F}_{41 / 2}^{0}\end{array}$ & $\begin{array}{l}6 \\
6\end{array}$ & 0.116 & $\begin{array}{l}0.437 \\
0.554\end{array}$ & 1. $\overline{272}$ & $\begin{array}{l}\text { (1. } 081) \\
1.330\end{array}$ & $\begin{array}{l}\text { 1. } 186 \\
\text { 1. } 214\end{array}$ \\
\hline $6 d$ & 3353.675 & & $\left\{\begin{array}{l}a^{4} \mathrm{P}_{01 / 2}-y^{2} \mathrm{~S}_{01 / 2} \\
a^{4} \mathrm{H}_{41 / 2}-r \\
{ }^{4} \mathrm{~F}_{41 / 2}^{0}\end{array}\right.$ & & & & & & \\
\hline $\begin{array}{r}7 d \\
10 c\end{array}$ & $\begin{array}{l}3353.352 \\
3352.868\end{array}$ & $\begin{array}{l}29812.37 \\
29816.67\end{array}$ & $a^{4} \mathrm{H}_{31 / 2}-q^{2} \mathrm{~F}_{21 / 2}^{\circ}$ & & & & & & \\
\hline $\begin{array}{l}15, \mathrm{~V} \\
12, \mathrm{~V} \\
3 c \\
1 \\
2 h\end{array}$ & $\begin{array}{l}3352.592 \\
3352.282 \\
3351.819 \\
3351.511 \\
3351.33\end{array}$ & $\begin{array}{l}29819.13 \\
29821.88 \\
29826.00 \\
29828.74 \\
29830.36\end{array}$ & $\begin{array}{l}a{ }^{4} \mathrm{~F}_{31 / 2}-v{ }^{4} \mathrm{~F}_{31 / 2}^{0} \\
a^{4} \mathrm{P}_{11 / 2}-x^{4} \mathrm{~S}_{11 / 2}^{0} \\
a^{4} \mathrm{H}_{41 / 2}-q^{4} \mathrm{~F}_{31 / 2}^{0} \\
b^{4} \mathrm{D}_{21 / 2}-m^{2} \mathrm{~F}_{21 / 2}^{0}\end{array}$ & 6 & 0.093 & 0.140 & 1. $79^{*}$ & (1. 721) & 1. 814 \\
\hline $\begin{array}{c}7 c \\
4 \\
40 c, \mathrm{~V} \\
200, \text { III } \\
4\end{array}$ & $\begin{array}{l}3350.689 \\
3350.048 \\
3349.524 \\
3349.068 \\
3348.082\end{array}$ & $\begin{array}{l}29836.06 \\
29841.77 \\
29846.44 \\
29850.50 \\
29859.29\end{array}$ & $\begin{array}{l}a^{4} \mathrm{G}_{41 / 2}-r^{2} \mathrm{G}_{41 / 2}^{\circ} \\
a^{2} \mathrm{D}_{21 / 2}-s^{4} \mathrm{G}_{21 / 2}^{\circ} \\
a^{4} \mathrm{H}_{61 / 2}-s^{4} \mathrm{G}_{51 / 2}^{0} \\
a^{4} \mathrm{~F}_{31 / 2}-x^{4} \mathrm{G}_{41 / 2}^{0} \\
a^{4} \mathrm{~F}_{31 / 2}-w^{4} \mathrm{~F}_{21 / 2}^{0}\end{array}$ & $\begin{array}{l}4 \\
4\end{array}$ & & $\begin{array}{c}0 h \\
w\end{array}$ & $\begin{array}{l}1.14 \\
0.958\end{array}$ & $\begin{array}{l}(1.22) \\
(1.235)\end{array}$ & $\begin{array}{l}\text { 1. } 25 \\
1.134\end{array}$ \\
\hline
\end{tabular}


TABLE 4.-First spectrum of columbium (Cb I) - Continued

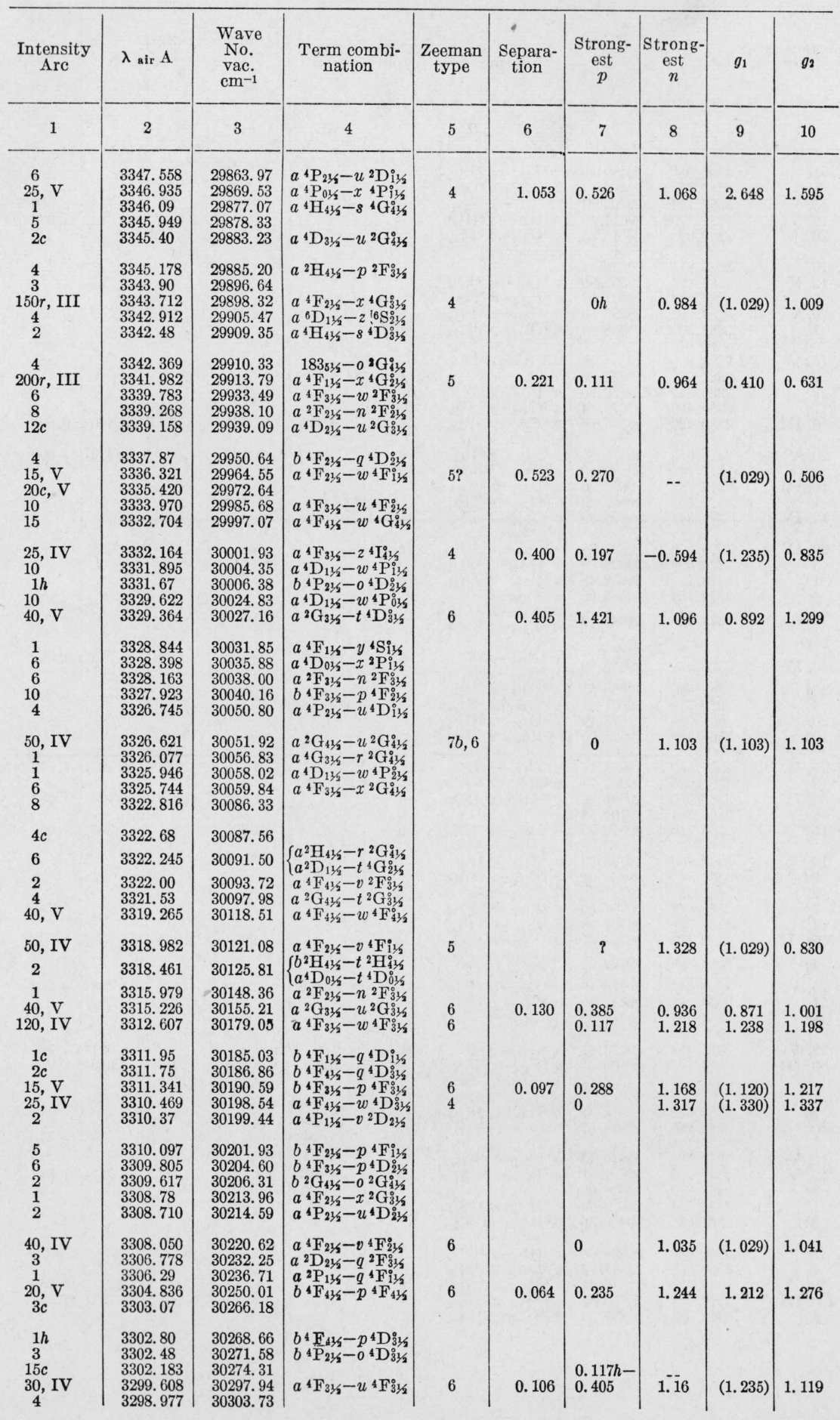


TABLE 4.-First spectrum of columbium (Cb I)-Continued

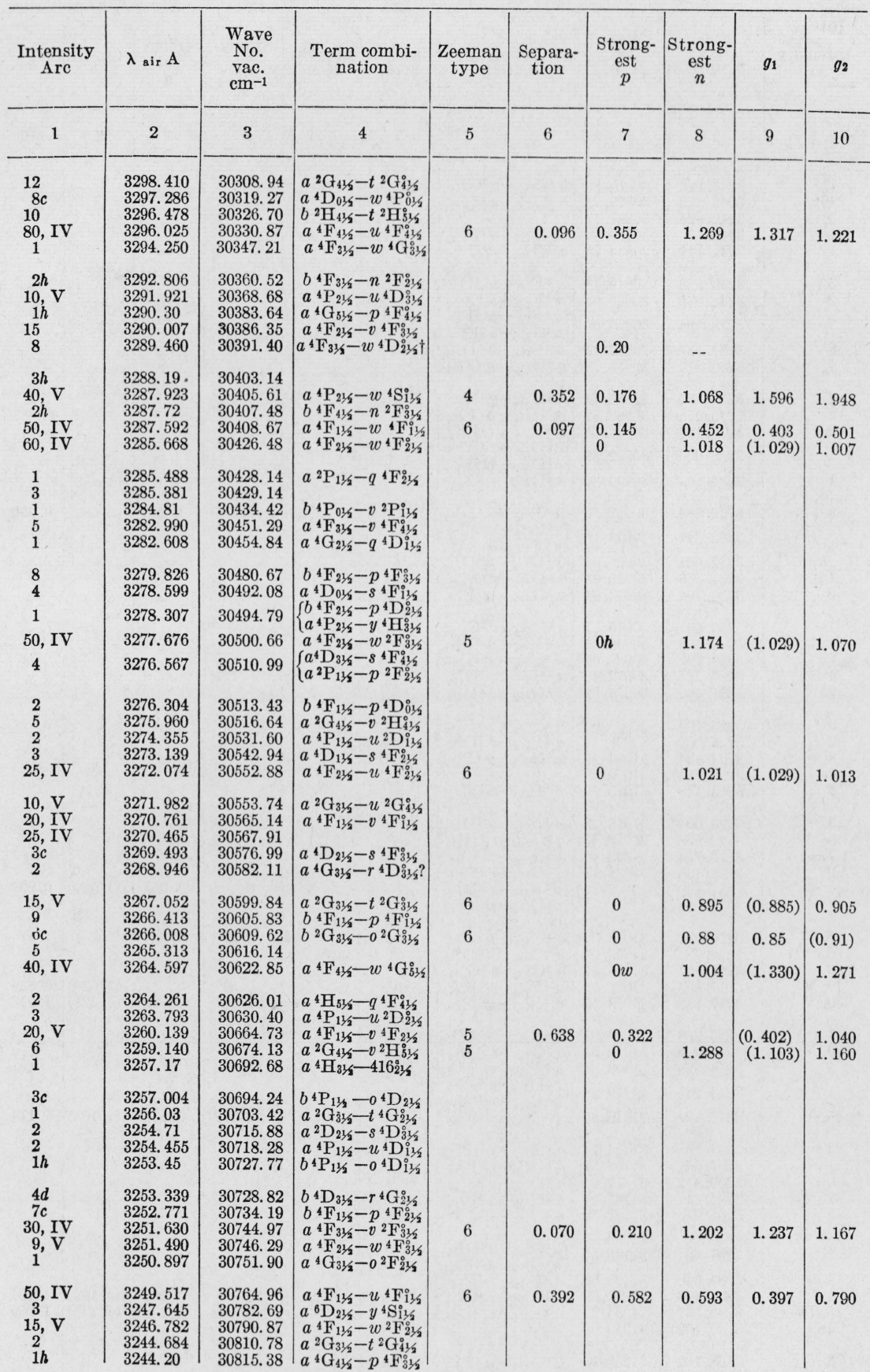


TABLE 4.-First spectrum of columbium (Cb I) - Continued

\begin{tabular}{|c|c|c|c|c|c|c|c|c|c|}
\hline $\begin{array}{l}\text { Intensity } \\
\text { Arc }\end{array}$ & $\lambda$ air $A$ & $\begin{array}{l}\text { Wave } \\
\text { No. } \\
\text { vac. } \\
\mathrm{cm}^{-1}\end{array}$ & $\begin{array}{l}\text { Term combi- } \\
\text { nation }\end{array}$ & $\begin{array}{c}\text { Zeeman } \\
\text { type }\end{array}$ & $\begin{array}{l}\text { Separa- } \\
\text { tion }\end{array}$ & $\begin{array}{l}\text { Strong- } \\
\text { est } \\
p\end{array}$ & $\begin{array}{c}\text { Strong- } \\
\text { est } \\
n\end{array}$ & $g_{1}$ & $g_{2}$ \\
\hline 1 & 2 & 3 & 4 & 5 & 6 & 7 & 7 & 8 & 10 \\
\hline $\begin{array}{l}1 \\
4 \\
3 \\
1 \\
1\end{array}$ & $\begin{array}{l}3243.725 \\
3243.322 \\
3242.928 \\
3242.67 \\
3241.111\end{array}$ & $\begin{array}{l}30819.89 \\
30823.72 \\
30827.47 \\
30829.92 \\
30844.75\end{array}$ & $\mid \begin{array}{l}b^{4} \mathrm{P}_{21 / 2}-p^{2} \mathrm{G}_{31 / 2}^{0} \\
a^{4} \mathrm{H}_{31 / 2}-q^{4} \mathrm{~F}_{21 / 2}^{1} \\
a^{4} \mathrm{H}_{41 / 2}-q^{4} \mathrm{~F}_{41 / 2}^{\circ} \\
a^{2} \mathrm{D}_{21 / 2}-w^{2} \mathrm{P}_{11 / 2}^{\circ}\end{array}$ & & & & & & \\
\hline $\begin{array}{l}3 \\
2\end{array}$ & $\begin{array}{l}3240.582 \\
3238.975\end{array}$ & $\begin{array}{l}30849.78 \\
30865.09\end{array}$ & $\mid \begin{array}{l}a^{4} \mathrm{~F}_{31 / 2}-w^{4} \mathrm{D}_{31 / 2}^{0} \\
a^{4} \mathrm{~F}_{21 / 2}-u^{4} \mathrm{~F}_{31 / 2}^{3}\end{array}$ & & & & & & \\
\hline 3 & 3238.394 & 30870.63 & $\left\{\begin{array}{l}a^{4} \mathrm{~F}_{11 / 2}-w^{4} \mathrm{~F}_{21 / 2} \\
a^{4} \mathrm{G}_{21 / 3}-0^{2} \mathrm{~F}_{21 / 2}^{0}\end{array}\right.$ & & & & & & \\
\hline $\begin{array}{l}8 \\
1\end{array}$ & $\begin{array}{l}3237.187 \\
3236.810\end{array}$ & $\begin{array}{l}30882.14 \\
30885.73\end{array}$ & $\mid \begin{array}{l}a^{4} \mathrm{P}_{1 / 2}-u^{4} \mathrm{D}_{21 / 2}^{2} \\
a^{4} \mathrm{G}_{31 / 2}-p^{4} \mathrm{~F}_{21 / 2}^{2}\end{array}$ & & & & & & \\
\hline $\begin{array}{l}2 \\
2 \\
2\end{array}$ & $\begin{array}{l}3236.704 \\
3236.078 \\
3235.182\end{array}$ & $\begin{array}{l}30886.74 \\
30892.72 \\
30901.28\end{array}$ & $\begin{array}{l}a^{4} \mathrm{H}_{41 / 2}-q^{4} \mathrm{~F}_{31 / 2}^{0} \\
a^{2} \mathrm{H}_{51 / 2}-p^{4} \mathrm{~F}^{4} \mathrm{~F}_{1 / 2} \\
a^{4} \mathrm{P}_{21 / 3}-s^{2} \mathrm{~F}_{21 / 2}^{4}\end{array}$ & & & & & & \\
\hline 1 & 3235.066 & 30902.38 & $\left\{\begin{array}{l}a^{6} \mathrm{D}_{11 / 2}-x^{4} \mathrm{G}_{21 / 2} \\
a^{2} \mathrm{D}_{212}-411_{313}^{2}\end{array}\right.$ & & & & & & \\
\hline 1 & 3234.65 & 30906.36 & $a^{4} \mathrm{H}_{31 / 2}-p^{2} \mathrm{~F}_{21 / 2}^{2}$ & & & & & & \\
\hline 3 & 3232.984 & 30922. 29 & $a^{4} \mathrm{P}_{01 / 2}-u^{4} \mathrm{D}_{01 / 2}^{0}$ & 6 & 2. 592 & 1. 296 & 1. 296 & 2. 592 & 0.000 \\
\hline 2 & 3232.891 & 30923.18 & $\left\{\begin{array}{l}a^{6} \mathrm{D}_{41 / 2}-v^{4} \mathrm{~F}_{31 / 2}^{1} \\
a^{4} \mathrm{~F}_{11 / 2}-w^{4} \mathrm{D}_{01 / 3}^{0}\end{array} \mid\right.$ & & & & & & \\
\hline $\begin{array}{l}2 h \\
1 \\
2\end{array}$ & $\begin{array}{l}3232.661 \\
3231.336 \\
3230.794\end{array}$ & $\begin{array}{l}30925.37 \\
30938.05 \\
30943.24\end{array}$ & $\mid \begin{array}{l}b^{4} \mathrm{D}_{11 / 2}-n^{4} \mathrm{D}^{1} 1 / 2 \\
a^{4} \mathrm{D}_{21 / 2}-r^{4} \mathrm{~F}_{11 / 2}^{11} \\
a^{4} \mathrm{H}_{41 / 2}-p^{2} \mathrm{~F}_{31 / 2}^{\circ}\end{array}$ & & & & & & ( \\
\hline $\begin{array}{l}1 \\
1 \\
4 \\
4 \\
2 h\end{array}$ & $\begin{array}{l}3230.53 \\
3229.455 \\
3229.19 \\
3227.711 \\
3227.49\end{array}$ & $\begin{array}{l}30945.77 \\
30956.07 \\
30958.61 \\
30972.80 \\
30974.92\end{array}$ & $\begin{array}{l}a^{4} \mathrm{H}_{51 / 3}-r^{2} \mathrm{G}_{41 / 2}^{\circ} \\
a^{2} \mathrm{P}_{01 / 2}-w^{2} \mathrm{P}_{11 / 2}^{11} \\
a^{4} \mathrm{~F}_{21 / 2}-w^{4} \mathrm{D}_{21 / 2}^{2} \\
a^{4} \mathrm{H}_{31 / 2}-s^{2} \mathrm{G}_{41 / 2}^{4} \\
a^{4} \mathrm{G}_{41 / 2}-q^{4} \mathrm{D}_{31 / 2}^{0}\end{array}$ & & & & & & \\
\hline $\begin{array}{l}9 \\
2 \\
8 \\
3 c \\
1\end{array}$ & $\begin{array}{l}3225.194 \\
3225.009 \\
3224.434 \\
3223.751 \\
3223.448\end{array}$ & $\begin{array}{l}30996.97 \\
30998.74 \\
31004.27 \\
31010.85 \\
31013.76\end{array}$ & $\begin{array}{l}a^{4} \mathrm{~F}_{11 / 2}-u^{4} \mathrm{~F}_{21 / 2}^{\circ} \\
a^{2} \mathrm{P}_{11 / 2}-s^{2} \mathrm{D}_{21 / 2}^{21} \\
a^{4} \mathrm{G}_{21 / 2}-p^{4} \mathrm{~F}_{21 / 2}^{1} \\
a^{4} \mathrm{P}_{21 / 2}-s^{2} \mathrm{~F}_{31 / 2}^{\circ}\end{array}$ & & & & & & \\
\hline $\begin{array}{l}3 \\
6 \\
7 \\
2\end{array}$ & $\begin{array}{l}3223.004 \\
3222.958 \\
3222.754 \\
3221.436\end{array}$ & $\begin{array}{l}31018.03 \\
31018.47 \\
31020.44 \\
31033.13\end{array}$ & $\begin{array}{l}a^{4} \mathrm{P}_{01 / 2}-u^{4} \mathrm{D}_{11 / 2}^{\circ} \\
a^{2} \mathrm{G}_{31 / 2}-v^{2} \mathrm{H}_{41 / 2} \\
a^{6} \mathrm{D}_{11 / 2}-y^{4} \mathrm{~S}_{11 / 2}^{0} \\
a^{2} \mathrm{G}_{41 / 2}-r{ }^{4} \mathrm{~F}_{31 / 2}^{3}\end{array}$ & & & & & & \\
\hline $15, \mathrm{~V}$ & 3221.126 & 31036.11 & $\left\{\begin{array}{l}a^{4} \mathrm{~F}_{21 / 2}-x^{2} \mathrm{D}_{11 / 1}^{0} \\
a^{4} \mathrm{G}_{31 / 2}-p^{4} \mathrm{~F}_{31 / 2}^{\circ}\end{array}\right.$ & 5 & & 0 & 1. 055 & $(1.029)$ & 0.998 \\
\hline $15, \mathrm{~V}$ & $\begin{array}{l}3220.927 \\
3219.681\end{array}$ & $\begin{array}{l}31038.03 \\
31050.04\end{array}$ & $\begin{array}{l}a^{4} \mathrm{G}_{41 / 2}-p{ }^{4} \mathrm{~F}_{41 / 2}^{0} \\
a^{4} \mathrm{G}_{31 / 3}-p p^{4} \mathrm{D}_{21 / 3}^{2}\end{array}$ & 6 & & 0.27 & 1. 20 & $(1.23)$ & 1.17 \\
\hline $\begin{array}{c}6 \\
20, \mathrm{~V}\end{array}$ & $\begin{array}{l}3218.797 \\
3217.865\end{array}$ & $\begin{array}{l}31058.57 \\
31067.57\end{array}$ & $\begin{array}{l}a^{2} \mathrm{G}_{31 / 2}-t^{4} \mathrm{G}_{31 / 2}^{3} \\
a^{4} \mathrm{~F}_{212}-v^{2} \mathrm{~F}^{3} 21\end{array}$ & 6 & & 0.507 & & & \\
\hline $30, \mathrm{~V}$ & 3217.288 & $\begin{array}{l}31057.57 \\
31073.13\end{array}$ & $\mid \begin{array}{l}a \\
a^{4}{ }^{4} \mathrm{~F}_{11 / 3}-v^{2}-w^{4} \mathrm{~S}_{11 / 2}^{6}\end{array}$ & $\begin{array}{l}0 \\
6\end{array}$ & & $-0.42^{*}$ & 1.832 & $(1.721)$ & $\begin{array}{l}0.029 \\
1.943\end{array}$ \\
\hline$\frac{1}{10, \mathrm{~V}}$ & $\begin{array}{l}3215.959 \\
3215.229 \\
3213.884\end{array}$ & $\begin{array}{l}31085.97 \\
31093.03 \\
31106.04\end{array}$ & $\begin{array}{l}b{ }^{4} \mathrm{P}_{11 / 2}-m^{2} \mathrm{~F}_{21 / 2}^{0} \\
a^{2} \mathrm{G}_{41 / 2}-t^{4} \mathrm{G}_{41 / 2} \\
a^{4} \mathrm{~F}_{11 / 2}-w^{4} \mathrm{D}_{11 / 2}^{0}\end{array}$ & & & & & & \\
\hline 1 & 3213.24 & 31112.28 & $\left\{\begin{array}{l}a^{6} \mathrm{D}_{31 / 2}-v{ }^{4} \mathrm{~F}_{21 / 2}^{0} \\
b^{4} \mathrm{P}_{21 / 2}-r{ }^{4} \mathrm{G}_{21 / 2}^{2}\end{array}\right.$ & & & & & & \\
\hline $15, \mathrm{~V}$ & 3210.29 & 31140.86 & $a^{2} \mathrm{G}_{41 / 2}-q^{2} \mathrm{~F}_{31 / 2}^{0}$ & 4 & & $0.172 *$ & 0.851 & (1. 103) & 1.174 \\
\hline $\begin{array}{l}3 \\
1 h \\
5 \\
1 \\
3\end{array}$ & $\begin{array}{l}3208.86 \\
3207.64 \\
3206.812 \\
3206.11 \\
3205.975\end{array}$ & $\begin{array}{l}31154.74 \\
31166.59 \\
31174.64 \\
31181.47 \\
31182.78\end{array}$ & $\begin{array}{l}a^{4} \mathrm{G}_{21 / 2}-p{ }^{4} \mathrm{~F}_{31 / 2}^{0} \\
a^{4} \mathrm{D}_{21 / 2}-r^{4} \mathrm{~F}_{21 / 2}^{2} \\
a^{6} \mathrm{D}_{012}-y^{4} \mathrm{~S}_{11 / 2} \\
a^{2} \mathrm{G}_{31 / 2}-s^{4} \mathrm{~F}_{411 / 2}^{0} \\
a^{2} \mathrm{G}_{31 / 2}-400_{21 / 2}^{0}\end{array}$ & & & & & & \\
\hline $2 c$ & 3204.66 & 31195.57 & $\left\{\begin{array}{l}a^{4} \mathrm{G}_{31 / 2}-q^{4} \mathrm{D}_{31 / 2}^{0} ? \\
a^{4} \mathrm{G}_{41 / 3}-n^{2} \mathrm{~F}_{31 / 6}^{0}\end{array}\right.$ & & & & & & \\
\hline $\begin{array}{l}4 \\
4 \\
15, I V \\
3 c\end{array}$ & $\begin{array}{l}3204.340 \\
3201.498 \\
3200.537 \\
3200.102\end{array}$ & $\begin{array}{l}31198.69 \\
31226.39 \\
31235.76 \\
31240.01\end{array}$ & $\begin{array}{c}a^{4} \mathrm{~F}_{41 / 2}-z^{2} \mathrm{I}_{51 / 2}^{0} \\
a^{2} \mathrm{G}_{41 / 2}-s^{4} \mathrm{G}_{31 / 2}^{0} \\
a^{4} \mathrm{~F}_{31 / 2}-w^{2} \mathrm{D}_{21 / 2}^{0}\end{array}$ & $\begin{array}{l}7 b, 4 \\
5\end{array}$ & & $\begin{array}{l}0 \\
0\end{array}$ & $\begin{array}{l}1.100 \\
1.268\end{array}$ & $\begin{array}{l}(1.103) \\
(1.235)\end{array}$ & $\begin{array}{l}\text { 1. } 104 \\
1.209\end{array}$ \\
\hline $\begin{array}{l}3 \\
1 h \\
5 \\
1 \\
3\end{array}$ & $\begin{array}{l}\text { 3198. } 182 \\
3197.94 \\
3196.18 \\
3194.64 \\
3194.367\end{array}$ & $\begin{array}{l}31258.76 \\
31261.12 \\
31278.34 \\
31293.42 \\
31296.09\end{array}$ & 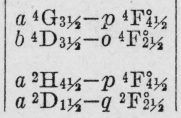 & & & & & & \\
\hline
\end{tabular}


TABLE 4.-First spectrum of columbium (Cb I) - Continued

\begin{tabular}{|c|c|c|c|c|c|c|c|c|c|}
\hline $\begin{array}{c}\text { Intensity } \\
\text { Arc }\end{array}$ & $\lambda_{\text {air }} \mathrm{A}$ & $\begin{array}{l}\text { Wave } \\
\text { No. } \\
\text { vac. } \\
\mathrm{cm}^{-1}\end{array}$ & $\begin{array}{l}\text { Term combi- } \\
\text { nation }\end{array}$ & $\begin{array}{c}\text { Zeeman } \\
\text { type }\end{array}$ & $\begin{array}{l}\text { Separa- } \\
\text { tion }\end{array}$ & $\begin{array}{l}\text { Strong- } \\
\text { est } \\
p\end{array}$ & $\begin{array}{c}\text { Strong- } \\
\text { est } \\
n\end{array}$ & $g_{1}$ & $g_{2}$ \\
\hline 1 & 2 & 3 & 4 & 5 & 6 & 7 & 8 & 9 & 10 \\
\hline $\begin{array}{l}4 c \\
2 \\
3 \\
4 \\
40, \mathrm{IV}\end{array}$ & $\begin{array}{l}3193.003 \\
3192.73 \\
3190.490 \\
3189.560 \\
3187.497\end{array}$ & $\begin{array}{l}31309.46 \\
31312.14 \\
31334.12 \\
31343.26 \\
31363.54\end{array}$ & $\begin{array}{l}a^{6} \mathrm{D}_{31 / 2}-x^{4} \mathrm{G}_{41 / 2}^{0} \\
a^{4} \mathrm{~F}_{21 / 2}-v^{2} \mathrm{~F}_{31 / 2}^{0} \\
a^{2} \mathrm{D}_{21 / 2}-8^{2} \mathrm{G}_{31 / 2}^{1} \\
a^{4} \mathrm{~F}_{41 / 2}-v^{4} \mathrm{D}_{31 / 2}^{\circ}\end{array}$ & 4 & & $w$ & 1.145 & $(1.330)$ & 1. 383 \\
\hline $\begin{array}{l}15, \mathrm{~V} \\
2 \\
1 \\
4 c \\
5\end{array}$ & $\begin{array}{l}3186.550 \\
3185.55 \\
3184.77 \\
3182.399 \\
3182.063\end{array}$ & $\begin{array}{l}31372.86 \\
31382.71 \\
31390.39 \\
31413.78 \\
31417.10\end{array}$ & $\begin{array}{l}a^{4} \mathrm{P}_{01 / 2}-w^{4} \mathrm{~S}_{1 / 2}^{0} \\
a^{2} \mathrm{G}_{31 / 2}-r^{4} \mathrm{~F}_{21 / 2}^{2} \\
a^{2} \mathrm{~F}_{31 / 2}-0^{4} \mathrm{D}_{21 / 2}^{2} \\
b^{2} \mathrm{H}_{41 / 2}-0^{2} \mathrm{G}_{41 / 6}^{21 / 6} \\
a^{4} \mathrm{~F}_{21 / 2}-w^{4} \mathrm{D}_{31 / 3}^{3}\end{array}$ & 4 & 0.700 & 0.355 & 1. 603 & 2. 653 & 1. 953 \\
\hline $\begin{array}{l}1 h \\
2 \\
2 \\
1\end{array}$ & $\begin{array}{l}3181.11 \\
3180.79 \\
3178.632 \\
3178.17\end{array}$ & $\begin{array}{l}31426.51 \\
31429.67 \\
31451.01 \\
31455.58\end{array}$ & 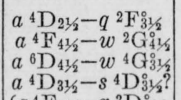 & & & & & & \\
\hline 3 & 3175.68 & 31480.24 & $\left\{\begin{array}{l}a^{4} \mathrm{~F}_{11 / 2}-x^{2} \mathrm{D}_{11 / 2}^{\circ} \\
b^{4} \mathrm{D}_{11 / 2}-469_{21 / 2}^{\circ}\end{array}\right.$ & & & & & & \\
\hline $\begin{array}{c}4 \\
12, \mathrm{~V}\end{array}$ & $\begin{array}{l}3173.677 \\
3172.511 \\
3172.304\end{array}$ & $\begin{array}{l}31500.11 \\
31511.69 \\
31513.74\end{array}$ & $\begin{array}{l}a^{4} \mathrm{~F}_{21 / 1}-w^{2} \mathrm{D}^{0} 11 / 2 \\
a^{4} \mathrm{~F}_{11 / 2}-v^{2} \mathrm{~F}_{21 / 2}^{\circ} \\
a^{4} \mathrm{~F}_{413 / 2}-w^{2} \mathrm{G}_{31 / 2}^{3}\end{array}$ & 5 & 0.437 & 0.212 & 1.452 & 0.360 & 0.797 \\
\hline $\begin{array}{c}10 c \\
6\end{array}$ & $\begin{array}{l}3171.425 \\
3171.168\end{array}$ & $\begin{array}{l}31522.48 \\
31525.03\end{array}$ & $\left\{\begin{array}{l}a^{6} \mathrm{D}_{41 / 1}-x^{4} \mathrm{G}_{51 / 2}^{0} \\
a^{4} \mathrm{G}_{4 / 2}-q^{2} \mathrm{G}_{31 / 2}^{0} \\
a^{2} \mathrm{G}_{41 / 2}-r^{4} \mathrm{~F}^{6}{ }_{41 / 6}^{6}\end{array}\right.$ & & & & & & \\
\hline $\begin{array}{l}4 \\
8 \\
2 \\
2 h \\
1 h\end{array}$ & $\begin{array}{l}3170.163 \\
3168.599 \\
3168.141 \\
3166.59 \\
3166.49\end{array}$ & $\begin{array}{l}31535.03 \\
31550.60 \\
31555.15 \\
31570.60 \\
31571.60\end{array}$ & 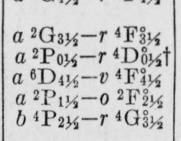 & & & 0 & 0.726 & & \\
\hline $\begin{array}{l}1 \\
2\end{array}$ & $\begin{array}{l}3166.230 \\
3164.488\end{array}$ & $\begin{array}{l}31574.19 \\
31591.57\end{array}$ & $\begin{array}{l}a^{4} \mathrm{P}_{21 / 2}-t^{4} \mathrm{~F}_{31 / 2}^{0} \\
a^{2} \mathrm{D}_{21 / 2}-p^{2} \mathrm{~F}_{21 / 2}^{2}\end{array}$ & & & & & & \\
\hline 1 & 3164.148 & 31594.97 & $\left\{\begin{array}{l}a^{2} \mathrm{~F}_{31 / 2}-u^{2} \mathrm{H}^{2}{ }^{2} / 1 / 2 \\
a^{2} \mathrm{G}_{31 / 2}-t^{4} \mathrm{G}_{41 / 3}\end{array}\right.$ & & & & & & \\
\hline $\begin{array}{l}1 \\
8\end{array}$ & $\begin{array}{l}\text { 3161. } 49 \\
3161.194\end{array}$ & $\begin{array}{l}31621.53 \\
31624.49\end{array}$ & $\begin{array}{l}a^{6} \mathrm{D}_{21 / 2}-w^{4} \mathrm{~F}^{21 / 3} \\
a^{2} \mathrm{G}_{41 / 3}-s^{4} \mathrm{D}_{31 / 2}^{3}\end{array}$ & & & & & & \\
\hline $\begin{array}{l}3 \\
6 \\
2 h \\
2 \\
2\end{array}$ & $\begin{array}{l}3160.60 \\
3159.322 \\
3159.21 \\
3158.962 \\
3157.73\end{array}$ & $\begin{array}{l}31630.44 \\
31643.13 \\
31644.35 \\
31646.84 \\
31659.18\end{array}$ & $\begin{array}{c}a^{2} \mathrm{D}_{11 / 2}-w^{2} \mathrm{P}_{11 / 2}^{i} \\
b^{4} \mathrm{P}_{21 / 3}-0^{4} \mathrm{~F}_{21 / 2}^{2} \\
a^{2} \mathrm{G}_{31 / 1}-s^{4} \mathrm{D}_{21 / 5}^{21} \\
a^{4} \mathrm{P}_{21 / 2}-x^{4} \mathrm{H}_{31 / 2}^{13}\end{array}$ & 6 & 0.434 & 0.651 & 1. 165 & 0.948 & 1. 382 \\
\hline $\begin{array}{r}2 \\
10\end{array}$ & $\begin{array}{l}3154.321 \\
3153.98\end{array}$ & $\begin{array}{l}31693.40 \\
31696.82\end{array}$ & $a^{2} \mathrm{D}_{21 / 2}-q^{4} \mathrm{~F}_{31 / 2}^{0}$ & & & 0 & 0.97 & & \\
\hline $\begin{array}{l}30, \mathrm{IV} \\
6 \\
2\end{array}$ & $\begin{array}{l}3151.870 \\
3148.710 \\
3146.863\end{array}$ & $\begin{array}{l}31718.04 \\
31749.87 \\
31768.51\end{array}$ & $\begin{array}{l}a^{4} \mathrm{~F}_{31 / 2}-v^{4} \mathrm{D}_{21 / 2}^{\circ} \\
a^{2} \mathrm{D}_{21 / 2}-p^{2} \mathrm{~F}_{31 / 2}^{3} \\
a^{4} \mathrm{D}_{11 / 2}-s^{4} \mathrm{D}_{213 / 2}\end{array}$ & $\begin{array}{l}4 \\
4\end{array}$ & & 0 & $\begin{array}{l}0.998 \\
0.976\end{array}$ & $\begin{array}{l}(1.235) \\
(1.206)\end{array}$ & $\begin{array}{l}1.330 \\
1.140\end{array}$ \\
\hline $\begin{array}{l}5 \\
1 h \\
1 \\
1 h \\
2\end{array}$ & $\begin{array}{l}3145.920 \\
3144.45 \\
3143.44 \\
3142.926 \\
3142.644\end{array}$ & $\begin{array}{l}31778.03 \\
31792.9 \\
31803.1 \\
31808.30 \\
31811.16\end{array}$ & $\begin{array}{l}a^{2} \mathrm{H}_{41 / 3}-q^{2} \mathrm{G}_{31 / 2}^{0} \\
b^{4} \mathrm{P}_{11 / 2}-v^{2} \mathrm{P}_{01 / 2}^{0} \\
a^{4} \mathrm{~F}_{21 / 2}-w^{2} \mathrm{D}_{21 / 2}^{21} \\
b^{4} \mathrm{D}_{21 / 2}-n^{4} \mathrm{D}_{31 / 2}^{\circ} \\
a^{2} \mathrm{G}_{41 / 2}-411_{31 / 2}^{\circ}\end{array}$ & & & & & & \\
\hline $\begin{array}{c}2 \\
1 \\
15, \mathrm{~V} \\
1 \\
1\end{array}$ & $\begin{array}{l}3142.384 \\
3138.888 \\
3136.972 \\
3136.49 \\
3134.835\end{array}$ & $\begin{array}{l}31813.79 \\
31849.22 \\
31868.67 \\
31873.6 \\
31890.40\end{array}$ & $\begin{array}{l}a^{4} \mathrm{P}_{113 / 2}-t^{4} \mathrm{~F}_{11 / 2}^{0} \\
a^{4} \mathrm{P}_{21 / 2}-r^{2} \mathrm{~F}_{21 / 2}^{2} \\
a^{4} \mathrm{~F}_{113}-v^{4} \mathrm{D}_{01 / 2}^{0} \\
a^{6} \mathrm{D}_{41 / 2}-w^{4} \mathrm{~F}_{41 / 2}^{11} \\
a^{4} \mathrm{P}_{11 / 2}-u^{4} \mathrm{G}_{21 / 2}\end{array}$ & 4 & & 0 & 0.381 & $(0.402)$ & 0.444 \\
\hline $\begin{array}{l}2 \\
1 \\
15\end{array}$ & $\begin{array}{l}3134.620 \\
3133.89 \\
3133.088\end{array}$ & $\begin{array}{l}31892.58 \\
31900.00 \\
31908.18\end{array}$ & $\begin{array}{l}a^{2} \mathrm{~F}_{21 / 2}-m^{2} \mathrm{~F}_{21 / 2}^{0} \\
a^{4} \mathrm{P}^{2} \mathrm{P}_{21 / 3}-t^{2} \mathrm{D}_{11 / 2}\end{array}$ & & & & & & \\
\hline $\begin{array}{c}15, \mathrm{~V} \\
1\end{array}$ & $\begin{array}{l}3133.088 \\
3132.881\end{array}$ & $\begin{array}{l}31908.18 \\
31910.28\end{array}$ & $\begin{array}{l}a^{2} \mathrm{G}_{31 / 2}-q^{2} \mathrm{~F}_{21 / 2}^{2} \\
a^{6} \mathrm{D}_{31 / 2}-v \\
a^{4} \mathrm{~F}_{41 / 2}^{0} \mathrm{D}_{21 / 2}-s^{4} \mathrm{D}_{316}\end{array}$ & 4 & & 0 & 0.833 & $(0.885)$ & 0.927 \\
\hline 2 & 3132. 589 & 31913. 26 & $b^{4} \mathrm{P}_{21 / 2}-n^{4} \mathrm{D}_{21 / 2}^{1}$ & & & & & 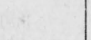 & \\
\hline $\begin{array}{l}2 h \\
4 \\
2 \\
3 \\
15, \mathrm{~V}\end{array}$ & $\begin{array}{l}3131.61 \\
3129.554 \\
3125.197 \\
3125.12 \\
3122.646\end{array}$ & $\begin{array}{l}31923.2 \\
31944.21 \\
31988.74 \\
31989.5 \\
32014.87\end{array}$ & $\begin{array}{l}b^{4} \mathrm{~F}_{31 / 2}-0^{4} \mathrm{D}_{21 / 2}^{0} \\
a^{4} \mathrm{~F}_{11 / 2}-w^{2} \mathrm{D}_{1 / 2}^{\circ} \\
a^{4} \mathrm{P}_{11 / 2}-t^{4} \mathrm{~F}_{21 / 2}^{\circ} \\
a^{4} \mathrm{P}_{21 / 2}-t^{4} \mathrm{D}_{11 / 2}^{0} \\
a^{4} \mathrm{~F}_{31 / 2}-v^{4} \mathrm{D}_{31 / 2}^{0}\end{array}$ & 6 & 0.157 & 0.548 & 1.307 & 1. 229 & 1.386 \\
\hline
\end{tabular}


TABLE 4.-First spectrum of columbium (Cb I) - Continued

\begin{tabular}{|c|c|c|c|c|c|c|c|c|c|}
\hline $\begin{array}{l}\text { Intensity } \\
\text { Arc }\end{array}$ & $\lambda_{\text {air }} \mathrm{A}$ & $\begin{array}{l}\text { Wave } \\
\text { No. } \\
\text { vac. } \\
\mathrm{cm}^{-1}\end{array}$ & $\begin{array}{c}\text { Term combi- } \\
\text { nation }\end{array}$ & $\begin{array}{c}\text { Zeeman } \\
\text { type }\end{array}$ & $\begin{array}{c}\text { Separa- } \\
\text { tion }\end{array}$ & $\begin{array}{c}\text { Strong- } \\
\text { est } \\
p\end{array}$ & $\begin{array}{l}\text { Strong- } \\
\text { est } \\
n\end{array}$ & $g_{1}$ & $g_{2}$ \\
\hline 1 & 2 & 3 & 4 & 5 & 6 & 7 & 8 & 9 & 10 \\
\hline${ }_{5}^{1 h}$ & $\begin{array}{l}3122.13 \\
3121.964\end{array}$ & $\begin{array}{l}32020.2 \\
32021.86\end{array}$ & $\begin{array}{l}b^{4} \mathrm{P}_{21 / 2}-469_{21 / 3}^{\circ} \\
a^{2} \mathrm{D}_{11 / 2}-414_{11 / 3}^{19}\end{array}$ & 6 & 0.483 & 0.724 & 1.180 & 0.939 & 1. 422 \\
\hline $2 h$ & 3121.66 & 32024.98 & $\left\{\begin{array}{l}a a^{2} \mathrm{P}_{13 / 3}-n^{2} \mathrm{~F}_{21 / 1}^{\circ} \\
b^{4} \mathrm{~F}_{41 / 3}-0^{4} \mathrm{D}_{31 / 6}^{0}\end{array}\right.$ & & & & & & \\
\hline $\begin{array}{l}3 h \\
5\end{array}$ & $\begin{array}{l}3121.35 \\
3120.878\end{array}$ & $\begin{array}{l}\text { 32028.2 } \\
32033.01\end{array}$ & $a^{4} \mathrm{~F}_{43 / 2}-z^{2}{ }^{2} \mathrm{H}_{3 / 2}^{3}$ & & & & & & \\
\hline $\begin{array}{c}3 \\
6 \\
1 \\
15, \mathrm{~V} \\
1\end{array}$ & $\begin{array}{l}3120.276 \\
3119.402 \\
3117.65 \\
3116.366 \\
3114.934\end{array}$ & $\begin{array}{l}32039.19 \\
32048.16 \\
32066.2 \\
32079.38 \\
32094.13\end{array}$ & 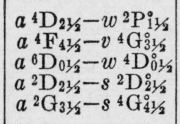 & 4 & & 0 & 1.194 & (1.206) & 1. 214 \\
\hline${ }_{2 h}^{1}$ & $\begin{array}{l}\text { 3114. } 51 \\
3114.38\end{array}$ & $\begin{array}{l}32098.5 \\
32099.8\end{array}$ & 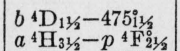 & & & & & & \\
\hline 4 & 3113.061 & 32113.44 & $\left\{\begin{array}{l}a^{4} \mathrm{P}_{01 / 2}-\bar{t}^{4} \mathrm{~F}_{1 / 2}^{1} \\
b^{4} \mathrm{~F}_{413}-u^{2} \mathrm{H}^{2}\end{array}\right.$ & & & & & & \\
\hline $\begin{array}{l}3 \\
2 h\end{array}$ & $\begin{array}{l}3112.630 \\
3112.08\end{array}$ & $\begin{array}{l}32117.89 \\
32123.6\end{array}$ & $\begin{array}{l}a^{4} \mathrm{D}_{33 / 2}-411_{21} 1 / 2 \\
b^{4} \mathrm{P}_{21 / 5}-0^{4} \mathrm{~F}_{3 / 2}\end{array}$ & & & & & & \\
\hline $\begin{array}{c}1 \\
2 \\
20, \mathrm{~V} \\
5 \\
3\end{array}$ & $\begin{array}{l}3111.79 \\
3111.558 \\
3111.446 \\
3109.735 \\
3108.07\end{array}$ & $\begin{array}{l}32126.6 \\
32128.95 \\
32130.11 \\
32147.78 \\
32165.0\end{array}$ & 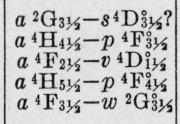 & 4 & 0.201 & 0.100 & 0.730 & 1. 031 & 1. 232 \\
\hline $\begin{array}{l}1 \\
2 h\end{array}$ & $\begin{array}{l}3105.79 \\
3104.86\end{array}$ & $\begin{array}{l}32188.61 \\
32198.26\end{array}$ & 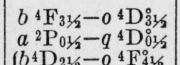 & & & & & & \\
\hline 1 & 3103. 39 & 32213.5 & 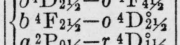 & & & & & & \\
\hline $\begin{array}{l}1 h \\
2 \\
5\end{array}$ & $\begin{array}{l}3101.69 \\
3101.064 \\
3100.97\end{array}$ & $\begin{array}{l}32231.2 \\
32237.67 \\
32238.6\end{array}$ & 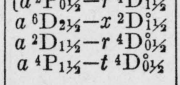 & & & & & & \\
\hline 3 & 3100.163 & 32247.04 & $\left\{\begin{array}{l}a^{4} \mathrm{~F}_{113 / 2}-w^{2} \mathrm{D}_{21 / 2}^{2} \\
b^{4} \mathrm{~F}_{21 / 2}-0^{4} \mathrm{D}_{11 / 2}^{2}\end{array}\right.$ & & & & & & \\
\hline $\begin{array}{c}2 \\
2 \\
3 h \\
10, \mathrm{~V}\end{array}$ & $\begin{array}{l}\text { 3098. } 98 \\
3098.780 \\
3098.300 \\
3096.490\end{array}$ & $\begin{array}{l}32259.4 \\
32261.43 \\
32266.43 \\
32285.29\end{array}$ & 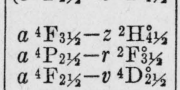 & 6 & 0.331 & 0.813 & 1.198 & 1.034 & 1. 363 \\
\hline $\begin{array}{l}2 \\
5 \\
1\end{array}$ & $\begin{array}{l}3095.22 \\
3094.37 \\
3093.65\end{array}$ & $\begin{array}{l}32298.5 \\
32307.4 \\
32314.9\end{array}$ & 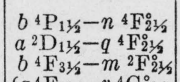 & & & & & & \\
\hline 2 & 3090.13 & 32351.7 & $\left\{\begin{array}{l}a^{4} \mathrm{~F}_{41 / 3}-v^{4} \mathrm{G}_{41 / 2}^{01 / 2} \\
a^{4} \mathrm{H}_{41 / 2}-p^{4} \mathrm{~F}_{41 / 3}^{0}\end{array}\right.$ & & & & & & \\
\hline 9 & 3088.05 & 32373.5 & $a^{4} \mathrm{~F}_{41 / 2}-u^{2} \mathrm{~F}_{3 / 2}$ & & & & & & \\
\hline $\begin{array}{l}1 h \\
4 \\
2 \\
1 h \\
4 h\end{array}$ & $\begin{array}{l}3087.75 \\
3087.625 \\
3086.475 \\
3085.67 \\
3084.94\end{array}$ & $\begin{array}{l}32376.7 \\
32377.98 \\
32300.04 \\
32398.5 \\
32406.2\end{array}$ & 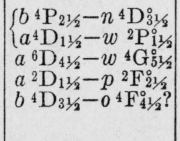 & & & & & & \\
\hline $\begin{array}{r}2 \\
1 \\
10 \\
3 \\
3\end{array}$ & $\begin{array}{l}3083.828 \\
3083.607 \\
3082.859 \\
3077.800 \\
3075.816\end{array}$ & $\begin{array}{l}32417.85 \\
32420.17 \\
32428.03 \\
32481.33 \\
32502.28\end{array}$ & $\begin{array}{l}a^{4} \mathrm{D}_{21 / 2}-414_{1 / 2}^{0} \\
a^{4} \mathrm{H}_{31 / 2}-n^{2} \mathrm{~F}_{21 / 2} \\
a^{4} \mathrm{~A}_{21 / 2}-t^{4} \mathrm{D}_{21 / 2} \\
a^{4} \mathrm{P}_{21 / 2}-x^{2} \mathrm{P}_{11 / 2}\end{array}$ & & & & & & \\
\hline $\begin{array}{r}3 \\
10 \\
7 \\
1 \\
1\end{array}$ & $\begin{array}{l}3074.447 \\
3072.397 \\
3072.296 \\
3071.77 \\
3071.34\end{array}$ & $\begin{array}{l}32516.76 \\
32538.45 \\
32539.52 \\
32545.1 \\
32549.6\end{array}$ & $\begin{array}{l}a^{4} \mathrm{P}_{11 / 2}-r^{2} \mathrm{~F}_{21 / 2}^{0} \\
a^{4} \mathrm{P}_{013}-t^{4} \mathrm{D}_{01 / 2}^{0} \\
a^{4} \mathrm{~F}_{41 / 2}-y^{2} \mathrm{H}_{51 / 2}^{5} \\
a^{2} \mathrm{G}_{41 / 2}-q^{4} \mathrm{~F}_{41 / 2}^{0} \\
a^{4} \mathrm{~F}_{31 / 2}-x^{4} \mathrm{P}_{21 / 2}^{2}\end{array}$ & & & & & & \\
\hline $\begin{array}{r}10 \\
3 \\
2 \\
10 \\
8\end{array}$ & $\begin{array}{l}3069.023 \\
3066.41 \\
3061.78 \\
3061.232 \\
3061.100\end{array}$ & $\begin{array}{l}32574.42 \\
32602.0 \\
32651.3 \\
32657.12 \\
32658.53\end{array}$ & $\begin{array}{l}a^{4} \mathrm{~F}_{11 / 2}-v^{4} \mathrm{D}_{11 / 2}^{\circ} \\
a^{2} \mathrm{G}_{41 / 2}-q^{4} \mathrm{~F}_{31 / 2}^{1} \\
a^{2} \mathrm{D}_{21 / 2}-0^{2} \mathrm{~F}_{21 / 2} \\
a^{4} \mathrm{P}_{11 / 2}-t^{4} \mathrm{D}_{11 / 2}^{0} \\
a^{2} \mathrm{G}_{41 / 2}-p^{2} \mathrm{~F}_{31 / 2}^{0}\end{array}$ & & & & 1.057 & & 1. 129 \\
\hline
\end{tabular}


TABLE 4.-First spectrum of columbium (Cb I)-Continued

\begin{tabular}{|c|c|c|c|c|c|c|c|c|c|}
\hline $\begin{array}{l}\text { Intensity } \\
\text { Arc }\end{array}$ & $\lambda_{\text {air }} \mathrm{A}$ & $\begin{array}{l}\text { Wave } \\
\text { No. } \\
\text { vac. } \\
\mathrm{cm}^{-1}\end{array}$ & $\begin{array}{l}\text { Term combi- } \\
\text { nation }\end{array}$ & $\begin{array}{c}\text { Zeeman } \\
\text { type }\end{array}$ & $\begin{array}{l}\text { Separa- } \\
\text { tion }\end{array}$ & $\begin{array}{c}\text { Strong- } \\
\text { est } \\
p\end{array}$ & $\begin{array}{c}\text { Strong- } \\
\text { est } \\
n\end{array}$ & $g_{1}$ & $g_{2}$ \\
\hline 1 & 2 & 3 & 4 & 5 & 6 & 7 & 8 & 9 & 10 \\
\hline $\begin{array}{r}5 \\
9 \\
1 \\
3 \\
10\end{array}$ & $\begin{array}{l}3060.39 \\
3056.608 \\
3054.47 \\
3054.14 \\
3053.086\end{array}$ & $\begin{array}{l}32666.1 \\
32706.52 \\
32729.4 \\
32733.0 \\
32744.25\end{array}$ & $\begin{array}{l}a^{4} \mathrm{~F}_{21 / 2}-y^{2} \mathrm{P}_{11 / 2} \\
a^{4} \mathrm{~F}_{11 / 2}-v^{4} \mathrm{D}_{21 / 2}^{0} \\
a^{4} \mathrm{P}_{21 / 2}-w^{4} \mathrm{P}_{11 / 2}\end{array}$ & 4 & & $0.133 h$ & $\begin{array}{l}0.904 \\
1.497\end{array}$ & (1. 596) & 1.662 \\
\hline $\begin{array}{l}6 \\
5\end{array}$ & $\begin{array}{l}3052.988 \\
3052.73\end{array}$ & $\begin{array}{l}32745.30 \\
32748.1\end{array}$ & $a^{2} \mathrm{P}_{1 / 2}-v^{2} \mathrm{P}_{11 / 2}^{0}$ & 6 & & 0 & 1. 215 & (1.175) & 1.255 \\
\hline $\begin{array}{r}8 \\
6 \\
20\end{array}$ & $\begin{array}{l}3051.990 \\
3051.666 \\
3048.093\end{array}$ & $\begin{array}{l}32756.01 \\
32759.48 \\
32797.88\end{array}$ & $\begin{array}{l}a^{2} \mathrm{H}_{51 / 2}-u^{2} \mathrm{H}_{51 / 2}^{\circ} \\
a^{4} \mathrm{~F}_{1 / 1}-y^{2} \mathrm{P}_{01 / 2}^{\circ} \\
a^{4} \mathrm{P}_{21 / 2}-w^{4} \mathrm{P}_{21 / 2}^{6}\end{array}$ & $\begin{array}{l}6 \\
4 \\
7 b, 6\end{array}$ & & $\begin{array}{l}0 \\
0 \\
0\end{array}$ & $\begin{array}{l}1.08 \\
0.381 \\
1.598\end{array}$ & $\begin{array}{l}(1.10) \\
(0.402) \\
(1.596)\end{array}$ & $\begin{array}{l}1.06 \\
0.440 \\
1.600\end{array}$ \\
\hline $\begin{array}{l}5 \\
5 \\
5 \\
5 \\
1\end{array}$ & $\begin{array}{l}3045.546 \\
3041.89 \\
3041.660 \\
3041.358 \\
3040.67\end{array}$ & $\begin{array}{l}32825.31 \\
32864.8 \\
32867.25 \\
32870.51 \\
32877.9\end{array}$ & $\begin{array}{l}a{ }^{4} \mathrm{~F}_{41 / 1}-v^{4} \mathrm{G}_{53 / 2}^{\circ} \\
a^{2} \mathrm{G}_{41 / 2}-r^{2} \mathrm{G}_{41 / 2}^{2} \\
a^{4} \mathrm{P}_{01 / 2}-t^{2} \mathrm{D}_{11 / 2}^{1} \\
a^{2} \mathrm{D}_{11 / 2}-s^{2} \mathrm{D}_{21 / 2}^{\circ}\end{array}$ & & & 0 & 0.960 & & \\
\hline $\begin{array}{r}3 \\
20 \\
7 \\
5 \\
3\end{array}$ & $\begin{array}{l}3040.25 \\
3039.68 \\
3039.19 \\
3033.396 \\
3029.23\end{array}$ & $\begin{array}{l}32882.5 \\
32888.7 \\
32894.0 \\
32956.78 \\
33002.1\end{array}$ & $\begin{array}{l}a{ }^{4} \mathrm{P}_{11 / 2}-t^{2} \mathrm{D}_{21 / 2}^{0} \\
a^{4} \mathrm{P}_{21 / 2}-t^{4} \mathrm{D}_{31 / 2}^{0} \\
a^{4} \mathrm{P}_{01 / 2}-t^{4} \mathrm{D}_{11 / 2}^{\circ} \\
a^{2} \mathrm{G}_{31 / 2}-p^{2} \mathrm{~F}_{21 / 2}^{6}\end{array}$ & & & & & & \\
\hline $\begin{array}{l}4 \\
7 \\
7 \\
1 \\
2\end{array}$ & $\begin{array}{l}3029.17 \\
3028.69 \\
3027.89 \\
3027.16 \\
3023.38\end{array}$ & $\begin{array}{l}33002.8 \\
33008.0 \\
33016.7 \\
33024.7 \\
33066.0\end{array}$ & 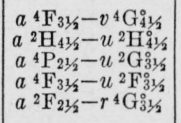 & & & & & & \\
\hline $\begin{array}{l}20 \\
3 h \\
2 h \\
1 \\
8\end{array}$ & $\begin{array}{l}\text { 3020. } 668 \\
3019.780 \\
3019.34 \\
\text { 3016. } 258 \\
\text { 3015. } 24\end{array}$ & $\begin{array}{l}33095.65 \\
33105.38 \\
33110.2 \\
33144.04 \\
33155.2\end{array}$ & 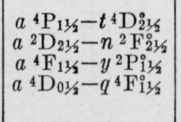 & 4 & 0.389 & 0.146 & 0.804 & 1. 777 & 1. 388 \\
\hline $3 h$ & 3013.27 & 33176.9 & $\left\{\begin{array}{l}a^{6} \mathrm{D}_{31 / 2}-v^{4} \mathrm{D}_{21 / 2}^{\circ} \\
a^{2} \mathrm{D}_{21 / 2}-p^{4} \mathrm{D}_{31 / 2}^{3}\end{array}\right.$ & & & & & & \\
\hline 5 & 3012.546 & 33184.87 & $\left\{\begin{array}{l}a a^{4} \mathrm{P}_{01 / 2}-x^{2} \mathrm{P}_{01 / 2}^{\circ} \\
a^{6} \mathrm{D}_{41 / 3}-w^{2} \mathrm{G}_{41 / 3}\end{array}\right.$ & 6 & & 0 & 1.608 & $(2.650)$ & 0.566 \\
\hline $\begin{array}{l}1 \\
3 \\
9\end{array}$ & $\begin{array}{l}\text { 3007. } 284 \\
3006.964 \\
3005.141\end{array}$ & $\begin{array}{l}33242.94 \\
33246.46 \\
33266.64\end{array}$ & $\begin{array}{l}a^{4} \mathrm{~F}_{41 / 2}-v^{2} \mathrm{G}_{31 / 2}^{5} \\
a^{4} \mathrm{~F}_{23 / 2}-v^{4} \mathrm{G}_{3}^{\circ} 3 / 2\end{array}$ & 4 & & 0 & 0.994 & (1.029) & 1. 013 \\
\hline $\begin{array}{l}1 \\
4 \\
7 h \\
1 \\
5\end{array}$ & $\begin{array}{l}3004.49 \\
3000.72 \\
3000.11 \\
2998.312 \\
2998.220\end{array}$ & $\begin{array}{l}33273.8 \\
33315.6 \\
33322.4 \\
33342.41 \\
33343.43\end{array}$ & $\begin{array}{l}a{ }^{4} \mathrm{D}_{21 / 1}-s^{2} \mathrm{D}_{21 / 5}^{2} \\
a^{2} \mathrm{D}_{21 / 2}-n^{2} \mathrm{~F}_{31 / 2}^{3} \\
a^{4} \mathrm{~F}_{31 / 2}-y^{2} \mathrm{H}_{131}^{\circ} \\
a^{4} \mathrm{~F}_{31 / 2}-v^{2} \mathrm{D}_{21 / 2}^{43}\end{array}$ & & & & & & \\
\hline $\begin{array}{l}6 h \\
5 \\
1 \\
6 \\
6\end{array}$ & $\begin{array}{l}2996.49 \\
2992.084 \\
2989.63 \\
2988.789 \\
2988.692\end{array}$ & $\begin{array}{l}33362.7 \\
33411.80 \\
33439.2 \\
33448.64 \\
33449.72\end{array}$ & 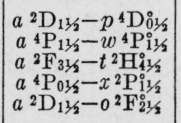 & & & & & & \\
\hline $\begin{array}{c}15 \\
3 h \\
1 \\
4 \\
5\end{array}$ & $\begin{array}{l}2987.286 \\
2986.84 \\
2986.02 \\
2984.077 \\
2983.13\end{array}$ & $\begin{array}{l}33465.47 \\
33470.5 \\
33479.6 \\
33501.47 \\
33512.1\end{array}$ & 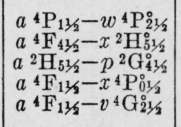 & 4 & & $w$ & 1. 382 & (1. 721) & 1.586 \\
\hline $\begin{array}{r}15 \\
1 \\
5 \\
1 \\
3\end{array}$ & $\begin{array}{l}2981.636 \\
2981.28 \\
2974.54 \\
2973.83 \\
2972.47\end{array}$ & $\begin{array}{l}33528.88 \\
33532.9 \\
33608.9 \\
33616.9 \\
33632.3\end{array}$ & $\begin{array}{l}a^{4} \mathrm{~F}_{41 / 3}-u^{4} \mathrm{D}_{31 / 3}^{\circ} \\
a^{4} \mathrm{~F}_{21 / 2}-x^{4} \mathrm{~S}_{1 / 2}^{i} \\
a^{2} \mathrm{H}_{413 / 2}-p^{2} \mathrm{G}_{31 / 2}^{\circ}\end{array}$ & 4 & & 0 & 1. 227 & $(1.330)$ & 1.389 \\
\hline $\begin{array}{r}1 \\
10 \\
5\end{array}$ & $\begin{array}{l}2969.594 \\
2965.48 \\
2963.682\end{array}$ & $\begin{array}{l}33664.84 \\
33711.5 \\
33731.99\end{array}$ & $\begin{array}{l}a^{4} \mathrm{~F}_{11 / 5}-y^{2} \mathrm{~S}_{01 / 1}^{0} \\
a^{4} \mathrm{P}_{01 / 1}^{0}-w^{4} \mathrm{P}_{1 / 1}^{0} \\
a^{4} \mathrm{P}_{01 / 3}-w^{4} \mathrm{P}_{01 / 2}^{0}\end{array}$ & $\begin{array}{l}4 \\
6\end{array}$ & 0.946 & $\begin{array}{l}0.462 \\
0\end{array}$ & $\begin{array}{l}1.186 \\
2.601\end{array}$ & $\begin{array}{c}2.605 \\
(2.650)\end{array}$ & $\begin{array}{l}1.659 \\
2.552\end{array}$ \\
\hline 1 & 2962.288 & 33747.86 & $\left\{\begin{array}{l}a^{2} \mathrm{D}_{11 / 3}-p^{4} \mathrm{D}_{21 / 5}^{2} \\
a^{6} \mathrm{D}_{11 / 3}-y^{2} \mathrm{P}_{013 / 6}^{\circ}\end{array}\right.$ & & & & & & \\
\hline 3 & 2959,973 & 33774,25 & $\left|a^{4} \mathrm{~F}_{31 / 2}-u^{2} \mathrm{D}_{21 / 3}^{2}\right|$ & 5 & & 0 & 1,326 & (1. 235) & 1,162 \\
\hline
\end{tabular}


564 Journal of Research of the National Bureau of Standards

TABLE 4.-First spectrum of columbium (Cb I)-Continued

\begin{tabular}{|c|c|c|c|c|c|c|c|c|c|}
\hline $\begin{array}{l}\text { Intensity } \\
\text { Arc }\end{array}$ & $\lambda_{\text {air }} \mathrm{A}$ & $\begin{array}{l}\text { Wave } \\
\text { No. } \\
\text { vac. } \\
\mathrm{cm}^{-1}\end{array}$ & $\begin{array}{l}\text { Term combi- } \\
\text { nation }\end{array}$ & $\begin{array}{c}\text { Zeeman } \\
\text { type }\end{array}$ & $\begin{array}{l}\text { Separa- } \\
\text { tion }\end{array}$ & $\begin{array}{c}\text { Strong- } \\
\text { est } \\
p\end{array}$ & $\begin{array}{c}\text { Strong- } \\
\text { est } \\
n\end{array}$ & $g_{1}$ & $g_{2}$ \\
\hline 1 & 2 & 3 & 4 & 5 & 6 & 7 & 8 & 9 & 10 \\
\hline $\begin{array}{l}5 \\
2 \\
1 d \\
2 \\
6\end{array}$ & $\begin{array}{l}2955.444 \\
2950.70 \\
2949.15 \\
2948.060 \\
2945.74\end{array}$ & $\begin{array}{l}33826.01 \\
33880.4 \\
33898.2 \\
33910.73 \\
33937.4\end{array}$ & 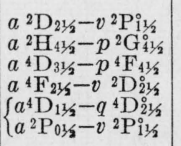 & 4 & & 0 & 1. 079 & (1. 206) & 1. 291 \\
\hline 1 & 2942.10 & 33979.4 & $\left\{\begin{array}{l}a^{4} \mathrm{D}_{21 / 1}-p^{4} \mathrm{~F}_{21 / 2}^{0} \\
a^{2} \mathrm{P}^{2}-m^{2} \mathrm{~F}^{6}\end{array}\right.$ & & & & & & \\
\hline $\begin{array}{l}15 \\
5 \\
1 h \\
4\end{array}$ & $\begin{array}{l}2938.067 \\
2934.980 \\
2932.93 \\
2929.662\end{array}$ & $\begin{array}{l}34026.06 \\
34061.85 \\
34085.6 \\
34123.67\end{array}$ & $\begin{array}{l}a^{4} \mathrm{~F}_{3}-u^{4} \mathrm{D}_{21 / 2}^{\circ} \\
a^{2} \mathrm{G}_{31 / 2}-0^{2} \mathrm{~F}_{21 / 2}^{\circ} \\
a^{2} \mathrm{G}_{41 / 2}-p^{4} \mathrm{D}_{31 / 2}^{0} \\
a^{2} \mathrm{~F}_{21 / 2}-0^{3} \mathrm{G}_{31 / 2}^{0}\end{array}$ & 4 & & $u r$ & 1. 084 & (1. 235) & 1. 296 \\
\hline $2 h$ & 2929.13 & 34129.9 & $\left\{\begin{array}{l}a^{4} \mathrm{D}_{21 / 2}-p^{4} \mathrm{~F}_{31 / 2}^{0} \\
b^{4} \mathrm{~F}^{4}-n^{4} \mathrm{D}_{315}^{0}\end{array}\right.$ & & & & & & \\
\hline $\begin{array}{r}8 \\
10 \\
8 \\
5\end{array}$ & $\begin{array}{l}2925.360 \\
2924.824 \\
2923.025 \\
2916.500\end{array}$ & $\begin{array}{l}34173.85 \\
34180.11 \\
34201.15 \\
34277.66\end{array}$ & $\begin{array}{l}a^{4} \mathrm{~F}_{41 / 2}-s^{2} \mathrm{~F}_{31 / 2}^{\circ} \\
a^{4} \mathrm{~F}_{31 / 2}-u^{4} \mathrm{D}_{31 / 2}^{0}\end{array}$ & $\begin{array}{l}5 \\
6\end{array}$ & & $0^{--}$ & $\begin{array}{l}1.958 \\
1.306 \\
1.180\end{array}$ & $\begin{array}{l}(1.330) \\
(1.235)\end{array}$ & $\begin{array}{l}\text { 1. } 151 \\
1.377\end{array}$ \\
\hline $\begin{array}{l}1 \\
2 \\
2 \\
2 \\
2\end{array}$ & $\begin{array}{l}2915.18 \\
2915.059 \\
2913.135 \\
2911.08 \\
2910.69\end{array}$ & $\begin{array}{l}34293.2 \\
34294.61 \\
34317.26 \\
34341.5 \\
34346.1\end{array}$ & $\begin{array}{l}b^{4} \mathrm{~F}_{31 / 2}-n^{4} \mathrm{D}_{31 / 2}^{0} \\
a^{6} \mathrm{D}_{41 / 2}-y^{2} \mathrm{H}_{51 / 2}^{0} \\
a^{4} \mathrm{D}_{11 / 2}-p^{4} \mathrm{~F}_{21 / 2} \\
a^{4} \mathrm{~F}_{21 / 2}-u^{2} \mathrm{D}^{2} 21 / 2 \\
a^{2} \mathrm{G}_{31 / 2}-p^{4} \mathrm{~F}_{31 / 2}^{2}\end{array}$ & & & 0 & 1.16 & & \\
\hline $\begin{array}{r}5 \\
3 \\
10 \\
1 \\
4\end{array}$ & $\begin{array}{l}2908.88 \\
2906.422 \\
2903.650 \\
2901.692 \\
2897.605\end{array}$ & $\begin{array}{l}34367.4 \\
34396.52 \\
34429.35 \\
34452.58 \\
34501.18\end{array}$ & $\begin{array}{l}a^{4} \mathrm{P}_{21 / 2}-r^{4} \mathrm{~F}_{31 / 2}^{0} \\
a^{4} \mathrm{~F}_{21 / 2}-u^{4} \mathrm{D}_{11 / 2}^{1} \\
a^{4} \mathrm{G}_{21 / 2}-r^{4} \mathrm{G}_{31 / 2}^{3}\end{array}$ & 4 & & $\begin{array}{l}w \\
0 h\end{array}$ & $\begin{array}{l}1.03 \\
0.799\end{array}$ & $(1.029)$ & 1.182 \\
\hline $\begin{array}{l}6 \\
6 \\
5 h \\
3 \\
1\end{array}$ & $\begin{array}{l}2897.345 \\
2896.998 \\
2896.24 \\
2894.90 \\
2894.77\end{array}$ & $\begin{array}{l}34504.27 \\
34508.40 \\
34517.4 \\
34533.4 \\
34534.9\end{array}$ & $\begin{array}{l}a^{4} \mathrm{P}_{21 / 2}-q^{2} \mathrm{~F}_{31 / 2}^{0} \\
a^{4} \mathrm{P}_{21 / 2}-s^{4} \mathrm{D}_{21 / 2}^{1} \\
b^{4} \mathrm{~F}_{41 / 2}-0^{4} \mathrm{~F}_{41 / 2}^{0}\end{array}$ & & & & & & \\
\hline $\begin{array}{c}3 h \\
6 \\
10 \\
6 \\
12\end{array}$ & $\begin{array}{l}2893.47 \\
2890.174 \\
2889.898 \\
2887.294 \\
2884.968\end{array}$ & $\begin{array}{l}34550.5 \\
34589.87 \\
34593.18 \\
34624.38 \\
34652.29\end{array}$ & $\begin{array}{l}a^{4} \mathrm{P}_{21 / 2}-s^{4} \mathrm{G}_{31 / 2}^{0} \\
a^{4} \mathrm{~F}_{21 / 2}-u^{4} \mathrm{D}_{21 / 2}^{\circ} \\
a^{2} \mathrm{D}_{11 / 2}-v^{2} \mathrm{P}_{11 / 2}^{\circ} \\
a^{2} \mathrm{H}_{51 / 2}-t^{2} \mathrm{H}_{51 / 2}^{\circ}\end{array}$ & 6 & 0.101 & 0.450 & 1. 049 & $(1.10)$ & 1.00 \\
\hline $\begin{array}{l}1 \\
1 \\
1 \\
8 \\
3\end{array}$ & $\begin{array}{l}2883.89 \\
2883.626 \\
2879.97 \\
2879.492 \\
2878.818\end{array}$ & $\begin{array}{l}34665.2 \\
34668.42 \\
34712.4 \\
34718.19 \\
34726.32\end{array}$ & $\begin{array}{l}a^{2} \mathrm{G}_{41 / 2}-0^{4} \mathrm{~F}_{31 / 2}^{0} \\
a^{4} \mathrm{G}_{51 / 2}-0^{4} \mathrm{~F}_{41 / 2}^{0} \\
a^{4} \mathrm{~F}_{31 / 2}-s^{2} \mathrm{~F}_{21 / 2}^{0} \\
a^{4} \mathrm{~F}_{41 / 2}-u^{4} \mathrm{G}_{41 / 2}^{4} \\
a^{2} \mathrm{G}_{31 / 2}-n^{2} \mathrm{~F}_{31 / 2}^{0}\end{array}$ & & & & & & \\
\hline $\begin{array}{c}3 \\
15 \\
2 h \\
1 h\end{array}$ & $\begin{array}{l}2878.16 \\
2874.564 \\
2873.68 \\
2872.93\end{array}$ & $\begin{array}{l}34734.3 \\
34777.70 \\
34788.4 \\
34797.5\end{array}$ & $\begin{array}{l}a^{4} \mathrm{~F}_{411 / 2}-t^{4} \mathrm{~F}_{31 / 2}^{0} \\
a^{4} \mathrm{~F}_{11 / 2}-u^{4} \mathrm{D}_{01 / 2}^{0} \\
a^{4} \mathrm{G}_{41 / 2}-n^{4} \mathrm{~F}_{41 / 2}^{0} \\
a^{4} \mathrm{G}_{41 / 2}-t^{2} \mathrm{H}_{51 / 2}^{0}\end{array}$ & 5 & 0.379 & 0.183 & 0.588 & 0.398 & 0.019 \\
\hline $\begin{array}{l}3 \\
5 \\
2\end{array}$ & $\begin{array}{l}2871.238 \\
2870.654 \\
2868.96\end{array}$ & $\begin{array}{l}34817.99 \\
34825.07 \\
34845.6\end{array}$ & $\begin{array}{l}a{ }^{4} \mathrm{~F}_{31 / 2}-s^{2} \mathrm{~F}_{31 / 2}^{0} \\
b^{4} \mathrm{~F}_{21 / 2}-475_{11 / 2}^{1}\end{array}$ & & & & & & \\
\hline 8 & 2866.672 & 34873.44 & $\left\{\begin{array}{l}a^{4} \mathrm{~F}_{11 / 2}-u^{4} \mathrm{~d}^{1 / 1} \\
a^{4} \mathrm{~F}_{21 / 2}-y^{4} \mathrm{H}_{31 / 3}^{0}\end{array}\right.$ & 6 & 0.794 & 1.191 & 0.800 & 0.403 & 1. 197 \\
\hline 20 & 2864.324 & 34902.03 & & & & 0.130 & 0.936 & & \\
\hline $\begin{array}{r}1 \\
2 \\
15 \\
10 \\
10\end{array}$ & $\begin{array}{l}2862.49 \\
2861.76 \\
2859.962 \\
2858.974 \\
2857.294\end{array}$ & $\begin{array}{l}34924.4 \\
34933.3 \\
34955.26 \\
34967.34 \\
34987.89\end{array}$ & $\begin{array}{l}a^{4} \mathrm{~F}_{21 / 2}-t^{2} \mathrm{~F}_{31 / 2}^{0} ? \\
a^{2} \mathrm{D}_{21 / 2}-0^{4} \mathrm{D}_{31 / 2}^{0} \\
a^{4} \mathrm{~F}_{41 / 2}-u^{4} \mathrm{G}_{51 / 2}^{0} \\
a^{4} \mathrm{P}_{21 / 2}-s^{4} \mathrm{D}_{31 / 2}^{0}\end{array}$ & & & & & & \\
\hline $\begin{array}{r}12 \\
3\end{array}$ & $\begin{array}{l}2854.168 \\
2853.264\end{array}$ & $\begin{array}{l}35026.21 \\
35037.31\end{array}$ & $\begin{array}{l}a^{4} \mathrm{~F}_{41 / 2}-t^{4} \mathrm{~F}_{41 / 2}^{0} \\
a^{4} \mathrm{~F}_{11 / 2}-u^{4} \mathrm{D}_{21 / 2}^{2}\end{array}$ & 6 & 0.080 & 0.296 & 1. $322^{*}$ & $(1.330)$ & 1. 250 \\
\hline 15 & 2851.978 & 35053.11 & $\left\{\begin{array}{l}a^{2} \mathrm{H}_{41 / 2}-t^{2} \mathrm{H}_{51 / 2}^{0} \\
a^{2} \mathrm{G}_{31 / 3}-q^{2} \mathrm{G}_{31 / 6}^{5}\end{array}\right.$ & 5 & & $0 h$ & 1.28 & $(0.93)$ & 0.99 \\
\hline $\begin{array}{r}20 \\
2\end{array}$ & $\begin{array}{l}2851.446 \\
2845.52\end{array}$ & $\begin{array}{l}35059.65 \\
35132.6\end{array}$ & $\mid \begin{array}{l}a^{2} \mathrm{D}_{21 / 2}-m{ }^{2} \mathrm{~F}^{2} 2 / 5 \\
a^{4} \mathrm{~F}_{31 / 2}-t^{4} \mathrm{~F}_{21 / 3}\end{array}$ & & & & & & \\
\hline
\end{tabular}


TABLE 4.-First spectrum of columbium (Cb I)-Continued

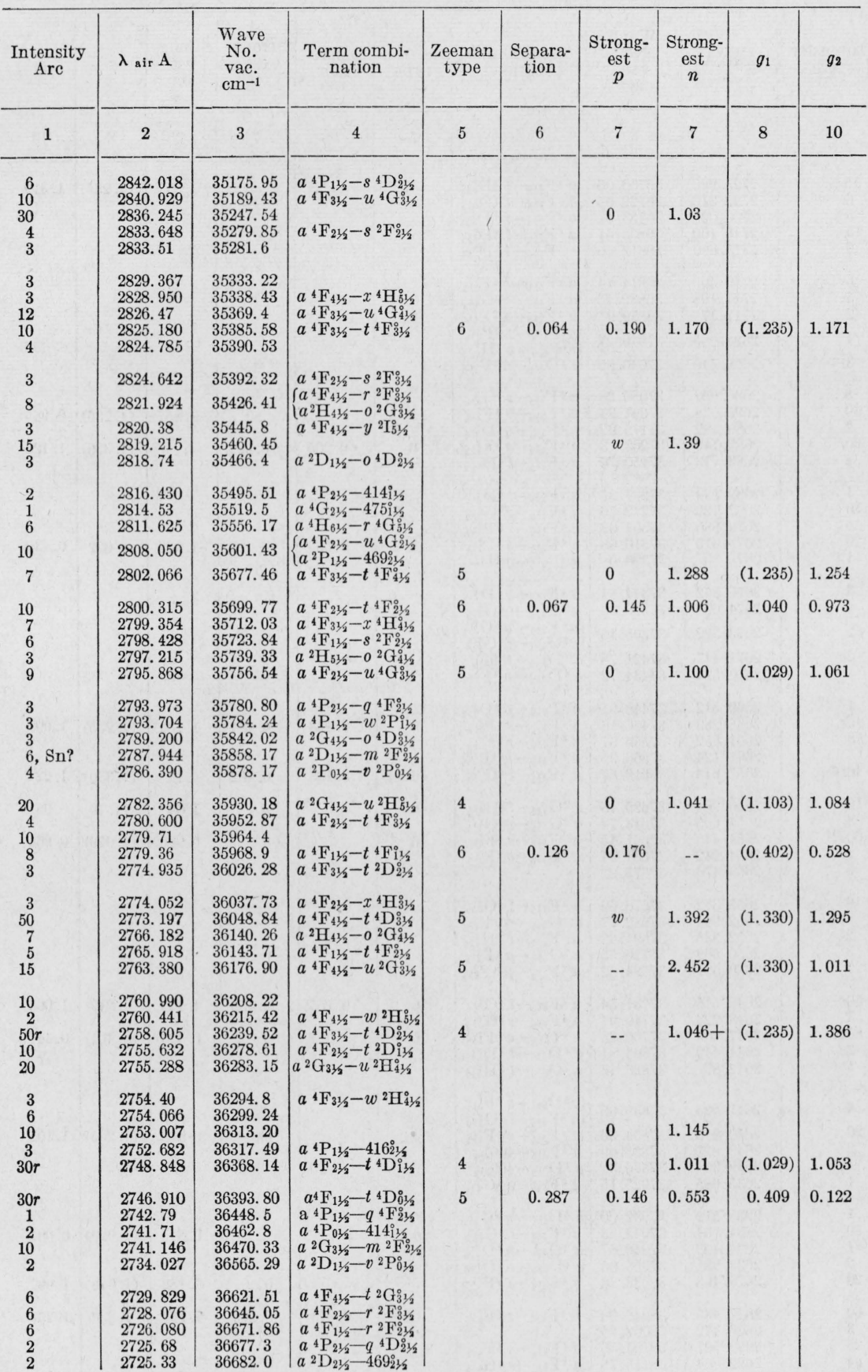


TABLE 4.-First spectrum of columbium (Cb I)-Continued

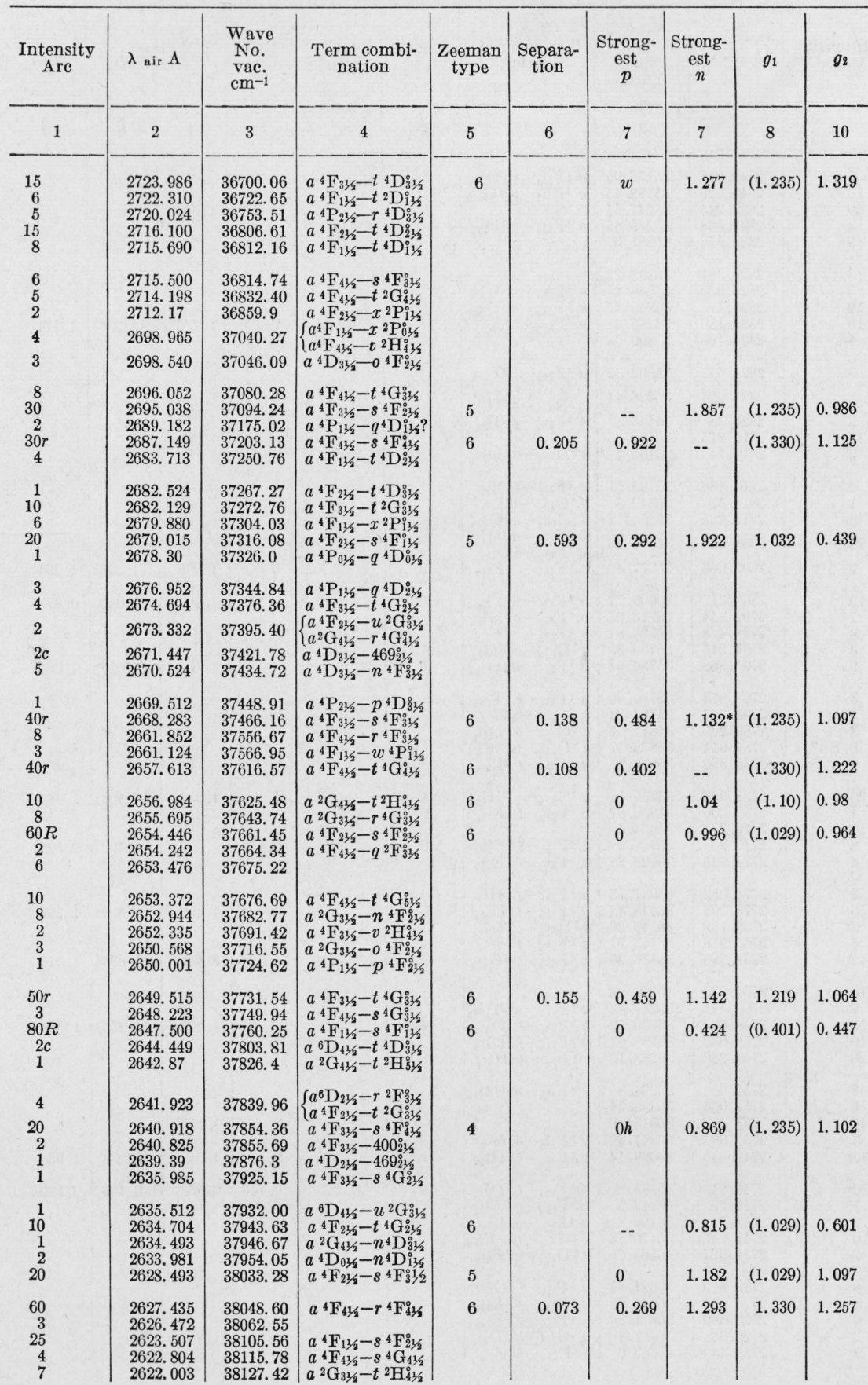


TABLE 4.-First spectrum of columbium (Cb I)-Continued

\begin{tabular}{|c|c|c|c|c|c|c|c|c|c|}
\hline $\begin{array}{c}\text { Intensity } \\
\text { Arc }\end{array}$ & $\lambda_{\text {air }} \mathbf{A}$ & $\begin{array}{l}\text { Wave } \\
\text { No. } \\
\text { vac. } \\
\mathrm{cm}^{-1}\end{array}$ & $\begin{array}{l}\text { Term combi- } \\
\text { nation }\end{array}$ & $\begin{array}{c}\text { Zeeman } \\
\text { type }\end{array}$ & $\begin{array}{c}\text { Separa- } \\
\text { tion }\end{array}$ & $\begin{array}{l}\text { Strong- } \\
\text { est } \\
p\end{array}$ & $\begin{array}{c}\text { Strong- } \\
\text { est } \\
n\end{array}$ & $g_{1}$ & $g_{2}$ \\
\hline 1 & 2 & 3 & 4 & 5 & 6 & 7 & 8 & 9 & 10 \\
\hline $\begin{array}{c}8 \\
1 c \\
4 \\
30 \\
3\end{array}$ & $\begin{array}{l}2620.585 \\
2618.178 \\
2617.046 \\
2616.476 \\
2614.444\end{array}$ & $\begin{array}{l}38148.05 \\
38183.12 \\
38199.63 \\
38207.95 \\
38237.65\end{array}$ & $\begin{array}{l}a^{4} \mathrm{~F}_{41 / 2}-s^{4} \mathrm{D}_{31 / 2}^{0} \\
a^{4} \mathrm{D}_{31 / 2}-0^{4} \mathrm{~F}_{41 / 2}^{0} \\
a^{2} \mathrm{G}_{41 / 2}-0^{2} \mathrm{G}_{31 / 2}^{0} \\
a^{4} \mathrm{~F}_{31 / 2}-r^{4} \mathrm{~F}_{31 / 2}^{0}\end{array}$ & 6 & 0.109 & 0.322 & 1. 182 & 1. 236 & 1. 127 \\
\hline $\begin{array}{r}15 \\
1 \\
20 \\
1 \\
6\end{array}$ & $\begin{array}{l}2612.377 \\
2612.028 \\
2610.268 \\
2609.118 \\
2608.836\end{array}$ & $\begin{array}{l}38267.90 \\
38273.01 \\
38298.82 \\
38315.70 \\
38319.84\end{array}$ & $\begin{array}{l}a^{4} \mathrm{~F}_{31 / 2}-t^{4} \mathrm{G}_{41 / 2}^{\circ} \\
a^{2} \mathrm{~F}_{31 / 2}-n^{2} \mathrm{G}_{41 / 2}^{0} \\
a^{4} \mathrm{~F}_{21 / 2}-t^{4} \mathrm{G}_{31 / 2}^{0} \\
a^{4} \mathrm{~F}_{31 / 2}-q^{2} \mathrm{~F}_{31 / 2}^{0} \\
a^{4} \mathrm{~F}_{31 / 2}-s^{4} \mathrm{D}_{21 / 2}^{0}\end{array}$ & $7 b, 4$ & & 0 & 1. 229 & $\begin{array}{l}(1.235) \\
(1.029)\end{array}$ & 1. 086 \\
\hline $\begin{array}{l}1 \\
6 \\
8 \\
7 \\
3\end{array}$ & $\begin{array}{l}2604.225 \\
2603.306 \\
2602.011 \\
2601.832 \\
2601.597\end{array}$ & $\begin{array}{l}38387.68 \\
38401.23 \\
38420.34 \\
38422.98 \\
38426.45\end{array}$ & $\begin{array}{l}a^{4} \mathrm{~F}_{11 / 2}-t^{4} \mathrm{G}_{21 / 2}^{\circ} \\
a^{4} \mathrm{~F}_{31 / 2}-s^{4} \mathrm{G}_{31 / 2}^{\circ} \\
a^{4} \mathrm{~F}_{21 / 2}-400_{21 / 2}^{\circ}\end{array}$ & & & & & & \\
\hline $\begin{array}{r}10 \\
4 \\
4 \\
50 \\
5\end{array}$ & $\begin{array}{l}2597.138 \\
2594.685 \\
2592.911 \\
2592.190 \\
2589.264\end{array}$ & $\begin{array}{l}38492.43 \\
38528.81 \\
38555.17 \\
38565.89 \\
38609.47\end{array}$ & $\begin{array}{l}a^{4} \mathrm{~F}_{21 / 2}-s^{4} \mathrm{G}_{21 / 2}^{0} \\
a^{4} \mathrm{~F}_{41 / 2}-s^{4} \mathrm{G}_{51 / 2}^{0}\end{array}$ & 4 & & -. & 0.979 & $\begin{array}{l}\text { (1.029) } \\
(1.330)\end{array}$ & $\begin{array}{l}0.929 \\
1.267\end{array}$ \\
\hline $\begin{array}{r}1 \\
8 \\
15 \\
7 \\
50\end{array}$ & $\begin{array}{l}2588.36 \\
2583.219 \\
2583.103 \\
2581.194 \\
2578.734\end{array}$ & $\begin{array}{l}38622.9 \\
38699.82 \\
38701.56 \\
38730.18 \\
38767.12\end{array}$ & $\begin{array}{l}a^{4} \mathrm{~F}_{21 / 2}-r^{4} \mathrm{~F}_{21 / 2}^{0} \\
a^{4} \mathrm{~F}_{31 / 2}-r^{4} \mathrm{~F}_{41 / 2}^{0} \\
a^{2} \mathrm{G}_{31 / 2}-0^{2} \mathrm{G}_{31 / 2}^{0} \\
a^{6} \mathrm{D}_{01 / 2}-w^{4} \mathrm{P}_{01 / 2}^{0} \\
a^{4} \mathrm{~F}_{31 / 2}-s^{4} \mathrm{G}_{41 / 2}^{0}\end{array}$ & $\begin{array}{l}6 \\
7 b, 5\end{array}$ & & 0 & $\begin{array}{l}0.898 \\
1.244\end{array}$ & $\begin{array}{l}(0.885) \\
(1.235)\end{array}$ & $\begin{array}{l}0.911 \\
1.238\end{array}$ \\
\hline $\begin{array}{r}8 \\
4 \\
7 \\
1 \\
15\end{array}$ & $\begin{array}{l}2578.203 \\
2578.086 \\
2576.592 \\
2572.80 \\
2572.099\end{array}$ & $\begin{array}{l}38775.10 \\
38776.87 \\
38799.35 \\
38856.5 \\
38867.12\end{array}$ & $\begin{array}{l}a^{4} \mathrm{~F}_{21 / 2}-r^{4} \mathrm{~F}_{31 / 2}^{\circ} \\
a^{4} \mathrm{~F}_{31 / 2}-s^{4} \mathrm{D}_{31 / 2}^{\circ} \\
a^{6} \mathrm{D}_{21 / 2}-s^{4} \mathrm{~F}_{21 / 2}^{\circ} ? \\
a^{4} \mathrm{~F}_{11 / 2}-400_{21 / 2}^{\circ}\end{array}$ & & & & & & \\
\hline $\begin{array}{r}7 \\
6 \\
20 \\
20 \\
3\end{array}$ & $\begin{array}{l}2571.054 \\
2570.782 \\
2569.030 \\
2567.510 \\
2567.281\end{array}$ & $\begin{array}{l}38882.91 \\
38887.03 \\
38913.55 \\
38936.58 \\
38940.05\end{array}$ & $\begin{array}{l}a^{4} \mathrm{~F}_{21 / 2}-q^{2} \mathrm{~F}_{31 / 2}^{0} \\
a^{4} \mathrm{~F}_{21 / 2}-s^{4} \mathrm{D}_{21 / 2}^{0} \\
a^{2} \mathrm{G}_{41 / 2}-0^{2} \mathrm{G}_{41 / 2}^{0} \\
a^{4} \mathrm{~F}_{11 / 2}-s^{4} \mathrm{G}_{21 / 2}^{0} \\
a^{4} \mathrm{P}_{21 / 2}-0^{4} \mathrm{D}_{21 / 2}^{\circ}\end{array}$ & $\begin{array}{l}7 b, 6 \\
5\end{array}$ & 0.513 & $\begin{array}{l}0 \\
--\end{array}$ & $\begin{array}{l}1.104 \\
1.689\end{array}$ & $\begin{array}{c}(1.103) \\
0.407\end{array}$ & $\begin{array}{l}1.105 \\
0.920\end{array}$ \\
\hline $\begin{array}{r}30 \\
8 \\
4 \\
1 \\
20\end{array}$ & $\begin{array}{l}2565.410 \\
2564.735 \\
2564.265 \\
2559.05 \\
2558.936\end{array}$ & $\begin{array}{l}38968.45 \\
38978.71 \\
38985.85 \\
39065.29 \\
39067.03\end{array}$ & $\begin{array}{l}a^{4} \mathrm{~F}_{21 / 2}-s^{4} \mathrm{G}_{31 / 2}^{0} \\
a^{4} \mathrm{~F}_{31 / 2}-411_{31 / 3}^{\circ} \\
a^{4} \mathrm{P}_{01 / 2}-v^{2} \mathrm{P}_{11 / 2}^{0} \\
a^{4} \mathrm{~F}_{11 / 2}^{0}-r^{4} \mathrm{~F}_{21 / 2}^{0}\end{array}$ & 5 & 0.508 & 0.258 & 1. 634 & (1.029) & 0.872 \\
\hline $\begin{array}{r}2 \\
4 \\
3 \\
12 \\
3\end{array}$ & $\begin{array}{l}2558.835 \\
2557.41 \\
2555.107 \\
2554.103 \\
2553.752\end{array}$ & $\begin{array}{l}39068.57 \\
39090.3 \\
39125.57 \\
39140.95 \\
39146.33\end{array}$ & $\begin{array}{l}a^{4} \mathrm{~F}_{41 / 2}-q^{4} \mathrm{~F}_{41 / 2}^{0} \\
a^{4} \mathrm{~F}_{41 / 5}-s^{2} \mathrm{G}_{41 / 2}^{0} \\
a^{4} \mathrm{~F}_{41 / 2}-q^{4} \mathrm{~F}_{31 / 2}^{0}\end{array}$ & & & & & & \\
\hline $\begin{array}{r}2 \\
7 \\
2 \\
10 \\
3\end{array}$ & $\begin{array}{l}2550.878 \\
2549.904 \\
2544.723 \\
2543.980 \\
2543.252\end{array}$ & $\begin{array}{l}39190.43 \\
39205.40 \\
39285.22 \\
39296.69 \\
39307.94\end{array}$ & $\begin{array}{l}a^{6} \mathrm{D}_{31 / 2}-t^{4} \mathrm{G}_{31 / 2}^{0} \\
a^{4} \mathrm{P}_{21 / 2}-0^{4} \mathrm{D}_{31 / 2}^{0} \\
a^{2} \mathrm{H}_{51 / 2}-n_{2}{ }^{2} \mathrm{G}_{41 / 2}^{0}\end{array}$ & & & & & & \\
\hline $\begin{array}{l}1 \\
5 \\
2 \\
1 \\
1\end{array}$ & $\begin{array}{l}2542.925 \\
2541.972 \\
2541.704 \\
2539.464 \\
2539.128\end{array}$ & $\begin{array}{l}39313.00 \\
39327.73 \\
39331.88 \\
39366.57 \\
39371.78\end{array}$ & $\begin{array}{l}a^{6} \mathrm{D}_{31 / 2}-s^{4} \mathrm{~F}_{41 / 2}^{0} ? \\
a^{4} \mathrm{P}_{21 / 2}-m^{2} \mathrm{~F}_{21 / 2}^{\circ} ? \\
a^{4} \mathrm{~F}_{21 / 2}-s^{4} \mathrm{D}_{31 / 2}^{\circ} \\
a^{6} \mathrm{D}_{41 / 2}-t^{4} \mathrm{G}_{41 / 2}^{0}\end{array}$ & & & & , & & \\
\hline $\begin{array}{l}6 \\
1 \\
4\end{array}$ & $\begin{array}{l}2538.055 \\
2535.259 \\
2527.277\end{array}$ & $\begin{array}{l}39388.42 \\
39431.86 \\
39556.39\end{array}$ & $\begin{array}{l}a^{4} \mathrm{~F}_{41 / 2}-r^{2} \mathrm{G}_{41 / 2} \\
a^{6} \mathrm{D}_{41 / 2}-t^{4} \mathrm{G}_{51 / 2}^{0}\end{array}$ & & & & & & \\
\hline $\begin{array}{r}10 \\
7\end{array}$ & $\begin{array}{l}2524.985 \\
2524.006\end{array}$ & $\begin{array}{l}39592.29 \\
39607.65\end{array}$ & $\begin{array}{l}\left\{\begin{array}{l}a^{4} \mathrm{~F}_{11 / 2}-q^{2} \mathrm{~F}_{21 / 2}^{0} \\
a^{4} \mathrm{~F}_{31 / 2}-q^{4} \mathrm{~F}_{21 / 2}^{2}\end{array}\right. \\
a^{4} \mathrm{P}_{11 / 2}-0^{4} \mathrm{D}_{21 / 5}^{0}\end{array}$ & 5 & & -. & 1. 296 & $(0.402)$ & 0.760 \\
\hline $\begin{array}{r}3 \\
2 \\
10 \\
2 \\
1\end{array}$ & $\begin{array}{l}2521.924 \\
2521.880 \\
2520.507 \\
2519.018 \\
2516.878\end{array}$ & $\begin{array}{l}39640.34 \\
39641.04 \\
39662.63 \\
39686.07 \\
39719.81\end{array}$ & $\begin{array}{l}a^{4} \mathrm{P}_{11 / 2}-0{ }^{4} \mathrm{D}_{11 / 2}^{0} \\
a^{2} \mathrm{H}_{41 / 2}-n^{2} \mathrm{G}_{41 / 2}^{\circ} \\
a^{4} \mathrm{~F}_{31 / 2}-q^{4} \mathrm{~F}_{11 / 2}^{0}\end{array}$ & & & & & & \\
\hline
\end{tabular}


TABLE 4.-First spectrum of columbium (Cb I) - Continued

\begin{tabular}{|c|c|c|c|c|c|c|c|c|c|}
\hline $\begin{array}{c}\text { Intensity } \\
\text { Arc }\end{array}$ & $\lambda_{\text {sir }} \mathrm{A}$ & $\begin{array}{l}\text { Wave } \\
\text { No. } \\
\text { vac. } \\
\mathrm{cm}^{-1}\end{array}$ & $\begin{array}{c}\text { Term combi- } \\
\text { nation }\end{array}$ & $\begin{array}{c}\text { Zeeman } \\
\text { type }\end{array}$ & $\begin{array}{l}\text { Separa- } \\
\text { tion }\end{array}$ & $\begin{array}{c}\text { Strong- } \\
\text { est } \\
p\end{array}$ & $\begin{array}{c}\text { Strong- } \\
\text { est } \\
n\end{array}$ & $g_{1}$ & $g_{2}$ \\
\hline 1 & 2 & 3 & 4 & 5 & 6 & 7 & 8 & 9 & 10 \\
\hline $\begin{array}{l}4 \\
3 \\
1 \\
2 \\
3\end{array}$ & $\begin{array}{l}2515.775 \\
2514.784 \\
2514.750 \\
2513.273 \\
2511.573\end{array}$ & $\begin{array}{l}39737.22 \\
39752.88 \\
39753.42 \\
39776.78 \\
39803.70\end{array}$ & $\begin{array}{l}a^{4} \mathrm{P}_{21 / 2}-p^{2} \mathrm{G}_{31 / 2}^{0} ? \\
a^{4} \mathrm{~F}_{31 / 2}-q^{4} \mathrm{~F}_{31 / 2}^{0} \\
a^{6} \mathrm{D}_{41 / 2}-r^{4} \mathrm{~F}_{41 / 2}^{\circ}\end{array}$ & & & & & & \\
\hline $\begin{array}{r}6 \\
30 \\
4 \\
2 \\
2\end{array}$ & $\begin{array}{l}2507.134 \\
2504.648 \\
2502.944 \\
2499.282 \\
2497.464\end{array}$ & $\begin{array}{l}39874.17 \\
39913.74 \\
39940.91 \\
39999.43 \\
40028.55\end{array}$ & $\begin{array}{l}a^{4} \mathrm{~F}_{21 / 2}-414_{11 / 2}^{\circ} \\
a^{4} \mathrm{~F}_{41 / 2}-r^{4} \mathrm{D}_{31 / 2}^{0} \\
a^{4} \mathrm{P}_{01 / 2}-0^{4} \mathrm{D}_{11}^{11} \\
a^{4} \mathrm{P}_{11 / 2}-m^{2} \mathrm{~F}_{21 / 2} \\
a^{4} \mathrm{~F}_{21 / 2}-416_{21 / 2}^{\circ}\end{array}$ & 4 & & $w$ & 1.12 & (1.33) & 1.39 \\
\hline $\begin{array}{r}3 \\
2 \\
1 \\
8 \\
15\end{array}$ & $\begin{array}{l}2494.801 \\
2489.112 \\
2479.351 \\
2476.478 \\
2474.655\end{array}$ & $\begin{array}{l}40071.27 \\
40162.85 \\
40320.95 \\
40367.73 \\
40397.47\end{array}$ & 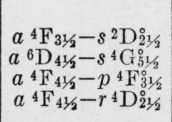 & 4 & & 0 & 0.698 & (1.235) & 1.450 \\
\hline $\begin{array}{r}2 \\
25 \\
1 \\
3 \\
20\end{array}$ & $\begin{array}{l}2473.752 \\
2469.072 \\
2468.064 \\
2467.630 \\
2466.727\end{array}$ & $\begin{array}{l}40412.21 \\
40488.80 \\
40505.34 \\
40512.46 \\
40527.29\end{array}$ & $\begin{array}{l}a{ }^{4} \mathrm{~F}_{11 / 2}-q^{4} \mathrm{~F}_{11 / 2}^{\circ} \\
a^{4} \mathrm{~F}_{31 / 2}-q^{4} \mathrm{D}_{21 / 2}^{2} \\
a^{4} \mathrm{P}_{21 / 2}-r^{4} \mathrm{G}_{31 / 2}^{8} \\
a^{4} \mathrm{~F}_{41 / 2}-q^{4} \mathrm{D}_{31 / 2}^{\circ}\end{array}$ & & & & & & \\
\hline $\begin{array}{r}10 \\
2 \\
10 \\
5 \\
20\end{array}$ & $\begin{array}{l}2466.318 \\
2465.709 \\
2464.432 \\
2463.631 \\
2462.889\end{array}$ & $\begin{array}{l}40534.02 \\
40544.02 \\
40565.03 \\
40578.22 \\
40590.44\end{array}$ & $\begin{array}{l}a^{4} \mathrm{~F}_{11 / 2}-r^{4} \mathrm{D}_{01 / 2}^{0} \\
a^{4} \mathrm{P}_{21 / 2}-n^{4} \mathrm{~F}_{21 / 2}^{2} \\
a^{4} \mathrm{~F}_{31 / 2}-r^{4} \mathrm{D}_{31 / 2}^{01} \\
a^{4} \mathrm{P}_{21 / 2}-0^{4} \mathrm{~F}_{21 / 2}^{21} \\
a^{4} \mathrm{~F}_{41 / 2}-p^{4} \mathrm{~F}_{41 / 2}^{1}\end{array}$ & 6 & & -- & 1. 259 & (1.330) & 1.188 \\
\hline $\begin{array}{r}10 \\
5 \\
1 \\
3 \\
2\end{array}$ & $\begin{array}{l}2461.757 \\
2458.575 \\
2456.83 \\
2455.872 \\
2454.163\end{array}$ & $\begin{array}{l}40609.11 \\
40661.66 \\
40690.5 \\
40706.41 \\
40734.75\end{array}$ & $\begin{array}{l}a^{4} \mathrm{~F}_{41 / 2}-p^{4} \mathrm{D}_{31 / 2}^{0} 0 \\
a^{6} \mathrm{D}_{21 / 2}-w^{2} \mathrm{P}_{11 / 2}^{0} ? \\
a^{4} \mathrm{P}_{11 / 2}-v^{2} \mathrm{P}_{01 / 2}^{01} \\
a^{4} \mathrm{~F}_{31 / 2}-0^{2} \mathrm{~F}_{21 / 2}^{0}\end{array}$ & & & & & & - \\
\hline 20 & 2453.367 & 40747.97 & $\left\{\begin{array}{l}a^{6} \mathrm{D}_{21 / 2}-411_{31 / 2}^{\circ} \\
a^{4} \mathrm{~F}_{416}-n^{2} \mathrm{~F}_{31}^{\circ}\end{array}\right.$ & & & & & & \\
\hline $\begin{array}{r}20 \\
4 \\
10 \\
1\end{array}$ & $\begin{array}{l}2453.084 \\
2447.413 \\
2446.130 \\
2445.913\end{array}$ & $\begin{array}{l}4075267 \\
40847.09 \\
40868.51 \\
40872.14\end{array}$ & $\begin{array}{l}a^{4} \mathrm{~F}_{21 / 2}-r^{4} \mathrm{D}_{11 / 2} \\
a^{4} \mathrm{P}_{21 / 2}-u^{4} \mathrm{D}_{21 / 2} \\
a^{4} \mathrm{~F}_{31 / 3}-p^{4} \mathrm{~F}_{21 / 2}^{2} \\
a^{4} \mathrm{P}_{11 / 2}-0^{4} \mathrm{~F}_{11 / 2}^{2}\end{array}$ & & & & & & \\
\hline $\begin{array}{r}20 \\
8 \\
2 \\
5 \\
3\end{array}$ & $\begin{array}{l}2445.066 \\
2443.529 \\
2441.021 \\
2440.392 \\
2439.715\end{array}$ & $\begin{array}{l}40886.30 \\
40912.01 \\
40954.04 \\
40964.60 \\
40975.97\end{array}$ & $\begin{array}{l}a^{4} \mathrm{~F}_{21 / 2}-q^{4} \mathrm{D}_{11 / 2}^{\circ} \\
a^{4} \mathrm{P}_{21 / 2}-468_{21 / 2}^{\circ} \\
a^{4} \mathrm{~F}_{21 / 2}-r^{4} \mathrm{D}_{21 / 2}^{2}\end{array}$ & & & & & & \\
\hline $\begin{array}{r}2 \\
3 \\
9 \\
10 \\
6\end{array}$ & $\begin{array}{l}2439.445 \\
2437.924 \\
2437.161 \\
2436.329 \\
2434.962\end{array}$ & $\begin{array}{l}40980.50 \\
41006.07 \\
41018.90 \\
41032.90 \\
41055.95\end{array}$ & $\begin{array}{l}a^{4} \mathrm{P}_{01 / 2}-n^{4} \mathrm{D}_{01 / 2}^{0} \\
a^{4} \mathrm{P}_{01 / 2}-v^{2} \mathrm{P}_{01 / 2}^{0} \\
a^{4} \mathrm{~F}_{31 / 2}-p^{4} \mathrm{~F}_{31 / 2}^{2} \\
a^{4} \mathrm{~F}_{31 / 2}-p^{4} \mathrm{D}_{21 / 2}^{2} \\
a^{4} \mathrm{~F}_{21 / 2}-q^{4} \mathrm{D}_{21 / 2}^{13}\end{array}$ & & & & & & - \\
\hline 4 & 2434.878 & 41057.36 & $a^{4} \mathrm{P}_{21 / 2}-0^{4} \mathrm{~F}_{31 / 2}^{\circ}$ & & & & & & \\
\hline $\begin{array}{r}1 \\
10 \\
2 \\
3 \\
3\end{array}$ & $\begin{array}{l}2434.310 \\
2433.680 \\
2430.442 \\
2428.848 \\
2428.096\end{array}$ & $\begin{array}{l}41066.94 \\
41077.59 \\
41132.29 \\
41159.28 \\
41172.03\end{array}$ & $\begin{array}{l}a{ }^{4} \mathrm{P}_{11 / 2}-n^{4} \mathrm{D}_{11 / 2}^{0} \\
a^{4} \mathrm{~F}_{21 / 2}-r{ }^{4} \mathrm{D}_{31 / 2}^{\circ} \\
a^{4} \mathrm{P}_{01 / 2}-0{ }^{4} \mathrm{~F}_{11 / 2}^{0}\end{array}$ & & & & & & \\
\hline $\begin{array}{l}3 \\
1 \\
9 \\
3 \\
5\end{array}$ & $\begin{array}{l}2428.047 \\
2427.714 \\
2427.536 \\
2427.105 \\
2426.957\end{array}$ & $\begin{array}{l}41172.86 \\
41178.51 \\
41181.52 \\
41188.84 \\
41191.35\end{array}$ & $\begin{array}{l}a^{4}_{31/2}-q^{4} \mathrm{D}_{31 / 2}^{0} \\
a^{4} \mathrm{~F}_{11 / 2}-q^{4} \mathrm{D}_{0}^{1 / 2} \\
a^{4} \mathrm{~F}_{31 / 2}-n^{2} \mathrm{~F}_{21 / 2}^{31}\end{array}$ & & & & & & \\
\hline $\begin{array}{l}6 h \\
5 \\
4 \\
3 \\
2\end{array}$ & $\begin{array}{l}2426.637 \\
2425.741 \\
2423.996 \\
2422.900 \\
2420.461\end{array}$ & $\begin{array}{l}41196.78 \\
41212.00 \\
41241.66 \\
41260.32 \\
41301.89\end{array}$ & 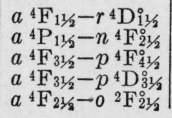 & & & & & & \\
\hline
\end{tabular}


TABLE 4.-First spectrum of columbium (Cb I) - Continued

\begin{tabular}{|c|c|c|c|c|c|c|c|c|c|}
\hline $\begin{array}{l}\text { Intensity } \\
\text { Arc }\end{array}$ & $\lambda_{\text {air }} A$ & $\begin{array}{l}\text { Wave } \\
\text { No. } \\
\text { vac. } \\
\mathrm{cm}^{-1}\end{array}$ & $\begin{array}{l}\text { Term combi- } \\
\text { nation }\end{array}$ & $\begin{array}{c}\text { Zeeman } \\
\text { type }\end{array}$ & $\begin{array}{l}\text { Separa- } \\
\text { tion }\end{array}$ & $\begin{array}{c}\text { Strong- } \\
\text { est } \\
p\end{array}$ & $\begin{array}{c}\text { Strong- } \\
\text { est } \\
n\end{array}$ & $g_{1}$ & $g_{2}$ \\
\hline 1 & 2 & 3 & 4 & 5 & 6 & 7 & 8 & 9 & 10 \\
\hline $\begin{array}{l}7 \\
5 c \\
6 \\
4 \\
3\end{array}$ & $\begin{array}{l}2420.144 \\
2419.976 \\
2419.804 \\
2418.792 \\
2418.490\end{array}$ & $\begin{array}{l}41307.30 \\
41310.16 \\
41313.10 \\
41330.38 \\
41335.55\end{array}$ & $\begin{array}{l}a^{4} \mathrm{~F}_{21 / 2}-p^{4} \mathrm{~F}_{11 / 2}^{0} \\
a^{4} \mathrm{P}_{21 / 2}-n^{4} \mathrm{D}_{31 / 2}^{\circ} \\
a^{4} \mathrm{~F}_{21 / 2}-p^{4} \mathrm{D}_{11 / 2}^{1} \\
a^{4} \mathrm{~F}_{11 / 2}-q^{4} \mathrm{D}_{11 / 2}^{\circ}\end{array}$ & & & & & & \\
\hline $\begin{array}{l}2 c \\
7 \\
5 \\
3 h \\
2\end{array}$ & $\begin{array}{l}2414.762 \\
2414.206 \\
2412.645 \\
2410.792 \\
2408.056\end{array}$ & $\begin{array}{l}41399.35 \\
41408.89 \\
41435.68 \\
41467.53 \\
41514.64\end{array}$ & $\mid \begin{array}{l}a^{4} \mathrm{~F}_{31 / 2}-n^{2} \mathrm{~F}_{31 / 2}^{0} \\
a^{4} \mathrm{~F}_{11 / 2}-r^{4} \mathrm{D}_{21 / 2}^{\circ} \\
a^{4} \mathrm{~F}_{21 / 2}-p^{4} \mathrm{~F}_{21 / 2}^{\circ} \\
a^{4} \mathrm{P}_{11 / 2}-n^{4} \mathrm{D}_{21 / 2}^{\circ}\end{array}$ & & & & & & \\
\hline $\begin{array}{c}10 \\
4 \\
4 \\
8 c\end{array}$ & $\begin{array}{l}2406.942 \\
2403.918 \\
2403.107 \\
2402.339\end{array}$ & $\begin{array}{l}41533.85 \\
41586.09 \\
41600.12 \\
41613.42\end{array}$ & $\begin{array}{l}a^{4} \mathrm{~F}_{21 / 2}-p^{4} \mathrm{~F}_{31 / 2}^{\circ} \\
a^{4} \mathrm{~F}_{21 / 2}-p^{4} \mathrm{D}_{21 / 2}^{\circ}\end{array}$ & & & - & & & \\
\hline 3 & 2401.873 & 41621.49 & $\left\{\begin{array}{l}a^{6} \mathrm{D}_{31 / 4}-s^{2} \mathrm{D}_{21 / 1}^{2} \\
a^{4} \mathrm{P}_{11 / 3}-469_{21 / 2}^{2}\end{array}\right.$ & & & & & & \\
\hline $\begin{array}{l}5 \\
5 \\
2 \\
4 \\
5\end{array}$ & $\begin{array}{l}2400.910 \\
2399.718 \\
2395.851 \\
2394.395 \\
2394.058\end{array}$ & $\begin{array}{l}41638.19 \\
41658.87 \\
41726.10 \\
41751.47 \\
41757.35\end{array}$ & $\begin{array}{l}a^{4} \mathrm{~F}_{11 / 2}-p^{4} \mathrm{D}_{01 / 2}^{0} \\
a^{4} \mathrm{~F}_{31 / 2}-q^{2} \mathrm{G}_{31 / 2}^{0} \\
a^{4} \mathrm{~F}_{11 / 2}-p^{4} \mathrm{~F}_{11 / 2}^{1} \\
a^{4} \mathrm{~F}_{11 / 2}-p^{4} \mathrm{D}_{1 / 2}^{1}\end{array}$ & & & & V & & \\
\hline $\begin{array}{r}4 \\
4 \\
3 \\
15 \\
3\end{array}$ & $\begin{array}{l}2390.454 \\
2382.246 \\
2373.074 \\
2368.860 \\
2368.64\end{array}$ & $\begin{array}{l}41820.30 \\
41964.38 \\
42126.56 \\
42201.49 \\
42205.41\end{array}$ & & & & & & & \\
\hline $\begin{array}{l}1 \\
3 \\
5 h \\
4 h \\
2\end{array}$ & $\begin{array}{l}2366.715 \\
2365.940 \\
2364.325 \\
2363.852 \\
2363.716\end{array}$ & $\begin{array}{l}42239.74 \\
42253.57 \\
42282.43 \\
42290.89 \\
42293.32\end{array}$ & $\mid \begin{array}{l}a^{4} \mathrm{P}_{11 / 2}-475_{1}^{\circ} 1 / 2 \\
a^{6} \mathrm{D}_{41 / 2}-q^{4} \mathrm{D}_{31 / 2}^{\circ} \\
a^{4} \mathrm{D}_{31 / 2}-n^{2} \mathrm{G}_{41 / 2}^{0} \\
a^{4} \mathrm{~F}_{21 / 2}-q^{2} \mathrm{G}_{3}^{0} 1 / 2\end{array}$ & & & & & & \\
\hline $\begin{array}{l}4 \\
3 \\
2 \\
3 \\
5\end{array}$ & $\begin{array}{l}2363.062 \\
2362.50 \\
2360.794 \\
2359.679 \\
2358.134\end{array}$ & $\begin{array}{l}42305.03 \\
42315.1 \\
42345.67 \\
42365.67 \\
42393.43\end{array}$ & $\begin{array}{l}a^{4} \mathrm{~F}_{41 / 2}-u^{2} \mathrm{H}_{41 / 2}^{\circ} \\
a^{6} \mathrm{D}_{41 / 2}-p^{4} \mathrm{~F}_{41 / 2}^{0} \\
a^{4} \mathrm{~F}_{41 / 2}-0^{4} \mathrm{D}_{31 / 2}^{01}\end{array}$ & & & & & & \\
\hline $\begin{array}{r}2 \\
9 \\
10 \\
6 \\
3\end{array}$ & $\begin{array}{l}2357.910 \\
2354.470 \\
2353.80 \\
2353.510 \\
2352.131\end{array}$ & $\begin{array}{l}42397.46 \\
42459.39 \\
42471.5 \\
42476.71 \\
42501.61\end{array}$ & $\begin{array}{l}a^{6} \mathrm{D}_{11 / 2}-r^{4} \mathrm{D}_{21 / 2}^{0} a^{2} \\
a^{2} \mathrm{G}_{41 / 2}-n^{2} \mathrm{G}_{41 / 2}^{0} \\
a^{4} \mathrm{~F}_{21 / 2}-v^{2} \mathrm{P}_{11 / 2}^{0}\end{array}$ & & & & & & \\
\hline $\begin{array}{c}2 \\
4 \\
6 \\
10 c \\
10 c\end{array}$ & $\begin{array}{l}2352.055 \\
2351.677 \\
2350.034 \\
2348.756 \\
2347.171\end{array}$ & $\begin{array}{l}42502.99 \\
42509.82 \\
42539.54 \\
42562.68 \\
42591.42\end{array}$ & $\begin{array}{c}a^{6} \mathrm{D}_{41 / 2}-n^{2} \mathrm{~F}_{31 / 2}^{0} \\
a^{4} \mathrm{P}_{01 / 2}-475_{11 / 2}^{\circ}\end{array}$ & & & & & & \\
\hline $\begin{array}{c}10 c \\
8 c \\
15 c \\
3 \\
10\end{array}$ & $\begin{array}{l}2346.679 \\
2344.640 \\
2344.517 \\
2341.152 \\
2340.277\end{array}$ & $\begin{array}{l}42600.34 \\
42637.39 \\
42639.63 \\
42700.92 \\
42716.87\end{array}$ & $\begin{array}{l}a^{6} \mathrm{D}_{31 / 2}-q^{4} \mathrm{D}_{31 / 2}^{0} \\
a^{6} \mathrm{D}_{31 / 2}-p^{4} \mathrm{~F}_{41 / 2}^{\circ} ?\end{array}$ & & & , & & & \\
\hline $\begin{array}{c}8 \\
4 \\
2 \\
20 c \\
7\end{array}$ & $\begin{array}{l}2340.149 \\
2338.730 \\
2338.38 \\
2337.744 \\
2336.410\end{array}$ & $\begin{array}{l}42719.21 \\
42745.12 \\
42751.5 \\
42763.16 \\
42787.57\end{array}$ & $\begin{array}{l}a^{6} \mathrm{D}_{31 / 2}-p^{4} \mathrm{D}_{31 / 2}^{0} \\
a^{4} \mathrm{~F}_{31 / 2}-0^{4} \mathrm{D}_{21 / 2}^{\circ}\end{array}$ & & & & & & \\
\hline $\begin{array}{r}10 \\
2 \\
5 \\
4 \\
3\end{array}$ & $\begin{array}{l}2333.650 \\
2329.543 \\
2328.222 \\
2328.077 \\
2326.964\end{array}$ & $\begin{array}{l}42838.16 \\
42913.68 \\
42938.03 \\
42940.70 \\
42961.24\end{array}$ & $\begin{array}{l}a^{4} \mathrm{~F}_{41 / 3}-p^{2} \mathrm{G}_{31 / 2}^{\circ} \\
a^{6} \mathrm{D}_{21 / 2}-q^{4} \mathrm{D}_{31 / 2}^{\circ} \\
a^{2} \mathrm{G}_{31 / 2}-n^{2} \mathrm{G}_{41 / 2}^{\circ}\end{array}$ & & & & & & \\
\hline $\begin{array}{l}4 \\
1 \\
3 \\
8 \\
4\end{array}$ & $\begin{array}{l}2324.600 \\
2323.959 \\
2323.654 \\
2322.992 \\
2321.489\end{array}$ & $\begin{array}{l}43004.93 \\
43016.79 \\
43022.43 \\
43034.69 \\
43062.54\end{array}$ & $\begin{array}{l}a^{4} \mathrm{~F}_{31 / 2}-0^{4} \mathrm{D}_{31 / 2}^{0} \\
a^{6} \mathrm{D}_{21 / 2}-p^{4} \mathrm{D}_{31 / 2}^{1}\end{array}$ & & & & & & \\
\hline
\end{tabular}


Table 4.-First spectrum of columbium (Cb I)-Continued

\begin{tabular}{|c|c|c|c|c|c|c|c|c|c|}
\hline $\begin{array}{l}\text { Intensity } \\
\text { Arc }\end{array}$ & $\lambda_{\text {sir }} \mathrm{A}$ & $\begin{array}{l}\text { Wave } \\
\text { No. } \\
\text { vac. } \\
\mathrm{cm}^{-1}\end{array}$ & $\underset{\text { nation }}{\text { Term combi- }}$ & $\begin{array}{l}\text { Zeeman } \\
\text { type }\end{array}$ & $\begin{array}{l}\text { Separa- } \\
\text { tion }\end{array}$ & $\begin{array}{l}\text { Strong- } \\
\text { est } \\
p\end{array}$ & $\begin{array}{c}\text { Strong- } \\
\text { est } \\
n\end{array}$ & $g_{1}$ & $g_{2}$ \\
\hline 1 & 2 & 3 & 4 & 5 & 6 & 7 & 8 & 9 & 10 \\
\hline $\begin{array}{l}5 \\
3 \\
4 \\
7 \\
3\end{array}$ & $\begin{array}{l}2318.432 \\
2316.271 \\
2314.359 \\
2311.676 \\
2307.759\end{array}$ & $\begin{array}{l}43119.33 \\
43159.55 \\
43195.21 \\
43245.33 \\
43318.72\end{array}$ & $a^{4} \mathrm{~F}_{21 / 2}-0{ }^{4} \mathrm{D}_{21 / 2}^{0}$ & & & & & & \\
\hline $\begin{array}{l}4 \\
3 \\
8 \\
6 \\
6\end{array}$ & $\begin{array}{l}2305.975 \\
2305.29 \\
2300.854 \\
2300.295 \\
2293.274\end{array}$ & $\begin{array}{l}43352.24 \\
43365.12 \\
43448.72 \\
43459.27 \\
43592.32\end{array}$ & $a^{4} \mathrm{~F}_{21 / 2}-0^{4} \mathrm{D}_{1 / 2}^{\circ}$ & & & & & & \\
\hline $\begin{array}{r}5 h \\
5 h \\
4 h \\
1 \\
2 \\
15\end{array}$ & $\begin{array}{l}2289.840 \\
2287.504 \\
2283.377 \\
2280.912 \\
2279.414 \\
2277.426\end{array}$ & $\begin{array}{l}43657.68 \\
43702.26 \\
43781.24 \\
43828.55 \\
43857.35 \\
43895.63\end{array}$ & $\begin{array}{l}a^{4} \mathrm{~F}_{31 / 2}-p^{2} \mathrm{G}_{41 / 2}^{\circ} \\
a^{4} \mathrm{~F}_{31 / 2}-r^{4} \mathrm{G}_{21 / 2}^{2}\end{array}$ & & & & & & \\
\hline $\begin{array}{l}4 c \\
9 c \\
4 \\
4 \\
3\end{array}$ & $\begin{array}{l}2276.223 \\
2274.770 \\
2269.653 \\
2269.535 \\
2268.59\end{array}$ & $\begin{array}{l}43918.83 \\
43946.88 \\
44045.95 \\
44048.24 \\
44066.6\end{array}$ & $a^{4} \mathrm{~F}_{41 / 2}-r^{4} \mathrm{G}_{41 / 2}^{0}$ & & & & & & \\
\hline $\begin{array}{r}2 \\
4 \\
3 \\
20 \\
160\end{array}$ & $\begin{array}{l}2265.489 \\
2265.217 \\
2264.354 \\
2260.854 \\
2257.886\end{array}$ & $\begin{array}{l}44126.89 \\
44132.19 \\
44149.01 \\
44217.35 \\
44275.47\end{array}$ & 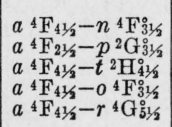 & & & & & & \\
\hline $\begin{array}{r}6 \\
5 \\
150 \\
8 \\
2\end{array}$ & $\begin{array}{l}2256.075 \\
2255.791 \\
2254.564 \\
2253.802 \\
2252.09\end{array}$ & $\begin{array}{l}44311.01 \\
44316.59 \\
44340.70 \\
44355.69 \\
44389.4\end{array}$ & $\begin{array}{l}a^{4} \mathrm{~F}_{31 / 2}-r^{4} \mathrm{G}_{31 / 2}^{\circ} \\
a^{4} \mathrm{~F}_{41 / 2}-n^{4} \mathrm{~F}_{41 / 2}^{0} \\
a^{4} \mathrm{~F}_{31 / 2}-n^{4} \mathrm{~F}_{21 / 2}^{2} \\
a^{4} \mathrm{~F}_{31 / 2}-0^{4} \mathrm{~F}_{21 / 2}^{2}\end{array}$ & & & & & & \\
\hline $\begin{array}{c}4 c \\
100 c \\
80 \\
8 c \\
90 c\end{array}$ & $\begin{array}{l}2252.51 \\
2250.308 \\
2247.997 \\
2246.421 \\
2246.176\end{array}$ & $\begin{array}{l}44400.8 \\
44424.56 \\
44470.22 \\
44501.42 \\
44506.26\end{array}$ & $\begin{array}{l}a^{4} \mathrm{~F}_{21 / 2}-r^{4} \mathrm{G}_{21 / 2}^{0} \\
a^{4} \mathrm{~F}_{41 / 2}-n^{4} \mathrm{D}_{31 / 2}^{0}\end{array}$ & & & & & & \\
\hline $\begin{array}{l}20 \\
20 \\
8 h \\
80 c \\
10\end{array}$ & $\begin{array}{l}2242.958 \\
2242.294 \\
2241.855 \\
2238.518 \\
2236.22\end{array}$ & $\begin{array}{l}44570.11 \\
44583.31 \\
44592.04 \\
44658.51 \\
44704.40\end{array}$ & $\begin{array}{l}a^{4} \mathrm{~F}_{31 / 2}-r^{4} \mathrm{G}_{41 / 2}^{\circ} \\
a^{4} \mathrm{~F}_{21 / 2}-0^{4} \mathrm{~F}_{11 / 2}^{0} \\
a^{4} \mathrm{~F}_{31 / 2}-n^{4} \mathrm{D}_{21 / 2}^{\circ}\end{array}$ & & & & & & \\
\hline 9 & 2233. 172 & 44765.40 & $a^{4} \mathrm{~F}_{31 / 2}-469_{21 / 3}^{\circ}$ & & & & & & \\
\hline $80 c$ & 2232.545 & 44777.97 & $\left\{\begin{array}{l}a^{4} \mathrm{~F}^{3} 31 / 2-n^{4} \mathrm{~F}^{3} \mathrm{~F}_{31 / 2} \\
a^{4} \mathrm{~F}_{21 / 2}-n^{4} \mathrm{D}_{11 / 1}\end{array}\right.$ & & & & & & \\
\hline $\begin{array}{r}8 \\
30 \\
4\end{array}$ & $\begin{array}{l}2231.428 \\
2229.65 \\
2228.39\end{array}$ & $\begin{array}{l}44800.39 \\
44836.11 \\
44861.46\end{array}$ & $\begin{array}{l}a^{4} \mathrm{~F}_{31 / 2}-t^{2} \mathrm{H}_{41 / 2}^{0} \\
a^{4} \mathrm{~F}_{11 / 2}-n^{4} \mathrm{D}_{01 / 3}^{01 / 2} \\
a^{4} \mathrm{~F}_{11 / 2}-v^{2} \mathrm{P}_{01 / 2}^{0}\end{array}$ & & & & & & \\
\hline $100 c$ & 2228.032 & 44868.69 & $\left\{\begin{array}{l}a^{4} \mathrm{~F}_{11 / 2}-r{ }^{4} \mathrm{G}_{21 / 2}^{0} \\
a^{4} \mathrm{~F}_{31 / 2}-0^{4} \mathrm{~F}_{31 / 2}^{2}\end{array}\right.$ & & & & & & \\
\hline $\begin{array}{c}150 c \\
10 \\
15 \\
50\end{array}$ & $\begin{array}{l}2227.706 \\
2227.280 \\
2226.927 \\
2225.343\end{array}$ & $\begin{array}{l}44875.23 \\
44883.82 \\
44890.93 \\
44922.88\end{array}$ & $\begin{array}{l}a^{4} \mathrm{~F}_{41 / 2}-0^{4} \mathrm{~F}_{41 / 3}^{0} \\
a^{4} \mathrm{~F}_{21 / 2}-r^{4} \mathrm{G}_{31 / 2}^{1} \\
a^{4} \mathrm{~F}_{21 / 2}-n{ }^{4} \mathrm{~F}_{21 / 2}^{0}\end{array}$ & & & & & & \\
\hline $\begin{array}{c}6 \\
60 c \\
70 c \\
3 \\
15\end{array}$ & $\begin{array}{l}2225.235 \\
2223.672 \\
2220.184 \\
2218.357 \\
2217.872\end{array}$ & $\begin{array}{l}44925.06 \\
44956.62 \\
45027.25 \\
45064.33 \\
45074.19\end{array}$ & $\begin{array}{l}a^{4} \mathrm{~F}_{21 / 2}-0{ }^{4} \mathrm{~F}_{21 / 2}^{0} \\
a^{4} \mathrm{~F}_{1 / 2}-0{ }^{4} \mathrm{~F}_{11 / 2}^{1}\end{array}$ & & & & & & \\
\hline $\begin{array}{c}30 c \\
40 c \\
50 c \\
7 \\
6\end{array}$ & $\begin{array}{l}2215.54 \\
2214.034 \\
2211.46 \\
2210.622 \\
2210.442\end{array}$ & $\begin{array}{l}45121.6 \\
45152.31 \\
45204.9 \\
45222.00 \\
45225.68\end{array}$ & $\begin{array}{l}a^{4} \mathrm{~F}_{31 / 2}-n^{4} \mathrm{D}_{31 / 2}^{0} \\
a^{4} \mathrm{~F}_{11 / 2}-n^{4} \mathrm{D}_{11 / 2}^{0} \\
a^{4} \mathrm{~F}_{21 / 2}-n^{4} \mathrm{D}_{21 / 2}^{2}\end{array}$ & & & & & & \\
\hline
\end{tabular}


TABLE 4.-First spectrum of columbium (Cb I)-Continued

\begin{tabular}{|c|c|c|c|c|c|c|c|c|c|}
\hline $\begin{array}{l}\text { Intensity } \\
\text { Arc }\end{array}$ & $\lambda_{\text {air }} \mathrm{A}$ & $\begin{array}{l}\text { Wave } \\
\text { No. } \\
\text { vac. } \\
\mathrm{cm}^{-1}\end{array}$ & $\begin{array}{l}\text { Term combi- } \\
\text { nation }\end{array}$ & $\begin{array}{c}\text { Zeeman } \\
\text { type }\end{array}$ & $\begin{array}{l}\text { Separa- } \\
\text { tion }\end{array}$ & $\begin{array}{c}\text { Strong- } \\
\text { est } \\
p\end{array}$ & $\begin{array}{c}\text { Strong- } \\
\text { est } \\
n\end{array}$ & $g_{1}$ & $g_{2}$ \\
\hline 1 & 2 & 3 & 4 & 5 & 6 & 7 & 7 & 8 & 10 \\
\hline $\begin{array}{r}2 \\
12 \\
3 \\
3 \\
4\end{array}$ & $\begin{array}{l}2205.230 \\
2204.617 \\
2203.555 \\
2201.916 \\
2200.218\end{array}$ & $\begin{array}{l}45332.56 \\
45345.16 \\
45367.01 \\
45400.78 \\
45435.81\end{array}$ & $\begin{array}{l}a^{4} \mathrm{~F}_{21 / 2}-469_{21 / 2}^{\circ} \\
a^{4} \mathrm{~F}_{21 / 2}-n^{4} \mathrm{~F}_{31 / 2}^{\circ} \\
a^{4} \mathrm{~F}_{11 / 2}-n^{4} \mathrm{~F}_{21 / 2}^{2} \\
a^{4} \mathrm{~F}_{11 / 2}-o^{4} \mathrm{~F}_{21 / 2}^{\circ} \\
a^{4} \mathrm{~F}_{21 / 2}-0^{4} \mathrm{~F}_{31 / 2}^{0}\end{array}$ & & & & & & \\
\hline $\begin{array}{r}3 \\
10 \\
8 \\
7 \\
4\end{array}$ & $\begin{array}{l}2195.83 \\
2193.805 \\
2193.011 \\
2188.944 \\
2178.225\end{array}$ & $\begin{array}{l}45526.6 \\
45568.61 \\
45585.11 \\
45669.80 \\
45894.51\end{array}$ & $\begin{array}{l}a^{4} \mathrm{~F}_{31 / 2}-0^{4} \mathrm{~F}_{41 / 2}^{0} \\
a^{4} \mathrm{~F}_{11 / 2}-n^{4} \mathrm{D}_{21 / 2}^{\circ}\end{array}$ & & & & & & \\
\hline $\begin{array}{l}3 \\
8 \\
5 \\
5\end{array}$ & $\begin{array}{l}2178.07 \\
2175.555 \\
2166.77 \\
2161.54\end{array}$ & $\begin{array}{l}45897.8 \\
45950.83 \\
46137.1 \\
46248.7\end{array}$ & $a^{4} \mathrm{~F}_{21 / 2}-475_{1 / 2}^{\circ}$ & & & & & & \\
\hline
\end{tabular}

Of particular interest is the large number of intersystem transitions, doublet-quartet combinations being second in abundance only to quartet-quartet. As in V I [7] doublet-sextet combinations appear in $\mathrm{Cb} \mathrm{I}$, but more abundantly. These latter include the transition $a^{6} \mathrm{D}-z^{2} \mathrm{G}^{\circ}$, a conspicuous five-line multiplet, all lines of which show fully resolved Zeeman patterns. The wavelengths are 3867.918, $3815.507,3803.879,3771.848$, and $3753.171 \mathrm{~A}$. See figures 1 and 2.

TABLE 5.-Distribution of classified $\mathrm{Cb}$ r lines

\begin{tabular}{|c|c|c|c|}
\hline Item & Number & $\begin{array}{l}\text { Percent- } \\
\text { age of } \\
\text { total } \\
\text { number }\end{array}$ & $\begin{array}{l}\text { Percent- } \\
\text { age of } \\
\text { total } \\
\text { intensity }\end{array}$ \\
\hline Lines in table 4 & 3,313 & Percent & Percent \\
\hline Classified lines & 2,836 & 85.6 & $93 . \overline{4}$ \\
\hline Level transitions & 3,035 & & 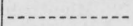 \\
\hline Sextet-sextet transitions & 137 & 4. 5 & 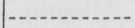 \\
\hline Quartet-quartet transitions & 1,276 & 42.1 & 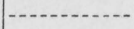 \\
\hline Doublet-doublet transitions & . 405 & 13. 3 & (-............. \\
\hline $\begin{array}{l}\text { Quartet-sextet transitions. } \\
\text { Doublet-quartet transitions }\end{array}$ & $\begin{array}{l}253 \\
814\end{array}$ & $\begin{array}{r}8.3 \\
26.8\end{array}$ & (-n-n \\
\hline Doublet-sextet transitions & 57 & 1. 9 & 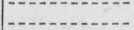 \\
\hline Transitions involving one unassigned level & 92 & 3.0 & 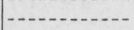 \\
\hline Lines classified two ways & 191 & -....- & 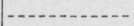 \\
\hline Lines classified three ways & 3 & $-\ldots+n$ & -................ \\
\hline $\begin{array}{l}\text { Zeeman patterns measured } \\
\text { Zeeman patterns interpreted }\end{array}$ & $\begin{array}{l}911 \\
815\end{array}$ & 89.5 & (1) \\
\hline & & & \\
\hline
\end{tabular}

\section{TERMS OF THE Cb I SPECTRUM}

The established terms of $\mathrm{Cb}$ I, or energy states of neutral columbium, are described in table 6, which is prepared according to the same plan as table 2 for $\mathrm{Cb}$ II. All level values are relative to $4 d^{4} 5 s a^{6} \mathrm{D}_{01 / 2}=0.00$. The total number of levels is 364, comprising 103 belonging to 58 doublets, 197 which make up 55 quartets, 56 which are associated with 13 sextets, and 8 unassigned levels. Levels associated with almost all terms come in regular order, that is, those of highest $j$-value are highest. There are five inverted doublets and five partially inverted quartets. Fifteen terms are incomplete. Instances of 
good agreement with the interval rule are in the minority. This is to be expected in the spectrum of an atom with a moderately heavy nucleus with level separations large enough that most terms overlap, a situation which leads to mutual perturbation and displacement of levels of the same $j$-value associated with sharing of $g$-values. Attention is called to a few noteworthy instances of such perturbation. Levels $a^{2} \mathrm{P}_{11 / 2}$, and $a^{2} \mathrm{D}_{11 / 2}$ have $g$-values 1.175 and 0.953 as compared with Landé $g$ 's, 1.333 and 0.800 , respectively. The sums are almost identical, 2.128 and 2.133. Levels $a^{2} \mathrm{P}_{01 / 2}$ and $a^{2} \mathrm{D}_{21 / 2}$ have nearly Landé $g$-values. The levels of $j$-values $2 \frac{1}{2}, 3 \frac{1}{2}$, and $4 \frac{1}{2}$ belonging to $a^{4} \mathrm{G}$ and $b^{4} \mathrm{~F}$ perturb one another to such an extent that it is difficult to distinguish between them on the basis of combinations or $g$-values although there is no doubt that these levels comprise the terms in question. The levels $b{ }^{4} \mathrm{~F}_{11 / 2}$ and $a^{4} \mathrm{G}_{51 / 2}$ being unique values are not so affected and again show nearly Landé $g$ 's. A very marked perturbation occurs in the levels $x^{6} \mathrm{D}_{01 / 2}^{\circ}$ and $y{ }^{4} \mathrm{D}_{01 / 2}^{\circ}$, the $g$-values being 2.441 and 1.141 , respectively instead of 3.333 and 0.000 theoretically expected. It may be assumed that these levels originate in the same electron configuration. Hence $x^{6} \mathrm{D}$ is assigned to $d^{4} p$ instead of $d^{3} s p$, interchanging the assignment with $y^{6} \mathrm{D}^{\circ}$, as given in the earlier paper [5]. The term formerly classified $z^{4} \mathrm{D}^{\circ}$ is now designated $z^{6} \mathrm{~F}^{\circ}$, with an additional level, $j=41 / 2$, found, but with the level of highest $j$ missing. The $g$-values favor the new assignment, and there is no other possibility for this sextet, which is expected to show strong combinations. These are almost the only instances of changes in earlier assignments. In using the scheme of designating intermediate odd terms in ascending order by letters beginning with $z$, the discovery of a large number of new terms has necessitated a reassignment of such letters for identification. Relative level values as set up in the earlier publication have been retained. Calculations based on additional wavelength data indicate negligible corrections.

TABLE 6.-Terms of the Cb I spectrum

\begin{tabular}{|c|c|c|c|c|c|}
\hline $\begin{array}{l}\text { Electron } \\
\text { configu- } \\
\text { ration }\end{array}$ & $\begin{array}{c}\text { Term } \\
\text { symbol }\end{array}$ & Level & $\begin{array}{l}\text { Differ- } \\
\text { ence }\end{array}$ & $\underset{\boldsymbol{g}}{\text { Obserred }}$ & Combinations \\
\hline$d{ }^{4} s\left({ }^{b} \mathrm{D}\right)$ & $\begin{array}{l}a^{6} \mathrm{D}_{01 / 2} \\
a^{6} \mathrm{D}_{11 / 2} \\
a^{6} \mathrm{D}_{21 / 2} \\
a^{6} \mathrm{D}_{31 / 2} \\
a^{6} \mathrm{D}_{41 / 2}\end{array}$ & $\begin{array}{r}0.00 \\
154.19 \\
391.99 \\
695.25 \\
1050.26\end{array}$ & $\begin{array}{l}154.19 \\
237.80 \\
303.26 \\
355.01\end{array}$ & $\begin{array}{l}\text { 3. } 323 \\
1.863 \\
\text { 1. } 652 \\
1.582 \\
1.549\end{array}$ & 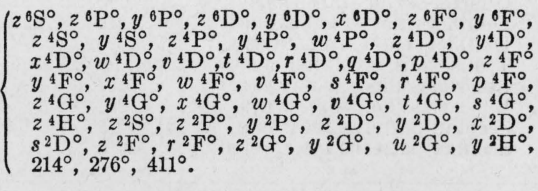 \\
\hline$d^{3} \mathrm{~S}^{2}$ & $\begin{array}{l}a^{4} \mathrm{~F}_{11 / 2} \\
a^{4} \mathrm{~F}_{21 / 2} \\
a^{4} \mathrm{~F}_{31 / 2} \\
a^{4} \mathrm{~F}_{4 / 3}\end{array}$ & $\begin{array}{l}1142.79 \\
1586.90 \\
2154.11 \\
2805.36\end{array}$ & $\begin{array}{l}444.11 \\
567.21 \\
651.25\end{array}$ & $\begin{array}{l}0.402 \\
1.029 \\
1.235 \\
1.330\end{array}$ & 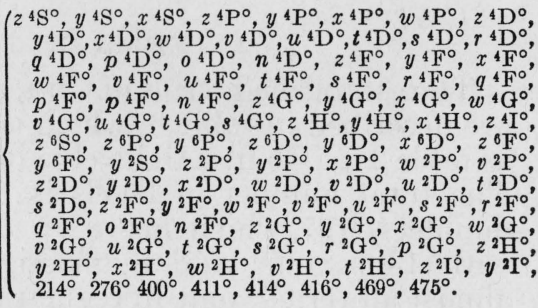 \\
\hline
\end{tabular}


TABLE 6.-Terms of the Cb I spectrum-Continued

\begin{tabular}{|c|c|c|c|c|c|}
\hline $\begin{array}{l}\text { Electron } \\
\text { configu- } \\
\text { ration }\end{array}$ & $\begin{array}{c}\text { Term } \\
\text { symbol }\end{array}$ & Level & $\begin{array}{l}\text { Differ- } \\
\text { ence }\end{array}$ & $\underset{g}{\text { Observed }}$ & Combinations \\
\hline$d^{3} s^{2}$ & $\begin{array}{l}a{ }^{4} \mathrm{P}_{01 / 6} \\
a^{4} \mathrm{P} 1 / 1 / 2 \\
a^{4} \mathrm{P}^{4} \mathrm{P}_{2 / 2}\end{array}$ & $\begin{array}{l}4998.17 \\
5297.92 \\
5965.45\end{array}$ & $\begin{array}{l}299.75 \\
667.53\end{array}$ & $\begin{array}{l}2.650 \\
1.721 \\
1.596\end{array}$ & 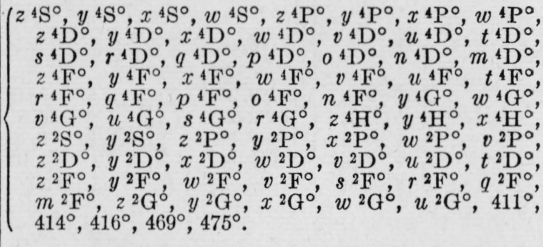 \\
\hline$d^{4} s\left({ }^{(5 D)}\right.$ & $\begin{array}{l}a^{4} \mathrm{D}_{01 / 5} \\
a^{4} \mathrm{D}_{11 / 5} \\
a^{4} \mathrm{D}_{21 / 5} \\
a^{4} \mathrm{D}_{31 / 2}\end{array}$ & $\begin{array}{l}8410.90 \\
8705.32 \\
9043.14 \\
9497.52\end{array}$ & $\begin{array}{l}294.42 \\
337.82 \\
454.38\end{array}$ & $\begin{array}{l}0.06 \\
1.197 \\
1.360 \\
1.420\end{array}$ & 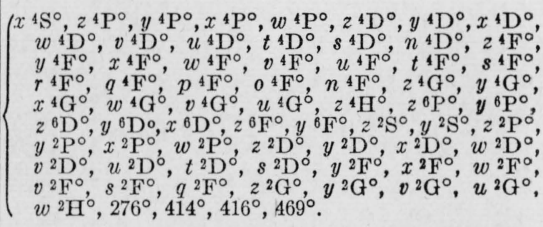 \\
\hline$d^{3} s^{2}$ & $\begin{array}{l}a^{2} \mathrm{I}_{31 / 5} \\
a^{2} \mathrm{G}_{4 / 2}\end{array}$ & $\begin{array}{l}8827.00 \\
9328.88\end{array}$ & 501.88 & $\begin{array}{l}0.885 \\
1.103\end{array}$ & 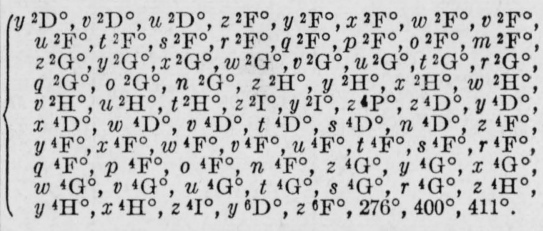 \\
\hline$d^{3} s^{2}$ & $\begin{array}{l}a{ }^{2} \mathrm{D}_{11 / 6} \\
a^{2} \mathrm{D}_{2 / 5}\end{array}$ & $\begin{array}{r}9439.08 \\
10237.51\end{array}$ & 798.43 & $\begin{array}{l}0.953 \\
1.206\end{array}$ & 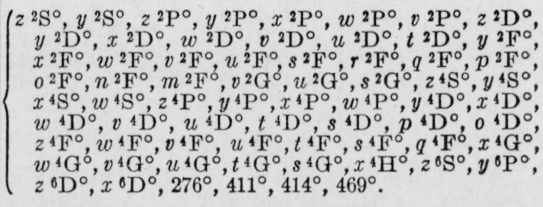 \\
\hline$d^{3} s^{2}$ & $\begin{array}{l}a{ }^{2} \mathrm{P}_{01 / 6} \\
a^{2} \mathrm{P}_{1 / 2}\end{array}$ & $\begin{array}{l}10126.06 \\
11318.09\end{array}$ & 1192.03 & $\begin{array}{l}0.66 \\
1.175\end{array}$ & 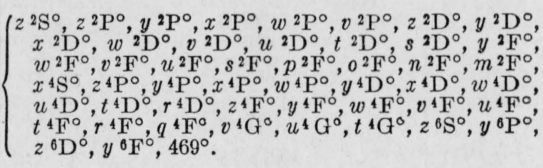 \\
\hline$d^{b}$ & $a{ }^{b} \mathrm{~S}_{21 / 6}$ & 11344.70 & & 1. 99 & $\left\{\begin{array}{c}z^{\mathrm{B}} \mathrm{P}^{\circ}, y^{6} \mathrm{P}^{\circ}, y{ }^{\circ} \mathrm{F}^{\circ}, z^{4} \mathrm{~S}^{\circ}, y^{4} \mathrm{P}^{\circ}, w{ }^{4} \mathrm{P}^{\circ}, v^{4} \mathrm{D}^{\circ}, w^{4} \mathrm{D}^{\circ}, z^{2} \mathrm{P}^{\circ}, x^{2} \mathrm{P}^{\circ}, y^{2} \mathrm{D}^{\circ}, v^{2} \mathrm{~F}^{\circ} .\end{array}\right.$ \\
\hline$d^{4} \&\left({ }^{3} \mathrm{H}\right)$ & $\begin{array}{l}a^{4} \mathrm{H}_{31 / 2} \\
a^{4} \mathrm{H}_{41 / 2} \\
a^{4} \mathrm{H}_{51 / 2} \\
a^{4} \mathrm{H}_{61 / 2}\end{array}$ & $\begin{array}{l}10922.74 \\
11044.08 \\
11247.88 \\
11524.65\end{array}$ & $\begin{array}{l}121.34 \\
203.80 \\
276.77\end{array}$ & $\begin{array}{l}0.690 \\
0.984 \\
1.12 \\
1.22\end{array}$ & 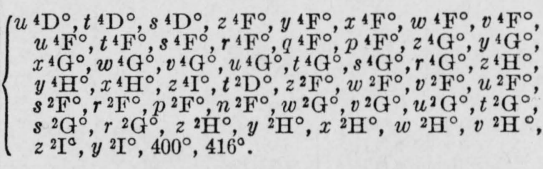 \\
\hline$d^{4} s\left({ }^{3} G\right)$ & $\begin{array}{l}a^{4}{ }^{4} \mathrm{G}_{21 / 2} \\
a^{4} \mathrm{G}_{31 / 2} \\
a^{4} \mathrm{G}_{41 / 5} \\
a^{4} \mathrm{G}_{51 / 5}\end{array}$ & $\begin{array}{l}12018.25 \\
12136.86 \\
12357.70 \\
13012.20\end{array}$ & $\begin{array}{l}118.61 \\
220.84 \\
654.50\end{array}$ & $\begin{array}{l}0.742 \\
1.081 \\
1.23 \\
1.26\end{array}$ & 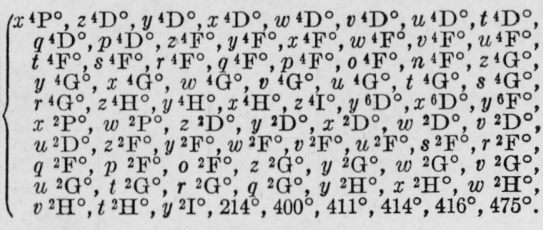 \\
\hline$d^{3} s^{2}$ & $\begin{array}{l}a^{2} \mathrm{H}_{41 / 2} \\
a^{2} \mathrm{H}_{515}\end{array}$ & $\begin{array}{l}12102.12 \\
12502.97\end{array}$ & 400.85 & $\begin{array}{l}0.93 \\
1.10\end{array}$ & 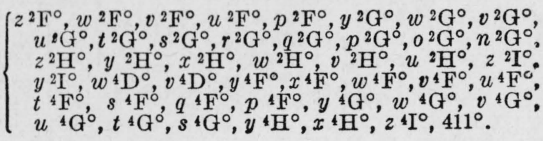 \\
\hline
\end{tabular}


574 Journal of Research of the National Bureau of Standards

TABLE 6.-Terms of the Cb I spectrum-Continued

\begin{tabular}{|c|c|c|c|c|c|}
\hline $\begin{array}{l}\text { Electron } \\
\text { configu- } \\
\text { ration }\end{array}$ & $\begin{array}{c}\text { Term } \\
\text { symbol }\end{array}$ & Level & $\begin{array}{l}\text { Differ- } \\
\text { ence. }\end{array}$ & $\begin{array}{c}\text { Observed } \\
g\end{array}$ & Combinations \\
\hline$d^{4} s\left({ }^{3} \mathrm{~F}\right)$ & $\begin{array}{l}b^{4} \mathrm{~F}_{11 / 2} \\
b^{4} \mathrm{~F}_{21 / 2} \\
b^{4} \mathrm{~F}_{31 / 2} \\
b^{4} \mathrm{~F}_{41 / 2}\end{array}$ & $\begin{array}{l}12288.25 \\
12692.12 \\
12982.38 \\
13145.71\end{array}$ & $\begin{array}{l}403.87 \\
290.26 \\
163.33\end{array}$ & $\begin{array}{l}0.402 \\
0.852 \\
1.120 \\
1.224\end{array}$ & 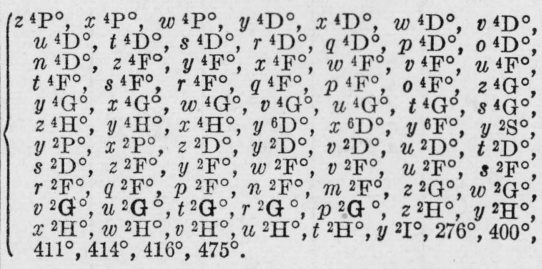 \\
\hline$d^{3} s^{2}$ & $\begin{array}{l}a{ }^{2} \mathrm{~F}_{21 / 2} \\
a^{2} \mathrm{~F}_{31 / 2}\end{array}$ & $\begin{array}{l}13404.77 \\
13515.20\end{array}$ & 110.43 & $\begin{array}{l}0.860 \\
1.130\end{array}$ & 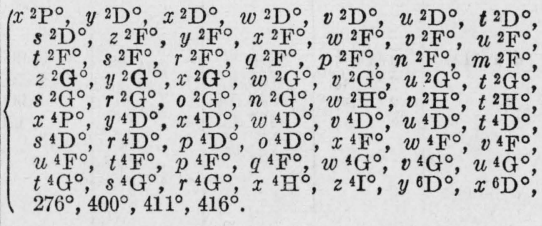 \\
\hline$d^{4} s\left({ }^{3} \mathrm{P}\right)$ & $\begin{array}{l}b{ }^{4} \mathrm{P}_{01 / 2} \\
b{ }^{4} \mathrm{P}_{11 / 2} \\
b^{4} \mathrm{P}_{21 / 2}\end{array}$ & $\begin{array}{l}13629.15 \\
14211.30 \\
14899.26\end{array}$ & $\begin{array}{l}582.15 \\
687.96\end{array}$ & $\begin{array}{l}2.64 \\
1.71 \\
1.54\end{array}$ & 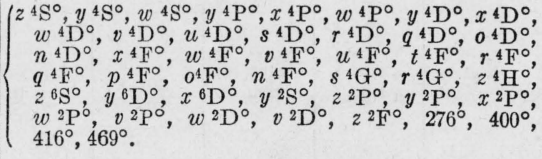 \\
\hline$d^{4} s\left({ }^{3} \mathrm{D}\right)$ & $\begin{array}{l}b^{4} \mathrm{~A}_{01 / 5} \\
b^{4} \mathrm{D}_{1 / 3} \\
b^{4} \mathrm{D}_{21 / 5} \\
b^{4} \mathrm{D}_{31 / 2}\end{array}$ & $\begin{array}{l}15460.77 \\
15439.25 \\
15467.08 \\
15282.35\end{array}$ & $\begin{array}{r}-21.52 \\
27.83 \\
-184.73\end{array}$ & $\begin{array}{l}0.04 \\
1.21 \\
1.42 \\
1.43\end{array}$ & 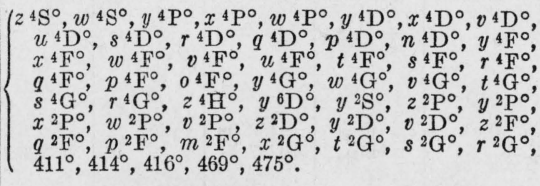 \\
\hline$d^{4} s\left({ }^{3} \mathrm{H}\right)$ & $\begin{array}{l}b^{2} \mathrm{H}_{4 / 1} \\
b^{2} \mathrm{H}_{51 / 2}\end{array}$ & $\begin{array}{l}16828.52 \\
17476.22\end{array}$ & 647.70 & $\begin{array}{l}1.04 \\
1.01\end{array}$ & $\left\{\begin{array}{l}w^{2} \mathrm{~F}^{\circ}, r^{2} \mathrm{~F}^{\circ}, g^{2} \mathrm{~F}^{\circ}, p^{2} \mathrm{~F}^{\circ}, m{ }^{2} \mathrm{~F}^{\circ}, z^{2} \mathrm{G}^{\circ}, y^{2} \mathrm{G}^{\circ}, \\
x^{2} \mathrm{G}^{\circ}, w^{2} \mathrm{G}^{\circ}, v^{2} \mathrm{G}^{\circ}, u^{2} \mathrm{G}^{\circ}, t^{2} \mathrm{G}^{\circ}, s^{2} \mathrm{G}^{\circ}, r^{2} \mathrm{G}^{\circ}, \\
p^{2} \mathrm{G}^{\circ}, 0^{2} \mathrm{G}^{\circ}, z^{2} \mathrm{H}^{\circ}, y^{2} \mathrm{H}^{\circ}, x^{2} \mathrm{H}^{\circ}, w^{2} \mathrm{H}^{\circ}, v^{2} \mathrm{H}^{\circ}, \\
u^{2} \mathrm{H}^{\circ}, t^{2} \mathrm{H}^{\circ}, z^{2} \mathrm{I}^{\circ}, y^{2} \mathrm{I}^{\circ}, q^{4} \mathrm{D}^{\circ}, y 4 \mathrm{~F}^{\circ}, x^{4} \mathrm{~F}^{\circ}, t^{4} \mathrm{~F}^{\circ}, \\
r^{4} \mathrm{~F}^{\circ}, q^{4} \mathrm{~F}^{\circ}, w^{4} \mathrm{G}^{\circ}, v v^{4} \mathrm{G}^{\circ}, u^{4} \mathrm{G}^{\circ}, t^{4} \mathrm{G}^{\circ}, s^{4} \mathrm{G}^{\circ}, \\
y^{4} \mathrm{H}^{\circ}, x^{4} \mathrm{H}^{\circ}, 411^{\circ} .\end{array}\right.$ \\
\hline$d^{4} s\left({ }^{3} \mathrm{G}\right)$ & $\begin{array}{l}b^{2} \mathrm{G}_{31 / 2} \\
b^{2} \mathrm{G}_{41 / 2}\end{array}$ & $\begin{array}{l}16918.78 \\
18035.97\end{array}$ & 1117. 19 & $\begin{array}{l}0.88 \\
0.97\end{array}$ & 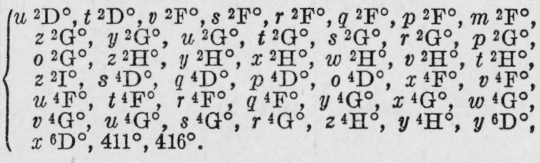 \\
\hline & $183 b 1 / 2$ & 18332.04 & & & $\left\{\begin{array}{l}y^{2} \mathrm{G}^{\circ}, w^{2} \mathrm{G}^{\circ}, t^{2} \mathrm{G}^{\circ}, s^{2} \mathrm{G}^{\circ}, r^{2} \mathrm{G}^{\circ}, o^{2} \mathrm{G}^{\circ}, n^{2} \mathrm{G}^{\circ}, \\
z^{2} \mathrm{H}^{\circ}, y \mathrm{y}^{2} \mathrm{H}^{\circ}, x^{2} \mathrm{H}^{\circ}, w^{2} \mathrm{H}^{\circ}, v^{2} \mathrm{H}^{\circ}, z^{2} \mathrm{I}^{\circ}, v^{4} \mathrm{~F}^{\circ}, \\
u^{4} \mathrm{~F}^{\circ}, s^{4} \mathrm{~F}^{\circ}, x^{4} \mathrm{G}^{\circ}, t^{4} \mathrm{G}^{\circ}, r^{4} \mathrm{G}^{\circ} .\end{array}\right.$ \\
\hline$d^{3} s p\left({ }^{5} \mathrm{~F}\right)$ & 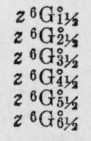 & $\begin{array}{l}16672.00 \\
16981.01 \\
17303.96 \\
17937.26 \\
18435.14 \\
18876.46\end{array}$ & $\begin{array}{l}309.01 \\
322.95 \\
633.30 \\
497.88 \\
441.32\end{array}$ & 0.00 & $e^{6 \mathrm{~F}}$. \\
\hline$d^{3} s p\left({ }^{5} \mathrm{~F}\right)$ & $\begin{array}{l}z^{6} \mathrm{~F}_{01 / 2}^{\circ} \\
z^{6} \mathrm{~F}_{11 / 2}^{\circ} \\
z^{6} \mathrm{~F}_{21 / 2}^{2} \\
z^{6} \mathrm{~F}_{31 / 2}^{3} \\
z^{0} \mathrm{~F}_{41 / 2}^{31} \\
z^{6} \mathrm{~F}_{51 / 2}^{0}\end{array}$ & $\begin{array}{l}18791.09 \\
19036.55 \\
19427.90 \\
19916.69 \\
20438.11\end{array}$ & $\begin{array}{l}245.46 \\
391.35 \\
488.79 \\
515.42\end{array}$ & $\begin{array}{c}-0.373 \\
1.145 \\
1.35 \\
1.39 \\
1.44\end{array}$ & $a^{6} \mathrm{D}, e^{6} \mathrm{D}, e^{6} \mathrm{~F}, a^{4} \mathrm{P}, a^{4} \mathrm{D}, a^{4} \mathrm{~F}, a^{2} \mathrm{G}$. \\
\hline$d^{3} s p\left({ }^{8} \mathrm{~F}\right)$ & 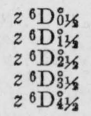 & $\begin{array}{l}19623.96 \\
19765.15 \\
19993.78 \\
20315.74 \\
20733.88\end{array}$ & $\begin{array}{l}141.22 \\
228.60 \\
321.96 \\
418.14\end{array}$ & $\begin{array}{l}3.01 \\
1.72 \\
1.56 \\
1.55 \\
1.54\end{array}$ & $a^{6} \mathrm{D}, e^{6} \mathrm{~F}, a^{4} \mathrm{P}, a^{4} \mathrm{D}, a^{4} \mathrm{~F}$. \\
\hline
\end{tabular}


TABLE 6.-Terms of the Cb I spectrum-Continued

\begin{tabular}{|c|c|c|c|c|c|}
\hline $\begin{array}{l}\text { Electron } \\
\text { configu- } \\
\text { ration }\end{array}$ & $\begin{array}{l}\text { Term } \\
\text { symbol }\end{array}$ & Level & $\begin{array}{l}\text { Differ- } \\
\text { ence }\end{array}$ & $\begin{array}{c}\text { Observed } \\
g\end{array}$ & Combinations \\
\hline$d^{3} s p\left({ }^{3} \mathrm{~F}\right)$ & $\begin{array}{l}z{ }^{4} \mathrm{D}_{0143}^{\circ} \\
z^{4} \mathrm{D}_{11 / 3}^{\circ} \\
z^{4} \mathrm{D}_{21 / 2}^{\circ} \\
z^{4} \mathrm{D}_{31 / 3}^{\circ}\end{array}$ & $\begin{array}{l}2010 \% .36 \\
20383.62 \\
2083 \% .98 \\
21512.18\end{array}$ & $\begin{array}{l}276.26 \\
454.36 \\
674.20\end{array}$ & $\begin{array}{r}-0.02 \\
1.26 \\
1.39 \\
1.44\end{array}$ & $a^{4} \mathrm{P}, a^{4} \mathrm{D}, a^{4} \mathrm{~F}, a^{4} \mathrm{G}, a^{6} \mathrm{D}, a^{2} \mathrm{G}$ \\
\hline$d^{4} p\left({ }^{5} \mathrm{D}\right)$ & $\begin{array}{l}z 4 \mathrm{P}_{01 / 2}^{0} \\
z^{4} \mathrm{P}_{11 / 2}^{011} \\
z \\
{ }^{4} \mathrm{P}_{21 / 2}^{0}\end{array}$ & $\begin{array}{l}22006.74 \\
23006.86 \\
23684.44\end{array}$ & $\begin{array}{r}1000.12 \\
677.58\end{array}$ & $\begin{array}{l}2.47 \\
1.61 \\
1.477\end{array}$ & $a^{4} \mathrm{P}, a^{4} \mathrm{D}, a^{4} \mathrm{~F}, a^{6} \mathrm{D}, a^{2} \mathrm{P}, a^{2} \mathrm{D}$ \\
\hline$d^{3} s p\left({ }^{3} \mathrm{~F}\right)$ & $\begin{array}{l}z^{4} \mathrm{G}_{21 / 2}^{0} \\
z^{4} \mathrm{G}_{31 / 2}^{0} \\
z^{4} \mathrm{G}_{41 / 2}^{\circ} \\
z^{4} \mathrm{G}_{51 / 2}^{0}\end{array}$ & $\begin{array}{l}22647.03 \\
23022.56 \\
23536.77 \\
24203.05\end{array}$ & $\begin{array}{l}375.53 \\
514.21 \\
666.28\end{array}$ & $\begin{array}{l}0.578 \\
0.98 \\
1.15 \\
1.25\end{array}$ & $a^{4} \mathrm{D}, a^{4} \mathrm{~F}, b^{4} \mathrm{~F}, a^{4} \mathrm{G}, a^{4} \mathrm{H}, a^{6} \mathrm{D}, a^{2} \mathrm{G}$. \\
\hline$d^{3} s p\left({ }^{3} \mathrm{~F}\right)$ & $\begin{array}{l}z^{4} \mathrm{~F}_{11 / 3}^{0} \\
z^{4} \mathrm{~F}_{21 / 2}^{0} \\
z^{4} \mathrm{~F}_{31 / 2}^{0} \\
z^{4} \mathrm{~F}_{41 / 3}^{0}\end{array}$ & $\begin{array}{l}23248.87 \\
23574.14 \\
24015.11 \\
24506.53\end{array}$ & $\begin{array}{l}330.27 \\
440.97 \\
491.42\end{array}$ & $\begin{array}{l}0.416 \\
1.061 \\
1.243 \\
1.336\end{array}$ & $\begin{array}{l}a^{4} \mathrm{P}, a^{4} \mathrm{D}, a^{4} \mathrm{~F}, b^{4} \mathrm{~F}, a^{4} \mathrm{G}, a^{4} \mathrm{H}, a^{6} \mathrm{D}, a^{2} \mathrm{P}, a^{2} \mathrm{D}, \\
a^{2} \mathrm{G} .\end{array}$ \\
\hline$d^{4} p\left({ }^{3} \mathrm{P}\right)$ & $\begin{array}{l}z^{2} \mathrm{D}_{11 / 2} \\
z^{2} \mathrm{D}_{21 / 2}^{\circ}\end{array}$ & $\begin{array}{l}23525.80 \\
24773.03\end{array}$ & 1247.23 & $\begin{array}{l}0.898 \\
1.30\end{array}$ & $a^{2} \mathrm{P}, a^{2} \mathrm{D}, a^{4} \mathrm{P}, a^{4} \mathrm{D}, b^{4} \mathrm{D}, a^{4} \mathrm{~F}, b^{4} \mathrm{~F}, a^{4} \mathrm{G}, a^{6} \mathrm{D}$. \\
\hline$d^{4} p\left({ }^{3} \mathrm{P}\right)$ & $z^{2} \mathrm{~S}_{01 / 2}$ & 23910.90 & & 2.123 & $a^{2} \mathrm{P}, a^{2} \mathrm{D}, a^{4} \mathrm{P}, a^{4} \mathrm{D}, a^{6} \mathrm{D}$ \\
\hline$d^{4} p\left({ }^{5} \mathrm{D}\right)$ & $\begin{array}{l}y^{0} \mathrm{~F}_{01 / 2}^{\circ} \\
y^{6} \mathrm{~F}_{11 / 2}^{\circ} \\
y^{0} \mathrm{~F}_{21 / 2}^{\circ} \\
y^{0} \mathrm{~F}_{31 / 2}^{0} \\
y^{6} \mathrm{~F}_{11 / 2}^{0} \\
y^{6} \mathrm{~F}_{31 / 2}^{0}\end{array}$ & $\begin{array}{l}23984.87 \\
24164.79 \\
24396.80 \\
24769.91 \\
25199.81 \\
25680.36\end{array}$ & $\begin{array}{l}179.92 \\
232.01 \\
373.11 \\
429.90 \\
480.55\end{array}$ & $\begin{array}{r}-0.601 \\
1.060 \\
1.306 \\
1.380 \\
1.427 \\
1.450\end{array}$ & $a^{6} \mathrm{~S}, a^{6} \mathrm{D}, e^{6} \mathrm{D}, a^{4} \mathrm{D}, a^{4} \mathrm{~F}, b^{4} \mathrm{~F}, a^{4} \mathrm{G}, a^{2} \mathrm{P}, a^{2} \mathrm{D}$. \\
\hline$d^{4} p\left({ }^{5} \mathrm{D}\right)$ & $\begin{array}{l}z^{6} \mathrm{P}_{11 / 2}^{0} \\
z^{6} \mathrm{P}_{21 / 2}^{0} \\
z^{6} \mathrm{P}_{31 / 2}^{0}\end{array}$ & $\begin{array}{l}24283.34 \\
24543.13 \\
24904.86\end{array}$ & $\begin{array}{l}259.79 \\
361.73\end{array}$ & $\begin{array}{l}2.382 \\
1.874 \\
1.703\end{array}$ & $a^{6} \mathrm{~S}, a^{6} \mathrm{D}, e^{6} \mathrm{D}, a^{4} \mathrm{P}, a^{4} \mathrm{D}, a^{4} \mathrm{~F}$. \\
\hline$d^{3} s p\left({ }^{3} \mathrm{P}\right)$ & 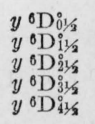 & $\begin{array}{l}25879.81 \\
26067.06 \\
26386.36 \\
26832.43 \\
27419.62\end{array}$ & $\begin{array}{l}187.25 \\
319.30 \\
446.07 \\
587.19\end{array}$ & $\begin{array}{l}3.22 \\
1.820 \\
1.610 \\
1.248 \\
1.422\end{array}$ & $\left\{\begin{array}{l}a^{6} \mathrm{D}, e^{6} \mathrm{D}, a^{4} \mathrm{P}, b^{4} \mathrm{P}, a^{4} \mathrm{D}, b^{4} \mathrm{D}, a^{4} \mathrm{~F}, b^{4} \mathrm{~F}, a^{2} \mathrm{~F}, \\
a^{2} \mathrm{G}, b^{2} \mathrm{G}, b^{2} \mathrm{H} .\end{array}\right.$ \\
\hline$d^{4} p\left({ }^{5} \mathrm{D}\right)$ & $\begin{array}{l}y^{4} \mathrm{~F}_{11 / 2}^{0} \\
y^{4} \mathrm{~F}_{21 / 2}^{\circ} \\
y^{4} \mathrm{~F}_{31 / 2}^{\circ} \\
y^{4} \mathrm{~F}_{41 / 2}^{\circ}\end{array}$ & $\begin{array}{l}25930.01 \\
26060.65 \\
26165.79 \\
26440.33\end{array}$ & $\begin{array}{l}130.64 \\
105.14 \\
274.54\end{array}$ & $\begin{array}{l}0.467 \\
1.085 \\
1.245 \\
1.334\end{array}$ & $\begin{array}{l}a^{4} \mathrm{P}, a^{4} \mathrm{D}, b^{4} \mathrm{D}, a^{4} \mathrm{~F}, b^{4} \mathrm{~F}, a^{4} \mathrm{G}, a^{6} \mathrm{D}, a^{2} \mathrm{P}, a^{2} \mathrm{G}, \\
a^{2} \mathrm{H}, b^{2} \mathrm{H} .\end{array}$ \\
\hline$d^{4} p\left({ }^{6} \mathrm{D}\right)$ & $\begin{array}{l}x^{6} \mathrm{D}_{01 / 2}^{\circ} \\
x^{6} \mathrm{D}_{11 / 2}^{\circ} \\
x^{6} \mathrm{D}_{21 / 2}^{\circ} \\
x^{6} \mathrm{D}_{31 / 2}^{\circ} \\
x^{6} \mathrm{D}_{41 / 2}^{\circ}\end{array}$ & $\begin{array}{l}26552.40 \\
26713.32 \\
26983.34 \\
27427.07 \\
27974.87\end{array}$ & $\begin{array}{l}160.92 \\
270.02 \\
443.73 \\
547.80\end{array}$ & $\begin{array}{l}2.441 \\
1.45 \\
1.618 \\
1.567 \\
1.542\end{array}$ & $a^{6} \mathrm{D}, a^{4} \mathrm{P}, b^{4} \mathrm{P}, a^{4} \mathrm{D}, a^{4} \mathrm{~F}, b^{4} \mathrm{~F}, a^{4} \mathrm{G}, a^{2} \mathrm{D}, b^{2} \mathrm{G}$. \\
\hline$d^{4} p\left({ }^{5} \mathrm{D}\right)$ & 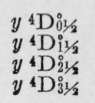 & $\begin{array}{l}26717.73 \\
26936.86 \\
27359.70 \\
27596.74\end{array}$ & $\begin{array}{l}219.13 \\
422.84 \\
237.04\end{array}$ & $\begin{array}{l}\text { 1. } 141 \\
1.292 \\
1.32 \\
1.422\end{array}$ & $\left\{\begin{array}{l}a^{4} \mathrm{P}, b^{4} \mathrm{P}, a^{4} \mathrm{D}, b^{4} \mathrm{D}, a^{4} \mathrm{~F}, b^{4} \mathrm{~F}, a^{4} \mathrm{G}, a^{6} \mathrm{D}, a^{2} \mathrm{P}, \\
a^{2} \mathrm{D}, a^{2} \mathrm{~F}, a^{2} \mathrm{G} .\end{array}\right.$ \\
\hline$d^{4} p\left({ }^{3} \mathrm{H}\right)$ & $\begin{array}{l}z^{2} \mathrm{G}_{31 / 2}^{3} \\
z^{2} \mathrm{G}_{41 / 2}^{0}\end{array}$ & $\begin{array}{l}26896.68 \\
27331.80\end{array}$ & 435.12 & $\begin{array}{l}1.013 \\
1.078\end{array}$ & $\begin{array}{l}a^{2} \mathrm{~F}, a^{2} \mathrm{G}, b^{2} \mathrm{G}, b^{2} \mathrm{H}, a^{4} \mathrm{P}, a^{4} \mathrm{D}, a^{4} \mathrm{~F}, b^{4} \mathrm{~F}, a^{4} \mathrm{G}, \\
a^{6} \mathrm{D} .\end{array}$ \\
\hline$d^{3} s p\left(c^{3} \mathrm{P}\right)$ & $\begin{array}{l}y \mathrm{AP}_{01 / 2} \\
y^{4} \mathrm{P}_{115}^{0} \\
y^{4} \mathrm{P}_{21 / 2}\end{array}$ & $\begin{array}{l}27498.94 \\
27782.57 \\
28445.33\end{array}$ & $\begin{array}{l}283.63 \\
662.76\end{array}$ & $\begin{array}{l}2.467 \\
1.660 \\
1.606\end{array}$ & $a^{4} \mathrm{P}, b^{4} \mathrm{P}, a^{4} \mathrm{D}, a^{4} \mathrm{~F}, a^{6} \mathrm{~S}, a^{6} \mathrm{D}, a^{2} \mathrm{P}, a^{2} \mathrm{D}$ \\
\hline & $27621 / 2$ & & & 1.370 & $b^{4} \mathrm{P}, a^{4} \mathrm{D}, b^{4} \mathrm{D}, a^{4} \mathrm{~F}, a^{6} \mathrm{D}, a^{2} \mathrm{D}, a^{2} \mathrm{~F}, a^{3} \mathrm{G}$ \\
\hline$d^{3} s p\left(c^{3} \mathrm{P}\right)$ & $\begin{array}{l}x{ }^{4} \mathrm{D}_{01 / 2}^{\circ} \\
x^{4} \mathrm{D}_{11 / 2}^{\circ} \\
x{ }^{4} \mathrm{D}_{21 / 2}^{\circ} \\
x \pm \mathrm{D}_{31 / 2}^{0}\end{array}$ & $\begin{array}{l}27666.46 \\
28079.09 \\
28549.42 \\
29209.42\end{array}$ & $\begin{array}{l}412.63 \\
470.33 \\
660.00\end{array}$ & $\begin{array}{l}0.222 \\
1.443 \\
1.472 \\
1.241\end{array}$ & $\left\{\begin{array}{l}a^{4} \mathrm{P}, b^{4} \mathrm{P}, a^{4} \mathrm{D}, b^{4} \mathrm{D}, a^{4} \mathrm{~F}, b^{4} \mathrm{~F}, a^{4} \mathrm{G}, a^{6} \mathrm{D}, a^{2} \mathrm{P}, a^{2} \mathrm{D}, \\
a^{2} \mathrm{G} .\end{array}\right.$ \\
\hline$d^{3} s p\left({ }^{3} \mathrm{~F}\right)$ & $\begin{array}{l}z^{2} \mathrm{~F}_{21 / 2}^{0} \\
z^{2} \mathrm{~F}_{31 / 2}^{0}\end{array}$ & $\begin{array}{l}27797.44 \\
28535.36\end{array}$ & 737.92 & $\begin{array}{l}1.16 \\
1.12\end{array}$ & $\begin{array}{l}a^{2} \mathrm{~F}, a^{2} \mathrm{G}, a^{2} \mathrm{H}, a^{4} \mathrm{P}, b^{4} \mathrm{P}, a^{4} \mathrm{D}, b^{4} \mathrm{D}, a^{4} \mathrm{~F}, b^{4} \mathrm{~F}, \\
a^{4} \mathrm{G}, a^{6} \mathrm{D} .\end{array}$ \\
\hline$d^{3} s p\left({ }^{3} \mathrm{~F}\right)$ & $\begin{array}{l}y^{2} \mathrm{G}_{31 / 2}^{1} \\
y^{2} \mathrm{G}_{41 / 2}^{\circ}\end{array}$ & $\begin{array}{l}27855.13 \\
28433.74\end{array}$ & 578.61 & $\begin{array}{l}0.92 \\
1.12\end{array}$ & $\begin{array}{l}a^{2} \mathrm{~F}, a^{2} \mathrm{G}, b^{2} \mathrm{G}, a^{2} \mathrm{H}, b^{2} \mathrm{H}, 183, a^{4} \mathrm{P}, a^{4} \mathrm{D}, a^{4} \mathrm{~F}, a^{4} \mathrm{G}, \\
a^{6} \mathrm{D} .\end{array}$ \\
\hline & $\begin{array}{l}z^{2} \mathrm{P}_{01 / 2}^{0} \\
z^{2} \mathrm{P}_{1 / 2}^{0}\end{array}$ & $\begin{array}{l}28442.16 \\
27918.85\end{array}$ & -523.31 & $\begin{array}{l}0.772 \\
1.450\end{array}$ & $a^{2} \mathrm{P}, a^{2} \mathrm{D}, a^{4} \mathrm{P}, b^{4} \mathrm{P}, a^{4} \mathrm{D}, b^{4} \mathrm{D}, a^{4} \mathrm{~F}, a^{6} \mathrm{~S}, a^{6} \mathrm{D}$ \\
\hline$d^{4} p\left({ }^{3} \mathrm{P}\right)$ & $z^{4} \mathrm{~S} i 1 / 2$ & 28208.48 & & 1. 794 & $a^{4} \mathrm{P}, b^{4} \mathrm{P}, b^{4} \mathrm{D}, a^{4} \mathrm{~F}, a^{6} \mathrm{~S}, a^{6} \mathrm{D}, a^{2} \mathrm{D}$ \\
\hline
\end{tabular}


TABLE 6.-Terms of the Cb I spectrum-Continued

\begin{tabular}{|c|c|c|c|c|c|}
\hline $\begin{array}{l}\text { Electron } \\
\text { configu- } \\
\text { ration }\end{array}$ & $\begin{array}{l}\text { Term } \\
\text { symbol }\end{array}$ & Level & $\begin{array}{l}\text { Differ- } \\
\text { ence }\end{array}$ & $\underset{g}{\text { Observed }}$ & Combinations \\
\hline$d^{3} s p\left({ }^{5} \mathrm{P}\right)$ & 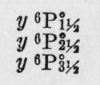 & $\begin{array}{l}28278.25 \\
28652.66 \\
28973.12\end{array}$ & $\begin{array}{l}374.41 \\
320.46\end{array}$ & $\begin{array}{l}1.981 \\
1.768 \\
1.701\end{array}$ & \}$a^{6} \mathrm{~S}, a^{6} \mathrm{D}, a^{4} \mathrm{P}, b^{4} \mathrm{P}, a^{4} \mathrm{D}, a^{4} \mathrm{~F}, a^{2} \mathrm{D}$ \\
\hline$d^{3} s p\left({ }^{3} \mathrm{G}\right)$ & $\begin{array}{l}z^{4} \mathrm{H}_{33 / 2}^{\circ} \\
z^{4} \mathrm{H}_{41 / 2}^{\circ} \\
z^{4} \mathrm{H}_{51 / 2}^{1} \\
z^{4} \mathrm{H}_{61 / 2}^{\circ}\end{array}$ & $\begin{array}{l}29271.99 \\
29519.05 \\
29846.50 \\
30191.25\end{array}$ & $\begin{array}{l}247.06 \\
327.25 \\
344.75\end{array}$ & $\begin{array}{l}0.890 \\
1.01 \\
1.15 \\
1.23\end{array}$ & $\left\{\begin{array}{l}a^{4} \mathrm{P}, b{ }^{4} \mathrm{P}, a^{4} \mathrm{D}, b^{4} \mathrm{D}, a^{4} \mathrm{~F}, b^{4} \mathrm{~F}, a^{4} \mathrm{G}, a^{4} \mathrm{H}, a^{6} \mathrm{D}, \\
a^{2} \mathrm{G}, b^{2} \mathrm{G} .\end{array}\right.$ \\
\hline$d^{4} p\left({ }^{3} \mathrm{H}\right)$ & $\begin{array}{l}y^{4} \mathrm{G}_{21 / 5}^{2} \\
y^{4} \mathrm{G}_{31 / 3}^{3} \\
y^{4} \mathrm{G}_{41 / 2}^{2} \\
y^{4} \mathrm{G}_{51 / 2}^{3}\end{array}$ & $\begin{array}{l}29359.58 \\
29762.70 \\
30117.32 \\
30657.60\end{array}$ & $\begin{array}{l}403.1 ? \\
354.62 \\
540.28\end{array}$ & $\begin{array}{l}0.693 \\
0.999 \\
1.276 \\
1.25\end{array}$ & $\left\{\begin{array}{l}a^{4} \mathrm{P}, a^{4} \mathrm{D}, b{ }^{4} \mathrm{D}, a^{4} \mathrm{~F}, b^{4} \mathrm{~F}, a^{4} \mathrm{G}, a^{4} \mathrm{H}, a^{6} \mathrm{D}, a^{2} \mathrm{G}, \\
b^{2} \mathrm{G}, a^{2} \mathrm{H} .\end{array}\right.$ \\
\hline$d^{3} s p\left({ }^{3} \mathrm{~F}\right)$ & $\begin{array}{l}y^{2} \mathrm{D}_{11 / 2}^{0} \\
y^{2} \mathrm{D}_{21 / 2}^{21 / 2}\end{array}$ & $\begin{array}{l}29622.73 \\
29775.80\end{array}$ & 153.07 & $\begin{array}{l}0.81 \\
1.348\end{array}$ & \}$^{a{ }^{2} \mathrm{P}, a^{2} \mathrm{D}, a^{2} \mathrm{~F}, a^{2} \mathrm{G}, a^{4} \mathrm{P}, a^{4} \mathrm{D}, b^{4} \mathrm{D}, a^{4} \mathrm{~F}, b^{4} \mathrm{~F},}$ \\
\hline$d^{4} p\left({ }^{3} \mathrm{~F}\right)$ & $\begin{array}{l}x^{4} \mathrm{~F}_{11 / 2}^{\circ} \\
x^{4} \mathrm{~F}_{21 / 2}^{\circ} \\
x^{4} \mathrm{~F}_{31 / 2}^{\circ} \\
x^{4} \mathrm{~F}_{41 / 2}^{0}\end{array}$ & $\begin{array}{l}29779.44 \\
29987.45 \\
30161.56 \\
30279.23\end{array}$ & $\begin{array}{l}208.01 \\
174.11 \\
117.67\end{array}$ & \begin{tabular}{l|}
0.42 \\
1.006 \\
1.18 \\
1.20
\end{tabular} & $\left\{\begin{array}{l}a^{4} \mathrm{P}, b^{4} \mathrm{P}, a^{4} \mathrm{D}, b^{4} \mathrm{D}, a^{4} \mathrm{~F}, b^{4} \mathrm{~F}, a^{4} \mathrm{G}, a^{4} \mathrm{H}, a^{6} \mathrm{D}, \\
a^{2} \mathrm{~F}, a^{2} \mathrm{G}, b^{2} \mathrm{G}, a^{2} \mathrm{H}, b^{2} \mathrm{H} .\end{array}\right.$ \\
\hline$d^{3} s p\left({ }^{5} \mathrm{P}\right)$ & $z^{6} \mathrm{~S}_{21 / 2}^{\circ}$ & 30059.60 & & & $a^{6} \mathrm{D}, a^{4} \mathrm{P}, b{ }^{4} \mathrm{P}, a^{4} \mathrm{~F}, a^{2} \mathrm{P}, a^{2} \mathrm{D}$. \\
\hline$d^{4} p\left({ }^{1} \mathrm{D}\right)$ & $\begin{array}{l}y^{2} \mathrm{~F}_{21 / 2}^{0} \\
y^{2} \mathrm{~F}_{31 / 2}^{0}\end{array}$ & $\begin{array}{l}30716.50 \\
31025.52\end{array}$ & 309.02 & $\begin{array}{l}0.891 \\
1.139\end{array}$ & $a^{2} \mathrm{P}, a^{2} \mathrm{D}, a^{2} \mathrm{~F}, a^{2} \mathrm{G}, a^{4} \mathrm{P}, a^{4} \mathrm{D}, a^{4} \mathrm{~F}, a^{4} \mathrm{G}$ \\
\hline$d^{3} s p\left({ }^{5} \mathrm{~F}\right)$ & $\begin{array}{l}x^{4} \mathrm{G}_{21 / 2}^{2} \\
x^{4} \mathrm{G}_{31 / 3}^{3} \\
x^{4} \mathrm{G}_{41 / 2}^{2} \\
x^{4} \mathrm{G}_{51 / 2}\end{array}$ & $\begin{array}{l}31056.60 \\
31485.20 \\
32004.63 \\
32572.72\end{array}$ & $\begin{array}{l}428.60 \\
519.43 \\
568.09\end{array}$ & $\begin{array}{l}0.630 \\
1.012 \\
1.160 \\
1.24\end{array}$ & $\left\{\begin{array}{l}a^{4} \mathrm{D}, a^{4} \mathrm{~F}, b{ }^{4} \mathrm{~F}, a^{4} \mathrm{G}, a^{4} \mathrm{H}, a^{6} \mathrm{~S}, a^{6} \mathrm{D}, a^{2} \mathrm{D}, a^{2} \mathrm{G}, \\
b^{2} \mathrm{G}, 183 .\end{array}\right.$ \\
\hline$d^{3} s p\left(c^{3} \mathrm{P}\right)$ & $y^{4} \mathrm{~S}_{1 / 2}^{0}$ & 31174.65 & & 1.957 & $a^{4} \mathrm{P}, b^{4} \mathrm{P}, a^{4} \mathrm{~F}, a^{6} \mathrm{D}, a^{2} \mathrm{D}$. \\
\hline$d^{3} s p\left({ }^{1} \mathrm{G}\right)$ & $\begin{array}{l}x^{2} \mathrm{~F}_{21 / 3}^{2} \\
x^{2} \mathrm{~F}_{31 / 3}^{2}\end{array}$ & $31 \overline{6} \bar{\gamma} .53$ & & 1.20 & \}$a^{2} \mathrm{D}, a^{2} \mathrm{~F}, a^{2} \mathrm{G}, a{ }^{4} \mathrm{D}$ \\
\hline$d^{3} s p\left({ }^{1} \mathrm{G}\right)$ & $\begin{array}{l}x^{2} \mathrm{G}_{31 / 2}^{\circ} \\
x^{2} \mathrm{G}=1 / 2\end{array}$ & $\begin{array}{l}31800.74 \\
32213.94\end{array}$ & 413.20 & $\begin{array}{l}0.906 \\
1.092\end{array}$ & $a^{2} \mathrm{~F}, a^{2} \mathrm{G}, b^{2} \mathrm{H}, a^{4} \mathrm{P}, b^{4} \mathrm{D}, a^{4} \mathrm{~F}, a^{4} \mathrm{H}$. \\
\hline$d^{4} p\left({ }^{1} \mathrm{G}\right)$ & $\begin{array}{l}w^{2} \mathrm{~F}_{21 / 3}^{\circ} \\
w^{2} \mathrm{~F}_{31 / 2}^{0}\end{array}$ & $\begin{array}{l}31933.68 \\
32087.58\end{array}$ & 153.90 & $\begin{array}{l}0.982 \\
1.074\end{array}$ & \}$_{b^{2} \mathrm{P}, a^{2} \mathrm{D}, a^{2} \mathrm{~F}, a^{2} \mathrm{G}, a^{4} \mathrm{H} .}$ \\
\hline$d^{3} s p\left({ }^{5} \mathrm{~F}\right)$ & $\begin{array}{l}w^{4} \mathrm{~F}_{11 / 2}^{0} \\
w^{4} \mathrm{~F}_{21 / 2}^{\circ} \\
w^{4} \mathrm{~F}_{31 / 2}^{\circ} \\
w^{4} \mathrm{~F}_{41 / 2}^{\circ}\end{array}$ & $\begin{array}{l}31551.46 \\
32013.40 \\
32333.18 \\
32923.87\end{array}$ & $\begin{array}{l}461.94 \\
319.78 \\
590.69\end{array}$ & $\begin{array}{l}0.501 \\
1.01 \\
1.199 \\
1.24\end{array}$ & $\left\{\begin{array}{l}a^{4} \mathrm{P}, b{ }^{4} \mathrm{P}, a^{4} \mathrm{D}, b{ }^{4} \mathrm{D}, a^{4} \mathrm{~F}, b{ }^{4} \mathrm{~F}, a^{4} \mathrm{G}, a^{4} \mathrm{H}, a^{6} \mathrm{D}, \\
a^{2} \mathrm{P}, a^{2} \mathrm{D}, a^{2} \mathrm{~F}, a^{2} \mathrm{G}, a^{2} \mathrm{H} .\end{array}\right.$ \\
\hline$d^{4} p\left({ }^{3} \mathrm{D}\right)$ & $\begin{array}{l}v^{4} \mathrm{~F}_{11 / 3}^{\circ} \\
v^{4} \mathrm{~F}_{21 / 2}^{\circ} \\
v^{4} \mathrm{~F}_{31 / 2}^{\circ} \\
v^{4} \mathrm{~F}_{41 / 2}^{\circ}\end{array}$ & $\begin{array}{l}3170 \% .94 \\
3180 \% .55 \\
3197.24 \\
32605.39\end{array}$ & $\begin{array}{r}99.61 \\
165.69 \\
632.15\end{array}$ & $\begin{array}{l}0.80 \\
1.048 \\
1.343 \\
1.216\end{array}$ & $\left\{\begin{array}{l}a^{4} \mathrm{P}, b{ }^{4} \mathrm{P}, a^{4} \mathrm{D}, b^{4} \mathrm{D}, a^{4} \mathrm{~F}, b^{4} \mathrm{~F}, a^{4} \mathrm{G}, a^{4} \mathrm{H}, a^{6} \mathrm{D}, \\
a^{2} \mathrm{P}, a^{2} \mathrm{D}, a^{2} \mathrm{~F}, a^{2} \mathrm{G}, b^{2} \mathrm{G}, a^{2} \mathrm{H}, 183 .\end{array}\right.$ \\
\hline$d^{4} p\left({ }^{3} \mathrm{G}\right)$ & $\begin{array}{l}u^{4} \mathrm{~F}_{11 / 2}^{0} \\
u^{4} \mathrm{~F}_{21 / 2}^{0} \\
u^{4} \mathrm{~F}_{31 / 2}^{0} \\
u^{4} \mathrm{~F}_{41 / 2}^{0}\end{array}$ & $\begin{array}{l}31907.74 \\
32139.78 \\
32451.99 \\
33136.30\end{array}$ & $\begin{array}{l}232.04 \\
312.21 \\
684.31\end{array}$ & $\begin{array}{l}0.791 \\
1.035 \\
1.115 \\
1.240\end{array}$ & $\left\{\begin{array}{l}a^{4} \mathrm{P}, b^{4} \mathrm{P}, a^{4} \mathrm{D}, b^{4} \mathrm{D}, a^{4} \mathrm{~F}, b{ }^{4} \mathrm{~F}, a^{4} \mathrm{G}, a^{4} \mathrm{H}, a^{2} \mathrm{P}, \\
a^{2} \mathrm{D}, a^{2} \mathrm{~F}, a^{2} \mathrm{G}, b^{2} \mathrm{G}, a^{2} \mathrm{H}, 183 .\end{array}\right.$ \\
\hline$d^{4} p\left({ }^{3} \mathrm{P}\right)$ & $\begin{array}{l}w^{4} \mathrm{D}_{01 / 2}^{0} \\
w^{4} \mathrm{D}_{11 / 2}^{1} \\
w^{4} \mathrm{D}_{21 / 2}^{\circ} \\
w^{4} \mathrm{D}_{31 / 2}^{\circ}\end{array}$ & $\begin{array}{l}32066.06 \\
32248.69 \\
32545.52 \\
33003.89\end{array}$ & $\begin{array}{l}182.63 \\
296.83 \\
458.37\end{array}$ & $\begin{array}{l}0.046 \\
1.184 \\
1.320 \\
1.341\end{array}$ & $\begin{array}{l}a^{4} \mathrm{P}, b{ }^{4} \mathrm{P}, a^{4} \mathrm{D}, b^{4} \mathrm{D}, a^{4} \mathrm{~F}, b^{4} \mathrm{~F}, a^{4} \mathrm{G}, a^{6} \mathrm{D}, a^{2} \mathrm{P} \\
a^{2} D, a^{2} F, a^{2} G, a^{2} \mathrm{H} .\end{array}$ \\
\hline$d^{4} p\left({ }^{3} \mathrm{H}\right)$ & $\begin{array}{l}z^{4} \mathrm{I}_{41 / 2}^{0} \\
z^{4} \mathrm{I}_{5 / 2}^{\circ} \\
z^{4} \mathrm{I}_{61 / 2}^{\circ} \\
z^{4} \mathrm{I}_{73 / 2}^{\circ}\end{array}$ & $\begin{array}{l}32156.00 \\
32382.24 \\
32672.39 \\
33116.36\end{array}$ & $\begin{array}{l}226.24 \\
290.15 \\
443.97\end{array}$ & $\begin{array}{l}0.835 \\
0.993 \\
1.08 \\
1.19\end{array}$ & $a a^{4} \mathrm{~F}, a^{4} \mathrm{G}, a^{4} \mathrm{H}, a^{2} \mathrm{~F}, a^{2} \mathrm{G}, a^{2} \mathrm{H}$. \\
\hline$d^{4} p\left({ }^{3} F\right)$ & $\begin{array}{l}w^{4} \mathrm{G}_{21 / 2}^{0} \\
w^{4} \mathrm{G}_{31 / 2}^{0} \\
w^{4} \mathrm{G}_{41 / 2}^{0} \\
w^{4} \mathrm{G}_{51 / 2}^{0}\end{array}$ & $\begin{array}{l}32501.33 \\
32802.44 \\
33428.20\end{array}$ & $\begin{array}{l}301.11 \\
625.76\end{array}$ & $\begin{array}{l}\text { 1. } 06 \\
1.21 \\
1.27\end{array}$ & $\left\{\begin{array}{l}a^{4} \mathrm{P}, a^{4} \mathrm{D}, b^{4} \mathrm{D}, a^{4} \mathrm{~F}, b^{4} \mathrm{~F}, a^{4} \mathrm{G}, a^{4} \mathrm{H}, a^{6} \mathrm{D}, a^{2} \mathrm{D}, \\
a^{2} \mathrm{~F}, a^{2} \mathrm{G}, b^{2} \mathrm{G}, a^{2} \mathrm{H} .\end{array}\right.$ \\
\hline$d^{3} s p\left({ }^{1} \mathrm{P}\right)$ & $\begin{array}{l}x^{2} \mathrm{D}_{1 / 3}^{\circ}{ }^{\circ} \\
x^{2} \mathrm{D}_{21 / 2}^{2}\end{array}$ & 32623.02 & & 1.00 & \}$a^{2} \mathrm{P}, a^{2} \mathrm{D}, a^{2} \mathrm{~F}, a^{4} \mathrm{P}, a^{4} \mathrm{D}, a^{4} \mathrm{~F}, a^{4} \mathrm{G}, a^{6} \mathrm{D}$ \\
\hline$d^{4} p\left({ }^{3} \mathrm{G}\right)$ & $\begin{array}{l}v^{2} \mathrm{~F}_{21 / 3}^{\circ} \\
v^{2} \mathrm{~F}_{31 / 5}^{2}\end{array}$ & $\begin{array}{l}32654.48 \\
32899.08\end{array}$ & 244.60 & $\begin{array}{l}0.830 \\
1.17\end{array}$ & 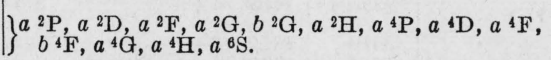 \\
\hline
\end{tabular}


TABLE 6.-Terms of the $\mathrm{Cb}$ I spectrum-Continued

\begin{tabular}{|c|c|c|c|c|c|}
\hline $\begin{array}{l}\text { Electron } \\
\text { configu- } \\
\text { ration }\end{array}$ & $\begin{array}{l}\text { Term } \\
\text { symbol }\end{array}$ & Level & $\begin{array}{l}\text { Differ- } \\
\text { ence }\end{array}$ & $\underset{g}{\text { Observed }}$ & Combinations \\
\hline$d^{3} s p\left({ }^{5} \mathrm{~F}\right)$ & 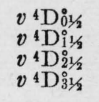 & $\begin{array}{l}33011.45 \\
33717.01 \\
33872.18 \\
34168.94\end{array}$ & $\begin{array}{l}705.56 \\
155.17 \\
296.76\end{array}$ & $\begin{array}{l}0.46 \\
1.230 \\
1.350 \\
1.390\end{array}$ & $\left\{\begin{array}{l}a^{4} \mathrm{P}, b^{4} \mathrm{P}, a^{4} \mathrm{D}, b{ }^{4} \mathrm{D}, a^{4} \mathrm{~F}, b^{4} \mathrm{~F}, a^{4} \mathrm{G}, a^{6} \mathrm{D}, a^{2} \mathrm{D}, \\
a^{2} \mathrm{~F}, a^{2} \mathrm{G}, a^{2} \mathrm{H} .\end{array}\right.$ \\
\hline$d^{3} s p(1 \mathrm{D})$ & $\begin{array}{l}w^{2} \mathrm{D}_{11 / 2}^{\circ} \\
w^{2} \mathrm{D}_{21 / 2}^{\circ}\end{array}$ & $\begin{array}{l}33086.98 \\
33389.87\end{array}$ & 302.89 & $\begin{array}{l}\text { 1. } 058 \\
1.212\end{array}$ & \}$a^{2} \mathrm{P}, a^{2} \mathrm{D}, a^{2} \mathrm{~F}, a^{4} \mathrm{P}, b^{4} \mathrm{P}, a^{4} \mathrm{D}, a^{4} \mathrm{~F}, a^{4} \mathrm{G}$ \\
\hline$d^{3} s p\left(c^{3} \mathrm{P}\right)$ & $\begin{array}{l}y{ }^{2} \mathrm{P}_{01 / 2}^{1 / 2} \\
y^{2} \mathrm{P}_{11 / 2}^{\circ}\end{array}$ & $\begin{array}{l}35902.24 \\
34252.96\end{array}$ & 350.72 & 0.442 & $a^{2} \mathrm{P}, a^{2} \mathrm{D}, a^{4} \mathrm{P}, b^{4} \mathrm{P}, a^{4} \mathrm{D}, b^{4} \mathrm{D}, a^{4} \mathrm{~F}, b^{4} \mathrm{~F}$. \\
\hline$d^{3} s p\left({ }^{1} \mathrm{H}\right)$ & $\begin{array}{l}z^{2} \mathrm{I}_{51 / 2}^{\circ} \\
z^{2} \mathrm{I}_{61 / 2}^{\circ}\end{array}$ & $\begin{array}{l}34004.08 \\
34323.20\end{array}$ & 319.12 & $\begin{array}{l}0.946 \\
1.08\end{array}$ & $a^{2} \mathrm{G}, b^{2} \mathrm{G}, a^{2} \mathrm{H}, b^{2} \mathrm{H}, 183, a^{4} \mathrm{~F}, a^{4} \mathrm{H}$. \\
\hline$d^{3} s p\left({ }^{1} \mathrm{H}\right)$ & $\begin{array}{l}w^{2} \mathrm{G}_{31 / 2}^{\circ} \\
w^{2} \mathrm{G}_{41 / 2}^{0}\end{array}$ & $\begin{array}{l}34319.09 \\
34235.04\end{array}$ & -84.05 & $\begin{array}{l}0.87 \\
1.10\end{array}$ & $\begin{array}{l}a^{2} \mathrm{~F}, a^{2} \mathrm{G}, a^{2} \mathrm{H}, b^{2} \mathrm{H}, 183, a^{4} \mathrm{P}, a^{4} \mathrm{~F}, b^{4} \mathrm{~F}, a^{4} \mathrm{G}, \\
a^{4} \mathrm{H}, a^{6} \mathrm{D} .\end{array}$ \\
\hline$d^{4} p\left({ }^{3} \mathrm{H}\right)$ & $\begin{array}{l}z^{2} \mathrm{H}_{41 / 2}^{\circ} \\
z^{2} \mathrm{H}_{51 / 2}^{\circ}\end{array}$ & $\begin{array}{l}34415.52 \\
34838.39\end{array}$ & 422.81 & $\begin{array}{l}0.819 \\
1.01\end{array}$ & $a^{2} \mathrm{G}, b^{2} \mathrm{G}, a^{2} \mathrm{H}, b^{2} \mathrm{H}, 183, a^{4} \mathrm{~F}, b{ }^{4} \mathrm{~F}, a^{4} \mathrm{G}, a^{4} \mathrm{H}$. \\
\hline$d^{3} s p\left({ }^{5} \mathrm{P}\right)$ & $\begin{array}{l}x^{4} \mathrm{P}_{01 / 2}^{\circ} \\
x^{4} \mathrm{P}_{11 / 2}^{\circ} \\
x^{4} \mathrm{P}_{21 / 2}^{\circ}\end{array}$ & $\begin{array}{l}34644.22 \\
34867.68 \\
34703.70\end{array}$ & $\begin{array}{r}223.46 \\
-163.98\end{array}$ & $\begin{array}{l}2.05 \\
1.587 \\
1.55\end{array}$ & $\left\{\begin{array}{l}a^{4} \mathrm{P}, b^{4} \mathrm{P}, a^{4} \mathrm{D}, b^{4} \mathrm{D}, a^{4} \mathrm{~F}, b^{4} \mathrm{~F}, a^{4} \mathrm{G}, a^{2} \mathrm{P}, a-\mathrm{D}, \\
a^{2} \mathrm{~F} .\end{array}\right.$ \\
\hline$d^{4} p\left({ }^{3} \mathrm{G}\right)$ & $\begin{array}{l}v^{4} \mathrm{G}_{21 / 2}^{0} \\
v^{4} \mathrm{G}_{31 / 2}^{\circ} \\
v^{4} \mathrm{G}_{41 / 2}^{0} \\
v^{4} \mathrm{G}_{51 / 2}^{0}\end{array}$ & $\begin{array}{l}34654.79 \\
34853.50 \\
35156.94 \\
35630.62\end{array}$ & $\begin{array}{l}198.71 \\
303.44 \\
473.68\end{array}$ & $\begin{array}{l}0.627 \\
1.000 \\
1.073 \\
1.160\end{array}$ & $\left\{\begin{array}{l}a^{4} \mathrm{P}, a^{4} \mathrm{D}, b{ }^{4} \mathrm{D}, a^{4} \mathrm{~F}, b^{4} \mathrm{~F}, a^{4} \mathrm{G}, a^{4} \mathrm{H}, a^{2} \mathrm{P}, a^{2} \mathrm{D}, \\
a^{2} \mathrm{~F}, a^{2} \mathrm{G}, b^{2} \mathrm{G}, a^{2} \mathrm{H}, b^{2} \mathrm{H} .\end{array}\right.$ \\
\hline$d^{3} s p\left(b^{3} \mathrm{P}\right)$ & $\begin{array}{l}v^{2} \mathrm{D}_{1134}^{\circ} \\
v^{2} \mathrm{D}_{21 / 2}^{\circ}\end{array}$ & $\begin{array}{l}34752.70 \\
35497.48\end{array}$ & 744.78 & $\begin{array}{l}0.948 \\
1.160\end{array}$ & \}$_{b^{4} \mathrm{~F} .}^{{ }^{2} \mathrm{P}, a^{2} \mathrm{D}, a^{2} \mathrm{~F}, a^{2} \mathrm{G}, a^{4} \mathrm{P}, b^{4} \mathrm{P}, a^{4} \mathrm{D}, b^{4} \mathrm{D}, a^{4} \mathrm{~F},}$ \\
\hline$d^{3} s p\left(c^{3} \mathrm{P}\right)$ & $y^{2} \mathrm{~S}_{01 / 2}$ & $3480 \% .57$ & & 2. 080 & $a^{2} \mathrm{D}, a^{4} \mathrm{P}, b^{4} \mathrm{P}, a^{4} \mathrm{D}, b{ }^{4} \mathrm{D}, a^{4} \mathrm{~F}, b{ }^{4} \mathrm{~F}$. \\
\hline$d^{3} s p\left({ }^{1} \mathrm{D}\right)$ & $\begin{array}{l}u^{2} \mathrm{~F}_{21 / 2}^{\circ} \\
u^{2} \mathrm{~F}_{31 / 2}^{\circ}\end{array}$ & $\begin{array}{l}35099.86 \\
35178.82\end{array}$ & 78.96 & $\begin{array}{l}0.868 \\
1.117\end{array}$ & $\left\{\begin{array}{l}a^{2} \mathrm{P}, a^{2} \mathrm{D}, a^{2} \mathrm{~F}, a^{2} \mathrm{G}, a^{2} \mathrm{H}, a^{4} \mathrm{P}, a^{4} \mathrm{D}, b^{4} \mathrm{D}, a^{4} \mathrm{~F}, \\
b^{4} \mathrm{~F}, a^{4} \mathrm{G}, a^{4} \mathrm{H} .\end{array}\right.$ \\
\hline$d^{3} s p\left(b^{3} \mathrm{P}\right)$ & $x$ 1 $\mathrm{S}_{\mathrm{i} 1 / 2}$ & 35119.65 & & 1.806 & $a^{4} \mathrm{P}, a^{4} \mathrm{D}, a^{4} \mathrm{~F}, a^{2} \mathrm{D}$ \\
\hline$d^{4} p\left({ }^{3} \mathrm{G}\right)$ & $\begin{array}{l}y^{2} \mathrm{H}_{41 / 2}^{\circ} \\
y^{2} \mathrm{H}_{51 / 2}^{0}\end{array}$ & $\begin{array}{l}35496.39 \\
35344.86\end{array}$ & -151.53 & $\begin{array}{l}0.954 \\
1.125\end{array}$ & $\begin{array}{l}a^{2} \mathrm{G}, b^{2} \mathrm{G}, a^{2} \mathrm{H}, b^{2} \mathrm{H}, 183, a^{4} \mathrm{~F}, b^{4} \mathrm{~F}, a^{4} \mathrm{G}, a^{4} \mathrm{H}, \\
a^{6} \mathrm{D} .\end{array}$ \\
\hline$d^{4} p\left({ }^{3} \mathrm{~F}\right)$ & $\begin{array}{l}u^{2} \mathrm{D}_{11 / 2}^{\circ} \\
u^{2} \mathrm{D}_{21 / 2}^{\circ}\end{array}$ & $\begin{array}{l}35829.46 \\
35928.35\end{array}$ & 98.89 & $\begin{array}{l}0.834 \\
1.17\end{array}$ & \}$^{a^{2} \mathrm{P}, a^{2} \mathrm{D}, a^{2} \mathrm{~F}, a^{2} \mathrm{G}, b^{2} \mathrm{G}, a^{4} \mathrm{P}, a^{4} \mathrm{D}, a^{4} \mathrm{~F}, b^{4} \mathrm{~F},}$ \\
\hline$d^{4} p\left({ }^{3} \mathrm{~F}\right)$ & $\begin{array}{l}u^{4} \mathrm{D}_{013}^{\circ}{ }^{\circ} \\
u^{4} \mathrm{D}_{11 / 2}^{\circ} \\
u^{4} \mathrm{D}_{21 / 2}^{\circ} \\
u^{4} \mathrm{D}_{31 / 2}^{\circ}\end{array}$ & $\begin{array}{l}35920.45 \\
36016.26 \\
36180.13 \\
36334.21\end{array}$ & $\begin{array}{r}95.81 \\
163.87 \\
154.08\end{array}$ & $\begin{array}{l}0.019 \\
1.195 \\
1.316 \\
1.378\end{array}$ & $\left\{\begin{array}{l}a_{3^{4}} \mathrm{P}, b^{4} \mathrm{P}, a^{4} \mathrm{D}, b^{4} \mathrm{D}, a^{4} \mathrm{~F}, b^{4} \mathrm{~F}, a^{4} \mathrm{G}, a^{4} \mathrm{H}, a^{6} \mathrm{~S}, \\
a^{2} \mathrm{P}, a^{2} \mathrm{D}, a^{2} \mathrm{~F} .\end{array}\right.$ \\
\hline$d^{3} s p\left({ }^{3} \mathrm{G}\right)$ & $\begin{array}{l}v^{2} \mathrm{G}_{31 / 2}^{0} \\
v^{2} \mathrm{G}_{41 / 2}^{\circ}\end{array}$ & $\begin{array}{l}\$ 6048.10 \\
36333.70\end{array}$ & 285.60 & $\begin{array}{l}0.925 \\
1.086\end{array}$ & $\begin{array}{l}a^{2} \mathrm{D}, a^{2} \mathrm{~F}, a^{2} \mathrm{G}, a^{2} \mathrm{H}, b^{2} \mathrm{H}, a^{4} \mathrm{P}, b^{4} \mathrm{P}, a^{4} \mathrm{D}, b^{4} \mathrm{D}, \\
a^{4} \mathrm{~F}, b^{4} \mathrm{~F}, a^{4} \mathrm{G}, a^{4} \mathrm{H} .\end{array}$ \\
\hline$d^{4} p\left({ }^{1} \mathrm{G}\right)$ & $\begin{array}{l}x^{2} \mathrm{H}_{41 / 2}^{\circ} \\
x^{2} \mathrm{H}_{51 / 2}^{\circ}\end{array}$ & $36 \overline{2} \overline{\bar{b}} . \overline{7 \gamma}$ & & 1.14 & \}$^{a^{2} \mathrm{G}, b^{2} \mathrm{G}, a^{2} \mathrm{H}, b^{2} \mathrm{H}, 183, a^{4} \mathrm{~F}, b^{4} \mathrm{~F}, a^{4} \mathrm{G}, a^{4} \mathrm{H} .}$ \\
\hline$d^{3} s p\left({ }^{5} \mathrm{P}\right)$ & $w^{4} \mathrm{~S}_{\mathrm{i} 1 / 3}$ & 36371.05 & & 1.948 & $a^{4} \mathrm{P}, b^{4} \mathrm{P}, b^{4} \mathrm{D}, a^{2} \mathrm{D}$ \\
\hline$d^{4} \mathrm{p}\left({ }^{3} \mathrm{H}\right)$ & $\begin{array}{l}y{ }^{4} \mathrm{H}_{31 / 5}^{\circ} \\
y^{4} \mathrm{H}_{41 / 2}^{\circ} \\
y^{4} \mathrm{H}_{51 / 2}^{4} \\
y^{4} \mathrm{H}_{61 / 2}^{\circ}\end{array}$ & $\begin{array}{l}36460.34 \\
36717.11 \\
36976.10 \\
37254.41\end{array}$ & $\begin{array}{l}256.77 \\
258.99 \\
278.31\end{array}$ & $\begin{array}{l}0.691 \\
0.970 \\
1.15 \\
1.23\end{array}$ & $a^{4} \mathrm{P}, a^{4} \mathrm{~F}, b^{4} \mathrm{~F}, a^{4} \mathrm{G}, a^{4} \mathrm{H}, a^{2} \mathrm{G}, b^{2} \mathrm{G}, a^{2} \mathrm{H}, b^{2} \mathrm{H}$. \\
\hline$d^{3} s p\left({ }^{3} \mathrm{G}\right)$ & $\begin{array}{l}t{ }^{2} \mathrm{~F}_{21 / 2}^{\circ} \\
t^{2} \mathrm{~F}_{31 / 5}^{0}\end{array}$ & $36 \overline{5} \overline{1} \overline{1.49}$ & & & \}$a^{2} \mathrm{~F}, a^{2} \mathrm{G}$ \\
\hline$d^{4} p\left({ }^{3} \mathrm{D}\right)$ & $\begin{array}{l}s^{2} \mathrm{~F}_{21 / 3}^{0} \\
s^{2} \mathrm{~F}_{31 / 3}^{0}\end{array}$ & $\begin{array}{l}36866.60 \\
36979.20\end{array}$ & 112. 60 & $\begin{array}{l}0.846 \\
1.14\end{array}$ & $\left\{\begin{array}{l}a^{2} \mathrm{P}, a^{2} \mathrm{D}, a^{2} \mathrm{~F}, a^{2} \mathrm{G}, b^{2} \mathrm{G}, a^{4} \mathrm{P}, a^{4} \mathrm{D}, a^{4} \mathrm{~F}, b^{4} \mathrm{~F}, \\
a^{4} \mathrm{G}, a^{4} \mathrm{H} .\end{array}\right.$ \\
\hline$d^{4} p\left(c^{3} \mathrm{~F}\right)$ & $\begin{array}{l}t^{4} \mathrm{~F}_{11 / 3}^{0} \\
t^{4} \mathrm{~F}_{21 / 3}^{\circ} \\
t^{4} \mathrm{~F}_{31 / 3}^{0} \\
t^{4} \mathrm{~F}_{415}^{0}\end{array}$ & $\begin{array}{l}37111.67 \\
37286.62 \\
37539.67 \\
37831.58\end{array}$ & $\begin{array}{l}174.95 \\
253.05 \\
291.91\end{array}$ & $\begin{array}{l}0.526 \\
0.964 \\
1.172 \\
1.260\end{array}$ & $\left\{\begin{array}{l}a^{4} \mathrm{P}, a^{4} \mathrm{D}, b{ }^{4} \mathrm{D}, a^{4} \mathrm{~F}, b^{4} \mathrm{~F}, a^{4} \mathrm{G}, a^{4} \mathrm{H}, a^{2} \mathrm{P}, a^{2} \mathrm{D}, \\
a^{2} \mathrm{~F}, a^{2} \mathrm{G}, b^{2} \mathrm{G}, a^{2} \mathrm{H}, b^{2} \mathrm{H} .\end{array}\right.$ \\
\hline$d^{4} p\left({ }^{3} \mathrm{~F}\right)$ & $\begin{array}{l}u^{4} \mathrm{G}_{21 / 5}^{\circ} \\
u^{4} \mathrm{G}_{31 / 5}^{\circ} \\
u^{4} \mathrm{G}_{41 / 5}^{\circ} \\
u^{4} \mathrm{G}_{51 / 5}^{0}\end{array}$ & $\begin{array}{l}37188.28 \\
37343.50 \\
37523.53 \\
37760.60\end{array}$ & $\begin{array}{l}155.22 \\
180.03 \\
237.07\end{array}$ & $\begin{array}{l}0.840 \\
1.058 \\
1.190 \\
1.25\end{array}$ & $\left\{\begin{array}{l}a^{4} \mathrm{P}, a^{4} \mathrm{D}, a^{4} \mathrm{~F}, b^{4} \mathrm{~F}, a^{4} \mathrm{G}, a^{4} \mathrm{H}, a^{3} \mathrm{P}, a^{2} \mathrm{D}, a^{2} \mathrm{~F}, \\
a^{2} \mathrm{G}, b^{2} \mathrm{G}, a^{2} \mathrm{H}, b^{2} \mathrm{H}, 183 .\end{array}\right.$ \\
\hline
\end{tabular}


TABLE 6.-Terms of the Cb I spectrum-Continued

\begin{tabular}{|c|c|c|c|c|c|}
\hline $\begin{array}{l}\text { Electron } \\
\text { configu- } \\
\text { ration }\end{array}$ & $\begin{array}{l}\text { Term } \\
\text { symbol }\end{array}$ & Level & $\begin{array}{l}\text { Differ- } \\
\text { ence }\end{array}$ & $\underset{g}{\text { Observed }}$ & Combinations \\
\hline$d^{4} \&\left({ }^{5} \mathrm{D}\right)$ & $\begin{array}{l}e^{6} \mathrm{D}_{01 / 2} \\
e^{6} \mathrm{D}_{11 / 2} \\
e^{6} \mathrm{D}_{21 / 2} \\
e^{6} \mathrm{D}_{31 / 2} \\
e^{6} \mathrm{D}_{41 / 2}\end{array}$ & $\begin{array}{l}37410.17 \\
37578.72 \\
37842.36 \\
38177.65 \\
38567.85\end{array}$ & $\begin{array}{l}168.55 \\
263.64 \\
335.29 \\
390.20\end{array}$ & & $z^{\circ} \mathrm{P}^{\circ}, y{ }^{\circ} \mathrm{D}^{\circ}, z{ }^{\circ} \mathrm{F}^{\circ}, y{ }^{6} \mathrm{~F}^{\circ}$. \\
\hline$d^{4} p\left({ }^{3} \mathrm{D}\right)$ & 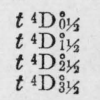 & $\begin{array}{l}37536.56 \\
37954.99 \\
38393.49 \\
38854.14\end{array}$ & $\begin{array}{l}418.43 \\
438.50 \\
460.65\end{array}$ & $\begin{array}{l}0.10 \\
1.051 \\
1.371 \\
1.305\end{array}$ & $\left\{\begin{array}{l}a^{4} \mathrm{P}, a^{4} \mathrm{D}, a^{4} \mathrm{~F}, b^{4} \mathrm{~F}, a^{4} \mathrm{G}, a^{4} \mathrm{H}, a^{6} \mathrm{D}, a^{2} \mathrm{P}, a^{2} \mathrm{D}, \\
a^{2} \mathrm{~F}, a^{2} \mathrm{G} .\end{array}\right.$ \\
\hline$d^{4} p\left({ }^{3} \mathrm{G}\right)$ & $\begin{array}{l}x{ }^{4} \mathrm{H}_{31 / 2}^{0} \\
x^{4} \mathrm{H}_{41 / 2}^{0} \\
x^{4} \mathrm{H}_{51 / 2}^{0} \\
x^{4} \mathrm{H}_{61 / 2}^{\circ}\end{array}$ & $\begin{array}{l}37624.53 \\
37866.06 \\
38149.76 \\
38513.85\end{array}$ & $\begin{array}{l}241.53 \\
277.70 \\
370.09\end{array}$ & $\begin{array}{l}0.706 \\
0.992 \\
1.089 \\
1.21\end{array}$ & $\begin{array}{l}a{ }^{4} \mathrm{P}, a^{4} \mathrm{~F}, b{ }^{4} \mathrm{~F}, a^{4} \mathrm{G}, a^{4} \mathrm{H}, a^{2} \mathrm{D}, a^{2} \mathrm{~F}, a^{2} \mathrm{G}, a^{2} \mathrm{H} . \\
b^{2} \mathrm{H} .\end{array}$ \\
\hline$d^{4} p\left({ }^{3} \mathrm{~F}\right)$ & $\begin{array}{l}r^{2} \mathrm{~F}_{21 / 4}^{\circ} \\
r^{2} \mathrm{~F}_{31 / 2}^{\circ}\end{array}$ & $\begin{array}{l}37814.64 \\
38231.85\end{array}$ & 417.21 & $\begin{array}{l}0.876 \\
1.111\end{array}$ & $\left\{\begin{array}{l}a^{2} \mathrm{D}, a{ }^{2} \mathrm{~F}, a^{2} \mathrm{G}, b^{2} \mathrm{G}, b^{2} \mathrm{H}, a^{4} \mathrm{P}, a^{4} \mathrm{~F}, b^{4} \mathrm{~F}, a^{4} \mathrm{G} \\
a^{4} \mathrm{H}, a^{6} \mathrm{D} .\end{array}\right.$ \\
\hline$d^{3} s p\left({ }^{3} \mathrm{D}\right)$ & $\begin{array}{l}t^{2} \mathrm{D}_{11 / 3}{ }^{1} \\
t^{2} \mathrm{D}_{21 / 2}\end{array}$ & $\begin{array}{l}37865.42 \\
38180.32\end{array}$ & 314. 90 & $\begin{array}{l}0.90 \\
1.077\end{array}$ & $a^{2} \mathrm{P}, a^{2} \mathrm{D}, a^{2} \mathrm{~F}, b^{2} \mathrm{G}, a^{4} \mathrm{P}, a^{4} \mathrm{D}, a^{4} \mathrm{~F}, b^{4} \mathrm{~F}, a^{4} \mathrm{H}$. \\
\hline$d^{3} s \cdot s$ & $\begin{array}{l}e^{6}{ }^{6} \mathrm{~F}_{01 / 5} \\
e^{\circ} \mathrm{F}_{11 / 2} \\
e^{6} \mathrm{~F}_{21 / 5} \\
e^{6} \mathrm{~F}_{31 / 5} \\
e^{6} \mathrm{~F}_{41 / 5} \\
e^{6} \mathrm{~F}_{51 / 2}\end{array}$ & $\begin{array}{l}37871.30 \\
38021.41 \\
38276.59 \\
38638.47 \\
39100.73 \\
39408.88\end{array}$ & $\begin{array}{l}150.11 \\
255.18 \\
361.88 \\
462.26 \\
308.15\end{array}$ &. & $z^{\circ} \mathrm{D}^{\circ}, z^{6} \mathrm{~F}^{\circ}, z^{6} \mathrm{G}^{\circ}$. \\
\hline$d^{4} p\left({ }^{3} \mathrm{D}\right)$ & $\begin{array}{l}x^{2} \mathrm{P}_{01 / 2}^{\circ} \\
x^{2} \mathrm{P}_{1 / 3}^{\circ}\end{array}$ & $\begin{array}{l}38182.96 \\
38446.78\end{array}$ & 263.82 & $\begin{array}{l}0.564 \\
1.29\end{array}$ & $\left\{\begin{array}{l}a^{2} \mathrm{P}, a^{2} \mathrm{D}, a^{2} \mathrm{~F}, a^{4} \mathrm{P}, b^{4} \mathrm{P}, a^{4} \mathrm{D}, b^{4} \mathrm{D}, a^{4} \mathrm{~F}, b^{4} \mathrm{~F}, \\
a^{4} \mathrm{G} .\end{array}\right.$ \\
\hline$d^{4} p\left({ }^{3} \mathrm{H}\right)$ & $\begin{array}{l}y^{2}{ }^{2} I_{51 / 2}^{\circ} \\
{ }^{2} I_{63 / 2}^{0}\end{array}$ & $\begin{array}{l}38251.28 \\
38583.04\end{array}$ & 331.76 & $\begin{array}{l}0.97 \\
1.09\end{array}$ & $a^{2} \mathrm{G}, a^{2} \mathrm{H}, b^{2} \mathrm{H}, a^{4} \mathrm{~F}, b^{4} \mathrm{~F}, a^{4} \mathrm{G}, a^{4} \mathrm{H}$. \\
\hline$d^{3} s p\left({ }^{1} \mathrm{G}\right)$ & $\begin{array}{l}w^{2} \mathrm{H}_{41 / 2}^{\circ} \\
w^{2} \mathrm{H}_{51 / 2}^{5}\end{array}$ & $\begin{array}{l}38448.77 \\
39020.81\end{array}$ & 572.04 & $\begin{array}{l}0.936 \\
1.084\end{array}$ & $\left\{\begin{array}{l}a{ }^{2} \mathrm{~F}, a^{2} \mathrm{G}, b{ }^{2} \mathrm{G}, a^{2} \mathrm{H}, b{ }^{2} \mathrm{H}, 183, a^{4} \mathrm{D}, a^{4} \mathrm{~F}, b{ }^{4} \mathrm{~F}, \\
a^{4} \mathrm{G}, a^{4} \mathrm{H} .\end{array}\right.$ \\
\hline$d^{4} p\left({ }^{3} \mathrm{D}\right)$ & $\begin{array}{l}w^{4} \mathrm{P}^{0} 1 / 1 / 2 \\
w^{4} \mathrm{P}_{11 / 2}^{1} \\
w^{4} \mathrm{P}_{21 / 2}^{1}\end{array}$ & $\begin{array}{l}38730.16 \\
38709.66 \\
38763.34\end{array}$ & $\begin{array}{r}-20.50 \\
53.68\end{array}$ & $\begin{array}{l}2.55 \\
1.660 \\
1.588\end{array}$ & $a^{4} \mathrm{P}, b^{4} \mathrm{P}, a^{4} \mathrm{D}, b^{4} \mathrm{D}, a^{4} \mathrm{~F}, b^{4} \mathrm{~F}, a^{6} \mathrm{~S}, a^{6} \mathrm{D}, a^{2} \mathrm{P}, a^{2} \mathrm{D}$. \\
\hline$d^{3} s p\left({ }^{3} G\right)$ & $\begin{array}{l}s^{4} \mathrm{~F}^{1} 1 / 2 \\
s^{4} \mathrm{~F}_{21 / 2}^{2} \\
s^{4} \mathrm{~F}_{31 / 2}^{3} \\
s^{4} \mathrm{~F}_{41 / 2}\end{array}$ & $\begin{array}{l}38903.00 \\
39248.30 \\
39620.13 \\
40008.52\end{array}$ & $\begin{array}{l}345.30 \\
371.83 \\
388.39\end{array}$ & $\begin{array}{l}0.448 \\
0.973 \\
1.094 \\
1.108\end{array}$ & $a^{4} \mathrm{D}, b^{4} \mathrm{D}, a^{4} \mathrm{~F}, b^{4} \mathrm{~F}, a^{4} \mathrm{G}, a^{4} \mathrm{H}, a^{2} \mathrm{D}, a^{2} \mathrm{G}, a^{2} \mathrm{H}, 183$. \\
\hline$d^{3} s p\left({ }^{3} \mathrm{H}\right)$ & $\begin{array}{l}u^{2} \mathrm{G}_{31 / 2} 1 / 2 \\
u^{2} \mathrm{G}_{41 / 2}^{1}\end{array}$ & $\begin{array}{l}\text { 38982. } 20 \\
39380.77\end{array}$ & 398.57 & $\begin{array}{l}\text { 1. } 010 \\
1.10\end{array}$ & $\left\{\begin{array}{l}a^{2} \mathrm{D}, a^{2} \mathrm{~F}, a^{2} \mathrm{G}, b^{2} \mathrm{G}, a^{2} \mathrm{H}, b^{2} \mathrm{H}, a^{4} \mathrm{P}, a^{4} \mathrm{D}, a^{4} \mathrm{~F}, \\
b{ }^{4} \mathrm{~F}, a^{2} \mathrm{G}, a^{4} \mathrm{H}, a^{6} \mathrm{D} .\end{array}\right.$ \\
\hline$d^{3} s p\left(d^{3} \mathrm{~F}\right)$ & $\begin{array}{l}t^{2} \mathrm{G}_{3}^{0} 1 / 2 \\
t^{2} \mathrm{G}_{4}^{0} 1 / 2\end{array}$ & $\begin{array}{l}39426.84 \\
39637.83\end{array}$ & 210.92 & $\begin{array}{l}0.91 \\
1.04\end{array}$ & $\left\{\begin{array}{l}a^{2} \mathrm{~F}, a^{2} \mathrm{G}, b{ }^{2} \mathrm{G}, a^{2} \mathrm{H}, b{ }^{2} \mathrm{H}, 183, a^{4} \mathrm{D}, b^{4} \mathrm{D}, a^{4} \mathrm{~F}, \\
a^{4} \mathrm{G}, a^{4} \mathrm{H} .\end{array}\right.$ \\
\hline$d^{3} s p\left({ }^{3} \mathrm{G}\right)$ & $\begin{array}{l}t^{4} \mathrm{G}_{21 / 2}^{2} \\
t^{4} \mathrm{G}_{31 / 2}^{2} \\
t^{4} \mathrm{G}_{41 / 2} \\
t^{4} \mathrm{G}_{51 / 2}^{1}\end{array}$ & $\begin{array}{l}39530.46 \\
39885.66 \\
40421.93 \\
40481.96\end{array}$ & $\begin{array}{r}355.20 \\
536.27 \\
60.03\end{array}$ & $\begin{array}{l}0.61 \\
1.085 \\
1.228 \\
1.18\end{array}$ & $\left\{\begin{array}{l}b^{4} \mathrm{D}, a^{4} \mathrm{~F}, b^{4} \mathrm{~F}, a^{4} \mathrm{G}, a^{4} \mathrm{H}, a^{6} \mathrm{D}, a^{2} \mathrm{P}, a^{2} \mathrm{D}, a^{2} \mathrm{~F}, \\
a^{2} \mathrm{G}, a^{2} \mathrm{H}, b^{2} \mathrm{H}, 183 .\end{array}\right.$ \\
\hline$d^{4} p(1 \mathrm{I})$ & $\begin{array}{l}v^{2} \mathrm{H}_{41 / 2}^{\circ} \\
v^{2} \mathrm{H}_{5}^{1} 3 / 2\end{array}$ & $\begin{array}{l}39845.51 \\
40003.00\end{array}$ & 157.49 & 1.152 & $\left\{\begin{array}{l}a^{2} \mathrm{~F}, a^{2} \mathrm{G}, b^{2} \mathrm{G}, a^{2} \mathrm{H}, b^{2} \mathrm{H}, 183, a^{4} \mathrm{~F}, b^{4} \mathrm{~F}, a^{4} \mathrm{G} \text {, } \\
a^{4} \mathrm{H} .\end{array}\right.$ \\
\hline$d^{3} s p\left({ }^{3} \mathrm{D}\right)$ & 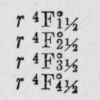 & $\begin{array}{l}39981.20 \\
40209.72 \\
40362.00 \\
40853.89\end{array}$ & $\begin{array}{l}228.52 \\
152.28 \\
491.89\end{array}$ & $\begin{array}{l}0.45 \\
0.886 \\
1.129 \\
1.250\end{array}$ & $\left\{\begin{array}{l}a^{4} \mathrm{P}, b^{4} \mathrm{P}, a^{4} \mathrm{D}, b^{4} \mathrm{D}, a^{4} \mathrm{~F}, b^{4} \mathrm{~F}, a^{4} \mathrm{G}, a^{4} \mathrm{H}, a^{6} \mathrm{D}, \\
a^{2} \mathrm{P}, a^{2} \mathrm{~F}, a^{2} \mathrm{G}, b^{2} \mathrm{G}, b^{2} \mathrm{H} .\end{array}\right.$ \\
\hline & $400_{21 / 2}^{\circ}$ & 40009.84 & & & $b^{4} \mathrm{P}, a^{4} \mathrm{~F}, b^{4} \mathrm{~F}, a^{4} \mathrm{G}, a^{4} \mathrm{H}, a^{2} \mathrm{~F}, a^{2} \mathrm{G}$. \\
\hline$d^{3} s p(1 \mathrm{~F})$ & $\begin{array}{l}q 2 \mathrm{~F}_{21 / 3}^{\circ} \\
q^{2} \mathrm{~F}_{31 / 2}^{\circ}\end{array}$ & $\begin{array}{l}40735.18 \\
40469.71\end{array}$ & -265.47 & $\begin{array}{l}0.91 \\
1.17\end{array}$ & $\left\{\begin{array}{l}a^{2} \mathrm{D}, a^{2} \mathrm{~F}, a^{2} \mathrm{G}, b^{2} \mathrm{G}, b^{2} \mathrm{H}, 183, a^{4} \mathrm{P}, b^{4} \mathrm{P}, a^{4} \mathrm{D}, \\
b^{4} \mathrm{D}, a^{4} \mathrm{~F}, b^{4} \mathrm{~F}, a^{4} \mathrm{G}, a^{4} \mathrm{H} .\end{array}\right.$ \\
\hline$d^{4} p\left(c^{3} \mathrm{~F}\right)$ & $\begin{array}{l}s^{4} \mathrm{D}_{01 / 2}^{0} \\
s^{4} \mathrm{D}_{13 / 2}^{0} \\
s^{4} \mathrm{D}_{21 / 2}^{211} \\
s^{4} \mathrm{D}^{3} 31 / 2\end{array}$ & $\begin{array}{l}40473.90 \\
40953.36\end{array}$ & 479.46 & $\begin{array}{l}\text { 1. } 29 \\
1.367\end{array}$ & $\left\{\begin{array}{l}a^{4} \mathrm{P}, b^{4} \mathrm{P}, a^{4} \mathrm{D}, b{ }^{4} \mathrm{D}, a^{4} \mathrm{~F}, b^{4} \mathrm{~F}, a^{4} \mathrm{G}, a^{4} \mathrm{H}, a^{2} \mathrm{D}, \\
a^{2} \mathrm{~F}, a^{2} \mathrm{G}, b^{2} \mathrm{G} .\end{array}\right.$ \\
\hline$d^{3} s p\left({ }^{3} \mathrm{H}\right)$ & 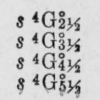 & $\begin{array}{l}40079.30 \\
40555.28 \\
40921.15 \\
41371.17\end{array}$ & $\begin{array}{l}475.98 \\
365.87 \\
450.02\end{array}$ & $\begin{array}{l}0.929 \\
1.089 \\
1.243 \\
1.25\end{array}$ & $\left\{\begin{array}{l}a^{4} \mathrm{P}, b^{4} \mathrm{P}, a^{4} \mathrm{D}, b^{4} \mathrm{D}, a^{4} \mathrm{~F}, b^{4} \mathrm{~F}, a^{4} \mathrm{G}, a^{4} \mathrm{H}, a^{6} \mathrm{D}, \\
a^{2} \mathrm{D}, a^{2} \mathrm{~F}, a^{2} \mathrm{G}, b^{2} \mathrm{G}, a^{2} \mathrm{H}, b^{2} \mathrm{H} .\end{array}\right.$ \\
\hline
\end{tabular}


TABLE 6.-Terms of the Cb I spectrum-Continued

\begin{tabular}{|c|c|c|c|c|c|}
\hline $\begin{array}{l}\text { Electron } \\
\text { configu- } \\
\text { ration }\end{array}$ & $\begin{array}{l}\text { Term } \\
\text { symbol }\end{array}$ & Level & $\begin{array}{l}\text { Differ- } \\
\text { ence }\end{array}$ & $\underset{g}{\text { Observed }}$ & Combinations \\
\hline \multirow[t]{5}{*}{$d^{3} s p(1 \mathrm{D})$} & $\begin{array}{l}w^{2} \mathrm{P}_{01 / 2}^{\circ} \\
w^{2} \mathrm{P}_{11 / 2}^{\circ}\end{array}$ & 41082.24 & & 1. 38 & \}$a^{2} \mathrm{P}, a^{2} \mathrm{D}, a^{4} \mathrm{P}, b^{4} \mathrm{P}, a^{4} \mathrm{D}, b^{4} \mathrm{D}, a^{4} \mathrm{G}$ \\
\hline & $411_{31 / 2}^{\circ}$ & 41139.95 & . & 0.88 & $\begin{array}{l}a^{4} \mathrm{P}, b{ }^{4} \mathrm{D}, a^{4} \mathrm{~F}, a^{4} \mathrm{G}, a^{2} \mathrm{D}, a^{2} \mathrm{~F}, a^{2} \mathrm{G}, b^{2} \mathrm{G}, a^{2} \mathrm{H}, \\
b^{2} \mathrm{H}, a^{0} \mathrm{D} .\end{array}$ \\
\hline & $414_{1 / 2}^{0}$ & 41460.97 & & 1. 42 & $a^{4} \mathrm{P}, b^{4} \mathrm{P}, a^{4} \mathrm{D}, b^{4} \mathrm{D}, a^{4} \mathrm{~F}, b^{4} \mathrm{~F}, a^{4} \mathrm{G}, a^{2} \mathrm{D}, a^{2} \mathrm{~F}$. \\
\hline & $41621 / 2$ & 41615.40 & & & $\begin{array}{l}a^{4} \mathrm{P}, b^{4} \mathrm{P}, a^{4} \mathrm{D}, b^{4} \mathrm{D}, a^{4} \mathrm{~F}, b^{4} \mathrm{~F}, a^{4} \mathrm{G}, a^{4} \mathrm{H}, a^{2} \mathrm{~F}, \\
b^{2} \mathrm{G} .\end{array}$ \\
\hline & $\begin{array}{l}q^{4} \mathrm{~F}_{13 / 2}^{0} \\
q^{4} \mathrm{~F}_{21 / 2}^{0} \\
q^{4} \mathrm{~F}_{31 / 2}^{0} \\
q^{4} \mathrm{~F}_{41 / 2}^{0}\end{array}$ & $\begin{array}{l}41554.86 \\
41746.34 \\
41930.87 \\
41873.91\end{array}$ & $\begin{array}{r}191.48 \\
184.53 \\
-56.96\end{array}$ & $\begin{array}{l}0.556 \\
1.25 \\
1.186\end{array}$ & $\left\{\begin{array}{l}a^{4} \mathrm{P}, b^{4} \mathrm{P}, a^{4} \mathrm{D}, b^{4} \mathrm{D}, a^{4} \mathrm{~F}, b^{4} \mathrm{~F}, a^{4} \mathrm{G}, a^{4} \mathrm{H}, a^{2} \mathrm{P} \\
a^{2} \mathrm{D}, a^{2} \mathrm{~F}, a^{2} \mathrm{G}, b^{2} \mathrm{G}, a^{2} \mathrm{H}, b^{2} \mathrm{H} .\end{array}\right.$ \\
\hline$d^{4} p\left({ }^{3} \mathrm{G}\right)$ & $\begin{array}{l}s^{2} \mathrm{G}_{31 / 2} \\
s^{2} \mathrm{G}_{41 / 2}^{\circ}\end{array}$ & $\begin{array}{l}415 \gamma 1.61 \\
41895.67\end{array}$ & 324.06 & & \}$a^{2} \mathrm{D}, a^{2} \mathrm{~F}, b^{2} \mathrm{G}, a^{2} \mathrm{H}, b^{2} \mathrm{H}, 183, b^{4} \mathrm{D}, a^{4} \mathrm{~F}, a^{4} \mathrm{H}$. \\
\hline$d^{4} p(1 \mathrm{~F})$ & $\begin{array}{l}p^{2} \mathrm{~F}_{21 / 2}^{\circ} \\
p^{2} \mathrm{~F}_{31 / 2}^{\circ}\end{array}$ & $\begin{array}{l}41829.11 \\
41987.35\end{array}$ & 158.24 & $\begin{array}{l}0.86 \\
1.135\end{array}$ & \}$^{a^{2} \mathrm{P}, a^{2} \mathrm{D}, a^{2} \mathrm{~F}, a^{2} \mathrm{G}, b^{2} \mathrm{G}, a^{2} \mathrm{H}, b^{2} \mathrm{H}, b^{4} \mathrm{D}, b^{4} \mathrm{~F},}$ \\
\hline$d^{4} p\left({ }^{3} \mathrm{~F}\right)$ & $\begin{array}{l}r^{2} \mathrm{G}_{31 / 2}^{\circ} \\
r^{2} \mathrm{G}_{41 / 2}^{\circ}\end{array}$ & $42 \overline{19} \overline{3} .70$ & & 1.15 & \}$_{a^{2} \mathrm{~F}, a^{2} \mathrm{G}, b^{2} \mathrm{G}, a^{2} \mathrm{H}, b^{2} \mathrm{H}, 183, b^{4} \mathrm{D}, a^{4} \mathrm{~F}, b^{4} \mathrm{~F},}^{a^{4} \mathrm{G}, a^{4} \mathrm{H} .}$ \\
\hline \multirow[t]{2}{*}{$d^{3} s p\left(b^{3} \mathrm{P}\right)$} & $\begin{array}{l}r{ }^{4} \mathrm{D}_{01 / 2}^{\circ} \\
r^{4} \mathrm{D}_{11 / 2}^{\circ} \\
r{ }^{4} \mathrm{D}_{2}^{\circ} 1 / 2 \\
r{ }^{4} \mathrm{D}_{31 / 2}^{\circ}\end{array}$ & $\begin{array}{l}41676.81 \\
42339.56 \\
42551.56 \\
42719.18\end{array}$ & $\begin{array}{l}662.75 \\
212.00 \\
167.62\end{array}$ & $\begin{array}{l}1.45 \\
1.39\end{array}$ & $a^{4} \mathrm{P}, b^{4} \mathrm{P}, b^{4} \mathrm{D}, a^{4} \mathrm{~F}, b^{4} \mathrm{~F}, a^{2} \mathrm{P}, a^{2} \mathrm{D}$ \\
\hline & $\begin{array}{l}s^{2} \mathrm{D}_{11 / 2} \\
s^{2} \mathrm{D}_{21 / 2}^{\circ}\end{array}$ & $42 \overline{10-9} .90$ & & 1. 21 & \}$_{a{ }^{2} \mathrm{P}, a^{2} \mathrm{D}, a^{2} \mathrm{~F}, b^{2} \mathrm{H}, b^{4} \mathrm{P}, a^{4} \mathrm{D}, b^{4} \mathrm{D}, a^{4} \mathrm{~F}, b^{4} \mathrm{~F},}$ \\
\hline$d^{3} s p\left({ }^{5} \mathrm{P}\right)$ & 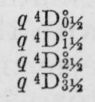 & $\begin{array}{l}42324.31 \\
42473.21 \\
42642.90 \\
43332.66\end{array}$ & $\begin{array}{l}148.90 \\
169.69 \\
689.76\end{array}$ & & $\left\{\begin{array}{l}a^{4} \mathrm{P}, b^{4} \mathrm{P}, b^{4} \mathrm{D}, a^{4} \mathrm{~F}, b^{4} \mathrm{~F}, a^{4} \mathrm{G}, a^{6} \mathrm{D}, a^{2} \mathrm{P}, b^{2} \mathrm{G}, \\
b^{2} \mathrm{H} .\end{array}\right.$ \\
\hline$d^{3} s p\left({ }^{3} \mathrm{D}\right)$ & $\begin{array}{l}p^{4} \mathrm{D}_{0}^{\circ} 1 / 2 \\
p^{4} \mathrm{D}_{11 / 2}^{\circ} \\
p^{4} \mathrm{D}_{21 / 2}^{\circ} \\
p^{4} \mathrm{D}^{\circ} 31 / 2\end{array}$ & $\begin{array}{l}42801.67 \\
42900.07 \\
4318 \% .00 \\
43414.44\end{array}$ & $\begin{array}{r}98.40 \\
286.93 \\
227.44\end{array}$ & & $\left\{\begin{array}{l}a^{4} \mathrm{P}, b^{4} \mathrm{P}, b^{4} \mathrm{D}, a^{4} \mathrm{~F}, b^{4} \mathrm{~F}, a^{4} \mathrm{G}, a^{6} \mathrm{D}, a^{2} \mathrm{D}, a^{2} \mathrm{~F}, \\
a^{2} \mathrm{G}, b^{2} \mathrm{G} .\end{array}\right.$ \\
\hline$d^{3} s p\left({ }^{3} \mathrm{D}\right)$ & $\begin{array}{l}0^{2} \mathrm{~F}_{21 / 2}^{0} \\
0^{2} \mathrm{~F}_{31 / 2}^{0}\end{array}$ & 42888.84 & & & \}$a^{2} \mathrm{P}, a^{2} \mathrm{D}, a^{2} \mathrm{G}, a^{4} \mathrm{~F}, a^{4} \mathrm{G}$ \\
\hline$d^{3} s p\left(d^{3} \mathrm{~F}\right)$ & $\begin{array}{l}p^{4} \mathrm{~F}_{11 / 2}^{0} \\
p^{4} \mathrm{~F}_{21 / 2}^{0} \\
p^{4} \mathrm{~F}_{31 / 2}^{0} \\
p^{4} \mathrm{~F}^{0} 11 / 2\end{array}$ & $\begin{array}{l}42894.24 \\
43022.60 \\
43173.00 \\
43995.80\end{array}$ & $\begin{array}{l}128.36 \\
150.40 \\
222.80\end{array}$ & $\begin{array}{l}\text { 1. } 22 \\
\text { 1. } 28\end{array}$ & $\left\{\begin{array}{l}a^{4} \mathrm{P}, b^{4} \mathrm{P}, a^{4} \mathrm{D}, b^{4} \mathrm{D}, a^{4} \mathrm{~F}, b^{4} \mathrm{~F}, a^{4} \mathrm{G}, a^{4} \mathrm{H}, a^{6} \mathrm{D}, \\
a^{2} \mathrm{G}, a^{2} \mathrm{H} .\end{array}\right.$ \\
\hline$d^{3} s p\left(d^{3} \mathrm{~F}\right)$ & $\begin{array}{l}n^{2} \mathrm{~F}_{21 / 2}^{\circ} \\
n^{2} \mathrm{~F}_{31 / 2}^{\circ}\end{array}$ & $\begin{array}{l}43342.88 \\
43553.17\end{array}$ & 212.29 & & \}$a^{2} \mathrm{D}, a^{2} \mathrm{~F}, a^{2} \mathrm{G}, b^{2} \mathrm{G}, b^{4} \mathrm{D}, a^{4} \mathrm{~F}, b^{4} \mathrm{~F}, a^{4} \mathrm{G}, a^{6} \mathrm{D}$. \\
\hline & $\begin{array}{l}q^{2} \mathrm{G}_{31 / 2}^{\circ} \\
q^{2} \mathrm{G}_{41 / 2}^{\circ}\end{array}$ & 43880.22 & & & $a^{2} \mathrm{G}, a^{2} \mathrm{H}, a^{4} \mathrm{~F}, a^{4} \mathrm{G}$. \\
\hline$d^{3} s p\left({ }^{3} \mathrm{D}\right)$ & $\begin{array}{l}v^{2} \mathrm{P}_{01 / 3}^{\circ} \\
v^{2} \mathrm{P}_{11 / 2}^{\circ}\end{array}$ & $\begin{array}{l}46004.29 \\
44063.49\end{array}$ & -1940.80 & 1. 27 & \}$a^{2} \mathrm{P}, a^{2} \mathrm{D}, a^{4} \mathrm{P}, b^{4} \mathrm{P}, b^{4} \mathrm{D}, a^{4} \mathrm{~F}$ \\
\hline & $\begin{array}{l}0^{4} \mathrm{D}_{01 / 2}^{\circ} \\
04 \mathrm{D}_{11 / 2}^{\circ} \\
0^{4} \mathrm{D}_{21 / 2}^{\circ} \\
0^{4} \mathrm{D}_{31 / 2}^{\circ}\end{array}$ & $\begin{array}{l}44939.08 \\
44905.54 \\
45170.90\end{array}$ & $\begin{array}{r}-33.54 \\
265.36\end{array}$ & & $a^{4} \mathrm{P}, b^{4} \mathrm{P}, a^{4} \mathrm{~F}, b^{4} \mathrm{~F}, a^{2} \mathrm{D}, a^{2} \mathrm{~F}, a^{2} \mathrm{G}, b^{2} \mathrm{G}, b^{2} \mathrm{H}$ \\
\hline$d^{3} s p\left({ }^{3} \mathrm{G}\right)$ & $\begin{array}{l}u^{2} \mathrm{H}_{i 1 / 2}^{\circ} \\
u^{2} \mathrm{H}_{51 / 2}^{\circ}\end{array}$ & $\begin{array}{l}45110.13 \\
45259.03\end{array}$ & 148.90 & 1.09 & \}$a^{2} \mathrm{~F}, a^{2} \mathrm{G}, b^{2} \mathrm{G}, a^{2} \mathrm{H}, b^{2} \mathrm{H}, 183, a^{4} \mathrm{~F}, a^{4} \mathrm{G}$. \\
\hline$d^{4} p\left(c^{3} \mathrm{~F}\right)$ & $\begin{array}{l}m^{2} \mathrm{~F}_{21 / 2}^{\circ} \\
m^{2} \mathrm{~F}_{31 / 2}^{\circ}\end{array}$ & 45297.30 & & 0.94 & \}$a^{2} \mathrm{D}, a^{2} \mathrm{~F}, a^{2} \mathrm{G}, b^{2} \mathrm{G}, b^{2} \mathrm{H}, a^{4} \mathrm{P}, b^{4} \mathrm{P}, b^{4} \mathrm{D}, b^{4} \mathrm{~F}$. \\
\hline$d^{4} p\left(c^{3} \mathrm{~F}\right)$ & $\begin{array}{l}p^{2} \mathrm{G}_{31 / 2}^{\circ} \\
p^{2} \mathrm{G}_{41 / 2}^{\circ}\end{array}$ & $\begin{array}{l}45719.07 \\
45982.56\end{array}$ & 263. 49 & 0.84 & $b^{2} \mathrm{G}, a^{2} \mathrm{H}, b^{2} \mathrm{H}, b^{4} \mathrm{P}, a^{4} \mathrm{~F}, b^{4} \mathrm{~F}$. \\
\hline$d^{3} s . p\left({ }^{3} \mathrm{~F}\right)$ & $\begin{array}{l}n{ }^{4} \mathrm{D}_{01 / 2}^{0} \\
n{ }^{4} \mathrm{D}_{11 / 2}^{1} \\
n{ }^{4} \mathrm{D}_{21 / 2}^{\circ} \\
n{ }^{4} \mathrm{D}_{31 / 2}\end{array}$ & $\begin{array}{l}45978.84 \\
46364.79 \\
46812.58 \\
47275.64\end{array}$ & $\begin{array}{l}385.95 \\
447.79 \\
463.06\end{array}$ & & $a^{4} \mathrm{P}, b^{4} \mathrm{P}, a^{4} \mathrm{D}, b^{4} \mathrm{D}, a^{4} \mathrm{~F}, b^{4} \mathrm{~F}, a^{2} \mathrm{G}$. \\
\hline
\end{tabular}


TABLE 6.-Terms of the $\mathrm{Cb}$ I spectrum-Continued

\begin{tabular}{|c|c|c|c|c|c|}
\hline $\begin{array}{l}\text { Electron } \\
\text { configu- } \\
\text { ration }\end{array}$ & $\begin{array}{l}\text { Term } \\
\text { symbol }\end{array}$ & Level & $\begin{array}{l}\text { Differ- } \\
\text { ence }\end{array}$ & $\underset{g}{\text { Observed }}$ & Combinations \\
\hline$d^{3} s . p\left({ }^{3} \mathrm{~F}\right)$ & 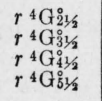 & $\begin{array}{l}46011.48 \\
46470.71 \\
46724.20 \\
47080.83\end{array}$ & $\begin{array}{l}459.23 \\
253.49 \\
356.63\end{array}$ & & $\begin{array}{l}a{ }^{4} \mathrm{P}, b^{4} \mathrm{P}, b^{4} \mathrm{D}, a^{4} \mathrm{~F}, a^{4} \mathrm{G}, a^{4} \mathrm{H}, a^{2} \mathrm{~F}, a^{2} \mathrm{G}, b^{2} \mathrm{G} \text {, } \\
183 .\end{array}$ \\
\hline \multirow[t]{3}{*}{$d^{3} s \cdot p\left({ }^{3} \mathrm{~F}\right)$} & 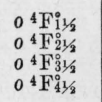 & $\begin{array}{l}46170.04 \\
46543.56 \\
47029.78 \\
47680.59\end{array}$ & $\begin{array}{l}373.52 \\
479.22 \\
657.81\end{array}$ & & $a^{4} \mathrm{P}, b^{4} \mathrm{P}, a^{4} \mathrm{D}, b^{4} \mathrm{D}, a^{4} \mathrm{~F}, b^{4} \mathrm{~F}, a^{4} \mathrm{G}, a^{2} \mathrm{G}$. \\
\hline & 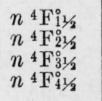 & $\begin{array}{r}46509.80 \\
46932.06 \\
47146.06\end{array}$ & $\begin{array}{l}422.26 \\
214.00\end{array}$ & & $a^{4} \mathrm{P}, b^{4} \mathrm{P}, a^{4} \mathrm{D}, a^{4} \mathrm{~F}, a^{4} \mathrm{G}, a^{2} \mathrm{G}$ \\
\hline & $469_{2 / 2}^{\circ}$ & 46919.48 & & & $a^{4} \mathrm{P}, b{ }^{4} \mathrm{P}, a^{4} \mathrm{D}, b{ }^{4} \mathrm{D}, a^{4} \mathrm{~F}, a^{2} \mathrm{P}, a^{2} \mathrm{D}$. \\
\hline \multirow[t]{4}{*}{$d^{3} s p\left({ }^{3} \mathrm{H}\right)$} & $\begin{array}{l}t^{2} \mathrm{H}_{i 1 / 2}^{\circ} \\
t^{2} \mathrm{H}_{51 / 2}^{13}\end{array}$ & $\begin{array}{l}46954.40 \\
47155.22\end{array}$ & 200.82 & 1.00 & $a{ }^{2} \mathrm{~F}, a^{2} \mathrm{G}, b^{2} \mathrm{G}, a{ }^{2} \mathrm{H}, b{ }^{2} \mathrm{H}, a^{4} \mathrm{~F}, a^{4} \mathrm{G}$. \\
\hline & $\begin{array}{l}0^{2} \mathrm{G}_{31 / 2}^{8} \\
0^{2} \mathrm{G}_{41 / 2}^{8}\end{array}$ & $\begin{array}{l}47528.49 \\
48242.44\end{array}$ & 713.95 & $\begin{array}{l}0.92 \\
1.10\end{array}$ & $a^{2} \mathrm{~F}, a^{2} \mathrm{G}, b^{2} \mathrm{G}, a^{2} \mathrm{H}, b^{2} \mathrm{H}, 183$. \\
\hline & $475^{\circ} 1 / 2$ & 47537.67 & & & $a^{4} \mathrm{P}, b^{4} \mathrm{D}, a^{4} \mathrm{~F}, b^{4} \mathrm{~F}, a^{4} \mathrm{G}$. \\
\hline & $\begin{array}{l}n^{2} \mathrm{G}_{31 / 2}^{\circ} \\
n^{2} \mathrm{G}_{41 / 2}^{3}\end{array}$ & $51 \bar{\gamma} 8 \overline{8} .30$ & & & $a^{2} \mathrm{~F}, a^{2} \mathrm{G}, a^{2} \mathrm{H}, 183, a{ }^{4} \mathrm{D}$. \\
\hline
\end{tabular}

The observed $g$-values are entered in column 5 of table 6 . These may be compared with Landé values by referring to the publication mentioned earlier [17]. The $g$-sums for the levels originating in the various possible electron configurations are compared with the Landé sums for the same set of levels in table 7 . The agreement is very close. Except in the case of the configuration $d^{3} s^{2}$, for which only one level is missing, several levels are missing from each configuration. The remarkable agreement of the sums supports the conclusion that the coupling is closely $L S$, deviations from Landé $g$ 's being accounted for by sharing in instances similar to those especially noted above.

TABLE 7.-Test of g-sum rule for $\mathrm{Cb} \mathbf{I}$

\begin{tabular}{|c|c|c|}
\hline Electron configuration & $\underset{\text { observed } g^{\prime} \text { s }}{\text { Sum }}$ & $\begin{array}{l}\text { Landé } \\
\text { sum }\end{array}$ \\
\hline $\begin{array}{l}d^{4} s^{4} \\
d^{3} s^{2}-d^{2} \\
d^{4} p^{2} \\
d^{3} s p \\
d^{5}\end{array}$ & $\begin{array}{r}39.811 \\
19.965 \\
135.279 \\
136.598 \\
1.99\end{array}$ & $\begin{array}{r}40.000 \\
20.000 \\
135.268 \\
136.582 \\
2.000\end{array}$ \\
\hline
\end{tabular}

All observed term combinations are listed in the last column of table 6 . These conform to the selection rules for transitions between energy states, any others that were observed being ruled out as fortuitous. As in $\mathrm{Cb}$ II and all other complex spectra, a considerable number of permitted transitions is not observed, because of low 
transition probability or superposition. The total number of listed term combinations is 1,042 , of which 195 are doublet-doublet, 284 quartet-quartet, 18 sextet-sextet, 375 doublet-quartet, 61 quartetsextet, 31 doublet-sextet, and 78 involving unidentified levels.

\section{ELECTRON CONFIGURATIONS, THEORETICAL AND OBSERVED TERMS}

The five outer electrons responsible for the chemical properties and optical spectrum of neutral columbium may be grouped in three possible ways to produce the low terms of $\mathrm{Cb}$, as follows:

$4 d^{5}:{ }^{2} \mathrm{D},{ }^{2}(\mathrm{PDFGH}),{ }^{4}(\mathrm{PF}),{ }^{2}$ (SDFGI), ${ }^{4}(\mathrm{DG}),{ }^{6} \mathrm{~S}$. $4 d^{4} 5 s:{ }^{2}(\mathrm{SDG}),{ }^{2,4}(\mathrm{PF}),{ }^{2}(\mathrm{SDFGI}),{ }^{2,4}(\mathrm{PDFGH}),{ }^{4,6}(\mathrm{D})$. $4 d^{3} 5 s^{2}:{ }^{2} \mathrm{D},{ }^{2}(\mathrm{PDFGH}),{ }^{4}(\mathrm{PF})$.

Of the $4 d^{5}$ terms only ${ }^{6} \mathrm{~S}$ has been found in $\mathrm{Cb}$ I. According to theoretical studies by Laporte [18], reasonably precise calculations can be made of the location of the others. The combinations should lie in the long wavelength visible or infrared and may be masked by bands. The ${ }^{6} \mathrm{D}$ term from $4 d^{4} 5 s$ is the lowest state of $\mathrm{Cb} \mathrm{I}$. The quartets have all been found except the relatively high ${ }^{4} \mathrm{P}$ and ${ }^{4} \mathrm{~F}$ in the second parentheses above. The doublets from this configuration are very incomplete. The low terms $b^{2} \mathrm{G}$ and $b^{2} \mathrm{H}$ have been identified, but are the only low terms about which some uncertainty is felt. The combinations are scattered and all Zeeman patterns unresolved. The assigned $j$ 's give the largest number of combinations. An alternative possibility is that the unassigned level, 18332.04, $j=5 \frac{1}{2}$, belongs to $b^{2} \mathrm{H}$, and that one of levels now assigned to $b^{2} \mathrm{G}$ or $b^{2} \mathrm{H}$ is part of the missing ${ }^{2} \mathrm{~F}$ from $4 d^{4} 5 s$. The only other possibility for 18332.04 is ${ }^{2} \mathrm{I}_{51 / 2}$. These terms must of course come from $4 d^{4} 5 s$. No other possibility exists, since all terms from $4 d^{3} 5 s^{2}$ have been found except ${ }^{2} \mathrm{D}$ listed first in the tabulation above and expected to be relatively high.

The observed $\mathrm{Cb}$ I spectrum is almost entirely accounted for by transitions involving the low terms just discussed and intermediate sets arising from $4 d^{4} 5 p$ and $4 d^{3} 5 s 5 p$. The predicted and observed terms from these configurations are displayed in table 8 . Although a considerable number of terms, particularly doublets, is still unknown, the remaining unclassified lines are generally scattered and weak. All recent efforts to extend the classification have yielded meager results so that further work has not been deemed profitable. All intermediate odd sextets are known. One of these, $\left(4 d^{3} 5 s 5 p\right) z^{6} \mathrm{G}^{\circ}$, combines only with a high even term $\left(4 d^{3} 5 s \cdot 6 s\right) e^{6} \mathrm{~F}$, but the combinations are intense and unmistakable with some support from Zeeman effect. Nearly all quartets are known, ${ }^{4} \mathrm{~S}$ and ${ }^{4} \mathrm{P}$ terms being hardest to find. In some instances more than the expected number are found, particularly among the ${ }^{4} \mathrm{~F}$ and ${ }^{4} \mathrm{G}$ terms. This indicates the appearance of terms from a $6 p$ electron. Some of these have been utilized in series calculations as will be shown in the next section. 
TABLE 8.- $\mathrm{Cb}$ I predicted and observed terms

\begin{tabular}{|c|c|c|c|}
\hline $\begin{array}{l}\text { Electron } \\
\text { configu- } \\
\text { ration }\end{array}$ & Limit $\mathrm{Cb}$ II & Predicted & Observed \\
\hline $4 d^{4} 5 s$ & $\begin{array}{c}{ }^{5 \mathrm{D}} \\
{ }^{3 \mathrm{P}} \\
{ }^{3 \mathrm{D}} \\
{ }^{3 \mathrm{~F}} \\
{ }^{3} \mathrm{G} \\
{ }^{3 \mathrm{H}} \\
1 \text { (SDFGI) } \\
3(\mathrm{PF}) \\
1 \text { (SDG) }\end{array}$ & $\begin{array}{c}{ }^{6} \mathrm{D},{ }^{4} \mathrm{D} \\
{ }^{4} \mathrm{P},{ }^{2} \mathrm{P} \\
{ }^{4} \mathrm{D},{ }^{2} \mathrm{D} \\
{ }^{4} \mathrm{~F},{ }^{2 \mathrm{~F}} \\
{ }^{4} \mathrm{G},{ }^{2} \mathrm{G} \\
{ }^{4} \mathrm{H},{ }^{2} \mathrm{H} \\
{ }^{2}(\mathrm{SDFGI}) \\
{ }^{4}(\mathrm{PF}),{ }^{2}(\mathrm{PF}) \\
{ }^{2}(\mathrm{SDG})\end{array}$ & 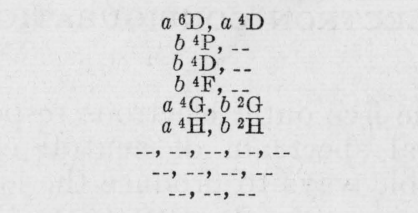 \\
\hline $4 d^{3} 5 s^{2}$ & & ${ }_{2(\mathrm{PDFGH}}^{{ }_{2} \mathrm{P},{ }^{4} \mathrm{~F}}$ & $\begin{array}{c}a^{4} \mathrm{P}, a^{4} \mathrm{~F} \\
a^{2} \mathrm{P}, a^{2} \mathrm{D}, a^{2} \mathrm{~F}, a^{2} \mathrm{G}, a^{2} \mathrm{H} \\
--\end{array}$ \\
\hline $4 d^{5}$ & & $\begin{array}{l}{ }^{6}(\mathrm{~S} \\
{ }^{2}(\mathrm{SDF}), 4(\mathrm{PF}) \\
{ }^{2}(\mathrm{PDFGH}) \\
{ }_{2 \mathrm{D}}\end{array}$ & 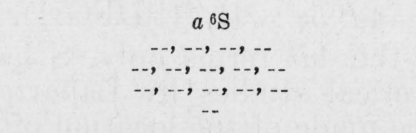 \\
\hline $4 d^{4} 5 p$ & $\begin{array}{l}{ }^{5} \mathrm{D} \\
3 \mathrm{P} \\
{ }^{3 \mathrm{D}} \\
3 \mathrm{~F} \\
{ }^{3} \mathrm{G} \\
{ }^{3} \mathrm{H} \\
1 \mathrm{~S} \\
1 \mathrm{D} \\
1 \mathrm{~F} \\
1 \mathrm{G} \\
1 \mathrm{I} \\
{ }^{3 \mathrm{P}} \\
{ }^{3 \mathrm{~F}} \\
1 \mathrm{~S} \\
1 \mathrm{D} \\
{ }_{1}^{1 \mathrm{G}}\end{array}$ & 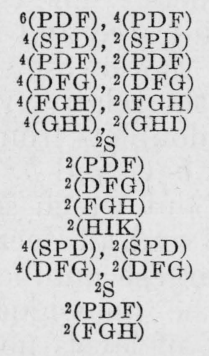 & 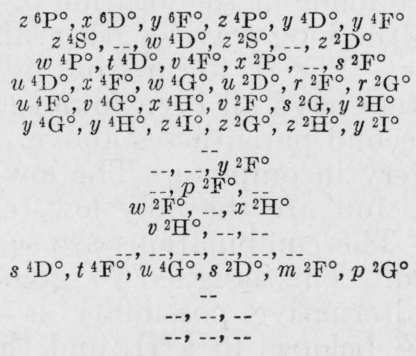 \\
\hline $4 d^{3} 5 s 5 p$ & $\begin{array}{l}{ }^{5 \mathrm{P}} \\
{ }^{3 \mathrm{P}} \\
{ }^{5 \mathrm{~F}} \\
{ }^{3 \mathrm{~F}} \\
{ }^{3} \mathrm{P} \\
{ }^{\mathrm{P}} \\
{ }^{3 \mathrm{D}} \\
{ }_{1 \mathrm{D}} \\
{ }^{1 \mathrm{~F}} \\
{ }^{3 \mathrm{~F}} \\
{ }^{1 \mathrm{G}} \\
{ }^{3} \mathrm{H} \\
{ }_{1 \mathrm{H}} \\
{ }^{3 \mathrm{D}}\end{array}$ & 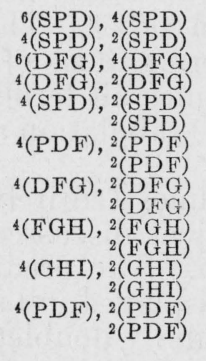 & 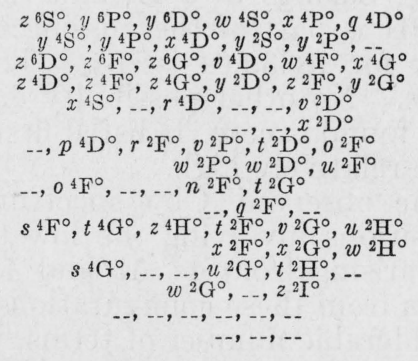 \\
\hline
\end{tabular}

The criteria which have been found useful in assigning the various terms to electron configurations and ionization limits are summarized as follows:

1. Order or relative position of terms of $\mathrm{Cb}$ II.- These terms constitute the ionization limits for $\mathrm{Cb}$, and terms correlated with the various limits are expected to be distributed in an analogous manner.

2. Intensities of multiplet combinations.-If the ionization limit of the low term is known, and it is certain in all instances in $\mathrm{Cb} \mathrm{I}$, the 
high term of the combination yielding the strongest line is expected to go to the same limit. In other words, it is in the same family.

3. The position of analogous terms in vanadium $\mathrm{I}$.-These spectra closely resemble each other, terms of similar origin appearing at about the same elevation above the lowest.

4. Assignment to give the best conformity with the g-sum rule.-This is largely a matter of picking out instances of $g$-sharing, of which selected examples have been given.

5. Hyperfine structure.-The association of the appearance of hyperfine structure with the configuration involving a single $s$-electron has been discussed earlier in the paper. It is to be noted that the numerous combinations of $a^{4} \mathrm{~F}$ and $a^{4} \mathrm{P}$ from $4 d^{3} 5 s^{2}$ are relatively sharp.

\section{SERIES AND IONIZATION POTENTIAL}

Two high even terms have been found in $\mathrm{Cb} \mathrm{I}$, of which the first, $4 d^{4} 6 s\left({ }^{5} \mathrm{D}\right) e^{6} \mathrm{D}$, is the second series member associated with $4 d^{4} 5 s\left({ }^{5} \mathrm{D}\right) a^{6} \mathrm{D}$, the lowest state of the atom. This series converges to $a^{5} \mathrm{D}$, the lowest state of the ion. The other high term, $4 d^{3} 5 s \cdot 6 s e^{6} \mathrm{~F}$, appears for the first time in this configuration, having no counterpart in the $4 d^{3} 5 s^{2}$ configuration because of the operation of the Pauli exclusion principle. Attempts to find high even quartets analogous to those known in VI proved futile. A few fragmentary terms, tentatively established, were not considered certain enough to be retained.

Three high odd terms, $n^{4} \mathrm{D}^{\circ},{ }^{4} \mathrm{~F}^{\circ}$, and $r^{4} \mathrm{G}^{\circ}$, all attributed to the configuration $4 d^{3} 5 s 6 p$, are associated with the lowest odd terms $z^{4} \mathrm{D}^{\circ}, z^{4} \mathrm{~F}^{\circ}$, and $z^{4} \mathrm{G}^{\circ}$ from $4 d^{3} 5 s 5 p$, and converge to $4 d^{3} 5 s a^{3} \mathrm{~F}$ in $\mathrm{Cb}$ II.

The ionization potential has been calculated both from the even sextet series and from these odd series. In general, four steps are required to calculate the ionization limit or elevation of the lowest state of the ion from the lowest state of the atom for a given set of levels in series when only two members of the series are known. First, the separation of the two members is found by subtracting the relative values. Second, this difference is located as closely as possible in a Rydberg interpolation table [19]. In the column adjoining on the left in the table is found the absolute value of the lower term or distance in wave-number units between this term and its ion limit. Third, the value of the lower term measured from the normal state is added to the number so found to give the elevation of the ion limit above the normal state of the atom. Finally, the value of the limiting term in the ion spectrum relative to its normal state is subtracted, and the remainder gives the required lowest ionization limit. It is converted to electron volts by dividing [20] by 8067. The results of these calculations for the available data are summarized in table 9 . 
TABLe 9.- $\mathrm{Cb}$ I series

\begin{tabular}{|c|c|c|c|c|c|c|}
\hline \multirow{2}{*}{ Term } & \multicolumn{2}{|c|}{ Electron } & \multirow{2}{*}{$n *$ for $5 s$} & \multirow{2}{*}{ Limit } & \multirow{2}{*}{$\mathrm{Cb}$ II } & \multirow{2}{*}{$\begin{array}{c}\text { Ioniza- } \\
\text { tion }\end{array}$} \\
\hline & $5 s$ & $6 s$ & & & & \\
\hline \multirow{2}{*}{$\begin{array}{l}a^{6} \mathrm{D}_{41 / 2} \\
a^{6} \mathrm{D}_{31 / 2} \\
a^{6} \mathrm{D}_{21 / 2} \\
a^{6} \mathrm{D}_{11 / 2} \\
a^{6} \mathrm{D}_{01 / 2} \\
\text { Mean }\end{array}$} & $\begin{array}{r}1050 \\
695 \\
392 \\
154 \\
0\end{array}$ & $\begin{array}{l}38568 \\
38178 \\
37842 \\
37579 \\
37410\end{array}$ & $\begin{array}{l}\text { 1. } 3910 \\
\text { 1. } 3915 \\
\text { 1. } 3920 \\
\text { 1. } 3925 \\
\text { 1. } 3927\end{array}$ & $\begin{array}{l}57762 \\
57363 \\
57021 \\
56751 \\
56579\end{array}$ & $\begin{array}{r}1225 \\
801 \\
438 \\
159 \\
0\end{array}$ & $\begin{array}{l}56537 \\
56562 \\
56582 \\
56592 \\
56579\end{array}$ \\
\hline & $\ldots$ & ..... & $\ldots . .$. & -... & $\ldots$ & 56571 \\
\hline \multirow{2}{*}{ Term } & \multicolumn{2}{|c|}{ Electron } & \multirow{2}{*}{$n^{*}$ for $5 p$} & \multirow{2}{*}{ Limit } & \multirow{2}{*}{$\mathrm{Cb}$ II } & \multirow{2}{*}{$\begin{array}{c}\text { Ioniza } \\
\text { tion }\end{array}$} \\
\hline & $5 p$ & $6 p$ & & & & \\
\hline $\begin{array}{l}z^{4} \mathrm{D}_{31 / 3}^{0} \\
z^{4} \mathrm{D}_{21 / 2}^{\circ} \\
z^{4} \mathrm{D}_{11 / 2}^{0} \\
z^{4} \mathrm{D}_{01 / 2}^{\circ}\end{array}$ & $\begin{array}{l}21512 \\
20838 \\
20384 \\
20107\end{array}$ & $\begin{array}{l}47276 \\
46813 \\
46365 \\
45979\end{array}$ & $\begin{array}{l}1.6216 \\
1.6163 \\
1.6161 \\
1.6189\end{array}$ & $\begin{array}{l}63242 \\
62844 \\
62399 \\
61979\end{array}$ & $\begin{array}{l}8320 \\
7901 \\
7506 \\
7506\end{array}$ & $\begin{array}{l}54922 \\
54944 \\
54893 \\
54473\end{array}$ \\
\hline Mean... & $\ldots$ & ..... & $\ldots$ & $\ldots$ & $\ldots$ & 54808 \\
\hline $\begin{array}{l}z^{4} \mathrm{~F}_{41 / 3}^{0} \\
z^{4} \mathrm{~F}_{31 / 3}^{0} \\
z^{4} \mathrm{~F}_{21 / 3}^{\circ} \\
z^{4} \mathrm{~F}_{11 / 2}^{0}\end{array}$ & $\begin{array}{l}24057 \\
24015 \\
23574 \\
23244\end{array}$ & $\begin{array}{l}47681 \\
47023 \\
46544 \\
46170\end{array}$ & $\begin{array}{l}\text { 1. } 6924 \\
\text { 1. } 6973 \\
\text { 1. } 6984 \\
\text { 1. } 7002\end{array}$ & $\begin{array}{l}62819 \\
62106 \\
61614 \\
61226\end{array}$ & $\begin{array}{l}8320 \\
7901 \\
7506 \\
7506\end{array}$ & $\begin{array}{l}54498 \\
54205 \\
54108 \\
53720\end{array}$ \\
\hline Mean & $\ldots$ & $\ldots$ & $-\ldots$ & $\ldots$ & $\ldots$ & 54133 \\
\hline $\begin{array}{l}z^{4} \mathrm{G}_{51 / 2}^{0} \\
z^{4} \mathrm{G}_{11 / 2}^{0} \\
z^{4} \mathrm{G}_{31 / 2}^{\circ} \\
z^{4} \mathrm{G}_{21 / 2}^{\circ}\end{array}$ & $\begin{array}{l}24203 \\
23537 \\
23023 \\
22647\end{array}$ & $\begin{array}{l}47081 \\
46724 \\
46471 \\
46011\end{array}$ & $\begin{array}{l}\text { 1. } 7012 \\
\text { 1. } 6920 \\
1.6844 \\
1.6868\end{array}$ & $\begin{array}{l}62121 \\
61867 \\
61699 \\
61212\end{array}$ & $\begin{array}{l}8320 \\
7901 \\
7506 \\
7506\end{array}$ & $\begin{array}{l}53800 \\
53966 \\
54193 \\
53707\end{array}$ \\
\hline Mean... & $\ldots$ & $-\ldots$ & - . & $\ldots$ & $-\ldots$ & 53916 \\
\hline
\end{tabular}

The mean ionization limit from 5 levels associated with $s$-type electrons is $56,571 \mathrm{~cm}^{-1}$, whereas the mean from 12 levels involving $p$-type electrons is $54,286 \mathrm{~cm}^{-1}$. The difference is probably due to the departure of one or other type of series from the simple Rydberg form. For V I Meggers and Russell [7] discarded this procedure, and calculated the ionization limit from values of the effective quantum number $n^{*}$ estimated from the spectra of flanking elements, Ti and $\mathrm{Cr}$. Columbium is flanked by $\mathrm{Zr}$ and $\mathrm{Mo}$, for which the effective quantum numbers of terms with highest multiplicity associated with $5 s$ are 1.47 and 1.36 , respectively [21]. The value thus indicated for $\mathrm{Cb} \mathrm{I}$ is 1.41 , corresponding to a term value of

$$
T=\left(R / n^{* 2}\right)=109737 / 1.41^{2}=54,868 \mathrm{~cm}^{-1}
$$

for $a^{6} \mathrm{D}_{01 / 2}$. This is in close agreement with the ionization value $54,286 \mathrm{~cm}^{-1}$ derived from the odd quartet terms. We consider a rounded mean of $54,600 \mathrm{~cm}^{-1}$ will represent the ionization value of $\mathrm{Cb}$ I with fair accuracy - it corresponds to a principal ionization potential of 6.77 volts. 


\section{REFERENCES}

11] W. F. Meggers, J. Wash. Acad. Sci. 14, 442 (1924).

[2] W. F. Meggers and C. C. Kiess, J. Opt. Soc. Am. 12, 432 (1926).

[3] A. S. King, Astrophys. J. 73, 441 (1931).

[4] W. F. Meggers and A. S. King, NBS J. Research 16, 385 (1936) RP881.

[5] W. F. Meggers and B. F. Scribner, NBS J. Research 14, 629 (1935) RP793

6] G. R. Harrison and F. Bitter, Phys. Rev. 5\%, 15 (1940).

[7] W. F. Meggers and H. N. Russell, NBS J. Research 17, 125 (1936) RP906;

W. F. Meggers and Charlotte E. Moore, 25, 84 (1940) RP1317.

[8] W. D. Wilkinson, Jr., Metal Progress 32, 661 (1937).

R. Franks, Trans. Am. Soc. Metals 27, 78 (1939).

R. Franks, W. O. Binder, and C. R. Bishop, Trans. Am. Soc. Metals 29, 35 (1941).

W. Peter, Archiv. Eisenhüttenw. 15, 364 (1942).

[9] A. U. Seybott, Metals Tech. TP1342 (Aug. 1941).

[10] W. F. Meggers and K. Burns, J. Opt. Soc. Am. 14, 453 (1927).

[11] S. S. Ballard, Phys. Rev. 46, 806 (1934).

[12] R. Jack, Proc. Roy. Irish Acad. [Sec. A] 30, 42 (1912).

[13] G. R. Harrison, J. Opt. Soc. Am. 25, 169 (1935), Rev. Sci. Instr. 9, 15 (1938).

[14] E. Back and A. Landé, Zeemaneffect und Multi lettstruktur der Spektrallinien, p. 168-169 (Julius Springer, Berlin, 1925).

[15] R. C. Gibbs and H. E. White, Phys. Rev. 31, 520 (1928).

A. Y. Eliason, Phys. Rev. 43, 745 (1933).

[16] R. J. Lang, Zeeman Verhandelingen, p. 44 (1935).

[17] C. C. Kiess and W. F. Meggers, BS J. Research 1, 641 (1928) RP23.

[18] O. Laporte, Phys. Rev. 61, 302 (1942).

[19] Rydberg Interpolation Table, Departments of Physics and Astronomy of Princeton University, Princeton, N. J. (1934).

[20] R. T. Birge, Rev. Modern Phys. 13, 233 (1941).

[21] R. F. Bacher and S. Goudsmit, Atomic Energy States (McGraw-Hill Book Co., Inc., New York, N. Y., 1932).

Washington, June 17, 1944. 